omprendre et gérer les finances de ma collectivité II In i ne 


\section{Comprendre et gérer les finances de ma collectivité}

\section{Nils Soguel}

Voici un manuel unique en son genre qui permet, pas à pas, de comprendre les finances de votre collectivité pour pouvoir les gérer. Un ouvrage complet qui aborde en détail, mais de manière accessible, les multiples aspects de la gestion financière: le processus budgétaire, les jeux d'acteurs, les comptes organisés selon les normes les plus récentes, la péréquation financière, les taxes et autres contributions. L'ouvrage apporte aussi des outils de diagnostic pour maîtriser les enjeux majeurs : mesurer le coût des prestations, installer un tableau de bord, saisir la dynamique de la dette, traquer l'influence de la conjoncture sur les finances, déceler - ou pratiquer - la tactique politique dans la présentation des comptes. Un ouvrage qui n'oublie pas d'offrir des pistes pour améliorer la situation financière si cela s'avère nécessaire. Les outils proposés sont systématiquement appliqués au cas d'une vraie collectivité; qui fournit des prestations à sa population et verse des salaires à son personnel; qui prélève des impôts et des taxes; qui investit et s'endette; bref qui vit. L'ouvrage s'inscrit ainsi résolument dans la réalité des collectivités locales suisses: cantons ou communes.

Nils Soguel est professeur ordinaire de finances publiques à l'Institut de hautes études en administration publique (IDHEAP) de l'Université de Lausanne. Il est également président du Conseil suisse de présentation des comptes publics, l'instance de normalisation des états financiers pour toutes les collectivités publiques suisses.
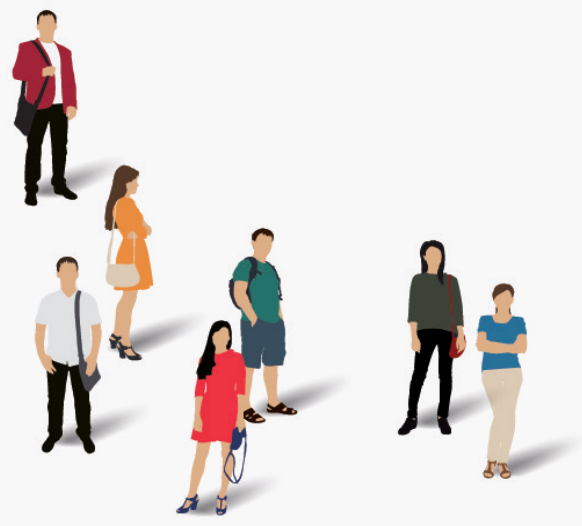
Comprendre et gérer les finances de ma collectivité 


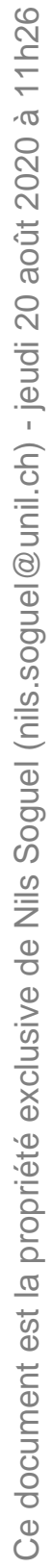




\section{Comprendre et gérer les finances de ma collectivité Nills Soguel}


Illustration de couverture: Katty2016, () shutterstock

EPFL PRESS est un label des Presses polytechniques et universitaires romandes (PPUR), qui publient principalement les travaux d'enseignement et de recherche de l'École polytechnique fédérale de Lausanne (EPFL) des universités et des hautes écoles francophones.

PPUR, EPFL - Rolex Learning Center, CP 119, CH-1015 Lausanne,

info@epflpress.org, tél.: +41216932130,fax: +41216934027.

www.epflpress.org

Première édition

(C) EPFL PRESS / Presses polytechniques et universitaires romandes, 2020

Édition imprimée: ISBN 978-2-88915-389-3

Livre numérique (PDF) : ISBN 978-2-88914-561-4

Tous droits réservés

Reproduction, même partielle, sous quelque forme ou sur quelque support que ce soit, interdite sans l'accord écrit de l'éditeur.

Imprimé en France 


\section{Sommaire}

$\begin{array}{ll}\text { Deux mots... } & 7\end{array}$

1 Introduction pour prendre l'ouvrage en main 9

2 Identifier les acteurs et les fondements de la gestion financière 11

3 Décrypter comment les comptes et les budgets sont organisés 39

$4 \quad$ Utiliser les imputations internes pour refléter les coûts des prestations 113

5 Comprendre le financement spécifique de certaines prestations 123

6 Connaître les mécanismes de transferts et de péréquation financière 145

7 Identifier les modalités de financement des investissements et d'emprunt 169

8 Déceler la tactique politique en matière de présentation des comptes 183

9 Porter un diagnostic sur la situation financière à l'aide d'indicateurs 191

10 Trouver les informations financières pour se comparer 215

11 Analyser l'origine des déficits: conjoncture ou structure 229

12 Analyser la dynamique de l'endettement: cercle vicieux ou vertueux 253

13 Projeter la situation financière à la limite 271

14 Améliorer la situation financière à l'avenir: quelques pistes 285

15 Portée stratégique des finances de ma collectivité 297

Bibliographie 301

$\begin{array}{ll}\text { Annexes } & 305\end{array}$

Index 323

Listes (figures, portraits, extraits, tableaux) 332

Abréviations $\quad 335$

Table des matières $\quad 337$

Biographie $\quad 340$ 


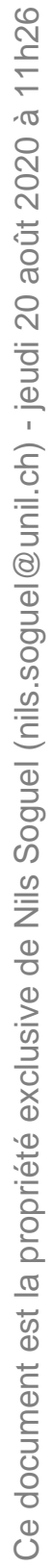




\section{Deux mots...}

... Un mot de remerciement. Gérer les finances d'une collectivité ne s'improvise pas. Proposer un ouvrage comme celui-ci ne s'improvise pas davantage. C'est un long travail de conception et d'écriture, qu'il faut soumettre au banc d'essai. Pour y parvenir, j'ai pu compter sur les participantes et les participants aux cours que j'ai donnés dans ce domaine à l'IDHEAP et ailleurs, depuis plus de vingt ans. Je leur en suis grandement reconnaissant. Je suis également redevable envers les responsables politiques et administratifs qui se sont adressés à moi pour me confier de nombreux mandats d'expertise, me faisant ainsi part de leurs expériences et de leurs besoins. Ces expertises, ces expériences, ces besoins sont devenus les éléments fondateurs de cet ouvrage. Par ailleurs, je remercie Yves Ammann, Antoine Brochet, Aurelia Buchs, Ramon Christen, Nicola Mauri, et Evelyn Munier de l'assistance qu'ils m'ont apportée dans l'élaboration du manuscrit. Mes remerciements s'adressent finalement à Florian Chatagny, Béatrice Hausmann, Allister Keane, Gilles Léchot, Francesco Re, André Schwaller, Alain Voirol, Michel Walthert et Sonja Ziehli pour leurs commentaires au sujet des passages que je leur ai soumis.

... Un mot sur l'auteur. Nils Soguel est professeur ordinaire de finances publiques à l'Institut de hautes études en administration publique (IDHEAP) de l'Université de Lausanne depuis 1995. Il a été professeur invité ou chargé de cours à l'University College London (UCL), aux universités de Bath (GB), Laval (Québec), Fribourg, Genève et à l'École polytechnique fédérale de Lausanne (EPFL). Ses enseignements, ses recherches et ses travaux d'expertise portent sur la gestion financière du secteur public, la santé financière et l'organisation des collectivités publiques, ainsi que le fédéralisme fiscal. Dans ces domaines, il a publié ou édité plusieurs ouvrages et articles pour des revues scientifiques. À partir de 1999, il publie et dirige chaque année le Comparatif des finances cantonales et communales de l'IDHEAP. Depuis 2008, il préside le Conseil suisse de présentation des comptes publics, l'instance de normalisation des états financiers pour toutes les collectivités publiques suisses. Il est titulaire d'un doctorat en sciences économiques de l'Université de Neuchâtel. 


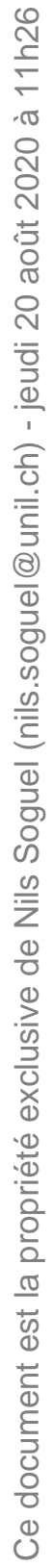




\section{Introduction pour prendre l'ouvrage en main}

Technique et politique. Dans une commune, dans un canton, la gestion financière consiste en un tiers de technique... et deux tiers de politique. Toutefois, pour maîtriser le volet politique, il faut auparavant maîtriser le volet technique. Tel est mon constat, sous forme de boutade, basé sur mon expérience, une expérience déjà longue. Cet ouvrage aborde ces deux aspects, mais dans une proportion inverse: de la technique surtout, mais sans occulter le volet politique.

Comprendre. L'ouvrage vous apporte de quoi comprendre les finances de votre collectivité afin de pouvoir les gérer: comprendre le contenu du budget, celui des comptes ; comprendre les différentes étapes du processus financier, du programme de législature à l'élaboration de la statistique financière; comprendre les mécanismes comptables fondamentaux, ceux de la péréquation financière ou des contributions causales; connaître les bases légales qui gouvernent l'ensemble. Les premiers chapitres -2 à $8-y$ sont consacrés.

Diagnostiquer. L'ouvrage vous apporte également des outils de diagnostic: pour déceler - ou pratiquer - la tactique politique dans la présentation des comptes, pour mettre en place un tableau de bord fondé sur divers indicateurs financiers, pour analyser la dynamique de la dette, pour déterminer si un éventuel déficit est dû à la conjoncture ou si le mal est plus profond. Voilà les thèmes des chapitres 9 à 12 .

Améliorer. Après avoir appréhendé la technique et posé un diagnostic, vient le temps de l'action. Le temps des politiques publiques est un temps long. L'action doit donc se concevoir dans le long terme, en se fixant un cadre financier, notamment pour savoir quel est le volume d'investissement et de charges que la collectivité peut se permettre. L'ouvrage vous offre plusieurs pistes pour améliorer ou maintenir la situation financière de la commune ou du canton. C'est l'objet des chapitres 13 à 15 .

Un livre pour vous. Vous êtes élue ou élu, responsable politique, responsable d'une unité dans l'administration, à l'échelon communal ou cantonal, voire fédéral. Bref, vous avez des responsabilités financières dans votre collectivité. Plus généralement, vous voulez maîtriser les techniques de gestion financière et pouvoir interpréter l'information financière pour fonder votre propre opinion. Dans tous les cas, ce livre est fait pour vous.

Pratique. Ce livre est fait pour vous et il est fait pour être utilisé... pas seulement lu. Il est un manuel destiné à un usage pratique. Il met donc l'accent sur la mise en ouvre, sur le passage de la théorie à la pratique. C'est pourquoi les outils de gestion financière qui sont présentés sont systématiquement appliqués au cas de MaCollectivité. Ainsi, la mécanique comptable est expliquée à l'aide des comptes de cette entité. Quant à eux, les instruments de diagnostic financier y sont également appliqués. 
MaCollectivité. MaCollectivité n'est pas un cas d'école. C'est une vraie collectivité qui existe, qui offre des prestations à sa population et verse des salaires à son personnel, qui prélève des impôts et des taxes, qui investit et s'endette, bref qui vit. Les chiffres et les calculs présentés vous permettent de saisir cette réalité.

Contexte suisse: La gestion des finances dépend beaucoup du contexte institutionnel. Or le contexte institutionnel suisse est différent de celui qui prévaut dans d'autres pays: le processus budgétaire diffère, les mécanismes de contrôle diffèrent, le modèle comptable diffère pour ne mentionner que ces quelques éléments. L'ouvrage s'inscrit résolument dans le contexte des collectivités locales helvétiques: cantons et communes. C'est la conséquence logique d'un ouvrage orienté sur la pratique. De ce fait, l'ouvrage s'adressera moins à celles et ceux en charge des finances d'une collectivité se trouvant ailleurs qu'en Suisse.

Sans crainte face aux chiffres. Le recours systématique à des chiffres facilite la compréhension. Les calculs nécessaires pour appliquer les outils proposés sont toujours présentés pas à pas et de manière transparente. Par conséquent, l'ouvrage reste accessible à tous, que vous débutiez dans le domaine ou que vous soyez déjà aguerri. Justement, il n'y a pas de crainte à nourrir par rapport aux chiffres. Les compétences de base en arithmétique suffisent. Et si vous souhaitez aller plus loin, vous trouverez, au gré des pages, des références à des publications ciblées. Compte tenu de la portée pratique du livre, les références bibliographiques se limitent à l'essentiel. Et elles sont, dans toute la mesure du possible, en langue française et accessibles sur l'Internet.

Une feuille de calcul à votre disposition. Afin de vous faciliter les calculs pour votre collectivité, une feuille de calcul électronique est à votre disposition. Le fichier est téléchargeable à l'adresse suivante: www.unil.ch/idheap/finances. Il vous suffit d'y introduire les informations provenant des comptes publiés par votre collectivité et vous obtiendrez les résultats des analyses présentées dans l'ouvrage. 


\section{Identifier les acteurs et les fondements de la gestion financière}

Ce chapitre présente le cadre légal, le cadre institutionnel et les acteurs impliqués dans le processus financier. Évidemment, au sein du système fédéraliste suisse, les cantons bénéficient d'une autonomie considérable en matière de gestion financière. Chaque canton a ainsi développé sa propre façon de gérer ses finances publiques. Chaque canton a également développé ses propres prescriptions applicables à la gestion financière des communes et, cas échéant, des autres entités publiques situées sur son territoire ${ }^{1}$. Face à cette diversité, nous dégageons ici les éléments partagés par la plus grande partie des collectivités publiques helvétiques. Pour cela, nous évoquons d'abord les fondements juridiques de la gestion financière en mettant l'accent sur les principes financiers et sur le droit des crédits. Ensuite viennent deux institutions spécifiques: les règles budgétaires et le référendum financier. Enfin nous égrenons les différentes phases du processus financier et le rôle joué par les différents acteurs.

Les fondements juridiques régissant la gestion financière sont dictés par la Constitution et par la loi sur les finances ${ }^{2}$. La même architecture juridique prévaut au niveau de la Confédération et pour chacun des vingt-six cantons. S'agissant des communes, les dispositions sont en principe contenues dans la législation sur les communes (loi sur les communes et ses règlements d'application).

Plus spécifiquement, les constitutions cantonales fixent les compétences des diverses autorités (Législatif, Exécutif) et les limites posées aux prélèvements fiscaux (constitutionnalité, légalité). Elles énumèrent les sources de financement qui peuvent être mobilisées. Elles déterminent également les composantes principales de la gestion financière avec notamment la planification financière et le budget. S’y ajoutent souvent des dispositions relatives à un possible frein aux déficits (ou aux dépenses ou encore à l'endettement). Ce que l'on qualifie de droit des crédits, c'està-dire les dispositions légales en matière d'autorisation de dépenses, s'y trouve également. Les constitutions fondent par ailleurs le contrôle des finances et la péréquation financière, ainsi que le partage des charges avec les communes (Lienhard et al. 2017, pp. 152-170).

1 Par exemple, dans le canton de Berne, les prescriptions légales s'appliquent à toutes les collectivités de droit public au sens de la loi sur les communes, telles que les communes politiques, les communes bourgeoises, les paroisses, les syndicats de communes ou encore les conférences régionales.

2 Finanzhaushaltgesetz | Legge sulle finanze | Financial management act of Parliament. 


\subsection{Principes financiers}

Divers principes régissent la gestion des finances publiques ${ }^{3}$. Leur éventail et leur dénomination varient selon les collectivités ${ }^{4}$. Quoi qu'il en soit, il faut distinguer trois groupes de principes: les principes d'ordre stratégique déterminant l'esprit dans lequel les ressources et en particulier les ressources financières doivent être gérées, les principes d'ordre opérationnel régissant la budgétisation et les principes d'ordre plus technique régissant la présentation du budget et des comptes. La figure 1 montre comment ces principes s'articulent. Ces principes sont complémentaires et visent un double objectif: (a) l'objectif d'assurer une gestion efficace, claire et honnête des ressources, (b) l'objectif de permettre au Législatif de contrôler, dans le cadre du processus financier, le plus efficacement possible l'action de l'Exécutif.

Figure 1 Articulation des principes financiers.

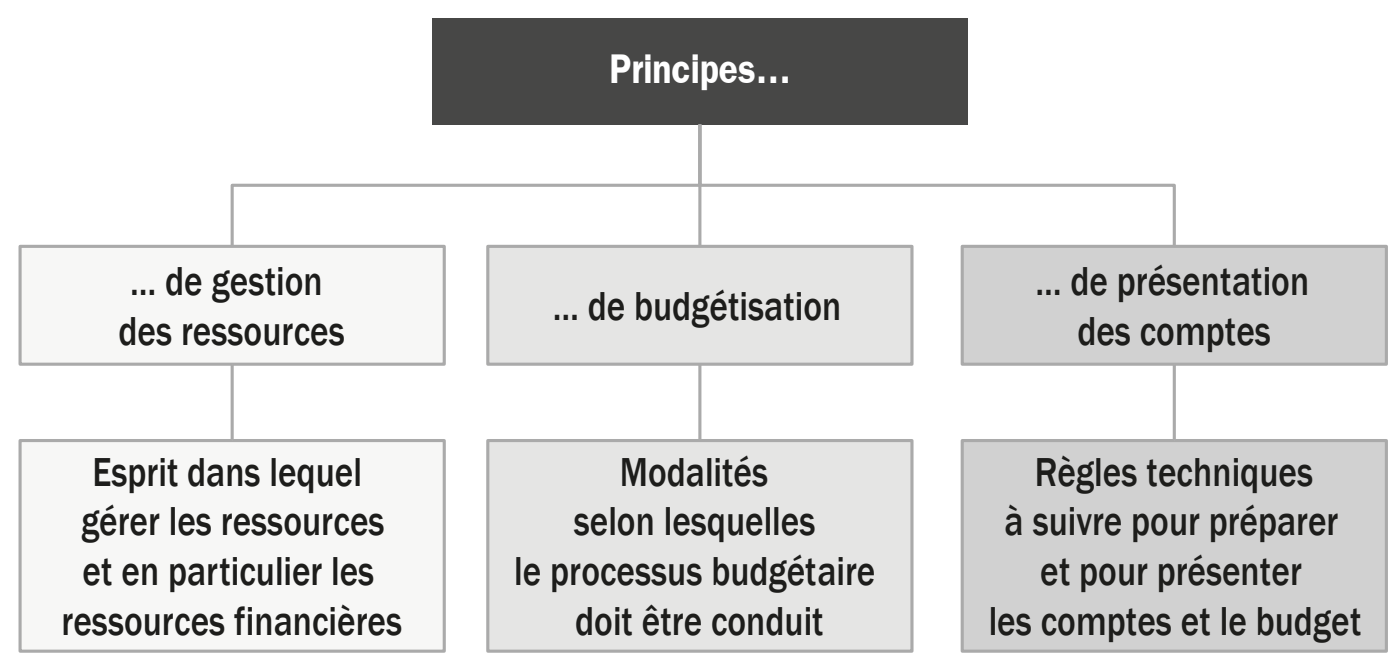

\subsubsection{Principes de gestion des ressources, notamment financières}

Souvent, l'esprit dans lequel la gestion des ressources et en particulier la gestion des ressources financières de la collectivité doit être conduite n'est pas explicitement formulé. La loi sur les finances de la Confédération énonce toutefois divers principes que nous développons ici: principe de la légalité, principe de l'urgence, et principes de l'emploi ménager, économe et efficace des fonds (art. 12, al. 4).

3 Finanzielle Grundsätze | Principi finanziari | Financial principles.

4 S'agissant de connaître les dispositions s'appliquant spécifiquement à une collectivité donnée, on se référera à la législation correspondante. L'ouvrage de Gilles (2009) présente l'évolution historique de ces principes, en se référant toutefois essentiellement au contexte français. 
Principe de la légalité5. De manière générale, ce principe exige que tout acte administratif soit fondé sur une base légale, c'est-à-dire qu'il trouve son fondement dans l'ordre juridique existant. Appliqué spécifiquement à la gestion financière, il implique que tout montant porté au budget de la collectivité repose sur une base juridique existante. Par conséquent, une dépense ne peut être engagée que pour financer une tâche prévue par la législation. De même, seules les recettes - impôts et taxes - disposant d'une base légale peuvent être prélevées et donc mises au budget, puis inscrites dans les comptes. En outre, seules les instances légalement prévues sont compétentes pour le faire et cela dans le cadre de la procédure prescrite par le législateur. Prenons l'exemple des dépenses pour les bâtiments scolaires. Dans beaucoup de cantons, la législation relative à l'enseignement obligatoire prévoit que les communes sont responsables de mettre à disposition l'infrastructure dont ont besoin les établissements scolaires. C'est donc en raison de cette base légale qu'une commune peut (et doit) consentir une dépense dans ce domaine.

Principe de l'urgence ${ }^{6}$. Ce principe veut qu'une collectivité alloue ses moyens en tenant compte du degré d'urgence des besoins à satisfaire. Les ressources financières devraient donc être prioritairement allouées aux besoins les plus urgents. Selon ce principe, on devrait cas échéant différer une dépense peu ou pas urgente, voire y renoncer.

Principe de l'emploi ménager des fonds ${ }^{7}$. Selon ce principe, les autorités devraient faire preuve de parcimonie dans l'utilisation des fonds publics. Autrement dit, elles devraient s'employer à minimiser le volume des dépenses.

Principe de l'emploi économe des fonds ${ }^{8}$. Selon ce principe, les autorités devraient organiser la fourniture des prestations publiques de manière à obtenir le meilleur rapport possible entre le volume de prestations fournies et les ressources financières engagées. Techniquement parlant, il s'agit de maximiser le rapport entre les outputs $\mathrm{du}$ processus de production et les inputs de ce processus. C'est pourquoi on parle aussi du principe de l'emploi efficient des fonds ou du principe de l'efficience.

Principe de l'emploi efficace des fonds' ${ }^{9}$. Les autorités doivent également s'attacher à utiliser les ressources financières de manière à atteindre les buts visés. Elles devraient par conséquent allouer les fonds aux activités et aux prestations qui concourent les mieux à atteindre les objectifs fixés en termes de politiques publiques. On parle souvent aussi d'effectivité. 


\subsubsection{Principes de budgétisation}

Pour passablement de collectivités, la manière de conduire le processus budgétaire n'est encadrée que par un nombre très restreint de principes. Toutefois, la démarche d'élaboration du budget répond implicitement à des principes que l'on trouve en revanche largement formulés dans la loi sur les finances de la Confédération (art. 31): principe de l'universalité, principe de la spécialité et principe de l'annualité. Nous ajoutons à cette liste le principe de l'unité, celui de la non-affectation des impôts et celui de la prudence, cela dans la mesure où le droit budgétaire de quelques cantons et de nombreux pays étrangers les mentionnent.

Principe de l'universalité ${ }^{10}$. Ce principe de budgétisation veut que chaque dépense et chaque recette, qu'elle concerne le fonctionnement ou l'investissement, figure dans le document budgétaire et subséquemment dans les comptes. Ce principe équivaut à celui de l'intégralité tel qu'énoncé dans certaines législations. Concrètement, ce principe interdit qu'une opération liée au fonctionnement ou à l'investissement soit enregistrée directement dans le bilan de la collectivité.

Principe de non-affectation des impôts ${ }^{11}$. Ce principe découle du principe d'universalité. Cette règle voudrait que les recettes d'un impôt principal - c'est-à-dire d'un impôt offrant un important rendement - ne soient pas légalement affectées au financement d'une dépense particulière et ce en amont du processus budgétaire. Des arguments à la fois pratiques et politiques peuvent être invoqués à l'appui de ce principe. Du point de vue pratique, cette règle empêche un gaspillage des ressources financières dans le cas où le rendement de l'impôt serait supérieur au coût de la tâche à laquelle il serait affecté (voir le principe de l'emploi ménager des fonds). Cette règle permet également d'écarter les risques d'exécution incomplète d'une tâche si le coût n'est pas couvert par les revenus affectés. Sur le plan politique, cette règle évite une dérive qui ne verrait se réaliser que des projets pour lesquels un financement spécifique (affecté) est prévu. Les contribuables pourraient ne vouloir payer que les impôts affectés au financement de tâches dont ils bénéficient directement. Ensuite et toujours du point de vue politique, affecter les impôts principaux réduirait la marge de manœuvre parlementaire: cela amputerait d'autant le volume des recettes et des dépenses sur lesquelles les parlementaires peuvent intervenir. Enfin, il est possible que les priorités budgétaires changent ou que les assiettes sur lesquelles sont fondés les impôts se modifient; on risque alors d'assister à des blocages budgétaires dus à des obligations légales d'affectation des recettes. Évidemment, ce principe ne s'applique pas aux taxes et aux redevances d'utilisation. En effet, cellesci visent précisément à financer spécialement le coût d'une prestation spécifique (par exemple la fourniture d'eau propre) et donc à mettre à charge des bénéficiaires de cette prestation le coût correspondant. C'est la raison pour laquelle quelques

10 Vollständigkeit | Universalità | Universality.

11 Verbot der Zweckbindung von Hauptsteuern | Divieto del vincolo delle entrate | No earmarking of main taxes. 
législations énoncent également le principe de l'utilisateur-payeur en parallèle à celui de la non-affectation des impôts.

Principe de l'unité ${ }^{12}$. Ce principe implique que, matériellement, l'ensemble des dépenses et des recettes de la collectivité figure dans un document unique soumis à l'approbation du Législatif. D'un point de vue formel, il implique également que ce document soit un seul et même document. Des impératifs pratiques, en particulier typographiques, nécessitent parfois dans les grandes collectivités que la masse des informations soit scindée en plusieurs fascicules. On considère toutefois qu'il n'y a pas là atteinte au principe de l'unité, pour autant que le contenu de ces différents fascicules soit présenté, discuté et adopté dans le cadre du même débat budgétaire. Présenter l'ensemble des dépenses et des recettes dans un seul document et délibérer au cours d'un seul débat budgétaire est nécessaire pour avoir la possibilité de procéder à des arbitrages budgétaires.

Principe de la prudence ${ }^{13}$. Selon ce principe, les charges et les engagements doivent être considérés dans le budget et dans les comptes dès que ces éléments sont envisageables et probables. En revanche, les revenus et les actifs ne doivent être considérés qu'à partir du moment où ils sont certains. Évidemment, ce principe induit un biais dans les résultats prévisionnels ou comptables, dans la mesure où il encourage à différer la prise en compte des revenus et des actifs, alors qu'il encourage à prendre en compte précocement les charges et les engagements. Appliqué avec trop de zèle, ce principe conduit à présenter systématiquement des pronostics et des résultats moins bons ou pires encore qu'ils ne sont en réalité. Dans ce cas, ce principe nuit à celui de la fiabilité (voir ci-après).

Principe de la spécialité ${ }^{14}$. Spécialiser l'autorisation budgétaire consiste à détailler cette autorisation afin que chaque montant porté au budget soit dépensé conformément à la volonté du Législatif. Cela signifie que l'Exécutif ne peut pas modifier la destination d'un montant budgétaire sans l'approbation préalable du Législatif. La règle de la spécialité (ou de la spécialisation) s'oppose à celle de l'abonnement, qui consiste en une adoption par le Législatif du budget sous la forme d'un montant global, avec pour corollaire une liberté laissée à l'Exécutif d'utiliser ce montant de manière discrétionnaire. On fait la distinction entre la spécialité qualitative, la spécialité quantitative et la spécialité temporelle.

- La spécialité qualitative a trait au propos de la dépense: le montant porté au budget ne peut être utilisé que pour l’objet prévu ou pour l'objectif prévu; cela oblige par conséquent à désagréger les montants portés au budget en fonction des différents objets et des différents objectifs poursuivis. On parle en l'occurrence de décomposition entre différentes lignes budgétaires.

12 Einheit | Unità | Unity.

13 Vorsicht|Prudenza|Prudence.

14 Spezifikation | Specificazione | Specification. 
- La spécialité quantitative exige le respect du plafond fixé par le montant budgétisé. L'autorisation de dépenser est donnée jusqu'à concurrence de ce montant, mais pas au-delà.

- La spécialité temporelle, enfin, veut que les montants budgétisés et votés ne puissent être dépensés que durant l'année de référence et pas au-delà, soit au cours d'une période de douze mois seulement.

Principe de l'annualité ${ }^{15}$. Le principe de l'annualité s'inscrit dans la suite logique du principe de la spécialité temporelle, avec lequel on le confond souvent. Si le principe de spécialité temporelle veut que les autorisations de dépenses portées au budget ne soient valables que pour une durée d'une année, le principe de l'annualité exige que, chaque année, un budget soit préparé et que, chaque année, des comptes soient présentés. Autrement dit, le principe de l'annualité fixe le rythme budgétaire. Historiquement, le principe de l'annualité fut le premier principe budgétaire mis en œuvre. Il découlait implicitement de l'obligation annuelle du vote de l'impôt par le Corps législatif représentant le peuple souverain en fonction du rythme de la vie sociale et économique, en particulier du rythme de la société agricole du XIXe siècle.

Au-delà de la raison historique, la règle de l'annualité permet toujours et encore un contrôle régulier (annuel) des finances de la collectivité et oblige l'Exécutif et l'administration à rendre compte à intervalles réguliers et rapprochés. Parfois, ce principe est explicitement complété dans la législation par le principe de l'antériorité, selon lequel le budget doit avoir été approuvé par le Législatif avant que l'Exécutif puisse en disposer.

La figure 2 montre qu'à l'heure actuelle, la plupart des pays - dont la Suisse et l'essentiel des pays membres de l'Organisation de coopération et de développement économiques (OCDE) - débutent leur année budgétaire le $1^{\text {er }}$ janvier. Ces pays calquent donc l'année budgétaire sur l'année civile. Il se trouve toutefois d'autres pays parmi les membres de l'OCDE pour commencer l'année budgétaire à un autre moment et donc être en décalage par rapport à l'année civile. Le Canada, le Japon et le Royaume-Uni débutent l'année budgétaire le $1^{\mathrm{er}}$ avril, la Nouvelle-Zélande le

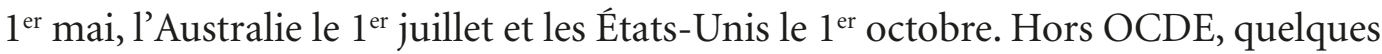
pays démarrent leur année budgétaire au cours d'un mois (par exemple le 21 mars en Iran ou le 11 septembre en Éthiopie).

En pratique, le principe de l'annualité associé à celui de la spécialité temporelle comporte des inconvénients. Ils induisent un cycle budgétaire qui pousse les administrations à adopter des comportements tactiques, notamment le fameux year-end spending spree ou feu d'artifice des dépenses de fin d'année. Par ailleurs, bien que le principe de l'annualité couplé avec celui d'unité permette, en principe, que l'ensemble des dépenses et des recettes soit réexaminé chaque année, en pratique on 
constate que le budget de l'année précédente sert de base au processus budgétaire de l'année concernée. On assiste ainsi à un phénomène de sédimentation ou d'incrémentation budgétaire qui pénalise les unités administratives qui n’ont pas épuisé l'entier des montants budgétaires mis à leur disposition.

Figure 2 Début de l'année budgétaire.

\section{Année civile}

Jan. Fév. Mars Avril Mai Juin Juil. Août Sept. Oct. Nov. Déc.

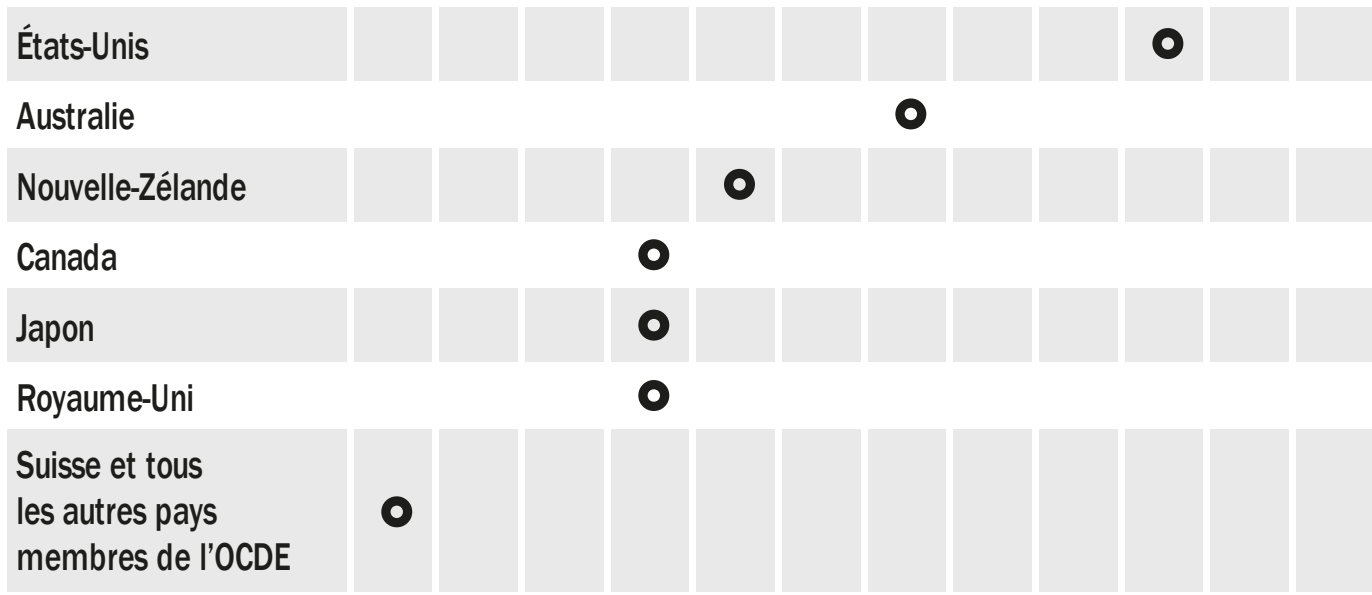

\subsubsection{Principes de présentation des comptes}

Bien que la liste des principes à respecter pour présenter les comptes puisse varier d'un canton à l'autre, la Conférence des directrices et directeurs cantonaux des finances $(\mathrm{CDF})$ recommande d'observer quelques grands principes ${ }^{16}$. Nous en reprenons la liste ici afin d'éclairer le sens de chaque principe. Par analogie, ces principes s'appliquent également pour préparer le document de présentation du budget.

Principe du produit brut ${ }^{17}$. Ce principe, aussi appelé principe de la non-contraction, découle du principe de l'universalité. Il concerne particulièrement les unités administratives qui, à la fois, réalisent des dépenses et encaissent des recettes. Dans ce cas, deux solutions sont a priori imaginables pour la présentation au budget, puis dans les comptes. Une première solution consisterait à contracter les recettes et les dépenses, puis à se contenter de faire apparaître le solde; on appliquerait alors une règle comptable fondée sur le produit net. Une deuxième solution consiste à présenter d'un côté l'intégralité des recettes et de l'autre l'intégralité des dépenses; cette

16 Recommandation 2, CDF (2008). Notons que ces principes correspondent largement à ceux qui sont énoncés dans le Code des obligations (art. 958 CO, état au $1^{\text {er }}$ avril 2017).

17 Bruttodarstellung | Espressione al lordo | Gross recognition. 
manière de faire respecter le principe du produit brut. Le principe du produit brut permet une gestion plus transparente et un contrôle parlementaire plus étroit. Il est en effet plus difficile d'exercer un contrôle lorsque l'information est agrégée et lorsqu'il n'est pas possible de connaître l'origine et le détail des recettes et des dépenses.

Principe de la comptabilité d'exercice ${ }^{18}$. Ce principe veut que toutes les charges et tous les revenus soient présentés dans la période qui les concerne. Il permet de mettre en œuvre à la fois le principe de l'annualité et le principe de la spécialité temporelle. En effet, ces deux principes nécessitent que l'on définisse clairement à quelle année se rattachent les opérations budgétaires. On pourrait ainsi croire que seuls les encaissements et les décaissements réalisés entre le $1^{\text {er }}$ janvier et le 31 décembre doivent être présentés dans le budget, puis dans les comptes de l'année considérée. Cela reviendrait à suivre le principe de la comptabilité de caisse. Une telle simplification n'est plus compatible avec le souhait de connaître le coût des prestations publiques offertes au cours d'une année et de savoir si ce coût est couvert par les revenus de cette même année. C'est pourquoi le principe de la comptabilité d'exercice, aussi qualifié de principe de l'échéance, de principe des droits constatés, ou encore de la périodicité, prévaut depuis plusieurs années. Selon cette règle, les charges sont budgétisées, puis comptabilisées lorsqu'elles sont dues et non lorsqu'elles sont payées. De même, les revenus sont présentés dès le moment où la collectivité peut économiquement y prétendre, par exemple lorsqu'ils sont facturés, et non lorsqu'ils sont encaissés. Autrement dit, dès qu'un tiers a un droit envers la collectivité ou dès que la collectivité a un droit envers un tiers, ce droit doit être constaté et présenté dans le budget, puis dans les comptes. Concrètement, cela implique par exemple de constituer des provisions ou d'enregistrer des opérations transitoires, ainsi que nous le détaillerons dans la section 3.3 consacrée au modèle comptable des cantons et des communes.

Principe de la continuité de l'exploitation ${ }^{19}$. Selon ce principe, il faut présenter les informations dans le budget et dans les comptes en partant de l'hypothèse que la collectivité poursuivra ses activités indéfiniment. Cela paraît aller de soi. Mais poser explicitement ce principe permet ensuite de procéder à l'estimation du patrimoine de la collectivité en se fondant sur la valeur d'acquisition de celui-ci. Si, au contraire, on partait du principe que la collectivité allait cesser ses activités dans un proche avenir, alors son patrimoine devrait plutôt être estimé sur la base de sa valeur vénale, puisque l'on s'attendrait à ce qu'il doive être bientôt vendu.

Principe de l'importance ${ }^{20}$. Ce principe met l'accent sur le fait que le budget et les comptes de la collectivité sont des sources d'information majeures. Par conséquent, toute information susceptible d'influencer le jugement ou la décision des

18 Periodenabgrenzung | Correlazione temporale | Accrual.

19 Fortführung | Continuità di esercizio | Going concern.

20 Wesentlichkeit | Essenzialità | Materiality. 
utilisateurs de ces documents doit être considérée comme importante. En revanche, une information non pertinente devrait être laissée de côté. Le cercle des utilisateurs est par définition vaste. Il englobe généralement évidemment les autorités de la collectivité - le Législatif et l'Exécutif. Mais il englobe aussi les citoyens-contribuables, les bailleurs de fonds, les organes statistiques ou encore les milieux de la recherche. Ce principe est essentiel. Il permet aux personnes en charge de la préparation du budget et des comptes et aux organes de révision de décider quels montants doivent être présentés séparément et quel est le montant à partir duquel les erreurs et les omissions doivent impérativement être évitées. Déterminer si l'information véhiculée par un montant est importante ou non nécessite une bonne dose d'expertise. En effet, l'analyse doit tenir compte à la fois de l'importance du montant concerné et de la nature de la transaction à présenter dans les documents. Par exemple, un montant de quelques milliers de francs est important et doit être présenté séparément lorsque la collectivité est de taille modeste. Par contre, son importance est marginale dans une collectivité plus grande. Les charges de transferts fournissent un autre exemple dans la mesure où certaines peuvent concerner des dédommagements à d'autres entités ou des versements dans le cadre d'une péréquation financière. Dans la mesure où la nature économique de ces deux types de transferts est fondamentalement différente, une présentation séparée est en principe nécessaire.

Principe de la clarté ${ }^{21}$. Par principe, les informations doivent être clairement présentées dans le budget et dans les comptes. Le respect de cet impératif doit assurer que des personnes disposant d'un niveau raisonnable de connaissances puissent comprendre le contenu de ces documents. Par conséquent, l'information financière doit être présentée de manière à en faciliter l'accès et l'usage. Ce principe n'autorise cependant pas à omettre des informations au prétexte qu'elles seraient complexes et donc difficiles à comprendre. Il exige simplement que les informations soient présentées de manière méthodique et logique, sans velléité d'offuscation ou de dissimulation. Par exemple, une collectivité qui recourt à des instruments financiers dérivés doit les présenter méticuleusement et en détail dans l'annexe à ses comptes, dans la mesure où ses instruments sont complexes et difficilement compréhensibles.

Principe de la fiabilité ${ }^{22}$. Par principe, les informations présentées dans le budget et dans les comptes doivent être précises. Elles doivent refléter fidèlement et sincèrement la réalité des faits. Certaines législations complètent d'ailleurs ce principe par celui de la sincérité ou de la neutralité. Les normes comptables internationales indiquent d'ailleurs que les comptes doivent offrir une true and fair view de la situation financière de la collectivité. L'arbitraire doit donc être exclu au profit de l'objectivité de l'information. Par conséquent, les informations doivent refléter la réalité 
économique plutôt que les aspects juridiques des faits. C'est pourquoi, par exemple, une collectivité qui contracte un leasing pour un véhicule devra présenter ce véhicule comme faisant partie de son patrimoine au bilan, dans la mesure où ce véhicule est utilisé pour fournir des prestations à la population, et cela même si d'un point de vue juridique le propriétaire reste la société de leasing. Pour compléter ce principe, des législations incluent également le principe de traçabilité. Selon ce principe, les montants enregistrés dans les comptes doivent avoir une trace, c'est-à-dire être fondés sur une pièce justificative, par exemple une facture justifiant une charge d'achat de biens ou de services. Dans cette même veine, le principe de la comptabilité en partie double est énoncé ici ou là.

On trouve parfois également la mention du principe de la régularité. Selon ce dernier, les opérations comptables doivent être conformes aux pratiques ainsi qu'à la législation et aux règlements en vigueur. La coexistence du principe de fiabilité et du principe de régularité est source de problème lorsque la législation autorise, voire préconise, des pratiques qui nuisent à la fiabilité des comptes. Mentionnons le cas des amortissements supplémentaires. Cette opération, purement comptable, relève de la tactique politique; mais elle est autorisée par plusieurs législations cantonales. Dans ces cantons, elle peut s'effectuer sans enfreindre le principe de la régularité. Par contre, elle nuit à la présentation sincère et neutre de la situation financière de la collectivité. Donc, elle enfreint le principe de la fiabilité de l'information véhiculée par les comptes. Nous reviendrons sur cet aspect dans le chapitre 8 consacré à la tactique politique en matière de présentation des comptes.

Principe de la comparabilitée ${ }^{23}$. Ce principe veut que l'information véhiculée par le budget et par les comptes d'une collectivité pour une période donnée soit comparable avec l'information apportée pour des périodes précédentes et pour des périodes futures, ainsi que pour d'autres collectivités. Autrement dit, l'information doit être comparable à travers le temps et entre collectivités. Pour cela, les normes et les pratiques de budgétisation et de comptabilisation doivent être déployées de manière constante et uniforme. Par exemple, cela permet de comparer le coût des prestations de l'année avec le coût des années précédentes.

Principe de la permanence des méthodes ${ }^{24}$. Pour garantir le respect du principe de la comparabilité, il faut que les méthodes de préparation et de présentation de l'information, dès le moment où elles ont été adoptées, soient appliquées de manière constante par la suite. Ce principe veut également que, dans des situations identiques, les mêmes méthodes soient mises en œuvre. La collectivité doit par conséquent renoncer à modifier ses pratiques, sauf lorsque des motifs raisonnables le commandent. En cas de changement, la raison ainsi que les effets de ce changement doivent être dûment documentés. 


\subsection{Droit des crédits}

Afin de mettre en œuvre les principes financiers et en particulier le principe de spécialité, la législation prévoit divers types d'autorisations financières. Ces divers types d'autorisations forment ce que l'on appelle le droit de crédit ou la législation sur les crédits $^{25}$. Dans un État démocratique comme la Suisse, une collectivité publique ne peut pas librement disposer de ses ressources. La décision d'utiliser des ressources, que ce soit pour une dépense courante ou pour une dépense d'investissement, est soumise à une procédure d'autorisation, dans la mesure où toute dépense implique de consacrer une partie du patrimoine financier de la collectivité dans le but d'accomplir une tâche publique. C'est pourquoi le droit des crédits fixe qui peut décider, de quel type de dépense, et selon quel critère.

Les autorisations financières s'articulent autour de deux grandes catégories de crédits, comme le schématise la figure 3 : les autorisations de procéder à une dépense et les autorisations de contracter un engagement.

Figure 3 Articulation des crédits.

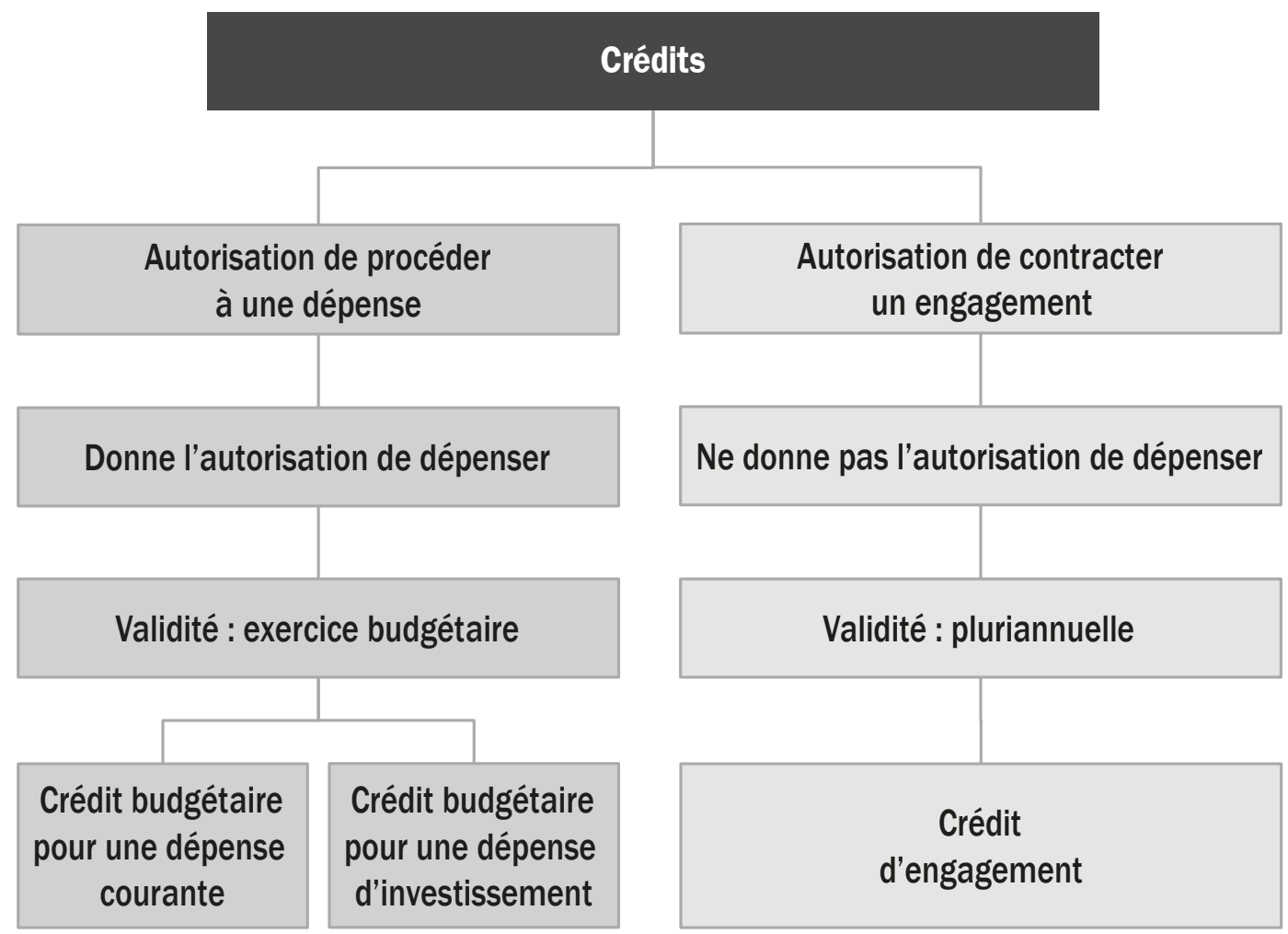




\subsubsection{Autorisation de procéder à une dépense et crédit budgétaire}

Lorsqu'une autorisation de procéder à une dépense est octroyée à l'Exécutif, ce dernier a le droit de dépenser la somme autorisée au cours de l'exercice à venir. Par conséquent, la durée de validité - ou spécialité temporelle - de cette autorisation est limitée à un seul exercice.

Crédit budgétaire ${ }^{26}$. L'autorisation peut porter sur une dépense courante, par exemple sur le paiement de salaires ou sur l'achat de biens et de services. Elle peut aussi porter sur une dépense d'investissement, par exemple sur le paiement de factures pour la construction d'une route ou pour l'achat d'un bâtiment scolaire, autrement dit sur une dépense qui aura une utilité sur plusieurs exercices.

On parle en l'occurrence de crédit budgétaire parce que le montant est porté au budget de la collectivité (soit dans la section de fonctionnement, soit dans la section des investissements). Il s'agit donc d'une autorisation donnée pour une année par le Législatif à l'Exécutif de procéder, jusqu'à un montant déterminé, à charge d'une position budgétaire déterminée, à une dépense concernant un but précis et cela au cours de l'exercice concerné.

Crédit bloqué. Il se peut qu'au moment de l'approbation du budget par le Législatif, la base légale sur laquelle devrait s'appuyer la dépense fasse encore défaut. C'est en particulier le cas lorsque le délai référendaire par rapport à une nouvelle loi n'est pas échu ou que le vote populaire sur un éventuel référendum n'a pas encore eu lieu. Dans ce cas, le crédit peut être voté par le Législatif, mais il reste bloqué jusqu’à l'entrée en vigueur de la base légale. Tant que le crédit est bloqué, il ne peut pas être dépensé par l'Exécutif. Ce dispositif permet de respecter le principe de la légalité, tout en limitant les retards dans la mise en ouvre des dispositions légales. Suivant les cas, un crédit bloqué peut porter sur une dépense courante ou sur une dépense d'investissement.

Crédit supplémentaire ${ }^{27}$. En cours d'année, le crédit budgétaire octroyé par le Législatif peut s'avérer insuffisant. Dans ce cas, un crédit supplémentaire doit être demandé afin de compléter le crédit budgétaire de base et de respecter ainsi le principe de la spécialité quantitative. Un tel crédit doit être dûment motivé et sollicité préalablement à la dépense auprès du Législatif. Toutefois, si le besoin était imprévisible, qu'il est urgent et indispensable, l'Exécutif peut sous certaines conditions l'octroyer, puis le faire valider rétroactivement par le Législatif. Suivant les cas, un crédit supplémentaire peut porter sur une dépense courante ou sur une dépense d'investissement.

Dépassement de crédit. En vertu du principe de la spécialité quantitative, l'Exécutif n'est pas autorisé à dépenser au-delà des crédits alloués par le Législatif. Donc tout

26 Budgetkredit | Credito di preventivo | Budgetary appropriation.

27 Nachtragskredit | Credito aggiuntivo | Supplementary appropriation. 
dépassement de crédit est à éviter. Cependant, cela est parfois admis lorsque l'excès de dépenses est couvert par des recettes directement liées à la dépense elle-même ou qu'il est compensé par une dépense inférieure au crédit octroyé pour un autre type de dépenses. Par ailleurs, un dépassement peut apparaître sous l'effet des opérations de clôture des comptes, notamment liées aux opérations de régularisation de passifs (passifs transitoires). Dans ce cas, il est trop tard pour l'éviter. Un dépassement de crédit peut porter sur une dépense courante ou sur une dépense d'investissement.

Report de crédit. Diverses raisons, notamment techniques et procédurales, peuvent expliquer qu'un crédit alloué par le Législatif pour un investissement, un projet ou une activité importante n'ait pu être utilisé au cours de l'exercice qui s'achève. Sous certaines conditions, l'Exécutif peut autoriser le transfert du reliquat de crédit d'une année sur l'autre. Il faut pour cela que le solde du crédit résulte d'un retard dans l'avancement des travaux. Les conditions peuvent parfois être plus strictes s'agissant d'un investissement: l'Exécutif ou l'administration doivent avoir déjà engagé la dépense, et la dépense ne pas avoir encore été facturée par le prestataire. Dans ce cas, le report de crédit s'effectue grâce à un passif de régularisation et apparaît en tant que tel au bilan de la collectivité. Suivant les cas, un report de crédit peut donc porter sur une dépense courante ou sur une dépense d'investissement.

\subsubsection{Autorisation de contracter un engagement et crédit d'engagement}

Dans la mesure où la validité des crédits budgétaires est limitée à une année, le droit des crédits se doit d'offrir un mécanisme permettant à l'Exécutif d'engager la collectivité pour des projets dont la durée de réalisation dépasse l'année budgétaire. Ce mécanisme est le crédit d'engagement.

Crédit d'engagement ${ }^{28}$. Il permet au Législatif de donner la compétence à l'Exécutif d'engager la collectivité sur un horizon temporel pluriannuel. Toutefois, contrairement au crédit budgétaire, il ne donne pas à l'Exécutif l'autorisation de procéder à une dépense. En obtenant du Législatif un crédit d'engagement, l'Exécutif est, par exemple, à même de signer des contrats avec une entreprise de construction pour bâtir un bâtiment scolaire même si la construction s'étend sur plusieurs années. Toutefois, afin de pouvoir payer l'entreprise, il faudra qu'année après année, l'Exécutif demande au Législatif qu'il lui octroie le crédit budgétaire nécessaire au décaissement.

La figure 4 montre comment s'articule un crédit d'engagement avec les crédits budgétaires successifs en prenant l'exemple de la construction d'un nouveau bâtiment scolaire. Le crédit d'engagement octroyé par le Législatif au cours de l'Année t s'élève à CHF 55 mio. Les travaux démarrent au cours de l'exercice $t+1$ et l'Exécutif 
obtient un crédit budgétaire de CHF 10 mio pour payer les factures des travaux réalisés au cours de cette année-là. En $t+2$, le crédit budgétaire octroyé s'élève à CHF 25 mio. Les travaux s'achèvent en $t+3$, année pour laquelle le crédit budgétaire se monte à CHF 15 mio. Par conséquent, la construction du bâtiment aura duré trois ans comme prévu, mais aura coûté CHF 5 mio de moins que prévu dans le crédit d'engagement - dans l'hypothèse où l'intégralité des crédits budgétaires, mais pas davantage, ait été dépensée.

Un crédit d'engagement est généralement sollicité par l'Exécutif au cas par cas. Il peut donc être sollicité en cours d'année et en dehors de la procédure budgétaire proprement dite. En vertu de la spécialité qualitative, le crédit est lié à une réalisation, par exemple un investissement, un projet spécifique ou encore une subvention. En vertu de la spécialité quantitative, la possibilité donnée à l'Exécutif d'engager la collectivité vis-à-vis de tiers porte sur un montant déterminé. Le crédit est périmé dès lors que son objet est réalisé.

Dans certaines circonstances, par exemple pour des dépenses de moindre importance, il est possible de recourir uniquement à la voie du crédit budgétaire, sans avoir à solliciter au préalable un crédit d'engagement. La notion d'importance est concrètement liée à un seuil fixé dans la législation. On parle en l'occurrence d'un seuil d'activation, ainsi que nous le détaillerons dans la section 3.3 consacrée au modèle comptable des cantons et des communes.

Figure 4 Articulation entre crédit d'engagement et crédits budgétaires.

Exemple de la construction d'un nouveau bâtiment scolaire.

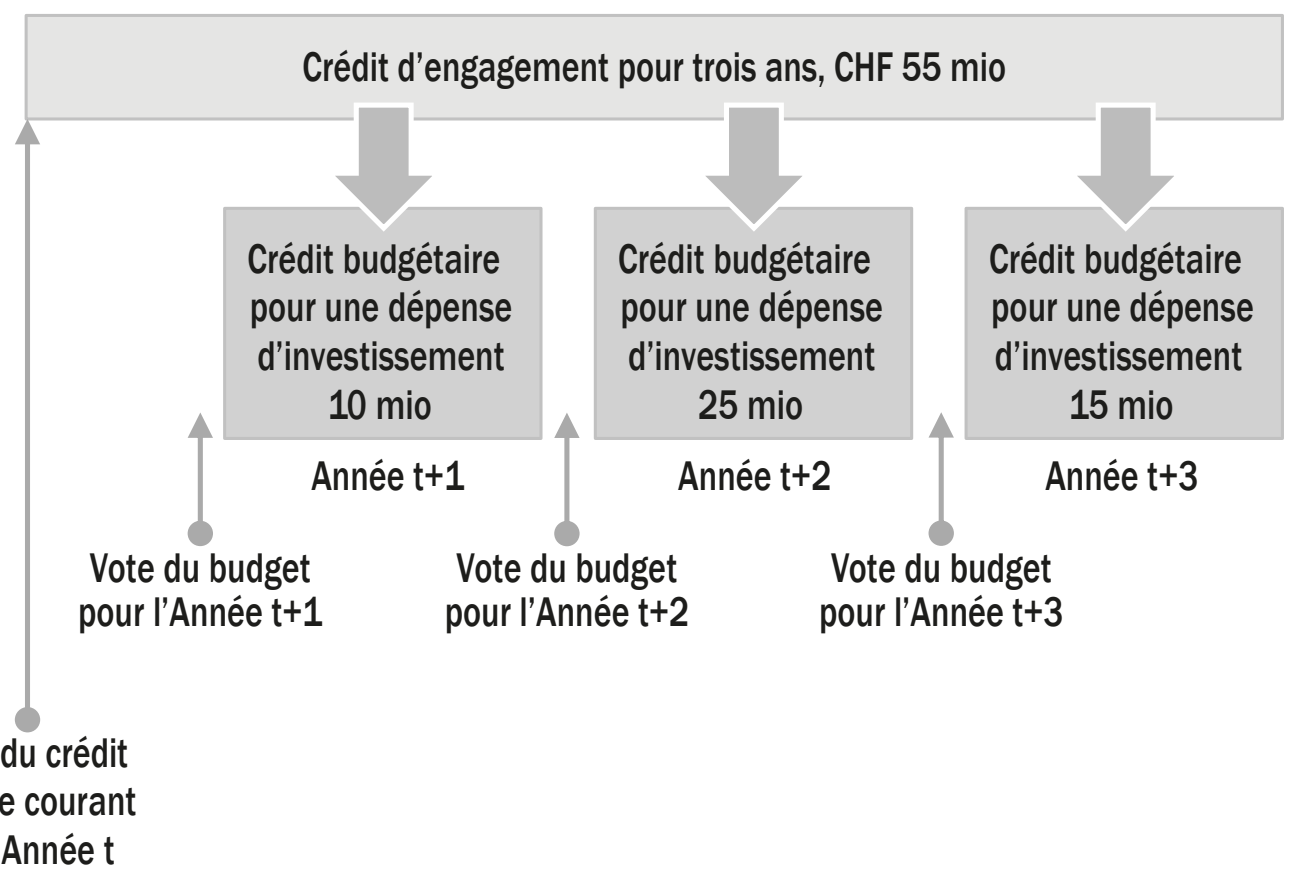


Crédit d'objet. À travers un crédit d'objet, le Législatif autorise l'Exécutif à engager la collectivité pour une réalisation spécifique, par exemple l'achat ou la construction d'un bâtiment à usage public, ou encore l'octroi d'une subvention d'investissement. L'autorisation de dépenser doit être obtenue par l'Exécutif chaque année sous la forme d'un crédit budgétaire en fonction des décaissements prévus au cours de l'année correspondante.

Crédit cadre. Le crédit cadre est, comme le crédit d'objet, un crédit d'engagement. Il s'en distingue par le fait qu'il porte sur la réalisation d'un programme, donc sur la mise en place d'un ensemble de mesures. Par ailleurs, il autorise l'Exécutif, et dans certains cas l'administration, à répartir le crédit d'engagement entre les diverses composantes du programme dans les limites de l'objectif fixé par le Législatif et du montant global prévu. Ici également, l'Exécutif doit chaque année obtenir un crédit budgétaire pour pouvoir procéder aux dépenses prévues au cours de l'année correspondante.

Crédit additionne ${ }^{29}$. Un crédit d'engagement peut s'avérer insuffisant pour réaliser l'objet ou le programme prévu. Dans ce cas, l'Exécutif peut demander au Législatif l'octroi d'un crédit additionnel. Hormis dans les cas d'urgence, cette demande doit être introduite avant tout nouvel engagement.

\subsection{Règles budgétaires}

Des règles budgétaires de plus en plus contraignantes sont introduites dans la législation. Les appellations sont diverses: frein à l'endettement, frein aux déficits, frein aux dépenses, etc. Leur objectif est de remédier à la fois à une asymétrie institutionnelle et à une asymétrie comportementale. L'asymétrie institutionnelle provient du fait que la compétence pour décider des dépenses n'est pas légalement accordée au même niveau institutionnel que celle de décider des recettes. En effet, le Législatif bénéficie d'une marge de manœuvre significative pour moduler, en particulier à la hausse, le niveau des crédits budgétaires. Par contre, les propositions de hausses d'impôts peuvent être combattues par voie référendaire et ce n'est donc plus le Législatif qui décide, mais la population (Lienhard et Marti Locher 2015). Par conséquent, la population peut s'opposer à une hausse de la pression fiscale pour financer une augmentation des dépenses quand bien même cela conduirait à un déficit et à un surcroît d'endettement. Ces règles budgétaires imposent plus qu'avant au Législatif de se préoccuper des équilibres financiers.

L'asymétrie comportementale est de nature plus politique et nous amène à introduire la situation conjoncturelle dans la réflexion. En effet, tant l'Exécutif que le Législatif tolèrent les déficits lorsque la situation économique est mauvaise, voire les aggravent en décidant un surcroît de dépenses dans l'espoir de stimuler l'économie. 
Par contre, ils ne s'efforcent pas de dégager des excédents lorsque l'économie est en expansion. Cette asymétrie engendre une élévation graduelle du niveau de la dette. Rendre plus contraignantes les règles budgétaires permet également de résoudre cette seconde asymétrie.

Depuis longtemps la législation évoque l'idée que les finances des collectivités doivent être équilibrées. Mais, jusqu'à récemment et dans la plupart des cantons, les dispositions légales en sont restées à ce niveau de généralité. Le canton de SaintGall a été le premier, en 1929, à muscler cette disposition en limitant drastiquement la possibilité pour le Législatif d'accepter un budget déficitaire ou de réduire les impôts. À l'heure actuelle, tous les cantons sauf un (Appenzell Rhodes-Intérieures) ont renforcé la contrainte légale, à des degrés divers et selon des modalités diverses - fédéralisme et autonomie cantonale obligent (Yerly 2014). Ce durcissement de la règle rencontre pour ainsi dire à chaque fois le soutien massif de la population, en particulier en votation populaire.

Certains cantons ont ancré une règle détaillée déjà dans leur constitution, d'autres dans la loi sur les finances (qu'il est plus facile pour le Législatif de modifier). La règle s'applique parfois au budget, parfois aux comptes annuels et parfois aux deux documents. En général, la règle exige un équilibre ou un quasi-équilibre (c'est-àdire tolère un déficit, mais limité à 2 ou 3\%). Cependant, l'exigence d'équilibre peut concerner l'ensemble des transactions ou seulement celles qui concernent le fonctionnement (c'est-à-dire en excluant les dépenses et les recettes d'investissement). En outre, la règle inclut parfois un mécanisme de sanction en cas de non-respect, par exemple une augmentation automatique de la charge fiscale. Certains résultats indiquent que les cantons dotés de règles plus strictes ont des déficits et des dettes significativement plus faibles (Feld et Kirchgässner 2008).

Pour ce qui est de la Confédération, en 2001, le peuple a approuvé - à une majorité de près de $85 \%$ des votants - l'introduction d'une règle détaillée de frein à l'endettement dans la Constitution (art. 126, extrait 1). Appliquée depuis 2003, la règle impose d'équilibrer le budget sur un cycle économique. Pour atteindre cet objectif, les dépenses sont, chaque année, plafonnées au niveau des recettes annuelles, mais en tenant compte de la situation conjoncturelle. L'ajustement est obtenu en multipliant les recettes par un facteur reflétant la situation économique. Techniquement, ce facteur s'obtient en rapportant le produit intérieur brut potentiel (c'est-à-dire la valeur de la production qui serait obtenue en situation de plein-emploi des facteurs de production) au PIB réel (production effectivement réalisée). Lorsque l'économie est en récession et que l'écart de production est négatif, le facteur qui multiplie les recettes est supérieur à un. De cette manière, le plafond des dépenses autorisées est plus élevé que les recettes réelles, permettant un déficit. Lorsque l'économie est en expansion, l'écart de production est positif et le facteur multipliant les recettes est inférieur à un. Ainsi, le plafond des dépenses autorisées est inférieur aux recettes réelles, ce qui oblige à dégager un excédent. Dans des circonstances exceptionnelles, 
toutefois, une majorité qualifiée du Parlement a le pouvoir d'augmenter le plafond des dépenses. En tenant mécaniquement compte du cycle conjoncturel, la règle budgétaire de la Confédération permet aux stabilisateurs automatiques de fonctionner et offre même au Conseil fédéral et au Parlement une certaine marge de manœuvre pour prendre des mesures anticycliques (Conseil fédéral 2000).

\section{Extrait 1 Constitution fédérale, art. 126 "Gestion des finances"}

"1 La Confédération équilibre à terme ses dépenses et ses recettes.

2 Le plafond des dépenses totales devant être approuvées dans le budget est fixé en fonction des recettes estimées, compte tenu de la situation conjoncturelle.

3 Des besoins financiers exceptionnels peuvent justifier un relèvement approprié du plafond des dépenses cité à l'al. 2. L'Assemblée fédérale décide d'un tel relèvement conformément à l'art. 159, al. 3, let. c.

4 Si les dépenses totales figurant dans le compte d'État dépassent le plafond fixé conformément aux al. 2 ou 3, les dépenses supplémentaires seront compensées les années suivantes.

5 La loi règle les modalités."

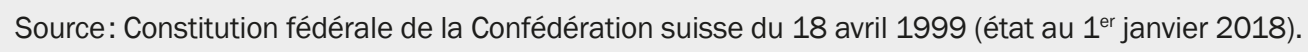

\subsection{Référendum financier et droit d'initiative}

Le référendum financier est une déclinaison du référendum législatif. Dans les cantons et les communes qui le connaissent, il permet -à partir d'un montant donné-de soumettre de manière obligatoire ou facultative une décision de dépense du Législatif au vote populaire. Le seuil de déclenchement, le caractère obligatoire ou facultatif du référendum, et dans ce second cas le nombre de signatures nécessaires pour obtenir le référendum, ainsi que le délai pour collecter ces signatures sont réglés par le droit cantonal et, pour l'échelon communal, en partie par le droit communal. Pour donner l'exemple des cantons, le seuil de déclenchement varie entre CHF 250000 et 25 mio pour les dépenses non récurrentes et entre CHF 50000 et 400000 pour les dépenses récurrentes. Lorsque la possibilité d'un référendum facultatif existe, suivant les cantons, le nombre de signatures à recueillir va de 100 à 10000.

Certaines études indiquent que, là où les référendums financiers obligatoires existent, les dépenses sont significativement plus faibles (Funk et Gathmann 2011). À titre d'exemple anecdotique, la population des Grisons a, par deux fois, refusé en votation populaire un crédit d'engagement pour financer la candidature du canton à l'organisation des Jeux olympiques d'hiver: d'abord en 2013 pour les Jeux de 2022, puis en 2017 pour les Jeux de 2026. 
L'instrument n'existe pas à l'échelon fédéral. Des propositions visant son introduction reviennent régulièrement. Jusqu'ici, elles ne sont pas parvenues à rallier une majorité parlementaire. Les opposants craignent que cela ralentisse le processus décisionnel pour une utilité toute relative, étant donné que les dépenses de la Confédération sont essentiellement des dépenses récurrentes de transferts au profit d'autres entités publiques, notamment les cantons (voir le chapitre 6 dédié aux transferts et à la péréquation financière).

Par rapport à ce débat, il convient de rappeler que les citoyens peuvent également user de leur droit d'initiative pour influencer les budgets des collectivités publiques, même lorsque le référendum ne peut pas être actionné. L'initiative populaire «Économiser dans l'armée et la défense générale - pour davantage de paix et d'emplois d'avenir», appelée également «initiative en faveur d'une redistribution des dépenses», en donne un exemple frappant. Déposée en 1997 avec les 100000 signatures nécessaires, cette initiative proposait d'inscrire dans la Constitution fédérale un nouvel article exigeant une réduction de moitié des dépenses militaires de la Confédération dans un délai de dix ans. Soumise au peuple en 2000 , elle a été rejetée par $62 \%$ des électeurs, bien qu'elle ait été acceptée dans quatre cantons romands.

\subsection{Processus financier et principaux acteurs}

Le processus financier peut varier d'une collectivité à l'autre. C'est pourquoi la figure 5 esquisse la séquence d'un processus type et les documents qui s'y rapportent: le programme de législature, le plan financier, les crédits d'engagement, le budget, la préparation des comptes, la vérification et l'approbation des comptes, ainsi que la statistique financière. Pour ordonnancer le processus, la figure indique à quel moment chaque étape intervient et cela par référence à l'année financière considérée que nous désignons comme étant l'Année t. Chaque étape est exposée ci-après.

Cette figure met également en évidence le rôle joué par les différents acteurs du processus, en particulier l'Exécutif, le Législatif et, dans certains cas, les citoyens. On pourrait croire que le processus dans les collectivités publiques suisses ressemble à ce qui se passe dans d'autres pays. Néanmoins, certaines particularités exercent une influence majeure. Mentionnons les instruments de la démocratie directe avec le droit de référendum et d'initiative. Notons également la prééminence du Législatif sur le pouvoir exécutif ou le fait que les membres des Exécutifs, cantonaux et communaux sont presque partout élus au suffrage universel, alors que dans d'autres pays les membres des Exécutifs sont nommés par le chef de l'Exécutif, qu'il s'agisse d'un président ou d'un maire. Par conséquent, dans les collectivités suisses, le membre de l'Exécutif en charge des finances (le «ministre des finances») et les autres membres 
responsables des politiques publiques dites substantielles comme l'éducation ou la santé (les «ministres dépensiers») ont des rôles clairement identifiés dans le processus. De ce fait, lorsqu'ils cherchent une réélection, c'est leur bilan dans leur fonction respective qui peut être pris en considération par le corps électoral. Cela incite les membres de l'Exécutif à jouer leur rôle spécifique et stimule automatiquement un système de contrôle et d'équilibrage entre les ministres dépensiers d'une part et le ministre des finances d'autre part. Ce mécanisme s'observe aussi bien au sein d'un Exécutif cantonal que d'un Exécutif communal.

Figure 5 Processus financier et organes décisionnels.

\begin{tabular}{|c|c|c|c|c|c|}
\hline & $\begin{array}{c}\text { Année } t-1 \\
\text { ou avant }\end{array}$ & Année t-1 & Année $t$ & Année t+1 & $\begin{array}{l}\text { Année } t+1 \\
\text { et au-delà }\end{array}$ \\
\hline $\begin{array}{l}\text { Programme de législature } \\
\text { et plan financier }\end{array}$ & Exécutif & & & & \\
\hline $\begin{array}{l}\text { Planification des projets et } \\
\text { demande de crédits d'engagement }\end{array}$ & Exéc & cutif & & & \\
\hline $\begin{array}{l}\text { Décision d'octroi des } \\
\text { crédits d'engagement }\end{array}$ & $\begin{array}{l}\text { Légis } \\
\text { et citc }\end{array}$ & $\begin{array}{l}\text { slatif } \\
\text { oyens }\end{array}$ & & & \\
\hline Modification des lois d'imposition & & $\begin{array}{l}\text { Législatif } \\
\text { et citoyens }\end{array}$ & & & \\
\hline $\begin{array}{l}\text { Préparation d'une proposition } \\
\text { de budget }\end{array}$ & & Exécutif & & & \\
\hline $\begin{array}{l}\text { Adoption du budget et octroi } \\
\text { des crédits budgétaires }\end{array}$ & & Législatif & & & \\
\hline Exécution du budget & & & Exécutif & & \\
\hline $\begin{array}{l}\text { Octroi de crédits } \\
\text { supplémentaires éventuels }\end{array}$ & & & Législatif & & \\
\hline Préparation des comptes & & & & Exécutif & \\
\hline Vérification des comptes & & & & $\begin{array}{l}\text { Organe de } \\
\text { révision }\end{array}$ & \\
\hline Approbation des comptes & & & & Législatif & \\
\hline $\begin{array}{l}\text { Élaboration de la } \\
\text { statistique financière }\end{array}$ & & & & \multicolumn{2}{|c|}{$\begin{array}{l}\text { Département fédéral } \\
\text { des finances }\end{array}$} \\
\hline
\end{tabular}




\section{Programme de législature et plan financier pluriannuel}

Dans les faits, le processus financier commence avant même le début des discussions sur l'allocation des ressources financières. En effet, de plus en plus souvent, la législation exige que l'Exécutif nouvellement élu se mette d'accord sur des objectifs politiques communs et sur un programme d'actions à entreprendre prioritairement. Ce document couvre la législature qui commence. Parfois, il va au-delà de cet horizon temporel pour permettre à l'Exécutif de développer une vision à plus long terme. Pour concrétiser son action, l'Exécutif sollicite souvent les unités administratives $^{30}$ afin qu'elles conçoivent et chiffrent des projets permettant de réaliser les objectifs gouvernementaux. Ces projets sont ensuite rassemblés dans un plan financier pluriannuel ${ }^{31}$ qui accompagne le programme gouvernemental. Généralement, ce plan financier est actualisé d'année en année afin de tenir compte notamment de l'évolution des perspectives socio-économiques prévues pour les années à venir ou pour intégrer des changements de priorités. Ce plan financier pluriannuel est donc glissant ou roulant. Le programme de législature et le plan financier sont portés à la connaissance du Législatif. Toutefois, ce dernier n'a pas à les accepter ou à les refuser. Il n'y a donc pas de vote et le Législatif n'est pas lié par ces documents. Ces documents sont donc avant tout des documents d'orientation.

\section{Planification des projets et demande de crédits d'engagement}

La réalisation des projets requis en particulier par un programme de législature ou prévu dans le plan financier nécessite une préparation spécifique. Dès lors que ces projets sont importants et s'étendent sur plusieurs années, le droit des crédits exige l'obtention d'un crédit d'engagement.

Pour illustrer le travail administratif que cela implique, reprenons l'exemple du projet de construction d'un nouveau collège. L'unité administrative concernée - ici la direction des écoles - doit préparer un rapport justifiant le projet, détaillant tous les aspects techniques et planifiant ses implications financières (dépenses pour la construction, contributions éventuellement à recevoir pour la construction, charges et revenus opérationnels liés). Dans les grandes collectivités, et en particulier les cantons, ce rapport est soumis pour consultation aux autres directions concernées par le projet. La direction des finances donne également son avis. Pour cela, elle évalue en particulier si le projet répond aux principes financiers (légalité, emploi efficace des fonds, etc.). Ensuite, l'Exécutif examine le projet sur la base du rapport de planification et des commentaires. Dès lors que le projet dépasse la compétence financière de l'Exécutif, le Législatif est sollicité pour qu'il octroie un crédit d'engagement sur la base du rapport de planification.

30 Par unités administratives, on entend au gré des situations les départements, les directions, les services, les offices, ou encore les dicastères, en fonction des appellations en vigueur. Par la suite, pour simplifier, nous utiliserons souvent le terme de directions.

31 Finanzplan | Piano finanziario | Multi-year financial plan. 


\section{Décision sur les demandes de crédits d'engagement}

Lorsque le Législatif est saisi d'une demande de crédit d'engagement par l'Exécutif, les commissions parlementaires compétentes analysent le projet. Cela peut être la commission thématique permanente concernée ou une commission spécifiquement chargée du dossier. La commission des finances est également consultée. Ces commissions transmettent leur opinion au plénum du Législatif pour décision. En fonction des règles légales en vigueur et selon le montant de la dépense prévue, la décision du Législatif - si elle est positive - est exécutoire ou peut être attaquée par la voie d'un référendum populaire. Une fois le crédit d'engagement accepté, que ce soit par le Législatif ou par les citoyens après un référendum, il doit être mis en œuvre année après année au travers des crédits budgétaires.

\section{Préparation du budget}

Le budget est la concrétisation des dispositions légales en vigueur, qu'il s'agisse des lois régissant les prestations publiques à offrir ou des lois fiscales. En effet, il n'est guère imaginable de modifier le cadre légal en même temps que l'on prépare le budget. Modifier une loi implique l'éventualité d'un référendum législatif et donc impose des délais qui ne sont pas compatibles avec le calendrier financier et avec la nécessité de respecter le principe de l'antériorité du budget. En comparaison, dans les pays qui ne connaissent pas le droit de référendum, le gouvernement est davantage libre de modifier les lois pour les adapter aux nécessités budgétaires, que ce soit pour modifier le niveau des dépenses ou celui des recettes. Pour les collectivités publiques suisses, les grandes décisions concernant les politiques publiques et leur exécution sont prises bien avant le début de la préparation du budget. Par conséquent, la marge de manœuvre à disposition au moment de la préparation du budget est bien plus limitée.

Cela étant précisé, le budget annuel (pour l'Année t) est généralement préparé en même temps que l'on met à jour le plan financier glissant pour les années suivantes avec un horizon habituel de trois ou quatre ans $(t+1$ à $t+3$ ou $t+4)$. Pour élaborer le budget et mettre à jour le plan financier, le plan financier préexistant est pris en compte. Cependant, ce dernier n'est pas contraignant. Dans les collectivités où la préparation du budget est décentralisée, c'est-à-dire dans les collectivités d'une certaine taille, les derniers développements de la situation sont pris en compte par l'Exécutif dans des directives qu'il donne aux directions pour élaborer leurs demandes de crédits budgétaires. Pour élaborer ces directives, l'Exécutif, avec le soutien de la direction des finances, tient compte de l'évolution du cadre légal (par exemple obligeant la collectivité à fournir de nouvelles prestations ou à assumer de nouvelles charges), de la démographie, de la situation conjoncturelle. L'Exécutif tient également compte de ses priorités, en particulier lorsqu'il poursuit un programme d'économie. Il intègre les prévisions de recettes et plus particulièrement de recettes fiscales. Ce sont ces prévisions de recettes qui déterminent l'enveloppe budgétaire dans laquelle devront finalement s'inscrire l'ensemble des crédits budgétaires pour les dépenses courantes. 
Compte tenu de leur rôle spécifique, les responsables des finances sont généralement prudents. Ils ont tendance à sous-estimer les recettes fiscales pour tenter de limiter les appétits budgétaires de leurs collègues membres de l'Exécutif et responsables des autres directions (Chatagny et Soguel 2012). Malgré cela, lorsque la direction des finances collecte les différentes demandes de crédits et les consolide, le total des demandes excède systématiquement les recettes prévues, ne respecte pas les directives budgétaires ou enfreint la règle budgétaire prévue par la législation (voir ci-dessus la section 2.3 consacrée aux règles budgétaires). Il faut généralement plusieurs cycles de négociations entre la direction des finances et les autres directions (par exemple la direction des écoles, celle de la police, etc.) pour mettre sous toit le budget. L'Exécutif lui-même s'implique lorsque les responsables administratifs des directions ne parviennent pas à trouver une solution satisfaisante.

D'un point de vue formel, le budget est préparé selon les normes de présentation prévues dans la législation. Les mêmes normes s'appliqueront lors de la clôture de l'exercice pour la présentation des comptes (voir la section 3.3 traitant du modèle comptable des cantons et des communes).

La préparation du budget telle que nous l'avons décrite peut donner l'impression que ce processus est un processus ascendant et incrémental: ascendant - bottom-up - parce que fondé sur les demandes budgétaires venant de la base, c'est-à-dire des directions; incrémental parce que les demandes de crédits budgétaires pour l'Année $t$ sont fondées sur les crédits budgétaires approuvés par le Législatif pour l'année précédente ( $\mathrm{t}-1)$ et adaptés à la marge. Il en va encore ainsi dans de nombreuses collectivités. Cette manière de procéder est parfois remise en cause. C'est le cas lorsque des inefficiences significatives sont mises à jour, lorsqu'il est nécessaire de dégager des ressources pour financer de nouveaux projets ou lorsque des difficultés financières forcent l'Exécutif à revoir systématiquement les programmes existants. Selon Aschwanden et Gerny (2013, dans la Neue Zürcher Zeitung), c'est ce qui s'est passé en 2013 lorsque seize cantons ont éprouvé des difficultés très importantes à équilibrer leurs budgets et n'ont pas eu d'autres options que de procéder à un ajustement structurel.

Le mouvement que l'on a appelé celui de la «nouvelle gestion publique»a incité de nombreuses collectivités à adopter une budgétisation fondée sur la performance. L'idée est que le Législatif n'encadre plus l'action de l'Exécutif en définissant uniquement les intrants (inputs) et donc les ressources du processus administratif. $\mathrm{Au}$ lieu de cela, le Législatif devrait formuler plus explicitement ses exigences en matière de prestations (outputs) à fournir par l'Exécutif et l'administration, sous l'angle des caractéristiques à la fois qualitatives et quantitatives des prestations. Par exemple, un Législatif peut vouloir fixer des objectifs quantitatifs à la direction de la santé en matière de vaccination en milieu scolaire. Ces exigences déterminent ensuite les crédits budgétaires à accorder. Le processus budgétaire s'en trouve ainsi davantage axé sur les résultats. 
En parallèle, le renforcement des règles budgétaires a conduit de nombreuses collectivités, mais surtout les cantons, à adopter un processus budgétaire descendant - top-down. En effet, les règles budgétaires du type frein à l'endettement corsètent de plus en plus le budget et la loi interdit pratiquement tout déficit. Par conséquent, l'enveloppe définie par les recettes prévues en tout début de processus est devenue beaucoup plus contraignante par rapport au temps où les règles budgétaires étaient plus accommodantes. Pour s'assurer que l'équilibre budgétaire exigé par la règle soit atteint, l'Exécutif procède à une allocation budgétaire initiale et répartit d'emblée l'enveloppe des recettes prévues entre les différentes directions. Cette approche offre également une meilleure adéquation entre le programme de législature et le budget. À partir de là, chaque direction est responsable d'affecter l'allocation qui lui est faite par l'Exécutif - mais pas plus - aux différentes tâches qu'elle doit accomplir.

\section{Adoption du budget}

Le débat budgétaire au sein du Législatif intervient à l'automne. Les discussions préliminaires sont menées en commissions. Schématiquement, la commission financière adopte généralement une position défensive et tente de maintenir, voire d'améliorer, le résultat prévisionnel proposé par l'Exécutif; tandis que les commissions thématiques militent pour développer les prestations publiques placées sous leur égide, comme l'éducation, la santé ou la culture, avec à la clé le souhait de leur allouer davantage de moyens que ceux qui ont été initialement proposés. Il revient au plénum du Législatif de s'accorder et de voter le budget courant décembre. Comme l'évoque l'extrait 2, le budget voté par le Législatif ne diffère finalement guère de la proposition que lui fait l'Exécutif.

Extrait 2 Au mois de décembre, la messe est dite...

"Le budget est au Grand Conseil ce que l'Avent est aux chrétiens. Une liturgie spéciale de décembre quand les jours sont courts et les discussions longues.

Limité à la rare présence simultanée des sept conseillers d'État à leur pupitre, le décorum est simple. Le nombre de rapports de minorités présentés décide de la complexité du rituel. Mais finalement seule compte l'ambiance, ce qui fait la sévérité des empoignades."

Source: Busslinger (2006, dans 24 Heures).

S'il n'est pas possible au Législatif de s'accorder sur un projet et de le voter avant la fin de l'année, l'Exécutif ne dispose d'aucun budget pour fonctionner à partir du $1^{\text {er }}$ janvier. Appliquer à la lettre le principe de l'antériorité impliquerait qu'aucune dépense ne puisse être effectuée. En réalité, la législation prévoit ce cas de figure. 
Dans certains cantons, elle autorise l'Exécutif à engager une dépense à la condition expresse que cette dépense soit indispensable à l'activité de l'administration ou à la réalisation des investissements en cours. Dans d'autres cantons, la législation autorise l'Exécutif à procéder, mois après mois, à des dépenses pour un montant équivalant au douzième des montants figurant au budget de l'année précédente, c'est-àdire de l'Année t-1. Cette condition dite des «douzièmes provisoires" permet d'une certaine manière de respecter le principe d'antériorité, puisque le budget de l'Année $\mathrm{t}-1$ a été précédemment accepté et voté par le Législatif (en décembre t-2). Dans la majorité des cas, l'Exécutif transmet au Législatif une nouvelle proposition de budget pour l'Année $t$ au cours de la première moitié de l'Année t. Cette nouvelle proposition est généralement acceptée par le Législatif.

Le Législatif a donc le dernier mot en matière budgétaire. En théorie, cela lui confère plus de pouvoir que l'Exécutif dans l'allocation des ressources. Cependant, le calendrier est serré et empêche le Législatif de modifier significativement la proposition qui lui est transmise (Pfäffli 2011). Les contingences du calendrier transfèrent de facto une bonne partie du pouvoir budgétaire à l'Exécutif et à l'administration. Si en cours de préparation du budget, un changement de loi fiscale s'avère nécessaire pour boucler le budget, ce changement législatif est soumis au référendum facultatif, voire obligatoire. Cette particularité donne alors le dernier mot de facto aux citoyens avec un droit de veto sur le budget. Par exemple, fin 2016, l'électorat du canton de Lucerne a rejeté l'augmentation du taux d'imposition proposée par le Législatif avec, par conséquent, la proposition de budget 2017. L’Exécutif a entamé l'exercice 2017 sans budget. Un accord n'a été trouvé qu'à la mi-septembre 2017 au sein du Législatif. L'accord comprenait une baisse des dépenses afin de satisfaire l'exigence d'équilibre budgétaire imposée par la législation et cela sans hausse du taux d'imposition. Entre-temps toutefois, l'Exécutif a dû se limiter aux seules dépenses découlant des contrats déjà signés (Däniken 2017 dans la Luzerner Zeitung). Évidemment, la menace d'un référendum en matière fiscale incite l'Exécutif et l'administration à proposer un budget acceptable aux yeux du Législatif. Cependant, il existe toujours une asymétrie entre le pouvoir conféré d'une part au Législatif et à l'Exécutif et d'autre part aux citoyens. En effet, en cas de référendum fiscal, ces derniers n'ont le choix qu'entre accepter ou refuser la hausse d'impôt et donc accepter ou refuser en bloc la proposition de budget qui l'accompagne. Les citoyens ne peuvent pas décider de modifier individuellement les crédits budgétaires proposés. Cela étant, deux mécanismes permettent aux citoyens d'influencer en amont et directement l'allocation budgétaire: le référendum financier pour s'opposer à un crédit d'engagement ou l'initiative populaire pour modifier la législation avec pour conséquence une hausse ou une baisse des dépenses de mise en œuvre de la législation concernée. 


\section{Exécution du budget et crédits supplémentaires éventuels}

Il appartient à l'Exécutif, à proprement parler, d'exécuter le budget, c'est-à-dire de le mettre en œuvre. En effet, les crédits budgétaires obtenus donnent à l'Exécutif le droit de dépenser les montants prévus dans les limites et aux fins prescrites, entre le $1^{\text {er }}$ janvier et le 31 décembre de l'Année $t$.

L'exécution du budget se déroule rarement comme prévu. Le coût de certains inputs ou la demande de certaines prestations publiques peuvent augmenter. Dans ce cas, les crédits budgétaires initiaux peuvent ne pas suffire. Certes, l'Exécutif dispose d'une certaine marge de manœuvre pour réaffecter les crédits initiaux. Cependant, passé ces limites, des moyens supplémentaires peuvent être nécessaires. C'est à ce moment que l'Exécutif doit solliciter des crédits supplémentaires auprès du Législatif.

Il se peut également que les crédits budgétaires octroyés se révèlent supérieurs aux besoins. Dans ce cas, en fin d'année, une partie des crédits reste inutilisée. Cela devrait être un phénomène normal dans la mesure où les crédits budgétaires sont une autorisation donnée à l'Exécutif de dépenser, non pas une obligation. Mais on observe souvent que les directions accélèrent le rythme de leurs dépenses en fin d'année afin d'utiliser dans toute la mesure du possible la totalité des crédits à leur disposition.

\section{Préparation des comptes}

La fin de l'exercice budgétaire marque le début des travaux de préparation des comptes annuels également appelés états financiers annuels. D'un point de vue formel, les comptes sont préparés par la direction des finances selon les normes de présentation prescrites par la législation. Ces normes sont les mêmes que celles qui sont appliquées pour la présentation du budget (voir la section 3.3 consacrée au modèle comptable des cantons et des communes). Cela implique de suivre les règles de la comptabilité d'exercice, de représenter fidèlement la réalité économique de la collectivité. Pour cela, il faut suivre les règles de comptabilisation des actifs, des passifs, des revenus et des charges. Mais la préparation des comptes donne lieu également à des opérations comptables visant à satisfaire une autre rationalité que celle qui consiste à présenter la situation financière telle qu'elle est. Cette autre rationalité correspond à des impératifs de tactique politique et à la volonté des responsables des finances de présenter la situation financière plutôt telle qu'ils voudraient qu'elle soit. Pensons aux opérations de préfinancement ou aux «amortissements» supplémentaires lorsqu'ils sont autorisés par la législation (voir le chapitre 8 consacré à la tactique politique en matière de présentation des comptes). Tant que ces opérations restent dans les limites fixées par la loi sur les finances, on doit les qualifier de comptabilité créatrice ou d'artifice comptable (Clémenceau et Soguel 2018). Si elles enfreignent les prescriptions, on est en présence d'opérations illégales et irrégulières. Les responsables des finances cherchent naturellement souvent à exploiter les possibilités d'artifices offertes par la législation. D'abord ces possibilités peuvent directement les aider à satisfaire les exigences légales d'équilibre budgétaire, surtout si la 
règle budgétaire est stricte. Ensuite, un déficit excessif comme un excédent important peuvent placer les responsables des finances dans une situation politiquement délicate. Un déficit peut laisser entendre aux citoyens que les responsables ont perdu le contrôle de la situation financière. Quant à un excédent, il peut donner l'impression aux citoyens qu'ils ont payé plus d'impôts que nécessaire et les inciter à revendiquer une amélioration des prestations publiques ou une baisse de la pression fiscale (par exemple en faisant usage de leur droit d'initiative). Si elles aboutissent, ces revendications menacent l'équilibre budgétaire, surtout si l'excédent provient d'une conjoncture particulièrement favorable, autrement dit si l'excédent est conjoncturel plutôt que structurel (voir le chapitre 11 consacré à l'analyse de l'origine conjoncturelle ou structurelle des déficits). Cette éventualité hypothèque les chances que la collectivité parvienne à équilibrer ses comptes à l'avenir. Elle risque aussi de peser sur le score électoral du responsable des finances s'il est candidat à une réélection (Clémenceau et Soguel 2017).

\section{Vérification des comptes}

La législation sur les finances, ainsi que la législation sur le contrôle des finances, exigent que les comptes soient vérifiés, révisés. Cette même législation prescrit les critères de vérification à utiliser par l'organe de contrôle. Elle requiert explicitement un audit de conformité, afin de pouvoir attester vis-à-vis du Législatif que les principes en vigueur ont été respectés dans la tenue, la préparation et la présentation des comptes. De cette façon, le réviseur doit certifier l'absence de fraude et vérifier si les états financiers respectent la loi. Cependant, dans la plupart des collectivités, la loi n'exige aucunement du réviseur qu'il certifie que les comptes donnent une image fidèle de la situation financière. Ce paradoxe doit être mentionné, puisque souvent la législation permet aux responsables financiers qui préparent les comptes de recourir à des artifices à des fins de tactique politique. Le rapport d'audit établi par le réviseur est ensuite envoyé au Législatif pour accompagner les comptes.

La législation précise également à quelle instance la fonction de contrôle financier est confiée. Dans les communes de taille modeste, la fonction est parfois encore confiée à des réviseurs non professionnels. Elle peut également être attribuée à une commission extra-parlementaire. Lorsque la taille de la collectivité s'accroît, ce sont des spécialistes qui s'en occupent. Dans les grandes collectivités - villes, cantons et Confédération - un organe spécifique assume la fonction, sous des appellations diverses: contrôle ou inspectorat des finances ${ }^{32}$. Selon les cas, le responsable de l'organe est nommé par l'Exécutif ou par le Législatif. Cet organe est habituellement rattaché administrativement à la direction des finances. Cependant, dans son travail, il est soumis uniquement à la loi. Légalement, il est donc fonctionnellement indépendant lorsqu'il agit en tant que réviseur des comptes au profit du Législatif. Cette 
fonction est celle d'une révision dite «externe» (c'est-à-dire "externe» à l'Exécutif et à l'administration). Tout au long de l'année, cet organe fonctionne également au profit de l'Exécutif pour contrôler que l'administration respecte les principes en vigueur dans le cadre de l'exécution du budget. À ce titre, il agit en tant qu'auditeur interne, à l'image de ce qui se fait dans le secteur privée ${ }^{33}$.

\section{Approbation des comptes}

Dès qu'ils sont vérifiés, les comptes sont soumis par l'Exécutif au Législatif pour approbation. Presque toujours, cette approbation s'apparente à une simple formalité. En effet, à ce stade, il n'y a plus d'enjeu.

\section{Élaboration de la statistique financière}

Nous associons au processus financier une ultime étape, celle qui consiste à élaborer la statistique financière. C'est là un élément souvent ignoré, mais important pour contribuer à une bonne gestion des collectivités publiques. Cette étape consiste à rendre comparables et disponibles les informations contenues dans les comptes de l'ensemble des communes et des cantons ainsi que de la Confédération. Cette opération s'étend généralement sur plusieurs années après la présentation des comptes par les collectivités.

Comme nous le verrons dans la section 3.3.1 consacrée au développement historique de la comptabilité publique, la présentation des états financiers est censée être harmonisée entre toutes ces entités. En réalité, c'est loin d'être le cas. Le Département fédéral des finances a la lourde tâche d'élaborer une statistique financière à l'échelon national. Cela suppose d'harmoniser le périmètre considéré. En effet, et pour citer quelques exemples, certains cantons intègrent les hôpitaux dans leurs comptes, d'autres non. Il en va de même pour les universités ou les offices régionaux de placement. Surmonter ces difficultés, garantir que la statistique financière helvétique satisfasse les exigences de qualité requise et soit conforme aux directives internationales (FMI 2014) demande donc beaucoup d'efforts et de temps de la part de tous les acteurs. Mais c'est le prix à payer pour obtenir des informations statistiques fiables dans un système aussi décentralisé que le système suisse.

33 Seuls deux cantons - Genève et Vaud - ont décidé de se doter d'une Cour des comptes (Rechnungshof | Corte dei conti | Court of auditors) aux côtés de l'organe de contrôle déjà existant. L'idée était de disposer d'une autorité plus indépendante pour surveiller le fonctionnement de l'État. Tandis que les membres de la Cour genevoise sont élus au suffrage universel direct, ceux de la Cour vaudoise sont élus par le Législatif cantonal. Singulièrement, la tâche principale de la Cour des comptes vaudoise consiste à procéder à des audits de performance plutôt qu'à vérifier la conformité des comptes. 


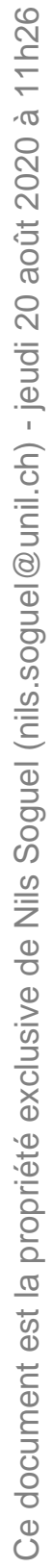




\section{Décrypter comment les comptes et les budgets sont organisés}

À ce stade, le moment est venu d'aborder les conventions qui régissent la préparation et la présentation des comptes de la collectivité. Ce sont les mêmes conventions qui prévalent pour la présentation du budget. Conscient que ces conventions sont souvent considérées comme rébarbatives et incompréhensibles pour les non-initiés, nous avons conclu qu'il fallait les approcher d'une manière naturelle.

C'est pourquoi nous vous proposons d'abord de marcher dans les pas de l'histoire de la comptabilité. En effet, la comptabilité est, à ses débuts, d'une simplicité enfantine; ses développements sont intervenus graduellement.

Puis, nous ferons un détour par la comptabilité de l'entreprise. En effet, la présentation des comptes et du budget dans les collectivités publiques s'est peu à peu rapprochée des pratiques de l'entreprise. Elle continue d'ailleurs à s'en rapprocher.

Il sera alors temps d'en venir aux conventions que la collectivité doit appliquer. L'objectif est d'en rester à un degré de détail qui permette au lecteur d'appréhender l'information apportée par ces documents afin de pouvoir ensuite l'analyser.

\subsection{Approche de la comptabilité dans les pas de son histoire}

À travers les siècles, les comptables ont adapté leur technique pour faire face à la complexité croissante des relations économiques. Nous présentons ici les étapes qui ont marqué l'évolution des méthodes comptables.

Selon les principes élémentaires de gestion, «gérer c'est prévoir» et, par ailleurs, il n'y a pas de bonne prévision sans une bonne connaissance de la situation actuelle. La gestion a par conséquent toujours nécessité l'établissement et l'analyse de deux catégories de documents administratifs: (a) un relevé des opérations réalisées et (b) une prévision des opérations à venir. La comptabilité au sens traditionnel assure l'enregistrement des opérations réalisées. Prévoir les opérations à venir relève d'un autre document: le budget. L'un ne va pas sans l'autre et les deux documents se sont développés sous une influence réciproque. Les aspects budgétaires ont conduit à des aménagements des modalités comptables, tandis que ces mêmes modalités ont façonné l'élaboration du budget. Ces interrelations ont déterminé l'évolution historique - et logique - de la comptabilité. À partir des méthodes élémentaires de comptabilité simple, on est passé à la comptabilité primitive en partie double, pour aboutir à la comptabilité moderne ${ }^{34}$.

34 Les informations relatives aux développements historiques de la technique comptable sont tirées de Fourastier et Kovacs (1995) et de Vlaeminck (1956). 


\subsubsection{Comptabilité simple}

\section{Une affaire de recettes et de dépenses}

On désigne du nom de comptabilité simple l'enregistrement chronologique des recettes et des dépenses découlant d'encaissements ou de décaissements d'argent. On parle aussi de comptabilité de type caisse ou de comptabilité camérale. C'est le stade le plus élémentaire de la comptabilité.

Illustrons son fonctionnement par l'exemple du ménage des Thénardier ${ }^{35}$ qui souhaite garder la mémoire de ses dépenses. Chacun des membres apporte son salaire et reçoit de l'argent de poche. Un montant sert à s'acquitter des factures communes et un autre est alloué à l'épargne. Chacun de ces mouvements monétaires donne lieu à une écriture comme indiqué au tableau 1.

Il n'y a pas de système plus primitif: les montants figurent sur une seule colonne; les recettes et les dépenses se suivent sans classement. Il apparait d'emblée plus commode de classer recettes et dépenses en deux colonnes distinctes. On trouve au tableau 2 tous les éléments essentiels d'une technique comptable, aussi rudimentaire soit-elle:

- un enregistrement des mouvements de valeurs en unités monétaires courantes, ici en francs suisses (CHF);

- un classement, faisant la distinction entre recettes et dépenses; puis dans une version plus évoluée, introduisant des rubriques comme les salaires ou l'argent de poche;

- une vérification, puisque la différence entre recettes et dépenses doit se trouver dans le porte-monnaie du ménage - ici CHF 1000.

Si l'un de ces trois éléments manquait, on ne pourrait pas parler d'une comptabilité, mais tout au plus d'un aide-mémoire. Même la plus sophistiquée des comptabilités se fonde sur ces trois éléments et ses objectifs restent les mêmes: garder en mémoire, classer les opérations, contrôler les résultats.

Tableau 1 Les recettes et les dépenses des Thénardier.

Mois d'avril 1820, en CHF.

\begin{tabular}{llc}
\hline Date & Libellé & Montant \\
\hline 20 avril & Reçu salaire de $\mathrm{M}^{\mathrm{me}} \mathrm{T}$. & +3000 \\
25 avril & Argent de poche pour $\mathrm{M}^{\mathrm{me}} \mathrm{T}$. & -500 \\
25 avril & Argent de poche pour M. T. & -500 \\
27 avril & Reçu salaire de M. T. & +3000 \\
30 avril & Paiement de factures & -3000 \\
30 avril & Acheté un bon du Trésor & -1000 \\
\hline & Solde dans le porte-monnaie & +1000 \\
\hline
\end{tabular}

35 Victor Hugo nous pardonnera l'emprunt de ses personnages pour mettre en lumière les rigueurs peu romantiques de la comptabilité. 


\section{Déjà les Incas et les Romains...}

L'ancienneté des méthodes de comptabilité simple est attestée par de nombreux documents. Les archéologues ont retrouvé des «quipous» parmi les témoignages de la civilisation des Incas. Il s'agit de ficelles nouées représentant des nombres, la couleur de la corde désignant la nature des éléments dénombrés (têtes de bétail, sacs de blé, etc.). Chaque ficelle représente ainsi un compte qui enregistre les recettes (nouage) et les dépenses (dénouage).

À l'époque romaine, on trouve également des traces du système de comptabilité simple à double colonne de type recettes-dépenses. Pline s'y réfère dans un passage de son «Histoire naturelle» où il philosophe sur la Fortune: «Les hommes lui attribuent toutes les chances et tous les insuccès; sur le compte ouvert aux mortels, elle remplit entièrement à elle seule l'une et l'autre colonne.»

Cependant, les plus anciens documents comptables que l'on a retrouvés ne remontent pas au-delà de 1100 après J.-C. Suite à l'effondrement de l'empire romain, il a en effet fallu attendre que les croisades redonnent au commerce son ampleur passée. L'accroissement des échanges a rendu à nouveau nécessaire la tenue d'une comptabilité. On a principalement retrouvé des témoignages de l'existence d'une comptabilité simple dans les villes commerçantes de l'époque et celles où se tenaient les grandes foires commerciales.

\subsubsection{Avènement de la comptabilité en partie double}

\section{Nécessité d'enregistrer les opérations de crédit}

Jusqu'au XIII ${ }^{e}$ siècle, le crédit était resté peu développé et limité à un cercle restreint de correspondants. Le chef d'entreprise pouvait se contenter de tenir une liste de ses créances et de ses dettes, et un aide-mémoire était tenu en marge du compte de caisse. Ni les créances, ni les dettes n'apparaissaient par conséquent dans la comptabilité. Cet aide-mémoire était utile dans le cadre de la préparation du budget, puisqu'il indiquait les recettes et les dépenses à venir - c'est-à-dire lorsque les débiteurs ou l'entreprise rembourseraient leurs dettes.

Tableau 2 Une colonne pour les recettes, une autre pour les dépenses. Mois d'avril 1820, en CHF.

\begin{tabular}{llcc}
\hline Date & Libellé & Recettes & Dépenses \\
\hline 20 avril & Salaire, $\mathbf{M}^{\mathrm{me}}$ T. & 3000 & \\
25 avril & Argent de poche, $\mathbf{M}^{\mathrm{me}} \mathrm{T}$. & & 500 \\
25 avril & Argent de poche, M. T. & & 500 \\
27 avril & Salaire, M. T. & 3000 & \\
30 avril & Factures & & 3000 \\
30 avril & Bon du Trésor & 6000 & 5000 \\
\hline & Total & & \\
\hline
\end{tabular}


L'expansion commerciale qui suivit les conquêtes maritimes s'accompagna d'un fort développement du crédit. Le chef d'entreprise vit sa liste de créanciers et de débiteurs s'allonger. Il lui devint de plus en plus difficile de connaitre à tout moment l'état de son crédit. Il se déchargea alors de cette tâche sur le comptable, c'est-à-dire sur le membre de son personnel qui avait déjà l'habitude de tenir des états financiers.

Le comptable utilisa la technique comptable qu'il connaissait déjà: de la même manière que le compte "caisse» enregistrait les opérations de l'entreprise, il ouvrit un compte de tiers pour chacun des correspondants de l'entreprise et y porta les relations financières qui liaient les deux entités. Ce type de compte indique, toujours sur deux colonnes, d'une part ce qui a été prêté et d'autre part ce qui a été rendu - ou avancé (tableau 3):

- Lorsque le correspondant est un débiteur, on trouve dans la colonne de gauche, le montant qu'il doit à l'entreprise.

- Lorsque le correspondant est un créancier, la colonne de droite indique quel est son avoir par rapport à l'entreprise.

Tableau 3 Compte du Débiteur X.

Mois d'avril 1820, en CHF.

\begin{tabular}{llcc}
\hline Date & Libellé & Doit (débit) & Avoir (crédit) \\
\hline 10 avril & Prêt à $X$ & 1000 & \\
15 avril & Remboursement de X & & 500 \\
17 avril & Prêt à X & 2000 & \\
\hline & Total & 3000 & 500 \\
\hline
\end{tabular}

Ces expressions de doit et avoir - on parle également de débit et de crédit-ont depuis lors été retenues pour intituler les colonnes des comptes. Cette règle s'applique à tous les comptes, y compris au compte de caisse. On constate que dans la logique comptable, la situation du correspondant prévaut: il est soit le débiteur et dans ce cas il doit de l'argent à l'entreprise, soit le créancier et il a alors un avoir - ou un crédit - par rapport à l'entreprise. Cela s'explique par le fait qu'à l'époque, le compte de tiers servait à renseigner le correspondant sur l'état de sa créance ou de sa dette. Il fallait donc que le correspondant comprenne aisément sa situation. En lui signifiant son doit et son avoir, on lui évitait de devoir prendre le point de vue de l'entreprise.

L'introduction des comptes de tiers eut un impact important. Il fallut cependant attendre la fin du XIII ${ }^{\mathrm{e}}$ siècle pour que des liens se tissent entre le compte de caisse traditionnel et les nouveaux comptes de tiers. Jusqu'ici, le remboursement d'un prêt donnait lieu à deux opérations distinctes et sans relation: la première dans le compte de caisse, la seconde dans le compte du débiteur. C'est à Gênes que l'on eut l'idée de faire jouer en même temps les deux comptes grâce à une seule écriture, 
mais une écriture double. Ainsi, lorsque le débiteur X rembourse CHF 500 sur la dette de CHF 1000 qu'il avait contractée, le comptable inscrit CHF 500 au doit du compte de caisse (en augmentation de l'encaisse initiale) et CHF 500 à l'avoir du compte du débiteur X (en réduction de la dette). Pour matérialiser le lien entre les deux comptes, on indique dans chaque compte quel est l'autre compte touché (tableau 4). Les liens entre les comptes sont habituellement exprimés par la préposition «à». Dans notre cas, l'opération s'énonce de la manière suivante "Caisse à Débiteur X». Notons que le compte dont le doit est touché est toujours mentionné en première position. Le principe de lecture de gauche à droite est respecté, même dans les comptes.

Tableau 4 Le débiteur rembourse sa dette: deux comptes sont touchés. Mouvement des comptes Caisse et Débiteur X, en CHF.

\begin{tabular}{llr|l}
\hline & \multicolumn{2}{c}{ Caisse } \\
\hline & & Doit & Avoir \\
\hline 17 avril & En caisse & 7000 & \\
20 avril & Débiteur X & 500 &
\end{tabular}

\begin{tabular}{lrr|lll}
\hline \multicolumn{5}{c}{ Débiteur $X$} \\
\hline 17 avril & Solde dû & Avoir & \\
& 1000 & 500 & Caisse & 20 avril
\end{tabular}

Pour compléter l'exemple, imaginons dans le tableau 5 que l'entreprise rembourse CHF 1000 à son créancier Y dont l'avoir s'élevait à CHF 8000. Le libellé de l'opération sera «Créancier Y à Caisse» pour CHF 1000. Le niveau de la dette de l'entreprise (la créance de $\mathrm{Y}$ ) et celui de l'encaisse se réduisent, tous deux, de CHF 1000.

Tableau 5 L'entreprise rembourse son créancier.

Mouvement des comptes Créancier Y et Caisse, en CHF.

\begin{tabular}{|c|c|c|c|c|c|}
\hline \multicolumn{6}{|c|}{ Créancier Y } \\
\hline & & Doit & Avoir & & \\
\hline 22 avril & Caisse & 1000 & 8000 & Solde & 20 avril \\
\hline \multicolumn{6}{|c|}{ Caisse } \\
\hline & & Doit & Avoir & & \\
\hline 20 avril & En caiss & 7500 & 1000 & Créan & 22 avril \\
\hline
\end{tabular}


Ce procédé donne naissance à l'une des propriétés fondamentales de la comptabilité en partie double: Ne jamais porter une somme au doit d'un compte, sans porter une somme égale à l'avoir d'un autre compte, et inversement! C'est le principe de la double écriture de signe contraire ${ }^{36}$.

\section{Généralisation des comptes de patrimoine}

Le compte de tiers représente, nous l'avons mentionné, un compte de créance ou un compte de dette. Il n'enregistre donc pas les entrées et les sorties de caisse, mais renseigne sur l'état de la fortune de l'entreprise. Il est un compte de patrimoine $\boldsymbol{e}^{37}$.

Cette multiplication des comptes a incité les comptables à tenir des comptes pour tous les biens de l'entreprise, comme les immeubles ou les stocks. On parle ici de comptes de valeurs. Les comptes de tiers et les comptes de valeurs offrent une description comptable exhaustive du patrimoine.

Il fallait assujettir cette nouvelle catégorie de comptes à la règle antérieure de l'écriture double. Un problème s'est alors posé: les comptables ont constaté que la valeur du patrimoine variait selon les circonstances, conduisant ainsi à des aberrations, comme le montre l'exemple suivant.

Retrouvons les Thénardier, dans leur auberge cette fois. Ils achètent un tonneau de vin pour un montant de CHF 5000 et le revendent pour une somme de CHF 6000, soit avec un bénéfice de CHF 1000. En introduisant un compte de patrimoine «Vin » qui renseigne sur l'état du stock (ce qu'il y a en cave), le libellé de l'opération d'achat est clairement «Vin à Caisse» pour CHF 5000. Le problème surgit avec la seconde opération. Physiquement, le compte Vin devrait indiquer que le vin a été vendu en totalité; il devrait donc pouvoir être crédité de CHF 5000. Or la valeur de la vente est CHF 6000. Si l'on enregistre ce montant, le doit et l'avoir comportent deux sommes différentes, qui se réfèrent pourtant à un même tonneau de vin, et qui empêchent qu'on le solde. On se trouve donc confronté à une impasse lorsque l'on essaie de plier l'ensemble des comptes de patrimoine à la règle générale de la double écriture. En utilisant le seul compte de caisse de la comptabilité simple, il n'y avait pas de problème: contrairement au vin, la monnaie ne change pas de prix entre l'achat du vin et sa vente. Il reste donc bien CHF 1000 en caisse. Cependant, le vin lui change de prix; c'est ce changement de prix qui doit permettre aux Thénardier de «gagner leur vie». Pour que la comptabilité reflète bien la réalité, il ne faut pas sortir CHF 6000 du compte Vin, mais CHF 5000. Il y a alors un déséquilibre entre l'écriture de caisse et celle de magasin (ou de cave).

36 On attribue au religieux franciscain et mathématicien Luca Bartolomes Pacioli, dit Luca di Borgo (1445-1517), d'avoir diffusé le principe de l'écriture double, connu depuis sous le nom de «comptabilité en partie double».

37 Notons que le compte Caisse est également un compte de patrimoine, puisque l'encaisse est l'un des éléments de la fortune d'une entreprise. 


\section{Création du compte de Pertes et profits}

Il fallait trouver une solution pour marier l'ensemble des comptes de patrimoine (tiers et valeurs) en respectant la règle de l'écriture double. Un comptable dont on ignore encore le nom n'a pas hésité à créer un compte spécial dont le rôle était de «mettre les autres comptes au diapason». Ce nouveau compte devait absorber les différences. Dans notre exemple, on ne peut sortir du compte Vin davantage que le montant porté au doit, soit CHF 5000. Il faut donc porter ailleurs la différence de CHF 1000 si l'on entend respecter la règle de l'égalité des débits et des crédits. Ce montant sera enregistré à l'avoir du compte spécial, de même que toutes les différences analogues qui se manifesteront.

À l'évidence, ce compte n'est pas un compte de patrimoine. Sa nature économique apparaît lorsque l'on réfléchit à la façon dont il est alimenté. Il n'enregistre pas l'achat ou la vente de patrimoine, mais il absorbe par construction toutes les différences qui ne peuvent être imputées aux comptes de patrimoine. Il est donc un compte de variations de valeurs du patrimoine. Ce compte, qui n'est autre que le compte de Pertes et profits, est un document fondamental dans la gestion de l'entreprise, souvent abrégé $\boldsymbol{P} \boldsymbol{P}$.

On peut vérifier cette importance dans l'exemple - complet - des Thénardier (tableau 6): (1) l'achat pour CHF 5000 (Vin à Caisse), (2) la vente pour CHF 6000 (Caisse) dont 5000 à l'avoir du compte Vin et 1000 de bénéfice (avoir de Pertes et profits). La règle de la double écriture est respectée, puisque le même montant a été enregistré au doit et à l'avoir (tous comptes confondus).

- Le compte de Pertes et profits - ou PP - joue donc dès que l'entreprise enregistre une recette qui n'a pas pour contrepartie une réduction correspondante de la valeur du patrimoine.

- Il joue également si l'entreprise effectue une dépense qui ne conduit pas à une augmentation du patrimoine; c'est le cas lorsque l'entreprise verse les salaires de son personnel ou paie ses impôts.

Tableau 6 Le compte de Pertes et profits enregistre la variation de valeur. Relations entre les comptes Vin, Caisse et Pertes et profits, en CHF.

\begin{tabular}{|c|c|c|c|c|c|}
\hline \multicolumn{6}{|c|}{ Vin } \\
\hline & & Doit & Avoir & & \\
\hline (1) & Caisse & 5000 & 5000 & Caisse & (2) \\
\hline \multicolumn{6}{|c|}{ Caisse } \\
\hline & & Doit & Avoir & & \\
\hline (2) & $\begin{array}{l}\text { Solde } \\
\text { Vin \& PP }\end{array}$ & $\begin{array}{l}6500 \\
6000\end{array}$ & 5000 & Caisse & (1) \\
\hline \multicolumn{6}{|c|}{ Pertes et profits } \\
\hline & & Doit & Avoir & & \\
\hline & & & 1000 & Caisse & (2) \\
\hline
\end{tabular}


Une remarque sémantique s'impose ici: le libellé du compte PP s'articule comme le contenu des colonnes, les pertes à gauche au débit, les profits à droite au crédit. Le principe de l'écriture double, partant d'un libellé de colonnes imposé par les relations avec les correspondants, permet de retrouver le point de vue de l'entreprise lorsqu'il s'agit des pertes et des profits de cette dernière.

L'apparition historique d'un compte de Pertes et profits date de 1340, toujours à Gênes. Il fallut cependant attendre la découverte de l'imprimerie pour que la nouvelle technique comptable soit largement diffusée.

\section{Le Grand livre et le journal}

Depuis la Renaissance, la technique comptable s'est encore perfectionnée. Un autre document dont nous n'avons pas encore véritablement parlé jusqu'ici est apparu à côté des comptes proprement dits ou comptes du Grand livre : c'est le journal.

On qualifie les comptes de patrimoine et celui de Pertes et profits de comptes du Grand livre parce qu'ils étaient tenus sur un seul registre. Chaque compte enregistre les opérations relatives à une valeur donnée: Caisse, Débiteur X, Créancier Y, Vin, Pertes et profits, ou autres. Le Grand livre classe donc les valeurs par nature.

Cette approche se distingue totalement de l'enregistrement chronologique des opérations du couple Thénardier. Le Grand livre est inutilisable lorsqu'il s'agit de retrouver une écriture mal classée ou dont on ne connaît que la date.

Cette lacune a rendu nécessaire un relevé général des écritures par ordre chronologique en plus du Grand livre. Ce document, qui recense par date les opérations, c'est le journal (tableau 7). Il enregistre tous les mouvements du Grand livre sans classement par nature. Chaque enregistrement - ou article - mentionne la date, un descriptif (par exemple «achat de vin»), le compte dont le doit joue (Vin) suivi de la préposition «à» et du compte dont l'avoir joue (Caisse), et enfin la(les) somme(s). En principe, les montants sont indiqués dans deux colonnes distinctes, une pour le doit, l'autre pour l'avoir; cela permet une vérification arithmétique des balances. Par convention, on regroupe sous le libellé «Suivants» tous les comptes dont le doit - ou l'avoir - joue au cours d'une même écriture; puis la rubrique «Suivants» est détaillée en mentionnant les comptes dont elle fait la synthèse; cela évite de passer deux articles ou davantage pour résumer une seule opération.

Tableau 7 Quelques articles d'un journal.

Achat et vente de vin.

\begin{tabular}{|c|c|c|c|c|c|c|}
\hline Date & Libellé & & & & Doit & Avoir \\
\hline 20 avril & Achat de vin & Vin & à & Caisse & 5000 & 5000 \\
\hline \multirow[t]{3}{*}{25 avril } & Vente de vin & Caisse & aux & Suivants & 6000 & \\
\hline & & & & Vin & & 5000 \\
\hline & & & & PP & & 1000 \\
\hline 30 avril & Balance & & & & 11000 & 11000 \\
\hline
\end{tabular}




\section{Le bilan}

Nous avons vu que l'essor de la comptabilité en parties doubles repose sur la mission donnée au comptable de tenir les comptes de tiers - débiteurs ou créanciers - et de facto de tenir des prévisions de recettes et de dépenses. Il s'agit là d'une tâche de nature budgétaire. Pour mieux la remplir, les comptables ont synthétisé les montants devant être reçus des débiteurs et les montants devant être payés aux créanciers à l'intérieur d'un même document: le bilan.

Le solde des comptes de patrimoine (comptes de tiers et comptes de valeurs) donne par définition la valeur des avoirs (à ne pas confondre avec avoir ou crédit) et des dettes de l'entreprise. À la clôture de l'exercice comptable (généralement à la fin de l'année civile), il suffit de reprendre la liste des soldes - ou balances - de chaque compte et d'y ajouter le solde du compte de Pertes et profits. On trouve alors sur deux colonnes: d'un côté, l'état des avoirs, c'est l'actif; de l'autre côté, les dettes, c'est le passif (tableau 8). La comptabilité fournit ainsi un moyen de dresser les budgets pour l'exercice suivant. Les deux colonnes d'un tel budget furent appelées Bilan, par déformation du mot italien bilancia signifiant balance, lui-même dérivé du latin bilanx (double plateau).

\section{Tableau 8 On reporte au bilan les soldes des comptes de patrimoine et de PP. Bilan au 31 décembre 1820, en CHF.}

\begin{tabular}{|c|c|c|c|}
\hline \multicolumn{4}{|c|}{ Bilan } \\
\hline & Actif & Passif & \\
\hline Caisse & 7500 & 7000 & Créancier Y \\
\hline Débiteur X & 500 & 1000 & PP \\
\hline \multirow[t]{2}{*}{$\operatorname{Vin}^{\mathrm{a}}$} & 0 & & \\
\hline & 8000 & 8000 & \\
\hline
\end{tabular}

Une remarque fondamentale s'impose ici. Nous avons déjà abordé un premier principe de la comptabilité en partie double, celui de la double écriture. Ici, un second principe apparait, celui de la double détermination du résultat. En effet, le résultat de l'exercice - perte ou bénéfice - correspond non seulement au solde du compte de Pertes et profits, mais aussi à la différence entre l'actif et le passif du bilan. Si le total de l'actif est supérieur à celui du passif, l'exercice se solde par un bénéfice; inversement, un passif supérieur à l'actif traduit une perte.

La tenue des comptes de patrimoine et de PP ne suffit pas encore à établir un bilan complet. Outre ces dettes ou ces créances connues avec précision, donc certaines, l'entreprise est confrontée à d'autres éléments moins solides. Imaginons qu'un procès soit en cours; si elle perd le procès, l'entreprise devra s'acquitter d'une certaine somme; si elle le gagne, elle sera créancière d'un montant peut-être important. D'abord d'une portée limitée, ces dettes et créances incertaines ont pris de l'importance dès 1800 . 
Le développement du secteur des assurances amena actuaires et mathématiciens à s'interroger sur les aspects comptables de ces éléments incertains.

On a donc cherché à compléter l'information comptable, jusqu'ici rétrospective, avec des éléments prospectifs afin de parvenir à un bilan complet qui tienne compte de ces incertitudes. Des comptes de nature «budgétaire» ont été instaurés. Ces comptes peuvent être classés en deux catégories (tableau 9):

- Les provisions tirent leur définition de la notion de risque. À l'actif du bilan figure une créance de CHF 500 sur X; or on peut craindre que X soit insolvable et ne puisse rembourser sa dette en totalité. Pour faire face à ce risque, on inscrira au passif une somme, par exemple $\mathrm{CHF} 300$, qui ramènera la valeur nette de la créance à CHF 200. Il s'agit en fait d'une provision pour débiteurs douteux - ou d'un compte «Provision pour débiteurs douteux». Ces CHF 300 constituent une perte anticipée par l'entreprise. Cette perte doit donc être portée au doit du compte PP. L'article de journal qui alimente la provision est le suivant: «PP à Provision sur débiteurs douteux: $300 »$.

L'entreprise en procès avec un de ses créanciers qui lui réclame CHF 7500 alors qu'elle estime ne lui devoir que CHF 7000 constituera également une provision. Elle portera au passif la dette qu'elle considère certaine (7000) et créera pour la part incertaine (500) une provision. Cette provision est elle aussi alimentée par débit du compte PP.

En débitant de manière «anticipée» le compte de Pertes et profits, la dépense - ou l'absence de recettes - future est mise à la charge de l'exercice qui a fait naître la probabilité de dette - ou le risque sur débiteurs.

- Les réserves s'inscrivent dans la logique à long terme de maintien et de développement des activités. Le(s) propriétaire(s) peut(vent) souhaiter assurer à l'entreprise une marge de sécurité, par exemple pour faire face aux aléas de la conjoncture. Dans ce cas, ils pourront renoncer à prélever leur bénéfice et décider qu'une réserve soit créée et alimentée par les bénéfices. Notre bilan imaginaire montrait un bénéfice de CHF 1000. À la suite de la création des deux provisions ci-dessus (CHF 300 et 500), le bénéfice ne se monte plus qu’à CHF 200. Une réserve est créée pour ce montant: «PP à Réserve: 200 ».

Tableau 9 Les provisions et les réserves figurent aussi au bilan.

Bilan au 31 décembre 1820.

\begin{tabular}{lr|rl}
\hline & \multicolumn{3}{c}{ Bilan } \\
\hline & Actif & Passif & \\
\hline Caisse & 7500 & 7000 & Créancier Y \\
Débiteur X & 500 & 300 & Provision pour débiteurs douteux \\
& & 500 & Provision pour créances douteuses \\
& & 200 & Réserve \\
\cline { 2 - 3 } & 8000 & 8000 & \\
\hline
\end{tabular}


Après l'introduction des provisions et des réserves, le système comptable ne connâ̂tra plus de changement d'ampleur comparable. Certes, la comptabilité a continué d'évoluer, mais elle n'a plus subi de révolution comme celle du passage à la partie double. L'étape suivante conduit à s'intéresser à la comptabilité telle qu'elle est appliquée aujourd'hui dans l'entreprise.

\subsection{Détour par la comptabilité de l'entreprise}

Le chapitre précédent a montré comment la technique comptable s'est peu à peu perfectionnée. Dans cette section, nous approfondissons la notion de patrimoine et de résultat; nous décrivons le fonctionnement des comptes pour aboutir à la détermination du résultat; nous analysons également le bilan et sa structure.

\subsubsection{Représentation schématique}

En schématisant, on peut imaginer le patrimoine de l'entreprise comme un réservoir qui a des échanges de valeurs avec l'extérieur. La différence entre les flux qui entrent et ceux qui sortent influence la valeur du patrimoine. On parle de produits ou de revenus pour les flux de valeurs qui entrent et de charges pour les flux qui sortent (figure 6).

Figure 6 L'entreprise: un patrimoine et des échanges.

Représentation schématique.

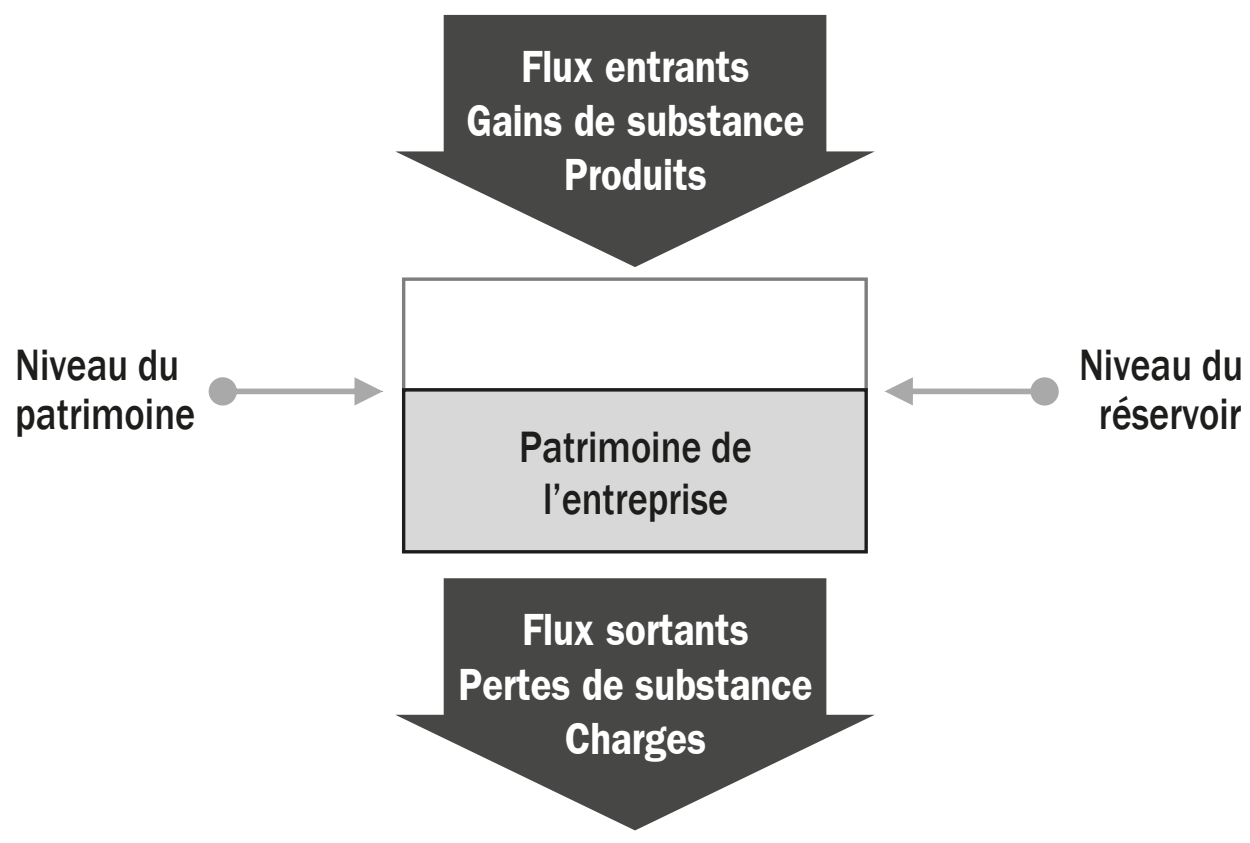


Ces flux peuvent être de l'argent, des marchandises, des services, etc. Bien que de natures différentes, tous peuvent être exprimés en unités monétaires. Ce mode de mesure présente une caractéristique fâcheuse par rapport à d'autres unités comme le litre, le mètre ou le kilogramme: il varie dans le temps et très souvent dans le sens d'une dépréciation - on pourrait parler «d'évaporation » - du patrimoine, en particulier sous l'effet de l'inflation.

Des flux peuvent aussi se produire à l'intérieur du réservoir sous forme de variation dans la composition du patrimoine. Par exemple, l'achat d'un immeuble provoque une augmentation du poste «Immeubles» à l'actif du bilan tandis que, toujours à l'actif, le compte «Banque» s'en trouve réduit d'autant.

Pour inventorier les flux et les éléments du patrimoine, la comptabilité recourt à des comptes. Le compte est traditionnellement un tableau; il prend la forme d'un fichier lorsque la comptabilité est informatisée. On y enregistre les entrées et les sorties de valeurs relatives à un même objet. Le compte est identifié par un nom - ou intitulé - et par un numéro. Ce numéro est capital pour le traitement informatique et obéit souvent aux règles d'un plan comptable (nous en reparlerons).

L'objet recensé par le compte peut être un élément du patrimoine:

- Un tiers, un correspondant avec lequel l'entreprise a des échanges: un fournisseur, un client, une banque, l'État. Le solde des comptes de tiers indique soit une créance, soit une dette de l'entreprise.

- Les comptes dit de valeurs sont d'autres comptes de patrimoine: ils enregistrent, par exemple, les opérations relatives aux immeubles, aux machines, aux stocks.

Le niveau du réservoir symbolise le total des comptes de patrimoine.

Certains comptes ne "vivent» que par l'activité et par la gestion de l'entreprise.

- Les charges de l'entreprise sont aussi un objet de compte, que ce soit des achats, des frais de personnel, des frais généraux. Ces flux de valeurs représentent des pertes de substance. S'ils ne sont pas compensés par des flux entrants équivalents, le niveau du réservoir diminue. Remarquons que ces comptes résultent d'une subdivision, d'un éclatement du doit du compte de Pertes et profits.

- Certains comptes recensent les produits ou les revenus de l'entreprise: ventes de produits, revenus de titres, etc. Ces flux de valeurs sont des gains de substance. Il s'agit aussi de subdivisions du compte PP, mais de l'avoir (profits) cette fois.

Une entreprise qui ne «vivrait» pas n'aurait pas besoin de ces comptes; c'est pourquoi on les qualifie de comptes de gestion.

La règle de l'écriture double et la distinction entre les comptes de gestion et de patrimoine permettent de différencier les opérations qui modifient la valeur de l'entreprise de celles qui n'impliquent pas de changement de la valeur du patrimoine. Une réorganisation du patrimoine n'implique que des comptes de patrimoine; la valeur globale de l'entreprise ne change pas; les mouvements entre les comptes 
n'entraînent aucune variation du résultat. Cela se produit par exemple lorsqu'un client règle sa dette: on transforme un actif (créance) en un autre actif (encaisse) ${ }^{38}$.

Les flux influencent le résultat et, par voie de conséquence, le niveau du patrimoine. Ils sont enregistrés dans les comptes de gestion (comptabilisation du flux) et ont une contrepartie dans les comptes de patrimoine (comptabilisation du changement de niveau du réservoir). À l'intérieur de ces opérations, on peut distinguer les opérations externes et les opérations internes. Les premières sont celles qui reflètent les échanges de l'entreprise avec d'autres agents économiques. C'est le cas des ventes aux clients (débiteurs à ventes) ou des achats de matières à des fournisseurs (achats à créanciers). Les opérations internes ne concernent aucun autre agent économique que l'entreprise. Elles correspondent à des corrections d'évaluation d'éléments du patrimoine. Nous avons déjà parlé des provisions pour faire face au risque (section 3.1.2). Il convient d'y ajouter les amortissements qui constatent la dépréciation de certains actifs sous l'effet de l'usure et de l'obsolescence (section 3.2.2).

\subsubsection{La vie des comptes}

Le solde des comptes de patrimoine est reporté, celui des comptes de gestion est viré

La période comptable - ou exercice - correspond généralement à une période d'un an, du $1^{\text {er }}$ janvier au 31 décembre. La parabole hydraulique de la figure 6 prend alors tout son sens comptable.

L'entreprise débute chaque exercice sur la base de l'état du patrimoine de l'exercice précédent (niveau initial du réservoir). L’objet recensé par chaque compte de patrimoine existe: c'est la caisse, un immeuble ou une créance. Par conséquent, le solde de l'exercice précédent est reporté à nouveau dans le compte du nouvel exercice. La créance de Y d'un montant de CHF 7000 ne s'éteint pas avec l'exercice, elle se perpétue sur l'exercice suivant, comme en témoigne le compte Créancier Y dans le tableau 10.

Tableau 10 Les soldes des comptes de patrimoine sont reportés à nouveau.

Bouclement et ouverture du compte Créancier Y.

\begin{tabular}{|c|c|c|c|c|c|}
\hline \multicolumn{6}{|c|}{ Créancier Y } \\
\hline & & Doit & Avoir & & \\
\hline 22 avril & Caisse & 1000 & 8000 & Solde & 20 avril \\
\hline \multirow[t]{3}{*}{31 déc. } & Solde pour balance & 7000 & & & \\
\hline & & 8000 & 8000 & & \\
\hline & & & 7000 & Solde à nouveau ${ }^{a}$ & $1^{\mathrm{er}}$ jan. \\
\hline
\end{tabular}

${ }^{\text {a }}$ Ce solde figurait au passif du bilan de clôture de l'année (cf. tableau 8 et tableau 9). Notons que la situation peut être plus compliquée. Pensons au débiteur qui escompte le montant de sa facture.
L'escompte correspond à une perte de substance qui doit être enregistrée dans un compte de charges. On s'écarte dès lors d'un simple réaménagement patrimonial. 
Par contre, au $1^{\text {er }}$ janvier, aucun flux n'a encore eu lieu; le paiement des salaires, les achats de matières premières auront lieu pendant l'exercice à venir. Pas de flux, pas de montant. Les comptes de gestion qui enregistrent les flux (ventes, salaires, achat de matières, etc.) sont remis à zéro à la clôture à l'instar du compte de Pertes et profits dont ils sont issus. Pour y parvenir et respecter la règle de l'écriture double, tous les comptes de gestion sont virés à PP. Le solde de PP (bénéfice ou perte) est ensuite viré au Bilan (tableau 11). Nous verrons comment ces opérations s'enchaînent (tableau 12). Il nous faut avant cela parler de l'influence du niveau du stock sur le résultat.

Tableau 11 Les comptes de gestion sont virés, y compris le PP. Bouclement et virement du compte de Pertes et profits, en CHF.

\begin{tabular}{|c|c|c|c|}
\hline \multicolumn{4}{|c|}{ Pertes et profits } \\
\hline & Doit & Avoir & \\
\hline \multirow[t]{2}{*}{31 déc. } & 1000 & 1000 & Caisse $^{a}$ \\
\hline & 1000 & 1000 & \\
\hline
\end{tabular}

\section{Influence du stock et rôle de l'inventaire}

Dans l'exemple des Thénardier (section 3.1.2), nous faisions l'hypothèse que le même tonneau de vin était acheté et vendu d'un seul tenant. En réalité, le stock est constitué par achats successifs. Les prix varient immanquablement d'un achat à l'autre. Le problème se pose alors de connaître le prix d'achat du vin vendu, dès lors que le vin appartient à des lots achetés à des périodes différentes, est acquis auprès de fournisseurs différents, etc. Procéder comme nous l'avons décrit précédemment obligerait le comptable à calculer un prix qui reflète le prix non pas d'un lot, mais de tous les lots auxquels appartenait le vin vendu: un travail long et fastidieux.

Ce problème a été résolu de manière expéditive. Le comptable ne met à jour les comptes de stock qu'une fois par exercice et cela à la fin de l'exercice, plutôt que de tenir ces comptes à jour à chaque vente. L'achat figure au crédit du compte Caisse comme avant. La contrepartie n'est toutefois plus le compte Stock de vin, mais le débit d'un compte "Achat de vin». Ce compte est un compte de gestion issu du compte de Pertes et profits. Comme ce compte enregistre toutes les charges relatives aux achats de vin, on le classe dans la catégorie des comptes de charges. Au moment de la vente, on porte le montant de la transaction au doit de Caisse (pas de changement) et à l'avoir d'un compte "Vente de vin». Ce compte résulte lui aussi de l'éclatement de PP, mais il enregistre tous les produits. Il appartient donc à une catégorie de comptes appelée comptes de produits. En fin d'exercice, le résultat sera l'excédent des produits sur les charges (ou inversément). Ce résultat doit 
être corrigé pour tenir compte de l'évolution du stock. On peut en effet imaginer qu'aucun achat n'a été effectué pendant l'exercice, mais que l'entreprise a vécu "sur ses réserves» - en consommant ses stocks. Le résultat devra donc tenir compte de cette réduction du niveau des stocks et être revu à la baisse. Dans l'hypothèse contraire d'une entreprise qui achète davantage qu'elle ne vend, les stocks, donc le patrimoine, s'accroissent. Le résultat devra en conséquence être revu à la hausse. En résumé, une augmentation des stocks doit être considérée comme un produit, une diminution comme une charge.

Tableau 12 Procédure de bouclement des comptes: un cas d'école.

L'exemple de l'auberge des Thénardier.

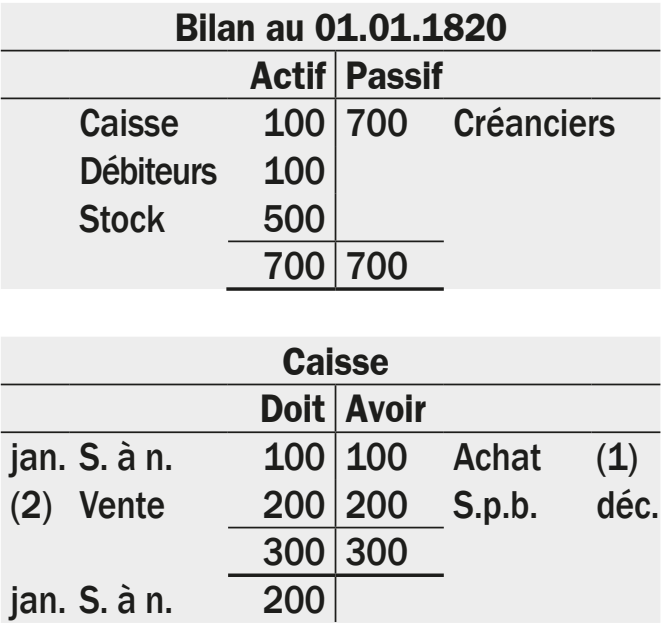

\begin{tabular}{lr|ll}
\multicolumn{3}{r|}{ Bilan au 31.12.1820 } \\
\hline & Actif & Passif \\
\hline Caisse & 200 & 900 Créanciers \\
Débiteurs & 700 & 600 PP \\
Stock & 600 & \\
\cline { 2 - 4 } & 1500 & 1500 \\
\hline
\end{tabular}

\begin{tabular}{ll|l|l}
\multicolumn{4}{c}{ Débiteur } \\
\hline & Doit & Avoir \\
\hline jan. S. à n. & 100 & & \\
(2) Vente & 600 & 700 & S.p.b. déc. \\
\cline { 2 - 3 } & & 700 & 700 \\
jan. S. à n. & 700 &
\end{tabular}

\begin{tabular}{lr|r|}
\multicolumn{3}{c}{ Stock de vin } \\
\hline & Doit & Avoir \\
\hline jan. S. à n. & 500 & 600 \\
(5) Résultat Inventaire déc. & 100 & \\
\cline { 2 - 3 } & 600 & 600 \\
\cline { 2 - 3 } jan. S. à n. & 600 & \multicolumn{1}{|c}{}
\end{tabular}

\begin{tabular}{|l|l|l|}
\multicolumn{2}{c}{ Achat de vin } \\
\hline & Doit & Avoir \\
\hline (1) Suivants & 300 & 300 \\
\cline { 2 - 3 } & 300 & 300 \\
& & 300 \\
& &
\end{tabular}

\begin{tabular}{|c|c|c|c|}
\hline \multicolumn{4}{|c|}{ Créancier } \\
\hline & Doit & Avoir & \\
\hline \multirow[t]{3}{*}{ déc. S.p.b. } & 900 & $\begin{array}{l}700 \text { S. à n. } \\
200 \text { Achat }\end{array}$ & $\begin{array}{l}\text { jan, } \\
\text { (1) }\end{array}$ \\
\hline & 900 & 900 & \\
\hline & & $\overline{900}$ S. à n. & jan. \\
\hline
\end{tabular}

\section{Résultat sur vin}

\begin{tabular}{lll|lll}
\hline & Doit & Avoir & & \\
\hline (3) Achat & 300 & 800 & Vente & $(4)$ \\
(6) PP & 600 & 100 & Stock & (5) \\
\cline { 2 - 3 } & & 900 & 900 & & \\
\cline { 2 - 3 } & & \multicolumn{3}{l}{} &
\end{tabular}

\begin{tabular}{|c|c|c|}
\hline \multicolumn{3}{|c|}{ Vente de vin } \\
\hline & Doit & Avoir \\
\hline \multirow[t]{2}{*}{ (4) Résultat } & 800 & 800 Suivants (2) \\
\hline & 800 & 800 \\
\hline
\end{tabular}

Pertes et profits

\begin{tabular}{ll|l}
\hline & Doit & Avoir \\
\hline (7) Bilan & 600 & 600 \\
& 600 & 600 \\
\cline { 2 - 3 } & & \multicolumn{2}{l}{} \\
& & \multicolumn{2}{l}{}
\end{tabular}


Pour déterminer comment a évolué le niveau du stock, il faut procéder à un inventaire. La comparaison de deux inventaires, en début et en fin d'exercice, indique si le stock a enflé ou s'il s'est contracté. Le rapprochement de ces trois éléments clés que sont les charges d'achats, les produits de ventes et l'évolution du stock s'effectue dans un nouveau compte, le compte de résultats. Il n'est qu'une étape intermédiaire pour parvenir à constituer le compte de Pertes et profits.

Nous allons maintenant envisager un cas d'école qui montre l'enchaînement des opérations de clôture. Nous retrouvons ici l'auberge des Thénardier. Le tableau 12 présente la situation initiale telle qu'elle figure au bilan au $1^{\text {er }}$ janvier, soit une encaisse de CHF 100, des débiteurs pour CHF 100, un stock de vin valant CHF 500 et une dette de CHF 700. Au cours de l'exercice, les Thénardier achètent du vin pour CHF 300, dont 100 au comptant et le reste à terme (1). Ils vendent du vin pour CHF 800, dont 200 au comptant et le reste à terme (2). À la clôture, l'inventaire montre que le stock de vin vaut CHF 600.

$\mathrm{Au} 1^{\text {er }}$ janvier, tous les comptes actifs et passifs figurant au bilan sont ouverts à nouveau (solde à nouveau, s. à n.). Au cours de l'exercice, les opérations d'achat et de vente sont comptabilisées suivant les articles du journal (tableau 13). À la clôture, la valeur d'inventaire du stock final (CHF 600) est portée à l'avoir du compte Stock de vin, en regard et en comparaison du stock initial (CHF 500). Notons qu'il s'agit d'une donnée exogène à la comptabilité, par conséquent cette écriture n'a pas de contrepartie: il s'agit d'une exception à la règle de la double écriture, c'est la seule. La différence du compte Stock (100) est virée au compte Résultat sur vin (5), de même que le solde des comptes Achat (3) et Vente de vin (4). Le solde du compte Résultats est lui viré à PP (6), dont le solde est viré au bilan (7). Les soldes des comptes de patrimoine (solde pour balance, s.p.b.) sont portés au bilan.

Tableau 13 Journal de l'an 1820.

Articles relatifs aux opérations des Thénardier, en CHF.

\begin{tabular}{|c|c|c|c|c|c|c|}
\hline \multirow{2}{*}{$\frac{\text { Date }}{(1)}$} & \multicolumn{4}{|l|}{ Libellé } & \multirow{2}{*}{$\begin{array}{l}\text { Doit } \\
300\end{array}$} & \multirow{2}{*}{$\begin{array}{l}\text { Avoir } \\
100 \\
200\end{array}$} \\
\hline & Achat de vin & Achat & aux & $\begin{array}{l}\text { Suivants } \\
\text { Caisse } \\
\text { Créanciers }\end{array}$ & & \\
\hline (2) & Vente de vin & $\begin{array}{l}\text { Suivants } \\
\text { Caisse } \\
\text { Débiteurs }\end{array}$ & à & Vente & $\begin{array}{l}200 \\
600\end{array}$ & 800 \\
\hline 31 déc. & Bouclement & & & & & \\
\hline (3) & Virement du solde d'Achat de vin & Résultat & à & Achat & 300 & 300 \\
\hline (4) & Virement du solde de Vente de vin & Vente & à & Résultat & 800 & 800 \\
\hline (5) & Correction de la hausse du stock & Stock & à & Résultat & 100 & 100 \\
\hline (6) & Virement du solde de Résultat & Résultat & à & PP & 600 & 600 \\
\hline (7) & Virement du bénéfice & PP & à & Bilan & 600 & 600 \\
\hline & Balance & & & & 3500 & 3500 \\
\hline
\end{tabular}


Une remarque s'impose ici. Bien que cet exemple ne soit qu'un cas d'école, il met en ouvre les cinq séries de comptes que l'on trouve dans toute comptabilité évoluée (figure 7):

- Les deux premières séries résultent de l'éclatement du bilan: il s'agit des comptes actifs et des comptes passifs.

- Les trois autres séries sont des subdivisions du compte de Pertes et profits: il s'agit des comptes de charges et des comptes de produits, qui à la clôture transitent par des comptes de résultats pour être finalement virés à PP (lui-même étant un compte de résultats).

Figure 7 Une comptabilité, cinq séries de comptes.

Décomposition du bilan et du compte de Pertes et profits.

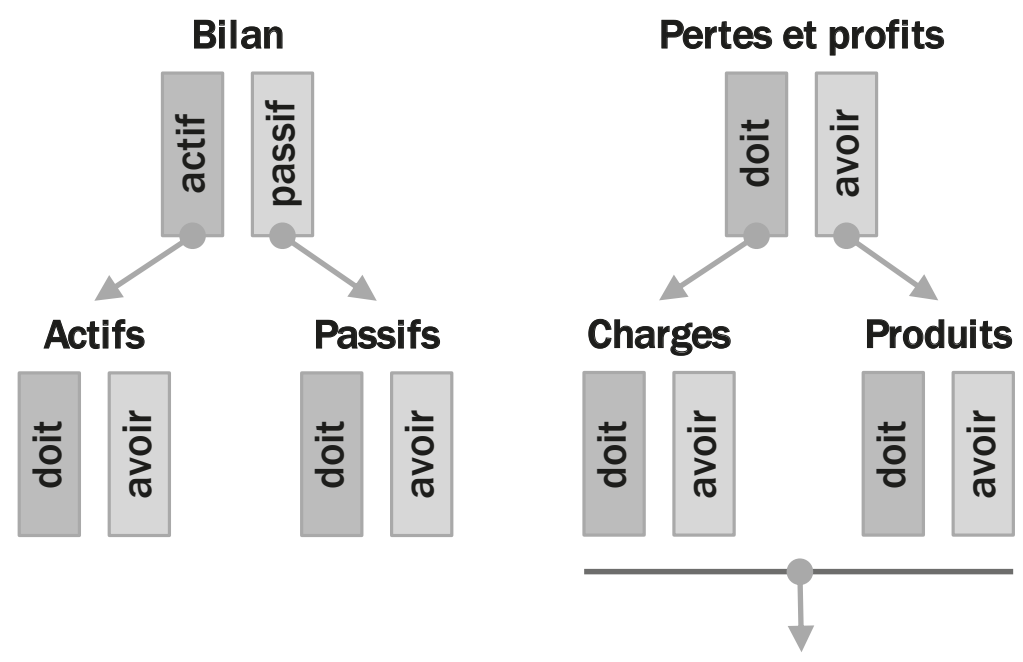

Résultats, y compris Pertes et profits

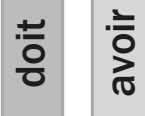

\section{Les comptes de correction}

$\mathrm{Au}$ terme de la section précédente, le résultat correspond à la différence entre les produits et les charges, compte tenu de la variation du stock. C'est le flux net entre les gains et les pertes de substance. On parle généralement de résultat brut d'exploitation.

Cette approximation serait correcte si l'équipement était indestructible et techniquement indémodable, si les débiteurs étaient toujours solvables ou si les marchandises stockées étaient toujours vendables au prix habituel. Ce n'est pas le cas en réalité; la comptabilité doit en tenir compte et corriger le résultat. 
- Nous avons déjà vu le cas des provisions. Ces comptes figurent au passif du bilan. Ils sont créés par exemple pour faire face au risque d'insolvabilité des débiteurs ou de détérioration du stock. Nous avions indiqué que ces pertes potentielles anticipées étaient portées au doit du compte de PP. Dans une comptabilité plus évoluée, elles viennent au doit d'un compte de charges qui s'intitule parfois «Dotation aux provisions». Ainsi, l'article de journal nécessaire pour alimenter une provision est du type «Dotation aux provisions à Provision pour débiteurs douteux». À la clôture, le solde du compte «Dotation... » est évidemment viré au compte de Pertes et profits, tandis que le solde du compte passif «Provision...» est reporté.

- Au cours de l'exercice, l'équipement de l'entreprise - bâtiments, machines, outillage - s'use ou devient obsolète. La valeur des comptes de patrimoine qui répertorient ces éléments diminue donc avec le temps. La comptabilité constate cette diminution en amortissant ces équipements. Cela peut se faire par un amortissement direct: l'amortissement est porté à l'avoir du compte concerné (machine, immeuble, etc.) en diminution immédiate de la valeur du patrimoine. L'amortissement peut également être indirect; dans ce cas, on crée un compte passif Amortissements alimenté selon le même schéma que les provisions. Un compte de charges «Dotation aux amortissements » grève le résultat. Lorsque l'on amortit un bien, on passe l'article «Dotation aux amortissements à Amortissements » (dans le cas de l'amortissement direct: Dotation... à Immeuble, par exemple). Cela revient à mettre à la charge de l'exercice la «consommation» de l'équipement au même titre que la consommation de matières premières ou de salaires. Les montants amortis devraient donc refléter précisément la dépréciation économique de l'équipement. Ils sont en réalité fixés selon des modalités variables. L'amortissement est par exemple linéaire sur la durée de vie de l'équipement (somme constante). Il peut aussi être dégressif (pourcentage constant appliqué à la valeur résiduelle de l'équipement). Nous y consacrons la section 3.3.5.

- La dernière catégorie de comptes qui permet de corriger le résultat de l'exercice est celle des comptes de régularisation. Ces comptes ne constatent pas, comme le font les comptes d'amortissement et les provisions, une évolution de la valeur, mais un décalage dans le temps par rapport aux limites de l'exercice. En effet, certaines charges ou certains produits correspondent à une période qui précède l'exercice ou s'étend au-delà, par exemple une facture d'électricité ou d'assurance qui «déborde» de l'exercice. Il s'agit donc de recadrer les charges et les produits afin que les comptes reflètent strictement l'exercice. On recourt pour cela à des comptes transitoires actifs qui soulagent le résultat lorsque des charges ne regardent pas l'exercice ou à des comptes transitoires passifs pour grever l'exercice si ces dépenses n'ont pas encore eu lieu. Dans le cas des primes d'assurances payées d'avance, on passe l'article suivant "Actifs transitoires à Primes d'assurances " pour l'équivalent des montants payés et qui débordent de l'exercice. Le résultat est ainsi corrigé, de même que le patrimoine de l'entreprise. 
Ces comptes actifs ou passifs transitoires figurent en effet au bilan à la fin de l'exercice, typiquement au 31 décembre de l'année concernée. Ils sont soldés au début de l'exercice suivant par l'extourne des opérations qui leur a donné naissance (par exemple «Primes d'assurance à Actifs transitoires») ${ }^{39}$. Ainsi, l'exercice qui commence supporte lui aussi les charges et les revenus qui lui incombent.

\subsubsection{Le bilan et sa structure}

Le bilan se présente sous la forme d'un inventaire, à une date donnée. Il récapitule l'ensemble des ressources (postes du passif) dont dispose l'entreprise, et la manière dont elle les a utilisées (postes de l'actif ou emplois). Il exprime donc la situation financière de l'entreprise de façon statique.

Nous avons indiqué les différentes masses qui figurent dans le bilan de l'entreprise. Il convient de compléter ces informations par d'autres, connexes, sur lesquelles le bilan reste muet. Tel est le cas des engagements reçus hors bilan (promesses de ventes, commandes fermes de clients, cautions dont l'entreprise peut bénéficier, etc.) et des engagements donnés hors bilan (promesses d'achat, privilèges de créanciers sur certains biens comme les hypothèques ou les nantissements, cautions données par l'entreprise, etc.).

\section{Les ressources}

Le passif du bilan enregistre l'ensemble des ressources dont dispose l'entreprise. Ces ressources se répartissent entre les capitaux propres et les dettes de l'entreprise. La distinction entre ces deux catégories est à la fois juridique et financière: les dettes devront être remboursées, contrairement aux capitaux propres.

- Les capitaux propres sont des ressources en permanence à la disposition de l'entreprise. Elles sont constituées par les apports des propriétaires ou des associés de l'entreprise (capital) et par la fraction du bénéfice qui reste dans l'entreprise (réserves $)^{40}$.

- Les autres ressources sont fournies par des tiers (fournisseurs, banques, etc.) pour une période plus ou moins longue. On distingue généralement les capitaux empruntés à long terme de ceux qui sont empruntés à court terme (exigibles dans moins d'un an).

\section{Les emplois}

L'actif du bilan renseigne sur les emplois économiques des capitaux. Ces emplois peuvent être durables ou temporaires.

39 Une extourne est donc une écriture qui est l'exact inverse de l'écriture initiale.

40 Certains s'étonnent de voir le capital figurer au passif du bilan. Ces personnes imaginent que le capital devrait être comme de l'argent en caisse et qu'il devrait donc être porté à l'actif. En vérité, le compte Capital est un compte de tiers qui enregistre la dette de l'entreprise envers ses fondateurs; à la différence que cette dette n'est pas exigible. 
Les emplois durables concernent les immeubles, le matériel, le mobilier, etc. Ils représentent des biens immobilisés dans l'entreprise parce qu'ils constituent des moyens d'exploitation, l'outil de travail. Les vendre perturberait l'exploitation ou la rendrait impossible. Il faut donc qu'ils restent dans l'entreprise. Ce sont les actifs immobilisés.

Les emplois temporaires ou cycliques concernent des biens qui sont constamment transformés au cours de la vie de l'entreprise: les stocks sont transformés, puis vendus, donnant naissance à des créances qui sont finalement encaissées. Ces actifs sont désignés sous le terme d'actifs circulants et comprennent:

- les valeurs d'exploitation: marchandises en stock, emballage en stock;

- les valeurs réalisables: créances sur les clients et sur les débiteurs;

- les valeurs disponibles: effets à recevoir, titres, avoirs en banque, espèces.

Au cours du cycle d'exploitation, les valeurs d'exploitation se transforment peu à peu en valeurs réalisables qui elles-mêmes deviennent des valeurs disponibles. Ces dernières permettent de régler les dettes à leur échéance.

\section{Les grandes masses}

Pour analyser le bilan, il faut regrouper les différents postes en masses aussi homogènes que possible. Puis on les classe dans un ordre décroissant de liquidité (actif) ou d'exigibilité (passif). La figure 8 indique les grandes masses les plus utilisées pour catégoriser les actifs et les passifs.

\section{Figure 8 Représentation de l'actif et du passif par grandes masses.}

Ordre décroissant de liquidité et d'exigibilité.

Actif

Actif circulant

Actif immobilisé ${ }^{a}$

\begin{tabular}{|l|}
\hline $\begin{array}{l}\text { Valeurs disponibles } \\
\text { ou réalisables }\end{array}$ \\
\hline Valeurs d'exploitation \\
\hline Valeurs immobilisées \\
\hline
\end{tabular}

\begin{tabular}{|l|}
\hline Disponible \\
\hline Réalisable \\
\hline Stocks \\
\hline Immobilisations \\
\hline
\end{tabular}

\section{Passif}

\begin{tabular}{|l|}
\hline $\begin{array}{l}\text { Capitaux étrangers } \\
\text { (ou Dettes) }\end{array}$ \\
\hline Capitaux propres \\
\hline
\end{tabular}

\begin{tabular}{|l|}
\hline Dettes à court terme \\
\hline Dettes à moyen \\
et à long terme \\
\hline Capitaux propres \\
\hline
\end{tabular}

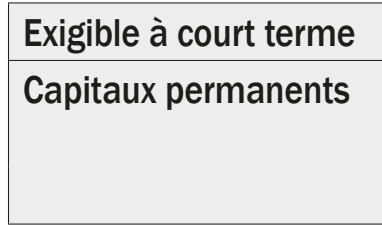

a La masse Actif immobilisé correspond aux montants nets à l'actif, c'est-à-dire après déduction des amortissements qui, en cas d'amortissement indirect, figurent dans un fonds au passif. 


\section{Le découvert}

Lorsque l'exercice se solde par un déficit, la perte est portée en diminution des réserves accumulées au cours des exercices précédents (Réserve à $\mathrm{PP}$ ). Si cette situation se répète, les réserves s'épuisent. Lorsque les réserves ont disparu, il faut se résoudre à capitaliser la perte à l'actif du bilan (ou, alternativement au passif avec un signe négatif); on parle alors de Découvert au bilan. Cette capitalisation s'effectue par l'opération suivante: Bilan à $\mathrm{PP}$, ou plus précisément Découvert à $\mathrm{PP}$ - Découvert étant un compte actif. Dès le moment où le volume du découvert dépasse celui des capitaux propres, l'entreprise se trouve en situation de surendettement, puis de faillite. En effet, le remboursement de ses créanciers ne peut plus être assuré par réalisation (liquidation) de l'actif (figure 9).

Figure 9 La capitalisation de la perte aboutit à un découvert.

Situation de faillite virtuelle.

Pertes et profits

Doit

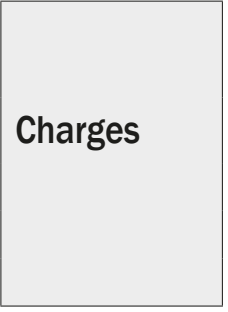

Avoir

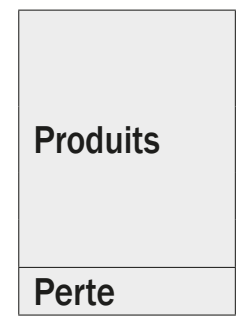

Bilan

Actif

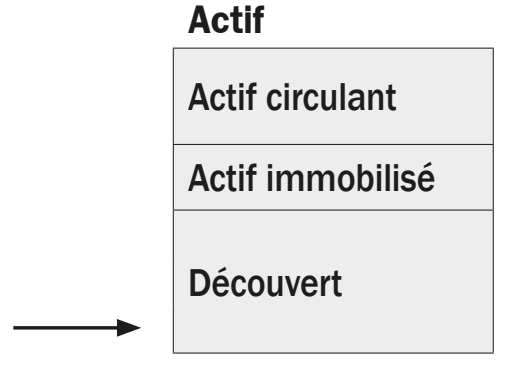

Passif

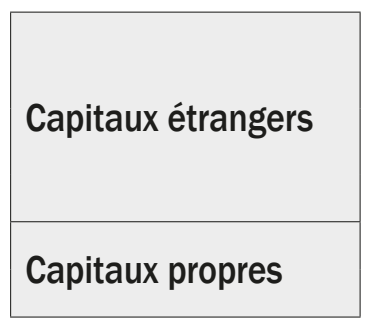

\section{Éléments de lecture et d'analyse du bilan}

Le simple examen du bilan donne une idée de la dimension de l'entreprise. Le total du bilan renseigne sur la taille de l'entreprise. Un total de quelques centaines de milliers de francs correspond au bilan d'une PME. Le bilan d'une grande entreprise s'articule en millions de francs, celui d'une multinationale de grande taille en milliards. C'est le cas de Nestlé avec un total de CHF 137,0 mrd à fin 2018. Par comparaison, à ce moment-là, le bilan de la Confédération suisse s'élevait à 167,2 mrd, celui du canton de Vaud à $8,8 \mathrm{mrd}$, celui du canton de Neuchâtel à 2,7 mrd et celui de la Ville de Lausanne à $3,2 \mathrm{mrd}^{41}$.

Il n'existe pas de norme pour juger la structure de l'actif d'une entreprise. Cette structure dépend en effet de la nature de l'activité de l'entreprise. Dans l'industrie, l'actif immobilisé est souvent important - de 30 à 90\% selon les branches; dans le commerce, il l'est beaucoup moins - souvent inférieur à $25 \%$. Par contre, la comparaison de la structure de l'actif d'une entreprise avec celle des firmes de la même branche est instructive, comme l'est l'analyse de l'évolution de la structure de l'actif sur plusieurs exercices.

41 Chiffres tirés des comptes 2018 de ces entités tels qu'ils sont mis à disposition sur leur site Internet. 
Il n'existe pas davantage de structure type pour le passif. Cependant, l'indépendance de l'entreprise face à ses créanciers est d'autant plus grande que ses capitaux propres sont importants. Dans le cas de Nestlé, les capitaux propres se montaient à CHF 58,4 mrd. Ils financent ainsi près de la moitié de l'actif.

Cette observation nous amène à parler d'une relation fondamentale entre certaines masses de l'actif et du passif. Cette relation est valable quelles que soient la taille et la branche d'activité de l'entreprise: les capitaux permanents doivent être supérieurs à l'actif immobilisé.

En effet, les immobilisations doivent être financées par des capitaux exigibles à long terme ou par des capitaux propres. Elles ne peuvent être financées de façon sûre par des crédits à court terme, susceptibles de ne pas être reconduits. Si l'on n'observe pas cette règle, la raréfaction des capitaux à court terme met l'entreprise en cessation de paiement.

La figure 10 montre cette relation. Elle montre que l'inégalité [Capitaux permanents $>$ Actif immobilisé] est équivalente à une seconde inégalité [Actif circulant $>$ Dettes à court terme].

La différence est appelée fonds de roulement. Le fonds de roulement se calcule donc de deux façons: soit Capitaux permanents-Actif immobilisé, soit Actif circulant-Dettes à court terme. Il doit toujours être positif ${ }^{42}$.

Notons que, dans le cas des collectivités publiques, on se trouve parfois confronté à une absence de capitaux propres, c'est-à-dire à un découvert du bilan suite à l'accumulation de déficits. Les actifs immobilisés doivent être alors entièrement financés par les dettes à moyen et à long terme pour éviter une impasse financière.

Figure 10 La règle fondamentale d'équilibre du financement.

Le fonds de roulement doit être positif.

\begin{tabular}{|l|l|l|}
\hline \multicolumn{1}{|l|}{ Actif } & \multicolumn{1}{c|}{ Passif } \\
& Dettes à court terme \\
\hline Actif circulant & $\begin{array}{c}\text { Fonds de } \\
\text { roulement }\end{array}$ & \\
\hline & & Capitaux permanents \\
\hline
\end{tabular}

\section{L'autofinancement}

Nous avons vu qu'il existe un lien mécanique à travers la double détermination du résultat entre le compte de Pertes et profits d'une part et le bilan d'autre part.

42 Les entreprises dont la rotation du stock est très rapide (pluriannuelle) financent parfois leurs valeurs d'exploitation par de l'exigible à court terme. C'est le cas des entreprises actives dans la grande distribution, comme les chaînes de supermarchés. On est alors en présence d'un fonds de roulement négatif. Mais c'est l'exception. 
Cette charnière entre les deux éléments est fondamentale. Pour maintenir ou étendre son activité, ou encore pour rémunérer les capitaux qu'elle utilise, l'entreprise cherche à réaliser un profit. Il importe donc de connaître comment le profit concourt au maintien ou à l'expansion de l'entreprise. En effet, plutôt que d'être, par exemple, distribué aux actionnaires sous forme de dividende, le bénéfice peut être retenu dans l'entreprise. Le profit représente alors une source de financement interne. Il s'ajoute à d'autres éléments pour constituer l'autofinancement de l'entreprise. Outre le profit non distribué, certaines charges et certaines pertes ne donnent également pas lieu à des flux de trésorerie (dépenses). Certes, ces charges et ces pertes grèvent le résultat, mais elles alimentent des fonds qui restent à la disposition de l'entreprise et concourent au maintien des ressources. Ce sont principalement:

- les dotations aux fonds d'amortissement;

- les dotations aux provisions pour dépréciation d'immobilisations;

- les dotations aux provisions pour pertes et charges à échéance lointaine (provisions pour grosse réparation à effectuer dans plusieurs années) ou à des provisions sans objet (provisions «factices»).

Le total de ces dotations et du profit non distribué constitue l'autofinancement, que l'on qualifie aussi de financement interne. Il évoque le flux net de trésorerie en direction de l'entreprise, c'est-à-dire la différence entre les recettes et les dépenses d'un exercice ${ }^{43}$.

L'autofinancement peut donc être envisagé sous deux angles distincts:

- par addition: comme nous l'avons déjà indiqué, l'autofinancement correspond à la somme du bénéfice non distribué et des dotations aux amortissements et aux provisions;

- par soustraction: il correspond à la différence entre les flux financiers entrants et sortants de l'entreprise au cours de son activité.

L'autofinancement représente l'épargne de l'entreprise (figure 11). Ces ressources internes peuvent être consacrées au financement de l'investissement ou à l'augmentation du fonds de roulement. Elles sont donc un des moteurs de la croissance de l'entreprise.

L'autofinancement présente l'avantage d'éviter à l'entreprise de s'endetter trop lourdement. Il préserve ainsi l'autonomie vis-à-vis des banques et, plus généralement, du marché des capitaux. Il permet donc à l'entreprise de bénéficier d'un processus décisionnel plus rapide, en évitant de l'exposer à des clauses limitatives, telles que des covenants $^{44}$, qui pourraient interférer avec les projets d'investissements de l'entreprise.

43 Parfois l'autofinancement, tel que nous l'avons défini, est qualifié d'autofinancement brut. On le distingue alors de l'autofinancement net. Ce dernier ne correspond qu'à la part du profit non distribuée et qui reste à la disposition de l'entreprise sous forme de report à nouveau, de réserve ou d'augmentation de capital.

44 Un covenant est une clause de sauvegarde figurant dans un contrat de prêt qui, en cas de non-respect des dispositions ou des objectifs du contrat, peut entraîner l'obligation de rembourser le prêt de manière anticipée. 
Figure 11 L'autofinancement, c'est l'épargne de l'entreprise.

Flux net de trésorerie émanant du compte de Pertes et profits.

\section{Pertes et profits}

Charges et pertes donnant lieu à des sorties de trésorerie

Dotations aux amortissements et aux provisions

Profit (solde de PP)

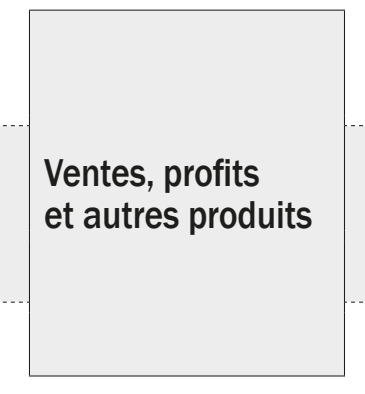

Autofinancement

Profit distribué

Toutefois, l'autofinancement comporte également des désavantages. Il accroît le risque supporté par les actionnaires par rapport à celui qui est supporté par les bailleurs de fonds externes. En compensation, les actionnaires requièrent une rémunération plus élevée. Finalement, contrairement aux intérêts payés aux bailleurs de fonds externes, les dividendes - qui représentent la rémunération des actionnaires ne sont pas fiscalement déductibles.

La structure de financement optimale d'une société doit donc se composer à la fois de fonds propres, apportés notamment par l'autofinancement, mais également de fonds étrangers. En fonction du secteur d'activité, cette structure peut se voir significativement modifiée.

\subsubsection{Plan comptable}

Le droit suisse laisse aux entreprises une grande liberté pour présenter leurs comptes. Le code des obligations fixe un certain nombre de normes relatives à la comptabilité commerciale et à la présentation des comptes. Il indique notamment les principes à suivre, la structure minimale de l'actif et du passif du bilan, la structure minimale du compte de résultat et le contenu minimal de l'annexe.

Il n'existe toutefois encore aucun plan comptable détaillé et obligatoire, à l'exception de ceux qui sont introduits spécifiquement par le législateur pour un nombre restreint de catégories d'entreprises: les banques, les négociants en valeurs mobilières, les placements collectifs de capitaux, les entreprises de chemins de fer et de transport concessionnaires.

Actuellement, la plupart des petites et moyennes entreprises utilisent le plan comptable PME proposé dès 1947 par l'Union suisse des arts et métiers (USAM). Ce plan a été modifié en partie et simplifié en 2015 afin de respecter les dispositions légales. Il n'a toutefois pas force de loi. 


\subsection{Modèle comptable des cantons et des communes suisses}

\subsubsection{Développements historiques de la comptabilité publique en Suisse et ailleurs}

En Europe, la comptabilité publique naît avec les États. Cette forme d'organisation collective se développe à partir du XIII siècle. Elle impose, sur un territoire donné, une administration militaire, judiciaire et fiscale. L'apparition et les développements de la comptabilité publique s'expliquent par des impératifs de contrôle. Une des priorités est d'assurer la perception des impôts. Cette priorité est déjà présente sous les anciens régimes dès la fin du Moyen Âge et elle survit, notamment aux révolutions anglaises ou françaises. À cette époque, pour remédier à une organisation étatique encore embryonnaire, le souverain mandate des agents privés - les trésoriers - et leur confie la mission de collecter les fonds nécessaires au financement du royaume. De par leur fonction, les trésoriers sont des personnages autant influents qu'indispensables aux souverains successifs. Ils bénéficient d'une large autonomie et, donc, de diverses possibilités de fraude: omission de recettes, retards d'enregistrement, faux emplois de fonds, pertes diverses. Assez rapidement, les souverains cherchent à mieux contrôler leurs activités pour augmenter les rentrées fiscales. Cela se traduit par de nouvelles exigences par rapport à la qualité des rapports - les comptes - que les trésoriers doivent remettre au souverain en même temps que leur caisse.

De ce point de vue, l'idée d'enregistrer les opérations d'encaissement et de décaissement en partie double est intéressante. Les marchands de l'époque connaissent ce système depuis longtemps. Ils peuvent évidemment dégager le résultat de chaque période d'exploitation. Mais, plus intéressant pour le souverain, ce système permet de s'assurer de la validité des comptes produits par les trésoriers. Les historiens comme Nikitin (2001) nous rapportent que les premières tentatives d'introduction de la partie double ont lieu en Espagne (dès 1592), dans les Provinces-Unies des Pays-Bas (1604), en Suède (1623) ou en France (entre 1716 et 1726). En France, sous le règne de Louis XVI et de Jacques Necker, son ministre des finances ${ }^{45}$, ces tentatives s'accompagnent d'un mouvement de centralisation et d'étatisation à travers la réduction du nombre de centres de collecte des impôts et à travers la subordination directe de ces centres au Trésor royal.

En matière de comptabilité, les États ont continué de tirer parti des expériences du capitalisme d'abord marchand (XVI ${ }^{e}-X{ }^{2} I^{e}$ siècles), puis industriel (dès la fin du $\mathrm{XVIII}^{\mathrm{e}}$ siècle), puis financier (dès le milieu du XIXe siècle). Peu à peu, ils définissent leurs propres normes et pratiques pour finalement aboutir à des manuels et autres

45 Jacques Necker ne fut pas la seule personnalité à avoir contribué à améliorer la gestion des finances publiques françaises au XVIII ${ }^{e}$ siècle. Toutefois, dans la mesure où cet ouvrage s'intéresse plus particulièrement à la gestion des finances publiques en Suisse, il faut rappeler que Necker est né à Genève, où il y a fait ses études et conduit une carrière bancaire et politique. Après avoir servi Louis XVI entre 1776 et 1790, avec deux interruptions, il démissionne et se retire à Coppet. 
traités de comptabilité publique (Legay 2010). Ce mouvement s'effectue toutefois de manière non concertée entre États.

En Suisse, ce mouvement conduit à une diversité intercantonale considérable. Différentes tentatives ont lieu pour harmoniser les pratiques à la fois horizontalement - entre cantons - et verticalement - entre communes, cantons et Confédération.

En 1878, puis en 1892, la Société suisse de statistique s'essaye à formuler des exigences minimales pour réduire cette diversité. Ses travaux sont marqués par l'opposition entre les partisans de la comptabilité camérale inspirée de la pratique allemande et les tenants de la comptabilité en partie double. Il faut attendre la fin des années 1940 pour que plusieurs cantons introduisent un plan comptable relativement similaire, construit autour de deux comptes : un compte ordinaire enregistrant les dépenses opérationnelles à financer de façon récurrente et un compte extraordinaire enregistrant les dépenses d'investissement à financer préalablement ou antérieurement. La Confédération, quant à elle, développe son propre système principalement fondé sur un compte financier enregistrant l'ensemble des dépenses. Pour ce qui les concerne, et dans plusieurs cantons, les communes recourent à une comptabilité dont le compte de résultats forme le noyau, se rapprochant ainsi des pratiques du secteur privé. Les trois niveaux institutionnels suivent ainsi leur propre voie jusqu'à la fin des années 1960.

Mais cette situation devient difficilement tenable compte tenu des besoins de coordination et de péréquation financière entre collectivités. En 1970, un nouveau chantier d'harmonisation comptable s'ouvre à l'instigation de la Conférence des directrices et directeurs cantonaux des finances (CDF, portrait 1) $)^{46}$. Ce chantier trouve son aboutissement en 1977 dans la publication par la Conférence d'une recommandation à l'attention des cantons et des communes. Cette recommandation est développée dans un "Manuel de comptabilité publique» (CDF 1982) ${ }^{47}$. Le modèle comptable proposé est aujourd'hui connu sous l'abréviation $\mathrm{MCH} 1$, pour Modèle Comptable Harmonisé de $1^{\text {re }}$ génération. Cette appellation reflète bien l'objectif: promouvoir un mouvement d'harmonisation en matière de présentation du budget et des comptes.

La mise en œuvre du MCH1 est assez laborieuse. En effet, il est laissé au bon vouloir de chaque canton de traduire les recommandations de la Conférence dans sa propre législation et dans la loi sur les finances des communes afin de lui conférer force obligatoire. Or la traduction n'est pas toujours fidèle au modèle. Par ailleurs, le processus est soumis au calendrier et à la faisabilité politique propre à chaque canton. Bref, il faut attendre le milieu des années 1990 pour que la quasi-totalité des cantons et des communes appliquent le $\mathrm{MCH} 1$ sous une forme ou sous une autre. Pendant ce temps, la Confédération utilise toujours principalement son modèle fondé sur son compte financier.

46 Konferenz der kantonalen Finanzdirektorinnen und Finanzdirektoren | Conferenza delle direttrici e dei direttori cantonali delle finanze | Conference of the cantonal finance ministers.

47 Handbuch des Rechnungswesens der öffentlichen Haushalte HRM1 | Manuale di contabilità degli enti pubblici MCA1 | Handbook of public sector entities accounting HAM1. 


\section{Portrait 1 Conférence des directrices et directeurs cantonaux des finances}

La Conférence des directrices et directeurs cantonaux des finances (CDF) est un organe intercantonal de liaison entre les cheffes et chefs des départements des finances des vingt-six cantons suisses. Elle vise à coordonner la politique des cantons en matière financière et fiscale dès lors qu'une coordination est dans l'intérêt commun des cantons. La CDF a également pour but de favoriser la collaboration entre cantons d'une part et entre les cantons et la Confédération d'autre part dans le domaine des finances publiques. Son rôle est finalement d'être une plate-forme d'échange d'information au service des cantons sur les questions financières de portée nationale. Ses principaux organes sont l'assemblée plénière réunissant les vingt-six responsables cantonaux des finances et le comité de sept membres élus parmi les membres de l'assemblée plénière. Cette dernière se réunit aussi souvent que nécessaire, mais au moins une fois l'an pour son assemblée ordinaire.

Source: Statuts de la Conférence des directrices et directeurs cantonaux des finances, du 20 mai 2018 (état au 29 septembre 2017).

Toutefois, il devient de plus en plus pressant pour la Confédération d'évoluer et d'abandonner sa vision camérale. Le mouvement de "gestion par enveloppe budgétaire et mandat de prestation (GMEB)» initié au cours des années 1990 dans la mouvance de ce que l'on a appelé alors la «nouvelle gestion publique» nécessite un cadre comptable davantage orienté vers une vision entrepreneuriale. Par ailleurs, des normes comptables internationales se développent pour les collectivités publiques (International Public Sector Accounting Standards, IPSAS, portrait 2). Leur objectif est de rendre plus facile la compréhension des états financiers, en particulier pour les bailleurs de fonds (des Robert et Colibert 2008; Müller-Marqués Berger 2018). Mais ces normes obligent à passer à une comptabilisation respectant les droits constatés (c'est-à-dire une comptabilité d'exercice) et le principe de la fiabilité (section 2.1.3).

Dans les cantons, de nombreuses voix réclament un nouvel effort d'harmonisation. En effet, la mise en œuvre du MCH1 a tout de même maintenu une grande diversité de pratiques.

À cela s'ajoute la nécessité pour la Suisse de produire une statistique de ses finances publiques respectant les normes du Fonds monétaire international (FMI 2001). Il faut aussi pouvoir élaborer les comptes nationaux dans le respect du Système européen des comptes nationaux et régionaux (Eurostat 1996). La Suisse s'y est en effet engagée en concluant les accords bilatéraux II avec l'Union européenne.

Dès le début des années 2000, la Confédération travaille à son Nouveau Modèle Comptable (NMC; AFF 2006) ${ }^{48}$. Elle l'applique pour la première fois pour présenter 
son budget 2007. À ce moment-là, certains cantons manifestent leur volonté d'appliquer les normes IPSAS avec le moins d'exceptions possible. C'est le cas de Genève pour son budget 2008 et de Zurich pour son budget 2009. La CDF se doit d'agir. Elle entame le remaniement du MCH1 en 2003. Le 25 janvier 2008, elle remplace officiellement le MCH1 par son nouveau «Manuel - Modèle comptable harmonisé pour les cantons et les communes $\mathrm{MCH} 2 »(\mathrm{CDF} 2008)^{49}$. Ce modèle de $2^{\mathrm{e}}$ génération reste une recommandation. Il offre explicitement aux collectivités plusieurs possibilités de choix afin de leur permettre de le mettre en ouvre d'une manière qui convienne le mieux à leurs besoins. C'est pourquoi le $\mathrm{MCH} 2$ est compatible avec le NMC de la Confédération, avec les normes IPSAS, voire avec certaines pratiques préexistantes. Le $\mathrm{MCH} 2$ constitue donc surtout une évolution par rapport au MCH1. Hormis principalement des classifications comptables contraignantes, il laisse passablement de liberté aux collectivités. C'est pourquoi tant celles qui souhaitent appliquer le principe de la fiabilité que les adeptes d'une approche plus tactique de la présentation des comptes s'y retrouvent.

\section{Portrait 2 International Public Sector Accounting Standards}

Les International Public Sector Accounting Standards (IPSAS) sont des normes comptables applicables dans le secteur public. Elles sont destinées aux collectivités aussi bien du niveau national que local, aux agences, aux établissements publics et parapublics et aux institutions internationales. Ces normes sont établies par un comité de normalisation fondé en 1997: I'International Public Sector Accounting Standards Board (IPSASB). Les IPSAS sont largement inspirées des International Financial Reporting Standards (IFRS) qui sont le référentiel comptable applicable aux sociétés cotées sur un marché européen (anciennement nommées International Accounting Standards, IAS). L'IPSASB adapte les IFRS aux spécificités du secteur public lorsqu'il le juge nécessaire. Cependant, il cherche autant que possible à garantir un alignement sur les IFRS. À ce jour, I'IPSASB a publié près de quarante normes, ainsi qu'un cadre conceptuel d'ensemble (conceptual framework).

L'IPSASB n'a pas de pouvoir légal pour exiger qu'une collectivité publique applique Ies IPSAS. Toutefois, pour de nombreux pays, ces normes sont une source d'inspiration lorsqu'il s'agit de concevoir leur propre référentiel comptable. À l'heure actuelle, rares sont encore les pays qui appliquent les IPSAS sans restriction. En Suisse, la Confédération dès 2007 , ainsi que les cantons de Genève dès 2008 et de Zurich dès 2009 s'efforcent d'appliquer les IPSAS dans leur intégralité.

Source: www.ipsasb.org.

49 Handbuch - Harmonisiertes Rechnungslegungsmodell für die Kantone und die Gemeinden HRM2 | Manuale - Modello contabile armonizzato di cantoni e comuni MCA2 | Handbook - Harmonized Accounting Model for cantons and municipalities HAM2. Le MCH2 bénéficie d'améliorations régulières. Sa version la plus à jour est disponible sur le site Internet du Conseil suisse de présentation des comptes publics à l'adresse www.srs-cspcp.ch. 
La liberté laissée aux cantons s'étend au calendrier d'introduction. En effet, la Conférence préconise «de mettre en œuvre la présente recommandation technique aussi rapidement que possible, soit au cours des dix prochaines années». On constate, avec la figure 12, que dix années auront effectivement été nécessaires aux cantons pour y parvenir; pour les communes, le délai aura été plus long. Mais ce laps de temps reste toutefois inférieur à celui qui aura été nécessaire pour parvenir à l'introduction du MCH1.

Ce bref historique montre que l'harmonisation des comptes publics en Suisse s'effectue pas à pas. La souveraineté des cantons en matière de gestion financière et l'absence de bases légales ne permettent pas à la Confédération d'imposer une pratique uniforme aux échelons cantonaux et communaux. Tout au plus, la loi sur les finances de la Confédération (LFC), du 7 octobre 2005, précise-t-elle que «le Conseil fédéral s'emploie à harmoniser les normes de présentation des comptes de la Confédération, des cantons et des communes» (art. 48, al. 4, état au $1^{\text {er }}$ janvier 2016). De son côté, la CDF - en sa qualité d'organe intercantonal de liaison - ne peut offrir que des recommandations non contraignantes, par définition.

Figure 12 Introduction du $\mathrm{MCH} 2$ dans les cantons et dans les communes.

\section{État au 31 décembre 2019*.}

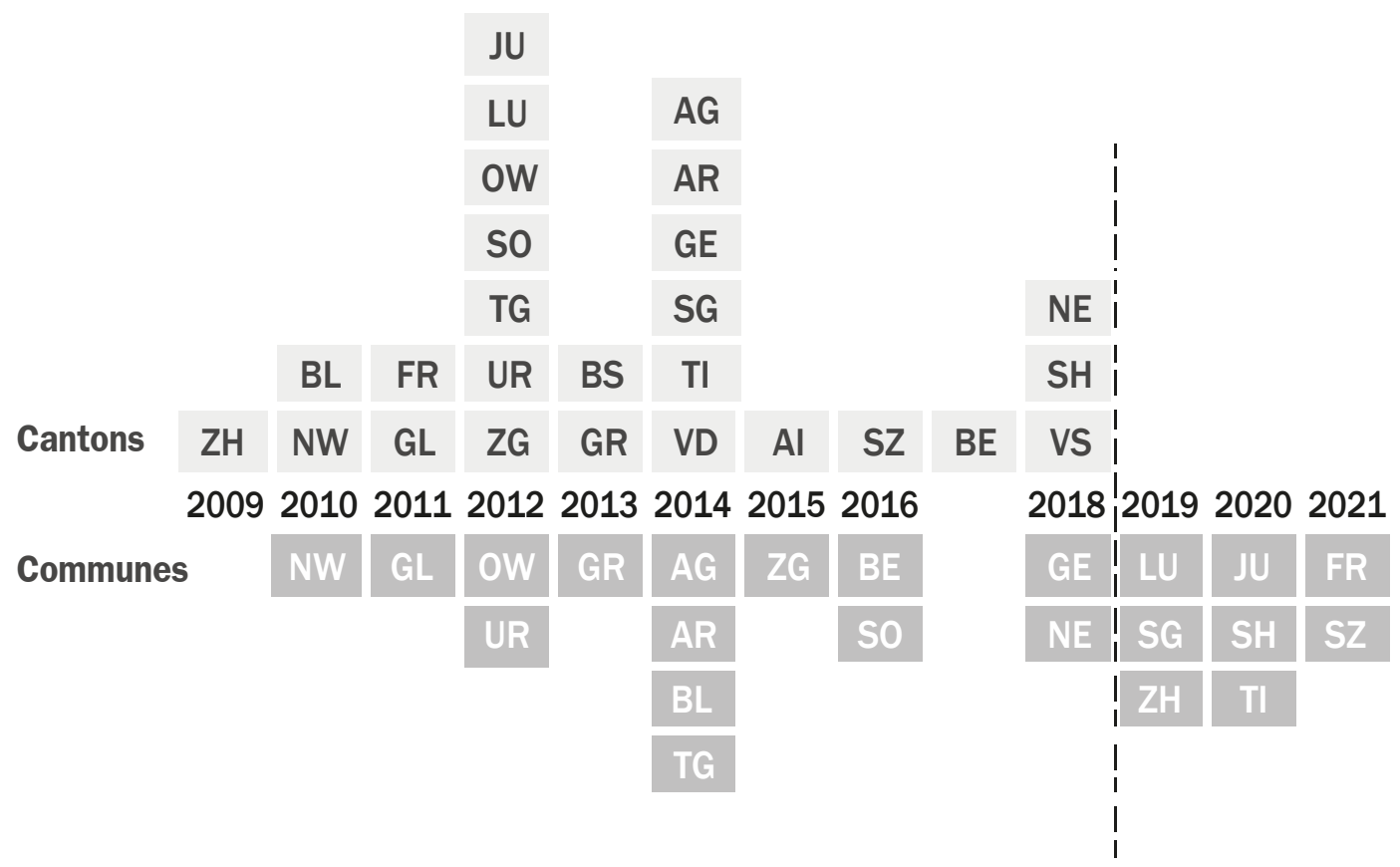

Source des données: Conseil suisse de présentation des comptes publics, www.srs-cspcp.ch.

* À cette date, les informations pour les communes des cantons de VD et VS ne sont pas encore disponibles. Les cantons d'Al et BS ne fixent pas de calendrier à leurs communes. 
Cela dit, en comparaison internationale, les collectivités suisses n'ont pas à rougir de la qualité de la présentation de leurs budgets et de leurs comptes. Certes, les normes IPSAS font beaucoup parler d'elles et de nombreuses organisations internationales les appliquent (INTERPOL, OTAN, OCDE, plusieurs agences onusiennes, etc.). Mais rares sont encore les pays qui appliquent une comptabilité d'engagement en suivant sans exception les normes IPSAS.

\subsubsection{Exigences faites au modèle comptable}

Les documents financiers préparés et publiés par une collectivité publique doivent pouvoir s'appuyer sur une systématique de présentation unifiée et cohérente. On parle de modèle comptable pour évoquer cette systématique. L'adjectif «comptable» est toutefois réducteur. En effet, le modèle ne s'applique pas seulement pour élaborer les comptes et les présenter. Les collectivités suisses l'appliquent également pour préparer leur budget et leur plan financier. Cela explique que la systématique doive répondre à plusieurs exigences:

- Permettre le contrôle et la gestion des recettes et des dépenses publiques soit par le peuple, soit par ses représentants dans les organes législatifs. Il s'agit d'une mission historique de la comptabilité publique, en particulier dans un système démocratique. Concrètement, le modèle de présentation doit permettre de contrôler le respect des principes financiers (section 2.1), du droit des crédits (sections 2.2 et 2.4) et des règles budgétaires (section 2.3).

- Servir de support à la prévision et à la planification. Les politiques publiques quelles qu'elles soient - éducation, santé, sécurité, par exemple - doivent être conçues dans une perspective de long terme. Le modèle comptable doit permettre de planifier les moyens à mettre en œuvre et les financements nécessaires. Par conséquent, la systématique du modèle doit faciliter la préparation du budget et la planification financière et leur servir de support.

- Permettre de déterminer le coût des prestations publiques. Le Manuel MCH1 (CDF 1982, p. 55) soulignait la nécessité pour un modèle comptable public de faciliter l'application du principe de l'utilisateur-payeur. Le dispositif doit fournir les informations nécessaires au calcul du prix de revient des prestations. La tarification des prestations pourra ensuite s'effectuer sur une base plus objective. Notons que l'on peut s'écarter de la règle de couverture des coûts, selon laquelle le prix de mise à disposition (prix de vente, taxe, etc.) devrait être proportionné au coût de revient - et cela pour des raisons de justice sociale et redistributive. On doit alors être conscient que l'écart entre prix de mise à disposition et coût de revient implique un financement par le contribuable. Ce financement résulte alors d'une démarche volontaire et réfléchie et non pas d'une ignorance des coûts. Connaître le coût des activités et des prestations a également un intérêt pour la gestion proprement dite, pour s'assurer de son efficacité. D’ailleurs le 
MCH1 évoque joliment que le modèle doit « développer le mode de penser selon les coûts» (voir le chapitre 4 consacré au mécanisme des imputations internes permettant de mieux refléter le coût des prestations et le chapitre 5 consacré au financement spécifique de certaines prestations).

- Servir de base aux comparaisons. Les collectivités sont de plus en plus intéressées à se comparer aux autres. Ces comparaisons - ou benchmarking - visent, entre autres, à allouer plus efficacement les ressources financières entre les différentes missions des collectivités, à mieux maîtriser les coûts des prestations publiques et à mieux choisir le mode de fourniture de ces prestations. La systématique retenue doit donc offrir des décompositions et des classifications permettant ce type d'analyses (voir le chapitre 10 consacré aux sources d'information permettant à une collectivité de se comparer).

- Faciliter la collaboration et la péréquation. Le fédéralisme helvétique implique un nombre et un volume considérables de transferts entre collectivités publiques. Ces mécanismes de péréquation des ressources, de compensation et de partage de charges doivent pouvoir s'appuyer sur des données comparables et fiables. Une plus grande transparence en matière de charges et de revenus facilite la collaboration et la confiance entre collectivités - par exemple au sein des associations de communes - et la péréquation - par exemple la disposition des collectivités aisées à partager leurs ressources fiscales avec les collectivités plus modestes (voir le chapitre 6 dédié aux transferts et à la péréquation financière).

- Permettre l'analyse macroéconomique. Les dépenses et les recettes de l'État influencent le développement économique du pays. Elles sont par exemple une des composantes importantes du produit intérieur brut. Conduire une politique macroéconomique efficace et de manière coordonnée entre collectivités constitue un défi majeur dans un état fédéral. Un modèle comptable ne permettra pas de lutter contre les aléas conjoncturels. Par contre, bien conçu, il facilitera l'analyse des décisions budgétaires pour déterminer si elles sont pro- ou anticycliques. Il permettra également de mieux connaître l'influence de ces aléas sur les dépenses et les recettes des collectivités (voir le chapitre 11 consacré à l'analyse de l'origine conjoncturelle ou structurelle des déficits).

- Faciliter la protection des créanciers. Le modèle retenu doit également permettre aux bailleurs de fonds potentiels d'une collectivité publique - une banque, une caisse de pension, une société d'assurance - d'évaluer le risque pris en lui octroyant un prêt. Par conséquent, le modèle doit permettre d'analyser la situation financière de la collectivité et d'ainsi protéger les intérêts des créanciers (voir le chapitre 9 consacré au diagnostic de la situation financière à l'aide d'indicateurs).

Le modèle comptable harmonisé développé par la CDF répond largement à ces exigences. Pour y parvenir, il recourt à la même systématique que la comptabilité 
de l'entreprise privée, c'est-à-dire selon une systématique de comptabilité commerciale. Cependant, il adapte la systématique aux besoins des collectivités publiques. C'est pourquoi les principaux éléments du modèle sont naturellement le compte de résultats - qui est assimilable au compte de Pertes et profits de l'entreprises privée - et le bilan. S'y ajoute un compte destiné à renseigner sur les dépenses pour constituer l'infrastructure de la collectivité et les recettes que la collectivité obtient spécifiquement pour cela; c'est le compte des investissements. La structure de ces trois éléments - compte de résultats, compte des investissements et bilan - est dictée par un plan comptable méthodique. Ce plan comptable et la classification fonctionnelle qui l'accompagne sont au cœur du dispositif, comme l'illustre la figure 13. À partir de là, la CDF a développé vingt recommandations visant à ce que les collectivités appliquent le plan comptable de manière uniforme. Si besoin est, nous renverrons ponctuellement notre lectrice et notre lecteur à ces recommandations. Ces dernières sont assez générales et leur mise en œuvre se heurte souvent à des problèmes pratiques. Pour pallier ces difficultés, la CDF en lien avec le Département fédéral des finances a institué le Conseil suisse de présentation des comptes publics (SRS-CSPCP, portrait 3$)^{50}$. Le Conseil diffuse des compléments aux recommandations. Ces compléments font intégralement partie du $\mathrm{MCH} 2$. Le Conseil répond en outre aux questions qui lui sont fréquemment posées (FAQ) dès le moment où ces questions touchent un nombre important de collectivités.

\section{Figure 13 Un plan comptable méthodique est au cœur du modèle.}

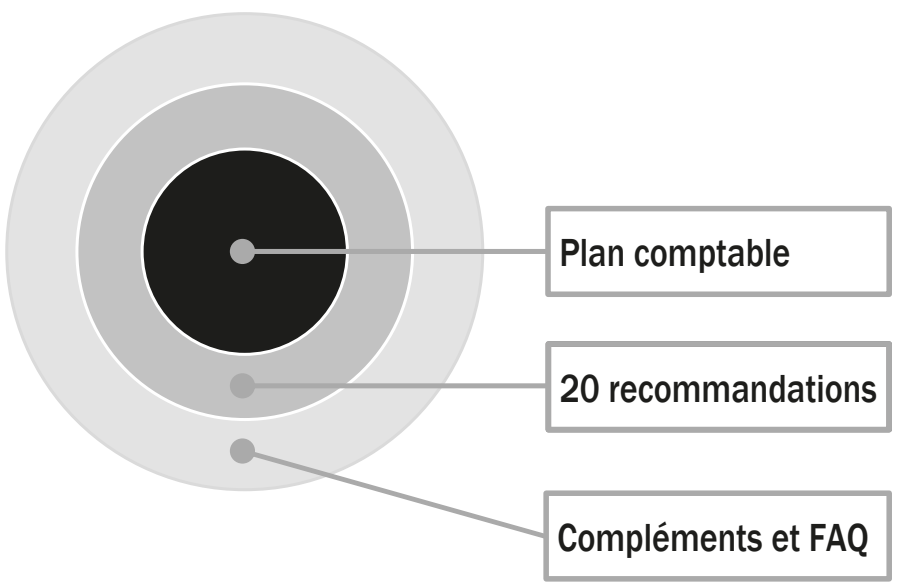

50 Schweizerisches Rechnungslegungsgremium für den öffentlichen Sektor (SRS) | Commissione svizzera per la presentazione della contabilità pubblica (CSPCP) | Swiss Public Sector Financial Reporting Advisory Committee (SRS). 


\section{Portrait 3 Conseil suisse de présentation des comptes publics}

Le Conseil suisse de présentation des comptes publics (SRS-CSPCP) a été créé en 2008 par la volonté du Département fédéral des finances et de la Conférence des directrices et directeurs cantonaux des finances. Mission lui a été donnée de promouvoir l'harmonisation, la comparabilité et la transparence dans la présentation des comptes des collectivités publiques suisses.

Pour ce faire, le Conseil doit apporter des compléments aux recommandations du $\mathrm{MCH} 2$ lorsque cela s'avère nécessaire. Si le $\mathrm{MCH} 2$ doit être modifié, il lui appartient de soumettre une proposition en ce sens à la Conférence des directrices et directeurs cantonaux des finances. Il offre également des éclaircissements en réponse aux questions pratiques que pose la présentation des comptes publics.

Il joue un rôle d'observateur en diffusant une information systématique sur la manière dont les cantons et les communes mettent en œuvre le $\mathrm{MCH}$. Finalement, dans le cadre des consultations lancées par le IPSASB, il prend position, au nom de l'ensemble des collectivités publiques suisses, au sujet des propositions de nouvelles normes comptables internationales.

Source: www.srs-cspcp.ch.

Évidemment, les cantons font usage de leur souveraineté lorsqu'ils traduisent les recommandations de la CDF dans leur législation et dans la législation applicable aux communes. Cependant, l'essentiel du $\mathrm{MCH} 2$ se retrouve dans les bases légales cantonales. Cela nous permet ici de vous présenter le modèle comptable standard, tout en mentionnant, lorsque cela est nécessaire, les spécificités que l'on peut rencontrer dans tel ou tel canton.

\subsubsection{Patrimoine administratif et patrimoine financier au bilan}

Le MCH1 a introduit une définition stricte de ce qu'est une dépense. Une dépense est une sortie de fonds soumise au droit des crédits. Il s'agit donc d'un décaissement sur lequel s'exerce le contrôle démocratique du Législatif et du peuple. Cette définition conduit à subdiviser l'actif du bilan ${ }^{51}$ de la collectivité entre un patrimoine administratif dont les mutations sont soumises au droit des crédits et un patrimoine financier qui ne dépend pas du droit des crédits. Sans l'indiquer explicitement, le $\mathrm{MCH} 2$ maintient cette définition de ce qu'est une dépense et, par conséquent, la distinction entre patrimoine administratif et patrimoine financier ${ }^{52}$. 
Patrimoine administratif ${ }^{53}$. La législation oblige les collectivités à fournir différentes prestations. Les cantons et les communes doivent se doter d'une infrastructure pour produire ces prestations et pour ainsi remplir leurs obligations légales. Une partie des actifs des collectivités est donc formé d'éléments indispensables à l'accomplissement des tâches publiques. L'ensemble de ces actifs constitue ce que l'on appelle le patrimoine administratif de la collectivité. Ces actifs se rapprochent par leur nature des immobilisations qui figurent au bilan de l'entreprise privée (figure 14). Une entreprise privée qui vendrait ses actifs immobilisés verrait son exploitation perturbée. De la même manière, une collectivité qui céderait son patrimoine administratif ne pourrait plus remplir ses obligations légales. Par exemple, si la législation cantonale relative à l'enseignement obligatoire prévoit que les communes sont responsables de mettre à disposition l'infrastructure dont ont besoin les établissements scolaires, les communes doivent acquérir les bâtiments scolaires nécessaires et les faire figurer dans leur patrimoine administratif. En effet, elles ne peuvent pas les vendre sous peine d'enfreindre la loi $^{54}$.

Figure 14 Distinction au bilan entre patrimoine administratif et patrimoine financier. MaCollectivité, 31 décembre de l'Année t, en KCHF.

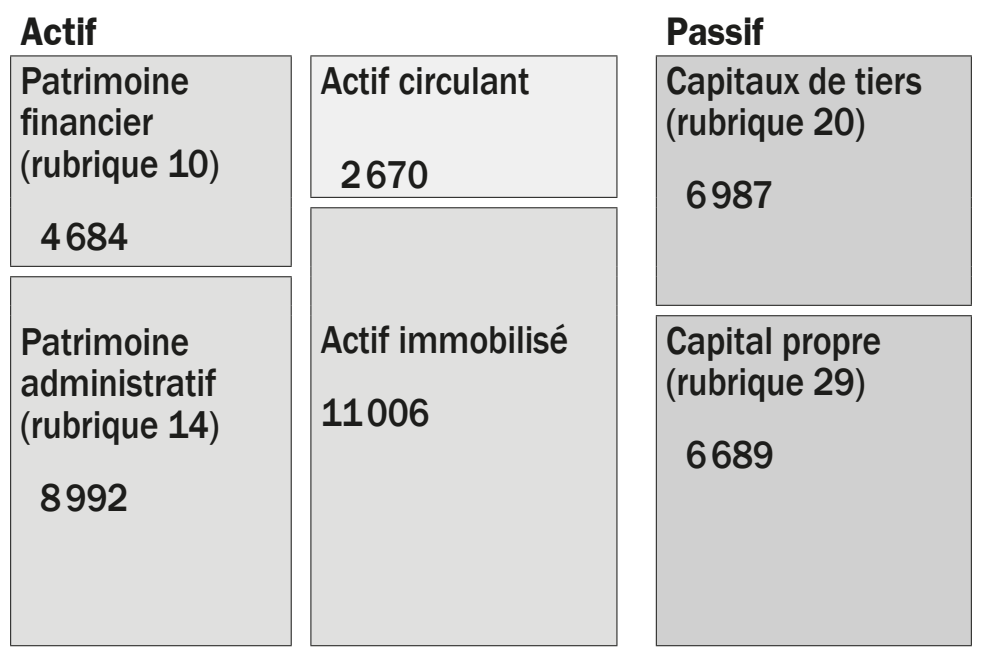

Le patrimoine administratif comprend donc tous les actifs qui servent directement à exécuter les tâches que l'entité doit accomplir en vertu de dispositions du droit public (Constitution, loi, ordonnance, règlement, etc.). On y trouve des immobilisations corporelles et incorporelles. On y trouve également des participations permanentes, des prêts et des contributions (autrement dit des subventions à fonds perdus) octroyés à des tiers par la collectivité à des fins d'investissement. Englober

53 Verwaltungsvermögen | Beni amministrativi | Administrative assets.

54 Les communes peuvent évidemment renoncer à acquérir les bâtiments nécessaires et les louer ou s'en remettre à une autre commune ou encore à une association intercommunale qui elle se dotera de l'infrastructure nécessaire et la fera figurer dans son propre patrimoine administratif. 
ces participations, prêts et contributions dans le patrimoine administratif permet de montrer que la collectivité remplit ses obligations légales en matière de mise à disposition d'infrastructures pour la fourniture de prestations publiques, même si, pour y parvenir, elle délègue cette activité à un tiers. Le patrimoine administratif occupe la rubrique 14 du plan comptable. Dans le cas de MaCollectivité, sa valeur au bilan s'élève à KCHF 8992, soit près de CHF 9 mio, au 31 décembre de l'Année t.

Patrimoine financier ${ }^{55}$. À côté des actifs formant son patrimoine administratif, la collectivité possède généralement d'autres actifs qu'elle n'utilise pas pour remplir des obligations légales. Ces actifs sont détenus pour d'autres raisons, par exemple pour dégager un rendement dans le cas d'immeubles de rente - immeubles locatifs ou commerciaux - ou de papiers valeurs, ou tout simplement pour pouvoir effectuer des paiements dans le cas des comptes bancaires ou postaux ou encore de la caisse. L'ensemble de ces actifs constitue ce que l'on appelle le patrimoine financier de la collectivité. Il occupe la rubrique 10 du plan comptable. Dans le cas de MaCollectivité, sa valeur au bilan s'élève à KCHF 4684 au 31 décembre de l'Année t.

La figure 14 montre que la notion de patrimoine financier est plus large que celle d'actif circulant puisque le patrimoine financier englobe des actifs certes immobilisés, mais que la collectivité peut vendre.

Aliénabilité56 ${ }^{56}$ Le critère permettant de savoir si un actif appartient au patrimoine financier plutôt qu'au patrimoine administratif est celui de l'aliénabilité (expression utilisée par le MCH1, mais ne figurant plus en tant que telle dans le MCH2). La figure 15 offre un arbre de décision fondé sur ce critère. Si la collectivité peut vendre cet actif sans pour autant hypothéquer sa capacité à produire les prestations que la législation attend d'elle, alors l'actif en question est réputé aliénable et fait donc partie du patrimoine financier. Si la collectivité ne peut s'en séparer sans compromettre son offre de prestations, autrement dit si l'actif est indispensable pour produire les prestations publiques exigées par le droit public, alors l'actif est inaliénable et fait partie du patrimoine administratif. Nous avons indiqué ci-dessus que la distinction entre les deux types de patrimoine est importante du point de vue de l'application du droit des crédits. Par voie de conséquence, elle l'est également pour délimiter les compétences de l'Exécutif et pour opérer une distinction entre investissement et placement. Investissement ${ }^{57}$. Une sortie de fonds pour créer, acheter ou construire un actif nécessaire pour fournir une prestation répondant aux prescriptions du droit public tombe sous le coup du droit des crédits. Elle nécessite notamment le vote d'un crédit d'engagement, puis d'un crédit budgétaire. Le contrôle démocratique par le Législatif et, cas échéant, par la population sur ce type de décaissement est donc fort. La sortie de fonds est qualifiée de dépense d'investissement. Elle doit être présentée

55 Finanzvermögen | Beni patrimoniali | Non administrative assets.

56 Realisierbarkeit | Realizzabilità | Disposability.

57 Investitionen | Investimenti | Capital expenditure. 
dans un compte spécifique: le compte des investissements. Par conséquent, les actifs composant le patrimoine administratif sont le résultat des différents investissements réalisés par la collectivité - ces derniers faisant l'objet d'un report au bilan à la fin de chaque exercice. La notion d'investissement doit être comprise ici dans son sens économique: un investissement crée un capital productif, en l'occurrence un capital productif de prestations publiques.

Figure 15 Critère de l'aliénabilité pour distinguer le patrimoine administratif du patrimoine financier.

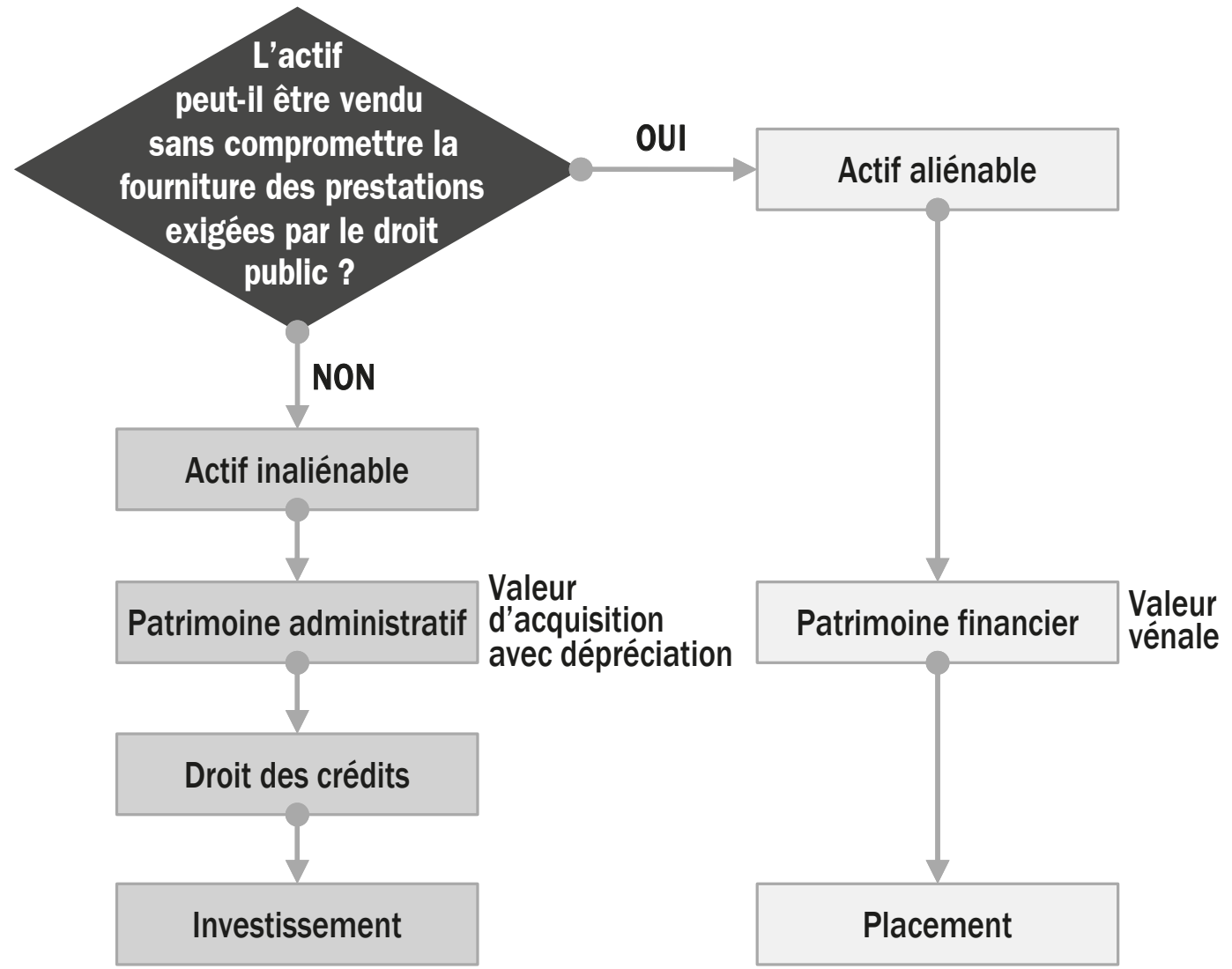

Placement ${ }^{58}$. Une sortie de fonds qui n'est pas réalisée pour créer une infrastructure nécessaire à la production des prestations publiques correspond à une opération de placement. Le choix de consacrer une partie des liquidités de la collectivité à acquérir un véhicule de placement mobilier (titres financiers, actions, obligations) ou immobilier (immeuble, terrain) relève de la gestion de la trésorerie et de la dette. Il s'agit d'un choix opérationnel qui est souvent laissé à la compétence de l'Exécutif et qui n'est donc pas soumis au droit des crédits. Le contrôle par le Législatif est 
donc plus limité que le contrôle sur les investissements. Les opérations de placement impactent directement les comptes du bilan, sans utiliser le compte des investissements. Par conséquent, les actifs composant le patrimoine financier sont le résultat des différents placements réalisés par la collectivité.

Dette nette. La distinction entre patrimoine administratif et patrimoine financier est également intéressante pour estimer le niveau d'endettement de la collectivité. Pour évaluer ce niveau, on peut de manière simpliste considérer uniquement les capitaux de tiers, c'est-à-dire la dette brute, au passif du bilan. Il suffit alors de se référer à la rubrique 20 du plan comptable. Dans le cas de MaCollectivité, la valeur au bilan des capitaux de tiers s'élève à KCHF 6987, soit près de CHF 7 mio, au 31 décembre de l'Année t. Mais on obtient alors une image biaisée de la réalité de l'endettement de la collectivité. En effet, les liquidités apportées par l'endettement sont pour partie utilisées à des fins de placements dans le patrimoine financier. Toutefois, dans la mesure où le patrimoine financier est aliénable, il peut être vendu afin d'obtenir des liquidités. À leur tour, ces liquidités peuvent permettre de rembourser la dette et de ramener l'endettement à ce qu'il est effectivement. Le vrai endettement de la collectivité, son endettement net (ou pour être exact, ses engagements nets), peut donc être calculé en soustrayant le patrimoine financier des capitaux de tiers. La figure 14 permet de mieux se rendre compte de l'utilité de connaître la valeur du patrimoine financier, donc aliénable, afin de pouvoir estimer les engagements nets. Ceux-ci s'élèvent à KCHF 2303 au 31 décembre de l'Année t, soit la différence entre KCHF 6987 et KCHF 4684.

Principes d'évaluation. Pour pouvoir calculer la dette nette de manière fiable, il faut connaître la vraie valeur du patrimoine financier. C'est pourquoi le patrimoine financier doit être présenté au bilan à sa valeur vénale. Par contre, dans la mesure où le patrimoine administratif est inaliénable, il n'est pas nécessaire de connaître sa valeur vénale. Le $\mathrm{MCH} 2$ prescrit donc qu'il doit être présenté au bilan à sa valeur d'acquisition tout en tenant compte de la dépréciation engendrée par l'usure et l'obsolescence. L'évaluation à la valeur d'acquisition permet d'analyser le coût des prestations offertes par la collectivité. Nous y reviendrons à la section 3.3.5 lorsque nous aborderons la thématique de l'amortissement. On trouve donc à l'actif du bilan de la collectivité deux types de patrimoine, évalués chacun de manière différente ${ }^{59}$.

Actifs hybrides. Certains actifs représentent pour une partie un capital productif de prestations publiques et pour une autre partie un placement. Pensons à un bâtiment scolaire dont un ou plusieurs étages seraient dévolus à des logements de rente. L'actif devrait alors être enregistré en fonction de son utilisation principale. Si l'utilisation pour produire des prestations publiques est quantitativement identique à l'utilisation comme placement, alors l'actif devrait être catégorisé dans le patrimoine administratif. Cela évite de surestimer le patrimoine financier et donc de sous-estimer la dette nette. Le principe de prudence est ainsi respecté. 
Transferts entre les patrimoines. Il arrive qu'un actif du patrimoine administratif tombe en désuétude et que la collectivité souhaite s'en défaire ou le réaffecter pour en tirer un rendement. Par exemple, un bâtiment scolaire vétuste est remplacé par un nouveau. L'actif doit alors être déclassé. Cela constitue une décision de transfert de l'actif du patrimoine administratif au patrimoine financier. Cette décision doit formellement être décrétée par le Législatif. Elle a pour effet de rendre aliénable l'actif concerné. Une décision inverse - transfert du patrimoine financier à l'administratif - intervient lorsque par exemple un immeuble de rente doit être affecté à un usage administratif. Dans la mesure où les disponibilités comme l'argent en caisse ou en compte postal ou bancaire appartiennent également au patrimoine financier, leur utilisation pour acheter ou construire un actif du patrimoine administratif correspond également à un transfert entre les deux patrimoines.

Dépense. En l'espèce, le pouvoir conféré par la loi au Législatif de décider du devenir d'un actif du patrimoine financier permet de donner une définition plus générale à la notion de dépense. Selon cette définition, une dépense correspond à l'utilisation du patrimoine financier pour remplir une tâche d'utilité publique, c'est-à-dire prescrite par le droit public. Par conséquent cette utilisation est soumise au droit des crédits, qu'il s'agisse d'une dépense courante ou d'une dépense d'investissement.

\subsubsection{Compte des investissements et compte de résultats}

Séparation entre dépenses d'investissement et dépenses pour le fonctionnement. Pour remplir une tâche d'utilité publique, il ne suffit pas de consentir des dépenses d'investissement pour créer un patrimoine administratif. La collectivité doit pouvoir disposer d'autres inputs pour fournir des prestations. Elle doit en particulier disposer d'un personnel qu'elle doit salarier, de biens et de services qu'elle doit acheter ou d'entités partenaires qu'elle doit subventionner. Les dépenses qui en découlent sont nécessaires au bon fonctionnement de la collectivité.

On voit ainsi apparaître une séparation claire entre les dépenses d'investissement et les dépenses pour le fonctionnement, c'est-à-dire les dépenses courantes. Le MCH1 a introduit cette distinction en recommandant de comptabiliser les premières dans le compte des investissements et les secondes dans le compte de fonctionnement. Le $\mathrm{MCH} 2$ maintient cette recommandation ${ }^{60}$. Toutefois, il rebaptise le compte de fonctionnement compte de résultats afin d'utiliser une appellation en cours dans les entreprises.

Compte administratif. Par contre, le $\mathrm{MCH} 2$ ne reprend pas du MCH1 la notion de compte administratif: dans le $\mathrm{MCH} 1$, le compte administratif chapeaute le compte des investissements et celui de fonctionnement dans la mesure où tous deux enregistrent l'ensemble des décaissements soumis au droit des crédits, donc l'ensemble des dépenses. 
Durée d'utilisation ${ }^{61}$. La distinction entre dépenses d'investissement et dépenses courantes repose sur le critère de la durée d'utilisation, comme l'indique la figure 16. Ne peuvent être considérées comme dépenses d'investissement que les dépenses contribuant à fournir à la population des prestations, donc une utilité pendant plus d'une année. Les dépenses qui n'apportent une utilité que pendant un seul exercice ou moins doivent être considérées comme des dépenses courantes. Les salaires versés appartiennent à cette seconde catégorie. En effet, ils ne permettent de s'assurer le concours des salariés que pendant la période de référence, par exemple un mois.

Figure 16 Critère de la durée d'utilisation pour distinguer entre dépenses courantes et dépenses d'investissement.

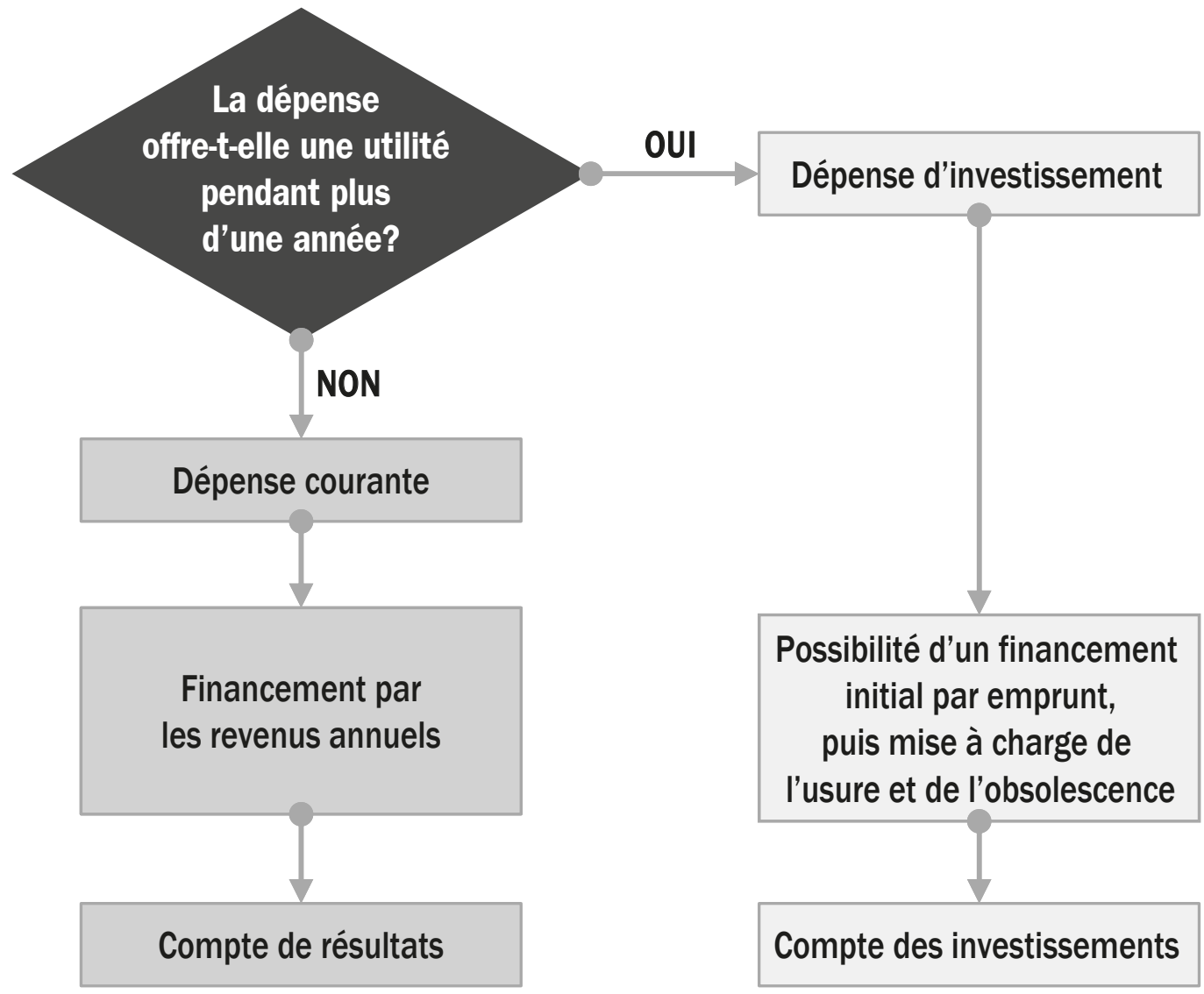

Financement. Cette distinction entre dépenses d'investissement et dépenses courantes permet de résoudre le problème du financement. Faut-il financer les dépenses qui ont une utilité éphémère de la même façon que les dépenses qui ont une utilité prolongée? Faut-il que l'achat d'un immeuble, comme l'achat de crayons et de gommes, soit 
financé par les recettes de l'exercice au cours duquel on a acquis ces objets? Le respect du principe de l'utilisateur-payeur impose de répondre «non» à ces questions. C'est pourquoi le $\mathrm{MCH} 2$, comme le MCH1 avant lui, prévoit des modalités de financement différenciées. Les charges enregistrées chaque année dans le compte de résultats doivent en principe être couvertes par les revenus de l'exercice: revenus fiscaux, taxes, revenus financiers, revenus de transferts, etc. Par contre, les dépenses d'investissement peuvent être provisoirement financées par un emprunt, mais les utilisateurs de l'infrastructure doivent aussi année après année payer pour l'usure et l'obsolescence de cette infrastructure et cela à travers une procédure d'amortissement (voir la section 3.3.5 ci-après consacrée à la dépréciation du patrimoine administratif).

\section{Compte des investissements}

Le compte des investissements ${ }^{62}$ a pour raison d'être de mettre en évidence le volume d'investissement à réaliser (budget) ou réalisé (comptes). Il renseigne donc sur l'importance des actifs administratifs créés au cours de l'exercice. Ces actifs seront, au moment de la clôture, répertoriés au bilan, dans le patrimoine administratif de la collectivité. Ils seront également graduellement amortis tout au long de leur durée d'utilité. Autrement dit, l'équivalent de leur usure et de leur obsolescence sera mis à charge du compte de résultats et réduira parallèlement leur valeur au bilan.

Le compte des investissements permet donc d'enregistrer et de présenter toutes les dépenses qui seront utiles pendant plusieurs exercices ${ }^{63}$. Ceci est le principe. En pratique, on évite de multiplier le nombre de petits montants à présenter dans le compte des investissements. De ce fait, les dépenses modestes sont enregistrées dans le compte de résultats en dépit du fait qu'elles peuvent avoir un caractère d'investissement. Pensons à des pièces de mobilier isolées, comme des chaises ou des tables, achetées pour remplacer du mobilier endommagé. Un autre exemple est donné par le remplacement d'un ordinateur. Il s'agit là d'une approche pragmatique qui tient compte du fait que cela fait partie d'une routine et que les montants en jeu ne sont pas significatifs au regard du critère de l'importance (voir la section 2.1.3).

Limite d'activation ${ }^{64}$. Toutefois, afin d'éviter que le choix de comptabiliser la dépense dans un compte plutôt que dans l'autre soit dicté par une possible tactique politique en matière de présentation des comptes, le $\mathrm{MCH} 2$ recommande que la collectivité se fixe explicitement une limite à partir de laquelle elle comptabilise une dépense dans le compte des investissements. On parle ici de limite d'inscription à l'actif ou de limite d'activation, dans la mesure où l'actif ainsi créé augmente le patrimoine administratif. La limite décidée par les cantons et les communes varie fortement ${ }^{65}$. Elle se situe parfois proche de zéro et oblige les collectivités à enregistrer un nombre important

62 Investitionsrechnung | Conto investimenti | Statement of capital expenditures.

63 Recommandation 10, CDF (2008).

64 Aktivierungsgrenze | Limite di iscrizione all'attivo | Threshold for capitalisation.

65 Le Conseil suisse de présentation des comptes publics recense, par canton, les limites d'activation en vigueur pour le canton lui-même et pour les communes du canton (www.srs-cspcp.ch). 
de transactions dans le compte des investissements. Parfois la limite est très élevée, réduisant d'autant le nombre d'enregistrements dans le compte des investissements et la nécessité pour l'Exécutif de solliciter du Législatif un crédit d'engagement, avec comme corollaire un travail réduit pour l'administration. La Conférence des autorités cantonales de surveillance des finances communales (CACSFC, portrait 4), se basant sur le $\mathrm{MCH} 2$, propose d'adapter la limite d'inscription à l'actif à la taille de la collectivité ${ }^{66}$. Ces limites sont reproduites dans le tableau 14.

\section{Portrait 4 Conférence des autorités cantonales de surveillance des finances communales}

La Conférence des autorités cantonales de surveillance des finances communales (CACSFC, KKAG en allemand) est une organisation intercantonale constituée sous forme d'association. Ses membres sont les services cantonaux exerçant une fonction de surveillant sur les communes concernant leurs finances, c'est-à-dire en principe les services des communes ou leurs équivalents. Les services sont représentés par les membres de leur personnel. Sa raison d'être est de faciliter l'échange de connaissances techniques et pratiques dans le domaine des finances et de la comptabilité communale.

Source: www.kkag-cacsfc.ch.

Tableau 14 Limite d'activation de la dépense.

Proposition de la Conférence des autorités cantonales de surveillance des finances communales.

\begin{tabular}{lc}
\hline Nombre d'habitants & Limite d'activation en CHF \\
\hline Jusqu'à 1000 & 25000 \\
De 1001 à 5000 & 50000 \\
De 5001 à 10000 & 75000 \\
Plus de 10000 & 100000 \\
\hline
\end{tabular}

Nature des dépenses et des recettes d'investissement. Outre le critère de la durée d'utilité et le montant de la limite d'activation, la dépense à comptabiliser dans le compte des investissements peut être de différentes natures. Il peut s'agir des éléments suivants:

- Une dépense pour acheter, réaliser ou améliorer une immobilisation corporelle comme un terrain, une route, un bâtiment, une machine ou encore un véhicule. Cette dépense est débitée de la rubrique 50 du plan comptable si elle est réalisée au profit de la collectivité elle-même, et de la rubrique 51 si elle est réalisée pour le compte d'un tiers et est ensuite payée par ce tiers; 
- Une dépense pour acheter, réaliser ou améliorer une immobilisation incorporelle comme un logiciel, une licence ou un brevet. La dépense est débitée de la rubrique 52;

- Un prêt octroyé par la collectivité à une autre entité (canton, commune, etc.), afin que ce soit cette entité qui procède à une dépense d'investissement en lieu et place de la collectivité prêteuse elle-même ${ }^{67}$. La sortie de fonds découlant du prêt est débitée de la rubrique 54 ;

- Une dépense pour acquérir une participation ou une part du capital social dans une entité tierce, qu'il s'agisse d'un concordat intercantonal, d'une association intercommunale, d'une entreprise ou d'une organisation publique ou privée. La sortie de fonds en découlant est débitée de la rubrique 55;

- Une contribution octroyée par la collectivitéà une autre entité (canton, commune, entreprise, etc.) dans le but qu'elle l'utilise pour financer un investissement. Le plan comptable parle en l'occurrence d'une subvention d'investissement. Cette subvention peut être décidée par la collectivité elle-même (rubrique 56, propres subventions d'investissement). Mais elle peut aussi être décidée et versée par une autre collectivité; alors la collectivité ne fait que transmettre et redistribuer la subvention en question (rubrique 57, subventions redistribuées). On peut s'étonner que les subventions - autrement dit les contributions - soient considérées comme des investissements et qu'elles soient par conséquent mises à l'actif du bilan de la collectivité dans son patrimoine administratif. En effet, les subventions ne fondent pas une propriété au sens formel. Qu'à cela ne tienne puisque leur but est identique à celui des investissements propres: la collectivité devrait s'équiper elle-même de l'infrastructure si elle n'en laissait pas le soin à d'autres.

Le tableau 15 présente l'ensemble des rubriques de dépenses du compte des investissements telles qu'elles sont prévues dans le plan comptable du MCH2 et le montant correspondant enregistré dans les comptes de MaCollectivité pour l'Année t. Il présente également les recettes d'investissement dont bénéficie la collectivité. Certaines de ces recettes représentent effectivement une entrée de fonds. Tel est le cas des remboursements venant de tiers (rubrique 61, en tant que contrepartie de la dépense enregistrée dans la rubrique 51), des remboursements de prêts (64, pour les prêts octroyés auparavant à travers la rubrique 54) ou de subventions (66, pour les subventions octroyées à travers la rubrique 56), des contributions ou subventions obtenues pour investir (63) ou pour les transférer à des tiers (67, en tant que contrepartie des subventions redistribuées enregistrées dans la rubrique 57). D'autres opérations ne sont pas à proprement parler des recettes, car elles ne correspondent pas à des entrées de fonds. Il s'agit des transferts du patrimoine administratif vers le patrimoine financier d'immobilisations corporelles (60)

67 Les collectivités octroient parfois des prêts à d'autres entités - publiques ou privées - sans que ces prêts soient dédiés au financement d'investissements réalisés en lieu et place des collectivités prêteuses. Ces prêts s'apparentent donc à des placements. Par conséquent, ils ne doivent en aucun cas transiter par le compte des investissements. Ils doivent être directement enregistrés dans les placements financiers à court terme (rubrique 102) ou à long terme (107) à l'actif du bilan. 
ou incorporelles (62) ou encore de participations (65). Ces opérations doivent être effectuées lorsque l'actif concerné n'est plus utilisé par la collectivité pour produire les prestations exigées par loi. Cela se produit notamment lorsqu'une nouvelle installation remplace l'installation existante ou lorsque la loi est modifiée pour abroger l'obligation correspondante. Ces transferts permettent donc de reclassifier les actifs à l'intérieur du bilan de la collectivité ${ }^{68}$. Mentionnons pour être complet les recettes extraordinaires (68), qui font écho aux dépenses extraordinaires (58). Ces rubriques ne peuvent que rarement être utilisées. Elles le seraient pour enregistrer des dépenses engendrées par des événements imprévisibles, sur lesquels la collectivité n'a pas d'emprise et qui sont sans lien avec l'activité opérationnelle. D'ailleurs, la lecture du tableau 15 montre que MaCollectivité n'a eu recours qu'à deux rubriques au cours de l'Année $\mathrm{t}$ : celle pour comptabiliser un investissement dans des immobilisations corporelles à hauteur de KCHF 1971, soit près de CHF 2 mio, et celle pour enregistrer les subventions acquises pour l'investissement à hauteur de KCHF 43.

Tableau 15 Rubriques du compte des investissements selon la classification par nature. MaCollectivité, Année t, en KCHF.

\begin{tabular}{|c|c|c|c|c|c|}
\hline $\mathrm{N}^{0}$ & Libellé & $\mathrm{KCHF}$ & $\mathbf{N}^{0}$ & Libellé & KCHF \\
\hline 5 & Dépenses d'investissement & 1971 & 6 & Recettes d'investissement & 43 \\
\hline 50 & Immobilisations corporelles & 1971 & 60 & $\begin{array}{l}\text { Transferts d'immobilisations } \\
\text { corporelles dans le PF }\end{array}$ & 0 \\
\hline 51 & $\begin{array}{l}\text { Dépenses d'investissement } \\
\text { pour le compte de tiers }\end{array}$ & 0 & 61 & $\begin{array}{l}\text { Remboursements de dépenses d'inves- } \\
\text { tissement pour le compte de tiers }\end{array}$ & 0 \\
\hline \multirow[t]{2}{*}{52} & $\begin{array}{l}\text { Immobilisations } \\
\text { incorporelles }\end{array}$ & 0 & 62 & $\begin{array}{l}\text { Transferts d'immobilisations } \\
\text { incorporelles dans le PF }\end{array}$ & 0 \\
\hline & & & 63 & Subventions d'investissement acquises & 43 \\
\hline 54 & Prêts & 0 & 64 & Remboursements de prêts & 0 \\
\hline 55 & Participations et capital social & 0 & 65 & Transferts de participations & 0 \\
\hline 56 & $\begin{array}{l}\text { Propres subventions } \\
\text { d'investissement }\end{array}$ & 0 & 66 & $\begin{array}{l}\text { Remboursements de propres } \\
\text { subventions d'investissement }\end{array}$ & 0 \\
\hline 57 & $\begin{array}{l}\text { Subventions d'investissement } \\
\text { redistribuées }\end{array}$ & 0 & 67 & $\begin{array}{l}\text { Subventions d'investissement } \\
\text { à redistribuer }\end{array}$ & 0 \\
\hline 58 & $\begin{array}{l}\text { Dépenses d'investissement } \\
\text { extraordinaires [e.o.] }\end{array}$ & 0 & 68 & $\begin{array}{l}\text { Recettes d'investissement } \\
\text { extraordinaires [e.o.] }\end{array}$ & 0 \\
\hline
\end{tabular}

68 Les rubriques disponibles à l'avoir du compte des investissements permettent de mettre en évidence les transferts d'actifs du patrimoine administratif vers le patrimoine financier. Par contre, il n'existe pas de rubrique spécifique au doit qui permette d'identifier les transferts d'actifs du patrimoine financier vers le patrimoine administratif. Un tel transfert se produit par exemple lorsqu'un immeuble de rendement est réaffecté pour accueillir les services administratifs de la collectivité. Comptablement, la valeur de l'actif transféré se confond avec les dépenses pour l'acquisition ou la construction de nouveaux actifs dans les rubriques du doit, et en particulier dans la rubrique 50 s'agissant d'un immeuble. Ce mélange des genres entre opérations monétaires et non monétaires est une faiblesse du plan comptable offert par le MCH2. 
Modalités de présentation au bilan. Le compte des investissements est donc structuré comme un compte de patrimoine - en principe un compte actif. En effet, il enregistre au doit les dépenses qui créent des actifs administratifs. Et à l'avoir sont portées les recettes reçues dans ce but.

À ce propos, le $\mathrm{MCH} 2$ laisse le choix entre deux options pour présenter au bilan les dépenses et les recettes d'investissement ${ }^{69}$. Une option consiste à inscrire le montant de l'investissement brut comme valeur à l'actif (c'est-à-dire le montant comptabilisé comme une dépense dans la rubrique 50 ou 52) et, parallèlement, le montant de la subvention reçue pour cet objet comme engagement financier à long terme au passif (rubrique 63). Cette option correspond à l'application stricte du produit brut. Si MaCollectivité pratiquait cette option elle présenterait un montant de KCHF 1971 à l'actif, dans son patrimoine administratif, et au passif un montant de KCHF 43.

L'autre option s'écarte du principe du produit brut. Elle consiste à activer le montant de l'investissement net (investissement déduction faite de la contribution d'investissement pour l'objet concerné). On applique donc une sorte de principe du produit net. C'est l'option qui prévalait dans le cadre du $\mathrm{MCH} 1$ et qui est encore en vigueur dans passablement de cantons, en particulier comme prescription à appliquer par les communes. Elle a le mérite de la simplicité, au détriment toutefois de la transparence. MaCollectivité pratique cette option et se contente d'activer un montant de KCHF 1928 (KCHF 1971 - KCHF 43).

Investissement net ${ }^{70}$. Le compte des investissements permet également de dégager l'investissement net de la collectivité, c'est-à-dire la différence entre les investissements consentis (rubrique 5) et les recettes obtenues pour les financer (6). Les dépenses sont généralement supérieures aux recettes. C'est le cas de MaCollectivité pour l'Année t: l'investissement net s'élève à KCHF 1928. Mais c'est parfois l'inverse, en particulier lorsque la dépense d'investissement et la recette correspondante interviennent au cours d'exercices comptables différents. Une subvention ou un remboursement peuvent être reçus dans l'exercice qui suit celui de la dépense. Dans cet exercice, l'investissement net peut alors être négatif.

\section{Compte de résultats}

Le compte de résultats ${ }^{71}$ est conçu comme le compte de Pertes et profits de l'entreprise privée. Il enregistre les opérations de consommation comme des charges avec, en contrepartie, des revenus ${ }^{72}$. Sa raison d'être est donc de renseigner sur le coût de fonctionnement de la collectivité et sur le degré de couverture des charges par les revenus. Autrement dit, le compte de résultats doit indiquer si les revenus - notamment fiscaux - vont suffire (budget) ou ont suffi (comptes) à couvrir le coût des prestations offertes. Un déficit - un excédent de charges - témoigne de revenus

69 Recommandation 10, CDF (2008).

70 Nettoinvestitionen | Investimenti netti | Net capital assets.

71 Erfolgsrechnung | Conto economico| Statement of financial performance (income statement).

72 Recommandation 04, CDF (2008). 
insuffisants ou de charges excessives. Un boni indique que les revenus excèdent les charges. De manière normative, le degré de couverture devrait être de 100\%: les revenus devraient être équivalents aux charges, ni plus, ni moins.

Le compte de résultats a également pour raison d'être de permettre la gestion des crédits budgétaires relatifs aux dépenses courantes. Au moment du budget, il présente l'ensemble des crédits budgétaires octroyé par le Législatif. Au moment des comptes, il permet de contrôler le niveau d'utilisation de ces crédits.

Il est donc un élément central du dispositif financier de la collectivité. C'est pourquoi il occupe, par le nombre de pages qui y sont consacrées, l'essentiel des états financiers. De plus, il se trouve presque toujours au début du fascicule du budget et de celui des comptes.

Charges par nature. Les charges comprennent les dépenses courantes, c'est-àdire les dépenses qui n'apportent une utilité que pendant un seul exercice, voire moins. Elles comprennent également des charges à caractère purement comptable, les charges dites non monétaires. Les amortissements en sont un exemple typique puisqu'ils ne s'accompagnent pas d'une sortie de fonds.

Le tableau 16 présente l'ensemble des rubriques de charges prévues dans le plan comptable du $\mathrm{MCH} 2$ et le montant correspondant enregistré dans les comptes de MaCollectivité pour l'Année $\mathrm{t}^{73}$. Sans surprise, on y trouve les charges de personnel (rubrique 30), celles qui sont liées à l'achat de biens et de services nécessaires à l'exploitation (31), les charges financières (34, qui englobe la rubrique 340 destinée à enregistrer les charges d'intérêts payés sur la dette). On y trouve également les charges de transferts qui souvent représentent une part importante dans le total des charges (36, qui englobe les dédommagements versés à d'autres entités 361 , les paiements à la péréquation financière 362 et les subventions 363). Les subventions redistribuées de la rubrique 37 font écho aux subventions reçues et à redistribuer de la rubrique de revenus 47 . Il doit donc y avoir parfaite égalité entre les montants de ces deux rubriques en charges et en revenus.

Trois rubriques sont intégralement dédiées à des charges sans sortie de fonds, c'est-à-dire à des charges non monétaires, donc purement comptables. La rubrique 33 enregistre les amortissements témoignant de l'usure et l'obsolescence du patrimoine administratif. La section 3.3.5 consacrée à la dépréciation du patrimoine administratif donne davantage d'information à ce sujet. La rubrique 35 recense les attributions aux financements spéciaux et fonds (35) effectuées lorsque des recettes affectées excèdent le coût qu'elles sont censées couvrir. La section 5.5 consacrée aux financements spéciaux s'y intéresse spécifiquement. La rubrique 39 renseigne sur les imputations internes, c'est-à-dire sur les charges supportées par

73 La rubrique 32 ne concerne que la Confédération. C'est pourquoi nous ne nous y arrêtons pas. Relevons toutefois que la présence des charges d'armement dans les charges par nature constitue une anomalie. C'est une concession faite aux besoins de présentation comptable de la Confédération. Au moment de la conception du plan comptable, on n'a pas souhaité enregistrer les dépenses d'armement dans le compte des investissements et donc les faire figurer à l'actif du bilan. Cela permettrait de mettre en évidence le stock d'infrastructure militaire accumulé. 
les directions qui recourent à des prestations sollicitées auprès d'autres directions. Par exemple, les directions qui recourent aux prestations de la direction des services informatiques peuvent se voir imputer le coût de ces prestations. Ces charges se retrouvent en correspondance avec des revenus pour les directions prestataires des services offerts à l'interne (49). L'égalité doit ainsi globalement être parfaite entre les montants de ces deux rubriques en charges et en revenus. Le chapitre 4 est consacré au mécanisme des imputations internes.

Tableau 16 Rubriques du compte de résultats selon la classification par nature. MaCollectivité, Année t, en KCHF.

\begin{tabular}{|c|c|c|c|c|c|}
\hline$N^{\circ}$ & Libellé & KCHF & $\mathrm{N}^{\circ}$ & Libellé & KCHF \\
\hline 3 & Charges & 14546 & 4 & Revenus & 14610 \\
\hline 30 & Charges de personnel & 2020 & 40 & Revenus fiscaux & 7651 \\
\hline 31 & $\begin{array}{l}\text { Charges de biens et services et } \\
\text { autres charges d'exploitation }\end{array}$ & 4076 & 41 & Patentes et concessions & 12 \\
\hline 32 & $\begin{array}{l}\text { Charges d'armement } \\
\text { (Confédération uniquement) }\end{array}$ & & 42 & Taxes et redevances & 5076 \\
\hline 33 & $\begin{array}{l}\text { Amortissements du patrimoine } \\
\text { administratif }\end{array}$ & 671 & 43 & Revenus divers & 17 \\
\hline 34 & Charges financières & 125 & 44 & Revenus financiers & 167 \\
\hline 35 & $\begin{array}{l}\text { Attributions aux financements } \\
\text { spéciaux [FSp] et fonds }\end{array}$ & 0 & 45 & $\begin{array}{l}\text { Prélèvements sur les finance- } \\
\text { ments spéciaux [FSp] et fonds }\end{array}$ & 23 \\
\hline 36 & Charges de transferts & 6393 & 46 & Revenus de transferts & 1373 \\
\hline 37 & Subventions redistribuées & 0 & 47 & Subventions à redistribuer & 0 \\
\hline 38 & Charges extraordinaires [e.o.] & 991 & 48 & Revenus extraordinaires & 21 \\
\hline 39 & Imputations internes & 270 & 49 & Imputations internes & 270 \\
\hline
\end{tabular}

Charges extraordinaires. Une rubrique n'a pas été évoquée jusqu'ici, celle des charges extraordinaires $(38)^{74}$. En fait, le $\mathrm{MCH} 2$ réunit dans cette seule rubrique deux types de charges très différentes, ainsi que l'illustre la figure 17.

On trouvera le plus souvent dans cette rubrique des charges qui n'ont pas de réalité économique et qui sont enregistrées dans une logique de tactique politique

74 Voir aussi le Complément à la Recommandation 04 élaboré par le Conseil suisse de présentation des comptes publics concernant les critères relatifs à la comptabilisation des éléments extraordinaires (www.srs-cspcp.ch). 
en matière de présentation des comptes. Le $\mathrm{MCH} 2$ parle ici - pudiquement - d'opération de politique budgétaire ${ }^{75}$. Il s'agit en particulier de charges que le $\mathrm{MCH} 2$, à l'instar du MCH1, qualifie d'amortissements supplémentaires (sous-rubriques 383 et 387). Ces charges n'ont toutefois aucun lien avec l'usure et l'obsolescence du patrimoine administratif. Elles n'ont donc pas de réalité économique, au contraire des «vrais» amortissements que l'on enregistre dans la rubrique $33^{76}$. Les attributions au capital propre (389) répondent également à des impératifs de tactique politique et correspondent à des mises en réserve, notamment sous la forme de préfinancements (compte 3893). Le montant de KCHF 991, soit près de CHF 1 mio, porté en charges par MaCollectivité répond donc à cette logique de politique budgétaire.

Figure 17 Les charges extraordinaires sont de nature très différente.

Opération de tactique politique et charge véritablement extraordinaire.

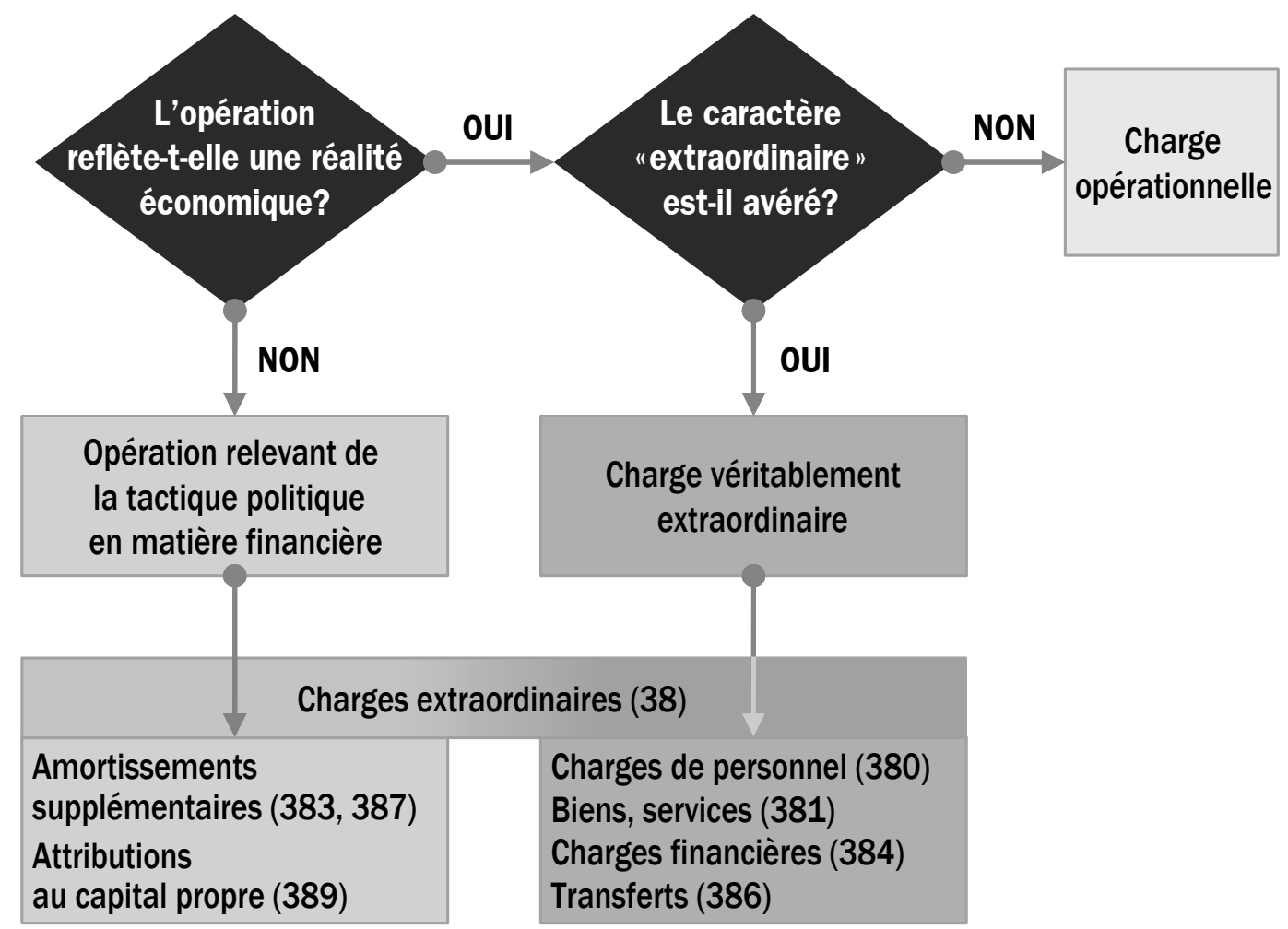

Le deuxième type de charges enregistrées dans la rubrique 38 découle d'événements véritablement extraordinaires. Pour que le caractère extraordinaire soit avéré, quatre conditions doivent être cumulativement remplies, faute de quoi les charges 
qui en découlent sont des charges tout bonnement opérationnelles. Ces conditions sont les suivantes:

a. L'événement et la charge qui en découlent ne pouvaient en aucune manière être prévus;

b. L'événement et la charge sont sans lien avec l'activité opérationnelle de la collectivité, c'est-à-dire qu'ils ne s'inscrivent pas dans les processus ordinaires de la collectivité;

c. Il était impossible, pour les autorités de la collectivité, de les influencer et de les contrôler d'une quelconque façon;

d. Le montant en jeu est important (voir le principe del'importance en section 2.1.3).

Le langage courant qualifie souvent d'extraordinaire une charge parfaitement ordinaire, dont seule l'ampleur du montant est inhabituelle. Par exemple, pour une collectivité, payer des cotisations sociales à la caisse de pension de ses employés est habituel. Il peut devenir nécessaire de combler un déficit de couverture de cette caisse en raison d'un manque de contributions de l'employeur par le passé. La charge à supporter peut certes s'avérer inhabituelle par son importance. Mais elle ne peut pas être considérée comme extraordinaire; en particulier à la lumière des conditions $a, b$ et $c$ ci-dessus. Par conséquent, les conditions posées pour pouvoir classer des charges dans les sous-rubriques 380 (charges extraordinaires de personnel), 381 (charges extraordinaires de biens et services), 384 (charges financières extraordinaires) ou 386 (charges extraordinaires de transferts) sont restrictives. D’ailleurs, MaCollectivité n'enregistre aucune charge de ce type au cours de l'Année t.

Revenus par nature. Les revenus par nature comprennent les revenus courants, c'est-à-dire les revenus qui sont destinés à financer les dépenses qui n’apportent une utilité que pendant un seul exercice, voire moins. Ils comprennent également des revenus à caractère purement comptable, c'est-à-dire des revenus qui ne s'accompagnent pas d'une entrée de fonds.

Le tableau 16 présente l'ensemble des rubriques de revenus prévues dans le plan comptable du $\mathrm{MCH}$ 2. La numérotation de la plupart des rubriques fait écho à celle des rubriques de charges. Sans surprise, on y trouve les revenus fiscaux, autrement dit les recettes fiscales (rubrique 40), les recettes provenant des patentes et concessions octroyées (41), les taxes et redevances encaissées (42), les autres revenus divers générés par l'activité d'exploitation (43), les revenus financiers (44, qui inclut les revenus générés par le patrimoine financier, dont les intérêts encaissés 440). On y trouve également les revenus de transferts (46, qui englobe les dédommagements reçus d'autres entités 461, les versements provenant de la péréquation financière 462 et les subventions 463). Les subventions à redistribuer (47) sont le pendant des subventions redistribuées (37).

Deux rubriques sont dédiées à des revenus sans entrée de fonds. La rubrique 45 recense les prélèvements aux financements spéciaux et fonds effectués lorsque les recettes affectées sont inférieures au coût qu'elles sont censées couvrir. L'opération 
s'apparente à un prélèvement sur réserve. La rubrique 49 renseigne sur les revenus liés à des imputations internes avec comme pendant, en charges, la rubrique 39.

Revenus extraordinaires. À l'instar de la rubrique des charges extraordinaires (38), la rubrique des revenus extraordinaires (48) réunit deux types d'éléments très différents. C'est pourquoi le diagramme de la figure 17 s'applique par analogie.

Tableau 17 Présentation échelonnée du compte de résultats. MaCollectivité, Année t, en KCHF.

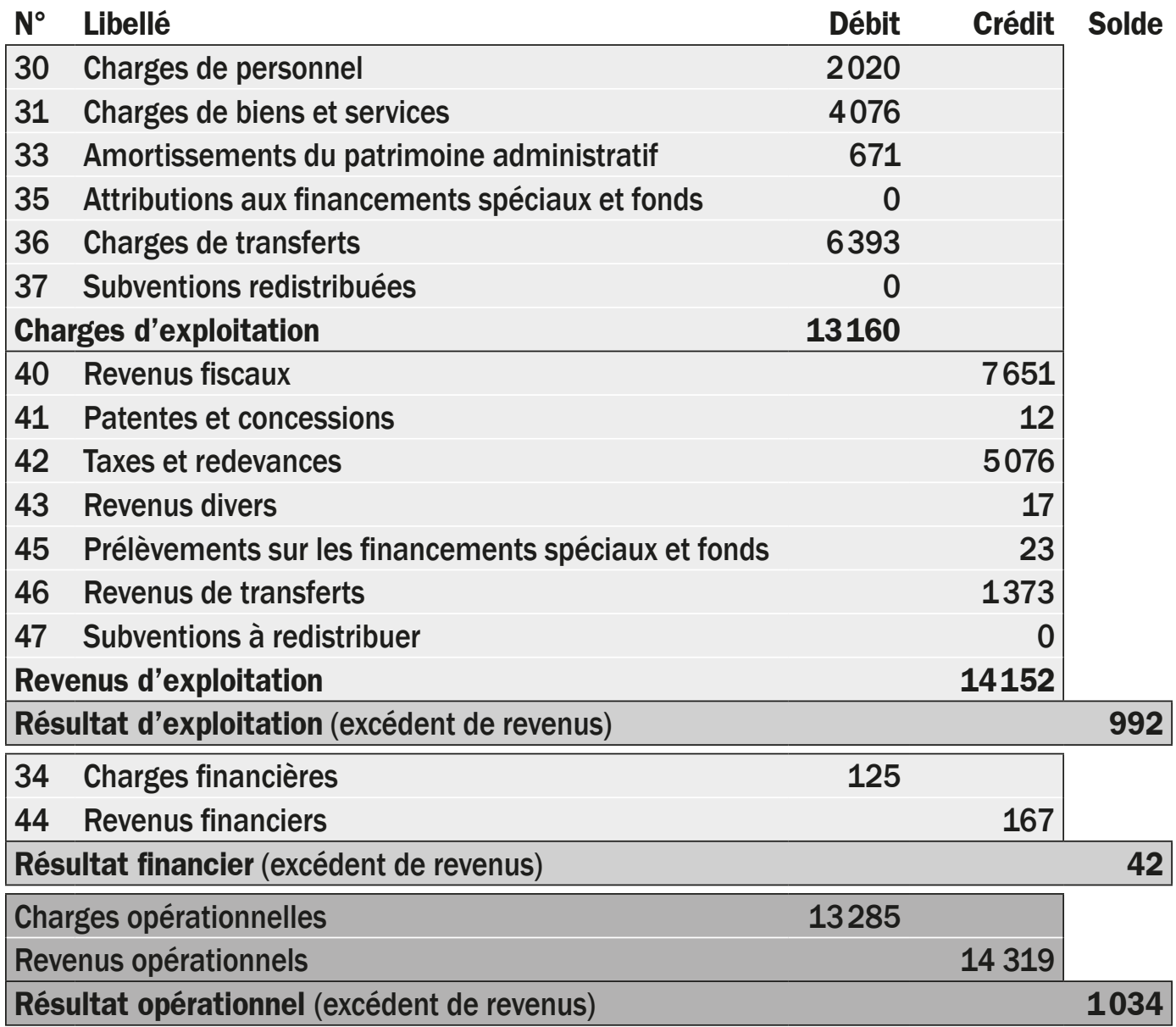

38 Charges extraordinaires

991

48 Revenus extraordinaires

21

Résultat extraordinaire (excédent de charges) 
Dans cette rubrique apparaîtront le plus souvent des revenus sans réalité économique, répondant à une logique de tactique politique en matière financière. Il s'agit des prélèvements sur le capital propre (489, dont les prélèvements sur les préfinancements et sur la réserve de politique budgétaire). Les quelques milliers de francs présentés dans la rubrique 48 par MaCollectivité répondent à cette logique (KCHF 21).

Il pourrait arriver que des revenus découlent d'événements véritablement extraordinaires. Pour que le caractère extraordinaire soit avéré, les quatre conditions mentionnées précédemment doivent être cumulativement remplies - faute de quoi les revenus qui en découlent sont des revenus tout bonnement opérationnels. Cela explique pourquoi les revenus fiscaux ne peuvent jamais être extraordinaires. Évidemment, certains impôts, comme l'impôt sur les successions ou donations, peuvent parfois générer des montants inhabituellement élevés. Mais collecter l'impôt est un processus opérationnel tout ce qu'il y a d'ordinaire pour une collectivité. À la lumière de la condition $b$ ci-dessus, le caractère extraordinaire doit donc être exclu dans ce cas. En réalité, on doit s'attendre à ce que les sous-rubriques 481 à 487 du plan comptable soient peu utilisées. MaCollectivité n'enregistre d'ailleurs aucun revenu de ce type au cours de l'Année t. Par conséquent, le total de la rubrique 48 (KCHF 21) découle uniquement d'opérations de politique budgétaire.

Solde du compte de résultats et présentation échelonnée. Le solde du compte de résultats renseigne sur le degré de couverture des charges. Un excédent de revenus par rapport aux charges accroît le capital propre au passif du bilan (rubrique 29), soit en augmentant l'excédent ou en réduisant le découvert du bilan (rubrique 299), soit en augmentant les financements spéciaux (rubrique 290) (voir la section 5.5.5 pour davantage d'informations). Cela correspond à une renonciation à consommer, c'est-à-dire à dépenser dans le cadre du fonctionnement courant. Autrement dit, dans son fonctionnement, la collectivité dégage une épargne. Cette épargne peut donc être utilisée pour financer l'investissement ou être placée dans le patrimoine financier.

Par contre, un excédent de charges réduit le capital propre au passif du bilan, soit en réduisant l'excédent ou en augmentant le découvert du bilan, soit en réduisant les financements spéciaux. Il s'agit d'une désépargne découlant du fonctionnement. Cette désépargne entame la substance patrimoniale de la collectivité. Cette situation peut s'expliquer de deux façons, soit (a) la collectivité vit au-dessus de ses moyens et est confrontée à des charges trop importantes, soit $(b)$ ses revenus - fiscaux notamment - sont trop faibles, on est alors en face d'une insuffisance de revenus.

Le $\mathrm{MCH} 2$ prévoit trois échelons de bouclement du compte de résultats, comme l'illustre le tableau 17. Le premier échelon dégage le résultat d'exploitation de la collectivité. Ce résultat correspond à la différence entre les revenus d'exploitation et les charges d'exploitation, soit un excédent de revenus de KCHF 992 au cours de l'Année t pour MaCollectivité. Le deuxième échelon renseigne sur le résultat 
financier, soit la différence entre les revenus financiers et les charges financières (KCHF 42). L'addition du résultat d'exploitation et du résultat financier conduit au résultat opérationnel (KCHF 1034 d'excédent de revenus). Celui-ci s'obtient également par différence entre les revenus opérationnels et les charges opérationnelles. Les revenus opérationnels sont composés des revenus d'exploitation et des revenus financiers; les charges opérationnelles correspondent à l'addition des charges d'exploitation et des charges financières. Ils n'incluent pas les éléments extraordinaires. Ces éléments apparaissent dans le troisième échelon qui renseigne sur le résultat extraordinaire (KCHF 970 d'excédent de charges). Le résultat total correspond à l'addition du résultat opérationnel et du résultat extraordinaire (autrement dit à la différence entre le total des revenus et le total des charges), soit KCHF 64 d'excédent de revenus.

Précédemment, le $\mathrm{MCH} 1$ ne préconisait pas l'échelonnement du compte de résultats (c'est-à-dire du compte de fonctionnement). L'échelonnement est donc une innovation du MCH2. Le principe de l'échelonnement n'apparaît pas dans les normes IPSAS. Mais il s'inspire des normes en vigueur dans le secteur privép ${ }^{77}$. Toutefois, ces normes ne connaissent que les deux premiers échelons. Elles excluent donc l'idée qu'il existe des éléments extraordinaires. Par contre, elles obligent à décomposer l'apport des opérations d'exploitation et des opérations financières au résultat.

Résultat d'exploitation ${ }^{78}$. Le résultat d'exploitation véhicule une information importante. Il permet de savoir si les finances de la collectivité sont fondamentalement équilibrées. Autrement dit, il montre si la collectivité couvre le coût des prestations que le droit public l'oblige à fournir avec les revenus que ce même droit public lui permet d'obtenir. En laissant en particulier de côté les éléments purement financiers, le résultat d'exploitation serait le résultat que la collectivité obtiendrait globalement si elle n'avait ni dette, ni patrimoine financier. Dans le cas de MaCollectivité, cet équilibre fondamental est largement assuré. En effet, l'excédent de revenus de KCHF 992 représente plus de 7\% des charges d'exploitation. Par contre, une commune ou un canton qui enregistrerait à ce stade un excédent de charges devrait $s^{\prime}$ interroger sur la manière de rééquilibrer la situation ${ }^{79}$.

77 La présentation échelonnée du résultat est requise par les normes suisses de présentation des comptes des petites et moyennes entités (Swiss GAAP RPC). Les normes internationales (International Financial Reporting Standards) se bornent à offrir un exemple de présentation échelonnée, sans pour autant rendre cette présentation contraignante.

78 Ergebnis aus betrieblicher Tätigkeit | Risultato operativo | Operating result.

79 Notons que les imputations internes ne figurent pas dans la présentation échelonnée du compte de résultats, ni en charges, ni en revenus, ni à l'échelon de l'exploitation, ni aux échelons suivants. On évite ainsi de compter à double les charges et les revenus imputés et de gonfler artificiellement le total des charges et des revenus. Il est d'ailleurs étonnant que les concepteurs du MCH n'aient pas renoncé à faire figurer les subventions redistribuées dans les charges d'exploitation (rubrique 37) et les subventions à redistribuer dans les revenus d'exploitation (47). En effet, ces subventions ne font que transiter par les comptes de la collectivité. Elles ne concernent pas l'exploitation, c'est-à-dire la fourniture de prestations par la collectivité. Et elles gonflent artificiellement le total des charges et des revenus. 
Résultat financier ${ }^{80}$. Le résultat financier aide à cerner les risques que les engagements au passif du bilan et les placements dans le patrimoine financier font peser sur la collectivité. En effet, une détérioration des conditions d'emprunt, notamment une hausse des taux d'intérêt débiteur, conduit à une dégradation du résultat financier. Il en va de même si les conditions de placement se péjorent en raison par exemple d'une baisse des taux créanciers ou des loyers des immeubles de rendement détenus par la collectivité. Le patrimoine financier peut également perdre de sa valeur. Or la correction à la baisse de la valeur de ce patrimoine entre dans les charges financières (en particulier à travers les rubriques 341 et 344).

Inversement, une amélioration des conditions d'emprunt, de placement, de location ou une augmentation de la valeur du patrimoine financier améliore le résultat financier. Dans le cas de MaCollectivité, on constate que la dimension financière apporte un léger excédent de revenus de KCHF 42.

Par conséquent, plus le patrimoine financier et les capitaux tiers de la collectivité sont importants, plus les opportunités de gains, mais aussi les risques de pertes sont élevés. Le résultat financier apporte donc une information sur une dimension que l'on pourrait qualifier de spéculative dans la gestion de la collectivité, dans la mesure où cette dimension est soumise, davantage que l'exploitation, à des influences qui échappent au contrôle de la collectivité.

Résultat opérationnel ${ }^{81}$, résultat extraordinaire ${ }^{82}$ et résultat total ${ }^{83}$. Le résultat opérationnel renseigne sur la performance de la collectivité au cours de l'exercice en faisant abstraction des éléments extraordinaires qui viennent brouiller l'image d'ensemble. Par rapport au résultat total, il permet de s'affranchir de l'influence des opérations de tactique politique. C'est ce que l'on constate dans le cas de MaCollectivité: les opérations de tactique politique créent un excédent de charges extraordinaires de KCHF 970 réduisant le résultat de KCHF 1034 d'excédent de revenus au niveau opérationnel à seulement KCHF 64 au total.

Dans cet exemple, les opérations extraordinaires contribuent donc à détériorer l'image que l'on peut globalement avoir de la performance financière de la collectivité. Évidemment, elles peuvent, suivant les cas, aussi être mises à profit pour améliorer l'image, notamment en recourant à des dissolutions de réserves ou de préfinancements qui viennent augmenter les revenus extraordinaires (voir le chapitre 8 consacré à la tactique politique en matière de présentation des comptes).

80 Ergebnis aus Finanzierung | Risultato finanziario | Financial result.

81 Operatives Ergebnis | Risultato operativo | Ordinary result.

82 Ausserordentliches Ergebnis | Risultato straordinario | Extraordinary result.

83 Gesamtergebnis | Risultato totale | Overall result. 


\subsubsection{Dépréciation du patrimoine administratif}

\section{Amortissement des immobilisations et des subventions d'investissement}

Une fois portée à l'actif, la valeur des dépenses d'investissement ordinaires et extraordinaires dans des immobilisations corporelles et incorporelles ${ }^{84}$, ainsi que des subventions d'investissement accordées ${ }^{85}$ doit être ajustée pour tenir compte de l'usure physique et de l'obsolescence technologique. Pour ce faire, une procédure d'amortissement doit être mise en place ${ }^{86}$.

L'idée générale est que la valeur au bilan reflète une estimation vraisemblable - puisque fondée sur la valeur d'acquisition -, mais aussi prudente - puisque revue à la baisse pour tenir compte d'une dépréciation économiquement justifiée.

Couverture ultérieure. La procédure d'amortissement permet de faire financer la dépense d'investissement par les générations d'utilisateurs qui profiteront de l'infrastructure ainsi créée et des prestations que cette infrastructure leur offrira. Le principe est donc celui de la couverture ultérieure. Le respect du principe de l'utilisateur-payeur et le souci de favoriser l'équité entre générations doivent conduire à rejeter la solution d'une couverture directe des dépenses d'investissement. La couverture directe consiste en effet à financer les dépenses de la période par les revenus du même exercice. Cela conduit également à rejeter le principe de la couverture préalable. Une telle couverture implique de faire préfinancer les investissements au cours des années qui précèdent la dépense - autrement dit, de faire supporter une charge à des contribuables qui ne bénéficient pas encore de l'infrastructure et des services concernés ou qui pourraient ne jamais en bénéficier s'ils venaient à quitter la collectivité avant la mise en service ${ }^{87}$.

La figure 18 illustre le mécanisme de l'amortissement reflétant l'usure et l'obsolescence de l'infrastructure. Elle montre la dépense initiale enregistrée dans le compte des investissements au cours de l'Année t et inscrite au bilan de clôture de la collectivité pour cette même année. Pour l'exemple, la dépense est de CHF 10 mio de francs pour un bâtiment administratif. La durée d'utilisation d'un bâtiment se situe généralement entre vingt-cinq et cinquante ans. Mais pour faciliter la représentation, nous écourtons cette durée à dix ans (qui correspond en réalité à la durée d'utilisation des biens meubles, des machines et des véhicules).

84 Rubriques de dépenses 50, 52, 580 et 582 correspondant aux rubriques 140 et 142 à l'actif du bilan.

85 Rubriques de dépenses 56 et 586 correspondant à la rubrique 146 à l'actif du bilan.

86 Recommandation 12, CDF (2008).

87 Lorsque des services doivent être financés spécialement, c'est-à-dire par une taxe, on admet généralement que la différence entre le coût d'acquisition historique de l'infrastructure d'une part et le coût de son futur renouvellement d'autre part fasse l'objet d'une couverture préalable. Pour ce faire, un fonds au passif du bilan est constitué et alimenté année après année (comptes 2091 ou 2910). Le montant de l'alimentation correspond à la différence entre les amortissements calculés par rapport à la valeur d'acquisition et l'amortissement qui aurait été mis à charge du compte de résultats s'il était calculé par rapport à la valeur de remplacement. Cette différence est mise à charge, non pas comme un amortissement (33), mais comme une attribution à un fonds (3501 ou 3511). Ce mécanisme est appliqué dans de nombreuses communes pour des prestations comme le traitement des eaux usées et la fourniture d'eau propre (voir également le chapitre 5 consacré aux financements spéciaux). 
Figure 18 Procédure d'amortissement avec couverture ultérieure.

Exemple d'une dépense de CHF 10 mio pour une immobilisation ayant une durée d'utilisation de dix ans.
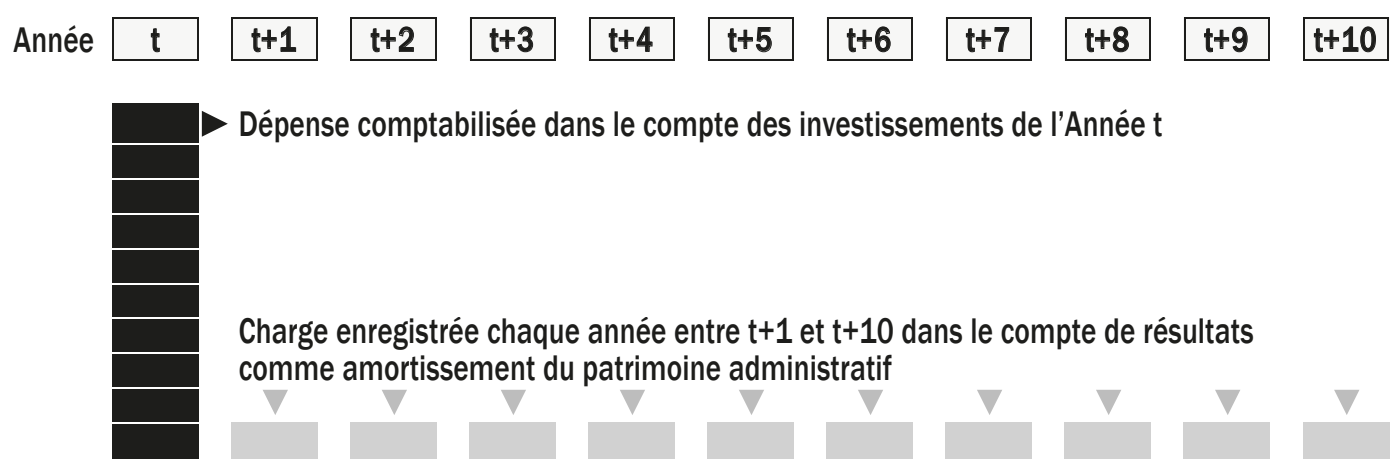

Charge enregistrée chaque année entre $t+1$ et $t+10$ dans le compte de résultats comme amortissement du patrimoine administratif

Valeur au bilan diminuant sous l'effet de l'amortissement
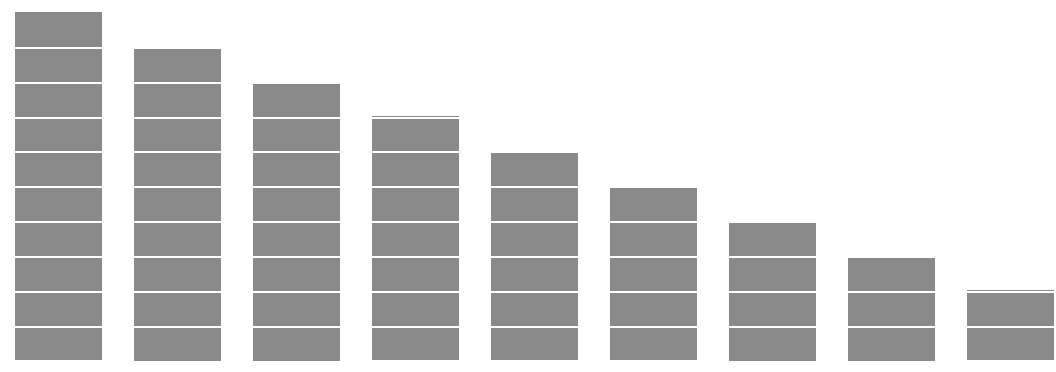

C'est pourquoi la figure montre ensuite l'amortissement de CHF 1 mio mis à charge du compte de résultats au cours de chacune des dix années suivantes, soit de $t+1$ à $t+10$. L'Année $t+1$ correspond à l'année de mise en service du bâtiment. C'est donc la première année pour laquelle on attend, notamment des contribuables, un apport de revenus suffisant pour couvrir le coût de l'usure et de l'obsolescence de cette infrastructure. Au total à la fin des dix années suivant l'investissement, les contribuables vont finalement avoir apporté les liquidités nécessaires au financement de l'investissement. Mais à l'Année $t$, la collectivité finance la dépense d'investissement, soit en utilisant son patrimoine financier - notamment ses liquidités -, soit en recourant à l'emprunt. Elle «fait donc la banque» vis-à-vis de ses contribuables en attendant qu'ils financent l'investissement initial via les tranches annuelles d'amortissement.

La figure montre enfin l'effet sur la valeur au bilan. Cette dernière rappelle le montant qui doit encore être mis à charge des utilisateurs. Elle diminue graduellement chaque année sous l'effet de la dépréciation économique, donc de l'amortissement. À la fin de l'Année t+10, la valeur au bilan n'est plus que de CHF 1 pour mémoire.

Amortissement linéaire et amortissement dégressif. Dans notre exemple, nous avons réparti linéairement le montant à amortir sur la durée d'utilisation de l'équipement. L'amortissement linéaire est l'une des deux possibilités prévues par le 
$\mathrm{MCH} 2$ et nécessite d'appliquer un taux d'amortissement constant sur la valeur d'acquisition, dans notre exemple 10\%. L'autre possibilité est l'amortissement dégressif. Le taux d'amortissement est également constant, mais il s'applique à la valeur résiduelle de l'actif au bilan. Par conséquent, à durée d'utilisation équivalente, la méthode de l'amortissement dégressif implique un taux plus élevé que celle de l'amortissement linéaire. À ce propos, le tableau 18 renseigne sur la durée d'utilisation et les taux d'amortissement recommandés par le $\mathrm{MCH} 2$ en fonction de l'actif concerné et de la méthode retenue. Il indique par exemple que pour une durée d'utilisation de dix ans (biens meubles, machines, véhicules), le taux d'amortissement selon la méthode dégressive est de $35,0 \%$, contre $10,0 \%$ pour la méthode linéaire.

Tableau 18 Durée d'utilisation et taux d'amortissement.

\begin{tabular}{|c|c|c|c|c|}
\hline \multirow[t]{2}{*}{ Catégories d'immobilisations } & & \multirow{2}{*}{$\begin{array}{c}\text { Durée } \\
\text { d'utilisation } \\
\text { en années }\end{array}$} & \multicolumn{2}{|c|}{$\begin{array}{c}\text { Taux d'amortissement } \\
\text { en } \%\end{array}$} \\
\hline & & & Linéaire & Dégressif \\
\hline Terrains & & \multicolumn{3}{|c|}{ Aucun amortissement } \\
\hline Bâtiments, terrains bâtis & & $25-50$ & $4,0-2,0$ & $15,0-8,0$ \\
\hline \multirow[t]{3}{*}{ Travaux de génie civil } & Route & $40-60$ & $2,5-1,7$ & $10,0-7,0$ \\
\hline & Canaux & $40-60$ & $2,5-1,7$ & $10,0-7,0$ \\
\hline & Ponts & $40-60$ & $2,5-1,7$ & $10,0-7,0$ \\
\hline Biens meubles, machines, véhicules & & $4-10$ & $25,0-10,0$ & $60,0-35,0$ \\
\hline $\begin{array}{l}\text { Immobilisations incorporelles (droit } \\
\text { de brevet, de raison de commerce, } \\
\text { d'édition, de concession, de licence } \\
\text { et autres droits d'utilisation, goodwill) }\end{array}$ & & 5 & 20,0 & 50,0 \\
\hline \multirow[t]{2}{*}{ Informatique } & Matériel & 3 & 33,3 & 60,0 \\
\hline & Logiciel & 5 & 20,0 & 50,0 \\
\hline Installations d'évacuation des eaux & & 15 & 6,5 & 25,0 \\
\hline Installations de traitement des déchets & & 40 & 2,5 & 10,0 \\
\hline
\end{tabular}

Source: Recommandation 12, CDF (2008).

La figure 19 permet de comparer l'effet des deux méthodes sur la valeur résiduelle de l'actif. On constate que l'amortissement dégressif engendre une dépréciation importante dès la mise en service de l'équipement et donc un amortissement initial plus important à charge du compte de résultats. Généralement, cette solution reflète moins bien le rythme de l'usure et de l'obsolescence que l'amortissement linéaire. Par conséquent, elle respecte moins l'équité entre générations de contribuables et d'utilisateurs. Mais des raisons de politique financière peuvent amener à 
retenir l'amortissement dégressif. En effet, l'importance de l'amortissement initial incite les membres des Exécutifs et des Législatifs à la retenue et à la prudence: les responsables qui décident d'un investissement doivent assumer l'essentiel de l'impact sur le compte de résultats au cours de leur propre mandat électif.

\section{Figure 19 Valeur résiduelle au bilan selon que l'amortissement est linéaire ou dégressif. Exemple d'un actif ayant une valeur initiale de CHF 10 mio et une durée d'utilisation de dix ans.}

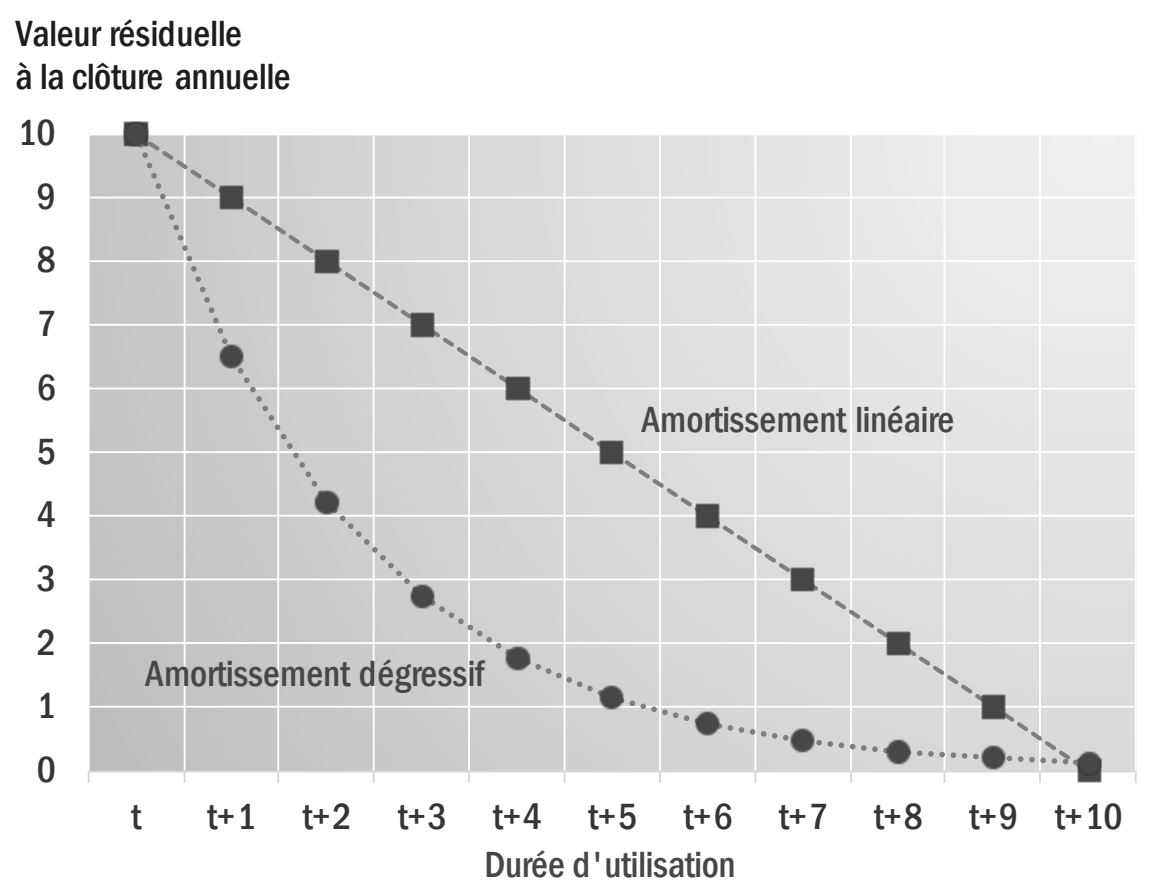

Notons que le choix entre amortissement linéaire ou dégressif est un choix de principe. Il est donc valable pour tous les investissements, quel que soit l'actif concerné. On ne peut amortir linéairement un actif et dégressivement un autre. Le taux d'amortissement est dicté par le type d'actifs, mais il est plus généralement dicté par la législation. Il est en outre consigné dans le crédit d'engagement voté par le Législatif ou dans la documentation à l'appui de la demande de crédit introduite par l'Exécutif. Il reste donc constant sur toute la durée d'utilisation et ne peut être modifié en cours de route, sous réserve d'une nouvelle décision du Législatif. Cela évite la tentation de vouloir le manipuler dans une logique de tactique politique, par exemple la tentation de le réduire pour éviter un excédent de charges d'exploitation ou de l'augmenter pour dissimuler un excédent de revenus.

Qu'elle s'opère à travers un amortissement linéaire ou dégressif, la couverture ultérieure offre ainsi trois avantages. D'abord, les collectivités de petites tailles doivent souvent procéder à des investissements lourds, mais ponctuels. Une 
couverture directe impliquerait qu'elles doivent massivement augmenter les recettes au cours de l'exercice touché par l'investissement. Ce faisant, elles pourraient avoir à accroître significativement et momentanément la pression fiscale sur leurs contribuables, quitte à réduire la pression l'année suivante. En comparaison, la couverture ultérieure offre l'avantage de lisser l'effort de financement par les contribuables et, plus généralement, les habitants. Elle permet à travers l'autofinancement d'assurer a posteriori un financement approprié des investissements.

Ensuite, la couverture ultérieure permet une comparaison pertinente du solde du compte de résultats à travers le temps. En effet, puisque la charge d'investissement se répartit sur tous les exercices qui suivent le moment de la dépense, la signification économique du résultat est maintenue et le principe de l'utilisateur-payeur est donc respecté.

Enfin, la couverture ultérieure permet de connaître le coût des prestations publiques, puisque la charge d'amortissement reflète aussi la consommation - ou l'usure et l'obsolescence-del'équipement. Cela facilite les comparaisons entre collectivités publiques. Opération non monétaire. L'opération comptable touche donc, au doit, le compte des amortissements du patrimoine administratif (compte 3300 pour les immobilisations corporelles et 3320 pour les immobilisations incorporelles ${ }^{88}$ ). La procédure est celle de l'amortissement direct puisque, à l'avoir, elle touche - en déduction la rubrique 140 qui recense les immobilisations corporelles (ou la rubrique 142 en cas d'immobilisations incorporelles), réduisant d'autant la valeur de ces actifs d'une clôture à l'autre. C'est ainsi que globalement des amortissements à hauteur de KCHF 671 ont été mis à charge du compte de résultats de MaCollectivité de l'Année $\mathrm{t}$ (tableau 16).

Pour ce qui concerne l'amortissement des subventions d'investissement accordées, l'opération touche, au doit, le compte des amortissements de ces subventions à l'intérieur de la rubrique 366 (compte 3660) dans la mesure où il s'agit par nature d'une charge de transferts ${ }^{89}$. À l'avoir - en déduction -, elle touche la rubrique 146 qui recense les subventions d'investissement.

La comptabilisation de l'usure et de l'obsolescence est donc une opération uniquement comptable. Elle ne donne lieu à aucun décaissement. C'est pourquoi on qualifie l'amortissement économique d'opération non monétaire.

Cette charge ne doit pas être confondue avec l'amortissement financier. L'amortissement financier consiste à rembourser un emprunt. Par conséquent, l'amortissement

Ces rubriques sont prévues pour les amortissements planifiés sur la durée d'utilisation de l'immobilisation. Lorsque la durée d'utilisation est raccourcie par suite de détériorations (par exemple en raison de la panne irrémédiable d'un véhicule), il faut alors comptabiliser des amortissements non planifiés en utilisant les comptes 3301 et 3321. La notion d'amortissements non planifiés ne doit pas être confondue avec celle d'amortissements supplémentaires. Les premiers sont économiquement justifiés par le fait que l’actif (le véhicule) est devenu inutilisable. Les seconds n'ont aucune raison d'être économique. Leur utilisation est une opération de tactique politique en matière de présentation des comptes.

89 Lorsque la durée d'utilisation est raccourcie par suite de détériorations, on enregistre des amortissements non planifiés en utilisant le compte 3661. 
financier implique un décaissement. L'opération ne touche que des comptes du bilan: au doit, les rubriques 201 et 206 qui recensent, dans les capitaux de tiers, l'emprunt ainsi remboursé et, à l'avoir, le compte des disponibilités utilisées à cette fin (par exemple 1001 Compte postal). Contrairement à l'amortissement économique ou comptable, l'amortissement financier est donc une opération monétaire.

Ces deux opérations - l'amortissement de l'immobilisation et l'amortissement financier de l'emprunt - peuvent être simultanées ou au contraire dissociées l'une de l'autre. Certains auteurs militent pour la simultanéité (Dafflon 1994). La dette contractée pour financer une infrastructure est remboursée au rythme de l'usure et de l'obsolescence de cette dernière. De cette manière, elle s'éteint à l'issue de la durée d'utilisation. Évidemment, l'argument ne tient que lorsque la collectivité contracte systématiquement un emprunt pour financer un objet. Or ce n'est bien souvent pas le cas. Une collectivité ayant atteint une certaine taille procède à des emprunts sans les lier directement à des objets particuliers. La dissociation des deux opérations permet de mettre en ouvre une stratégie de placement et d'emprunt et de minimiser le coût de financement (commission, émolument, négoce, intérêts actifs et passifs). Cette stratégie, pour être adéquate, ne doit pas être verrouillée en obligeant que les emprunts soient remboursés (amortissement financier) en fonction de l'amortissement comptable (voir à ce propos le chapitre 7 consacré aux modalités de financement des investissements et d'emprunt).

\section{Perte de valeur des prêts et participations}

La procédure d'amortissement en fonction de l'usure et de l'obsolescence ne concerne pas les prêts et les participations classés dans le patrimoine administratif $^{90}$. Initialement, les prêts et participations sont certes également portés au bilan à la valeur d'acquisition. Mais ensuite, le traitement de leur valeur au bilan diffère de celui qui est réservé aux immobilisations (et aux subventions d'investissement). En effet, la valeur des prêts et participations doit être réexaminée à intervalles réguliers sur la base de la valeur vénale (valeur de marché) et être corrigée si cette dernière se situe au-dessous de la valeur au bilan. Par conséquent, une opération ne doit être comptabilisée que si ces actifs ont perdu de leur valeur, sinon aucune opération n'est effectuée. Ici aussi, l'idée est que la valeur au bilan reflète une estimation vraisemblable, mais prudente.

En cas de perte de valeur d'un prêt, l'opération comptable touche, au doit, le compte de réévaluation (rubrique 364) et, à l'avoir, la rubrique 144, pour le montant de la perte de valeur. Lorsqu'il s'agit d'une participation, l'opération comptable touche le compte de réévaluation (rubrique 365) et la rubrique 145. Dans les deux cas, il s'agit d'une opération uniquement comptable et donc non monétaire, sans décaissement. 


\subsubsection{Correction de valeur du patrimoine financier}

Nous avons vu que la valeur au bilan des actifs composant le patrimoine administratif doit être dépréciée pour tenir compte de l'usure et de l'obsolescence des immobilisations et pour tenir compte de la perte de valeur des prêts et des participations. Par conséquent, au bilan, ces différents actifs ne peuvent que voir leur valeur diminuer.

Dans le cas du patrimoine financier, les valeurs au bilan sont corrigées en fonction de l'évolution de la valeur vénale des actifs (valeur de marché). Par conséquent, la correction peut se faire à la baisse si l'actif perd de sa valeur, comme à la hausse si sa valeur augmente ${ }^{91}$. Dans le cas des placements financiers à long terme (rubrique 107: actions, obligations, etc.), il faut adapter leur valeur au bilan, de manière à ce qu'elle corresponde à leur valeur vénale à chaque date de clôture. Dans le cas des immobilisations corporelles (rubrique 108), la valeur doit être adaptée tous les trois à cinq ans en se référant également à leur valeur vénale.

Il y a une logique à faire figurer la valeur vénale de ces actifs au bilan: les engagements nets de la collectivité - dette nette - peuvent ainsi être estimés de manière plus précise. Rappelons que les engagements nets correspondent aux capitaux de tiers (essentiellement formés de la dette brute), déduction faite du patrimoine financier - autrement dit, à ce qui resterait des capitaux de tiers si le patrimoine financier était intégralement réalisé (vendu) et que les liquidités ainsi dégagées étaient utilisées pour rembourser la dette de la collectivité.

En cas de moins-value d'un placement financier, l'opération comptable touche, au doit, le compte de réévaluation 3440 et, à l'avoir, la rubrique 107, pour le montant de la moins-value. En cas de moins-value d'une immobilisation corporelle, l'opération touche le compte de réévaluation 3441 et la rubrique 108.

En cas de plus-value, l'opération touche, au doit, les comptes actifs concernés (107 et 108) et, à l'avoir, les comptes 4440 et suivants, pour le montant de la plus-value.

Deux remarques s'imposent ici. D'abord, on voit que ces opérations de correction de valeur influencent le compte de résultats au niveau du résultat financier, soit du côté des charges (34), soit du côté des revenus (44). Ainsi, le résultat est susceptible d'être sensiblement influencé par les conditions du marché. Par conséquent, détenir des actifs financiers présentant une forte volatilité représente un risque pour la performance de la collectivité. Ensuite, les opérations de correction de valeur sont des opérations uniquement comptables et donc non monétaires. Elles n'impliquent ni décaissement, ni encaissement. Une éventuelle moins-value ou plus-value n'aura de conséquence monétaire qu'au moment où l'actif en question sera réalisé. 


\subsubsection{Tableau des flux de trésorerie}

Impact des activités sur la solvabilité à court terme. Nous avons vu précédemment que le compte de résultats nous permet de savoir dans quelle mesure la collectivité a pu couvrir ses coûts grâce aux revenus de l'exercice. De son côté, le compte des investissements nous indique quel effort la collectivité doit fournir elle-même pour financer ses investissements après avoir tenu compte des contributions externes obtenues, par exemple des subventions d'investissement. Nous avons également vu ci-dessus que certaines charges et certains revenus n'ont pas d'impact monétaire. Par conséquent, le compte de résultats et le compte des investissements n'offrent pas une base suffisante pour connaître l'impact des activités sur la trésorerie et plus généralement sur le bilan. Or l'évolution de la trésorerie est un élément important pour s'assurer de la solvabilité à court terme de la collectivité. C'est pourquoi le $\mathrm{MCH} 2$ préconise la présentation d'un élément additionnel: le tableau des flux de trésorerie ${ }^{92}$. Précédemment, le $\mathrm{MCH} 1$ avait introduit un tel élément, mais sous la forme embryonnaire d'un tableau de financement. Dans la mesure où ce tableau de financement est parfois encore utilisé, nous commençons par en rappeler les principes avant d'en venir au tableau des flux de trésorerie proprement dit.

\section{Tableau de financement selon MCH1}

La figure 20 montre de manière schématique comment le MCH1 décrit le financement de l'investissement net et quel est l'impact des opérations sur les grandes masses du bilan. En partant de la gauche, le compte des investissements dégage l'investissement net de la période - c'est-à-dire qu'il déduit de l'investissement brut l'ensemble des recettes acquises pour l'investissement. L'investissement net correspond donc à l'insuffisance de financement de l'investissement brut. Il s'agit du montant que la collectivité devra (budget) ou a dû (comptes) financer par ses propres moyens. L'insuffisance s'élève pour MaCollectivité à KCHF 1928, soit près de CHF 2 mio, à l'issue de l'Année $t$; c'est son investissement net.

Le fonctionnement courant de la collectivité est susceptible de dégager tout ou partie des moyens nécessaires pour financer l'investissement net. Pour en connaître le montant, il faut s'intéresser au compte de résultats, à droite dans notre schéma. Nous avons isolé les charges et les revenus monétaires des éléments non monétaires. Ces derniers sont composés des amortissements liés à l'usure et à l'obsolescence, soit KCHF 671. Les autres opérations non monétaires sont les opérations sur les financements spéciaux et les fonds (+rubrique 35 - rubrique 45), les imputations internes $(+39-49)$, ainsi que les opérations extraordinaires $(+38-48)$ et l'activation des prestations propres (dans le 43), soit KCHF 930. Compte tenu de cela, les revenus monétaires, autrement dit les revenus ayant occasionné une entrée de liquidités (ou d'équivalents de trésorerie), s'élèvent à KCHF 14279 (donc un peu 
plus de CHF 14 mio). Quant à elles, les charges monétaires s'élèvent à KCHF 12614 (soit plus de CHF 12 mio). Par différence, on voit que le fonctionnement courant a apporté des liquidités pour un montant de KCHF 1665 (près de CHF 2 mio).

Avec ce montant, MaCollectivité peut financer elle-même une partie de son investissement net. C'est donc l'autofinancement ${ }^{93}$ dégagé par son fonctionnement. Autrement et succinctement dit, l'autofinancement, c'est l'épargne dégagée par le fonctionnement au cours de l'Année t. Le montant de l'autofinancement s'obtient également en ajoutant aux éléments non monétaires l'excédent de revenus de l'exercice (KCHF $671+$ KCHF $930+$ KCHF 64).

Figure 20 Tableau de financement selon $\mathrm{MCH} 1$.

Présentation schématique du bouclement des comptes de MaCollectivité à la fin de l'Année t, en KCHF.

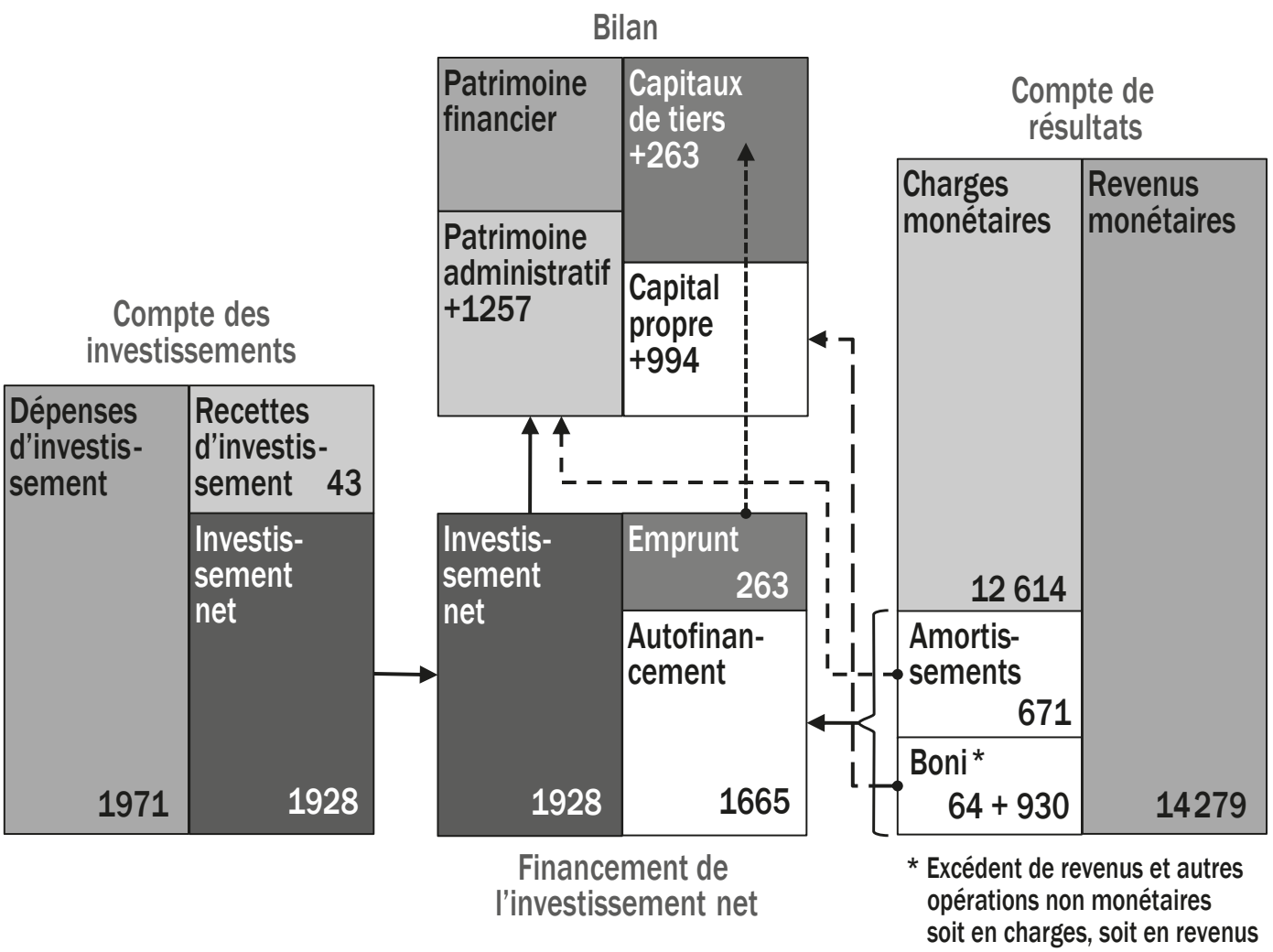

La partie centrale du schéma indique si l'autofinancement suffit à couvrir l'investissement net. Si l'autofinancement ne suffit pas, cela signifie que la collectivité a dû accroître son endettement, en particulier en augmentant son recours à des capitaux de tiers. C'est le cas de MaCollectivité. En effet, le besoin de financement de 
l'investissement net est supérieur de KCHF 263 à l'autofinancement ${ }^{94}$. Autrement dit, l'autofinancement de KCHF 1665 couvre environ 90\% des KCHF 1928 d'investissement net. À ce propos, mentionnons que la législation impose parfois aux collectivités de financer par elles-mêmes un pourcentage minimum de leurs investissements nets. Le pourcentage exigé se situe généralement en tout cas à $60 \%$ (voir également la section 9.3.1 consacrée aux indicateurs d'équilibres budgétaires).

La figure 20 indique de manière succincte comment les masses du bilan évoluent sous l'effet de ces opérations. La valeur du patrimoine administratif augmente du montant de l'investissement net, mais simultanément se réduit sous l'effet de l'usure et de l'obsolescence comme en témoigne l'amortissement. Au cours de l'Année t, le patrimoine administratif de MaCollectivité a donc progressé de KCHF 1257 (= KCHF 1928 - KCHF 671). Les autres opérations non monétaires, ainsi que l'excédent de revenus influencent pour l'essentiel le capital propre. Ici, le capital propre progresse de KCHF 994 (= KCHF 64 + KCHF 930). Finalement, l'emprunt augmente les capitaux de tiers de KCHF 263. Par conséquent, l'actif augmente du même montant que le passif (KCHF 1257), garantissant ainsi l'équilibre du bilan.

La figure 20 illustre le cas de MaCollectivité, avec un excédent de revenus ainsi qu'un déficit de financement des investissements supérieur à l'autofinancement. D’autres cas peuvent émerger. Nous nous bornons ici à mentionner quelques cas particulièrement remarquables:

- Si l'autofinancement est plus élevé que l'investissement net, la collectivité réduit son endettement.

- Lorsque l'excédent de charges au compte de résultats est particulièrement élevé, il se peut qu'il soit supérieur au montant des amortissements. Autrement dit, les revenus monétaires sont inférieurs aux charges monétaires. L' «épargne» dégagée par le fonctionnement est négative. Cela conduit à un autofinancement négatif. La collectivité doit alors s'endetter, non seulement pour financer son investissement net, mais également pour couvrir une partie de ses charges monétaires. C'est là une situation financière très problématique.

- Lorsque l'excédent de charges au compte de résultats reste modeste et inférieur au montant des amortissements, l'autofinancement est encore positif. Cela signifie que les revenus monétaires sont encore supérieurs aux charges monétaires. La collectivité doit toutefois s'endetter pour couvrir la quasi-totalité de son besoin de financement de l'investissement net.

- Il se peut que l'investissement net soit négatif, autrement dit que les recettes d'investissement reçues à l'Année t soient supérieures aux dépenses d'investissement.

94 Plutôt que d'emprunter et d'accroître sa dette brute et ses capitaux de tiers, la collectivité peut mobiliser son patrimoine financier afin de dégager les liquidités qui lui sont nécessaires pour financer son investissement net. Dans ce cas, certes la dette brute n'augmente pas, mais la dette nette augmente malgré tout parce que le patrimoine financier est réduit. Par conséquent, dans les deux cas la dette nette s’alourdit. 
Cela se produit lorsque les subventions d'investissement sont versées par l'entité subventionnante avec retard. La collectivité subventionnée n’a alors pas de besoin de financement et réduit son endettement grâce à son autofinancement auquel s'ajoute l'investissement net négatif.

\section{Tableau des flux de trésorerie selon $\mathrm{MCH} 2$}

Le $\mathrm{MCH} 2$ conseille de présenter un tableau des flux de trésorerie complet et plus détaillé, équivalent à celui des entreprises et répondant aux normes IPSAS ${ }^{95}$. Le but est de montrer les liquidités générées ou, au contraire, consommées par les différentes activités de la collectivité: (a) activité opérationnelle, (b) activités d'investissement et de placement et (c) activité de financement. La distinction effectuée sous le point $(b)$ entre l'activité d'investissement à proprement parler et celle de placement découle de celle qui est faite au bilan entre patrimoine administratif et patrimoine financier. Le tableau des flux de trésorerie renseigne ainsi sur les raisons de la variation des liquidités et des placements à court terme (caisse, poste, banque et équivalents de trésorerie) entre le $1^{\text {er }}$ janvier et le 31 décembre.

Activité opérationnelle. La première partie du tableau des flux de trésorerie renseigne sur la variation des liquidités et des placements à court terme engendrée spécifiquement par les encaissements et décaissements découlant de l'activité opérationnelle ${ }^{96}$. Le flux entrant est en particulier généré par les recettes fiscales, les taxes et redevances, tandis que le flux sortant est causé par les dépenses de personnel, d'achat de biens et services, ou encore de transferts. Deux méthodes peuvent être utilisées pour dégager le flux net lié à l'activité opérationnelle. La première, dite méthode directe, consiste à additionner les entrées de trésorerie et à soustraire les sorties de trésorerie. La seconde, dite méthode indirecte, reprend le solde du compte de résultats et le corrige de toutes les opérations non monétaires qui ont été passées et qui n'ont qu'un caractère comptable. Ainsi, on isole le flux de fonds provenant uniquement des opérations liées aux activités opérationnelles et ayant une influence sur la trésorerie. Tant la méthode directe que la méthode indirecte sont admises par le MCH2 et les normes IPSAS. Le tableau 19 pour MaCollectivité utilise la méthode indirecte. C'est la méthode retenue par la quasi-totalité des collectivités publiques en Suisse. Elle permet de faire explicitement le lien avec le solde du compte de résultats tel qu'il a été présenté en amont dans le rapport des comptes (KCHF 64). On découvre ainsi que l'activité opérationnelle de MaCollectivité a apporté un surcroît de trésorerie de KCHF 1989.

Notons que cette somme est largement supérieure à l'excédent de revenus opérationnels affiché par le compte de résultats (KCHF 1034, voir tableau 17). La différence s'explique par le fait que le compte de résultats, contrairement au tableau des flux de trésorerie, enregistre également des opérations non monétaires sans influence sur la 
liquidité. C'est pour cette raison que le tableau des flux de trésorerie parvient ici à une estimation proche de l'autofinancement dégagé par le tableau de financement selon $\mathrm{MCH} 1$ que nous venons de voir (KCHF 1665). Notons aussi que parfois, la différence peut être amplifiée par des encaissements et des décaissements provoqués par des événements réellement extraordinaires (c'est-à-dire ne relevant pas de la tactique politique). En effet, dans le tableau des flux de trésorerie, les encaissements et décaissements véritablement extraordinaires sont enregistrés comme des encaissements et des décaissements provenant de l'activité opérationnelle, alors que le compte de résultats les présente séparément dans le résultat extraordinaire. Cela étant, l'activité opérationnelle apporte en principe davantage de liquidités qu'elle n'en consomme.

Activité d'investissement. La partie suivante du tableau est dédiée à l'activité d'investissement proprement dite ${ }^{97}$. Elle renseigne sur la trésorerie consommée pour constituer du patrimoine administratif et sur l'apport de trésorerie lié à cette activité lorsque la collectivité encaisse une contribution de la part d'un tiers. Ici aussi, il existe deux méthodes pour déterminer le flux de trésorerie provenant de l'investissement. La méthode directe consiste à soustraire les décaissements des encaissements liés à cette activité. La méthode indirecte consiste à partir du solde du compte des investissements, c'est-à-dire de l'investissement net, puis à épurer ce solde des opérations n'ayant pas d'incidence sur les liquidités, notamment les transferts d'actifs entre le patrimoine financier et le patrimoine administratif (et vice versa). Les deux méthodes sont compatibles avec le $\mathrm{MCH} 2$ et les normes IPSAS. Le tableau 19 pour MaCollectivité utilise la méthode directe, méthode retenue par toutes les collectivités publiques en Suisse. On peut constater que l'activité d'investissement a consommé la trésorerie de MaCollectivité pour un montant de KCHF 1911. Ce montant est inférieur à celui de KCHF 1928 précédemment mis en évidence pour l'investissement net. La différence de KCHF 17 s'explique par la construction d'un abri forestier par le personnel de la voirie. Cette somme correspond donc à une prestation réalisée en propre. Elle a été comptabilisée comme une dépense d'investissement (bâtiments). Mais elle n'a pas donné lieu à une sortie de trésorerie dans le cadre de l'activité d'investissement. La sortie de trésorerie provoquée par l'activité d'investissement est également inférieure à l'investissement net lorsqu'un actif est transféré du patrimoine financier au patrimoine administratif pour être affecté à la fourniture de prestations publiques. Dans ce cas, l'actif est déjà propriété de la collectivité, comme un placement. Il n'y a donc pas non plus sortie de trésorerie.

De la même manière, le transfert d'un actif du patrimoine administratif au patrimoine financier, par exemple dans la perspective d'une vente à venir, n'aurait pas d'incidence sur les liquidités. Par conséquent, aucun flux n'apparaîtrait ni dans le flux de trésorerie provenant de l'activité d'investissement, ni dans le flux de trésorerie provenant de l'activité de placement. 
Le fait que le flux de trésorerie résultant de l'activité d'investissement soit négatif est conforme à la situation généralement observée dans les collectivités. Il est intéressant de comparer ce flux avec celui qui provient de l'activité opérationnelle. Cette comparaison offre une perspective alternative sur la faculté de la collectivité à financer son activité d'investissement grâce à son fonctionnement. C'est donc une façon alternative de considérer l'autofinancement. Dans le cas de MaCollectivité, le flux positif provenant de l'activité opérationnelle (KCHF 1989) compense à plus de 100\% le flux négatif causé par l'activité d'investissement (KCHF 1911). Ce pourcentage est donc sensiblement différent des quelque $90 \%$ qui pouvaient être calculés sur la base du tableau de financement selon MCH1 (figure 20).

Tableau 19 Tableau des flux de trésorerie préconisé par le MCH2.

Présentation simplifiée pour MaCollectivité et l'Année t, en KCHF.

+ Excédent de revenus du compte de résultats

KCHF

+ Amortissements du patrimoine administratif

+ Attributions à des comptes du capital propre

- Prélèvements sur des comptes du capital propre

- Activation de prestations propres

Correction de valeur de placements

- Correction de valeur de placements

+ Diminution des créances

- Augmentation des actifs de régularisation

+ Augmentation des engagements courants

+ Augmentation des provisions à court terme

- Diminution des passifs de régularisation $\quad-12$

- Diminution des financements spéciaux et fonds sous capitaux de tiers

- Dépenses pour des investissements nets avec incidence sur la trésorerie

Flux de trésorerie provenant de l'activité d'investissement [FTI]

Flux de trésorerie provenant de l'activité de placement [FTP]

Flux de trésorerie provenant de l'activité d'investissement et de placement [FTI+P] -1911

+ Augmentation des engagements financiers à court terme

- Remboursement d'engagements financiers à court terme

+ Augmentation des engagements financiers à long terme

- Remboursement d'engagements financiers à long terme 
Activité de placement. La suite du tableau est consacrée au flux de trésorerie provenant de l'activité de placement ${ }^{98}$. Elle renseigne sur les liquidités absorbées par les placements financiers et l'acquisition d'immobilisations corporelles appartenant au patrimoine financier (rubriques 107 et 108) ${ }^{99}$. Elle renseigne également sur les liquidités libérées par la vente de ces actifs. Une telle vente est possible puisque ces actifs, contrairement à ceux qui appartiennent au patrimoine administratif, ne sont pas utilisés par la collectivité pour remplir les obligations que lui confère le droit public. Au cours de l'Année t, aucune activité de placement n'a été réalisée dans MaCollectivité. C'est pourquoi le montant de CHF 0 est mentionné en regard de cette activité dans le tableau 19.

Activité d'investissement et de placement. Regrouper le flux de trésorerie provenant de l'activité d'investissement et le flux provenant de l'activité de placement permet de respecter la décomposition de l'actif en un patrimoine administratif et un patrimoine financier, tout en garantissant la conformité avec les pratiques du secteur privé et avec les normes IPSAS. En effet, ces normes ne connaissent pas une telle décomposition de l'actif. Dès lors, elles regroupent dans l'activité d'investissement à la fois les flux que le $\mathrm{MCH} 2$ subdivise entre investissement et placement ${ }^{100}$.

Activité de financement. Le tableau se termine avec le flux généré par l'activité de financement ${ }^{101}$. Cette activité apporte des liquidités lorsque la collectivité contracte des emprunts et elle consomme des liquidités au moment du remboursement d'emprunts. Par conséquent, cette partie du tableau renseigne sur l'évolution de la position de la collectivité vis-à-vis de ses bailleurs de fonds: un flux positif indique que la collectivité s'est engagée davantage vis-à-vis d'eux; un flux négatif indique que la collectivité a réduit ses engagements vis-à-vis d'eux. C'est cette seconde situation qui a prévalu au cours de l'Année $t$ : l'activité de financement a consommé la trésorerie de MaCollectivité à hauteur de KCHF 900 (près de CHF 1 mio). Ce montant s'explique principalement par le surcroît de remboursement d'engagements financiers à long terme. L'activité de financement joue donc un rôle d'ajustement par rapport aux autres activités. Elle doit maintenir la liquidité à un niveau permettant d'éviter toute impasse de trésorerie.

Variation des liquidités et placements à court terme. Au total, le flux de trésorerie provenant des différentes activités explique la variation des liquidités et des placements à court terme entre le début et la fin de l'exercice. Par conséquent, le total doit

98 Anlagentätigkeit | Attività di piazzamento | Investment activities.

99 Les placements financiers peuvent comprendre des actions, des titres de participation, des parts de fonds de placement, des bons de jouissance, des parts sociales de sociétés coopératives, des obligations, des hypothèques, des prêts. Les immobilisations corporelles peuvent comprendre notamment des terrains et des bâtiments.

100 Le MCH2 offre la possibilité d'une présentation alternative inspirée du tableau de financement prévu par le $\mathrm{MCH} 1$. Cette alternative présente indépendamment le flux de trésorerie provenant de l'activité d'investissement (reflété par le compte des investissements) et regroupe ensuite le flux de trésorerie provenant de l'activité de placement et de l'activité de financement. Toutefois, cette solution n'est pas conforme aux normes IPSAS.

101 Finanzierungstätigkeit | Attività finanziarie | Financing activities. 
correspondre à la différence entre le solde de la rubrique 100 à l'ouverture et à la clôture du bilan. C'est là un moyen de preuve que le tableau des flux de trésorerie est dénué d'erreur. Au total, la trésorerie de MaCollectivité s'est resserrée de KCHF 822,

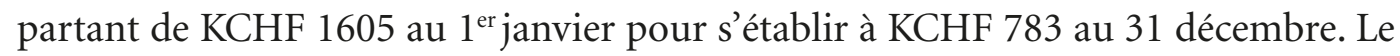
bilan nous l'indiquait déjà. Mais le tableau des flux de trésorerie nous fournit des explications sur l'origine de cette variation. D'une certaine manière, il revalorise l'information véhiculée par le bilan, sachant que ce document fait l'objet de peu d'attention, voire d'aucune attention, lors des discussions parlementaires.

Le tableau des flux de trésorerie nous aide à comprendre les besoins de trésorerie de la collectivité. Il laisse entrevoir la capacité de cette dernière à générer de la trésorerie à l'avenir. Il montre la capacité de la collectivité à adapter ses activités de placement et de financement pour saisir les opportunités qui se présentent sur les marchés financiers.

Cette présentation est donc assurément à la fois plus détaillée et plus précise que celle qui est offerte par le tableau de financement préconisé par le MCH1. Les flux de trésorerie ne dépendent pas des choix en matière de traitement comptable. Ils sont un fait découlant directement des sorties et des entrées de liquidités. Par conséquent, ils ne peuvent pas être biaisés par la tactique politique en matière de présentation des comptes.

Le tableau des flux de trésorerie préconisé par le $\mathrm{MCH} 2$ présente toutefois l'inconvénient de ne pouvoir être établi qu'a posteriori, lorsque les flux de trésorerie sont connus. Il ne peut pas être établi au moment de l'élaboration du budget sur la base des crédits alloués. Par contre, le tableau de financement selon MCH1 peut l'être. C'est l'une des raisons qui expliquent que de nombreuses collectivités y aient encore recours.

\subsubsection{Annexe aux comptes}

L'annexe aux comptes ${ }^{102}$ offre des informations dans différents domaines. Elle renseigne notamment sur les modalités qu'utilise la collectivité pour présenter ses comptes. Si une collectivité a renoncé à suivre l'une ou l'autre recommandation formulée par le $\mathrm{MCH} 2$, elle doit explicitement en faire mention dans l'annexe ${ }^{103}$. L'annexe doit présenter les indicateurs financiers prévus par le $\mathrm{MCH} 2$ (voir le chapitre 9 consacré au diagnostic de la situation financière à l'aide d'indicateurs). Elle doit également apporter de plus amples informations sur les changements ayant affecté, au cours de l'exercice, les immobilisations, le capital propre, les provisions, les participations et les garanties. En outre, l'annexe doit contenir tous les renseignements nécessaires afin de pouvoir apprécier l'état des finances, du patrimoine et du résultat. De ce point de vue, les exigences fixées par le $\mathrm{MCH} 2$ par rapport au contenu de l'annexe sont identiques à celles qui sont faites par les normes IPSAS. 


\subsubsection{Plan comptable des administrations publiques suisses}

Les collectivités organisent en principe l'information comptable en suivant trois classifications complémentaires: une classification organique, une classification par nature et une classification par fonction. Si les deux premières classifications sont utilisées depuis longtemps dans les collectivités, la classification fonctionnelle a peiné à faire son chemin, en particulier dans les collectivités de taille modeste. Or le $\mathrm{MCH} 2$ oblige maintenant toutes les collectivités, quelle que soit leur taille, à présenter leurs informations comptables selon la classification par nature et selon la classification fonctionnelle en appliquant un plan comptable normalisé ${ }^{104}$.

Classification organique. Dans toutes les collectivités, l'information comptable est structurée en fonction des organes, autrement dit en fonction de l'organigramme: c'est la classification organique ${ }^{105}$ des charges et des revenus dans le compte de résultats, des dépenses et des recettes dans le compte des investissements (par exemple charges de la direction des écoles ou de la direction des services informatiques). Les éléments sont donc classés en fonction des unités administratives (départements, services ou divisions). Parfois, on utilise le terme synonyme de classification administrative ou institutionnelle.

Cette classification est un instrument indispensable à la mise en œuvre du droit des crédits. En effet, elle sert de support à la compétence et à la responsabilité confiées de gérer les crédits et d'encaisser les recettes. Mais elle souffre d'une limitation importante: elle dépend de l'organisation de la collectivité. Or cette organisation change à travers le temps: les contours des directions évoluent de législature en législature au gré des découpages et redécoupages décidés par les membres de l'Exécutif. Par ailleurs, l'organigramme diffère souvent d'une collectivité à l'autre. Ainsi certains cantons comptent cinq départements, d'autres sept, avec des intitulés et des missions qui varient. L'organigramme diffère également selon le niveau institutionnel: l'organigramme de la Confédération ne ressemble à celui d'aucun canton, et celui d'un canton ne ressemble à celui d'aucune commune.

Classification par nature. Dans toutes les collectivités, l'information est également structurée selon la nature économique des opérations: c'est la classification par nature ${ }^{106}$ des charges (charges de personnel, charges de biens et services) et des revenus, des dépenses et des recettes, des actifs et des passifs. Cette classification permet de répondre à un double objectif.

D'abord, la classification par nature complète la classification organique pour pouvoir mettre en œuvre le droit des crédits. Elle permet d'allouer avec précision les crédits et de renforcer le contrôle sur leur utilisation.

Ensuite, elle offre les informations nécessaires à l'analyse des répercussions de l'action des collectivités publiques sur l'économie. Elle assure aussi que les données

104 Recommandation 03, CDF (2008).

105 Organische Gliederung | Classificazione istituzionale | Organic classification.

106 Artengliederung | Classificazione per genere di conto | Classification by nature. 
relatives aux finances publiques puissent aisément être intégrées dans la comptabilité nationale, ne serait-ce qu'en distinguant les ressources consacrées à la consommation courante $(3=$ charges $)$ et celles dévolues à l'investissement $(5=\text { dépenses })^{107}$.

La classification par nature contenue dans le $\mathrm{MCH} 2$, avec sa numérotation à quatre chiffres, a un caractère contraignant pour toutes les administrations publiques suisses. Il n'est donc pas permis à une collectivité de créer sa propre classification par nature, d'ajouter des rubriques ou encore de modifier la numérotation. Les numéros que nous avons mentionnés pour les comptes du bilan (figure 14), pour les rubriques du compte de résultats (tableau 16) et pour les rubriques du compte des investissements (tableau 15) sont ceux imposés par le plan comptable du MCH2 ${ }^{108}$.

Classification fonctionnelle. Pour offrir une parade aux limitations de la classification organique, pour satisfaire les besoins de comparaison et de collaboration, mais également de connaissance du coût des prestations, une troisième classification permet de structurer l'information de manière harmonisée selon les fonctions, tâches et prestations offertes par la collectivité: c'est la classification fonctionnelle ${ }^{109}$ des charges et des revenus, des dépenses et des recettes. Cette classification comprend dix fonctions principales, numérotées de 0 à 9 . Le tableau 20 en donne la liste et présente le compte de résultats et celui des investissements de MaCollectivité selon cette classification. Il permet de constater que la formation, avec une charge de plus de KCHF 2662, soit près de $\mathrm{CHF} 3$ mio, est la fonction qui engendre le coût de fonctionnement le plus élevé. Sans surprise, c'est la fonction «Finances et impôts» qui génère le plus de revenus. Avec la fonction "Culture, sport et loisirs, église», c'est également la formation qui a bénéficié de l'effort d'investissement le plus important avec une dépense supérieure à CHF 0,5 mio. Les totaux du tableau sont naturellement identiques à ceux qui sont obtenus en utilisant la classification par nature (voir le tableau 16 pour le compte de résultats avec un excédent de revenus de KCHF 64 et le tableau 15 pour le compte des investissements avec un investissement net de KCHF 1928).

À l'intérieur de chaque fonction, la classification fonctionnelle du $\mathrm{MCH} 2$ offre un degré de détail et une numérotation à trois positions. Par exemple, la fonction 2 «Formation» est détaillée pour circonscrire la tâche «Scolarité obligatoire» portant le numéro 21. Cette dernière est encore subdivisée de manière à cerner par exemple la prestation «École primaire» portant le numéro $212^{110}$. La classification fonctionnelle contenue dans le $\mathrm{MCH} 2$, avec sa numérotation à trois chiffres, a un caractère contraignant pour toutes les collectivités et les administrations publiques suisses. Il n'est donc pas permis à une collectivité de créer sa propre classification fonctionnelle, d'ajouter des fonctions ou des tâches ou encore de modifier la numérotation.

107 Les dépenses de consommation ne créent de la valeur ajoutée que pendant l'exercice courant. Les dépenses d'investissement contribuent à l'élargissement des capacités productives de l'économie; elles ont donc un impact sur la croissance économique pendant plusieurs années.

108 Pour les détails de la classification par nature, voir l'annexe A du Manuel MCH2, CDF (2008).

109 Funktionale Gliederung | Classificazione funzionale | Classification of the functions of government.

110 Pour les détails de la classification fonctionnelle, voir l'annexe B du Manuel MCH2, CDF (2008). 
Tableau 20 Compte de résultats et compte des investissements selon la classification fonctionnelle.

MaCollectivité, Année t, en KCHF.

\begin{tabular}{|lrrrr|}
\hline$N^{\circ}$ Libellé de la fonction & \multicolumn{1}{c}{ KCHF } & \multicolumn{1}{c}{ KCHF } & \multicolumn{1}{c}{ KCHF } \\
\hline Compte de résultats & Charges & Revenus & Résultat net \\
\hline 0 & Administration générale & 1170 & 176 & -994 \\
1 & Ordre et sécurité publics, défense & 350 & 289 & -61 \\
2 & Formation & 2662 & 165 & -2497 \\
3 & Culture, sport et loisirs, église & 326 & 222 & -104 \\
4 & Santé & 11 & 0 & -11 \\
5 & Prévoyance sociale & 2447 & 26 & -2421 \\
6 & Trafic et télécommunications & 1112 & 142 & -970 \\
7 & Protection de l'environnement & 2293 & 2084 & -209 \\
& et aménagement du territoire & & & \\
8 & Économie publique & 2553 & 2542 & -11 \\
9 & Finances et impôts & 1622 & 8964 & 7342 \\
\hline & Total & 14546 & 14610 & 64 \\
\hline Compte des investissements & Dépenses & Recettes & Résultat net \\
\hline 0 & Administration générale & 0 & 0 & 0 \\
1 & Ordre et sécurité publics, défense & 0 & 0 & 0 \\
2 & Formation & 715 & 0 & -715 \\
3 & Culture, sport et loisirs, église & 514 & 40 & -474 \\
4 & Santé & 0 & 0 & 0 \\
5 & Prévoyance sociale & 0 & 0 & 0 \\
6 & Trafic et télécommunications & 184 & 0 & -184 \\
7 & Protection de l'environnement & 354 & 0 & -354 \\
\hline 8 & et aménagement du territoire & & & \\
9 & Finances et impôts & 204 & 3 & -201 \\
\hline & Total & 0 & 0 & 0 \\
\hline
\end{tabular}

Systématique de numérotation. Pour pouvoir présenter les comptes selon chacune des trois classifications, les transactions doivent tout au long de l'année être saisies comptablement selon une systématique de numérotation rigoureuse. Le MCH2 exige que chacune des transactions touchant le compte de résultats et le compte des investissements soit classée à l'intérieur de chacun des trois axes. S'agissant des comptes du bilan, les transactions sont imputées et saisies en se limitant à la classification par nature. 
Les numérotations standardisées prévues par le $\mathrm{MCH} 2$ servent de support. Chaque charge, chaque revenu, chaque dépense et chaque recette est imputée à l'aide d'une clé d'imputation comportant un minimum de dix chiffres: les trois premiers chiffres permettent d'imputer la transaction dans la classification organique, les quatre suivants dans la classification par nature économique, les trois derniers dans la classification fonctionnelle. Le tableau 21 montre comment utiliser cette systématique en prenant comme exemple la comptabilisation dans le compte de résultats de la charge découlant de l'achat de fournitures pour l'enseignement. La contrepartie au bilan serait le compte par nature 1000 «Caisse» dans l'hypothèse d'un achat au comptant.

Tableau 21 Systématique de numérotation des transactions.

Exemple pour la comptabilisation dans le compte de résultats de l'achat de fournitures pour l'enseignement au degré primaire.

\begin{tabular}{|c|c|c|c|c|c|c|c|c|c|c|c|}
\hline \multicolumn{12}{|l|}{ Classification... } \\
\hline ... organique & $\begin{array}{l}\text { Direction: } \\
\text { Service: } \\
\text { Unité: }\end{array}$ & $\begin{array}{l}\text { Dép. Instruction publique } \\
\text { Service des écoles } \\
\text { École primaire }\end{array}$ & 5 & 2 & 1 & & & & & & \\
\hline ... par nature & $\begin{array}{l}\text { Classe de comptes: } \\
\text { Groupe de comptes: } \\
\text { Sous-groupe: } \\
\text { Compte: }\end{array}$ & $\begin{array}{l}\text { Charges } \\
\text { Biens et services } \\
\text { Matières et marchandises } \\
\text { Imprimés, publications }\end{array}$ & & & & & & & 2 & & \\
\hline ... fonctionnelle & $\begin{array}{l}\text { Fonction: } \\
\text { Tâche: } \\
\text { Subdivision: }\end{array}$ & $\begin{array}{l}\text { Formation } \\
\text { Scolarité obligatoire } \\
\text { Degré primaire }\end{array}$ & & & & & & & & & 2 \\
\hline Numéro à saisi & ur l'imputation: & & 5 & 2 & $1:$ & & & & & & 2 \\
\hline
\end{tabular}

Des chiffres supplémentaires peuvent être ajoutés en fonction des besoins. Par exemple, au-delà des quatre premiers chiffres de la classification par nature rendus obligatoires par le $\mathrm{MCH} 2$, les collectivités ajoutent souvent un cinquième, voire un sixième chiffre, pour pouvoir imputer les transactions sur des prestations très précises (notamment dans des domaines comme l'eau potable, l'épuration ou les déchets).

Cette systématique de numérotation permet ainsi d'utiliser l'information comptable pour répondre à diverses interrogations nécessaires à la gestion. C'est ce qu'illustre la figure 21. En croisant les classifications, il est par exemple possible de savoir combien MaCollectivité a dépensé globalement pour l'achat de matériel et plus spécifiquement pour le matériel scolaire utilisé par le Département de l'instruction publique dans l'enseignement au niveau primaire. 
Figure 21 L'information comptable répond à diverses interrogations de gestion.

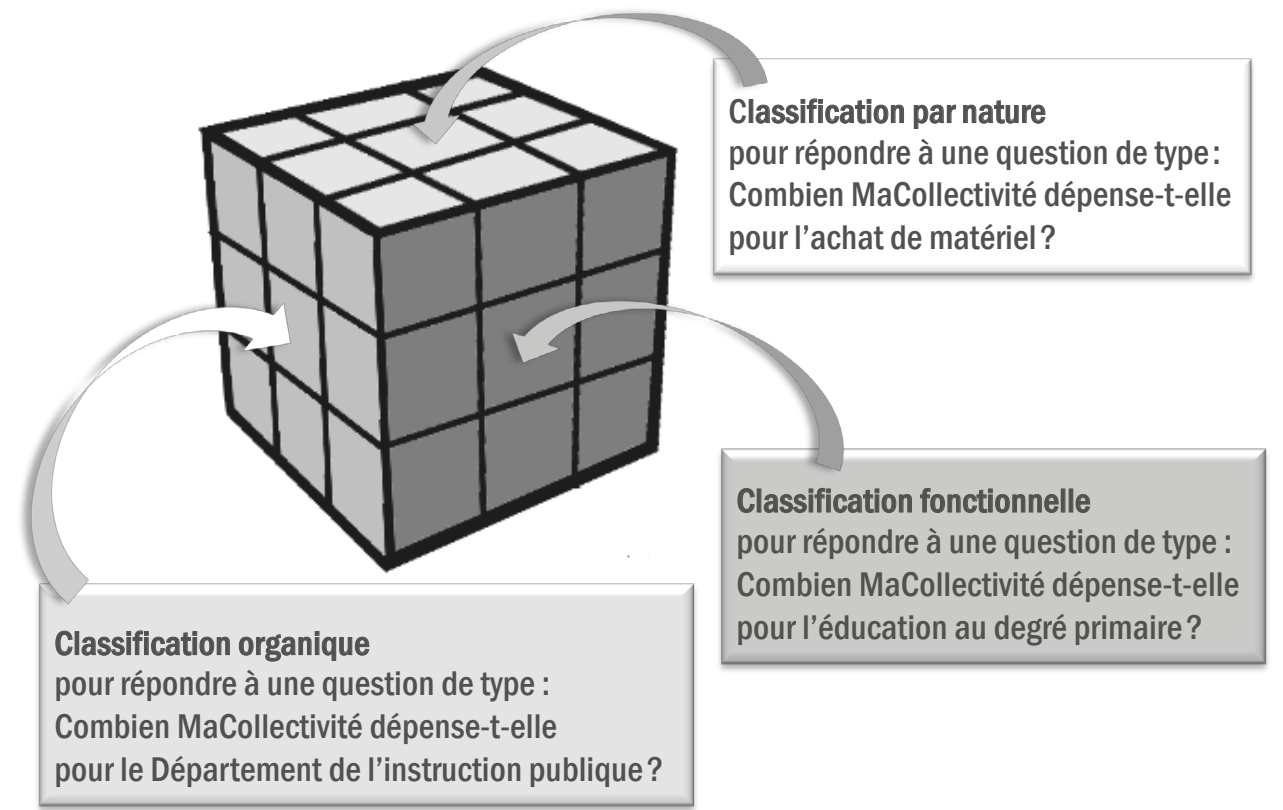

\subsection{Modèle comptable de la Confédération}

Contrairement aux cantons et aux communes, la Confédération offre peu de prestations et de services directement et spécifiquement à la population. La part relative des dépenses pour des prestations directes dans l'ensemble de son budget est faible. Par contre, environ deux tiers des dépenses fédérales sont des dépenses de transferts, par exemple au titre de la péréquation financière. Par ailleurs, le niveau des dépenses d'investissement de la Confédération est beaucoup moins volatil d'une année à l'autre que celui d'un canton ou d'une commune. Ces raisons expliquent que, jusqu'à récemment, la Confédération n'a pas appliqué un modèle comptable fondé sur la comptabilité d'exercice, séparant fonctionnement et investissement, et s'est contentée d'un modèle fondé sur le principe de caisse.

Compte de financement. Le compte de financement - anciennement le compte financier - était et reste au cœur de son modèle. C'est sur lui que s'appuie le frein fédéral à l'endettement, ancré dans la Constitution fédérale dès 2001 et appliqué dès 2003. Cet ancrage constitutionnel explique que le compte financier, rebaptisé compte de financement, a survécu à la réforme ultérieure du modèle comptable de la Confédération, ancré dans la loi sur les finances en 2005 et mis en ouvre en 2007. Cette réforme a toutefois permis à la Confédération de se doter, comme les autres administrations publiques suisses, d'un véritable compte de résultats et également d'un compte des investissements ainsi que d'un tableau des flux de trésorerie. Suite à cette réforme, la Confédération applique de facto un double modèle comptable: une 
comptabilité d'exercice avec son compte de résultats et une comptabilité de caisse avec son compte de financement ${ }^{111}$.

Le compte de financement subsiste pour répondre au besoin de la gestion budgétaire imposé par la Constitution. On y enregistre uniquement les opérations monétaires, c'est-à-dire l'ensemble des dépenses et des recettes de la Confédération. Il recense donc seulement les décaissements et les encaissements, que ce soit ceux qui sont liés aux opérations courantes ou aux investissements. Par conséquent, et contrairement au compte de résultats, les opérations non monétaires, purement comptables, telles que les amortissements, les régularisations (actifs et passifs transitoires), la constitution ou la dissolution de provisions, ne sont pas prises en considération. La présentation du compte de financement distingue toutefois les opérations courantes des opérations d'investissement. Elle distingue également les opérations ordinaires des opérations extraordinaires.

Solde de financement. Le solde du compte indique dans quelle mesure les décaissements d'un exercice sont couverts par les encaissements. On parle de solde de financement. Si les dépenses dépassent les recettes, le solde correspond au besoin d'emprunt de la Confédération. Dans le cas contraire, la Confédération peut rembourser une partie de sa dette; c'est la situation qui a prévalu par exemple en 2018 et que décrit le tableau 22: l'excédent de financement est de quelque CHF 3 mrd.

Tableau 22 Compte de financement de la Confédération. Comptes 2018, en mio CHF.

\begin{tabular}{|c|c|c|c|}
\hline Libellé & Débit & Crédit & Solde \\
\hline Recettes fiscales & 68121 & & \\
\hline Patentes et concessions & 1212 & & \\
\hline Recettes financières & 1121 & & \\
\hline Autres recettes courantes & 1789 & & \\
\hline Recettes d'investissement & 1270 & & \\
\hline Recettes ordinaires & 73513 & & \\
\hline Dépenses propres & & 10252 & \\
\hline Dépenses de transfert courantes & & 48120 & \\
\hline Dépenses financières & & 1203 & \\
\hline Dépenses d'investissement & & 11000 & \\
\hline Dépenses ordinaires & & 70575 & \\
\hline Solde de financement ordinaire (excédent) & & & 2938 \\
\hline Recettes extraordinaires & & 90 & \\
\hline Dépenses extraordinaires & 0 & & \\
\hline Solde de financement total & & & 3028 \\
\hline
\end{tabular}

Source: Compte d'État 2018 de la Confédération suisse, Section B2, p. 126.

111 À l'heure où nous écrivons ces lignes, la Confédération entame des réflexions pour simplifier son système et le rapprocher de celui des cantons et des communes. 
Ici, il faut souligner une différence fondamentale dans la manière dont la Confédération d'un côté et les cantons et communes de l'autre communiquent sur leurs budgets et sur leurs comptes. Tandis que la Confédération communique essentiellement son solde de financement, les autres collectivités suisses communiquent le solde de leur compte de résultats ${ }^{112}$. Si la Confédération communiquait de la même manière que les cantons et les communes, elle aurait mis l'accent sur un excédent de revenus de CHF 5,701 mrd correspondant au résultat total de son compte de résultats, plutôt que sur un excédent de financement de CHF 3,028 mrd correspondant au solde de son compte de financement. C'est là une différence fondamentale que le lecteur doit avoir à l'esprit en découvrant les informations relayées par les médias: même si les chiffres avancés de part et d'autre semblent comparables, ils ne le sont aucunement dans les faits.

Une autre différence importante doit être relevée. En vertu du frein fédéral à l'endettement, lorsque la conjoncture est équilibrée, autrement dit lorsque le produit intérieur brut correspond à son niveau normal, les dépenses doivent être intégralement couvertes par les recettes. Puisque les dépenses incluent les dépenses d'investissement, cela signifie que toutes les dépenses - y compris les dépenses d'investissement - doivent être financées par les recettes du même exercice. La Confédération doit donc opérer de facto une couverture directe de ses dépenses d'investissement, contrairement aux autres collectivités, qui opèrent une couverture ultérieure à travers la procédure d'amortissement et peuvent ainsi étaler leur financement sur plusieurs exercices ${ }^{113}$.

112 Toutefois, il est parfaitement possible de calculer le solde de financement d'un canton ou d'une commune. Nous verrons cela lorsque nous parlerons de la dynamique de l'endettement (section 12.5).

113 Cela étant, la Confédération investit de manière moins erratique que les cantons ou les communes. Par conséquent, elle peut absorber le financement annuel de ses investissements. Toutefois, les très grands projets infrastructurels, notamment dans le domaine des transports routiers et ferroviaires, ne sont pas compatibles avec le mécanisme de la couverture directe. Ce qui amène la Confédération à recourir à des comptes spéciaux, des «fonds ", gérés en marge du compte de financement, donc en marge du frein à l'endettement, même si les dépenses y relatives restent soumises au droit des crédits. D’autres domaines font également l'objet de comptes spéciaux. Pour le détail, voir AFF (2019b). 


\section{Utiliser les imputations internes pour refléter les coûts des prestations}

\subsection{Comptabilité générale et comptabilité de gestion}

En séparant fonctionnement et investissement et en appliquant le principe de la comptabilité d'exercice, le modèle comptable harmonisé offre une bonne image des coûts de fourniture des prestations fournies. La prise en compte de l'usure et de l'obsolescence de l'infrastructure à travers l'amortissement, de même que les constitutions et dissolutions de provisions, pour citer ces exemples, permettent d'estimer les coûts de manière plus exacte que ne le permettrait une comptabilité de caisse n'enregistrant que les encaissements et les décaissements. Le compte de résultats et en particulier le résultat opérationnel répondent donc globalement à l'exigence faite au modèle comptable de renseigner sur le coût de l'ensemble des prestations offertes par une collectivité.

Toutefois, les concepteurs du MCH ont poussé l'exigence plus loin. Ils ont imaginé que la comptabilité générale s'ouvre en direction de la comptabilité de gestion. Pour y parvenir, ils ont introduit dans le dispositif deux outils empruntés à la comptabilité de gestion: la classification fonctionnelle et le mécanisme des imputations internes. En effet, mieux servir les préoccupations de gestion s'inscrit dans la suite logique des améliorations apportées à la comptabilité générale.

Comptabilité générale ${ }^{114}$. La comptabilité générale - aussi appelée comptabilité financière - sert à élaborer les états financiers publiés par la collectivité. Elle génère donc une information pour des destinataires en dehors des unités administratives. Cette information doit montrer l'état des finances, du patrimoine et du résultat de la collectivité dans son ensemble. Pour y parvenir, la comptabilité générale est tenue d'observer les dispositions légales en vigueur, autrement dit les dispositions figurant dans la Constitution, la loi sur les finances et ses ordonnances d'application.

Comptabilité de gestion ${ }^{115}$. La comptabilité de gestion - aussi appelée comptabilité analytique ou comptabilité d'exploitation - sert à orienter les décisions dans les différents domaines d'intervention de la collectivité. Ce terme recouvre les analyses menées au sein de l'entité essentiellement pour apporter une information sur le coût de prestations particulières et pour confronter ce coût avec les revenus générés. Les analyses portent quasi uniquement sur l'activité opérationnelle. Les informations générées par la comptabilité de gestion sont donc à la fois plus sectorielles et plus détaillées que celles qui sont fournies par la comptabilité générale. Elles s'adressent par conséquent en priorité aux membres de l'Exécutif et aux directions des unités administratives. À l'inverse de la comptabilité générale, la comptabilité de

114 Finanzbuchhaltung | Contabilità generale | Financial accounting.

115 Betriebsbuchhaltung | Contabilità gestionale | Management accounting. 
gestion n'est régie par presque aucune disposition légale. Notons que la Conférence des directrices et directeurs cantonaux des finances (CDF) a fait des propositions au sujet d'un modèle de comptabilité analytique harmonisé pour les cantons et les communes (CDF 2002). Toutefois ces propositions n'ont pas été jusqu'ici suivies d'effets significatifs (Conseil fédéral 2009). Les collectivités de taille modeste renoncent généralement à se doter d'une comptabilité de gestion en plus de la comptabilité générale exigée d'elles par la législation. En effet, l'instrument est coûteux à mettre en place et à faire fonctionner année après année. Plutôt que de se doter d'une comptabilité de gestion systématique, ces collectivités établissent des décomptes ad hoc dans les domaines où il leur est nécessaire d'estimer le coût des prestations offertes.

\subsection{Imputations internes}

À l'heure actuelle, il n'existe donc pas de prescriptions applicables aux cantons et aux communes pour les obliger à mettre en place une comptabilité de gestion permettant de renseigner systématiquement sur le coût de chacune des prestations offertes. Par contre, à travers les imputations internes, la comptabilité générale offre un mécanisme permettant de refléter au mieux le coût des prestations échangées entre unités administratives à l'intérieur d'une même entité juridique. Grâce à cela, la comptabilité générale peut offrir une meilleure image du coût des prestations offertes aux ménages, aux entreprises et, plus généralement, au public.

Une imputation interne ${ }^{116}$ est une opération consistant à mettre à charge d'une unité administrative (ou d'une tâche) le coût d'une prestation fournie par une autre unité appartenant à la même collectivité. Simultanément, elle consiste à imputer le même montant aux revenus de l'unité qui fournit la prestation à l'interne. On parle parfois de prix de cession interne. Il s'agit donc d'une opération purement comptable, qui ne s'accompagne d'aucun mouvement de trésorerie, d'aucun décaissement, d'aucun encaissement.

Le plan comptable réserve la rubrique 39 pour la mise à charge et la rubrique 49 pour l'imputation en revenus. Ces deux rubriques se décomposent en diverses sous-rubriques présentées dans le tableau 23.

Les coûts les plus souvent imputés à l'interne sont les charges de personnel, les charges de biens et services et autres charges d'exploitation, ainsi que les charges d'intérêts et d'amortissement. En effet, ces charges sont fréquemment comptabilisées dans un premier temps de manière centralisée. Les imputations servent donc à les ventiler dans un second temps entre les unités ou les tâches qui utilisent effectivement la ressource correspondante. Certains revenus sont également comptabilisés de façon centralisée et doivent ensuite être ventilés, par exemple les revenus d'intérêts. 
Tableau 23 Importance des imputations internes dans le total des charges et des revenus. MaCollectivité, Année t, en KCHF.

\begin{tabular}{|c|c|c|c|c|c|}
\hline \multicolumn{2}{|c|}{$\mathrm{N}^{\circ}$ Libellé } & \multirow{2}{*}{$\begin{array}{c}\text { KCHF } \\
14546\end{array}$} & \multicolumn{2}{|c|}{$\mathrm{N}^{\circ}$ Libellé } & \multirow{2}{*}{$\begin{array}{c}\text { KCHF } \\
14610\end{array}$} \\
\hline 3 & Charges & & 4 & Revenus & \\
\hline$\ldots$ & dont... & & $\ldots$ & dont... & \\
\hline 39 & Imputations internes & 270 & 49 & Imputations internes & 270 \\
\hline 390 & $\begin{array}{l}\text { Approvisionnement en } \\
\text { matériel et marchandises }\end{array}$ & 0 & 490 & $\begin{array}{l}\text { Approvisionnement en } \\
\text { matériel et marchandises }\end{array}$ & 0 \\
\hline 391 & Prestations de service & 144 & 491 & Prestations de service & 144 \\
\hline 392 & $\begin{array}{l}\text { Baux à ferme, loyers, } \\
\text { frais d'utilisation }\end{array}$ & 0 & 492 & $\begin{array}{l}\text { Baux à ferme, loyers, } \\
\text { frais d'utilisation }\end{array}$ & 0 \\
\hline 393 & $\begin{array}{l}\text { Frais administratifs } \\
\text { et d'exploitation }\end{array}$ & 116 & 493 & $\begin{array}{l}\text { Frais administratifs } \\
\text { et d'exploitation }\end{array}$ & 116 \\
\hline 394 & $\begin{array}{l}\text { Intérêts et charges } \\
\text { financières théoriques }\end{array}$ & 10 & 494 & $\begin{array}{l}\text { Intérêts et charges } \\
\text { financières théoriques }\end{array}$ & 10 \\
\hline 395 & $\begin{array}{l}\text { Amortissements planifiés } \\
\text { et non planifiés }\end{array}$ & 0 & 495 & $\begin{array}{l}\text { Amortissements planifiés } \\
\text { et non planifiés }\end{array}$ & 0 \\
\hline 398 & Virements comptables & 0 & 498 & Virements comptables & 0 \\
\hline 399 & Autres imputations internes & 0 & 499 & Autres imputations internes & 0 \\
\hline
\end{tabular}

Il arrive fréquemment qu'une personne consacre son temps de travail à des activités bénéficiant à différentes unités administratives et à diverses prestations. Pour des raisons de clarté et de simplification comptable, les charges de personnel correspondantes (traitement et charges sociales) sont parfois d'abord saisies dans les rubriques comptables d'une seule unité, puis ventilées. Cette ventilation tire profit de la systématique de numérotation offerte par le plan comptable en classant l'imputation interne simultanément dans une unité administrative et dans une catégorie fonctionnelle. Le tableau 24 l'illustre avec une personne qui travaille dans l'unité Administration des prestations au sein de l'Administration centrale de MaCollectivité. Cette personne dédie une partie de son temps à la gestion des déchets. Puisque cette activité consomme un nombre d'heures important, une imputation interne se justifie. Le coût de cette prestation de service est estimé à KCHF 10 pour l'Année $t^{117}$. Ce montant est imputé auprès du Service de la propreté publique responsable de la gestion des déchets (484) comme une charge (3910) liée à cette fonction (730). Il est simultanément imputé à l'Administration des prestations (125) comme un revenu (4910) associé aux services généraux (022).

117 Ce montant de KCHF 10 apparaît dans les charges engendrées par la gestion des déchets. Ces charges sont présentées dans le tableau 27 (chapitre 5). 
Tableau 24 Imputations internes dans les unités administratives et dans les catégories fonctionnelles.

Exemple des prestations de service au profit de la gestion des déchets dans MaCollectivité.

Imputation en charges

\begin{tabular}{|c|c|c|c|c|c|c|c|c|c|c|}
\hline ... organique & $\begin{array}{l}\text { Direction: } \\
\text { Service: } \\
\text { Unité: }\end{array}$ & $\begin{array}{l}\text { Dép. Environnement et bâti } \\
\text { Propreté publique } \\
\text { Gestion des déchets }\end{array}$ & 4 & 8 & 4 & & & & & \\
\hline ... par nature & $\begin{array}{l}\text { Classe de comptes: } \\
\text { Groupe de comptes: } \\
\text { Sous-groupe: } \\
\text { Compte: }\end{array}$ & $\begin{array}{l}\text { Charges } \\
\text { Imputations internes } \\
\text { Prestations de service }\end{array}$ & & & 3 & 9 & 1 & 0 & & \\
\hline ... fonctionnelle & $\begin{array}{l}\text { Fonction: } \\
\text { Tâche: } \\
\text { Subdivision: }\end{array}$ & $\begin{array}{l}\text { Protection de l'environnement } \\
\text { Gestion des déchets } \\
\text { Gestion des déchets }\end{array}$ & & & & & & & 7 & 3 \\
\hline
\end{tabular}

Imputation en revenus

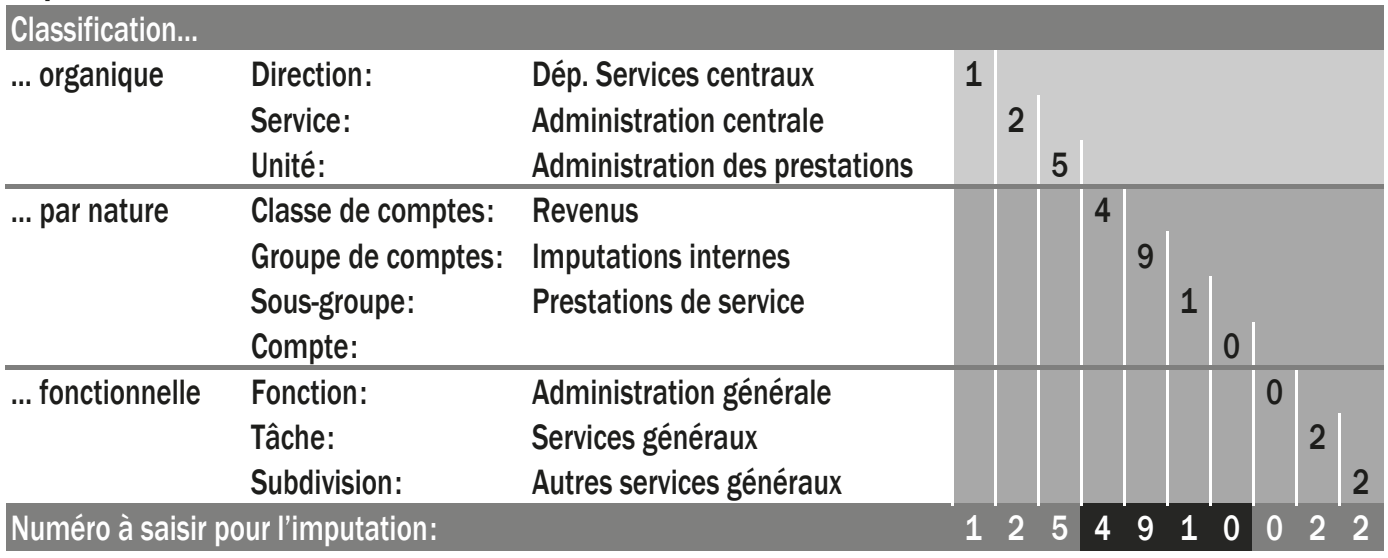

Le même mécanisme peut être mobilisé pour ventiler les charges des biens, de services et de marchandises lorsque les acquisitions sont effectuées et comptabilisées de manière centralisée (par exemple à travers un service de l'économat en charge de l'approvisionnement de l'ensemble des unités administratives). Le mécanisme est également utilisé pour répartir les intérêts passifs et autres charges financières quand la gestion des emprunts est confiée à une seule direction, généralement la direction des finances. La répartition s'effectue alors en fonction de l'importance de l'infrastructure utilisée et financée par l'emprunt ou de la valeur résiduelle de l'infrastructure au bilan. Parfois, les amortissements sont également comptabilisés dans un premier temps de manière centralisée, en particulier pour les bâtiments utilisés par plusieurs unités administratives. Leur ventilation intervient dans un second temps. 
Plutôt qu'une nature de charges considérée individuellement (charges de personnel, amortissements, etc.), c'est parfois un ensemble de charges découlant d'une prestation offerte par tout un service administratif qui est imputé à l'interne. Citons le cas des prestations offertes par une unité informatique, par un service des constructions ou par un atelier. Le mécanisme est identique à celui qui est exposé ci-dessus. Dans ce cas, la difficulté réside dans l'estimation du montant à imputer. Pour cela, il est nécessaire de procéder à une analyse de coûts, à un décompte, en dehors de la comptabilité générale. Ce thème fait l'objet de la section suivante.

Les imputations internes permettent donc d'accroître la transparence des coûts. Elles complètent l'estimation du coût de fonctionnement des unités administratives grâce à une meilleure ventilation des charges à l'intérieur de la classification organique. Elles offrent également un meilleur reflet du coût de fourniture des prestations publiques en ventilant les charges de manière plus réaliste à l'intérieur de la classification fonctionnelle.

Les imputations internes permettent de mieux présenter, à l'intérieur même de la comptabilité générale, le coût brut des unités et des prestations à travers la ventilation des charges. Mais elles offrent également une meilleure image du coût net grâce à la répartition des revenus. Par conséquent, on présente une information plus précise sur le degré de couverture des coûts par les revenus, non seulement dans les unités administratives, mais aussi pour les prestations offertes.

D'ailleurs, cet effort de transparence est indispensable lorsque les prestations sont financées par des contributions causales ${ }^{118}$. Cela concerne donc tous les domaines où un financement spécial est instauré, comme nous le verrons au chapitre 5. À l'échelon des communes, la gestion des déchets, celle de l'eau potable et des eaux usées sont par exemple touchées.

Au-delà du cas particulier des financements spéciaux, le recours aux imputations internes permet de conscientiser les services consommateurs de prestations internes sur les coûts que cela représente, des coûts qui prendraient la forme d'une véritable dépense si la prestation devait être achetée à l'extérieur. Une transparence accrue devrait donc favoriser un emploi plus ménager, plus économe et plus efficace des fonds, pour reprendre ici les principes de bonne gestion des ressources énoncés précédemment (section 2.1.1). Pour cette raison, les imputations internes ne doivent pas seulement être enregistrées au moment de l'établissement des comptes. Elles devraient aussi être inscrites au budget.

118 La législation en la matière dispose d'ailleurs souvent que «les bases de calcul qui servent à fixer le montant des taxes sont accessibles au public», pour reprendre ici les termes de la loi fédérale sur la protection de l'environnement (LPE, art. 60a, al. 4). Pour autant qu'elle existe, la comptabilité de gestion ne constitue pas, à notre avis, une base suffisante pour remplir cette obligation légale. Elle n’est pas codifiée par la législation, en particulier par la loi sur les finances. Par conséquent, elle n'est en principe pas soumise à un audit de conformité, pas plus qu'elle n'est soumise à l'acceptation du Législatif et n'est publiée. Par contre, elle devrait servir à déterminer les montants à imputer à l'interne. 
Finalement l'effort de transparence est important pour répondre à l'exigence faite au modèle comptable de servir de base aux comparaisons. Ici, les imputations internes, avec la classification fonctionnelle, jouent un rôle fondamental pour pouvoir comparer des collectivités qui sont organisées différemment. Imaginons une collectivité où tous les coûts sont comptabilisés d'emblée de manière éclatée dans la «bonne» tâche à l'intérieur de la classification fonctionnelle. Cette collectivité n'a donc pas besoin de procéder à des imputations internes pour connaître le véritable coût de ses services. Imaginons par ailleurs une collectivité qui comptabilise dans ses services transversaux (administration générale, finances, informatique, etc.) de manière centralisée un volume important de coûts et qui n'impute pas ensuite ces coûts sur les tâches concernées. Toutes choses égales par ailleurs, une comparaison de ces deux collectivités sur la base de leur comptabilité générale aboutira à un constat biaisé. En effet, elle montrerait forcément que les prestations offertes en finalité au public (éducation, santé, social, etc.) coûtent davantage dans la première collectivité (comptabilisant de manière éclatée) que dans la seconde (centralisée). Pour disposer d'une base de comparaison fiable, il faudrait que la seconde procède aux imputations internes nécessaires à la rendre comparable. Toutefois, pour un œil extérieur, à moins que les comptes montrent des imputations internes, il est difficile de savoir si l'enregistrement des charges et des revenus s'effectue de manière centralisée ou si l'enregistrement a lieu d'emblée de manière éclatée. En outre il est difficile de savoir si les imputations internes mises en place correspondent à la consommation réelle des unités administratives ou si, au contraire, cette consommation est sous-estimée, voire surestimée.

L'organisation et la taille de la collectivité déterminent donc l'ampleur des imputations internes à comptabiliser. Comme toute autre opération comptable, le bien-fondé d'une imputation interne repose sur le principe de l'importance. Si, par son montant, une charge ou un revenu est susceptible d'influencer le jugement ou la décision au sujet d'une unité administrative ou d'une prestation, alors une imputation interne doit avoir lieu. Dans les domaines où la législation et la jurisprudence exigent que les coûts soient couverts par des contributions causales, on imagine bien que la transparence des coûts est obligatoire pour ne pas biaiser les décisions. Les domaines où la législation n'introduit pas de telles exigences sont moins concernés. Toutefois, même dans ces domaines, le besoin croissant de transparence et de mâ̂trise des coûts milite en faveur d'imputations internes suffisamment poussées.

En réalité, les collectivités utilisent (encore) peu les imputations internes pour apporter davantage de transparence. Dans le cas de MaCollectivité, les imputations internes se limitent à KCHF 270 sur un total de charges de KCHF 14546, soit moins de $2 \%$. On peine à croire que les services échangés entre unités administratives soient si peu importants, à moins que l'on puisse être sûr que les charges sont enregistrées de manière éclatée. Dans les cantons, le pourcentage avoisine 6\% en moyenne sur les trente dernières années (Clémenceau et Soguel 2014). 


\section{3 Éléments d'analyse des coûts}

Ce chapitre consacré aux imputations internes nous offre l'opportunité d'aborder certains éléments d'analyse des coûts. Ces éléments sont utiles pour déterminer quels doivent être les montants à imputer. Le but est donc d'estimer le coût de revient - généralement le coût de revient complet - d'une tâche (ou d'une prestation) au sens de la classification fonctionnelle ${ }^{119}$.

Charges directes et indirectes. Le point de départ de l'analyse des coûts consiste à déterminer si une charge est directe ou si elle est indirecte. Cette distinction est illustrée grâce à la figure 22. Une charge est directe lorsqu'elle peut être comptablement affectée à une unité administrative ou à une tâche, sans ambiguité, ni répartition. C'est le cas de la charge (1) dans la figure. Par conséquent, si toutes les charges étaient directes, le mécanisme des imputations internes serait inutile. Le cas de la gestion des déchets dans MaCollectivité, qui sera traité ensuite dans le chapitre 5, nous fournit quelques exemples de charges directes. Dans ce chapitre-là, le tableau 27 montre que plusieurs charges sont directement affectées à l'unité administrative responsable de cette prestation (Unité A dans la figure) et, par conséquent, à cette tâche.

Figure 22 Distinction entre une charge directe et une charge indirecte.

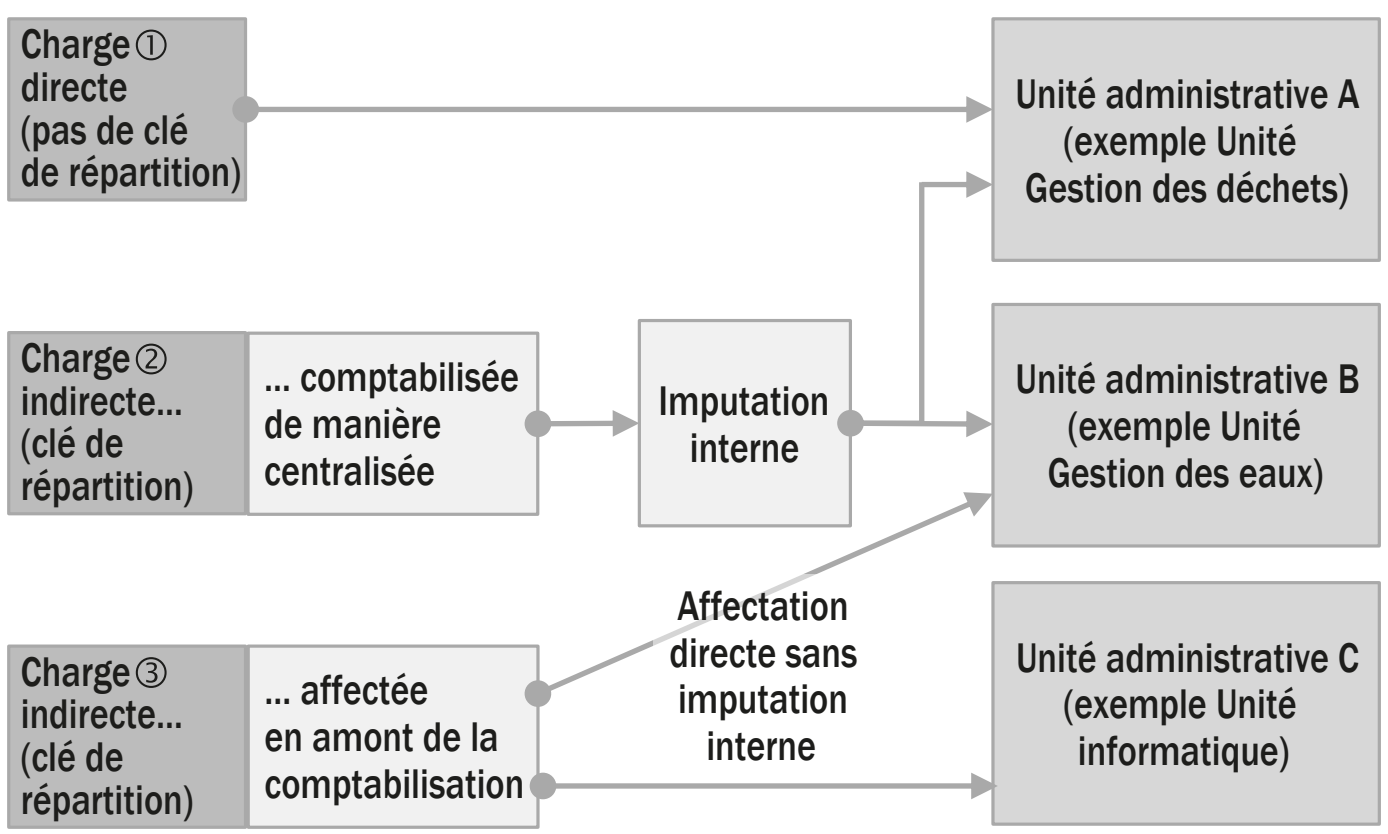

119 L'analyse des coûts peut poursuivre d'autres objectifs, par exemple l'objectif de déterminer le point mort d'exploitation. On fait appel alors à des éléments différents, notamment la distinction entre coûts fixes et coûts variables. Nous ne nous y intéressons pas ici. D’abord parce que ce type d'analyse est moins fréquent dans les administrations publiques. Ensuite parce qu'il est peu en lien avec la comptabilité générale et les imputations internes, contrairement à l'analyse du coût de revient complet. Pour un manuel de comptabilité de gestion voir par exemple Pasqualini (2019). 
Il s'agit des charges de personnel pour les employés œuvrant spécifiquement dans cette unité, des achats de biens et services réalisés par cette unité et de l'amortissement de l'infrastructure propre à cette unité.

En revanche, les charges indirectes ne peuvent pas être affectées sans autre. Une clé de répartition est nécessaire pour les imputer à une unité ou à une tâche. La personne travaillant au sein de l'Administration centrale et qui œuvre en partie pour l'unité responsable de la gestion des déchets nous en fournit un exemple. Sa charge salariale est indirecte (2), puisqu'elle est ventilée selon une clé de répartition. La clé peut se fonder sur le cahier des charges de la personne (et la ventilation du temps de travail qui y est prévue) ou, mieux, sur un relevé du temps effectivement passé à soutenir les activités de l'Unité A pour la gestion des déchets (et les activités de l'Unité B pour la gestion des eaux). Si les charges indirectes sont comptabilisées de manière centralisée, une imputation interne est ensuite nécessaire, comme nous l'avons illustré. Il arrive que la répartition s'effectue en amont de la comptabilisation proprement dite et que la comptabilisation des coûts se fasse ensuite immédiatement sur les unités concernées, sans recourir à une imputation interne. Quoi qu'il en soit, on reste en présence d'une charge indirecte, puisque l'on utilise aussi une clé de répartition. Cela peut être le cas, lorsqu'à réception d'une facture pour l'achat groupé de fournitures de bureau, 20\% de la charge (3) sont affectés et comptabilisés immédiatement dans l'Unité B, tandis que $80 \%$ sont enregistrés immédiatement dans l'Unité C.

Le fait de devoir utiliser une clé de répartition implique, de facto, une certaine approximation dans l'estimation du montant imputé, respectivement affecté. Une telle approximation n'existe pas lorsque les coûts sont directs.

Charges incorporables et non incorporables. Le principe de l'importance doit être appliqué afin de déterminer si une charge doit être imputée à l'interne ou non. Imputer les charges d'un montant insignifiant pour la gestion serait inefficient.

De plus, certaines charges, de par leur nature, ne devraient pas faire l'objet d'une imputation interne. Celles qui présentent un caractère exceptionnel ou qui ne relèvent pas de l'exploitation normale et courante sont généralement considérées comme des charges non incorporables au coût des prestations. Par conséquent, les charges catégorisées comme des charges extraordinaires dans la classification par nature du $\mathrm{MCH}$ (rubrique 38) ne devraient pas être imputées à l'interne. Dans les domaines où un financement spécial est instauré, il est rigoureusement exclu d'imputer à l'interne les charges extraordinaires relevant de la tactique politique en matière de présentation des comptes (amortissements supplémentaires, amortissements du découvert, préfinancements, etc.). Une imputation de telles charges biaiserait les résultats de l'analyse des coûts, qu'il s'agisse du coût d'une unité administrative ou du coût d'une tâche. Quant aux charges découlant d'événements véritablement extraordinaires, on ne devrait pas non plus les imputer à l'interne puisqu'elles ne relèvent pas de l'exploitation normale et courante. Les subventions 
à redistribuer ne devraient pas non plus faire l'objet d'une imputation interne. En effet, elles ne concourent en rien à la fourniture de prestations par la collectivité concernée puisqu'elles ne font que transiter par le compte de résultats de cette dernière à travers les rubriques de charges 37 et de revenus 47 .

En définitive, les rubriques de charges incorporables au coût des prestations sont bien circonscrites. Il s'agit des charges de personnel (30), des charges de biens et services et autres charges d'exploitation (31), des amortissements du patrimoine administratif (33), des charges financières (34) et des charges de transferts (36). Ce sont ces rubriques qui sont concernées par d'éventuelles imputations internes.

Centres de coûts homogènes et non homogènes. L'attribution des charges directes et indirectes aux unités administratives contribue à faire de celles-ci de véritables centres de responsabilité. C’est la logique qui généralement prévaut pour découper l'organisation de la collectivité: on s'attache à ce que les unités soient le plus homogènes possible, c'est-à-dire correspondent à des centres de responsabilité, chacun compétent pour fournir une prestation spécifique. Un centre est réputé homogène s'il est possible d'identifier une unité de référence unique pour mesurer de manière adéquate son niveau d'activité ou le volume de prestations offert. Si une telle unité de référence existe, on parlera d'unité d'œuvre. À l'inverse, un centre est dit non homogène s'il n'existe pas d'unité de mesure unique. On en sera alors réduit à utiliser une clé de répartition plus ou moins arbitraire pour répartir ses charges.

Centres prestataires et opérationnels. Dans la mesure où, à l'intérieur d'une collectivité, plusieurs unités fonctionnent comme des centres prestataires de services pour d'autres unités, leurs charges devraient être réparties sur les centres opérationnels fournissant in fine des services aux usagers. Le vocabulaire est vaste pour qualifier les centres prestataires: services centraux, services transversaux, services logistiques, services de support, etc. Dans la figure 22, l'Unité Informatique en est un exemple, alors que l'Unité Gestion des déchets et l'Unité Gestion des eaux sont des exemples de centres opérationnels. Notons toutefois qu'en pratique, la catégorisation entre centres prestataires et centres opérationnels est sujette à discussion. Ainsi l'Unité Gestion des eaux peut aussi être vue comme un centre prestataire si elle fournit de l'eau à l'école primaire.

La répartition des charges des centres prestataires est évidemment plus précise si elle peut se fonder sur une unité d'œuvre plutôt que sur une clé de répartition. Par exemple, dans la figure 23, la répartition des charges de l'Unité Gestion des eaux peut s'effectuer sur la base de l'unité d'œuvre du volume d'eau livré. On est donc en présence d'un centre homogène. Pour ce qui est de l'Unité Informatique, il est plus difficile d'identifier une unité unique qui permette de qualifier son activité et son offre de prestations de soutien. Ce centre est donc non homogène. Il faut par conséquent recourir à une clé de répartition comme le nombre de postes de travail dans les unités clientes ou le nombre d'heures dévolues à ces unités. 
Dans tous les cas, la ventilation donne lieu à une imputation interne puisque les charges à répartir - qu'elles soient directes ou indirectes - ont été comptabilisées préalablement dans les centres concernés.

La cascade de déversement des coûts esquissée dans la figure 23 peut évidemment s'allonger. Toutefois, le plus souvent, l'allocation des coûts se limite à une, voire deux étapes. La première est celle au cours de laquelle les coûts indirects sont affectés à une unité administrative ou à une fonction spécifique, ainsi qu'illustré dans la figure 22. Lorsque cette unité administrative est prestataire à l'interne, la seconde étape intervient pour répartir les charges accumulées dans ce centre de coûts sur les centres opérationnels (unités administratives selon la classification organisationnelle et tâches selon la classification fonctionnelle).

Figure 23 Imputation interne avec une unité d'œuvre ou avec une clé de répartition selon que le centre est homogène ou non.

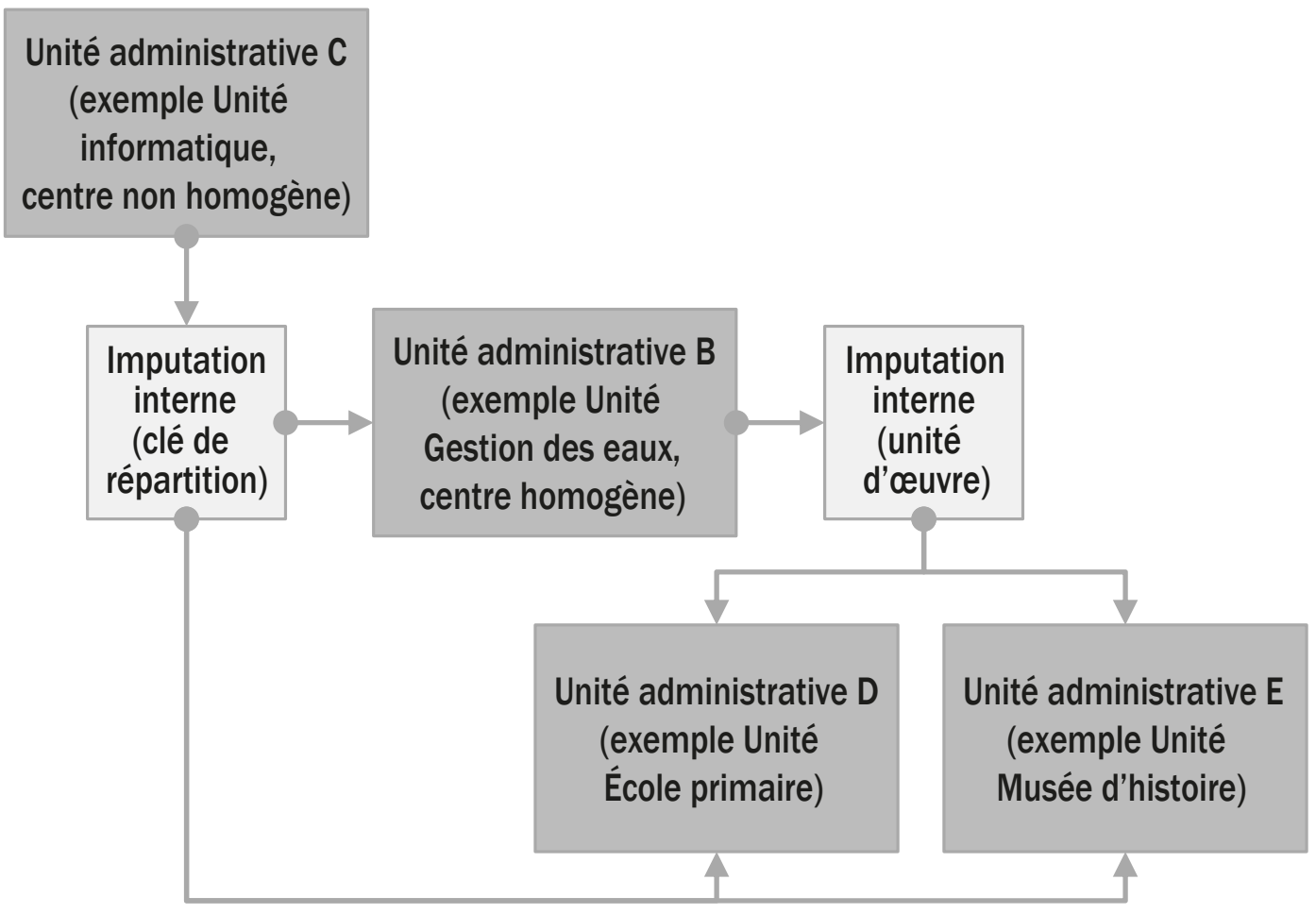




\section{Comprendre le financement spécifique de certaines prestations}

\subsection{Distinction entre impôts et contributions causales}

Dans bon nombre de domaines, la législation prévoit que le coût des prestations soit financé de manière particulière (Christen et Soguel 2018). Cela implique d'affecter certaines recettes à la fourniture de ces prestations. Diverses contributions causales sont prélevées auprès des utilisateurs de certaines prestations afin d'en financer spécialement le coût de fourniture. Pensons à la fourniture de l'eau, à l'épuration des eaux ou à l'enlèvement des ordures par les communes. En revanche, les recettes des impôts ne devraient pas être utilisées pour financer des prestations ciblées, en vertu du principe de non-affectation (voir la section 2.1.2 consacrée aux principes de budgétisation). Cependant, la segmentation entre le binôme «contributions causales | recettes affectées» et le binôme «impôts|recettes non affectées» n'est pas toujours respectée. En effet, certaines recettes fiscales sont bel et bien affectées et financent des prestations particulières. C'est pourquoi il vaut la peine de rappeler la distinction à opérer entre un impôt et une contribution causale au sens de la terminologie suisse, même si tous deux sont des prélèvements obligatoires ou forcés du fait du pouvoir régalien conféré à l'État. Pour ce faire, nous utilisons la classification de l'Administration fédérale des contributions (AFC 2018), illustrée par la figure 24.

Figure 24 Distinction entre impôts et contributions causales.

Classification de l'Administration fédérale des contributions.

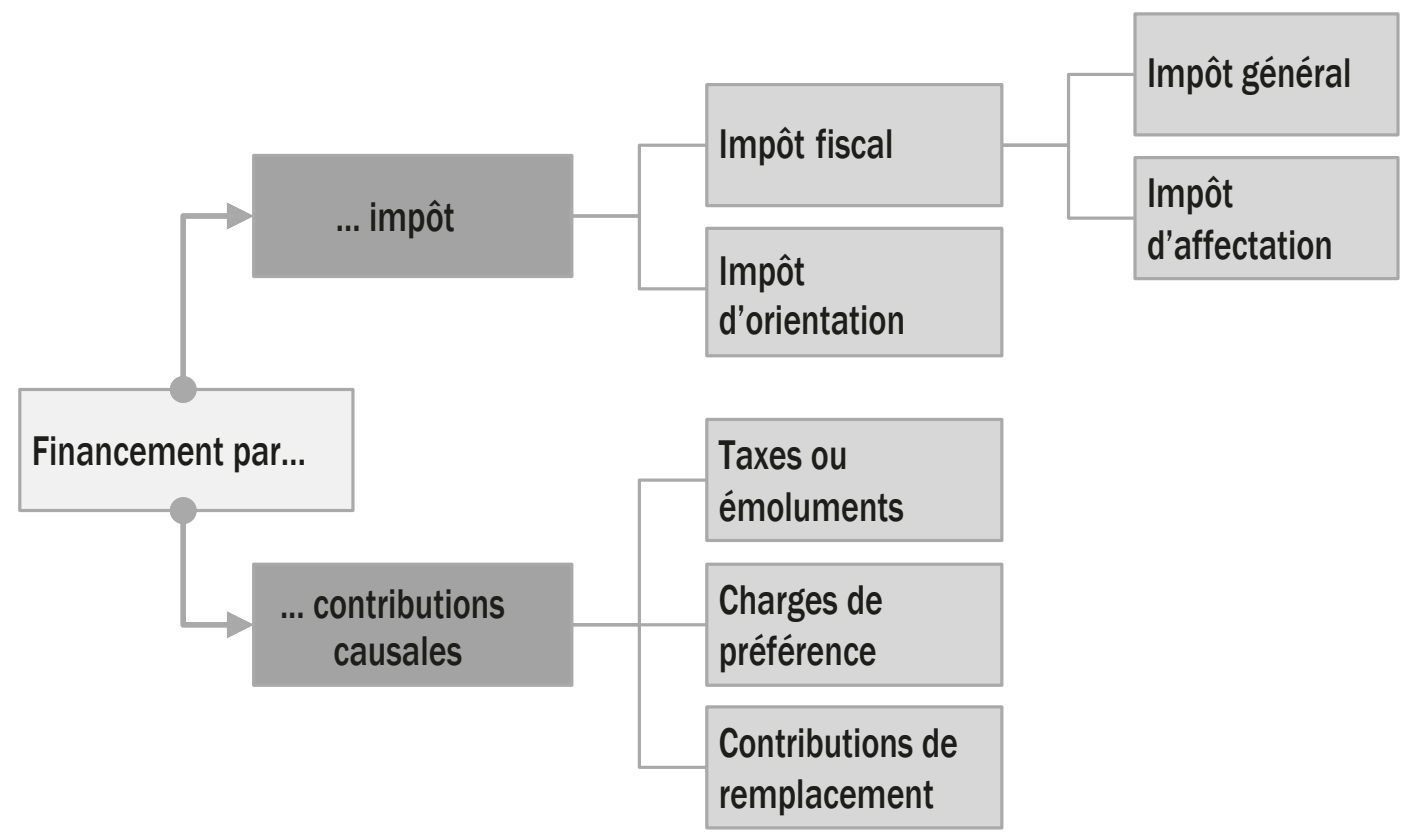


Impôt. En droit fiscal suisse, un impôt est un prélèvement obligatoire versé par les ménages ou les entreprises à une collectivité publique. Il est perçu uniquement en vertu de l'appartenance, fondée sur le droit, du contribuable à la collectivité concernée (par exemple une commune). Il est donc prélevé de manière inconditionnelle, c'est-à-dire indépendamment du fait que le contribuable bénéficie ou non de l'une ou l'autre des prestations offertes par la collectivité. Par contre, son montant est fonction de la situation économique du contribuable. En principe, le produit de l'impôt alimente le budget général de la collectivité. Il n'est donc pas affecté à financer une tâche particulière. Toutefois, et à titre d'exception au principe général de non-affectation, une partie des recettes d'un impôt est parfois consacrée au financement d'une tâche spécifique. L'impôt sur les maisons de jeu en est un exemple: il frappe le revenu brut des jeux, et ses recettes sont intégralement affectées au financement de l'assurance-vieillesse et survivants. On qualifie un tel impôt d'impôt d'affectation, par opposition à un impôt général comme l'impôt sur le revenu et la fortune frappant les personnes physiques. Dans les deux cas, on a affaire à des impôts fiscaux parce que leur objectif est de contribuer à alimenter le budget de la collectivité. Cependant, un impôt peut également avoir pour but d'inciter les ménages ou les entreprises à modifier leur comportement de consommation, d'investissement, etc. Il s'agit alors d'un impôt d'orientation dont les recettes ne sont pas nécessairement affectées à une tâche spécifique, mais qui souvent le sont. L'impôt sur le tabac en est un exemple. Il est prélevé en partie avec l'objectif de désinciter la consommation de tabac, mais l'objectif est également que ses recettes servent au financement de l'assurance-vieillesse et survivants. Les revenus fiscaux générés par les différents impôts sont comptabilisés dans la rubrique 40 de la classification par nature. Le tableau 25 montre que, pour MaCollectivité, les revenus fiscaux représentent avec KCHF 7651 la moitié des revenus de l'Année t (52\% pour être exact). Ces revenus fiscaux ne découlent pas d'impôts d'orientation, mais uniquement d'impôts fiscaux. Contribution causale. Comme l'appellation l'indique, une contribution causale est exigée par une collectivité publique dès lors qu'une personne physique ou morale bénéficie directement d'une prestation spécifique ou d'un avantage particulier. Le prélèvement d'une telle contribution se fonde donc sur un lien de causalité entre la prestation ou l'avantage offert d'une part et le paiement d'autre part. C'est là le principe de l'utilisateur-payeur ou encore du bénéficiaire-payeur. Le prélèvement respecte également une proportionnalité entre le montant prélevé et le coût de fourniture de la prestation ou la valeur qu'a l'avantage aux yeux du bénéficiaire.

Partant de là, une contribution causale peut revêtir différentes natures. Les taxes ou les émoluments sont prélevés en contrepartie d'une prestation de service de la part de la collectivité et de ses unités administratives. Ils sont également prélevés en contrepartie de l'utilisation du domaine public. Citons comme exemple les taxes d'écolage, les taxes pour l'enlèvement des ordures, les taxes de stationnement ou les émoluments pour des actes administratifs. 
Tableau 25 Importance des revenus fiscaux, ainsi que des taxes et redevances dans le total des revenus.

MaCollectivité, Année t, en KCHF.

\begin{tabular}{|llr|}
\hline$N^{\circ}$ & Libellé & KCHF \\
\hline 4 & Revenus & 14610 \\
\hline$\ldots$ & dont... & 7651 \\
40 & Revenus fiscaux & 6231 \\
400 & Impôts directs, personnes physiques & 501 \\
401 & Impôts directs, personnes morales & 898 \\
402 & Autres impôts directs & 21 \\
403 & Impôts sur la propriété et sur les charges & \\
{$[\ldots]$} & & 5076 \\
42 & Taxes et redevances & 192 \\
420 & Taxes de compensation & 73 \\
421 & Émoluments pour actes administratifs & 0 \\
422 & Taxes pour hôpitaux et établissements médicaux... & 0 \\
423 & Frais d'écolage et taxes de cours & 4651 \\
424 & Taxes d'utilisation et prestations de service & 92 \\
425 & Recettes sur ventes & 68 \\
426 & Remboursements & 0 \\
427 & Amendes & 0 \\
429 & Autres taxes
\end{tabular}

Les charges de préférence sont prélevées par une collectivité afin de couvrir les frais engendrés par la mise à disposition d'installations spécifiques. Le droit administratif suisse prévoit que deux conditions soient remplies pour qu'une personne doive payer une charge de préférence: d'abord, il faut que cette personne retire un avantage particulier des installations concernées, c'est-à-dire un avantage plus important que la moyenne des administrés; ensuite, l'avantage obtenu doit être de nature économique et accroître la valeur d'un bien dont la personne est propriétaire. Une charge de préférence peut ainsi être prélevée pour financer une station d'épuration des eaux, une route, des canalisations ou l'infrastructure de défense contre l'incendie.

Les contributions de remplacement occupent une catégorie à part. Elles ne sont pas exigées en contrepartie d'une prestation fournie à un administré. Elles frappent une personne dispensée d'une obligation ou d'un devoir de droit public. Ici, le lien de causalité est établi entre l'avantage que représente pour l'assujetti la libération de l'obligation primaire dont il devait s'acquitter et la contribution financière dont il doit s'acquitter. L'exemption de diverses obligations primaires donne lieu au 
prélèvement d'une contribution: exemption de l'obligation de servir, de l'obligation de construire une place de parc ou un abri de protection civile, etc.

Les contributions causales sont comptabilisées dans la rubrique 42 de la classification par nature, rubrique intitulée Taxes et redevances. Le tableau 25 montre que, pour MaCollectivité, les contributions causales représentent avec KCHF 5076 un tiers des revenus de l'Année t (35\% pour être exact, dans la rubrique 42). La majeure partie est constituée de contributions exigées en contrepartie de services autres que des actes administratifs, soit KCHF 4651 dans la rubrique 424 «Taxes d'utilisation et prestations de service».

\subsection{Principe d'équivalence et ses conditions de mise en œuvre}

La volonté de financer certaines prestations de façon particulière repose sur une justification économique - celle qui est donnée par le principe économique d'équivalence, plus communément qualifié, suivant le contexte, de principe de l'utilisateur-payeur ou du pollueur-payeur. Toutefois, cette volonté doit également être socialement acceptable.

Principe d'équivalence. Le principe d'équivalence consiste à mettre en balance l'utilité d'une prestation aux yeux du bénéficiaire d'une part et le prix que cette personne doit payer pour consommer cette prestation d'autre part. Pris au pied de la lettre, le principe d'équivalence veut que la collectivité fasse payer aux bénéficiaires des prestations offertes un montant équivalant à l'utilité que ces personnes retirent de ces prestations. L'idée est ici d'inciter les consommateurs de prestations à se comporter comme ils le feraient sur un marché. Dans ces conditions, les bénéficiaires sont également les payeurs des prestations et ils sont amenés à confronter le montant qu'ils paient à l'utilité qu'ils en retirent. La théorie prévoit qu'ils renoncent à consommer une prestation si le montant à payer est supérieur à l'utilité. Cet arbitrage entre montant à payer et utilité limite la demande de prestations publiques. Un tel arbitrage n'a pas lieu lorsqu'un service est fourni gratuitement, puisque dans ce cas l'utilité est toujours supérieure au prix qui est nul. En appliquant le principe d'équivalence, on évite une demande excessive et un gaspillage de ressources. Le corollaire est évidemment d'apporter des recettes au budget de la collectivité. Le principe de faire payer l'utilisateur pour des prestations collectives trouve une extension naturelle s'agissant de la consommation des ressources environnementales: le pollueur doit être le payeur pour les atteintes causées à l'environnement ou pour les mesures prises par les collectivités afin de remédier à ces atteintes.

Pour que le principe d'équivalence soit un mécanisme de financement approprié, plusieurs conditions doivent être observées. Ces conditions ont trait aux caractéristiques techniques de la prestation concernée et à l'acceptabilité sociale du principe (Dafflon 1994, pp. 107-111). 
Conditions techniques. Les conditions techniques sont au nombre de quatre.

- D'abord, il doit être possible d'identifier le bénéficiaire individuel de la prestation - personne physique ou morale. Par conséquent, la prestation doit s'adresser spécifiquement à un bénéficiaire et non à un groupe de bénéficiaires ou à l'ensemble de la population. Elle doit pouvoir être réservée au payeur. Si tel est le cas, alors l'offre de la prestation concernée est réputée divisible entre les utilisateurs.

- Ensuite, il doit être possible d'empêcher d'autres personnes que le payeur de bénéficier de la prestation. Il faut pouvoir déjouer les comportements de passagers clandestins. Des mesures techniques doivent pouvoir être instaurées à un coût raisonnable afin de contrôler l'accès à la prestation (péage, compteurs, contrôles sporadiques, etc.).

- De plus, la fourniture de la prestation à des usagers supplémentaires doit engendrer un coût supplémentaire. Imaginons au contraire que le coût de la prestation soit totalement indépendant du nombre d'utilisateurs, c'est-à-dire qu'il soit identique pour offrir la prestation à une personne ou à 10000 personnes. Dans ce cas, dès lors que la collectivité a supporté le coût pour un utilisateur, elle est capable de fournir les utilisateurs additionnels à un coût nul. On voit alors mal comment elle pourrait justifier de faire payer un prix aux utilisateurs additionnels. Cela dit, les prestations pour lesquelles le coût marginal (le coût additionnel par utilisateur) est nul sont rares. On cite parfois l'exemple des émissions radiotélévisées. Ces émissions impliquent un coût fixe initial important, mais dès lors que ce coût fixe est consenti, le coût marginal est nul. Pour la plupart des prestations, le coût marginal est non nul et le coût total augmente avec le nombre d'utilisateurs, même dans des domaines où le coût fixe initial est important. L'épuration des eaux implique par exemple un coût fixe important (collecteur, etc.). Mais le raccordement de chaque utilisateur supplémentaire au réseau a un coût, de même que l'épuration de chaque mètre cube d'eau usée supplémentaire. Dans ce cas, il devient techniquement possible de déterminer un coût non nul par utilisateur et par unité de prestation (ici le mètre cube).

- Enfin, les avantages offerts par la prestation fournie ne doivent pas outre mesure rejaillir sur d'autres personnes que celles qui paient pour cette prestation. En effet, si la prestation profite significativement à d'autres personnes que les payeurs, cela signifie d'une part que ces personnes bénéficient d'avantages sans en payer le prix et d'autre part que leurs préférences pour la prestation sont mal prises en compte puisqu'elles ne les manifestent pas à travers une décision de consommation révélée par le paiement d'un prix. Il faut donc avoir affaire à une prestation générant relativement peu d'effets externes. C'est le cas de la fourniture d'eau. La collectivité fournit en eau les ménages et les entreprises en mettant en place une infrastructure de production, de stockage et de distribution. Elle leur 
en fait payer le coût. Mais cette infrastructure offre des avantages externes dans le cadre de la lutte contre les incendies. Toutefois, ces avantages externes restent modérés en comparaison de ceux qui sont fournis par l'utilisation courante de l'infrastructure.

Acceptabilité sociale du mécanisme. Les éléments mentionnés ci-dessus sont des conditions techniquement nécessaires pour pouvoir financer une prestation de manière efficace en suivant le principe de l'utilisateur-payeur. Toutefois, dans la pratique, ces conditions ne sont pas toujours remplies. Par ailleurs, elles ne sont pas suffisantes pour garantir l'acceptabilité sociale de ce mode de financement. Cette acceptabilité sociale dépend de trois jugements de valeur au moins.

- D'abord, il doit être jugé acceptable que la fourniture de la prestation concernée soit réglée par un mécanisme de prix analogue à celui qui prévaut sur un marché. Cela nécessite d'admettre que le prix dicte le comportement de consommation des ménages et des entreprises. Cela implique de juger acceptable que la facturation d'un prix réduise, voire empêche, la consommation. On voit ici poindre la tension entre impératif d'efficacité et impératif d'équité. Dès lors que les impératifs d'équité sont jugés plus importants que les impératifs d'efficacité, le principe d'équivalence est moins acceptable ou son application doit s'accompagner de mesures redistributives. Mentionnons la mise à disposition dans certaines communes de sacs poubelle gratuits aux ménages avec enfants.

- Ensuite, il doit être socialement acceptable que la collectivité se finance en partie à travers le prélèvement d'un prix, plutôt qu'à travers le prélèvement d'un impôt.

- Enfin et par conséquent, il faut accepter qu'une partie du financement des activités de la collectivité n'intègre pas une dimension redistributive. Dans la mesure où l'on oppose le financement selon le principe d'équivalence au financement selon la capacité contributive des citoyens, cela implique d'accepter qu'une partie du financement du budget de la collectivité ne contribue pas à redistribuer les richesses.

La figure 25 offre un arbre de décision pour choisir entre un financement fondé sur le principe d'équivalence ou un financement fondé sur le principe de la capacité contributive. Si les critères techniques sont, au moins en partie, satisfaits et si le principe de l'équivalence est jugé socialement acceptable, alors le coût de la prestation considérée peut être financé par une contribution causale. Par contre, si les critères techniques ne sont pas du tout satisfaits et/ou si le principe d'équivalence est socialement inacceptable, alors il faudra opter pour un financement fondé sur la capacité contributive des citoyens. Par conséquent, l'impôt devra financer le coût de la prestation. 
Figure 25 Arbre de décision permettant de choisir entre un financement fondé sur le principe d'équivalence ou un financement fondé sur le principe de la capacité contributive.

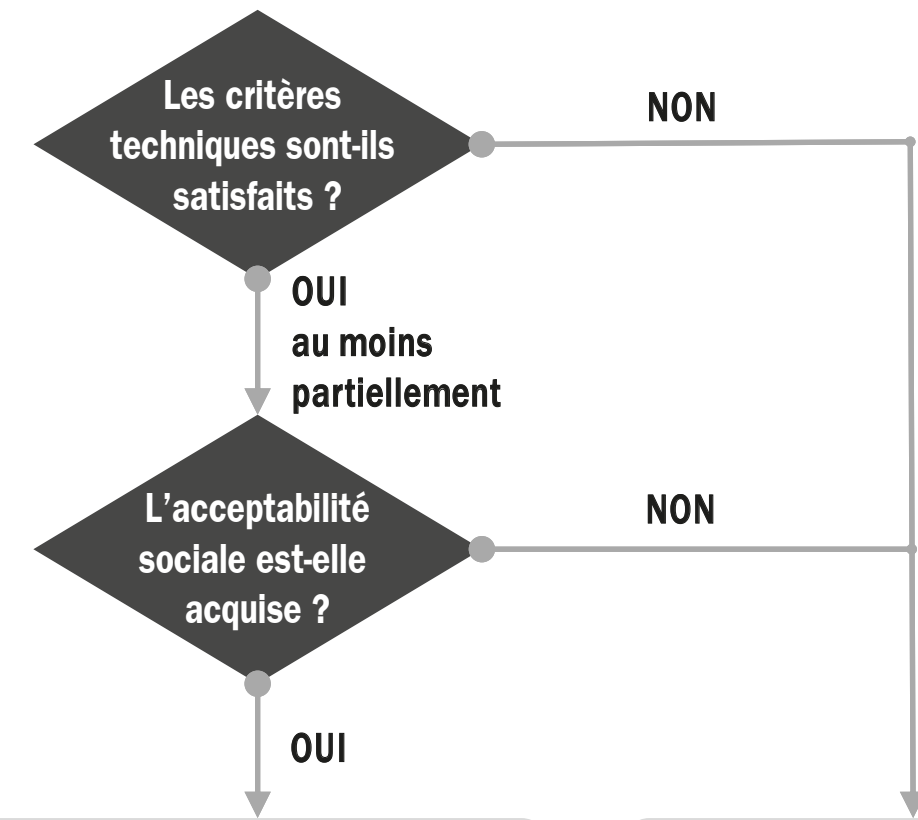

Principe d'équivalence (taxe, redevance, contribution, émolument)
Principe de la capacité contributive (impôt)

Source: Adapté de Dafflon (1994).

\subsection{Affectation tactique des recettes}

La conséquence logique d'une contribution causale en application du principe d'équivalence consiste à affecter les recettes à la couverture des coûts de la prestation concernée. Toutefois, l'affectation des recettes à une tâche particulière peut également répondre à un besoin tactique.

Favoriser l'acceptabilité politique. C'est en particulier le cas lorsqu'il s'agit de défendre politiquement l'introduction d'un nouvel impôt ou l'augmentation d'un impôt existant. L'impôt devient ainsi un impôt d'affectation. Mais cela vaut également dans le cas d'un impôt d'orientation. Proposer que les recettes d'un impôt soient affectées accroît l'acceptabilité politique. Cette dimension n'est pas négligeable dans un régime de démocratie directe. Et dans les faits, cet argument est souvent utilisé. Pensons au cas emblématique de l'impôt de défense nationale, prélevé dès 1941 et rebaptisé depuis impôt fédéral direct. Plus récemment, mentionnons l'augmentation successive du taux de la «taxe» sur la valeur ajoutée pour financer l'aménagement de l'infrastructure ferroviaire ou, temporairement, pour 
assainir l'assurance-invalidité ${ }^{120}$. Pour sa part, l'impôt sur le tabac est typiquement un impôt d'orientation donc les recettes sont affectées, dans ce cas particulier, quasi intégralement à l'assurance-vieillesse et survivants et à l'assurance-invalidité.

Verrouiller l'allocation budgétaire. Ancrer dans la législation qu'une contribution causale ou un impôt est d'emblée dévolu à une tâche particulière permet de soustraire ses recettes des discussions budgétaires actuelles et futures. L'objectif tactique poursuivi est d'empêcher que ces moyens soient détournés de leur utilisation au profit d'autres politiques publiques. L'avantage de cette entorse au principe de la non-affectation est de garantir une stabilité du financement dans la durée. Mais l'inconvénient est de verrouiller l'allocation budgétaire et d'empêcher une réallocation en fonction de priorités qui peuvent évoluer avec le temps. Par ailleurs, en cas de difficultés budgétaires, certains domaines peuvent être épargnés par les programmes d'assainissements parce qu'ils bénéficient de recettes affectées en suffisance. De ce fait, l'effort d'assainissement se concentre sur les secteurs qui dépendent des ressources libres du budget. Cela revient à postuler que les ressources affectées sont utilisées de manière parfaitement efficiente et qu'aucun gain de productivité n'est possible dans les domaines concernés. Il s'agit là d'un postulat fort. D'ailleurs, l'effet final de l'affectation des recettes sur l'efficacité n'est pas encore établi (Lee and Wagner 1991). Pour certains, l'application du principe d'équivalence incite les unités administratives à être performantes en répondant aux besoins des utilisateurs au moindre coût. Pour d'autres, les rigidités budgétaires qui découlent de l'affectation peuvent avoir l'effet inverse.

\subsection{Dispositions légales}

Dans de nombreux domaines, le législateur helvétique est parvenu à la conclusion que le principe de l'utilisateur-payeur, du bénéficiaire-payeur ou du pollueur-payeur doit être appliqué. Diverses dispositions légales ou réglementaires, fédérales, cantonales ou communales, en résultent.

Le droit fédéral oblige d'ailleurs parfois expressément les cantons à agir en ce sens. Par exemple, la loi fédérale sur la protection de l'environnement (LPE) prévoit que «les cantons veillent à ce que les coûts de l'élimination des déchets urbains [...] soient mis, par l'intermédiaire d'émoluments ou d'autres taxes, à la charge de ceux qui sont à l'origine de ces déchets» (art. 32a, al. 1, RS 814.20). La loi fédérale sur la protection des eaux (LEaux) a la même teneur s'agissant des eaux usées et du coût de leur épuration (art. 60a, al. 1, RS 814.01).

De leur côté, les cantons, à travers leurs propres lois ou ordonnances, peuvent exiger des communes situées sur leur territoire qu'elles mettent en place un tel mécanisme.

120 L'utilisation de la notion de «taxe» pour qualifier ce qui est un impôt sur la valeur ajoutée provient de la conversion de l'acronyme anglais VAT (value-added tax) en son équivalent français TVA. Or la traduction française correcte de tax est impôt. On doit donc faire attention aux faux-amis. 
Chaque commune doit alors se doter d'un règlement, voté par son Législatif et approuvé par le canton, avant de pouvoir facturer et encaisser les contributions causales destinées à couvrir ses coûts dans le domaine concerné.

Les dispositions fédérales et cantonales ont ainsi amené les communes à se doter de règlements, par exemple dans les domaines de la gestion des déchets, de l'épuration des eaux et de l'approvisionnement en eau potable. Ces règlements prévoient que le coût doit être couvert par des contributions causales. Implicitement ils imposent donc que le coût ne peut pas être couvert par des revenus fiscaux. Le Tribunal fédéral a d'ailleurs arrêté que le recours à l'impôt pour financer les prestations publiques dans le domaine de l'élimination des déchets n'est pas autorisé (ATF 2C_740/2009 du 04.07.2011). Par contre, ces règlements stipulent, le plus souvent explicitement, que le montant des contributions prélevées ne doit pas excéder le coût de fourniture. Par conséquent, ces contributions ne sauraient financer d'autres tâches.

Dans leur sphère de compétence et en fonction de leurs besoins, les communes se dotent de dispositions réglementaires similaires pour d'autres prestations. Mentionnons les parkings, le téléréseau, le réseau d'électricité ou le chauffage à distance.

Les règlements fixent les modalités du prélèvement en fonction du type de coûts à couvrir. Les charges de préférence et les taxes de raccordement financent les dépenses d'investissement. Les taxes de base et les taxes d'exploitation financent les coûts de fonctionnement.

Charge de préférence. Ainsi qu'il apparaît dans la figure 24, l'Administration fédérale des contributions sépare ce prélèvement des autres contributions causales (AFC 2018). Il est vrai que, contrairement aux autres contributions, la charge de préférence est perçue auprès des propriétaires fonciers même s'ils n'utilisent pas ou pas encore l'infrastructure publique à disposition, par exemple les canalisations de la commune. Cette contribution est exigible même si le bien-fonds n'est pas construit, mais qu'il est réputé raccordable. Elle est donc due indépendamment du fait qu'il y ait un raccordement effectif ou non à l'infrastructure concernée. Elle rémunère l'avantage que le propriétaire retire de savoir que le jour où il voudra raccorder son immeuble au réseau, ce réseau sera à disposition. L'assiette de calcul de la charge de préférence est généralement estimée à partir des caractéristiques techniques de l’immeuble raccordable ou raccordé. Grâce au prélèvement de cette contribution, la collectivité peut répartir la dépense infrastructurelle sur l'ensemble des propriétaires d'immeubles, que ces immeubles soient construits ou à construire. Cette solution est plus équitable que celle qui consisterait à faire supporter la dépense aux seuls propriétaires d'immeubles déjà bâtis et/ou aux contribuables.

Taxe de raccordement. Cette taxe sert en particulier à couvrir les coûts de raccordement des installations à l'infrastructure d'ensemble. Elle est perçue intégralement auprès des propriétaires de fonds bâtis. Elle est donc due dès qu'il y a un accès réel à l'infrastructure, indépendamment du fait qu'il y ait utilisation ou non de la prestation publique, par exemple une consommation d'eau. C'est pourquoi, dans la 
figure 24, elle appartient à la catégorie des taxes ou émoluments. La taxe rémunère l'avantage que le propriétaire retire de savoir que le jour où il voudra utiliser son accès au réseau et bénéficier ainsi de la prestation publique, il pourra le faire. À l'instar de la charge de préférence, l'assiette de calcul de la taxe de raccordement est généralement estimée à partir de caractéristiques techniques de l'immeuble raccordé.

Taxe de base. Cette taxe est parfois également appelée taxe forfaitaire. Dans la figure 24, elle appartient également à la catégorie des taxes ou émoluments. Elle sert à couvrir les coûts fixes inhérents à la fourniture de la prestation publique. Les coûts fixes incluent en particulier le coût de l'usure et de l'obsolescence des installations, le coût de la maintenance et de l'adaptation des équipements à de nouvelles exigences légales, les intérêts, ainsi que les charges concernant la gestion administrative et technique. Cette taxe est due par l'utilisateur indépendamment du volume effectivement consommé. Suivant la prestation, le montant de la taxe de base est donc déterminé en fonction du nombre d'utilisateurs, du nombre de ménages, du nombre d'accès ou de la puissance installée.

Taxe d'exploitation. Cette taxe est destinée à couvrir les coûts qui varient directement en fonction des quantités produites et qui sont donc directement provoqués par la consommation des utilisateurs. Selon les cas, ces coûts variables incluent les coûts de main-d'œuvre en particulier pour l'entretien et la production, les coûts énergétiques et les frais d'acquisition des autres biens et services. L'enjeu pour calculer le montant de la taxe consiste à déterminer et à mesurer l'unité de consommation. Pour prendre l'exemple de l'élimination des déchets, il s'agit de définir si la consommation se mesure au poids des déchets ou au nombre de sacs éliminés.

\subsection{Modalités comptables des financements spéciaux et des fonds}

\subsubsection{Distinction entre financements spéciaux et fonds}

Lorsqu'il a été décidé que les coûts de fourniture d'une prestation doivent être couverts par des recettes affectées, il est important de mettre en place un dispositif permettant de contrôler l'application des dispositions légales découlant de cette décision. Pour ce faire, le modèle comptable $\mathrm{MCH} 2$ offre un dispositif assez subtil qui mérite une attention particulière.

Le dispositif repose sur l'utilisation de financements spéciaux et de fonds ${ }^{121}$. Le plan comptable $\mathrm{MCH} 2$ offre une distinction entre ces deux cas de figure. Pour ce qui est du financement spécial, le Manuel $\mathrm{MCH} 2$ précise qu'il s'agit d'une allocation des recettes à une tâche spécifique en vertu d'une disposition légale. Par contre, le

121 Spezialfinanzierung | Finanziamenti speciali | Earmarked fund. Fonds | Fondi | Fund. Recommandation 08, CDF (2008). 
manuel ne donne pas de définition à la notion de fonds, parlant parfois de fonds affectés. On doit donc s'interroger sur les cas qui relèvent d'un financement spécial et ceux qui relèvent d'un fonds. Un rapport du Conseil fédéral (2015) apporte un éclairage sur cette distinction.

Financement spécial. On devrait parler de financement spécial dès le moment où (a) une contribution causale est prélevée et $(b)$ un équilibre annuel est recherché entre les contributions encaissées et les coûts à couvrir. En définitive, un financement spécial répond pleinement à la volonté de mettre en place le principe de l'utilisateur-payeur. Par exemple, on trouvera des financements spéciaux au niveau communal dans le domaine de la gestion des déchets, de l'eau usée ou de l'eau potable.

Fonds. On devrait réserver l'emploi de la notion de fonds (a) aux cas pour lesquels le financement n'est pas nécessairement assuré par une contribution causale, mais peut provenir d'un impôt d'affectation ou d'autres revenus affectés provenant d'autres entités, qu'il s'agisse de collectivités, d'individus ou d'entreprises ou (b) aux cas pour lesquels un équilibre annuel n'est pas exigé entre le financement obtenu et le coût à couvrir. En définitive, en recourant au mécanisme du fonds plutôt qu'à celui du financement spécial, on montre dans les comptes que l'on s'accorde davantage de flexibilité dans l'appariement entre les revenus affectés et les coûts à couvrir, pour faire face notamment aux pics et aux creux d'encaissements et de décaissements. Par exemple, on trouvera des fonds au niveau communal dans le domaine du financement des abris de protection civile ou, parfois, des parkings.

\subsubsection{Charges et revenus affectés dans la classification fonctionnelle}

Le dispositif du $\mathrm{MCH} 2$ tire parti de la systématique de numérotation offerte par le plan comptable et de la possibilité de classer simultanément une charge - ou un revenu - dans une rubrique par nature et dans une catégorie fonctionnelle.

C'est ce qu'illustre le tableau 26 en prenant l'exemple dans MaCollectivité des salaires versés au personnel du Service de la propreté publique directement en charge de la gestion des déchets. Dans les comptes, le montant de ces salaires est catégorisé dans la classification organique sur la base des trois premiers numéros de la clé d'imputation dans l'unité administrative 484. Le même montant est catégorisé dans la classification par nature sur la base des quatre numéros suivants - 3010 - parmi les salaires du personnel administratif et d'exploitation de MaCollectivité. Les trois derniers numéros - 730 - permettent de catégoriser ce même montant comme faisant partie du coût de la prestation de gestion des déchets à l'intérieur de la classification fonctionnelle. Le dispositif permet ainsi d'utiliser l'information comptable pour répondre à diverses interrogations nécessaires à la gestion, comme l'a schématisé la figure $21^{122}$. 
Tableau 26 Catégorisation des charges pour la gestion des déchets.

Exemple des salaires versés dans MaCollectivité au personnel

de l'unité administrative responsable de fournir la prestation.

\begin{tabular}{|c|c|c|c|c|c|c|c|c|c|c|c|}
\hline \multicolumn{12}{|l|}{ Classification... } \\
\hline ... organique & $\begin{array}{l}\text { Direction: } \\
\text { Service: } \\
\text { Unité: }\end{array}$ & $\begin{array}{l}\text { Dép. Environnement et bâti } \\
\text { Propreté publique } \\
\text { Gestion des déchets }\end{array}$ & 4 & 8 & 4 & & & & & & \\
\hline ... par nature & $\begin{array}{l}\text { Classe de comptes: } \\
\text { Groupe de comptes: } \\
\text { Sous-groupe: } \\
\text { Compte: }\end{array}$ & $\begin{array}{l}\text { Charges } \\
\text { Charges de personnel } \\
\text { Salaire du personnel... } \\
\text { administratif et d'exploitation }\end{array}$ & & & & 3 & 0 & 1 & 0 & & \\
\hline ... fonctionnelle & $\begin{array}{l}\text { Fonction: } \\
\text { Tâche: } \\
\text { Subdivision: }\end{array}$ & $\begin{array}{l}\text { Protection de l'environnement } \\
\text { Gestion des déchets } \\
\text { Gestion des déchets }\end{array}$ & & & & & & & & 7 & 3 \\
\hline Numéro à saisir & ur l'imputation: & & 4 & 8 & 4 & 3 & & 1 & & & 30 \\
\hline
\end{tabular}

En imputant tous les éléments nécessaires à la gestion des déchets dans la fonction 730 , on parvient à dégager le coût de revient de cette prestation. Cela revient à extraire de la classification fonctionnelle les informations spécifiques à la fonction 730. Cette extraction est présentée dans le tableau 27. On y voit apparaître aux côtés des salaires (KCHF 73), les autres charges imputées à cette fonction avec, en début de ligne, le rappel du numéro de la rubrique correspondante dans la classification par nature. Les autres charges sont les charges pour l'achat de biens et de services (KCHF 217), les amortissements des actifs concernés à l'intérieur du patrimoine administratif (KCHF 2), l'imputation interne des salaires du personnel de l'Administration générale pour le temps qu'il consacre à la tâche de gestion des déchets (KCHF 10). Au total, la gestion des déchets engendre un coût de KCHF 302 pour l'Année t.

Tableau 27 La gestion des déchets dégage un excédent de revenus, avant son attribution au capital propre.

MaCollectivité, Fonction 730, Année t, en KCHF, avant clôture.

\begin{tabular}{|c|c|c|c|c|}
\hline$N^{\circ}$ & Libellé & KCHF & $\mathrm{N}^{\circ}$ Libellé & $\mathrm{KCHF}$ \\
\hline 3 & Charges & 302 & Revenus & 362 \\
\hline 30 & Charges de personnel & 73 & 42 Taxes et redevances & 354 \\
\hline 31 & $\begin{array}{l}\text { Charges de biens et services et } \\
\text { autres charges d'exploitation }\end{array}$ & 217 & 44 Revenus financiers & 8 \\
\hline 33 & $\begin{array}{l}\text { Amortissements du patrimoine } \\
\text { administratif }\end{array}$ & 2 & & \\
\hline 39 & Imputations internes & 10 & & \\
\hline Exc & sédent de revenus & 60 & & \\
\hline
\end{tabular}


L'extraction montre aussi quels sont les revenus apportés par cette prestation. Les utilisateurs ont payé des taxes pour un montant de KCHF 354. Le mécanisme de catégorisation est le même que pour les charges. Il est illustré dans le tableau 28. La différence par rapport à la catégorisation des salaires présentée dans le tableau 26 se situe au niveau des quatre numéros relatifs à la classification par nature: le chiffre 4240 désignant les taxes d'utilisation et de prestations de service remplace le chiffre 3010 .

Aux taxes s'ajoutent des revenus financiers de KCHF 8. Au total, la gestion des déchets dégage des revenus à hauteur de KCHF 362 pour l'Année t. Le tableau 27 met ainsi en évidence un excédent de revenus pour cette prestation de KCHF 60, avant son attribution au capital propre telle que nous la décrirons plus loin.

D'un point de vue technique, il est possible de mettre en évidence la différence entre les revenus et les charges pour chaque fonction. Nous l'avons d'ailleurs fait précédemment dans le tableau 20. Cette information peut être intéressante et souhaitable pour la gestion. Pour les domaines soumis à une obligation légale d'équilibrer les charges et les revenus, cette information est indispensable. Elle permet de s'assurer que les taxes encaissées ont été utilisées uniquement pour financer la prestation concernée, ici la gestion des déchets. Parallèlement, on peut aussi vérifier que les revenus des taxes ont été suffisants pour couvrir l'ensemble des charges causées par cette prestation.

Tableau 28 Catégorisation des revenus pour la gestion des déchets.

Exemple des taxes payées à MaCollectivité par les utilisateurs.

\begin{tabular}{|c|c|c|c|c|c|c|c|c|c|c|c|c|}
\hline Classification... & & & & & & & & & & & & \\
\hline ... organique & Direction: & Dép. Environnement et bâti & 4 & & & & & & & & & \\
\hline & Service: & Propreté publique & & 8 & & & & & & & & \\
\hline & Unité: & Gestion des déchets & & & 4 & & & & & & & \\
\hline ... par nature & Classe de comptes: & Revenus & & & & 4 & & & & & & \\
\hline & Groupe de comptes: & Taxes et redevances & & & & & 2 & & & & & \\
\hline & Sous-groupe: & Taxes d'utilisation & & & & & & 4 & & & & \\
\hline & Compte: & Taxes d'utilisation & & & & & & & 0 & & & \\
\hline ... fonctionnelle & Fonction: & Protection de l'environnement & & & & & & & & 7 & & \\
\hline & Tâche: & Gestion des déchets & & & & & & & & & 3 & \\
\hline & Subdivision: & Gestion des déchets & & & & & & & & & & 0 \\
\hline Numéro à saisir & ur l'imputation: & & 4 & 8 & 4 & 4 & 2 & 4 & 0 & & 3 & 0 \\
\hline
\end{tabular}

\subsubsection{Financements spéciaux et fonds dans le bilan}

En réalité, pour une année donnée, comme l'Année t dans MaCollectivité, il est rare que revenus et charges s'équilibrent exactement. Soit les utilisateurs ont trop payé - comme illustré ici dans le cas de la gestion des déchets -, soit ils ont payé trop peu. 
L'excédent de revenus est assimilable à une créance des utilisateurs vis-à-vis de la collectivité: la collectivité a un engagement envers le financement spécial ou le fonds (créanciers) et, à travers lui, envers les utilisateurs. À l'inverse, un excédent de charges est assimilable à une dette des utilisateurs: la collectivité fait une avance aux utilisateurs et au financement spécial ou au fonds (débiteurs). Cet engagement ou cette avance doit être documenté, inventorié en fin d'année, c'est-à-dire que le financement spécial ou le fonds doit figurer dans le bilan de la collectivité.

La question est alors de déterminer si un financement spécial ou un fonds (créanciers) doit être assimilé à du capital propre ou à du capital de tiers. La réponse dépend de la législation, comme l'indique la figure 26. Si la collectivité est légalement seule compétente pour modifier la base légale concernée, par exemple si elle peut décider du niveau de la contribution causale, alors le financement spécial ou le fonds fait partie du capital propre. C'est aussi le cas s'il existe des dispositions de droit supérieur, mais que la collectivité dispose d'une marge de manœuvre considérable en matière d'application.

Figure 26 Selon la législation auquel il est soumis, un financement spécial ou un fonds doit être considéré comme du capital propre ou comme du capital de tiers.

\section{Financement spécial (FSp) ou fonds (Fds)}
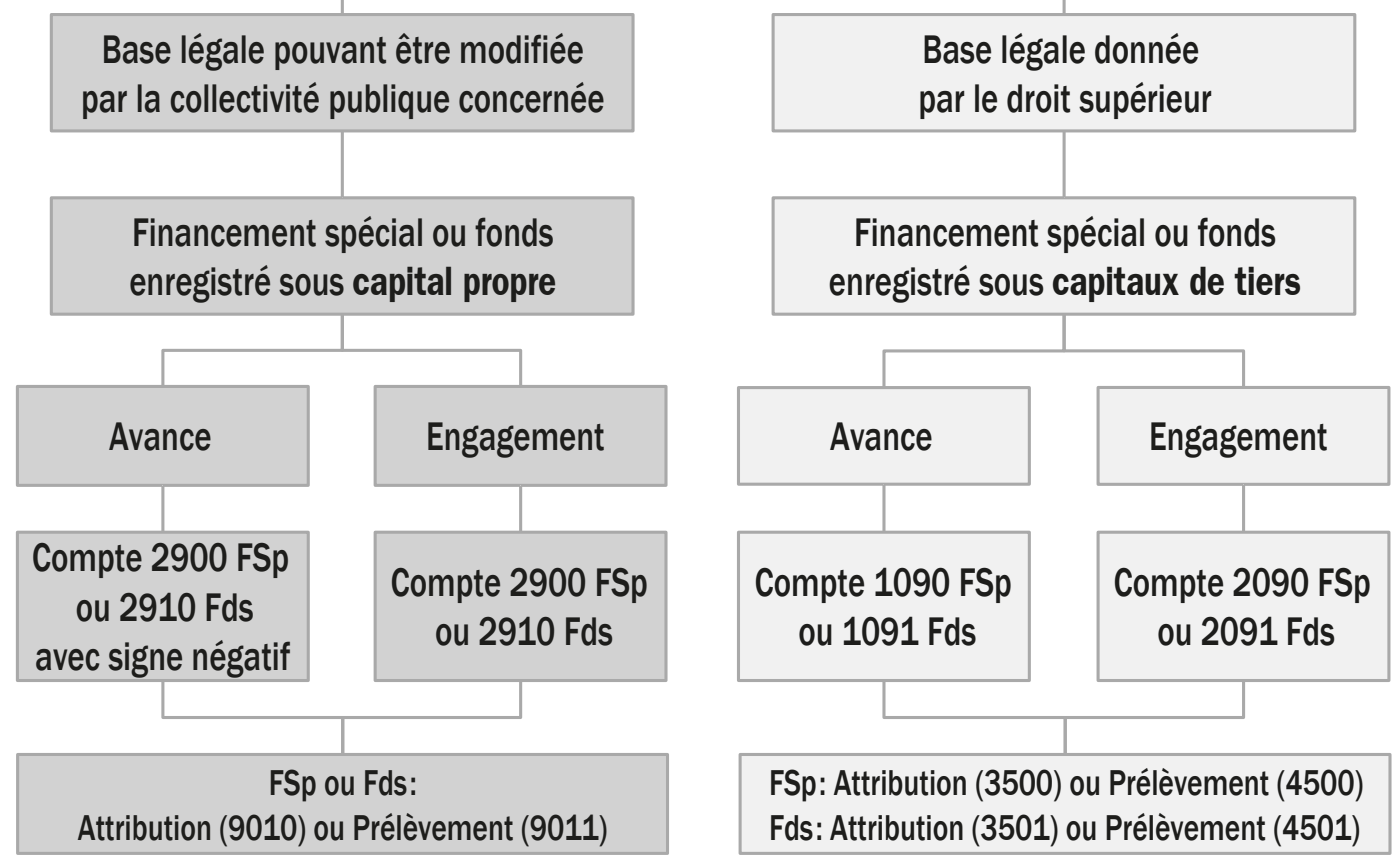
En revanche, si le droit de rang supérieur fixe des obligations, sans possibilité pour la collectivité de modifier les prescriptions ou avec une très faible marge de manœuvre, alors le financement spécial ou le fonds s'apparente à du capital étranger. Cela se justifie par le fait que la collectivité ne peut pas décider de modifier les conditions d'utilisation et d'alimentation. Par conséquent, elle n'a pas d'emprise sur l'engagement ou l'avance qui en découle. En outre, un financement spécial ou un fonds doit être considéré comme du capital de tiers s'il est en partie alimenté par d'autres revenus que des contributions causales. Il n'en reste pas moins que les revenus encaissés doivent obligatoirement être utilisés pour couvrir les charges imputées au financement spécial ou au fonds en question.

Lorsque la collectivité a un engagement envers un financement spécial (créancier), celui-ci figure au passif du bilan dans le compte 2090 «Engagements envers les financements spéciaux sous capitaux de tiers» ou dans le compte 2900 «Financements spéciaux sous capital propre». Lorsqu'il s'agit d'un fonds (créancier), il figure au passif dans le compte 2091 «Engagements envers les fonds sous capitaux de tiers» ou dans le compte 2910 «Fonds sous capital propre».

Lorsque la collectivité fait une avance à un financement spécial (débiteur) considéré comme du capital propre, ce financement spécial est présenté au passif avec un signe négatif au compte 2900. S'il s'agit d'un fonds (débiteur), il porte aussi un signe négatif dans le compte passif 2910. De cette manière, tous les éléments constitutifs du capital propre figurent au passif au bilan, même si certains doivent pour cela porter un signe négatif.

Par contre, si le financement spécial (débiteur) est considéré comme du capital de tiers, alors l'avance figure à l'actif du bilan, au compte 1090 «Créances envers les financements spéciaux sous capitaux de tiers». Pour un fonds (débiteur), l'avance est présentée au compte 1091 «Créances envers les fonds sous capitaux de tiers».

Le tableau 29 montre les différents financements spéciaux et fonds mis en place par MaCollectivité et présentés au passif de son bilan. Un seul fonds est enregistré sous capitaux de tiers: celui relatif aux abris de protection civile financé par des contributions de remplacement (compte 2091.0). Le classement sous capitaux de tiers se justifie dans la mesure où une commune ne peut utiliser ces recettes que si elle y est autorisée par l'autorité cantonale compétente. Par ailleurs, il s'agit d'un fonds dans la mesure où un équilibre annuel entre les encaissements et les décaissements n'est pas recherché.

Par contre, six financements spéciaux sont assimilés à du capital propre (comptes 2900.X). Cela est justifié puisque, dans ces domaines, la collectivité peut adapter les bases réglementaires en fonction de ses besoins. De plus, la collectivité doit tenter d'équilibrer dans toute la mesure du possible les charges et les revenus à la fin de l'exercice. Évidemment - en pratique, un équilibre exact ne peut pas être atteint chaque année, ce qui justifie l'existence d'une avance ou d'un engagement à faire apparaître au bilan. 
Tableau 29 Financements spéciaux et fonds au passif du bilan. MaCollectivité, Année t, en KCHF.

01.01. Attribution Prélèvement 31.12.

\begin{tabular}{|c|c|c|c|c|c|}
\hline $\mathbf{N}^{\circ}$ & Libellé & $\begin{array}{l}\text { Année t } \\
\text { KCHF }\end{array}$ & KCHF & $\mathrm{KCHF}$ & $\begin{array}{l}\text { Année } t \\
\text { KCHF }\end{array}$ \\
\hline 2 & Passif & 13274 & 19795 & 19393 & 13676 \\
\hline ... & dont... & & & & \\
\hline 209 & $\begin{array}{l}\text { Engagements envers les FSp } \\
\text { et les fonds sous CT }\end{array}$ & 348 & 0 & 26 & 322 \\
\hline ... & dont... & & & & \\
\hline 2091.0 & Fonds Abris PC & 201 & & 21 & 180 \\
\hline \multicolumn{6}{|l|}{ [...] } \\
\hline 290 & $\begin{array}{l}\text { Financements spéciaux [FSp] } \\
\text { sous CP }\end{array}$ & 1670 & 130 & 76 & 1724 \\
\hline 2900.1 & Financement spécial Déchets & 319 & 60 & & 379 \\
\hline 2900.2 & Financement spécial Eau potable & 379 & 10 & & 389 \\
\hline 2900.3 & Financement spécial Eau usée & 503 & 20 & & 523 \\
\hline 2900.4 & Financement spécial Téléréseau & 157 & 40 & & 197 \\
\hline 2900.5 & Financement spécial Électricité & 312 & & 27 & 285 \\
\hline 2900.6 & $\begin{array}{l}\text { Financement spécial Chauffage } \\
\text { à distance }\end{array}$ & 0 & & 49 & -49 \\
\hline$[\ldots]$ & & & & & \\
\hline
\end{tabular}

Le financement spécial concernant le chauffage à distance présente un solde débiteur à la fin de l'Année t (2900.6). En d'autres termes, il s'agit d'une avance de MaCollectivité en faveur de ce financement spécial qui est classé au passif du bilan, mais avec un signe négatif. Les autres financements spéciaux aussi enregistrés dans le capital propre, dont celui pour la gestion des déchets (2900.1), indiquent en début d'année comme en fin d'année un solde créancier. À l'instar du financement spécial «Déchets», ceux pour l'eau potable (2900.2), l'eau usée (2900.3) et le téléréseau (2900.4) ont vu en cours d'année leur solde créancier augmenter sous l'effet d'un excédent de revenus et d'une attribution de cet excédent au financement spécial. Les financements spéciaux relatifs à l'électricité (2900.5) et au chauffage à distance (2900.6) ont vu leur solde se réduire, pour devenir même débiteur dans le cas du chauffage à distance (signe négatif), sous l'effet d'un prélèvement pour couvrir un excédent de charges. 


\subsubsection{Attributions et prélèvements dans le cas des financements spéciaux et des fonds assimilés à du capital de tiers}

En fin d'exercice, il s'agit donc de présenter au bilan l'état du financement spécial ou du fonds. La procédure est différente selon que le financement spécial ou le fonds est assimilé à du capital propre ou à du capital de tiers.

Commençons par ce second cas, celui du capital de tiers. Il concerne typiquement le fonds 2091.0 enregistrant les excédents de charges et de revenus dans le domaine de la défense civile, en lien avec la construction d'abris de protection civile. Ce domaine correspond à la fonction 162 dans la classification fonctionnelle. Le tableau 29 montre que MaCollectivité a retiré KCHF 21 à la fin de l'Année t de ce fonds qui se trouve au passif du bilan sous le compte 2091 «Engagements envers les fonds sous capitaux de tiers». Un tel prélèvement permet de couvrir en partie l'excédent de charges en l'absence de contributions de remplacement facturées aux propriétaires des immeubles concernés au cours de l'Année t. Cette opération représente pour MaCollectivité un revenu à comptabiliser à l'avoir du compte 4501 «Prélèvements sur les fonds sous capitaux de tiers» (ou 4500 s'il s'agissait d'un financement spécial). La contrepartie est une diminution du fonds 2901.0 au passif du bilan, avec un solde au 31 décembre qui s'établit à KCHF 180. En l'occurrence, on parle de prélèvement sur le fonds puisque ce fonds a été constitué par les excédents de revenus réalisés dans ce domaine au cours des exercices antérieurs.

En effet, au cours des années antérieures, lorsque les charges ont été inférieures aux contributions - ici aux contributions de remplacement, un excédent de revenus est apparu. La collectivité a alors bénéficié d'une avance de la part de tiers - ici de la part des propriétaires d'immeubles. Pour rétablir un équilibre entre charges et revenus dans ce domaine spécifique, on a comptabilisé une charge au doit du compte 3501 «Attributions aux fonds sous capitaux de tiers» (ou 3500 s'il s'agit d'un financement spécial). La contrepartie a été une augmentation du fonds 2901.0 au passif du bilan. Dans le cas de MaCollectivité, au $1^{\text {er }}$ janvier de l'Année t, le solde du fonds s'élevait à KCHF 201. Cela montre que, par le passé, avant l'Année $t$, le fonds s'est vu attribué davantage qu'il n’y a été prélevé. Autrement dit, les propriétaires d'immeubles ont contribué davantage au domaine de la défense civile que ce domaine n'a coûté. Par conséquent, MaCollectivité a un engagement face à ces tiers.

Si cela avait été l'inverse, c'est-à-dire si les coûts de ce domaine avaient été supérieurs aux contributions, la collectivité aurait dû faire une avance à ce fonds. Cette avance serait alors présentée à l'actif du bilan dans le compte 1091 «Créances envers les fonds sous capitaux de tiers».

Le fait que les attributions et les prélèvements modifient la position de la collectivité face aux tiers - dans notre exemple, le groupe des propriétaires d'immeubles implique que ces opérations aient un impact sur le résultat de l'exercice. C'est bien ce qui se passe puisque les charges enregistrées dans le compte 3501 (ou 3500) et les 
revenus comptabilisés dans le compte 4501 (ou 4500) affectent le résultat d'exploitation à l'intérieur du compte de résultats. Sans ce prélèvement de KCHF 21 sur le fonds 2091.0, le résultat d'exploitation de MaCollectivité se serait élevé à KCHF 971 plutôt qu'à KCHF 992, tel qu'affiché dans le tableau 17 (section 3.3.4).

\subsubsection{Attributions et prélèvements dans le cas des financements spéciaux et des fonds assimilés à du capital propre}

Lorsque les financements spéciaux et les fonds sont assimilés à du capital propre, l'écart entre les charges et les revenus dans les domaines concernés ne peut pas être considéré comme le reflet d'une modification de la position de la collectivité face à des tiers. Par conséquent, il n'est pas envisageable de comptabiliser à la clôture des attributions et des prélèvements comme nous l'avons montré ci-dessus en utilisant les groupes de comptes 35 et 45 : ces opérations modifieraient le solde du compte de résultats, donc le niveau de l'excédent ou du découvert au bilan (rubrique 299).

On doit donc recourir à un mécanisme différent selon que les financements spéciaux ou les fonds sont considérés comme du capital propre ou qu'ils sont considérés comme des capitaux de tiers.

Le mécanisme proposé par le $\mathrm{MCH} 2$ permet de dégager, à la clôture, la contribution de chaque financement spécial ou fonds au résultat de la collectivité. Il consiste à transférer le solde de chaque financement spécial et de chaque fonds sous capital propre directement au bilan, sans passer par le compte de résultats. L'attribution de l'excédent de revenus et le prélèvement en cas d'excédent de charges sont comptabilisés dans le capital propre par le biais du groupe de comptes 901 "Clôture des financements spéciaux et fonds sous capital propre». Cette manière de procéder ne modifie pas le solde du compte de résultats et permet de montrer le résultat de chaque financement spécial et de chaque fonds appartenant au capital propre.

Excédent de revenus d'un financement spécial ou d'un fonds. Lorsque les revenus d'un financement spécial ou d'un fonds sont plus importants que les coûts à couvrir, un excédent de revenus existe. C'est ce qui arrive pour la gestion des déchets avec un excédent de revenus de KCHF 60 (tableau 27). L'excédent de revenus doit être porté au bilan à l'avoir du compte 2900 «Financements spéciaux sous capital propre» et plus spécifiquement à l'avoir du «Financement spécial Déchets» 2900.1. On y parvient en inscrivant au doit de la fonction concernée, ici la fonction 730 , un montant équivalent à l'excédent de revenus. Dans la classification par nature, le compte concerné est le compte 9010 «Clôture des financements spéciaux et des fonds sous capital propre, excédent de revenus». Ce dernier n'est ni un compte de revenus (rubrique 4), ni un compte de charges (rubrique 3). C'est un compte uniquement utilisé pour la clôture (rubrique 9). Par conséquent, cette opération n’a 
pas d'impact sur le solde du compte de résultats. Le mécanisme de catégorisation est illustré dans le tableau 30. On y voit que le montant est bien comptabilisé dans l'unité 484, dans le compte de clôture 9010 (donc ni en charges par nature, ni en revenus par nature), ainsi que dans la fonction 730 .

Cette opération d'attribution de l'excédent de revenus équilibre les revenus et les charges dans la fonction 730. C'est ce que montre le tableau 31. Parallèlement, elle accroît le montant du financement spécial 2900.1 au passif du bilan pour atteindre KCHF 379, comme l'indique la figure 27. À la fin de l'Année t, une opération similaire accroît le montant des financements spéciaux «Eau potable» 2900.2, «Eau usée » 2900.3 et «Téléréseau » 2900.4.

Tableau 30 Opération de clôture du domaine de la gestion des déchets.

Exemple dans MaCollectivité.

\begin{tabular}{|c|c|c|c|c|c|c|c|c|c|c|c|c|}
\hline \multicolumn{13}{|l|}{ Classification... } \\
\hline ... organique & $\begin{array}{l}\text { Direction: } \\
\text { Service: } \\
\text { Unité: }\end{array}$ & $\begin{array}{l}\text { Dép. Environnement et bâti } \\
\text { Propreté publique } \\
\text { Gestion des déchets }\end{array}$ & 4 & 8 & 4 & & & & & & & \\
\hline ... par nature & $\begin{array}{l}\text { Classe de comptes: } \\
\text { Groupe de comptes: } \\
\text { Sous-groupe: } \\
\text { Compte: }\end{array}$ & $\begin{array}{l}\text { Clôture des comptes } \\
\text { Compte de résultats } \\
\text { FSp et Fds sous capital propre } \\
\text { Excédent de revenus }\end{array}$ & & & & 9 & 0 & 1 & 0 & & & \\
\hline ... fonctionnelle & $\begin{array}{l}\text { Fonction: } \\
\text { Tâche: } \\
\text { Subdivision: }\end{array}$ & $\begin{array}{l}\text { Protection de l'environnement } \\
\text { Gestion des déchets } \\
\text { Gestion des déchets }\end{array}$ & & & & & & & & 7 & 3 & 0 \\
\hline Numéro à saisir & ur l'imputation: & & 4 & 8 & 4 & 9 & U & 1 & 0 & 7 & 3 & 0 \\
\hline
\end{tabular}

Tableau 31 Équilibre dans le domaine de la gestion des déchets après attribution du solde au capital propre. MaCollectivité, Fonction 730, Année t, en KCHF.

\begin{tabular}{|c|c|c|c|c|c|}
\hline$N^{\circ}$ & Libellé & KCHF & $\mathbf{N}^{\circ}$ & _ibellé & $\mathrm{KCHF}$ \\
\hline 3 & Charges & 362 & 4 & Revenus & 362 \\
\hline 30 & Charges de personnel & 73 & 42 & Taxes et redevances & 354 \\
\hline 31 & $\begin{array}{l}\text { Charges de biens et services } \\
\text { et autres charges d'exploitation }\end{array}$ & 217 & 44 & Revenus financiers & 8 \\
\hline 33 & $\begin{array}{l}\text { Amortissements du } \\
\text { patrimoine administratif }\end{array}$ & 2 & & & \\
\hline 39 & Imputations internes & 10 & & & \\
\hline 9010 & $\begin{array}{l}\text { Clôture des financements } \\
\text { spéciaux sous capital propre, } \\
\text { excédent de revenus }\end{array}$ & 60 & & & \\
\hline
\end{tabular}


Figure 27 L'attribution de l'excédent de revenus du domaine de la gestion des déchets augmente le financement spécial correspondant. MaCollectivité, Année t, en KCHF.

Passif du bilan Financement spécial Déchets 2900.1

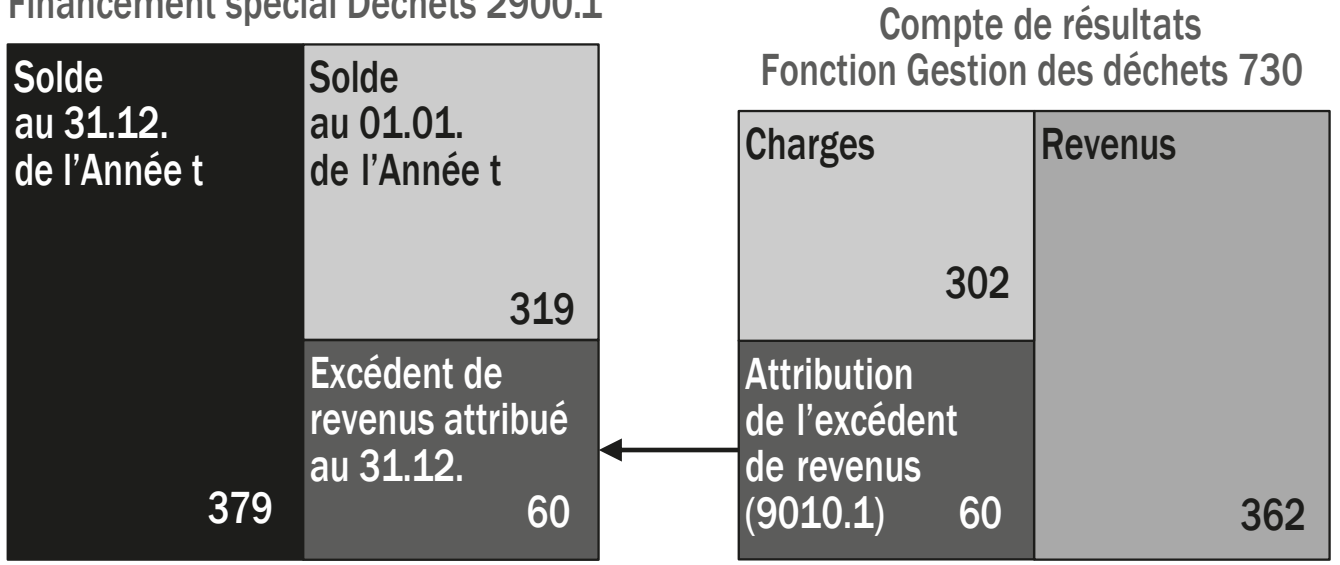

Excédent de charges d'un financement spécial ou d'un fonds. Lorsque les coûts à couvrir sont plus élevés que les revenus, le domaine concerné présente un excédent de charges. Pour rétablir l'équilibre entre revenus et charges, un prélèvement doit être effectué sur le financement spécial ou le fonds qui se trouve au passif du bilan, dans le capital propre. C'est ce qui arrive pour l'électricité et le chauffage à distance (tableau 29).

Le prélèvement est porté au bilan au doit du compte 2900 «Financements spéciaux sous capital propre» et plus spécifiquement au doit du «Financement spécial Électricité» 2900.5, respectivement au doit du «Financement spécial Chauffage à distance» 2900.6. On y parvient en inscrivant à l'avoir de la fonction concernée, ici la fonction 871 "Électricité», respectivement la fonction 879 "Autres énergies", un montant équivalent à l'excédent de charges. Dans la classification par nature, le compte concerné est le compte 9011 «Clôture des financements spéciaux et des fonds sous capital propre, excédent de charges ». Étant donné que ce dernier n'est ni un compte de revenus, ni un compte de charges, l'opération est sans effet sur le solde du compte de résultats.

Évidemment, un excédent de charges ne peut pas se répéter inlassablement puisque, dans le cas d'un financement spécial, les revenus doivent permettre de couvrir l'intégralité des coûts. Dans ce cas, le niveau de la contribution causale devrait alors être relevé pour que les utilisateurs paient effectivement suffisamment pour la prestation dont ils bénéficient. A contrario, un ajustement à la baisse de la contribution causale doit avoir lieu en cas d'excédents récurrents de revenus.

Décomposition du résultat. La figure 28 montre comment le résultat d'ensemble du compte de résultats, soit KCHF 64, se décompose entre le résultat des financements 
spéciaux, soit KCHF 54, et le résultat du «ménage général » hors financements spéciaux, soit, par différence, KCHF 10. La figure montre également que le résultat d'ensemble des financements spéciaux (901) découle de l'addition du résultat de chaque financement spécial individuel - un financement spécial pouvant présenter un excédent de revenus (compte 9010.X) ou de charges (9011.X). La figure montre également sur quel compte au passif du bilan l'excédent de revenus est attribué ou l'excédent de charges est prélevé. Il s'agit du compte 2900 pour les financements spéciaux et du compte 2990 pour celui du «ménage général».

Cette décomposition montre quel serait le résultat de la collectivité si elle n'avait pas à s'occuper des domaines financés par des revenus affectés, donc des domaines où la logique de fonctionnement est proche d'une logique de marché. Le résultat du «ménage général» hors financements spéciaux et fonds renseigne sur le degré de couverture des charges pour l'ensemble des fonctions autres que celles qui sont financées spécifiquement. Il s'agit donc du degré de couverture des opérations financées essentiellement par la fiscalité. Dans le cas de MaCollectivité, ces opérations présentent un excédent de revenus de KCHF 10.

Figure 28 Décomposition du résultat en tenant compte des variations des financements spéciaux (FSp). MaCollectivité, Année t, en KCHF.

FSp Déchets

$(9010.1 \rightarrow 2900.1) \quad 60$

FSp Eau potable

$(9010.2 \rightarrow 2900.2) \quad 10$

FSp Eau usée

$(9010.3 \rightarrow 2900.3) \quad 20$

FSp Téléréseau

$(9010.4 \rightarrow 2900.4) \quad 40$
FSp Électricité

$(9011.5 \rightarrow 2900.5) \quad-27$

FSp Chauffage à distance

$(9011.6 \rightarrow 2900.6) \quad-49$

Résultat d'ensemble des

financements spéciaux sous capital propre

$(901 \rightarrow 2900)$

Résultat du "ménage général ", hors financements spéciaux $(900 \rightarrow 2990)$

\section{4}

Resultat d'ensemble du compte de résultats

$(90 \rightarrow 29)$ 


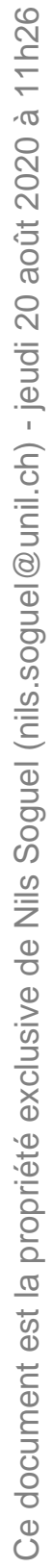




\section{Connaître les mécanismes de transferts et de péréquation financière}

\subsection{Types de transferts selon la classification par nature}

Les transferts constituent une part importante des charges, respectivement des revenus d'une collectivité. Les rubriques comptables 36 et 46 y sont consacrées. Le tableau 32 montre que, pour MaCollectivité, les transferts représentent près de la moitié des charges de l'Année $\mathrm{t}$ ( $44 \%$ pour être exact) et près de $10 \%$ des revenus. Derrière l'appellation «transferts» se cachent des éléments de natures fort différentes. On le constate à la lecture des sous-rubriques de la classification par nature.

Tableau 32 Importance des transferts dans le total des charges et des revenus. MaCollectivité, Année t, en KCHF.

\begin{tabular}{|c|c|c|c|c|c|}
\hline $\mathbf{N}^{\circ}$ & Libellé & $1000 \mathrm{CHF}$ & $N^{\circ}$ & Libellé & $.000 \mathrm{CHF}$ \\
\hline 3 & Charges & 14546 & 4 & Revenus & 14610 \\
\hline$\ldots$ & dont... & & $\ldots$ & dont... & \\
\hline 36 & Charges de transferts & 6393 & 46 & Revenus de transferts & 1373 \\
\hline 360 & $\begin{array}{l}\text { Parts de revenus } \\
\text { destinées à des tiers }\end{array}$ & 0 & 460 & $\begin{array}{l}\text { Parts à des revenus } \\
\text { de tiers }\end{array}$ & 0 \\
\hline 361 & $\begin{array}{l}\text { Dédommagements } \\
\text { à des collectivités publiques }\end{array}$ & 3905 & 461 & $\begin{array}{l}\text { Dédommagements } \\
\text { de collectivités publiques }\end{array}$ & 645 \\
\hline 362 & $\begin{array}{l}\text { Péréquation financière et } \\
\text { compensation des charges }\end{array}$ & 564 & 462 & $\begin{array}{l}\text { Péréquation financière et } \\
\text { compensation des charges }\end{array}$ & 610 \\
\hline 363 & $\begin{array}{l}\text { Subventions à des collectivités } \\
\text { publiques et à des tiers }\end{array}$ & 1924 & 463 & $\begin{array}{l}\text { Subventions de } \\
\text { collectivités et de tiers }\end{array}$ & 117 \\
\hline$[\ldots]$ & & & 469 & Autres revenus de transferts & 1 \\
\hline
\end{tabular}

* N'apparaissent pas dans ce tableau les comptes dédiés aux corrections de valeur des prêts (364) et des participations du patrimoine administratif (365), aux amortissements des subventions d'investissement lorsque celles-ci sont portées au passif du bilan (366), aux dissolutions de ces subventions (466) et aux autres charges de transferts (369). Ces comptes sont généralement peu utilisés. D'ailleurs, MaCollectivité ne les utilise pas au cours de l'Année $t$.

Parts. Les sous-rubriques 360 et 460 enregistrent les transferts qui découlent du partage des recettes de certains impôts entre plusieurs collectivités. Le plus souvent, ces transferts sont verticaux, entre deux échelons institutionnels différents. C'est, par exemple, le cas de l'impôt fédéral direct: la Confédération en partage les 
recettes avec les cantons. Il s'agit d'un droit que les cantons ont historiquement obtenu lorsqu'ils ont cédé à la Confédération le pouvoir d'empiéter sur leur périmètre fiscal. La Confédération comptabilise les montants payés aux cantons dans la sous-rubrique 360 (parts de revenus destinées à des tiers). Les cantons enregistrent les montants ainsi reçus dans la sous-rubrique 460 (parts à des revenus). Quant à MaCollectivité, elle ne partage aucun de ses impôts avec d'autres collectivités (360: CHF 0) et aucune collectivité ne partage ses impôts avec elle (460: CHF 0).

Dédommagements. Les sous-rubriques 361 et 461 enregistrent les transferts versés et reçus lorsqu'une collectivité publique fournit une prestation publique en lieu et place d'une autre collectivité, soit en totalité, soit en partie. Un dédommagement se distingue d'une subvention (voir ci-dessous) parce qu'il est versé en échange d'une contrepartie concrète, clairement identifiable et directement en lien avec le montant payé. Par conséquent, le montant du dédommagement est en rapport avec le coût de fourniture de la prestation concernée. De plus, ce type de transferts implique des administrations publiques (Confédération, cantons et concordats intercantonaux, communes et associations intercommunales, assurances sociales publiques). Les transferts au bénéfice des ménages, des entreprises, des institutions sans but lucratif ou des entités non résidentes en Suisse ne constituent pas des dédommagements, mais des subventions (autrement dit des contributions).

Le transfert peut être vertical si une tâche est déléguée à un autre niveau institutionnel que celui qui, du point de vue de la répartition des tâches, a la responsabilité d'offrir la prestation. C'est le cas lorsqu'un échelon inférieur remplit une fonction d'agence, autrement dit de sous-traitant, pour le compte de l'échelon supérieur. Par exemple, la Confédération dédommage les cantons et les communes pour le rôle qu'ils jouent dans le recensement de la population.

Le transfert peut également être horizontal entre collectivités d'un même niveau institutionnel. C'est le cas lorsqu'une collectivité - une commune ou un canton confie à une voisine, à une association de communes ou à un concordat intercantonal le soin d'offrir une prestation dont elle a la responsabilité. Par exemple, une commune verse un dédommagement à une association de communes à laquelle elle s'en remet pour le traitement des eaux usées.

On utilise donc en particulier ces rubriques pour présenter les transferts visant à corriger et à compenser un effet de débordement. Un tel effet intervient lorsqu'une collectivité fournit une prestation et que cette prestation n'est pas utilisée uniquement par les résidents de la collectivité, mais aussi par des résidents d'autres collectivités. Les infrastructures lourdes, comme les piscines, les théâtres, les universités, génèrent ce type d'effets. En dédommageant la collectivité qui supporte le coût opérationnel de ces infrastructures, les autres collectivités paient pour l'effet de débordement dont profitent leurs résidents. Par exemple, les flux financiers entre cantons au titre de l'accord intercantonal universitaire de 1997 répondent à cette logique. 
La collectivité qui dédommage comptabilise les montants payés dans la sousrubrique 361 (dédommagements à des collectivités publiques). La collectivité dédommagée enregistre les montants ainsi reçus dans la sous-rubrique 461 (dédommagements des collectivités publiques). Quant à MaCollectivité au cours de l'Année t, elle dédommage d'autres collectivités pour un montant supérieur aux dédommagements que lui versent d'autres collectivités. En effet, alors qu'elle paie KCHF 3905, elle n'encaisse que KCHF 645. Ces chiffres montrent que MaCollectivité bénéficie de davantage de prestations fournies par d'autres collectivités qu'elle ne leur en offre.

Péréquation financière et compensation des charges. Les sous-rubriques 362 et 462 enregistrent les transferts destinés à corriger les disparités entre collectivités. Il s'agit en particulier de disparités dans la répartition géographique de la substance fiscale. Mais il s'agit également de disparités dans les besoins de la population pour certaines prestations publiques et dans les coûts de production de ces prestations. La thématique de la péréquation financière mérite bien qu'on l'examine plus en détail. En effet, elle est compliquée à plusieurs égards. D’abord, il faut identifier les disparités qu'il est légitime de corriger et celles qui n'ont pas à l'être. Ensuite, les instruments de correction à mettre en place doivent être conçus pour satisfaire les objectifs de la péréquation. Nous y consacrerons les sections suivantes.

La collectivité pénalisée par les disparités reçoit une compensation et la comptabilise dans la sous-rubrique 462. La collectivité favorisée verse une compensation et l'enregistre dans la sous-rubrique 362. Quant à MaCollectivité au cours de l'Année $t$, elle bénéficie de davantage de transferts au titre de la péréquation financière qu'elle n'a à en verser. En effet, elle reçoit KCHF 610, mais ne paie que KCHF 564.

Subventions. Les sous-rubriques 363 et 463 enregistrent les transferts pour lesquels la collectivité subventionnante ne bénéficie pas d'une contrepartie concrète, clairement identifiable et directement liée au montant payé. Sur ce point, les subventions se distinguent donc des dédommagements. Elles s'en distinguent également par le fait que des entités autres que des administrations publiques peuvent les recevoir, soit des ménages, des entreprises ${ }^{123}$, des institutions à but non lucratif et des entités non résidentes en Suisse.

Certaines subventions peuvent avoir pour objectif d'inciter l'entité récipiendaire à modifier les choix qu'elle ferait en l'absence de telles contributions. Le but est donc d'orienter les choix vers des activités souhaitées par la collectivité subventionnante. De ce fait, l'obtention d'une contribution est généralement conditionnée à la réalisation d'une activité que le bénéficiaire devrait fournir selon des exigences qualitatives et quantitatives fixées par l'entité subventionnante. C'est le cas lorsqu'une

123 Les subventions aux entreprises représentent de véritables subventions au sens économique du terme. En effet, elles sont de nature à influencer le niveau des prix sur les marchés où les entreprises sont actives. Les subventions allouées aux collectivités publiques ou aux institutions à but non lucratif ne sont pas des subventions au sens économique du terme, mais véritablement des transferts. En effet, ces bénéficiaires-là ne vendent pas de biens et de services sur le marché. Le mécanisme des prix ne s’en trouve donc pas perturbé. 
collectivité verse une subvention découlant d'une garantie de couverture de déficit aux organisateurs d'une manifestation qui s'avère finalement déficitaire.

Il faut aussi considérer comme une subvention les versements que le niveau institutionnel supérieur exige du niveau inférieur au motif de partager ses charges avec lui. C'est ainsi que certains cantons demandent aux communes une subvention en prenant comme assise de calcul leurs dépenses en matière sociale, sanitaire ou scolaire. Les appellations utilisées - facture sociale, hospitalière ou scolaire ne doivent pas nous leurrer: si les dispositions légales en vigueur attribuent la responsabilité d'offrir la prestation à l'échelon cantonal, alors ces paiements ne peuvent pas être considérés comme un dédommagement ou comme une compensation des charges. Il s'agit bel et bien d'une subvention alimentant le budget général des cantons concernés, voire comblant une partie de leur excédent de charges.

Les subventions versées à des personnes, à des familles ou à des groupements publics ont également une visée redistributive marquée. En effet, bien souvent le montant de ces transferts sociaux dépend de la capacité économique des bénéficiaires. C'est le cas de l'aide fédérale et cantonale visant à réduire les cotisations à l'assurance maladie. C'est aussi le cas de l'aide accordée aux familles à revenu modeste dont les enfants fréquentent des structures d'accueil de la petite enfance. Ces aides doivent être enregistrées dans cette rubrique, même si elles sont versées directement à la compagnie d'assurance concernée ou à la structure d'accueil fréquentée.

La collectivité subventionnante comptabilise les montants payés dans la sousrubrique 363 (subventions à des collectivités publiques et à des tiers). L'entité subventionnée enregistre les montants reçus dans la sous-rubrique 463 (dédommagements de collectivités publiques et de tiers). Quant à MaCollectivité au cours de l'Année t, elle verse KCHF 1924 de subventions, alors qu'elle n'en reçoit que KCHF 117.

Les montants comptabilisés dans les rubriques 363 et 463 sont des subventions pour le fonctionnement. Les subventions pour l'investissement doivent être présentées dans le compte des investissements et dans les rubriques 56 pour les subventions accordées ( 57 si elles sont redistribuées), respectivement 63 pour les subventions reçues (67 si elles sont à redistribuer).

\subsection{Disparités et péréquation}

Les disparités entre collectivités peuvent avoir des origines diverses. Certaines disparités découlent de décisions prises par la collectivité, par ses autorités ou par sa population. Il s'agit donc de différences endogènes, dont la collectivité elle-même porte la responsabilité. D'autres disparités sont liées aux circonstances auxquelles la collectivité est confrontée et sur lesquelles elle n'a pas d'emprise, du moins pas à court et à moyen terme. Ce sont donc des disparités exogènes. 
Il est justifié de corriger les disparités exogènes par le biais d'une péréquation financière. Par contre, les différences endogènes ne peuvent pas être mises en avant pour revendiquer des mesures péréquatives. Dafflon (2007) ou Rühli et al. (2013) identifient diverses disparités exogènes - substance fiscale, besoins, coûts de production - et endogènes - structure de financement et préférences locales.

\subsubsection{Différences endogènes}

Structure de financement. La législation définit les impôts auxquels une collectivité peut recourir. Par ailleurs, le droit fédéral et la jurisprudence du Tribunal fédéral fixent un certain nombre de règles en matière de redevances d'utilisation. Toutefois, à l'intérieur de ce cadre, chaque collectivité dispose d'une autonomie fiscale. Cette autonomie lui permet de choisir par exemple de recourir à un impôt ou d'y renoncer. Pour un impôt donné, la collectivité peut choisir certaines modalités d'imposition (définition de l'assiette fiscale, du barème d'imposition, etc.). Elle peut également décider de facturer à l'utilisateur le coût des prestations dans une mesure plus ou moins importante. Par conséquent, des différences existent dans la manière dont les collectivités structurent le financement de leurs dépenses. Ces différences peuvent affecter la situation financière de chaque collectivité. Mais elles découlent de décisions prises par chaque entité. Elles n'ont donc pas à être prises en compte dans la péréquation financière.

Préférences locales. Quel que soit l'échelon institutionnel, la législation donne aux collectivités la compétence de décider si elles souhaitent ou non fournir une prestation et cela dans différents domaines. Même dans les domaines où la Confédération ou le canton obligent les communes à offrir certains services, ces dernières peuvent souvent décider de manière indépendante de fournir ces services selon des normes dépassant les exigences imposées. Pouvoir choisir d'offrir ou non une prestation et selon quel standard l'offrir permet de tenir compte des préférences locales, c'està-dire les préférences de la population locale. En conséquence, si une collectivité applique des standards élevés, c'est à elle d'en assumer les conséquences financières, même si cela conduit à une pression fiscale différente entre communes. On ne doit pas attendre de la péréquation financière qu'elle finance des coûts découlant des décisions prises localement.

\subsubsection{Disparités exogènes}

Substance fiscale. Au sein d'un même niveau institutionnel, les collectivités ne disposent pas de la même substance fiscale. Par exemple, le revenu imposable par habitant varie entre cantons et entre communes. Certaines entités ont la chance d'avoir des contribuables bénéficiant de ressources confortables sur lesquelles prélever l'impôt. D'autres ne peuvent disposer que d'une substance fiscale plus modeste. 
Toutes choses égales par ailleurs, pour financer leur budget, les collectivités à substance fiscale modeste doivent exercer une pression fiscale plus lourde sur leurs contribuables que celles où la substance fiscale est confortable. On s'accorde généralement sur le fait qu'une telle situation n'est pas équitable. En effet, les disparités en matière de substance fiscale sont liées aux circonstances locales. Les collectivités ne peuvent que difficilement influencer le niveau de leur substance fiscale, du moins à court et à moyen terme. Par conséquent, ces disparités justifient une péréquation des ressources.

Besoins. Dans le cadre du fédéralisme d'exécution, le droit supérieur oblige les collectivités à offrir des prestations dans plusieurs domaines où les situations locales peuvent être sensiblement différentes. Selon la prestation, une topographie plus difficile ou une composition sociale de la population plus défavorisée provoque davantage de dépenses, sans que la collectivité concernée n'y puisse rien. On considère habituellement que cela n'est pas équitable. En effet, les disparités en matière de besoins liés à la topographie ou à la composition sociale sont liées aux circonstances locales. Les collectivités peuvent difficilement agir pour les atténuer. La péréquation a donc un rôle à jouer pour compenser les collectivités confrontées à des dépenses plus importantes pour fournir des prestations que lui commande le droit supérieur. Il faut donc une compensation (ou péréquation) des charges.

S'agissant des besoins liés à la topographie, certaines entités doivent offrir davantage de prestations que d'autres, notamment en matière de protection contre les éléments naturels (par exemple besoins spécifiques en matière de protection contre les crues, d'endiguement, de ponts, de tunnels). Les collectivités concernées par ce phénomène doivent faire face à des charges particulières dans ces domaines spécifiques dès lors que la législation fédérale ou cantonale les oblige à prendre des mesures adéquates.

S'agissant des besoins liés à la composition sociale, certaines entités doivent offrir davantage de prestations que d'autres en matière d'aide sociale ou d'aides individuelles. Ce sont des collectivités dans lesquelles le nombre d'ayants droit est plus élevé qu'ailleurs. On associe souvent le phénomène à celui de l'urbanisation. Dans ce domaine également, dès lors que la législation fédérale ou cantonale oblige à offrir des prestations déterminées, les collectivités concernées doivent faire face à des charges particulières.

Coûts de production. Toujours dans le cadre du fédéralisme d'exécution, l'exécution des tâches confiées par le droit supérieur aux collectivités du niveau institutionnel inférieur peut s'avérer plus ou moins onéreuse. En effet, les circonstances de production de ces services délégués peuvent être plus ou moins favorables. La géographie des lieux, la densité de l'habitat ou encore le climat peuvent impliquer des coûts par habitant objectivement supérieurs dans les collectivités où il est techniquement plus compliqué de produire le service ${ }^{124}$. Pensons à l'approvisionnement

124 Le coût par habitant est un exemple de coût unitaire. Il peut également s'agir d'un coût par utilisateur ou d'un coût par unité de prestation offerte (coût par $\mathrm{m}^{2}$, par $\mathrm{m}^{3}$, par kilomètre, par tonne, etc.). 
en eau propre ou à l'épuration des eaux. En présence de services pour lesquels la décision de les offrir selon des normes minimales échappe aux entités concernées, on s'accorde généralement sur le fait que le surcoût lié à des circonstances de production défavorables doit être compensé, car cette situation n'est pas équitable. Une compensation (ou péréquation) des charges s'impose également dans ce domaine.

\subsubsection{Péréquation en cas de disparités exogènes}

La figure 29 schématise le lien entre les différentes disparités et une éventuelle péréquation. Elle rappelle que les différences entre collectivités découlant d'une structure de financement différente ou de préférences locales hétérogènes ne doivent pas donner lieu à une péréquation. En effet, ces différences proviennent de choix effectués par les collectivités concernées elles-mêmes.

Par contre, une péréquation s'impose lorsque les disparités découlent de facteurs exogènes, indépendants des décisions des collectivités concernées. À l'exemple du système péréquatif de la Confédération, les dispositifs en vigueur en Suisse sont souvent basés sur deux piliers fondamentaux: la péréquation des ressources et la péréquation des charges. La première se justifie par la volonté de réduire les disparités de substance fiscale entre les collectivités, entre les cantons ou entre les communes. La seconde découle de la volonté de compenser les collectivités confrontées à des coûts supplémentaires liés à leur topographie ou à leur structure socio-démographique.

Figure 29 Seules les disparités exogènes justifient une péréquation.

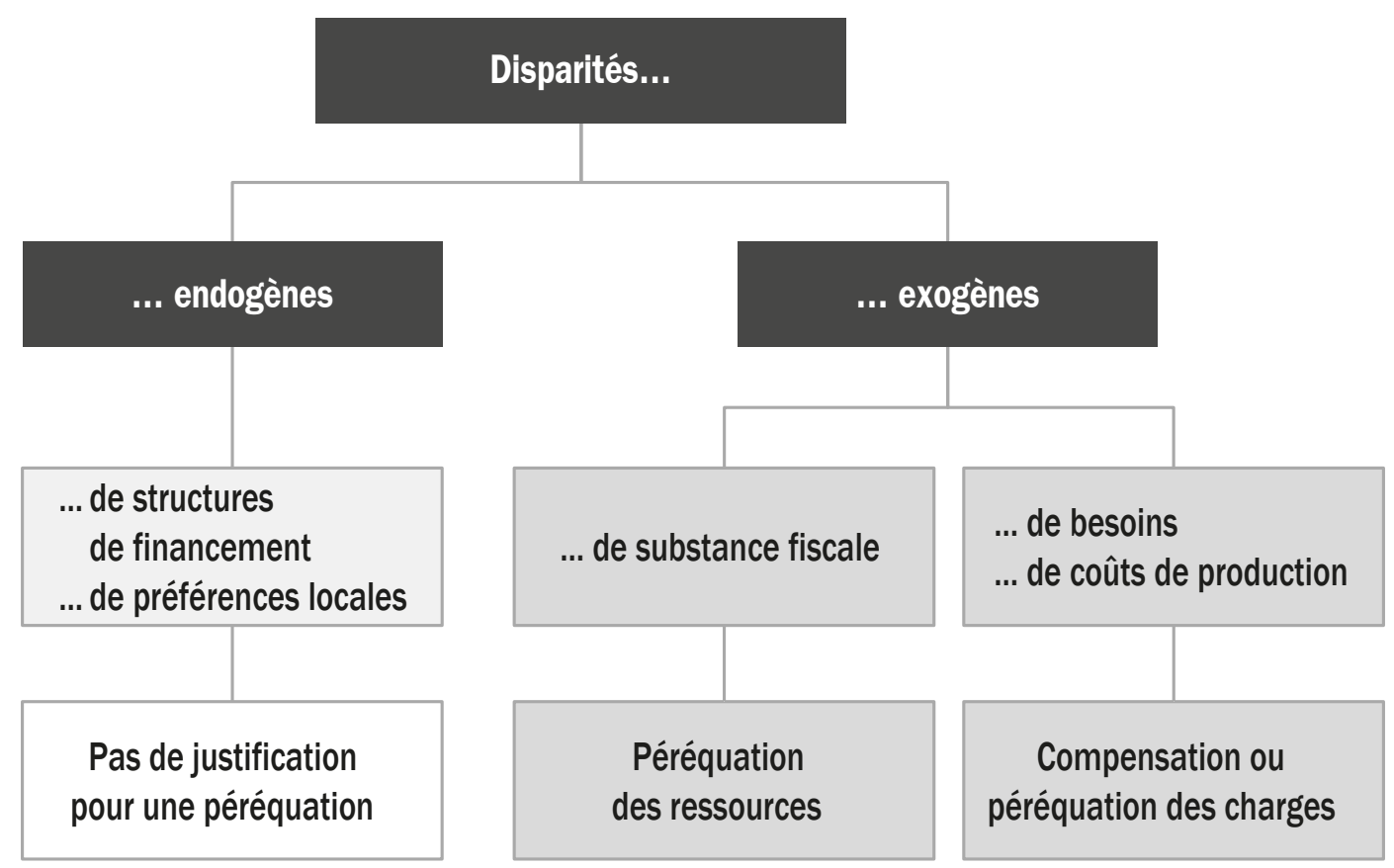




\subsection{Péréquation des ressources}

\subsubsection{Horizontalité}

Les disparités dans la répartition de la substance fiscale proviennent de circonstances locales favorables ou, au contraire, défavorables. Elles ne découlent pas d'une décision du niveau institutionnel supérieur. Par conséquent, la péréquation des ressources doit être essentiellement une affaire de solidarité horizontale, soit entre cantons, soit entre communes: les collectivités locales qui bénéficient de circonstances financières avantageuses aident celles qui sont confrontées à des conditions plus difficiles.

Le fait que la péréquation des ressources soit essentiellement horizontale présente d'autres avantages que celui - symbolique - de la solidarité. Du point de vue de l'économie politique, l'horizontalité des transferts financiers implique que les collectivités locales ne peuvent pas se tourner vers l'échelon supérieur pour obtenir une augmentation du volume financier de la péréquation. Autrement dit, une péréquation horizontale offre une meilleure cohérence interne: si les collectivités modestes souhaitent davantage de solidarité, elles doivent se tourner vers les collectivités plus aisées; si les entités plus aisées souhaitent moins de solidarité, elles doivent le négocier avec les entités plus modestes.

Par ailleurs, l'incidence péréquative des transferts horizontaux est plus forte que celle de transferts qui proviendraient de l'échelon supérieur. De manière imagée, «les transferts horizontaux rendent les collectivités locales riches moins riches et les pauvres moins pauvres». Par contre, «les transferts verticaux, s'ils rendent les collectivités locales pauvres moins pauvres, ne rendent pas les riches moins riches». Schématiquement, pour réduire les disparités de ressources dans une même mesure, une péréquation verticale nécessite un transfert deux fois plus important venant de l'échelon supérieur qu'une péréquation horizontale payée par les collectivités locales aisées.

\subsubsection{Potentiel fiscal}

\section{Point de départ}

Pour mettre en place une péréquation des ressources, il faut mesurer l'importance de la substance fiscale générée par les résidents. Ce potentiel fiscal conditionne les ressources que la collectivité peut espérer prélever. L'enjeu est donc d'estimer le potentiel offert par la substance fiscale de la collectivité. Cela permet ensuite d'identifier les collectivités disposant d'un potentiel modeste et les collectivités ayant un potentiel plus élevé. Les premières doivent bénéficier de la péréquation des ressources, tandis que les secondes doivent y contribuer.

Les recettes fiscales effectives s'observent facilement dans les comptes. Mais ces recettes donnent une image biaisée du potentiel fiscal d'une collectivité. En effet, elles ne dépendent pas seulement de la matière imposable, un élément exogène, du moins à court et à moyen terme. Elles dépendent également de la charge fiscale. La 
charge fiscale dépend du taux d'imposition, cas échéant, multiplié par un coefficient ${ }^{125}$. Or le taux ou le coefficient d'impôt sont en partie des facteurs endogènes puisqu'ils sont fixés par chaque collectivité selon des logiques qui lui sont propres.

Il est donc nécessaire de disposer d'une mesure non biaisée du potentiel fiscal pour que le dispositif péréquatif soit perçu comme juste et légitime. Un effort de normalisation doit être entrepris. D'abord, les différences de charge fiscale entre les collectivités doivent être neutralisées. Ensuite, les différentes composantes du potentiel fiscal (revenu imposable, fortune imposable, bénéfice imposable, etc.) doivent être réunies dans un seul et même indicateur. Enfin, il faut tenir compte de la taille des collectivités; autrement dit, le potentiel de ressources doit être exprimé par habitant.

Généralement, ces opérations permettent de mesurer le potentiel fiscal à travers un indice dont la marque 100 correspond à la limite entre collectivités financièrement modestes (dont l'indice est inférieur à 100) et collectivités aisées (dont l'indice est supérieur à 100). On parle ici d'indice des ressources.

\section{Composantes du potentiel fiscal}

Une collectivité peut tabler sur des ressources budgétaires de différentes natures (voir la section 3.3.4 consacrée au compte de résultats). Ce n'est pas pour autant que toutes doivent être prises en compte pour déterminer le potentiel fiscal. Quelques critères simples permettent de sélectionner les éléments à considérer.

Lien avec la capacité contributive des résidents. Les éléments à considérer dans le potentiel fiscal doivent logiquement être en lien avec la capacité contributive des personnes physiques ou morales résidant dans la collectivité. Ce critère justifie donc d'inclure les revenus fiscaux (40). Les patentes et concessions (41) doivent aussi être incluses. En effet, la possibilité pour une collectivité de bénéficier de revenus dans ce domaine découle de circonstances sur lesquelles elle n'a pas d'emprise. Il s'agit souvent de ce que l'on peut qualifier d'aubaines (par exemple une gravière exploitable sur le territoire communal). Le tableau 33 indique les montants concernés pour MaCollectivité et l'Année t. Ces montants représentent la moitié du total des revenus.

Par contre, ce critère implique d'exclure les taxes et redevances (42). Certes, des différences peuvent apparaître entre collectivités dans les montants prélevés. Toutefois, ces différences n'ont rien à voir avec des disparités de substance fiscale. Les taxes et les redevances sont perçues en contrepartie d'un avantage spécifique reçu par la personne qui s'en acquitte (approvisionnement en eau, collecte des déchets, etc.). Leur montant dépend directement du coût de fourniture de la prestation. Il est d'autant plus élevé qu'une collectivité fait face à des circonstances locales défavorables pour produire les prestations donnant lieu à des taxes et à des émoluments. Il irait donc à contre-emploi d'inclure ces montants, plus importants dans les collectivités

125 Selon les cantons, la notion de coefficient d'impôt a pour synonyme celle de quotité, de multiple ou de centimes additionnels. 
"défavorisées", dans le périmètre du potentiel. Par ailleurs, cela freinerait les collectivités dans l'application du principe pollueur-payeur (par exemple eaux usées) ou du principe utilisateur-payeur (eau). En effet, percevoir davantage de taxes et d'émoluments augmenterait le potentiel fiscal, donc réduirait les montants à recevoir de la péréquation ou augmenterait les montants à verser. Cela serait également en contradiction avec la législation fédérale sur la protection de l'environnement ${ }^{126}$.

Ce même critère du lien avec la capacité contributive des résidents implique d'exclure du périmètre les autres revenus non fiscaux découlant de prestations spécifiques offertes à un certain coût par les collectivités: les revenus divers (43) et les revenus financiers (44). Les revenus de transferts (46 et 47) doivent aussi être exclus. En effet, ils correspondent à des dédommagements et à des contributions pour des prestations fournies à d'autres entités. Ils peuvent aussi provenir du dispositif de péréquation financière. Finalement, les revenus découlant d'opérations purement comptables sur les financements spéciaux et fonds (45) ou extraordinaires (48), ainsi que les imputations internes (49) doivent également être exclus.

En définitive, seuls les revenus appartenant à la catégorie des revenus fiscaux et ceux qui découlent de patentes et de concessions doivent être inclus dans le périmètre du potentiel de revenu utilisé pour la péréquation des ressources.

Tableau 33 Revenus à intégrer dans l'estimation du potentiel fiscal. MaCollectivité, Année t, en KCHF.

\begin{tabular}{|llr|}
\multicolumn{1}{|l}{} & Libellé & $1000 \mathrm{CHF}$ \\
\hline 4 & Revenus & 14610 \\
\hline$\ldots$ & dont... & \\
40 & Revenus fiscaux & 7651 \\
400 & Impôts directs, personnes physiques & 6231 \\
401 & Impôts directs, personnes morales & 501 \\
402 & Autres impôts directs & 898 \\
403 & Impôts sur la propriété et sur les charges & 21 \\
41 & Patentes et concessions & 12 \\
410 & Patentes & 0 \\
411 & Banque nationale suisse & 0 \\
412 & Concessions & 12 \\
413 & Parts de revenus à des loteries, Sport-Toto, paris & 0 \\
\hline
\end{tabular}

126 L'art. 2 de la loi fédérale sur la protection de l'environnement (LPE) consacre le principe de causalité et prévoit que «celui qui est à l'origine d'une mesure prescrite par la présente loi en supporte les frais». Dans le domaine spécifique du financement des déchets, l'art. 32, al. 1, stipule: «Le détenteur des déchets assume le coût de leur élimination.» L'art. 32a, al. 1, précise: «Les cantons veillent à ce que les coûts de l'élimination des déchets urbains, pour autant que celle-ci leur soit confiée, soient mis, par l'intermédiaire d'émoluments ou d'autres taxes, à la charge de ceux qui sont à l'origine de ces déchets.» Ce même alinéa fixe relativement en détail les bases de calcul des taxes. 
Pas d'exception. Les impôts qui contribuent le plus au financement des budgets sont également ceux qui sont associés à une substance fiscale importante. Par conséquent, ils doivent impérativement être intégrés dans le périmètre du potentiel fiscal. Cela étant, même les impôts qui représentent une faible quote-part devraient également être inclus. En effet, les équilibres financiers sont tributaires de l'ensemble des revenus fiscaux. À la marge, chaque impôt, même peu important, est susceptible de modifier les équilibres. Par conséquent, il serait erroné et inéquitable de laisser de côté un impôt dans la définition du potentiel fiscal utilisé pour la péréquation.

Aussi les impôts aléatoires. On hésite parfois à inclure dans la mesure du potentiel fiscal les impôts dont le produit est aléatoire ou particulièrement irrégulier, surtout pour les collectivités de taille modeste. En effet, un impôt dont l'assiette fiscale est soumise au hasard des circonstances ne peut être considéré comme un impôt offrant un financement structurel. Plusieurs impôts classés sous la rubrique 402 (autres impôts directs) n'offrent souvent qu'un rendement aléatoire ou irrégulier: impôts sur les gains en capital (4022), droits de mutations (4023) ou impôts sur les successions et donations (4024). Malgré cela et compte tenu du critère précédent, ces impôts devraient également être inclus dans le périmètre. Toutefois, afin d'assurer la stabilité des transferts péréquatifs, un lissage sur quelques exercices devrait être introduit, en particulier pour tenir compte de la situation des collectivités de petite taille.

Définition uniforme. Il est important que la substance fiscale soit définie de manière uniforme. Pour chaque impôt, il faut donc s'assurer que le cercle des personnes assujetties, les composantes de la substance imposable, le montant des éventuelles déductions, soient définis de la même manière. À défaut, une correction doit être opérée afin que le montant de la substance fiscale prise en compte pour chaque collectivité soit comparable et ne soit pas biaisé. Il en va de même du barème d'imposition ou du coefficient d'impôt.

\section{Estimation du potentiel fiscal}

Le potentiel fiscal peut être estimé de trois manières. Une première possibilité consisterait à mesurer le potentiel à partir de données macroéconomiques, tel le produit intérieur brut (PIB) par habitant. À défaut d'information sur les PIB communaux, on dispose d'une statistique des PIB cantonaux. Cette dernière est toutefois moins fiable que les statistiques fiscales. C'est pourquoi les dispositifs péréquatifs en vigueur n'y recourent pas. Une deuxième possibilité consiste à baser l'estimation directement sur la substance fiscale. C'est l'approche retenue dans le cadre de la péréquation financière fédérale. Une troisième possibilité consiste à estimer les recettes fiscales dont bénéficieraient les collectivités si elles faisaient peser une charge fiscale harmonisée sur leurs contribuables. Les péréquations financières intercommunales recourent généralement à cette approche fondée sur l'estimation d'un revenu fiscal harmonisé. 
Estimation directe à partir de la substance fiscale. À l'échelon fédéral et intercantonal, la péréquation des ressources agrège les assiettes fiscales des différents impôts pris en compte dans le périmètre du potentiel fiscal. Les assiettes fiscales correspondent aux ressources imposables (revenu et fortune des personnes physiques, bénéfice des personnes morales) après avoir retranché les déductions fiscales admises. Cela offre une estimation du potentiel fiscal de chaque collectivité, également appelé «assiette fiscale agrégée». Cet agrégat permet de dégager un indice des ressources. En se fondant directement sur les assiettes fiscales, cette approche évite l'écueil soulevé par des barèmes d'imposition qui varient d'une collectivité à l'autre, en l'occurrence d'un canton à l'autre. Toutefois, on ne peut pas agréger l'assiette de l'impôt sur la fortune des personnes physiques avec celle de l'impôt sur le bénéfice des personnes morales par simple addition - pour prendre l'exemple de ces deux impôts. En effet, la fortune est quantitativement un multiple du bénéfice. Par conséquent, les différentes assiettes fiscales doivent être pondérées de manière à leur donner une importance similaire à chacun des impôts considérés dans les ressources fiscales des cantons. La démarche de pondération rend l'approche par la substance fiscale problématique à deux égards au moins. D’abord, toute pondération implique forcément une dose d'arbitraire. Ensuite, de par sa complexité, la pondération nuit à la transparence et à l'intelligibilité du dispositif.

Estimation à travers un revenu fiscal harmonisé. Cette approche ne se réfère pas à la substance fiscale, mais au revenu fiscal extrait de cette substance. Évidemment, le revenu fiscal extrait ne peut pas être utilisé directement pour estimer le potentiel fiscal, puisqu'il dépend arithmétiquement de la charge fiscale imposée par chaque collectivité. Pour contourner cette difficulté, on procède à une estimation du revenu fiscal qui serait dégagé par chaque collectivité si elle imposait une charge fiscale harmonisée. La démarche est facilitée dans le cadre des péréquations intercommunales puisque le montant des déductions et le barème fiscal (tranches de revenus, taux d'imposition par tranche) sont identiques sur l'ensemble du territoire cantonal. Les différences entre communes proviennent alors uniquement du coefficient d'impôt qu'elles choisissent.

Le tableau 34 illustre les étapes permettant de mesurer le potentiel fiscal. Évidemment, les étapes et les systèmes peuvent varier selon les cantons ${ }^{127}$. Toutefois, les principes sont généralement similaires. Le tableau recourt à un canton fictif composé de sept communes dont MaCollectivité (mentionnée par la lettre $\mathrm{M}$ dans la colonne 1). La deuxième colonne renseigne sur les revenus fiscaux encaissés par chaque commune en fonction du coefficient effectif qu'elle applique. Ce coefficient est mentionné dans la colonne $3^{128}$. Les revenus fiscaux de MaCollectivité s'élèvent à

127 Le mécanisme de certains systèmes est plus alambiqué. Voir par exemple Soguel (2004) pour une description du système vaudois.

128 Pour simplifier, nous considérons que chacune des communes applique un seul et même coefficient, quel que soit l'impôt concerné: impôt sur les personnes physiques (compte 400) et morales (401), autres impôts directs (402), impôts sur la propriété (403). Nous laissons de côté les revenus provenant des patentes et concessions (41). 
KCHF 7651 à l'Année t. Ils sont obtenus à l'aide d'un coefficient de 1,74. Seules les communes $\mathrm{K}$ et $\mathrm{J}$ imposent une charge fiscale plus importante avec un coefficient respectif de 1,85 et 1,92 .

La quatrième colonne renseigne sur les revenus qui auraient été encaissés avec un coefficient unitaire, c'est-à-dire avec un coefficient de 1 . On l'obtient en divisant les revenus par le coefficient. Pour MaCollectivité, ce calcul conduit à des revenus fiscaux de KCHF 4397 (KCHF 7651/1,74).

La ligne «Ensemble» au pied du tableau donne le total des colonnes [2] et [4]. En divisant le total de la colonne 2 par celui de la colonne 4, on obtient le coefficient harmonisé (colonne 5). Dans ce cas simplifié, le coefficient s'élève à 1,45. MaCollectivité impose donc plus lourdement ses contribuables que la charge fiscale moyenne pondérée reflétée par le coefficient harmonisé.

La sixième colonne indique quels seraient les revenus fiscaux encaissés si chaque commune pratiquait ce coefficient de 1,45, plutôt que son coefficient effectif ${ }^{129}$. MaCollectivité n'encaisserait que KCHF $6396($ KCHF $4397 \times 1,4546)$. La colonne 7 renseigne sur l'effectif de la population dans les communes. La colonne 8 indique le montant des revenus fiscaux harmonisés par habitant. Celui de MaCollectivité s'élèverait à CHF 2055. C'est le plus faible du canton après celui de J, de K et de L. Ce sont les quatre communes dont les revenus fiscaux harmonisés se situent au-dessous de la moyenne. Cette moyenne s'élève en effet à CHF 2168 (total des revenus fiscaux harmonisés rapporté au total des habitants du canton).

Tableau 34 Estimation du potentiel fiscal à travers le revenu fiscal harmonisé. MaCollectivité (M) et six autres communes, Année t, en KCHF.

\begin{tabular}{|c|c|c|c|c|c|c|c|c|}
\hline $\begin{array}{c}\text { Commune } \\
\text { [1] }\end{array}$ & $\begin{array}{c}\text { Revenus } \\
\text { fiscaux avec } \\
\text { coefficient } \\
\text { effectif } \\
\text { KCHF } \\
\text { [2] }\end{array}$ & $\begin{array}{c}\text { Coefficient } \\
\text { effectif } \\
{[3]}\end{array}$ & $\begin{array}{l}\text { Revenus } \\
\text { fiscaux avec } \\
\text { coefficient } \\
\text { unitaire } \\
\text { KCHF } \\
\text { [4] }\end{array}$ & $\begin{array}{c}\text { Coefficient } \\
\text { harmonisé } \\
{[5]}\end{array}$ & $\begin{array}{c}\text { Revenus } \\
\text { fiscaux avec } \\
\text { coefficient } \\
\text { harmonisé } \\
\text { KCHF } \\
\text { [6] }\end{array}$ & $\begin{array}{c}\text { Nombre } \\
\text { d'habitants }\end{array}$ & $\begin{array}{c}\text { Revenus } \\
\text { fiscaux } \\
\text { harmonisés } \\
\text { par habitant } \\
\text { CHF } \\
\text { [8] }\end{array}$ & $\begin{array}{l}\text { Indice } \\
\text { des } \\
\text { ressources }\end{array}$ \\
\hline$J$ & 732 & 1,92 & 381 & & 554 & 339 & 1634 & 75,4 \\
\hline K & 1852 & 1,85 & 1001 & & 1456 & 834 & 1746 & 80,5 \\
\hline L & 4372 & 1,50 & 2915 & & 4240 & 2271 & 1867 & 86,1 \\
\hline$M$ & 7651 & 1,74 & 4397 & & 6396 & 3113 & 2055 & 94,8 \\
\hline $\mathrm{N}$ & 2218 & 1,10 & 2016 & & 2933 & 1342 & 2186 & 100,8 \\
\hline 0 & 22336 & 1,38 & 16186 & & 23544 & 10286 & 2289 & 105,6 \\
\hline$P$ & 1549 & 1,42 & 1091 & & 1587 & 591 & 2685 & 123,8 \\
\hline Ensemble & 40710 & & 27987 & 1,4546 & 40710 & 18776 & 2168 & 100,0 \\
\hline
\end{tabular}

129 Notons que la somme des revenus fiscaux effectifs (colonne 2) est logiquement égale à la somme des revenus fiscaux harmonisés (colonne 6). 
Les revenus de MaCollectivité ne représentent que les 94,8\% de la moyenne. Ils sont bien plus faibles que les $123,8 \%$ de la commune P. Ces pourcentages constituent précisément l'indice des ressources qui apparait dans la dernière colonne. Pour chaque commune, le pourcentage est obtenu en rapportant les revenus fiscaux harmonisés par habitant à la moyenne de CHF 2168, qui représentent donc les 100\%.

Revenu fiscal harmonisé, gage de meilleure équité. L'approche fondée sur le revenu fiscal harmonisé répond mieux à l'impératif d'équité que celle qui est fondée directement sur la substance fiscale; cela pour deux raisons: d'abord parce que la fiscalité directe recourt à un barème progressif; ensuite parce que, à l'échelon local, la progressivité ne dépend pas d'une décision communale, mais d'une décision cantonale. En effet, plusieurs impôts utilisent un barème progressif, en particulier l'impôt sur le revenu et l'impôt sur la fortune des personnes physiques. Dans ce cas, même avec une substance fiscale d'un volume global identique entre collectivités, certaines communes peuvent extraire un revenu fiscal plus élevé que d'autres. Cela provient du fait que la substance fiscale se distribue différemment, c'est-à-dire plus ou moins uniformément entre les tranches du barème d'impôt.

Dafflon (2007) fournit un cas d'école que nous reproduisons dans le tableau 35. Ce tableau présente le cas de deux communes, $\mathrm{A}$ et $\mathrm{B}$. Chacune accueille trois contribuables dont la somme des revenus imposables est égale à CHF 15000 . En revanche, la distribution des revenus imposables varie selon la collectivité: les trois contribuables dans l'entité A ont un revenu imposable identique de CHF 5000, tandis que ceux de l'entité B ont des revenus imposables différents de CHF 1000, CHF 4000 et CHF 10000. Le barème prévu par la législation cantonale est progressif. Les deux premières colonnes du tableau indiquent le taux moyen d'imposition pour quatre niveaux de revenus imposables. Partant de là, les revenus fiscaux de l'entité A s'élèvent à CHF 450 et ceux de l'entité B à CHF 900. Autrement dit, la progressivité du barème implique des revenus fiscaux deux fois plus faibles dans l'entité $\mathrm{A}$, où la substance fiscale est distribuée uniformément, que dans l'entité $B$, où la substance fiscale est distribuée de manière moins uniforme, avec un contribuable dont le revenu imposable est particulièrement élevé (CHF 10 000). Cet exemple montre que si la substance fiscale est choisie comme base pour estimer le potentiel fiscal, les deux collectivités seront considérées comme égales (avec un potentiel de CHF 15000). $\mathrm{Si}$, en revanche, on utilise le revenu fiscal comme base, alors le potentiel fiscal de l'entité B est deux fois plus élevé que celui de l'entité A (CHF 900 contre CHF 450). Bien que trivial, cet exemple montre la nécessité de tenir compte de la distribution de la matière imposable à l'intérieur de l'assiette fiscale, dès le moment où l'on est en face d'une imposition progressive. Par conséquent, du point de vue de l'équité, l'approche fondée sur le revenu fiscal harmonisé doit être préférée à l'approche fondée directement sur la substance fiscale, lorsque l'on peut raisonnablement postuler que la distribution de la matière imposable à l'intérieur de l'assiette fiscale varie entre les collectivités. 
Tableau 35 Effet d'un barème progressif sur le potentiel fiscal à substance fiscale équivalente entre collectivités.

Exemple des collectivités fictives A et B.

Barème

Collectivité A

Collectivité B

\begin{tabular}{|c|c|c|c|c|c|}
\hline & & \\
\hline $\begin{array}{c}\text { Revenu } \\
\text { imposable }\end{array}$ & $\begin{array}{l}\text { Taux moyen } \\
\text { d'imposition }\end{array}$ & $\begin{array}{c}\text { Revenu } \\
\text { imposable }\end{array}$ & Impôt dû & $\begin{array}{c}\text { Revenu } \\
\text { imposable }\end{array}$ & Impôt dû \\
\hline CHF & $\%$ & CHF & $\mathrm{CHF}$ & CHF & $\mathrm{CHF}$ \\
\hline 1000 & 1,0 & & & & \\
\hline 4000 & 2,0 & 5000 & 150 & 1000 & 10 \\
\hline 5000 & 3,0 & 5000 & 150 & 4000 & 80 \\
\hline 10000 & 8,1 & 5000 & 150 & 10000 & 810 \\
\hline & & 15000 & 450 & 15000 & 900 \\
\hline
\end{tabular}

Source: Dafflon (2007, p. 42).

\subsubsection{Indice des ressources}

Les revenus fiscaux harmonisés par habitant mentionnés dans la colonne $8 \mathrm{du}$ tableau 34 sont déjà une mesure pertinente du potentiel fiscal. En effet, ils permettent une comparaison entre communes qui n'est plus biaisée, ni par des coefficients d'impôts différents, ni par des effectifs de population différents. Que ce soit dans le cadre de la péréquation fédérale ou des diverses péréquations intercommunales, on préfère utiliser l'indice des ressources de la colonne 9. Cet indice permet instantanément de se rendre compte de l'écart positif ou négatif qui sépare une collectivité de la moyenne. Autrement dit de savoir à quel point elle est financièrement forte ou faible. C'est ce qu'illustre la figure 30. Les indices des ressources y ont été reportés dans un ordre croissant. Les communes J, K, L et M (MaCollectivité) ont un indice des ressources inférieur à la moyenne de 100. Elles sont relativement modestes par rapport à la référence de 100 marquant le potentiel fiscal moyen par habitant. En revanche, les communes $\mathrm{N}, \mathrm{O}$ et $\mathrm{P}$ ont un indice des ressources supérieur à 100 . Ces collectivités sont donc relativement aisées par rapport à cette référence de 100.

Figure 30 Échelonnement des collectivités en fonction de l'indice des ressources. MaCollectivité (M) et six autres communes, Année t.

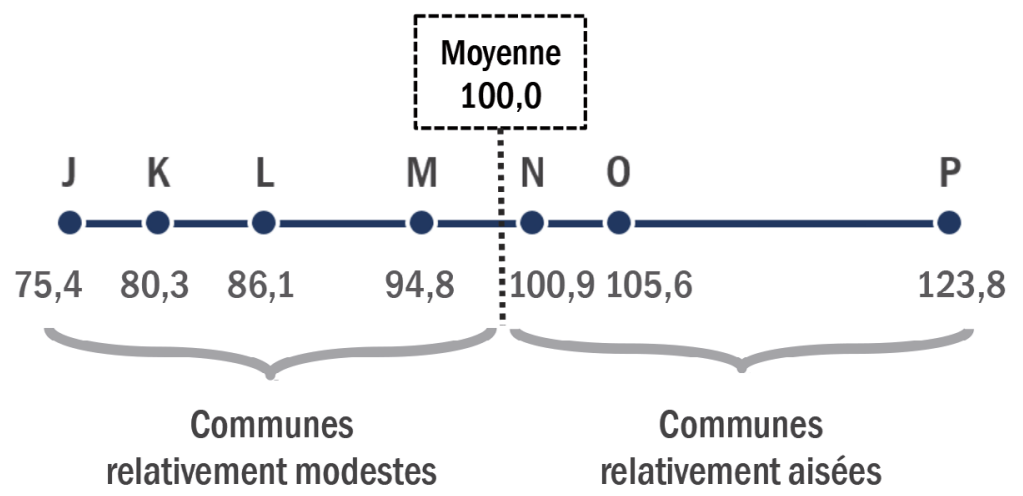




\subsubsection{Barème péréquatif}

Dans le cadre d'une péréquation horizontale, l'indice des ressources nous indique les collectivités qui doivent y contribuer et celles qui en bénéficient. Les collectivités contributrices ont un indice des ressources supérieur à 100, les collectivités bénéficiaires un indice inférieur à 100. Encore faut-il décider quelle est l'importance de la solidarité qui doit se mettre en place. Autrement dit, il faut décider à quel point la distribution de l'indice des ressources doit être corrigée pour être rendue plus équitable grâce à la péréquation.

Deux solutions extrêmes encadrent ce choix. Elles sont présentées dans la figure 31. L'axe horizontal de cette figure reprend celui de la figure précédente (figure 30). Il reflète donc l'indice des ressources avant péréquation. L'axe vertical mesure l'indice des ressources après péréquation. On y retrouve les sept communes, allant de J à $\mathrm{P}$.

Figure 31 Deux solutions péréquatives extrêmes:

système inopérant et système uniformisant.

MaCollectivité (M) et six autres communes, Année t.

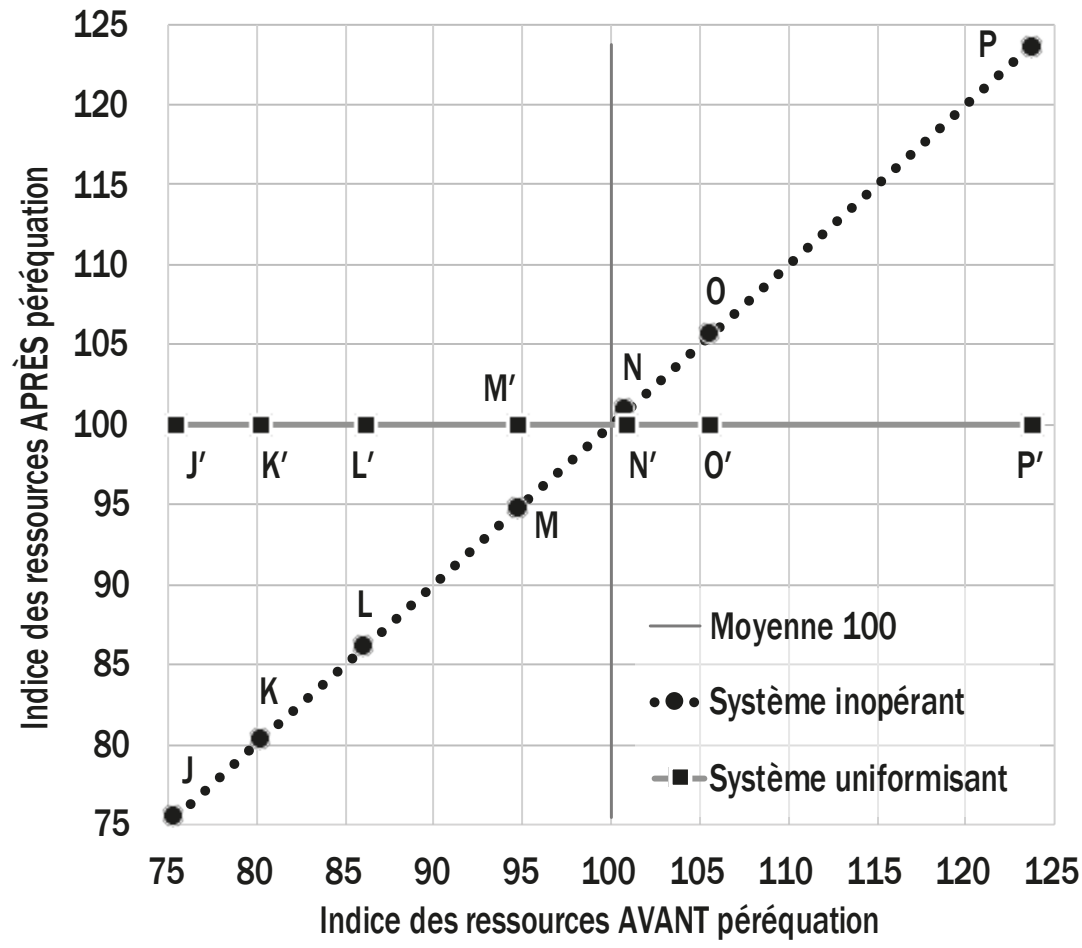

Système inopérant. La première solution extrême serait que les transferts ne modifient en rien la distribution des ressources. Autrement dit, l'indice des ressources de chaque collectivité serait le même avant et après la péréquation: l'indice des collectivités modestes ne serait pas relevé, tandis que celui des collectivités aisées ne 
serait pas abaissé. Le système n'aurait aucune incidence; il serait alors inopérant ${ }^{130}$. Pour MaCollectivité, l'indice, qui s'élève à 94,8 avant péréquation (axe horizontal), serait toujours de 94,8 après la péréquation (axe vertical). Il en va de même des six autres entités. Lorsque le système est inopérant, les collectivés - représentées par des marques rondes - se rangent sur une droite formant un angle de $45^{\circ}$ avec les deux axes. Cette droite passe par le point $[100 ; 100]$, c'est-à-dire par l'indice des ressources d'une collectivité qui serait exactement égal à la moyenne. La droite verticale foncée représente l'indice des ressources moyen (100) qui sépare, avant péréquation, les communes modestes (indice des ressources inférieur à 100) des communes aisées (indice des ressources supérieur à 100).

Système uniformisant. La seconde solution extrême serait que les transferts corrigent totalement la distribution et qu'après la péréquation l'indice de chaque collectivité se situe à 100. Le système serait alors totalement uniformisant. L'indice des ressources serait alors parfaitement égalisé par la péréquation. Celui des entités modestes serait relevé jusqu'à 100, à l'instar de celui de MaCollectivité. Pour cette dernière, cela correspond graphiquement à la marque carrée $\mathrm{M}^{\prime}$, découlant d'un indice de 94,8 avant péréquation et de 100 après. Pour l'entité $P$, cela correspond à la marque $\mathrm{P}^{\prime}$ puisque son indice avant péréquation s'élève à 123,8 et qu'il se situerait à 100 ensuite. Lorsque le système est uniformisant, les entités se rangent donc sur la droite horizontale grisée au niveau de l'indice 100,0 après péréquation.

Barème péréquatif. Ces deux extrêmes restent théoriques. En effet, on ne les trouve appliqués nulle part. Cependant, ils permettent de cerner dans quelles limites le barème péréquatif doit se situer. De fait, tant le barème de la péréquation fédérale que le barème des dispositifs cantonaux s'inscrivent dans ces limites. Évidemment, chaque barème à ses spécificités. Celui qui est présenté dans la figure 32 n'est qu'un exemple. Mais il permet d'illustrer certaines caractéristiques qui se retrouvent dans divers barèmes en vigueur. Les sept communes $\mathrm{J}$ à $\mathrm{P}$ sont marquées par des points blancs sur la courbe épaisse noire. Un premier constat est que la pente de la courbe n'est pas constante. Souvent les barèmes prévoient une dotation minimale après péréquation pour les collectivités financièrement les plus faibles ${ }^{131}$. C'est aussi le cas ici : la courbe est convexe et le dispositif assure un indice des ressources de 85 après péréquation à toutes les collectivités et en particulier aux collectivités dont l'indice avant péréquation est inférieur à 75 . L'idée est de garantir aux entités, même les plus modestes, le minimum de ressources nécessaire pour fournir les prestations que l'on attend d'une collectivité. Autrement dit, la péréquation apporte à ces entités une compensation égale à la différence entre leur indice avant péréquation et 85. Une commune qui se situerait à 75,0 recevrait une compensation de 10,0 points.

130 Cela impliquerait un système assez particulier où, par exemple, une collectivité recevrait un montant au titre de la péréquation des ressources, mais devrait simultanément contribuer à la péréquation exactement pour le même montant.

131 Parmi les cantons dont le système péréquatif prévoit une dotation minimale, on trouve AR, AI, BL, BE, GL, GR, JU, LU, NW, OW, SG, SH, SO, SZ, TG, TI, UR, VS, ZG et ZH (Rühli et al. 2013). 
Figure 32 Exemple de barème péréquatif.

MaCollectivité (M) et six autres communes, Année t.

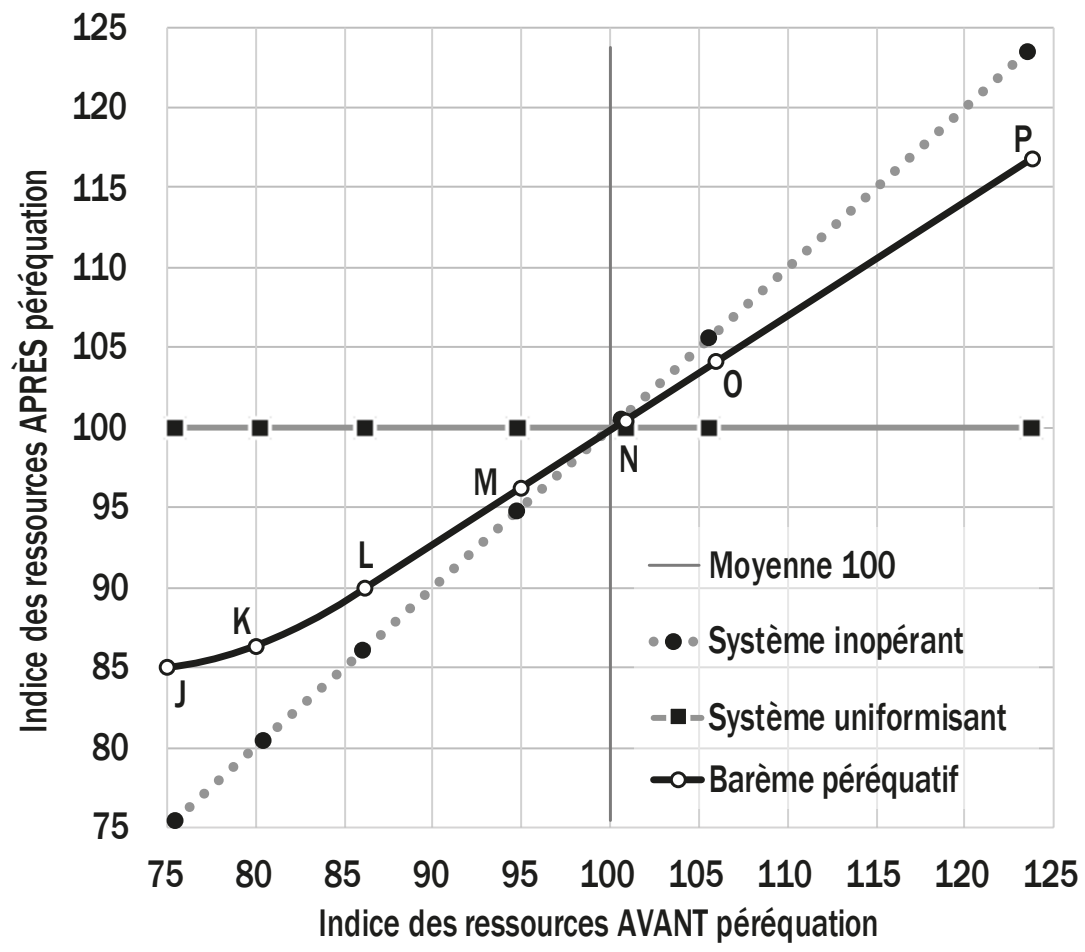

Plus la dotation minimale se situe à un niveau élevé, plus la solidarité avec les collectivités les plus modestes est importante. Toutefois, introduire une dotation minimale dans un dispositif péréquatif présente des risques. En effet, cela réduit l'incitation pour les collectivités bénéficiaires à prendre des initiatives pour se développer et à améliorer par elles-mêmes leur indice des ressources. Une entité ayant un indice initial particulièrement faible de 50 se verrait transférer 35 points pour l'assurer d'un indice de 85 après péréquation. Si cette entité améliore sa situation et porte son indice initial à 60 , elle sera "récompensée» par une baisse des transferts de 10 puisqu'elle ne recevrait plus que 25 points. Dans la zone de dotation minimale, l'augmentation de l'indice initial est donc totalement écrêtée: le taux d'écrêtage atteint $100 \%{ }^{132}$.

Au sortir de la zone de dotation minimale, le taux d'écrêtage passe au-dessous de $100 \%$ et tend vers $0 \%$ lorsque l'indice initial s'approche de 100 . C'est ce que montre la courbe: lorsque l'indice initial augmente, l'indice après péréquation augmente également, mais moins que proportionnellement. Cela signifie que la compensation

132 Le taux d'écrêtage marginal correspond à la baisse (en centimes) des revenus péréquatifs ou à la hausse des charges péréquatives quand le potentiel fiscal d'un canton ou d'une commune augmente de CHF 1. Le taux d'écrêtage est donc influencé par la pente du barème. 
se réduit graduellement pour disparaitre dès que la collectivité présente un indice de 100. Comme le montre la figure 32, l'entité J, la plus modeste de notre échantillon, reçoit une compensation de 9,8 points qui lui permet, partant d'un indice de 75,4 , de passer à 85,2 . Le tableau 36 donne pour chaque collectivité l'indice avant et après péréquation, ainsi que le nombre de points d'indice auquel cela correspond. MaCollectivité bénéficie à l'origine d'un indice de 94,8. Par conséquent, elle ne reçoit qu'une faible compensation de 1,4 point d'indice. Elle atteint ainsi un indice de 96,2.

À l'approche d'un indice initial des ressources de 100, certains barèmes prévoient également une zone neutre ${ }^{133}$. Tel n'est cependant pas le cas du barème présenté ici. Une telle zone est motivée par le constat que les collectivités qui s'y trouvent - par exemple celles entre 90 et 100 - ne sont plus tout à fait financièrement modestes. Par conséquent, on considère qu'elles n'ont pas besoin de recevoir de transferts péréquatifs. Lorsqu'une telle zone existe, la courbe du barème se confond avec celle du système inopérant sur l'intervalle concerné (par exemple entre 90 et 100) ${ }^{134}$. Introduire une zone neutre offre l'avantage de réduire le montant à transférer aux communes plus modestes et donc de limiter le montant à prélever auprès des communes aisées. Mais une zone neutre implique aussi des inconvénients. En ajoutant une rupture de pente au barème, elle peut inciter les collectivités à éviter d'entrer dans cette zone pour éviter de ne plus bénéficier de transferts péréquatifs. Une zone neutre peut aussi inciter les collectivités qui s'y trouvent à ne pas chercher à en sortir, en accroissant leur indice des ressources avant péréquation, de peur de devenir contributrices.

Tableau 36 Transferts péréquatifs à recevoir et à payer.

MaCollectivité (M) et cinq autres communes. Année t, en KCHF.

\begin{tabular}{|c|c|c|c|c|c|c|}
\hline \multirow{2}{*}{$\begin{array}{c}\text { Commune } \\
\text { [1] }\end{array}$} & \multirow{2}{*}{$\begin{array}{l}\text { Revenus } \\
\text { fiscaux } \\
\text { KCHF } \\
\text { [2] }\end{array}$} & \multirow{2}{*}{$\begin{array}{c}\text { Nombre } \\
\text { d'habitants } \\
\text { [3] }\end{array}$} & \multicolumn{2}{|c|}{$\begin{array}{l}\text { Indice des ressources... } \\
\ldots \text { avant } \ldots \text { après } \\
\text { péréquation }\end{array}$} & \multirow{2}{*}{$\begin{array}{l}\text { Points d'indice } \\
\text { à compenser } \\
\text { [6] }\end{array}$} & \multirow{2}{*}{$\begin{array}{c}\text { Transferts à recevoir } \\
\text { ou à payer [-] } \\
\text { KCHF } \\
{[7]}\end{array}$} \\
\hline & & & [4] & [5] & & \\
\hline J & 732 & 339 & 75,4 & 85,2 & 9,8 & 72 \\
\hline 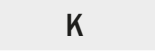 & 1852 & 834 & 80,5 & 86,8 & 6,3 & 114 \\
\hline L & 4372 & 2271 & 86,1 & 90,0 & 3,9 & 192 \\
\hline M & 7651 & 3113 & 94,8 & 96,2 & 1,4 & 94 \\
\hline $\mathrm{N}$ & 2218 & 1342 & 100,8 & 100,6 & $-0,2$ & -6 \\
\hline 0 & 22336 & 10286 & 105,6 & 103,9 & $-1,7$ & -375 \\
\hline$P$ & 1549 & 591 & 123,8 & 116,6 & $-7,1$ & -91 \\
\hline Ensemble & 40710 & 18776 & & & & 0 \\
\hline
\end{tabular}

133 Le barème péréquatif présente une zone neutre dans quelques cantons: AG, AR, JU, LU, NW, OW, TG, TI, UR et ZH (Rühli et al. 2013).

134 Parfois, l'intervalle de la zone neutre s'étend au-delà de 100. 
S'agissant des collectivités aisées, différentes formes de barème existent pour déterminer le montant des transferts à payer. À l'instar des systèmes fiscaux, la forme peut être proportionnelle, progressive ou dégressive. Ici, nous avons retenu la proportionnalité. En d'autres termes, le taux de contribution à la péréquation des communes aisées est constant. Nous utilisons un taux de 30\%: la compensation s'élève à $30 \%$ de la différence entre l'indice initial et la marque de 100 . Ainsi, la collectivité $\mathrm{P}$ avec un indice de 123,8 devrait contribuer à hauteur de 7,1 points $(30 \%$ de 23,8$)$ et voir ainsi son indice passer à 116,6.

De manière générale, au moment où l'on conçoit un barème péréquatif, on devrait éviter le plus possible les ruptures de pente afin de réduire le risque de fournir des incitations inopportunes aux collectivités. Plus la pente du barème est régulière, moins le risque est grand. Par conséquent, un barème parfaitement linéaire, donc une droite, serait préférable à la courbe convexe de la figure 32. En pratique, des considérations politiques empêchent généralement d'y parvenir.

\subsubsection{Montant des transferts péréquatifs}

Le montant des transferts à recevoir ou à payer est déterminé sur la base des points d'indice à compenser. Pour notre exemple, les points à compenser apparaissent dans la colonne 6 du tableau 36, comme différence entre la colonne 5 et la colonne 4. Ces deux colonnes sont données par barème péréquatif de la figure 32. Sur la base du total de la colonne 8 du tableau 34, nous savons que la valeur moyenne d'un point d'indice par habitant s'élève à CHF 21,68 (CHF 2168/100). Pour chaque commune, le montant du transfert est calculé en multipliant le nombre de points à compenser par la valeur du point et par le nombre d'habitants (colonne 7). MaCollectivité devrait donc recevoir un transfert de KCHF $94(1,4 \times$ CHF 21,68 $\times 3113)$.

Évidemment, le barème devrait être conçu et adapté de manière à ce que les transferts reçus soient intégralement financés par les transferts payés. C’est le cas dans notre exemple puisque le total de la colonne 7 est égal à zéro. Il est parfois difficile de faire en sorte que les montants se compensent. Cela conduit alors à instaurer un fonds de péréquation qui capitalise à travers le temps le trop payé et permet ainsi de faire face aux situations où les montants à recevoir excèdent les montants payés.

\subsection{Compensation (ou péréquation) des charges}

\subsubsection{Conditions}

La péréquation des charges veut que les collectivités reçoivent une compensation pour les coûts qu'elles supportent en vertu d'obligations que leur confère le droit supérieur. Les cantons doivent remplir des tâches que leur assigne le droit fédéral; 
les communes ont l'obligation d'assumer des tâches que leur assigne le droit cantonal. Lorsque, pour ces tâches, les besoins ou les coûts de production sont supérieurs à la normale, une péréquation se justifie. Le but est d'aider les collectivités délégataires qui se trouvent dans une situation particulière à couvrir leurs charges. Toutefois, quatre conditions devraient être satisfaites pour introduire une péréquation des charges dans un domaine particulier.

Obligation imposée par le droit supérieur. La première condition est que le droit supérieur - fédéral pour les cantons, cantonal pour les communes - oblige les collectivités délégataires à fournir des prestations dans le domaine concerné selon une norme minimale prescrite et à en assurer le financement. Cette condition exclut de compenser les charges dans les domaines où les délégataires sont compétentes pour décider d'offrir (ou non) une prestation. Il est donc exclu de compenser les communes par exemple pour les coûts de l'éclairage public municipal ou de l'entretien des parcs et jardins. Cette condition exclut aussi une compensation pour des coûts engendrés par un niveau de qualité supérieure à la norme imposée par le droit supérieur.

Disparités significatives. La seconde condition est (a) que des besoins spécifiques significativement différents entre collectivités délégataires puissent être identifiés, ou (b) qu'il est techniquement plus compliqué et onéreux de fournir le service concerné selon les normes minimales imposées par la Confédération ou le canton. Autrement dit, il faut déterminer si des délégataires se trouvent confrontées involontairement à des besoins ou à des conditions de production défavorables qui conduisent à des surcoûts pour leur budget.

Nombre significatif de collectivités délégataires touchées. La troisième condition est que, si des circonstances défavorables existent et que des surcoûts se manifestent, un nombre suffisamment élevé de collectivités délégataires soient confrontées au phénomène pour justifier une compensation systématique à travers le système de péréquation. En effet, un système doit être capable de traiter les cas ordinaires. Par contre, un système a généralement plus de difficultés à traiter les cas extraordinaires (souvent qualifiés d'aberrants en termes statistiques). Si le nombre de collectivités confrontées à des circonstances défavorables est très réduit, alors une solution «au cas par cas», en marge du système péréquatif, est préférable. Cela évite de complexifier exagérément le dispositif.

Surcoûts importants. Si les collectivités délégataires confrontées à des circonstances défavorables sont suffisamment nombreuses, encore faut-il que les surcoûts engendrés pour leur budget représentent un montant assez important. C'est à cette condition que la mise en place d'un mécanisme de compensation se justifie. En effet, un tel mécanisme implique des frais de gestion. Un rapport adéquat doit exister entre les surcoûts à compenser et les frais de gestion du dispositif péréquatif. 


\subsubsection{Verticalité}

Différentes conditions doivent donc être remplies pour qu'une péréquation des charges soit introduite. La plus importante est sans conteste le fait que le droit supérieur délègue aux collectivités locales la responsabilité de produire et de financer un service particulier, selon une norme minimale prescrite. Dans ce cas, le principe "qui commande paie» doit logiquement s'appliquer. L'échelon supérieur qui fixe la norme doit donc en assumer la conséquence financière. Par exemple, un canton doit compenser les charges des communes qui doivent supporter un coût plus élevé que les autres pour respecter la norme cantonale. En matière de compensation des charges, la péréquation doit donc être verticale, contrairement à la péréquation des ressources qui - quant à elle - doit être horizontale.

\subsubsection{Calcul}

$\mathrm{Si}$, à la lumière des critères mentionnés ci-dessus, une péréquation des charges doit être introduite, encore faut-il déterminer les montants compensatoires à verser. Ces montants devraient pouvoir être calculés sur la base de critères objectifs. De ce point de vue, la péréquation financière fédérale et son volet de compensation des charges ne sont pas exemplaires. En effet, les montants destinés à compenser les charges géo-topographiques et les charges socio-démographiques découlent surtout d'une négociation politique. Ils ne reposent guère sur des fondements objectivés ${ }^{135}$. Fixer les montants à verser de manière objective nécessite quelques précautions. Ces précautions doivent aussi permettre d'éviter le comportement tactique de la part de collectivités qui chercheraient à maximiser le montant des compensations obtenues. Elles s'inspirent notamment de la méthode des coûts standards utilisée dans le cadre du contrôle de gestion.

Identifier les inducteurs de coûts. La première précaution consiste à identifier les raisons qui sont à l'origine d'une charge excessive pour la collectivité délégataire. Il faut donc déterminer les variables qui exercent une influence significative sur le coût de la prestation. Prenons l'exemple des charges communales de déneigement. Nous faisons ici l'hypothèse que les conditions mentionnées précédemment sont remplies. Donc une péréquation verticale des charges doit être instaurée. La question est d'établir si l'altitude ou la densité de la population jouent effectivement un rôle. Pour le savoir, on peut recourir à des avis d'experts ou, mieux, à la méthode statistique de l'analyse de régression. Cette dernière offre l'avantage de quantifier (a) si une variable explicative influence réellement la variable à expliquer, c'est-à-dire si elle est un inducteur de coûts (cost driver) pour la tâche concernée, et (b) quelle est l'importance quantitative de cette influence. Pour procéder à cette analyse statistique, il faut

135 C'est pourquoi ces montants sont souvent remis en question. La controverse porte en particulier sur le poids relatif du pilier de compensation des charges socio-démographiques par rapport à celui de compensation des charges géo-topographiques. 
disposer d'une information fiable sur le coût de la prestation concernée, pour plusieurs communes et pour plusieurs années. Le troisième, voire le quatrième niveau de la classification fonctionnelle permet généralement d'obtenir une information suffisamment détaillée et comparable entre collectivités (voir la section 3.3.9 consacrée en particulier à la classification fonctionnelle). L'analyse statistique pourra déterminer que le coût du déneigement augmente, par exemple, de CHF 7,50 par habitant lorsque l'altitude augmente de 100 mètres. Notons que l'analyse de régression (multiple) permet potentiellement de mettre en évidence plusieurs inducteurs de coûts.

Fixer la norme. La seconde précaution consiste à fixer la valeur normale de chacun des inducteurs de coûts précédemment identifiés. Pour ce faire, on peut recourir à une valeur statistique. Dans notre exemple, ce pourrait être l'altitude moyenne des communes du canton concerné, par hypothèse 620 mètres. En choisissant cette norme, on postule que seules les communes situées à une altitude supérieure à la moyenne sont confrontées à des conditions climatiques induisant un coût additionnel en matière de déneigement. Fixer une valeur normale revient donc à décider de la valeur à partir de laquelle une collectivité reçoit une compensation des charges.

La manière de fixer la norme dépend de l'inducteur de coûts. Pour des questions d'objectivisation, il est conseillé de recourir à une grandeur statistique comme la moyenne ou la médiane. Par exemple, si l'inducteur se révèle être la densité de population de chaque commune, la norme retenue pourrait être la densité de population à l'échelon cantonal. Dans le domaine de l'aide sociale, une compensation des charges devrait être introduite si le droit cantonal confie la tâche aux communes et leur fixe des normes en matière de prise en charge. Si l'inducteur est le nombre de dossiers actifs auprès des services sociaux, la norme pourrait être le nombre de dossiers pour 100 habitants dans l'ensemble du canton. En effet, il est justifié de compenser les charges des communes qui doivent aider davantage de bénéficiaires que cette moyenne.

Calculer l'écart de quantité. La norme ayant été fixée, il devient possible de calculer l'écart entre la norme et la valeur de l'inducteur de coûts de chaque collectivité. Si la valeur observée se situe au-dessus de la norme, alors une compensation des coûts liés à cet écart de quantité se justifie. La péréquation devrait se limiter à compenser cet écart. Elle ne devrait pas compenser les charges supportées par les collectivités où les quantités induisant les coûts sont inférieures à la norme préétablie. Dans les collectivités où les quantités sont supérieures à la norme, la compensation ne devrait porter que sur les coûts provoqués par la part des quantités au-dessus de la norme. Elle ne devrait pas concerner les coûts provoqués par la production des quantités jusqu'au niveau de la norme. MaCollectivité se situe à une altitude de 970 mètres, donc son écart de quantité est de 350 mètres ( $970 \mathrm{~m}-620 \mathrm{~m})$.

Fixer un coût standard unitaire. On pourrait évidemment imaginer compenser les collectivités confrontées à une situation défavorable à hauteur du surcoût effectif 
qu'elles supportent. Cette méthode exposerait l'échelon supérieur qui verse le transfert à un risque de dérive des coûts. C'est pourquoi le recours à un coût standard unitaire s'impose. Cela implique de fixer a priori un montant forfaitaire par unité de prestation - ou par unité de quantité - au-delà de la norme. L'analyse de régression aide à décider du montant. Dans l'exemple du déneigement, elle signale un coût standard de CHF 7,50 par habitant et par 100 mètres. Parfois, des dispositions réglementaires ou des pratiques peuvent servir de base (coût standard par place de crèche par exemple).

Calculer la compensation des charges. Dès le moment où l'écart de quantité et le coût standard unitaire sont connus, il devient aisé de calculer par multiplication le montant à transférer aux collectivités. MaCollectivité se situant 350 mètres au-dessus de la norme et le coût standard s'élevant à CHF 7,50 par 100 mètres, la compensation par habitant pour le déneigement se monte à CHF 26,25 $(350 / 100 \times \mathrm{CHF} 7,50)$, soit KCHF 81,7 pour 3113 habitants.

Les collectivités pour lesquelles l'écart de production est négatif ne reçoivent aucune compensation. Dans notre exemple, il s'agit de toutes les entités dont l'altitude est inférieure à la norme de 620 mètres. 


\section{Identifier les modalités de financement des investissements et d'emprunt}

\section{1 Éventail des sources de financement des investissements}

Une collectivité publique devrait être capable de financer au moins l'intégralité de ses activités opérationnelles à l'aide de ses ressources propres. Ce financement interne devrait également lui permettre de financer en partie ou en totalité ses activités d'investissement. C'est la norme voulue par la règle d'or des finances publiques. Selon cette norme, une collectivité publique ne devrait recourir à un financement externe que pour financer ses investissements. L'emprunt est une forme de financement externe. Mais il en existe d'autres. La figure 33 donne une vue d'ensemble des différentes sources de financement - internes et externes - à la disposition de la collectivité pour ses investissements. Ce chapitre présente ces différents outils, leurs caractéristiques, leurs avantages et inconvénients. Cette analyse permet de dégager un arbre de décision pour déterminer l'outil de financement le plus adapté aux besoins de la collectivité. Cet arbre est présenté dans la figure 34 .

Figure 33 Sources de financement des investissements à la disposition des collectivités.

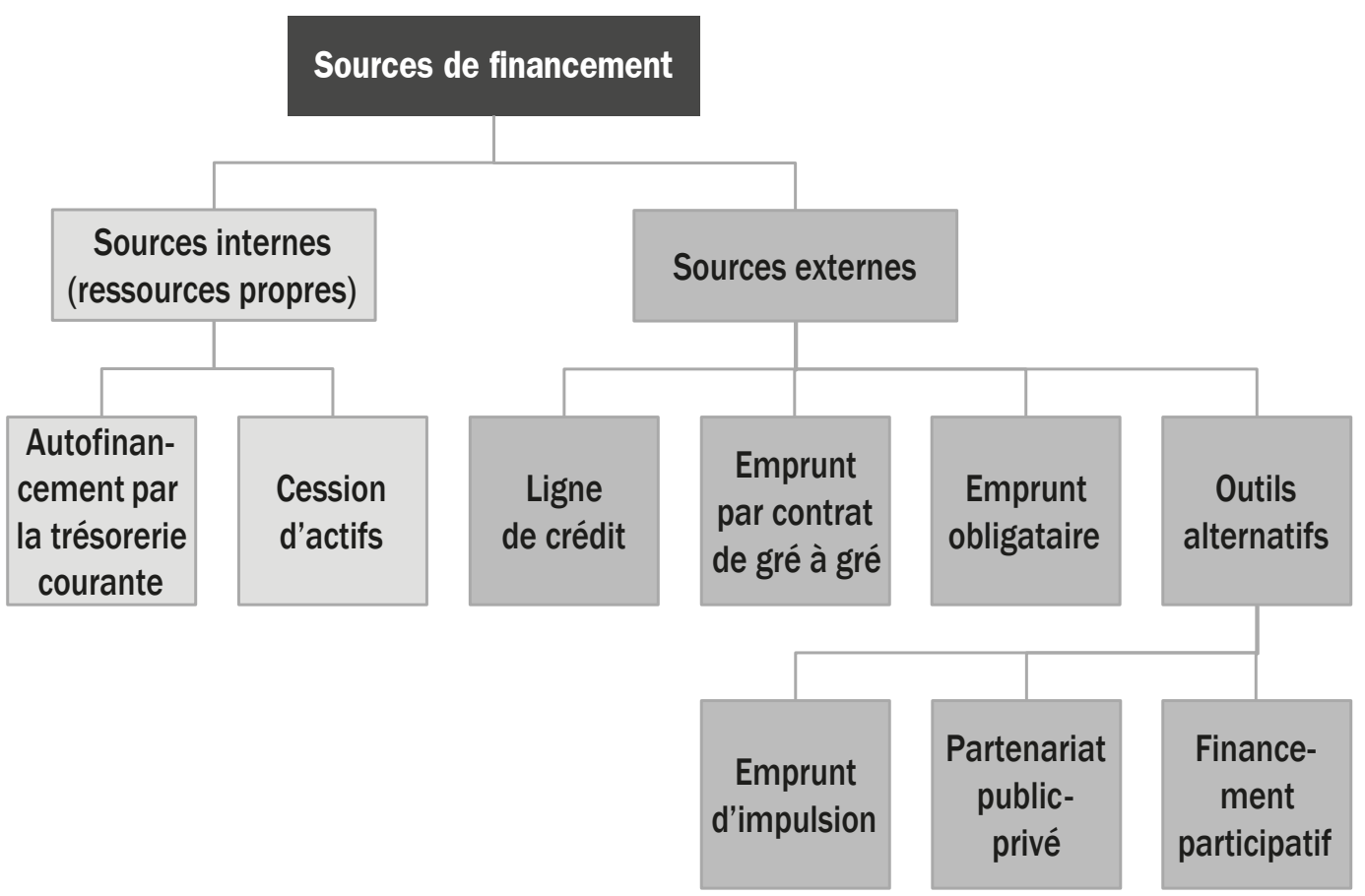




\subsection{Financement interne}

Autofinancement par la trésorerie courante. Grâce au financement interne, la collectivité se finance par ses propres moyens. Si l'on se concentre sur le financement des investissements, on pense naturellement à l'autofinancement apporté par la trésorerie et l'épargne courantes de la collectivité, c'est-à-dire par la différence entre les encaissements et les décaissements qui découlent de l'activité opérationnelle. Dans le cadre du $\mathrm{MCH} 2$, l'information à ce sujet nous est apportée par le tableau des flux de trésorerie. Pour MaCollectivité, ce financement interne s'élève à KCHF 1989 à l'Année $t$ (section 3.3.7, tableau 19). De manière moins précise, le tableau de financement selon $\mathrm{MCH} 1$ nous apporte la même information à travers la différence entre les revenus non monétaires et les charges non monétaires, soit à KCHF 1665 (section 3.3.7, figure 20). Ce montant correspond aussi à l'addition des amortissements (et des autres charges et revenus non monétaires) et de l'excédent de revenus ou de charges ${ }^{136}$. Cette source finance plus de $90 \%$ de l'investissement net de MaCollectivité ${ }^{137}$.

Cession d'actifs. La seconde source de financement interne est la vente d'actifs appartenant à la collectivité. Le processus de cession d'actifs est différent selon que le bien appartient au patrimoine administratif ou au patrimoine financier. Dans le cas d'un actif administratif, la décision de céder le bien doit être prise par le Législatif de la collectivité. C'est à ce dernier de décider si l'actif n'est plus nécessaire à l'accomplissement des tâches exigées par les dispositions de droit public. Sur la base de cette décision, l'actif peut être comptablement transféré du patrimoine administratif au patrimoine financier et la vente peut avoir lieu. Dans le tableau des flux de trésorerie, ce financement interne est visible dans les liquidités apportées par les activités de placement. Pour ce qui concerne MaCollectivité, on constate qu'elle n'a procédé à aucune vente d'actifs au cours de l'Année t puisque le flux de trésorerie provenant de l'activité de placement est égal à zéro (tableau 19).

Avantages et inconvénients du financement interne. Le financement interne a pour avantage majeur son faible coût ${ }^{138}$. En effet, contrairement au financement externe, il n'implique aucun paiement d'intérêt. Or les intérêts à payer peuvent contribuer à mettre en péril la soutenabilité financière d'une collectivité, ainsi que nous le verrons dans le chapitre consacré à la dynamique de l'endettement (chapitre 12).

Toutefois, le financement interne n'est pas toujours envisageable. La collectivité ne possède pas forcément d'actifs qu'elle pourrait céder. Par ailleurs, vouloir accroître l'autofinancement implique de dégager un excédent de revenus significatif

136 En l'occurrence, MaCollectivité réalise un excédent de revenus de KCHF 64. Cela montre que le coût des prestations offertes est intégralement financé par les revenus courants. Voir aussi l'indicateur de couverture des charges (I1) dans la section 9.3.1.

137 Voir l'indicateur financier I2 ${ }^{\# \#}$ dans la section 9.3.1.

138 Sous réserve d'éventuels taux d'intérêt négatifs. 
et récurrent, autrement dit de mettre en place un excédent structurel de revenus ${ }^{139}$. Cette stratégie nécessite donc de faire peser une fiscalité - ou une parafiscalité - plus importante que nécessaire sur les personnes physiques et morales.

De plus, le financement interne de l'investissement pose un problème de temporalité, en particulier s'il découle d'un excédent structurel de revenus, dans la mesure où cela équivaut à couvrir directement, au cours d'un même exercice, tout ou partie de la dépense d'investissement. La couverture directe de la dépense d'investissement ne permet pas de répartir l'effort de financement dans le temps, alors que l'infrastructure offrira ses services sur une longue période. Dans ces conditions, le financement interne n'est pas garant de l'équité intergénérationnelle (voir également le principe de couverture ultérieure dans la section 3.3.5). Le financement externe remédie à ces inconvénients.

\subsection{Financement externe}

En matière de financement externe, il faut distinguer le financement à court terme du financement à long terme. Cette distinction apparaît d'ailleurs également dans le tableau des flux de trésorerie dans la partie dédiée à l'activité de financement (voir le tableau 19 dans la section 3.3.7).

\subsubsection{Financement à court terme par une ligne de crédit}

La ligne de crédit est un instrument de financement externe à court terme, c'est-àdire qu'elle couvre un besoin de financement ne dépassant généralement pas une année. Elle est le plus souvent obtenue auprès d'établissements bancaires et consiste en une autorisation donnée à la collectivité de l'utiliser à son rythme et à sa guise. Elle correspond donc à un crédit ouvert et disponible. Son montant maximum et ses modalités font l'objet d'un contrat négocié à l'avance. La collectivité peut l'utiliser en cas de besoin urgent de trésorerie. Selon les cas, les modalités comprennent en général, outre le plafond de crédit, les modalités de remboursement, le taux d'intérêt et le délai de remboursement. Ces modalités contractuelles seront en principe négociées annuellement.

La ligne de crédit peut revêtir différentes formes. Elle peut correspondre à une avance à terme fixe. Dans ce cas, l'établissement bancaire crédite en une seule fois sur le compte courant de la collectivité le total du montant prêté. Le remboursement se fait également en une seule fois à l'échéance, avec possibilité de renouvellement du contrat. Le taux d'intérêt est fixe et les échéances des intérêts dépendent de la durée du crédit. Plus rarement, la ligne de crédit peut également se présenter sous

139 La notion d'excédent structurel est développée au chapitre 11 consacré au diagnostic de l'origine conjoncturelle ou structurelle des déficits et des excédents. 
la forme d'un crédit en compte courant. Ici l'établissement bancaire octroie l'autorisation à la collectivité de retirer du compte courant concerné les liquidités nécessaires à ses activités opérationnelles et de présenter un solde négatif dans le respect de la limite du crédit octroyé. Le crédit en compte courant se distingue de l'avance à terme fixe par le fait qu'il n'est pas limité dans le temps et que ses conditions, en particulier le taux d'intérêt, peuvent être modifiées unilatéralement et en tout temps par l'établissement bancaire prêteur.

Le principal inconvénient de la ligne de crédit est son coût, car le taux d'intérêt appliqué est relativement élevé. L'avantage est la rapidité avec laquelle elle permet d'obtenir des liquidités. Elle est donc particulièrement utile lorsqu'une entité est en attente de rentrées financières, par exemple fiscales, mais fait face à des dépenses courantes urgentes. Même si, en principe, la règle d'or exclut de recourir à un financement externe pour financer des dépenses courantes, des difficultés de trésorerie passagères justifient de solliciter une ligne de crédit. Mais cela doit rester une exception, limitée à des besoins de faible importance avec un horizon de court terme, c'est-à-dire pour moins d'une année. Cet instrument n'est pas adapté pour le financement à long terme des dépenses d'investissement. À l'inverse, un financement externe à long terme pour financer des dépenses courantes est définitivement à proscrire.

\subsubsection{Financement à long terme}

\section{Emprunt par contrat de gré à gré}

L'emprunt par contrat de gré à gré est un outil de financement à long terme couramment adopté par les collectivités publiques, en particulier à l'échelon local. Le contrat est conclu directement entre la collectivité et l'entité prêteuse. C'est pourquoi cet instrument est aussi qualifié d'emprunt privé. L'entité prêteuse est le plus souvent un établissement bancaire. Dans ce cas, on parle plus spécifiquement d'emprunt bancaire. Toutefois, d'autres acteurs, comme les caisses de pension ou les compagnies d'assurances, octroient également un volume significatif de prêts.

Dans la mesure où le contrat est passé de gré à gré, la collectivité doit pouvoir savoir quelles sont les entités intéressées à lui prêter des fonds. À moins d'être démarchée, il lui est a priori difficile de le savoir. C'est là qu'interviennent divers intermédiaires financiers et autres courtiers dont l'objectif et le rôle consistent à mettre en relation les collectivités avec les investisseurs intéressés. Des plates-formes de mise en réseau pair à pair via l'Internet (peer-to-peer) sont récemment apparues pour offrir ce type de service et réduire les coûts de transaction. Quel que soit le support technologique, l'idée est de permettre aux collectivités de comparer les différentes offres de crédit en toute transparence pour minimiser le coût du financement.

Le contrat de gré à gré s'adapte aux besoins de financement de la collectivité puisque ses termes - montant emprunté, devise dans laquelle s'effectue l'emprunt, 
maturité, amortissement financier, type de taux d'intérêt - sont négociables. À leur tour, les termes d'un contrat sont susceptibles d'influencer le taux d'intérêt de l'emprunt. Dans la mesure où cet outil est largement utilisé par les communes, mais aussi par les cantons, il vaut la peine de s'intéresser de plus près à ces différentes possibilités.

Maturité. La maturité désigne la durée du contrat d'emprunt. En principe, plus la maturité est longue, plus le taux d'intérêt sera important. Si la collectivité entend contracter afin de financer spécifiquement une dépense d'investissement, alors il est conseillé d'opter pour une maturité calquée sur la durée de vie de l'actif concerné. Ainsi, pour un bâtiment dont la durée d'utilisation est estimée à vingt-cinq ans, il faut s'attendre à d'importants travaux de rénovation et de mise à niveau au-delà de cet horizon. Il est donc préférable que la maturité de l'emprunt finançant cet objet n'excède pas cette durée.

De leur côté, les bailleurs de fonds suivent généralement des règles qui leur sont propres. Ces règles fixent notamment des durées maximales par type d'objets financés (par exemple, cent ans pour les immeubles de rendement appartenant au patrimoine financier). Par ailleurs, la pratique des prêteurs consiste à proposer un taux fixe pour une période allant généralement de deux à quinze ans, voire vingt ans.

Emprunter pour financer spécifiquement un actif est parfois recommandé pour les collectivités de taille modeste qui n'ont que quelques actifs importants. Pour les autres, il est plutôt conseillé de recourir à une stratégie visant à saisir les opportunités par le marché. Dans ce cas, le montant de l'emprunt et la maturité seront choisis en fonction des conditions offertes, notamment celles relatives au taux d'intérêt, sachant que le taux augmente généralement avec le montant et la durée de l'emprunt.

Amortissement financier. L'amortissement financier est le montant à rembourser à l'entité prêteuse. Il ne doit pas être confondu avec l'amortissement économique (ou comptable), qui correspond au coût annuel de l'usure et de l'obsolescence d'un actif et qui déprécie graduellement la valeur de l'objet au bilan (voir la section 3.3.5 consacrée à la dépréciation du patrimoine administratif). Le contrat peut prévoir un amortissement financier «à maturité». Dans ce cas, l'intégralité du montant emprunté sera remboursée à l'échéance du contrat. Alternativement, le contrat peut prévoir un amortissement financier annuel. Par exemple, il peut stipuler qu'un pourcentage du montant emprunté soit remboursé chaque année. Pour une petite collectivité, qui contracte un emprunt spécifique pour financer un objet, amortir annuellement permet d'avoir liquidé l'emprunt lorsque le contrat arrive à échéance. Cela permet également d'assurer la correspondance entre l'amortissement financier et l'amortissement économique. Quant à lui, l'amortissement financier «à maturité» implique soit de disposer de liquidités suffisamment importantes pour rembourser l'emprunt à l'échéance du contrat, soit de procéder à un refinancement, c'est-à-dire de contracter un nouvel emprunt apportant les liquidités nécessaires au 
remboursement. Évidemment, une telle stratégie présente un risque plus élevé que l'amortissement financier annuel, au cas où les taux d'intérêt évoluent défavorablement ou si la situation financière de la collectivité se détériore au point de rendre méfiants les investisseurs. Par ailleurs, un amortissement à maturité peut impliquer un taux d'intérêt plus élevé.

Type de taux d'intérêt. On distingue deux types de taux d'intérêt: le taux fixe et le taux variable. L'emprunt à taux fixe désigne un contrat dans lequel le taux d'intérêt est défini à la conclusion du contrat et reste constant tout au long de la durée de vie du crédit. Avec cette formule, la charge annuelle d'intérêts est identique pendant toute la durée du contrat.

A contrario, l'emprunt à taux variable (ou emprunt à taux révisable) désigne un contrat dans lequel le taux est lié à un taux d'intérêt de référence. Le taux d'intérêt de référence est souvent le LIBOR ou l'EURIBOR. Le LIBOR - London interbank offered rate ou taux interbancaire pratiqué à Londres - consiste en une moyenne des taux auxquels un échantillon de grandes banques internationales établies à Londres s'attendent à pouvoir emprunter «en blanc» (c'est-à-dire sans que le prêt soit gagé par des titres) auprès des autres banques de l'échantillon ${ }^{140}$. Le LIBOR est déterminé quotidiennement pour des emprunts en différentes devises: dollar américain, livre sterling, yen, euro et franc suisse. Le LIBOR pour l'euro reste peu utilisé. Il est en effet concurrencé par L'EURIBOR - Euro interbank offered rate - ou en français TIBEUR - taux interbancaire offert en euro. Ce dernier est analogue au LIBOR. Toutefois, il est construit à partir de taux émanant d'un échantillon de grandes banques établies en Europe pour des emprunts en euro. Le taux variable est donc un taux révisable par l'entité prêteuse sur la base de l'évolution du taux de référence auquel elle ajoute une marge. Cette marge est quant à elle fixe sur toute la durée du crédit. Elle est d'un point de pour cent, au plus. Si le taux LIBOR se situe à $2 \%$, le taux d'intérêt est donc de 3\%. La marge est parfois stipulée en points de base (basis points). Le point de base est une unité de mesure représentant un centième de point de pour cent. Une marge de 100 points de base signifie que, pour un taux LIBOR de $2 \%$, le taux d'intérêt s'établit à $3 \%$. Le recours à cette unité évite la confusion entre pourcentage et point de pourcentage. La fréquence de l'ajustement au taux LIBOR constitue également un terme à définir dans le contrat d'emprunt. La plupart du temps, on utilise trois ou six mois. Mais d'autres fréquences sont possibles, comme une fréquence mensuelle ou annuelle. Le taux d'intérêt change donc plusieurs fois au cours de la durée de l'emprunt.

Les collectivités publiques tendent à privilégier l'emprunt à taux fixe. Ce dernier leur permet de planifier précisément la charge d'intérêts à la fois pour établir

140 Le LIBOR reste encore utilisé. Cependant, son avenir est incertain, à l'heure où nous écrivons ces lignes. En effet, en 2012, il s'est avéré que, dès 2005, de grandes banques ont mis en place un dispositif pour le manipuler. Depuis, la supervision sur le mécanisme a été revue par l'autorité britannique de surveillance des marchés financiers. Toutefois, cette dernière a annoncé qu'elle ne défendrait pas le maintien du LIBOR au-delà de 2021. 
le budget et pour construire la planification financière. Au-delà de cette simplification administrative, un taux fixe facilite également la gestion financière car, avec lui, la collectivité ne s'expose pas au risque d'une augmentation des taux d'intérêt. Mais le choix du taux fixe prive également la collectivité de l'opportunité offerte par une baisse des taux d'intérêt. Opter pour un emprunt à taux variable permet à la collectivité de profiter d'une décrue du taux d'intérêt tout en faisant planer le risque d'une remontée du taux. Une stratégie d'emprunt fondée sur une mixité des taux permet généralement de tirer profit des baisses de taux tout en s'exposant de manière limitée aux évolutions défavorables. Un portefeuille composé pour 70 à $75 \%$ d'emprunts à taux fixes et pour 30 à $25 \%$ d'emprunts à taux variables semble correspondre à une stratégie plutôt défensive (Stenghele 2006).

Outre les termes du contrat d'emprunt, d'autres facteurs influencent le taux d'intérêt. L'environnement macroéconomique influence fortement le taux auquel une collectivité peut espérer emprunter. La politique monétaire suivie par la banque centrale, en particulier le taux directeur qu'elle impose, joue un rôle prédominant. Si la Banque nationale suisse (BNS) suit une politique accommodante avec un taux directeur bas, les emprunts seront moins coûteux, du moins à court terme. La nature des garanties fournies et le profil de risque de la collectivité influencent également les conditions qu'il est possible d'obtenir auprès des investisseurs ${ }^{141}$. Une collectivité présentant un risque d'avoir des difficultés à rembourser ses emprunts se verra offrir un taux d'intérêt moins favorable. Pour évaluer ce risque et la solvabilité de la collectivité, les créanciers se basent sur différents ratios, dont celui du taux d'endettement net ou de la dette brute par rapport aux revenus (voir la section 9.3 consacrée aux indicateurs financiers). La charge fiscale imposée par la collectivité est également utilisée pour évaluer le risque. Une charge fiscale relativement élevée réduit la marge de manœuvre à disposition et donc la capacité de la collectivité à s'extirper d'une situation financière difficile. Dans ce cas, l'investisseur prend davantage de risques à prêter des fonds et requiert un taux d'intérêt plus important, c'est-à-dire qu'il impose une prime de risque. Si la collectivité ne peut guère peser sur l'environnement macroéconomique, elle peut agir sur son profil de risque. Certes, il s'agit-là d'une opération qui prend du temps. Mais en améliorant son profil de risque, l'entité réduit sa prime de risque et par conséquent le coût de sa dette.

\section{Emprunt obligataire}

L'emprunt obligataire - ou emprunt par obligations - est un outil de financement permettant à la collectivité de lever des fonds directement sur le marché des capitaux. Il consiste à émettre des titres de créances, assimilables à des reconnaissances de dettes, remboursables à une date et pour un montant fixé à l'avance, et qui porte

141 En effet, de leur côté, les bailleurs de fonds doivent respecter les exigences de l'ordonnance fédérale sur les fonds propres et la répartition des risques des banques et des négociants en valeurs mobilières (ordonnance sur les fonds propres, OFR, RS 952.03). 
intérêt. Chaque emprunt obligataire a ses propres caractéristiques, en termes de taux d'intérêt - fixe ou variable -, de durée - généralement entre cinq et quinze ans - et de modalités de remboursement - au terme de la durée de l'emprunt ou par tranches.

L'avantage majeur de l'emprunt obligataire est d'offrir généralement des taux relativement attractifs et de diversifier les sources de financement. Toutefois, pour bénéficier de conditions intéressantes, divers obstacles doivent être surmontés - obstacles qui constituent autant d'inconvénients. D'abord, le volume d'émission doit être important (au minimum CHF 100 mio). Par ailleurs, la collectivité émettrice doit bénéficier d'une bonne image attestée à travers une notation réalisée par une agence spécialisée (Standard and Poor's, Moody's, Fitch Ratings, pour mentionner les trois grandes agences). La notation est censée refléter la bonité de l'emprunteur, la capacité de ce dernier à honorer ses engagements en temps et en heure et donc à rembourser le capital emprunté. Ces exigences impliquent un coût administratif élevé, à commencer par la nécessité de produire des états financiers passablement détaillés. Pour des obligations qui seront cotées en bourse, il est également nécessaire de réaliser un prospectus d'émission contenant toutes les conditions importantes liées au droit de créance.

Par conséquent, l'emprunt obligataire s'adresse à des emprunteurs aguerris et aux collectivités de grande taille. En Suisse, il est donc utilisé essentiellement par la Confédération, les cantons et les villes ${ }^{142}$.

\subsubsection{Outils alternatifs}

\section{Emprunt d'impulsion}

Dans le cadre de certains programmes d'impulsion, les collectivités peuvent bénéficier de prêts à intérêts réduits, voire sans intérêts. Par le passé, de nombreuses entités ont obtenu de tels prêts de la part des cantons et de la Confédération au titre de la loi fédérale sur l'aide aux investissements dans les régions de montagne (LIM, RS 901.1). Le relais a été pris par la nouvelle politique régionale (NPR) fondée sur la loi fédérale sur la politique régionale (LPR, RS 901.0). Cette dernière offre des facilités de financement similaires, ainsi que des contributions à fonds perdus. Bien qu'avantageux, ces financements s'adressent à des projets spécifiques et impliquent que l'entité souhaitant en bénéficier remplisse diverses conditions d'octroi. Ces conditions portent évidemment sur la nature du projet à financer. Dans le cadre de la NPR, il doit s'agir d'investissements qui améliorent la compétitivité et la création de valeur et cela dans des secteurs particuliers, notamment le tourisme et l'industrie. En outre, le canton doit participer à l'effort de financement au moins autant que la Confédération. Les montants alloués sont proportionnels aux effets attendus. L'entité qui sollicite le prêt doit engager des moyens propres dans le projet. Par

142 D’ailleurs, seules les villes de Berne, Bienne, Genève, Lausanne, Lugano, Saint-Gall, Winterthour et Zurich sont actuellement présentes sur le marché obligataire. 
conséquent, ces emprunts constituent une source de financement ponctuelle, qui plus est pour des projets que l'entité n'aurait souvent pas choisis spontanément de réaliser si elle avait eu à les financer intégralement elle-même.

\section{Partenariat public-privé}

Le partenariat public-privé (PPP) consiste en une collaboration entre une collectivité publique et une entreprise privée dans le cadre d'un projet d'infrastructure (hôpital, prison, stade, etc.). Cette collaboration comporte un mécanisme de financement dans la mesure où le prestataire privé est responsable à la fois de la conception, du financement, de la réalisation et de l'exploitation du projet concerné. La collaboration est réglée par contrat. L'avantage d'un tel partenariat pour la collectivité est de bénéficier d'un équipement collectif sans avoir à se procurer elle-même les liquidités pour le financer ${ }^{143}$. Par ailleurs, la collectivité bénéficie de l'expertise du spécialiste privé dans la gestion opérationnelle. Le plus souvent, la collectivité verse au partenaire privé un dédommagement dont le montant et la durée sont prévus contractuellement. En Suisse, seuls quelques projets font l'objet d'un véritable PPP et cela essentiellement à l'échelon cantonal.

\section{Financement participatif}

Le financement participatif (crowdfunding) se développe significativement grâce à l'essor des plates-formes collaboratives sur l'Internet. Ces plate-formes mettent en relation directe un emprunteur avec un grand nombre d'investisseurs potentiels. Elles apportent une meilleure visibilité au projet que la collectivité souhaite réaliser et font converger les financements d'un grand nombre de créanciers, qu'il s'agisse de particuliers ou d'entités diverses. C'est pourquoi on parle aussi de micro-financement. L'outil est donc plutôt réservé à des projets modestes suscitant un grand engouement populaire.

Le mode de rémunération varie selon le type de financement participatif. Le financement participatif par prêt (lending crowdfunding) se rapproche de l'emprunt par contrat de gré à gré. C'est donc celui qui peut intéresser plus particulièrement les collectivités publiques. Les investisseurs reçoivent également un intérêt. En évitant ainsi de recourir à un intermédiaire financier ou bancaire, la collectivité peut espérer bénéficier d'un taux d'intérêt plus favorable. Le financement participatif contre récompense (reward based-crowdfunding) donne lieu à une rémunération non financière des créanciers. Une collectivité peut envisager ce mode de rémunération pour des projets ciblés, par exemple à travers des entrées gratuites en échange du financement d'un projet culturel ou sportif. Reste le financement participatif caritatif où l'investisseur ne reçoit pas de rémunération, voire prête

143 Cela étant, la collectivité doit mentionner dans son bilan l'actif et l'engagement découlant du contrat de partenariat, ainsi que le prévoit le Conseil suisse de présentation des comptes publics (FAQ Partenariats publicprivé, du 10.09.2013). 
à fonds perdus. Dans ce dernier cas, il ne s'agit plus véritablement de prêts, mais davantage de donations.

\subsection{Quelques règles à garder en tête}

Emprunter comporte toujours une part de risque. Quelques règles simples permettent de réduire le risque ${ }^{144}$.

Diversifier. Il est important de diversifier le portefeuille de financements, les sources et les outils. Contracter systématiquement des emprunts à maturité longue et à taux fixe met certes la collectivité à l'abri d'une évolution à la hausse des taux. Mais cela ne lui permet pas de bénéficier d'une éventuelle décrue des taux. Par ailleurs, une telle stratégie n'offre pas la souplesse requise pour gérer efficacement la trésorerie. Il faut en effet pouvoir s'accommoder des excédents et des insuffisances de liquidités qui se manifestent régulièrement au gré des activités et des projets. À l'inverse, une stratégie axée sur l'emprunt à taux variable expose la collectivité à un risque excessif en cas de relèvement des taux. Il faut également diversifier les prêteurs. Cela évite de dépendre d'un seul établissement de crédit. Cela favorise aussi la concurrence et démontre que la collectivité est prête à faire jouer la concurrence pour se financer.

Faire jouer la concurrence. Les collectivités ont maintenant intégré la nécessité de faire jouer la concurrence pour obtenir des conditions d'approvisionnement favorables, en particulier pour acquérir des biens et des services. Les produits financiers doivent être traités de manière analogue. Une collectivité doit avoir le réflexe de solliciter plusieurs offres auprès de divers établissements de crédit pour minimiser le coût et le risque de son financement.

Comparer. Les prêteurs potentiels n'ont pas leur pareil pour tenter de se distinguer de la concurrence en offrant des produits particuliers. Or, pour véritablement tirer profit d'une mise en concurrence, la collectivité doit pouvoir comparer les offres qu'elle reçoit. À cette fin, la collectivité doit elle-même fixer les contours du produit financier dont elle a besoin. La première étape consiste donc à définir son besoin et décider d'une espèce de cahier des charges auquel les offres devront répondre. La figure 34 offre un arbre de décision permettant de choisir l'outil de financement adéquat. Si la collectivité aboutit au constat que l'emprunt de gré à gré est l'instrument adéquat, il lui reste à déterminer quels sont les termes qu'elle souhaite pour le contrat, en particulier la maturité, les modalités d'amortissement financier, le type de taux d'intérêt. La seconde étape consiste à exiger des offres conformes au cahier des charges, à déposer à la même date par tous les prêteurs potentiels. Les offres obtenues seront ainsi davantage comparables. 
Figure 34 Arbre de décision permettant de choisir un outil de financement.

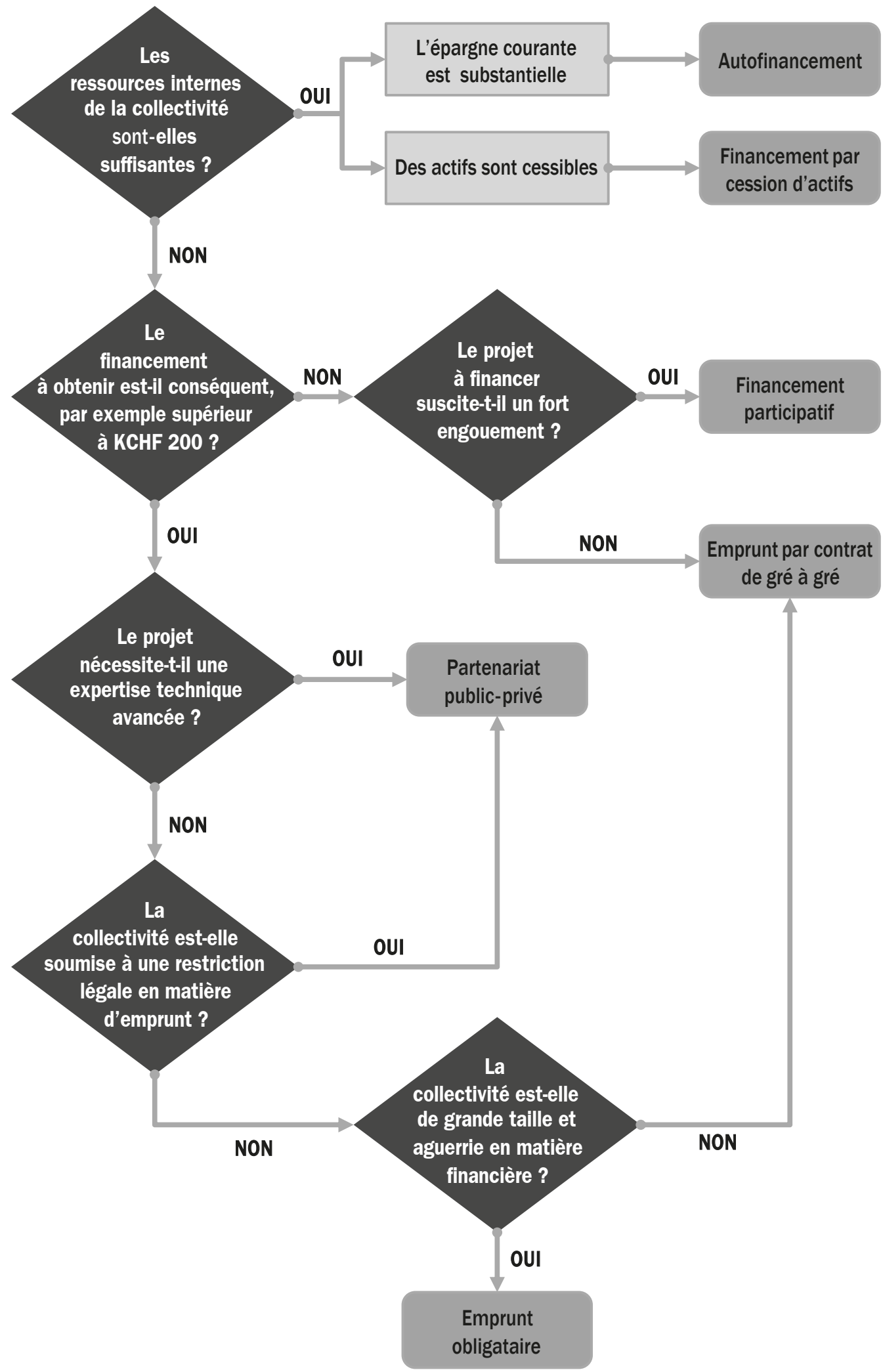


Ne pas succomber. Il peut arriver qu'un établissement de crédit propose un produit ne répondant pas exactement au cahier des charges, mais paraissant tout de même intéressant. La collectivité doit alors solliciter une cotation alternative auprès d'autres établissements pour un produit présentant les mêmes caractéristiques techniques et juridiques. Il ne faut jamais oublier que les établissements de crédit ne sont pas des organisations philanthropiques. Ces établissements sont soumis à des exigences de rentabilité. Par conséquent, une offre alléchante peut dissimuler une subtilité au détriment de l'emprunteur. Un taux d'intérêt sans rapport avec la réalité du marché ou avec les conditions obtenues par une collectivité voisine lors d'une transaction récente recèle probablement un piège dans lequel la collectivité qui cherche à emprunter ne doit pas tomber.

Demander conseil. Une asymétrie de compétences existe forcément entre l'expertise financière des établissements de crédit et celle de la collectivité qui cherche un financement. Les premiers emploient moult spécialistes de l'ingénierie financière. La collectivité, même relativement grande, ne se livre qu'à un nombre restreint d'opérations de financement. Elle n'a donc pas de personnes dédiées à l'activité de financement. Afin de réduire l'asymétrie, il est possible de recourir aux services d'entreprises spécialisées en matière de gestion de la dette. Là aussi, il est important de choisir une entreprise disposant des outils pour analyser les offres des établissements de crédits, leurs coûts et leurs risques. Les communes ne doivent pas hésiter à prendre conseil auprès de leurs associations faîtières ou auprès du service des communes de leur canton.

Gérer et ne pas spéculer. Il faut toujours se rappeler que les responsables d'une collectivité publique ont une mission de gestion. D’une certaine manière, gérer est tout le contraire de spéculer. Nous avons déjà évoqué le principe de la prudence (section 2.1.2). Ce principe vaut également pour la gestion de la trésorerie et de la dette. Il interdit de prendre des décisions en pariant sur l'évolution future et hypothétique des marchés en vue d'en tirer profit, tout en acceptant en parallèle le risque de perdre de l'argent si l'évolution est contraire aux espoirs. La gestion financière se doit au contraire de minimiser les risques. Par définition, elle devrait donc être circonspecte. D'entre deux produits, celui qui présente le risque le plus faible doit être retenu, même si cela implique un coût a priori supplémentaire pour la collectivité. Ce coût supplémentaire ne doit pas être considéré comme une perte, mais comme le prix à payer pour éviter un risque financier.

Comprendre ou renoncer. Il arrive que des collectivités suisses ou étrangères contractent des produits contenant des mécanismes spéculatifs, par exemple à travers un emprunt en devises étrangères ou une indexation des taux. Les responsables peuvent être séduits par les taux très performants que ces produits affichent facialement, c'est-à-dire dans les documents commerciaux remis par les prêteurs. Mais une évolution défavorable des marchés peut conduire à une explosion du coût du financement. Ces produits en deviennent alors toxiques. Certes, ces mécanismes 
sont mentionnés dans les offres faites par les établissements de prêt. Mais leur mention est souvent reléguée au chapitre des conditions particulières et leur présentation s'effectue à l'aide de formules mathématiques relativement hermétiques. Avant de choisir, il faut analyser l'ensemble des pièces au contrat. En cas de doute ou d'incompréhension concernant le produit offert et ses subtilités, le réflexe doit être d'écarter la proposition et de privilégier un instrument plus classique.

S'entendre sur une stratégie. Dans beaucoup de domaines, les administrations publiques exercent leurs activités dans un cadre contraignant. Par ailleurs, ces activités sont systématiquement soumises à autorisation et à contrôle. La gestion de la dette devrait également pouvoir s'exercer dans le cadre d'une stratégie explicite, discutée, approuvée et contrôlée. Certes, plusieurs collectivités, notamment des cantons, disposent de règles de base relatives au recours à l'emprunt. Mais rares sont encore celles qui sont dotées d'un cadre réglementaire pour l'ensemble de la gestion de la dette. Rares sont celles où la stratégie est élaborée par une commission d'experts, puis est soumise à l'approbation de l'Exécutif ou du Législatif. Une telle stratégie devrait notamment porter sur la répartition de la dette entre la dette à court terme et la dette à long terme, entre dette à taux fixe et dette à taux variable. Charge ensuite aux trésoriers d'opérer pour mettre en application la stratégie retenue. 


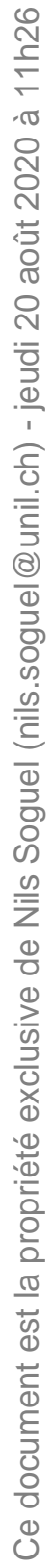




\section{Déceler la tactique politique en matière de présentation des comptes}

\subsection{Une volonté de fiabilité et de clarté}

La fiabilité et la clarté sont deux principes qu'une collectivité devrait respecter dans la préparation et la présentation de ses comptes (voir la section 2.1 consacrée aux principes financiers). En effet, les comptes sont censés offrir aux diverses parties prenantes une image transparente et par conséquent fiable de la situation financière. Le besoin de transparence est particulièrement élevé dans le secteur public. C'est en effet à travers les budgets et les comptes que s'effectuent les arbitrages entre les différentes interventions de l'État. C'est sur la base des budgets que le Parlement et, à travers lui, la population autorise le gouvernement à dépenser les deniers publics. C'est grâce aux comptes que l'on vérifie le bon usage de ces deniers.

Le principe de la comptabilité d'exercice répond à ce souhait de fiabilité et de clarté: les comptes doivent refléter fidèlement le résultat de l'exercice. Le principe concurrent, celui de la comptabilité de caisse, se contente d'enregistrer et de présenter les encaissements et les décaissements réalisés entre le $1^{\text {er }}$ janvier et le 31 décembre de l'Année t. Mais il ne permet pas de connaître le coût des prestations publiques offertes au cours de cette année-là et de savoir si ce coût est couvert par les revenus de cette même année. En effet, une partie des charges ou des revenus liés à ces prestations peut avoir été facturée et payée avant le $1^{\text {er }}$ janvier ou après le 31 décembre. Par conséquent, le solde financier - la différence entre encaissements et décaissements - ne reflète pas le résultat - la différence entre charges et revenus liés à la prestation. Pour rattacher les charges et les revenus à la bonne période - ici à l'Année t-, il est nécessaire d'enregistrer diverses opérations purement comptables. Pensons aux opérations de régularisation (actifs et passifs transitoires). Pensons également aux constitutions de provisions qui permettent de tenir compte des engagements non encore avérés, liés à un événement - un fait générateur - survenu au cours de l'Année t, et qui pourraient donner lieu à un décaissement ultérieur sans que cela soit encore parfaitement sûr; cela survient par exemple en cas de litige devant encore être tranché par un tribunal. Pensons finalement aux amortissements planifiés et non planifiés qui visent à enregistrer l'usure et l'obsolescence du patrimoine administratif intervenues au cours de l'Année $t$.

Les passifs transitoires, les constitutions de provisions et les amortissements augmentent le total des charges. Par conséquent, ces opérations péjorent le solde du compte de résultats. De leur côté, les actifs transitoires et les dissolutions de provisions améliorent le solde.

Évidemment, pour répondre au principe de fiabilité et de clarté, il est nécessaire que le montant de ces opérations reflète fidèlement la réalité. C'est en particulier le cas des opérations sur les provisions. Par définition, le montant et l'échéance des 
engagements qui justifient une provision sont incertains. Il est donc indispensable de procéder à une estimation, et cette estimation devrait être sincère.

\subsection{Une transparence qui comporte des risques}

Paradoxalement, et en particulier dans le secteur public, vouloir présenter les résultats financiers en toute transparence comporte des risques pour les équilibres budgétaires futurs. C'est notamment le cas lorsque les «comptes bouclent bien» et que le compte de résultats devrait afficher un excédent de revenus. Or les responsables des finances ne souhaitent souvent pas présenter un surplus trop important. En effet, aux yeux des contribuables, un surplus véhicule le message qu'ils ont payé trop d'impôts et de taxes par rapport aux prestations publiques dont ils ont bénéficié. Ou, alternativement, que la collectivité ne leur a pas fourni des prestations en suffisance par rapport aux recettes perçues. Si les surplus se succèdent et s'ils sont importants, les responsables financiers doivent raisonnablement s'attendre à des revendications émanant de divers acteurs: autres membres de l'Exécutif, membres du Législatif, citoyens, groupes de pression. Ces revendications peuvent aller en direction de nouvelles dépenses publiques ou d'une baisse de la pression fiscale.

Ceci est d'autant plus vrai que le débat politique se cristallise sur le compte de résultats et son solde. Le compte des investissements, le tableau des flux de trésorerie et le bilan captent beaucoup moins, voire pas du tout, l'attention. Cela s'explique aisément: le compte de résultats apporte l'essentiel de l'information nécessaire pour contrôler la bonne application du droit des crédits. D'un point de vue purement formel, il n'est donc pas surprenant que le compte de résultats figure bien en évidence en première partie du rapport des comptes. Et il en mobilise la plus grande partie des pages. De plus, les dispositions légales en matière de gestion financière, même si elles présentent des différences entre collectivités, convergent sur un point de principe: le compte de résultats doit être équilibré, si ce n'est annuellement, du moins à moyen terme. En d'autres termes, les charges courantes, c'est-à-dire le coût des prestations publiques, doivent être entièrement couvertes par les revenus courants.

Face à cette obligation de couverture des charges, on comprend aisément que les personnes en charge des finances cherchent à lutter contre les revendications qu'induirait un surplus, que ce surplus soit structurel, conjoncturel ou lié à des éléments extraordinaires ou inhabituels par leur montant (voir le chapitre 11 consacré à l'analyse de l'origine conjoncturelle ou structurelle des déficits). Pour y parvenir, ces responsables ont souvent deux avantages par rapport aux autres parties prenantes de la gestion financière. D'abord, ils ont une meilleure expertise en matière comptable. Ensuite, ils connaissent mieux le cadre légal. Cela leur permet de mettre assez facilement en place des artifices comptables pour réduire ou annihiler le surplus, et couper court à toute revendication qui pourrait menacer à l'avenir les équilibres budgétaires. 
Ce faisant, le résultat de comptes ne donne plus vraiment une image fiable de la performance financière de la collectivité. L'image devient arrangée à des fins tactiques. Elle permet de justifier le maintien d'une pression fiscale plus élevée ou de prestations publiques plus faibles qu'économiquement nécessaires. Si elles se répètent année après année, de telles opérations de cosmétique comptable finissent par dissimuler un surplus structurel ${ }^{145}$.

Évidemment, lorsque les comptes «bouclent mal», des artifices comptables peuvent aussi être utilisés afin de présenter un excédent de charges plus faible qu'il ne l'est en réalité. Ces artifices peuvent permettre aux responsables de sursoir à des mesures peu populaires pour rééquilibrer le budget à travers une hausse des revenus ou une meilleure maîtrise des charges. Mais dans ce cas aussi, le résultat des comptes ne donne plus vraiment une image fiable de la performance financière de la collectivité.

\subsection{Tension entre fiabilité et régularité}

On pourrait croire que les comptes présentent une image fiable de la situation financière dès lors que les opérations comptables sont conformes à la législation et aux règlements en vigueur. Mais un paradoxe apparaît lorsque la législation et les règlements autorisent, préconisent ou ne proscrivent pas des pratiques qui nuisent à la fiabilité des comptes. C'est le cas dans plusieurs cantons. Le modèle comptable $\mathrm{MCH} 2$ propose d'ailleurs explicitement certains "instruments de politique budgétaire » ${ }^{146}$. Bien que ces instruments et ces pratiques apparaissent légaux parce qu'ancrés dans la loi, il faut les qualifier d'artifices comptables. En effet, comme le rappellent Naser et Pendlebury (1992), on doit parler d'artifices comptables dès lors que des opérations visent à transformer les comptes afin qu'ils ne reflètent pas la situation telle qu'elle est, mais telle que les responsables souhaitent la présenter, soit en exploitant les règles existantes, soit en les ignorant. Avec cette cosmétique comptable, on modifie donc délibérément la performance et la situation financières de la collectivité pour qu'elles correspondent à celles que l'on veut communiquer et on renonce à en donner une image fidèle.

Ces artifices comptables permettent aux responsables financiers de présenter la situation financière de la collectivité de manière arrangée sans pour autant enfreindre la législation et le principe de régularité. Mais cela ne reste qu'une possibilité offerte par la législation; ce n'est pas une obligation. Par conséquent, une collectivité peut offrir une image fiable de sa situation financière si elle renonce à recourir à de tels artifices. Lorsqu'elle y recourt, elle n'enfreint cependant pas les dispositions légales. En revanche, lorsque les pratiques comptables dépassent le cadre légal, la présentation des comptes n’est ni fiable, ni légale.

145 Clémenceau et Soguel (2018) montrent d'ailleurs que gonfler les charges, en particulier à l'aide d'amortissements supplémentaires, pour faire apparaître un solde du compte de résultats plus faible qu'il ne l'est réellement, permet de dégager à l'avenir des surplus plus importants ou des déficits plus faibles.

146 Recommandation 04, CDF (2008). 
La figure 35 illustre ces différents modes de présentation des comptes. Ces modes vont d'une présentation fiable et fidèle à la réalité telle qu'on l'obtient notamment en suivant les normes IPSAS à une présentation illégale, en passant par une présentation, certes régulière, mais arrangée. Elle montre que la plupart des lois cantonales (et le $\mathrm{MCH} 2$ ) sont suffisamment flexibles pour permettre soit une présentation fiable, soit une présentation arrangée des faits économiques.

Évidemment, le droit public prévoit que l'action d'une collectivité doit être jugée à l'aune du respect du principe de la régularité. Il fournit ainsi aux organes de révision compétents les normes par rapport auxquelles ils doivent juger si les comptes sont régulièrement tenus et présentés. Cela explique pourquoi ces organes ne trouvent rien à redire lorsque des artifices comptables sont utilisés, dès lors qu'ils sont prévus par la législation. De la même manière, les membres de l'Exécutif, la commission des finances et le Législatif s'opposent rarement au recours à des artifices comptables par les responsables des finances. Comme l'évoque l'extrait 3, certes le principe de la fiabilité n'est pas respecté, mais la loi l'est.

Figure 35 Modes de présentation des faits économiques en fonction du référentiel comptable.

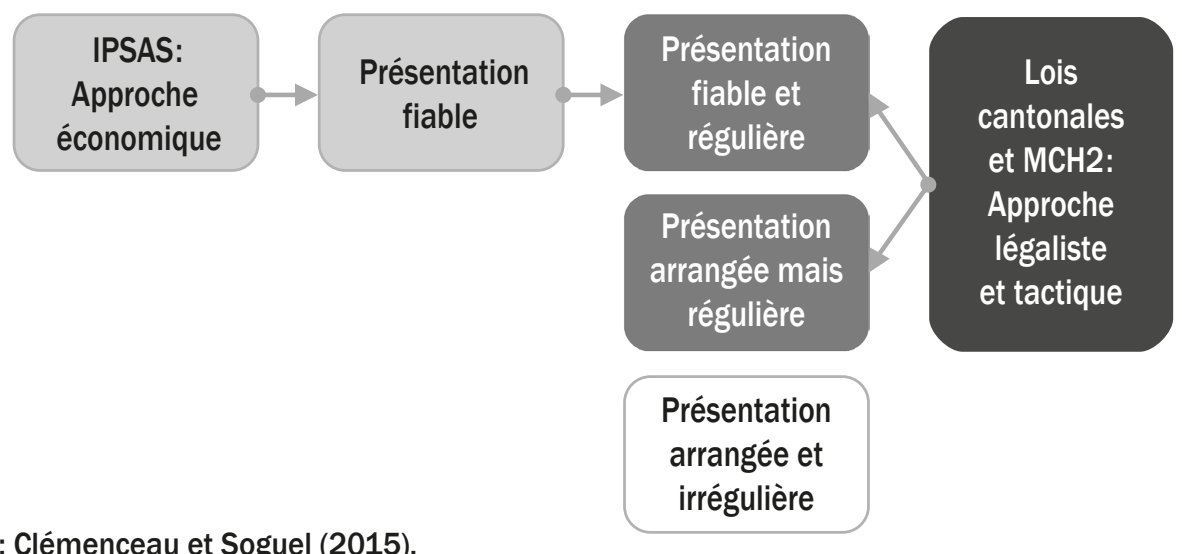

Source : Clémenceau et Soguel (2015).

\section{Extrait 3 La cosmétique comptable a lieu au vu et au sus de tous}

"Si la préparation du budget est toujours [...] l'occasion de coups de gueule au Parlement, l'examen des comptes est un rituel printanier beaucoup moins conflictuel. II commence en avril par la publication de cinq ouvrages volumineux dont on ne retient, la plupart du temps, que le résultat: un déficit est perçu comme un mauvais carnet, un bénéfice comme un bon. Or, souvent, le résultat est ajusté par des opérations comptables sur les constitutions/dissolutions de réserve ou par des écritures exceptionnelles [...]. Des opérations en tout bien tout honneur, puisque cette cosmétique comptable a lieu au vu et au su de tous."

Source: Mabut J.-F. (2014, dans La Tribune de Genève). 


\subsection{Cosmétique comptable}

Par rapport à la comptabilité de caisse, la comptabilité d'exercice facilite les artifices comptables. En effet, par définition, elle implique d'autoriser des opérations déconnectées des flux de trésorerie. Elle crée ainsi les conditions favorables pour enregistrer des opérations sans lien avec la réalité économique. Et ces opérations sont nombreuses. Nous abordons ici les opérations de cosmétique comptable les plus courantes et qui respectent les recommandations $\mathrm{MCH} 2$. Les pratiques qui ne suivent pas les recommandations $\mathrm{MCH} 2$ sont généralement également irrégulières en vertu des dispositions légales et réglementaires. Par conséquent, ces dernières devraient être détectées par les organes de révision et ne devraient donc pas entacher les comptes tels qu'ils sont présentés. Les opérations abordées ici sont les artifices comptables qui modifient la performance financière et la situation financière par rapport à la réalité. Ces opérations sont généralement passées lors de la clôture de comptes. Toutes affectent la performance financière, autrement dit le résultat total du compte de résultats (voir le tableau 17 relatif à la présentation échelonnée du compte de résultats). Certaines opérations sont assez aisément identifiables dans les comptes publiés, d'autres le sont plus difficilement.

\section{Instruments de politique budgétaire tolérés par le $\mathrm{MCH} 2$}

Les instruments de politique budgétaire ouvertement tolérés par le $\mathrm{MCH} 2$ sont les amortissements supplémentaires, l'amortissement du découvert du bilan, les constitutions et dissolutions de fonds, de préfinancements et d'autres réserves ${ }^{147}$. Les amortissements supplémentaires et ceux du découvert du bilan, ainsi que les constitutions de fonds, de préfinancements et d'autres réserves grossissent artificiellement le volume des charges. La hausse est artificielle parce qu'elle ne découle d'aucune utilisation de ressources productives, comme des ressources humaines, des biens et des services ou encore l'usure et l'obsolescence de l'infrastructure. Les dissolutions de fonds, de préfinancements et d'autres réserves gonflent artificiellement le volume des revenus. Ici la hausse est artificielle: elle ne reflète aucune ressource supplémentaire dont pourrait bénéficier la collectivité, comme des recettes fiscales ou des transferts.

La figure 36 indique quels sont les cantons qui autorisent ou proscrivent les amortissements supplémentaires ou les préfinancements, que ce soit au niveau du canton lui-même ou à l'échelon des communes. Quelques cantons s'autorisent à comptabiliser des amortissements supplémentaires, mais en interdisent l'usage à leurs communes. C'est le cas de Fribourg, de Schaffhouse et du Tessin. Concernant les préfinancements, environ la moitié des cantons s'autorisent à y recourir, tandis que les communes y sont autorisées dans tous les cantons, à l'exception de Fribourg,

147 Le Conseil suisse de présentation des comptes publics tient à jour un inventaire des instruments autorisés par la législation sur les finances des différentes collectivités suisses (www.srs-cspcp.ch). 
Lucerne, Schwyz et Tessin. Pour sa part, MaCollectivité a affecté ses revenus à des préfinancements pour un montant net de KCHF 970 à la fin de l'Année t. Ce faisant, l'excédent de revenus présenté se limite à KCHF 64, alors qu'en réalité, il s'élève à KCHF 1034. Cela représente tout de même plus de $6 \%$ du total des revenus.

Figure 36 Amortissements supplémentaires et préfinancements selon les cantons. État au 31 décembre 2019*.

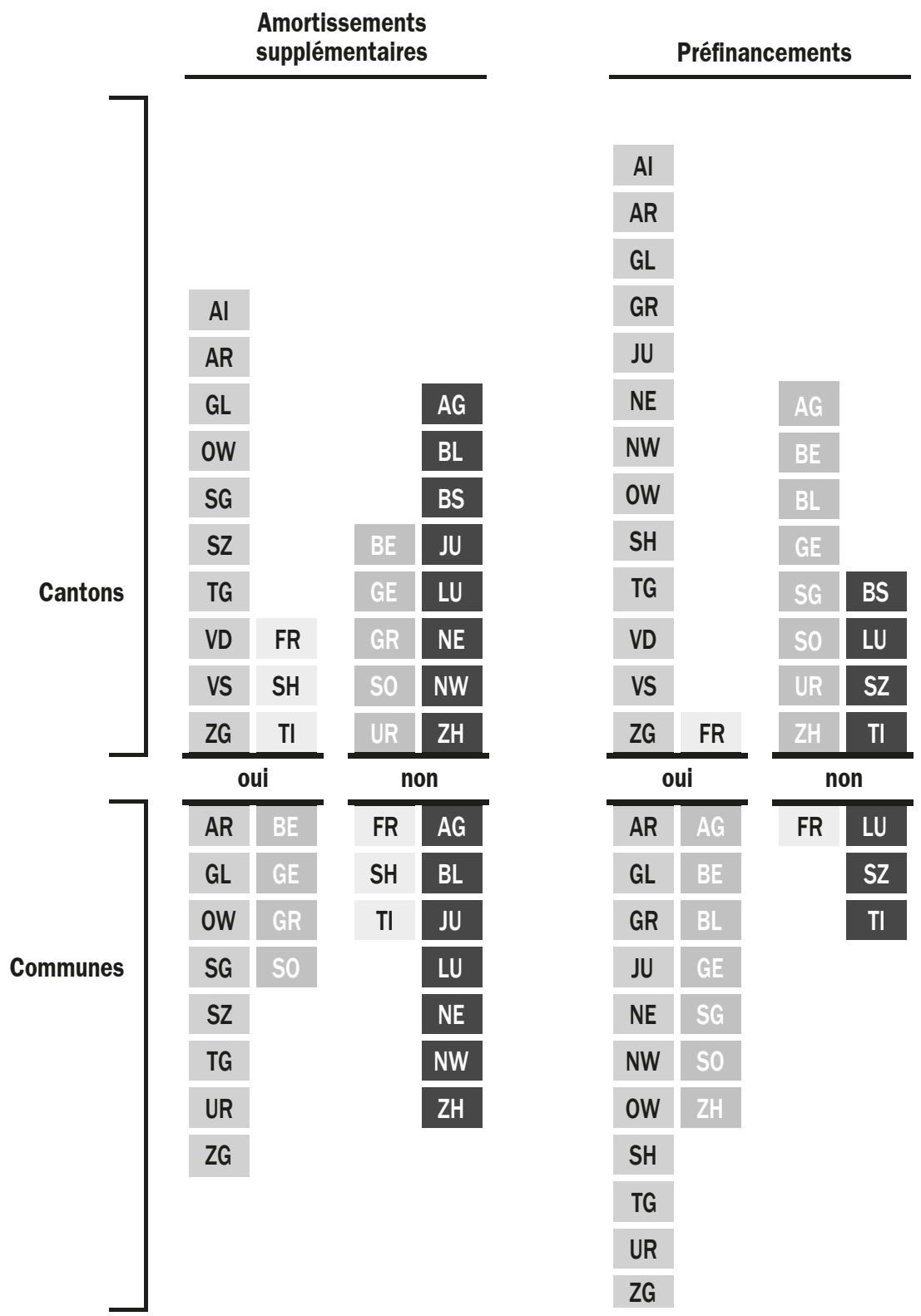

Source des données: Conseil suisse de présentation des comptes publics (www.srs-cspcp.ch).

* À cette date, les informations pour les communes des cantons de VD et VS ne sont pas encore disponibles. Les cantons d'Al et de BS ne fixent pas de prescription à leurs communes. 
Pour satisfaire malgré tout le principe de clarté, le $\mathrm{MCH} 2$ impose que ces opérations soient comptabilisées parmi les charges extraordinaires, respectivement les revenus extraordinaires (voir la section 3.3.4 consacrée au compte de résultats). Ces opérations de cosmétique comptable forment l'essentiel des charges et des revenus extraordinaires. On les retrouvera donc dans les rubriques 38 et 48 de la classification par nature, et plus particulièrement dans les sous-rubriques 383, 387 et 389 . Pour les responsables des finances, ces instruments sont d'autant plus commodes que les notions d'amortissements supplémentaires, d'amortissement du découvert au bilan, de préfinancements ou de fonds sont abstraites et difficilement comprises par les non-initiés.

Quoi qu'il en soit, ces rubriques 38 et 48 sont prises en compte pour dégager le résultat total du compte de résultats. Si l'on souhaite obtenir une image plus fiable de la performance financière de la collectivité, on se référera donc plus volontiers au résultat opérationnel, c'est-à-dire au résultat avant les opérations de cosmétique comptable. Malgré cela, on continue de s'intéresser avant tout au résultat total.

\section{Libertés prises avec la limite d'activation}

D’autres artifices comptables peuvent être utilisés sans qu'ils soient ouvertement tolérés par le $\mathrm{MCH} 2$. La coexistence d'un compte de résultats et d'un compte des investissements offre de ce point de vue des opportunités. Certes, le $\mathrm{MCH} 2$ recommande de fixer explicitement une limite d'activation à partir de laquelle une dépense devrait être comptabilisée dans le compte des investissements. Malgré cela, en pratique, le souhait de modifier délibérément le solde du compte de résultats conduit parfois à prendre des libertés avec cette limite. Prenons le cas d'une collectivité qui éprouve des difficultés à équilibrer son compte de résultats. Il est alors tentant d'enregistrer des dépenses dont la nature est hybride dans le compte des investissements, de manière à alléger les charges et améliorer le solde. À l'inverse, pour couper court aux revendications que pourrait faire naitre un excédent de revenus, ces mêmes dépenses pourraient être portées à charge du compte de résultats.

Ce type d'artifices est difficile à déceler de l'extérieur. Dans le cas de dépenses indûment portées au compte des investissements, un examen minutieux du tableau des immobilisations peut éventuellement permettre d'y parvenir. Ce tableau doit figurer dans l'annexe aux comptes. Encore faut-il qu'il présente un degré de détail élevé. À l'inverse, lorsque des dépenses sont indûment mises à charge du compte de résultats, l'artifice est bien difficile à identifier. En effet, le nombre de rubriques y est trop élevé et le détail publié dans les comptes est généralement insuffisant pour y parvenir.

\section{Opérations de bilan à bilan}

En dehors des opérations relatives à la gestion de la trésorerie et à la gestion de la dette, toutes les opérations devraient être enregistrées soit dans le compte de résultats, soit dans le compte des investissements. C'est la conséquence du droit des 
crédits. Autrement dit, toutes les opérations devraient influencer soit le résultat, soit le besoin de financement né de l'investissement net. Or l'expérience montre que certaines collectivités passent sciemment des opérations de bilan à bilan, c'est-à-dire en évitant le compte de résultats et le compte des investissements. Pour une collectivité qui doit provisionner ses engagements vis-à-vis de l'institution de prévoyance à laquelle elle affilie son personnel, il est tentant de constituer une provision au passif de son bilan en grevant directement le capital propre, également au passif. Elle évite ainsi de montrer clairement dans son compte de résultats les vraies charges patronales qui sont les siennes. La performance financière, l'autofinancement et le besoin de financement ne sont alors plus présentés de manière fiable. En effet, ces informations ne reflètent plus à quel point la collectivité est confrontée à des engagements pris dans le passé et dans quelle mesure la valeur au bilan de ses engagements doit être corrigée à la hausse ${ }^{148}$.

Rares sont les personnes à s'intéresser au bilan de la collectivité. C'est pourquoi ces opérations passent souvent inaperçues, surtout si elles sont de faible ampleur. Cela dit, les responsables financiers informent généralement lorsque les montants en jeu sont particulièrement importants. Il n'en demeure pas moins qu'il s'agit d'un artifice puisque le principe de fiabilité des comptes est mis à mal.

\section{Mésestimations}

Nous avons mentionné plus haut que la comptabilité d'exercice nécessite de recourir à diverses estimations dans le cadre des opérations sur les provisions, des opérations de régularisation et de délimitation des exercices, des corrections de valeurs au bilan. Ici, le risque n'est pas de voir apparaître un artifice comptable, mais que le montant soit estimé de manière biaisée: à la baisse si le compte de résultats menace de «mal boucler», à la hausse si l'on s'attend à ce que le compte «boucle bien ».

De l'extérieur, il est cependant difficile de jeter un regard critique sur les montants de ces opérations. On est alors obligé de s'en remettre aux contrôles effectués par l’organe de révision.

148 C'est pour s'affranchir d'un éventuel biais lié à ce type d'artifices que, dans le tableau de bord financier que nous proposons, l'indicateur des engagements nets supplémentaires mesure directement la variation de la valeur au bilan des engagements et du patrimoine financier (voir la section 9.3.1 consacrée aux indicateurs d'équilibres budgétaires). 


\section{Porter un diagnostic sur la situation financière à l'aide d'indicateurs}

\subsection{Notion d'indicateur et de tableau de bord}

L'information que nous donnent les comptes est une information relativement brute. Nous pouvons savoir quel est le volume des charges et des revenus, quels sont les montants investis. Nous pouvons également savoir si la collectivité bénéficie d'un excédent de revenus ou fait face à un excédent de charges, si elle peut autofinancer ses investissements ou si elle doit emprunter et combien. Une telle information est certes utile, mais elle reste rudimentaire. Afin de préciser le diagnostic sur la situation financière, il faut recourir à des indicateurs financiers.

Les indicateurs ${ }^{149}$, aussi appelés ratios, sont des outils courants d'évaluation, d'aide à la maitrise du risque et d'aide à la décision. Un indicateur met généralement en relation deux grandeurs, deux chiffres. Dès lors que l'une de ces grandeurs est mesurée en unités monétaires, l'indicateur devient financier. Par exemple, la dette par habitant est déjà un indicateur financier puisque le numérateur du ratio - la dette - est mesurée en francs.

Le regroupement de plusieurs indicateurs permet de mettre en place un tableau de bord financier. Le tableau de bord est d'autant plus utile que les indicateurs sont sélectionnés avec soin et organisés de manière logique. Les indicateurs doivent être suffisamment nombreux pour permettre un diagnostic nuancé. En même temps, leur nombre doit être suffisamment limité pour éviter de noyer l'information.

\subsection{Tableau de bord financier proposé}

\subsubsection{Structure du tableau de bord}

Chaque collectivité devrait se livrer à sa propre réflexion pour choisir les indicateurs qui reflètent le mieux les objectifs qu'elle poursuit ou les problèmes qu'elle rencontre. Cela étant, plusieurs tableaux de bord standards ont été proposés à l'attention des collectivités publiques suisses (Buschor et Jéquier 1985; Jeanrenaud et Soguel 1988; Dafflon 1994).

Le tableau de bord que nous proposons ici est calqué sur celui qui est utilisé par l'IDHEAP pour établir son comparatif annuel des finances des collectivités publiques suisses (portrait 5). Il recourt à une batterie de quinze indicateurs répondant à diverses préoccupations de gestion financière ${ }^{150}$. Cette batterie inclut les huit indicateurs prescrits par le Manuel MCH2. Ces derniers sont essentiellement centrés sur la problématique de l'endettement ${ }^{151}$. À l'intérieur du tableau de bord, les indicateurs sont répartis en trois groupes thématiques et un groupe auxiliaire (tableau 37).

149 Kennzahlen | Indicatori | Indicators.

150 Voir Soguel et Munier (2018) pour le détail.

151 Recommandation 18 et annexe C, CDF (2008). 


\section{Portrait 5 Comparatif des finances cantonales et communales de l'IDHEAP}

Depuis 1999, I'Institut de hautes études en administration publique (IDHEAP) élabore et publie chaque année le comparatif des finances cantonales et communales. L'objectif est d'analyser la situation financière des collectivités publiques à l'aide de divers indicateurs financiers. Les chiffres sont publiés pour l'ensemble des cantons, un grand nombre de villes et la Confédération. Une base de données contenant les valeurs des indicateurs pour toutes les années dès 1999 pour les cantons et la Confédération et dès 2001 pour les villes est à disposition pour être téléchargée depuis le site Internet de I'IDHEAP à I'Université de Lausanne (www.unil.ch/idheap/ comparatif). Une feuille de calcul est également disponible pour calculer aisément les différents indicateurs. Elle permet d'obtenir une note pour chaque indicateur en fonction de la valeur calculée, allant de 6 (situation excellente) à 1 (situation extrêmement préoccupante). II suffit pour cela de saisir quelques informations à partir des comptes de la collectivité.

Source: www.unil.ch/idheap/comparatif.

Tableau 37 Tableau de bord financier proposé et lien avec les indicateurs recommandés par le Manuel $\mathrm{MCH} 2$.

\begin{tabular}{|c|c|c|c|c|}
\hline \multirow[t]{2}{*}{ Groupes } & \multirow[t]{2}{*}{ Indicateurs du tableau de bord financier } & \multicolumn{3}{|c|}{ Indicateurs MCH2 } \\
\hline & & & $1^{\text {re }}$ priorité & $2^{\mathrm{e}}$ priorité \\
\hline \multirow{4}{*}{$\begin{array}{l}\text { Équilibres } \\
\text { budgétaires }\end{array}$} & Couverture des charges & I1 & & \\
\hline & Autofinancement de l'investissement net & 12 & $\#^{1}$ & \\
\hline & Engagements nets supplémentaires & 13 & & \\
\hline & Poids des intérêts nets & 14 & & \\
\hline \multirow{4}{*}{$\begin{array}{l}\text { Qualité } \\
\text { de la gestion } \\
\text { financière }\end{array}$} & Maîtrise des dépenses courantes par habitant & 15 & & \\
\hline & Effort d'investissement & 16 & & \\
\hline & Exactitude de la prévision fiscale & 17 & & \\
\hline & Intérêt moyen de la dette & 18 & & \\
\hline \multirow{2}{*}{$\begin{array}{l}\text { Importance de } \\
\text { l'endettement }\end{array}$} & Taux d'endettement net & 19 & \# & \\
\hline & Dette brute par rapport aux revenus & I10 & & \#\# \\
\hline \multirow{5}{*}{$\begin{array}{l}\text { Indicateurs } \\
\text { auxiliaires }\end{array}$} & Taux d'autofinancement & I11 & & \#\# \\
\hline & Part des charges d'intérêts & 112 & $\#^{2}$ & \\
\hline & Part du "service de la dette" & I13 & & \#\# \\
\hline & Proportion des investissements & 114 & & $\# \#^{3}$ \\
\hline & Dette nette par habitant & 115 & & \#\# \\
\hline
\end{tabular}

1 Indicateur intitulé "Degré d'autofinancement " dans le Manuel MCH2.

2 Indicateur analogue à l'indicateur du poids des intérêts nets.

3 Indicateur analogue à l'indicateur de l'effort d'investissement. 
Le premier groupe s'intéresse à la thématique des grands équilibres budgétaires de la collectivité. Les ratios qui le composent renseignent sur quatre enjeux clés: la collectivité parvient-elle à couvrir ses charges à l'aide de ses revenus, peut-elle suffisamment financer elle-même ses investissements, limite-t-elle le recours à des capitaux de tiers et la charge d'intérêts liée à la dette accumulée ne réduit-elle pas trop sa marge de manœuvre budgétaire?

Le second groupe aborde la thématique de la qualité de la gestion financière. Quatre aspects sont analysés: la collectivité maitrise-t-elle l'évolution de ses dépenses, produit-elle un effort d'investissement approprié, prévoit-elle ses revenus fiscaux avec exactitude et emprunte-t-elle à des conditions avantageuses?

Un troisième groupe traite de la thématique de l'importance de l'endettement et des engagements accumulés. Rappelons que la dette accumulée forme l'essentiel des engagements vis-à-vis des tiers. L'importance des engagements est saisie en les mettant en regard à la fois des revenus fiscaux et des revenus courants. Cela permet de déterminer pendant combien d'années les revenus fiscaux, respectivement les revenus courants, devraient être consacrés à rembourser les emprunts pour éteindre la dette.

Un quatrième groupe, plus hétérogène, réunit cinq indicateurs auxiliaires. Ces ratios, s'ils sont recommandés par le Manuel $\mathrm{MCH} 2$, sont pour la plupart traités dans le Manuel comme des indicateurs de deuxième priorité (\#\#).

Les trois indicateurs recommandés en première priorité (\#) par le Manuel MCH2 sont inclus dans le dispositif présenté ici. Il s'agit d'une part de la capacité de la collectivité à financer ses investissements par ses propres moyens, d'autre part du poids de la charge d'intérêts nets sur les revenus fiscaux et de l'importance de la dette nette par rapport à ces mêmes revenus fiscaux.

Chaque groupe a sa raison d'être. Il est important de savoir si les équilibres budgétaires peuvent être maintenus. Mais il est tout aussi important d'examiner la qualité de la gestion financière. En effet, un lien existe entre la qualité de la gestion financière et les équilibres budgétaires. À terme, la première influence les seconds. Entre deux collectivités qui peinent à se maintenir en équilibre, celle qui affiche une meilleure gestion financière se positionne plus favorablement. De même, pour une collectivité dont la situation financière est équilibrée, une gestion financière performante constitue une garantie pour l'avenir. Finalement, une collectivité qui, année après année, enregistre des déséquilibres verra son endettement enfler. En même temps, des engagements importants hypothèquent les équilibres futurs en raison des charges financières qui en résultent.

Le dispositif propose également, pour chaque indicateur, la possibilité d'évaluer la valeur calculée sur une échelle allant de 6 (excellente situation) à 1 (situation extrêmement problématique nécessitant des mesures correctives fortes). C'est un des avantages majeurs de notre proposition. En effet, noter les résultats sur une échelle uniforme permet de juger rapidement la situation, même sans être fin connaisseur 
des finances publiques. De plus, les notes obtenues pour chaque indicateur peuvent être agrégées à l'aide d'une moyenne. De son côté, le Manuel MCH2 donne certes quelques indications sur la manière d'évaluer individuellement le résultat des indicateurs qu'il recommande. Mais la manière d'évaluer le résultat change selon l'indicateur. L'échelle proposée n'est que qualitative. Par conséquent, il n'est pas possible d'agréger les résultats et de dégager une vision d'ensemble.

\subsubsection{Utilisation du tableau de bord}

Ce tableau de bord peut être utilisé de différentes manières. D’abord, il sert à évaluer la situation de la collectivité sur la base des comptes de cette dernière. Il permet donc de porter un diagnostic ponctuel en calculant les indicateurs au moment de la clôture. Il sert aussi à analyser l'évolution de la situation dans le temps en observant comment la valeur des indicateurs change de période en période.

Le tableau de bord permet également à la collectivité de se comparer dans l'espace, à d'autres collectivités. Pour cela, il faut calculer les indicateurs pour la collectivité avec laquelle on souhaite se comparer. On peut aussi recourir à la base de données du Comparatif de l'IDHEAP (portrait 5). Cette base de données comprend les indicateurs pour l'ensemble des cantons, pour de nombreuses villes et pour la Confédération.

Le tableau de bord est aussi utile au moment de la préparation du budget. D'abord parce que le diagnostic posé sur la base des comptes permet de se fixer des objectifs par rapport à certains indicateurs (par exemple maîtrise des dépenses, couverture des charges, effort d'investissement, autofinancement de l'investissement, engagements supplémentaires). Il permet également aux autorités - Exécutif, commission des finances, Législatif - de saisir facilement les conséquences des décisions budgétaires sur la situation financière générale de la collectivité. En effet, la plupart des indicateurs peuvent également être calculés avec les informations figurant dans le budget.

Le tableau de bord peut en outre faciliter la préparation du plan financier pluriannuel. Comparer la valeur des indicateurs au début et à la fin de la période de planification, basée sur des données prévisionnelles, permet de mettre en évidence les conséquences financières des grands projets et de déterminer si ces projets sont compatibles avec la situation financière de la collectivité.

Finalement, les indicateurs s'avèrent utiles pour informer au-delà du cercle des autorités de la collectivité, par exemple pour informer les médias, la population ou les bailleurs de fonds. En effet, ils offrent une synthèse de la situation financière et permettent de vulgariser le message. 


\subsection{Indicateurs}

\subsubsection{Indicateurs d'équilibres budgétaires}

\section{Couverture des charges - I1}

Cet indicateur renseigne sur l'état du compte de résultats. Il permet de savoir dans quelle mesure les revenus courants permettent de couvrir les charges courantes ${ }^{152}$ :

$$
\mathbf{I 1}=\frac{\text { revenus courants }}{\text { charges courantes }} \times 100
$$

Les charges devraient en principe être intégralement couvertes au moins à moyen terme, sous réserve de la situation conjoncturelle. La valeur de l'indicateur devrait donc être d'environ 100\% sur une période de quelques années. Un résultat inférieur à 100\% traduit un excédent de charges. Autrement dit, la collectivité vit au-dessus de ses moyens: ses revenus sont insuffisants ou ses charges sont trop élevées.

Les revenus excèdent les charges si le résultat est supérieur à 100\%. Cela est considéré comme une situation favorable. Mais, sur la durée, cela peut aussi indiquer une inadéquation entre la charge fiscale et les services offerts à la population. Il est toutefois vrai qu'un ratio supérieur à 100\% offre généralement davantage de marge de manœuvre pour faire face à l'avenir. Il faut donc évaluer différemment un excédent de charges et un excédent de revenus. D’après l'évaluation proposée par la figure 37, un pourcentage entre 100 et 103 correspond à une note de 6 . Au-dessous de 97,5\%, la note devient inférieure à 4. À l'opposé, à partir de $120 \%$, la note est de 4 , mais ne descend pas au-dessous.

\section{Figure 37 Évaluation de la couverture des charges - I1.}

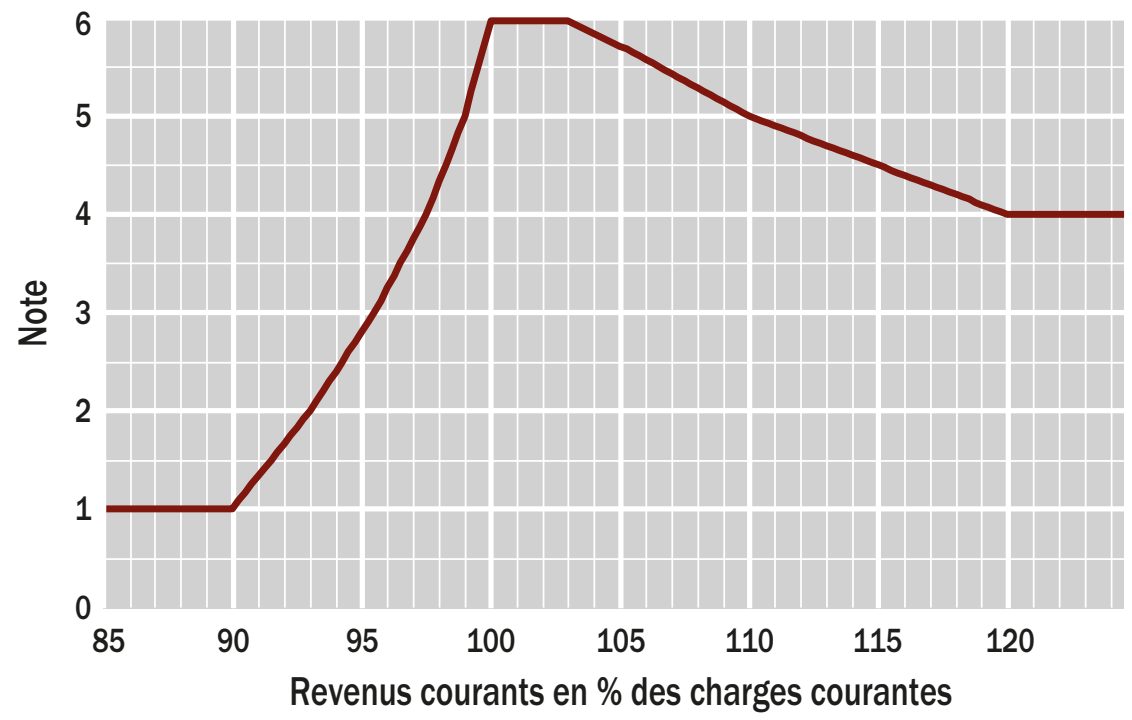

152 Les modalités de calcul des charges et des revenus courants sont précisées dans l'annexe 2. Soulignons cependant que les charges et les revenus extraordinaires enregistrés dans une logique de tactique politique doivent en être exclus. 
Avec des revenus courants de KCHF 14319 et des charges courantes de KCHF 13285 (hors opérations extraordinaires liées à de la tactique budgétaire dans la présentation des comptes), MaCollectivité présente une couverture des charges de $107,8 \%$ et obtient une note de 5,3.

\section{Autofinancement de l'investissement net - 12 ${ }^{\#}$}

Cet indicateur d'équilibre budgétaire est couramment utilisé. Il est d'ailleurs un indicateur $\mathrm{MCH} 2$ de première priorité (\#), sous la dénomination «degré d'autofinancement». Il renseigne sur la part des investissements nets que la collectivité peut financer par ses propres ressources grâce à son autofinancement, sans avoir recours à l'emprunt ${ }^{153}$ :

$$
\mathbf{I} 2^{*}=\frac{\text { autofinancement }}{\text { investissement net }} \times 100
$$

Pour l'investissement net, nous préconisons de considérer une moyenne sur quelques années, par exemple sur trois ans. On lisse ainsi les fortes variations que l'on observe dans les collectivités qui font face à des investissements relativement importants, mais de manière ponctuelle. Notons que le $\mathrm{MCH} 2$ ne suggère pas de lissage.

Un résultat inférieur à 100\% indique que l'autofinancement provenant du fonctionnement annuel ne suffit pas à financer les investissements nets et que la collectivité doit recourir à l'emprunt. Un résultat supérieur à 100\% montre que la collectivité peut financer davantage que ses investissements par ses propres ressources et réduire ainsi sa dette.

Des valeurs situées entre 80 et $70 \%$, voire $60 \%$ sont généralement considérées comme acceptables lorsque des investissements d'amélioration sont consentis à côté des investissements de renouvellement. Notons que la législation impose parfois aux collectivités de financer par elles-mêmes un pourcentage minimum de leurs investissements nets. Le pourcentage exigé se situe généralement en tout cas à $60 \%$.

Un pourcentage de 100 ou plus correspond à une note de 6 . Au-dessous de 70\%, la note devient inférieure à 4 (figure 38).

Avec un autofinancement de KCHF 1682 et des investissements nets de KCHF 1846 (moyenne des trois dernières années), MaCollectivité présente un résultat de $91,1 \%$ et obtient ainsi une note de 5,4 .

153 Les modalités de calcul de l'autofinancement et de l'investissement net sont précisées dans l'annexe 2. Elles sont plus complètes - et donc plus compliquées - que celles utilisées à la figure 20, page 99. 
Figure 38 Évaluation de l'autofinancement de l'investissement net - 12\#.

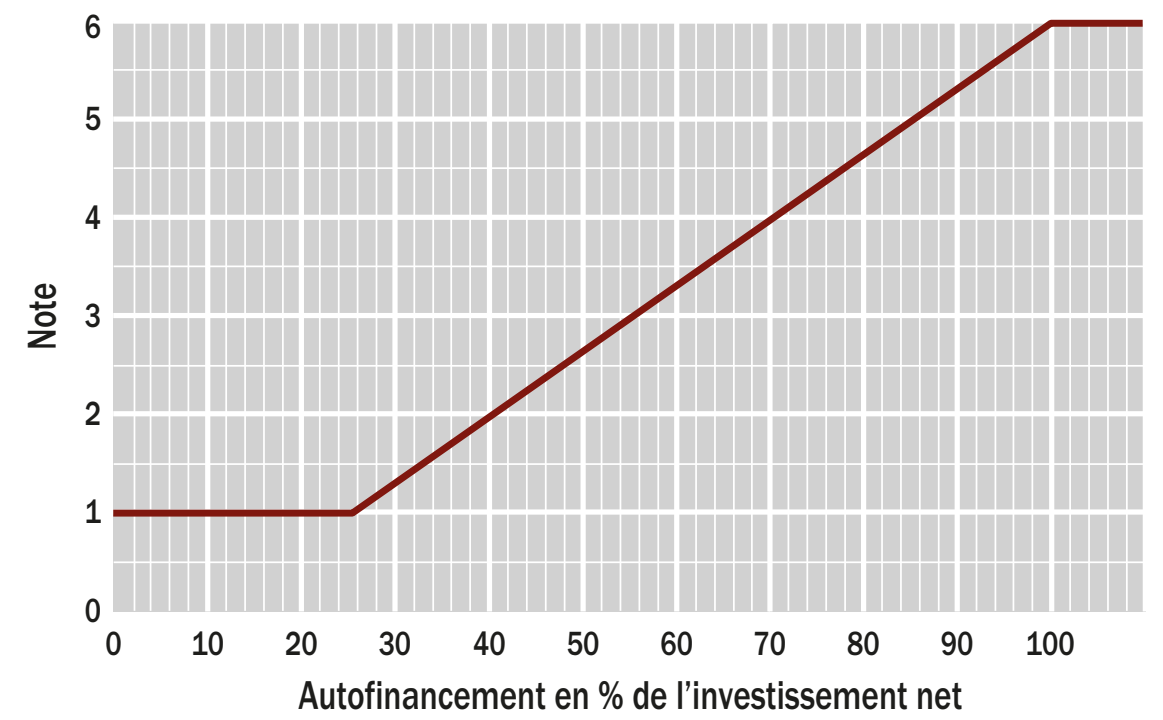

\section{Engagements nets supplémentaires - 13}

Cet indicateur mesure l'accroissement ou la diminution des engagements nets pendant l'exercice. Il chiffre l'évolution de l'ensemble des engagements nets (dettes et provisions notamment) au cours de l'exercice et il rapporte cette variation aux dépenses courantes de l'exercice, autrement dit à la surface financière de la collectivité ${ }^{154}$ :

$\mathbf{I} \mathbf{3}=\frac{\text { (engagements nets au 31.12.Année } \mathrm{t}-\text { engagements nets au 01.01.Année } \mathrm{t})}{\text { dépenses courantes de l'exercice }} \times 100$

Notons que l'évolution des engagements peut être influencée par d'autres facteurs que la nécessité ou non de recourir à l'emprunt pour financer les investissements, telle qu'elle est reflétée par l'indicateur d'autofinancement de l'investissement net. En effet, une collectivité peut procéder à des mutations à l'intérieur de son bilan sans que ces opérations soient enregistrées dans le compte de résultats ou dans celui des investissements. C'est un phénomène que l'on peut constater lorsqu'une collectivité recapitalise la caisse de pension de son personnel.

Un résultat inférieur à $0 \%$ indique que la collectivité s'est désengagée vis-à-vis de ses tiers en termes nets au cours de l'exercice. Le stock d'engagements est inférieur à ce qu'il était en début d'exercice, soit parce que la collectivité a réduit sa dette ou ses provisions, soit parce qu'elle a augmenté ses avoirs financiers. Cette situation correspond à une note de 6 (figure 39). 
Figure 39 Évaluation des engagements nets supplémentaires - I3.

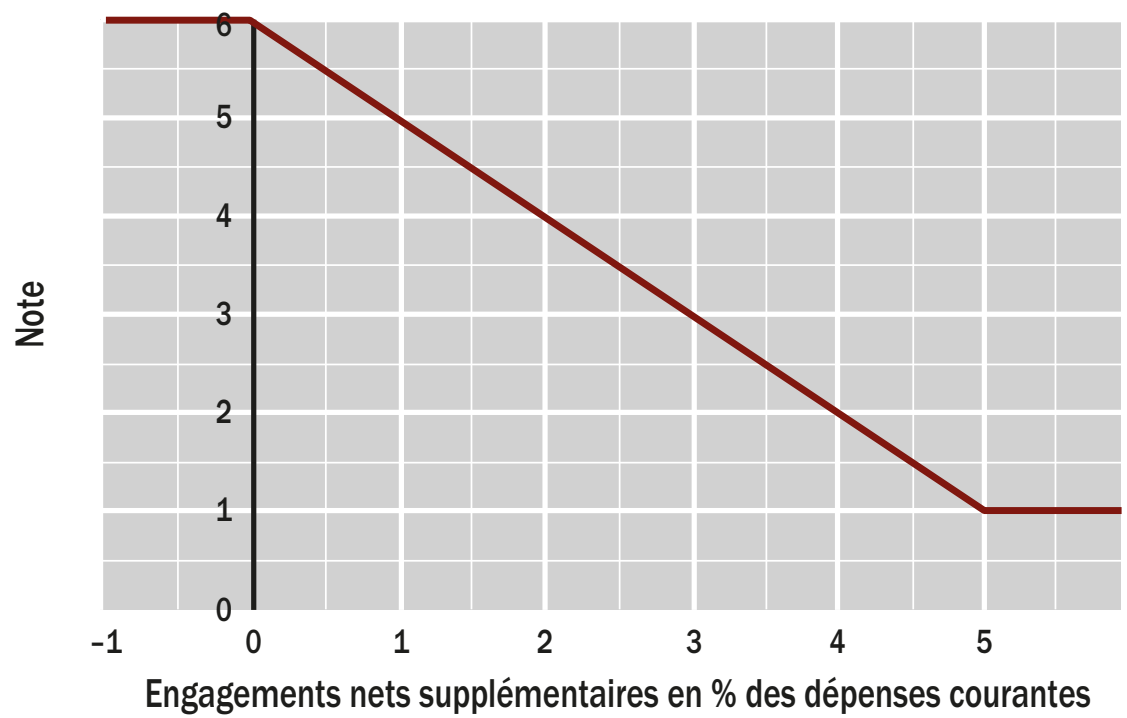

Par contre, un résultat supérieur à $0 \%$ signale une politique de croissance de l'endettement et du provisionnement. Les engagements nets sont supérieurs à ce qu'ils étaient en début de période. Si la croissance est supérieure à l'équivalent de 2,0\% des dépenses courantes, la note devient inférieure à 4 .

Avec des engagements nets supplémentaires de KCHF 223 et des dépenses courantes de de KCHF 12606, MaCollectivité présente un résultat de 1,8\% et obtient ainsi une note de 4,2. L'évaluation est légèrement différente de celle qui est obtenue pour l'autofinancement de l'investissement net (I2*). Cela montre que MaCollectivité a procédé à des mutations dans son bilan, sans les enregistrer dans son compte de résultats ou dans son compte des investissements.

\section{Poids des intérêts nets - 14}

Cet indicateur renseigne sur la part des revenus fiscaux devant être consacrée au paiement des charges financières, en particulier les charges liées à la dette, déduction faite des rendements obtenus sur les placements du patrimoine financier. Il est analogue à l'indicateur de la part des charges d'intérêts $\left(\mathrm{I} 12^{\#}\right)$, un indicateur de première priorité du $\mathrm{MCH} 2$. Toutefois, entre autres différences, le $\mathrm{MCH} 2$ compare le volume des intérêts nets à l'ensemble des revenus courants. Cet indicateur-ci se réfère aux revenus fiscaux fondamentaux de la collectivité, notamment ceux de l'impôt sur le revenu et la fortune des personnes physiques, ainsi que de l'impôt sur le bénéfice et le capital des sociétés. Ces revenus fiscaux fondamentaux constituent une part importante et constante des revenus. La collectivité peut exercer sur eux un certain contrôle ${ }^{155}$ : 


$$
\mathbf{I} 4=\frac{\text { intérêts nets }}{\text { revenus fiscaux fondamentaux }} \times 100
$$

Un poids élevé montre que la collectivité s'est lourdement endettée par le passé. Mais il présage aussi de mauvaises perspectives d'avenir. En effet, les revenus fiscaux consacrés au paiement des intérêts de la dette ne seront pas disponibles pour financer les prestations publiques. C'est pourquoi, avec des intérêts nets au-delà de 7,0\% des revenus fiscaux, la note passe en dessous de 4 (figure 40).

Un résultat inférieur à $0 \%$ indique que le rendement du patrimoine financier est supérieur aux charges financières liées principalement à l'endettement. La marge de manœuvre d'une telle collectivité ne subit aucune restriction dans ce domaine; bien au contraire, son patrimoine financier apporte des ressources additionnelles à son fonctionnement. La note s'élève donc logiquement à 6 .

Avec des intérêts nets négatifs de KCHF - 33 et des revenus fiscaux fondamentaux de KCHF 7575, MaCollectivité supporte un poids de -0,4\% et obtient ainsi une note de 6,0 .

Figure 40 Évaluation du poids des intérêts nets - 14.

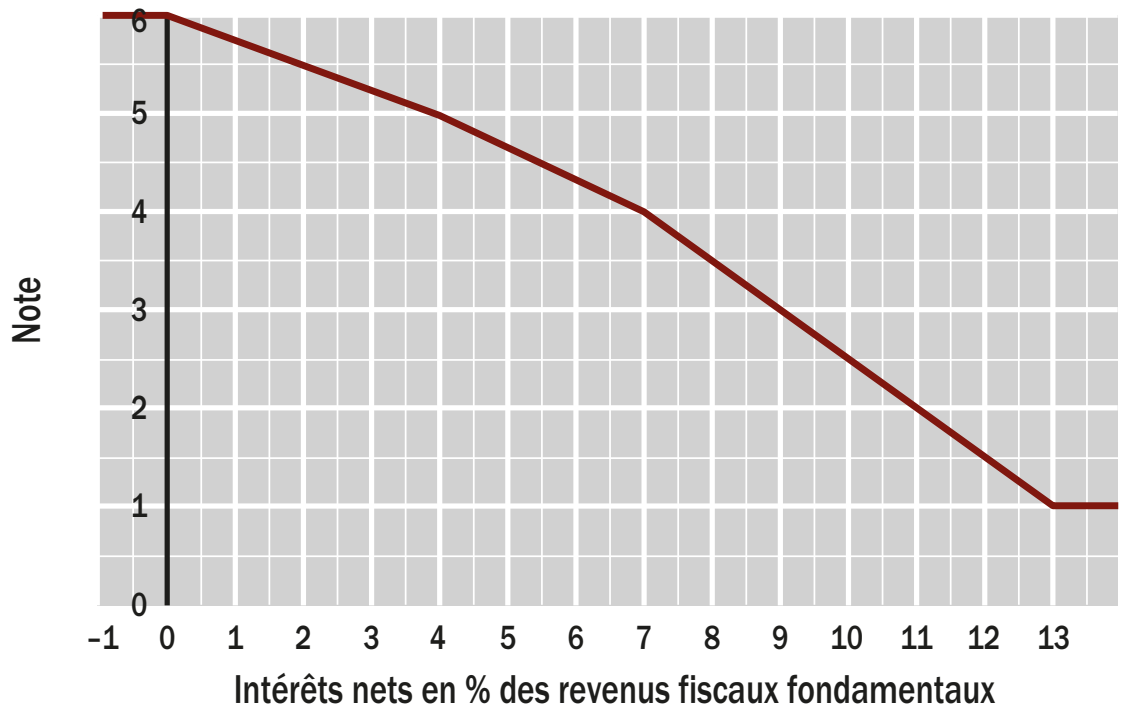

\subsubsection{Indicateurs de qualité de la gestion financière}

\section{Maîtrise des dépenses courantes par habitant - 15}

Cet indicateur renseigne sur l'aptitude de la collectivité et des responsables financiers de cette dernière à maîtriser l'évolution des dépenses courantes. Il mesure la hausse ou la baisse des dépenses par habitant par rapport aux dépenses de l'exercice précédent ${ }^{156}$ : 


$$
\mathbf{I 5}=\frac{\text { - dépenses de l'exercice précédent par habitant) }}{\text { dépenses de l'exercice précédent par habitant }} \times 100
$$

Rappelons que les dépenses courantes excluent les opérations comptables comme les amortissements, les attributions aux provisions ou aux financements spéciaux et fonds, et les imputations internes. Il convient également d'exclure les subventions redistribuées qui échappent au contrôle de la collectivité. Les dépenses courantes sont donc constituées d'éléments que les responsables financiers peuvent influencer.

Les résultats dépendent aussi de l'inflation puisque la variation des dépenses est mesurée en termes nominaux. La note évaluative doit donc être analysée avec indulgence en période de hausse marquée des prix. Cependant, l'impact de l'inflation doit être relativisé lorsque l'on compare des collectivités qui sont soumises aux mêmes conditions.

Un résultat inférieur à $0 \%$ indique que la collectivité a réduit ses dépenses par rapport à l'exercice précédent. Si le résultat est supérieur à $0 \%$, on assiste à un accroissement des dépenses. Tant que l'accroissement est inférieur à 1,0\%, la note correspondante est de 6 (figure 41). Si la hausse dépasse 3,0\%, la note passe en dessous de 4.

Avec une hausse des dépenses courantes de CHF 136 et des dépenses courantes de CHF 3913 par habitant à l'Année t-1, MaCollectivité présente une variation des dépenses courantes par habitant de 3,5\% et obtient ainsi une note de 3,5.

Figure 41 Évaluation de la maîtrise des dépenses courantes par habitant - 15.

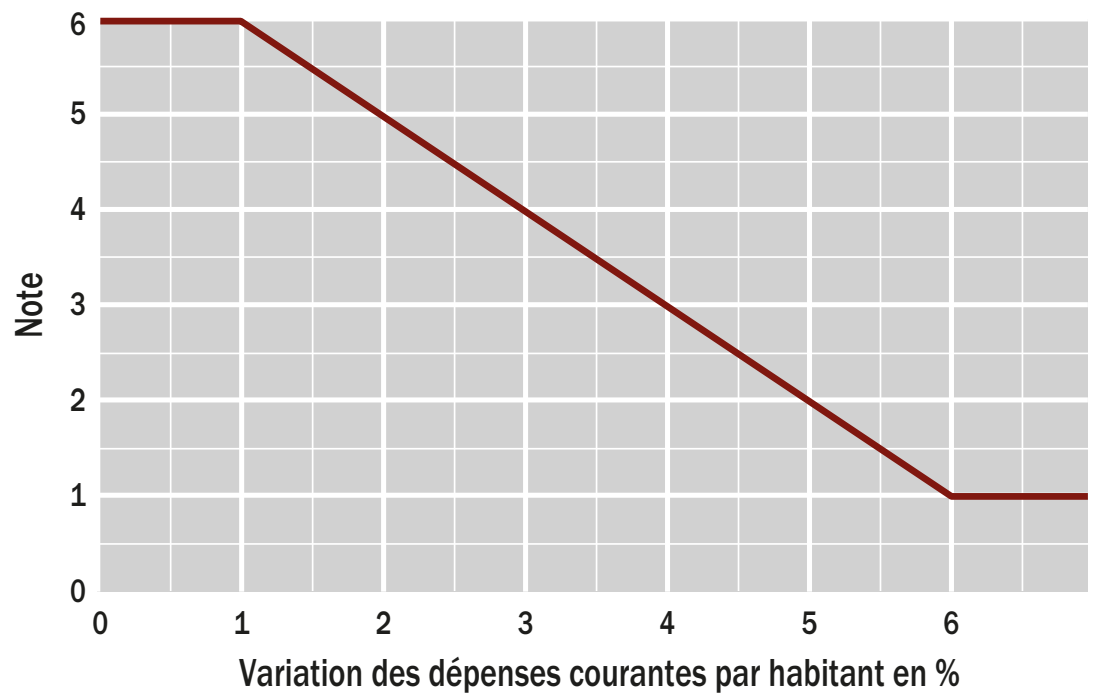

\section{Effort d'investissement - 16}

Cet indicateur mesure l'effort d'investissement déployé par la collectivité. Il renseigne donc sur l'ambition de la politique d'investissement en tenant compte de sa surface financière, appréhendée à travers ses dépenses courantes ${ }^{157}$ : 


$$
\mathbf{I} \mathbf{6}=\frac{\text { investissement net }}{\text { dépenses courantes }} \times 100
$$

Ce ratio est analogue, sans être identique à l'indicateur de la proportion des investissements (I14 ${ }^{\# \#}$, indicateur de deuxième priorité du MCH2). Il s'en distingue sur trois points. D'abord les dépenses d'investissement sont considérées ici nettes des contributions reçues. Cela évite de biaiser le résultat des collectivités qui doivent investir massivement afin de satisfaire une exigence légale et qui bénéficient pour cela d'une contribution. Ensuite, nous préconisons de considérer une moyenne sur quelques années, par exemple sur trois ans. On lisse ainsi les fortes variations que l'on observe dans les collectivités qui font face à des investissements relativement importants, mais de manière ponctuelle. Enfin, ici l'investissement net est rapporté aux seules dépenses courantes plutôt qu'aux dépenses totales, comme c'est le cas avec l'indicateur MCH2 relatif à la proportion des investissements (I14 ${ }^{\# \#) . ~ E n ~ e f f e t, ~}$ les dépenses totales incluent les dépenses courantes et les dépenses d'investissement. La formule de l'indicateur I6 évite le biais qui surgit si l'on porte les dépenses d'investissement à la fois au-dessus et au-dessous de la barre de fraction.

L'effort d'investissement peut être trop élevé ou trop faible en regard des possibilités et des besoins. La figure 42 reflète l'idée qu'il existe un volume idéal d'investissement. Une analyse historique des résultats des collectivités suisses nous montre que ce volume devrait se situer entre 7,0\% et 10,0\% par rapport aux dépenses courantes. La note correspondante est de 6. Lorsque la collectivité sous-investit - en deçà de $3,0 \%$ - ou surinvestit - au-delà de 14,0\% -, la note tombe en dessous de 4 .

Avec des investissements nets de KCHF 1846 (moyenne des trois dernières années) et des dépenses courantes de KCHF 12606, MaCollectivité présente un effort d'investissement de $14,6 \%$ et une note de 3,4 .

Figure 42 Évaluation de l'effort d'investissement - 16.

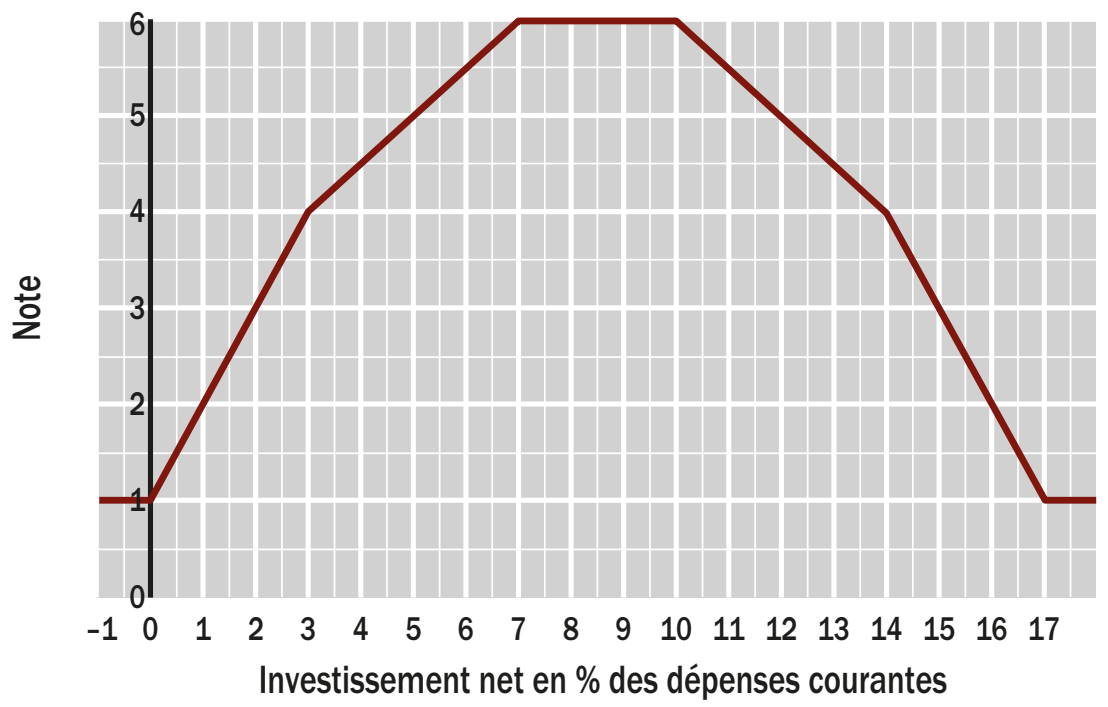




\section{Exactitude de la prévision fiscale - 17}

Cet indicateur renseigne sur l'aptitude de la collectivité à prévoir les revenus fiscaux avec exactitude. Le produit de certains impôts, comme l'impôt sur les gains immobiliers ou sur les successions, est difficile à prévoir. Par contre, on peut attendre des responsables qu'ils puissent mieux prévoir les revenus des impôts fondamentaux: impôt sur le revenu et la fortune des personnes physiques et impôt sur le bénéfice et le capital des sociétés. Cet indicateur mesure l'écart entre les revenus effectifs et les revenus budgétés en pourcentage des revenus effectifs pour ces deux types d'impôts ${ }^{158}$ :

$$
\mathbf{I 7}=\frac{\begin{array}{c}
\text { (revenus fiscaux fondamentaux budgétés } \\
\text { revenus fiscaux fondamentaux effectifs) }
\end{array}}{\text { rendamentaux effectifs }} \times 100
$$

Davantage de précision dans la prévision des revenus fiscaux permet d'inscrire l'élaboration du budget dans un cadre plus pertinent. Un résultat supérieur à $0 \%$ témoigne d'une surestimation: le budget prévoyait des recettes plus élevées que ce qui est finalement advenu. Au contraire, un résultat inférieur à $0 \%$ indique une sous-estimation. La nécessité de respecter le principe de prudence favorise une certaine sousestimation (voir la section 2.1.2 consacrée aux principes de budgétisation). C'est pourquoi une sous-estimation est considérée comme un résultat plus favorable qu'une surestimation: on préfere une «bonne surprise » à une «mauvaise surprise». Une sousestimation est donc évaluée moins sévèrement qu'une surestimation. Comme le reflète la figure 43, même au-delà de 10,0\%, une sous-estimation correspond à une note de 4 . Par contre, au-delà de 2,6\%, une surestimation conduit à une note inférieure à 4 .

Figure 43 Évaluation de l'exactitude de la prévision fiscale - 17.

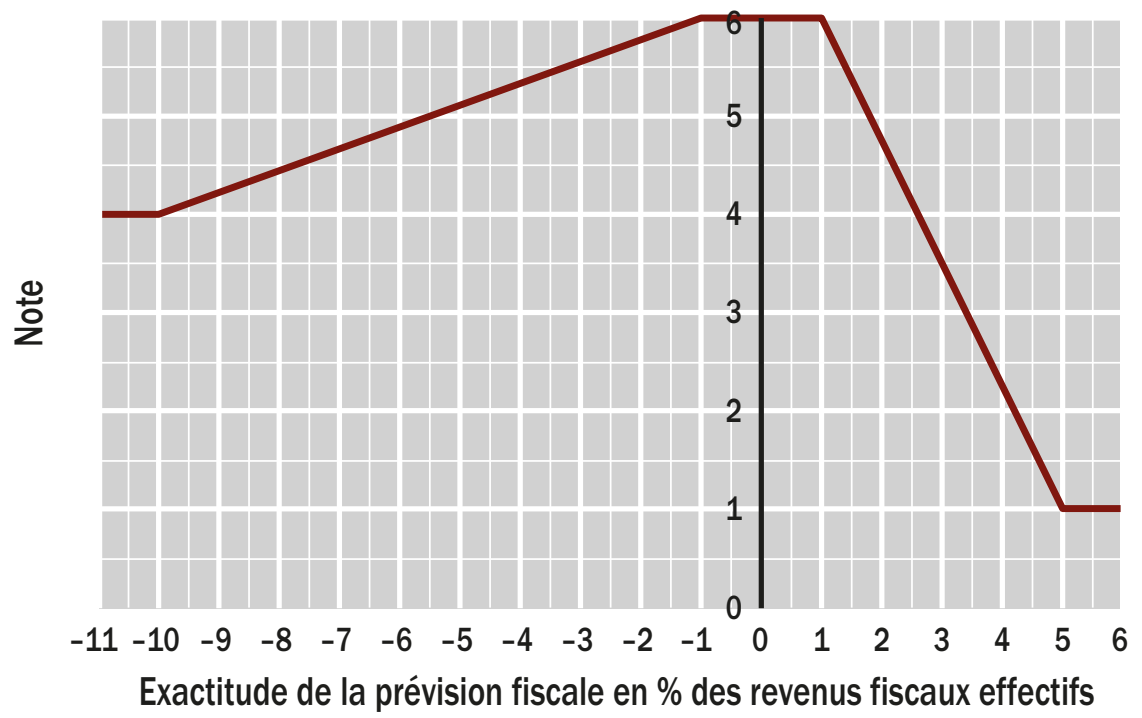

158 Les modalités de calcul des revenus fiscaux fondamentaux sont précisées dans l'annexe 2. 
Avec une sous-estimation de KCHF 360 et des revenus fiscaux fondamentaux effectifs de KCHF 6732, MaCollectivité présente une inexactitude de la prévision fiscale de $-5,3 \%$ et obtient ainsi une note de 5,0.

\section{Intérêt moyen de la dette - 18}

Cet indicateur renseigne sur la performance en matière de gestion de la trésorerie et de la dette. Une bonne gestion permet de limiter les coûts de la dette. Pour simplifier, nous nous intéressons ici au taux d'intérêt moyen (ou implicite) de la dette brute ${ }^{159}$ :

$$
\mathbf{I 8}=\frac{\text { intérêts passifs }}{\text { moyenne de la dette brute entre }} \times 100
$$

Cet indicateur est évidemment tributaire des performances antérieures de la gestion de trésorerie. Par exemple, il est tributaire du calendrier choisi pour les emprunts ou de la durée des emprunts. Quoi qu'il en soit, il reflète aussi l'habileté des responsables actuels à emprunter à des conditions avantageuses (voir à ce propos le chapitre 7 consacré aux modalités d'emprunt).

Un intérêt moyen de la dette inférieur à $2,5 \%$ correspond à la note 6 . La note tombe en dessous de 4 lorsque la valeur de l'indicateur passe au-dessus de 4,5\%.

Avec des intérêts passifs de KCHF 60 et une dette brute moyenne de KCHF 5050, MaCollectivité présente un intérêt moyen de la dette de 1,2\% et obtient ainsi une note de 6,0 .

Figure 44 Évaluation de l'intérêt moyen de la dette - 18.

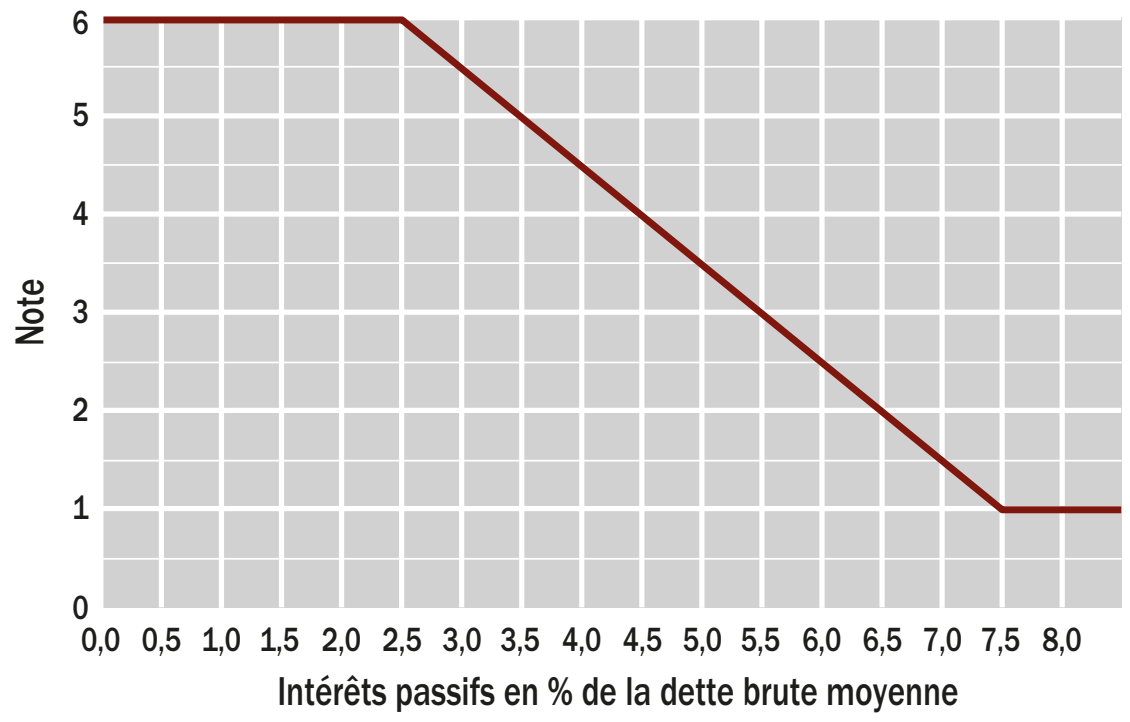

159 Les modalités de calcul des intérêts passifs et de la dette brute sont précisées dans l'annexe 2. 


\subsubsection{Indicateurs d'importance de l'endettement}

\section{Taux d'endettement net - 19"}

Indicateur $\mathrm{MCH} 2$ de première priorité (\#), ce ratio renseigne sur l'importance des engagements figurant au passif du bilan de la collectivité, déduction faite des actifs appartenant au patrimoine financier. On parle bien ici des engagements et pas seulement de l'endettement. Si les dettes constituent l'essentiel des engagements, d'autres éléments s'y ajoutent, comme les provisions, les passifs de régularisation ou les engagements envers les financements spéciaux et les fonds considérés comme des capitaux de tiers. Le titre de l'indicateur prête donc un peu à confusion. Mais c'est le titre retenu par le $\mathrm{MCH} 2$. C'est pourquoi nous l'utilisons également ici. L'indicateur met en relation le volume des engagements nets des actifs du patrimoine financier avec les revenus fiscaux ${ }^{160}$ :

$$
\mathbf{I}^{\#}=\frac{\text { engagements nets }}{\text { revenus fiscaux }} \times 100
$$

Bien qu'exprimé en pourcentage, le résultat peut aussi être compris comme le temps qu'il faudrait à la collectivité pour éteindre ses engagements si elle y consacrait l'intégralité de ses revenus fiscaux. Un résultat de 100\% signale que les revenus fiscaux d'une année complète devraient être intégralement dévolus au remboursement des engagements si la collectivité voulait éteindre son endettement. La note correspondante est de 5 (figure 45 ). Un résultat de $125 \%$ correspond à un an et trois mois et à la note $4.200 \%$ indique que le remboursement prendrait deux ans; c'est beaucoup et cela justifie la note 1 .

\section{Figure 45 Évaluation du taux d'endettement net - 19\#.}

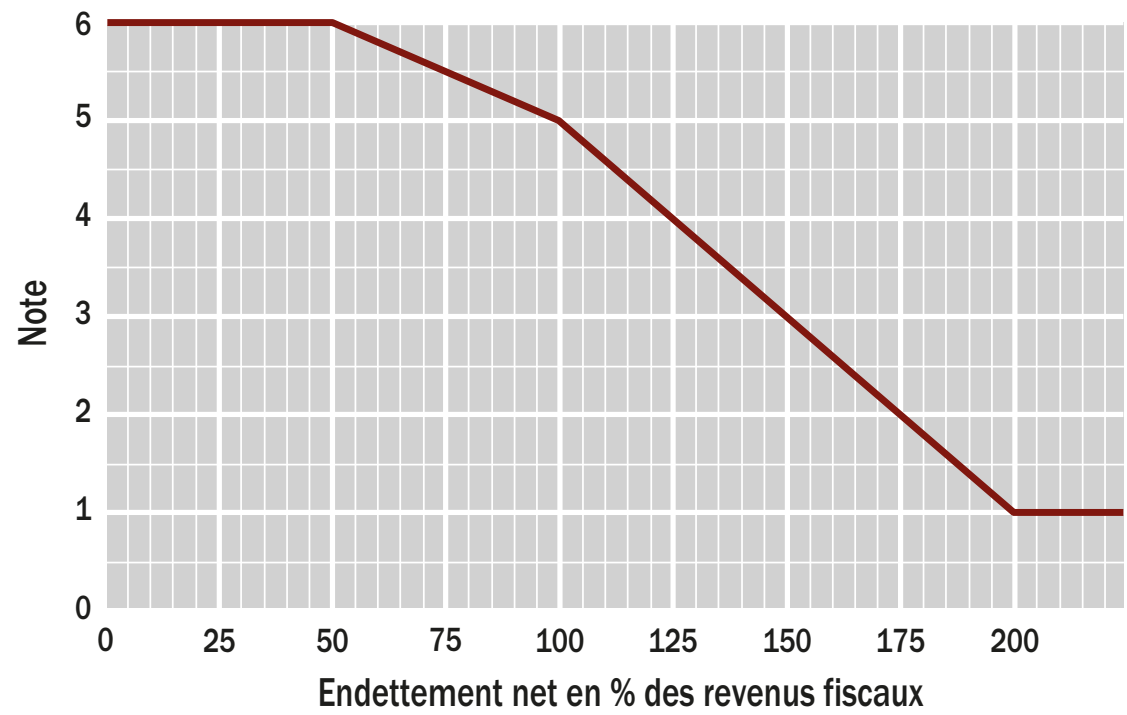

160 Les modalités de calcul des engagements nets et des revenus fiscaux sont précisées dans l'annexe 2. 
Un résultat inférieur à 100\% signifie que moins de douze mois suffiraient: 50\% représente six mois, $25 \%$ trois mois, $8 \%$ un peu moins d'un mois. Lorsque le résultat est inférieur à $50 \%$, la note est de 6 .

Avec des engagements nets de KCHF 2303 et des revenus fiscaux de KCHF 7651, MaCollectivité présente un taux d'endettement net de 30,1\% et obtient ainsi une note de 6,0. Elle ne devrait donc consacrer ses revenus fiscaux que pendant moins de quatre mois au remboursement de ses engagements nets pour en venir à bout.

\section{Dette brute par rapport aux revenus - $\mid 10^{\# \#}$}

Indicateur $\mathrm{MCH} 2$ de deuxième priorité (\#\#), ce ratio renseigne sur l'importance de la dette brute. Il est donc proche de l'indicateur mesurant le taux d'endettement (voir ci-dessus). Il s'en écarte par le fait que l'on considère ici la dette brute et pas les engagements nets. Ce faisant, il laisse donc de côté le patrimoine financier accumulé par la collectivité. Les engagements nets offrent une meilleure vue d'ensemble. Mais il faut être attentif au fait qu'en s'endettant pour réaliser en parallèle des placements, c'est-à-dire pour constituer un patrimoine financier, une collectivité court un risque spéculatif. Or le principe de prudence voudrait qu'on limite la spéculation, donc un endettement brut, même si c'est pour financer des placements. Cet indicateur répond à cet objectif. Il met en relation la dette brute avec les revenus courants ${ }^{161}$ :

$$
\mathbf{I 1 0}^{\# \#}=\frac{\text { dette brute }}{\text { revenus courants }} \times 100
$$

Le rapport est exprimé en pourcentage, mais le pourcentage peut être interprété comme le temps qu'il faudrait à la collectivité pour rembourser sa dette brute si elle y consacrait l'intégralité de ses revenus courants. Un résultat de $100 \%$ indique qu'il faudrait une année complète. La note correspondante est 4,5 (figure 46). Avec 125\%, un an et trois mois serait nécessaire; la note correspondante est $4,0.200 \%$ indique que cela prendrait deux ans, d'où la note de 1,0.

Un résultat inférieur à $100 \%$ signifie que moins de douze mois suffiraient. Un pourcentage de $25 \%$ représente trois mois; la note est alors de 6,0 .

Avec une dette brute de KCHF 6321 et des revenus courants de KCHF 14319, MaCollectivité présente un résultat de 44,1\%. Il lui faudrait consacrer ses revenus pendant un peu moins de six mois au remboursement de sa dette brute pour en venir à bout. Elle obtient ainsi une note de 5,6. 
Figure 46 Évaluation de la dette brute par rapport aux revenus - I10\#\#.

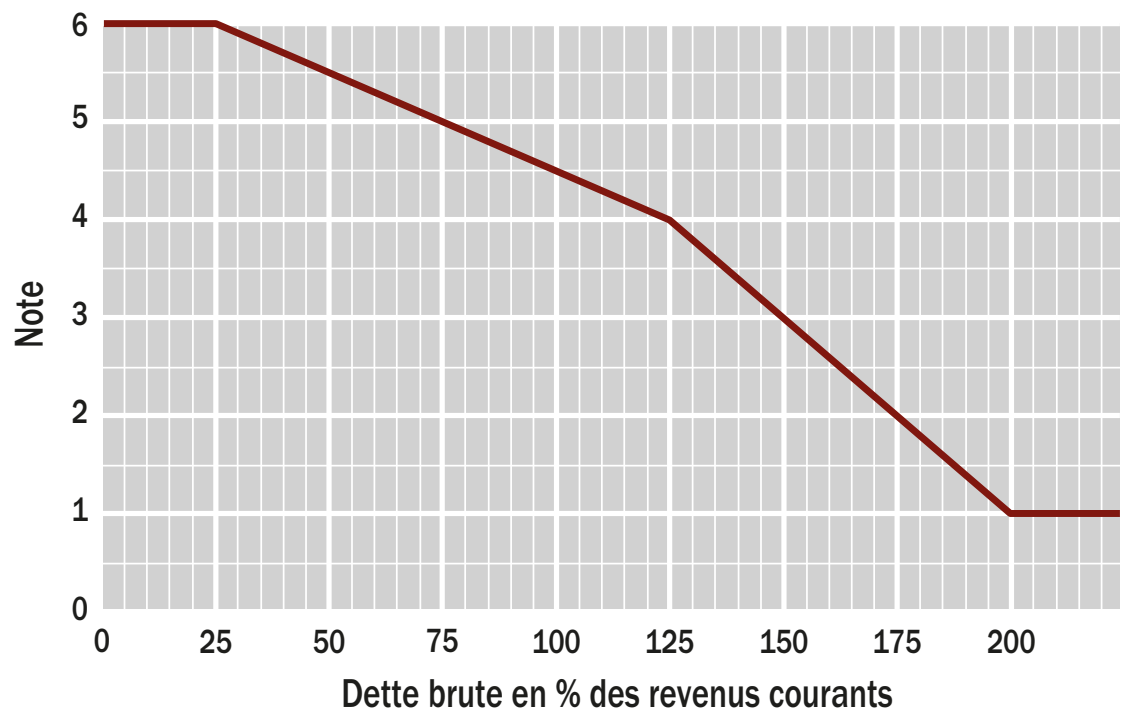

\subsubsection{Indicateurs auxiliaires}

\section{Taux d'autofinancement - I11 \#\#}

Indicateur $\mathrm{MCH} 2$ de deuxième priorité (\#\#), ce ratio informe sur la part des revenus courants qui reste en main de la collectivité pour financer par elle-même ses investissements, donc pour les autofinancer. Il indique dans quelle mesure les revenus peuvent être épargnés plutôt que consacrés à financer des dépenses courantes. Il rapporte donc l'autofinancement (c'est-à-dire l'épargne dégagée sur le fonctionnement) aux revenus courants ${ }^{162}$ :

$$
\text { I1 } 1^{\# \#}=\frac{\text { autofinancement }}{\text { revenus courants }} \times 100
$$

Si le taux d'autofinancement est faible, la collectivité devra réduire ses investissements ou, alternativement, recourir plus largement à des capitaux de tiers pour les financer. C'est pourquoi un résultat en dessous de $4,0 \%$ correspond à une note inférieure à 4 (figure 47). Parfois, le taux est même négatif. Cela se produit lorsque l'excédent de charges est plus important que les amortissements, obligeant la collectivité à s'endetter, non seulement pour financer ses investissements, mais aussi pour couvrir une partie de ses charges courantes. Cette situation mérite la note minimale de 1. À l'inverse, lorsque le taux est égal ou supérieur à 8,0\%, la note est de 6 .

Avec un autofinancement de KCHF 1682 et des revenus courants de KCHF 14319, MaCollectivité présente un taux d'autofinancement de 11,7\% et obtient ainsi une note de 6,0 . 
Figure 47 Évaluation du taux d'autofinancement $-111^{\# \# .}$

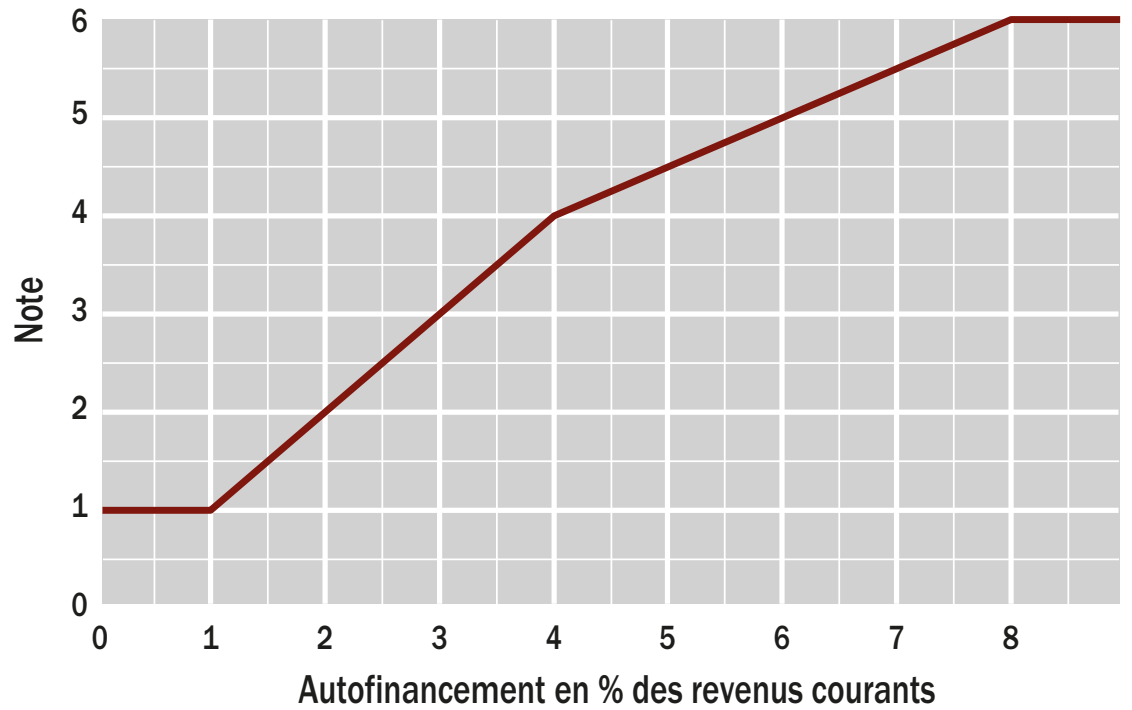

\section{Part des charges d'intérêts - I12"}

Cet indicateur $\mathrm{MCH} 2$ de première priorité (\#) est analogue, mais non identique, à celui qui mesure le poids des intérêts nets (I4). En effet, il s'intéresse aux seuls intérêts nets et exclut les autres charges et revenus liés à la gestion de la dette. En outre, cet indicateur-ci rapporte les intérêts nets, non pas aux revenus fiscaux, mais à l'ensemble des revenus courants. Autrement dit, il indique la part des revenus courants qui doit être consacrée au paiement des intérêts passifs nets des intérêts actifs ${ }^{163}$ :

$$
\mathbf{I 1 2}^{*}=\frac{\text { intérêts nets }}{\text { revenus courants }} \times 100
$$

Un poids élevé montre que la collectivité s'est lourdement endettée par le passé. Il présage de mauvaises perspectives d'avenir. En effet, les revenus courants consacrés au paiement des intérêts de la dette ne seront pas disponibles pour financer les prestations publiques. C'est pourquoi, lorsque la part excède $4 \%$, la note passe en dessous de 4 (figure 48).

Par contre, si les intérêts nets sont nuls ou négatifs, autrement dit, si les intérêts actifs compensent en totalité les intérêts passifs, la marge de manœuvre ne subit aucune restriction liée à sa dette; bien au contraire, son patrimoine financier lui apporte des ressources additionnelles. Logiquement, la note s'élève alors à 6 .

Avec des intérêts nets de KCHF 62 et des revenus courants de KCHF 14319, MaCollectivité présente une part des intérêts nets de $0,4 \%$ et obtient ainsi une note de 5,8.

163 Les modalités de calcul des intérêts nets et des revenus courants sont précisées dans l'annexe 2. Notons que cet indicateur (I12 $)$ et l'indicateur du poids des intérêts nets (I4) utilisent chacun une définition légèrement différente des intérêts nets. 
Figure 48 Évaluation de la part des charges d'intérêts - I12\#.

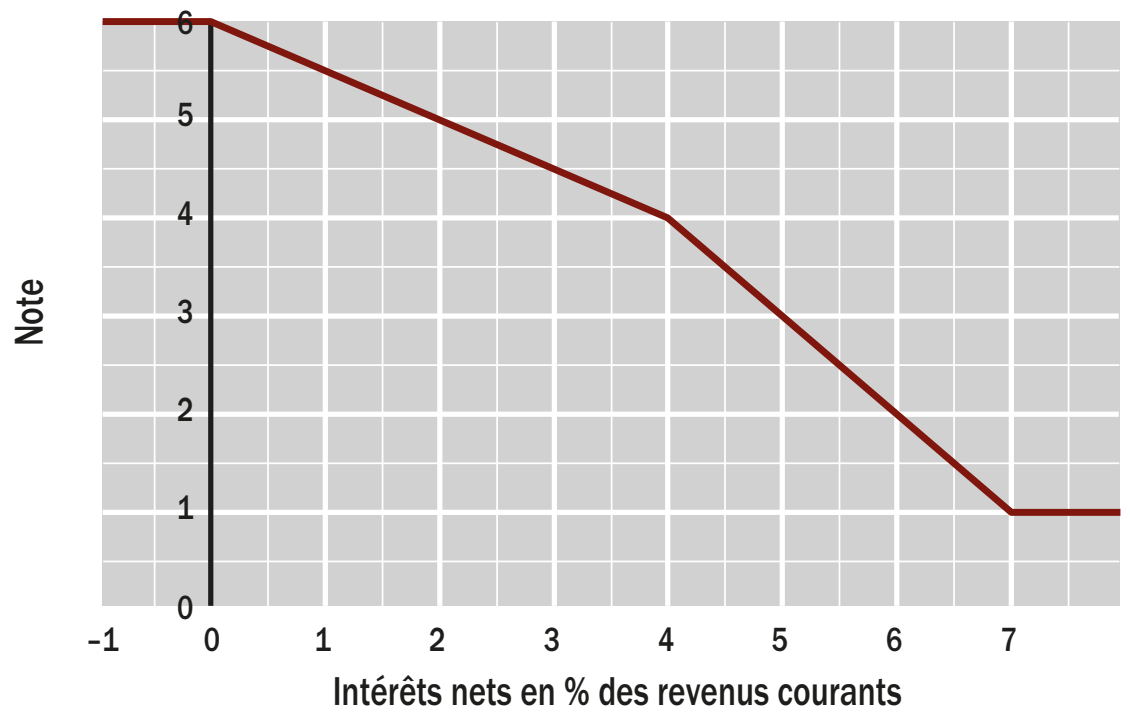

\section{Part du "service de la dette" - I13"\#}

Indicateur $\mathrm{MCH} 2$ de deuxième priorité (\#), ce ratio renseigne sur la part des revenus courants qui est absorbée par les charges d'intérêts nets et par les amortissements du patrimoine administratif. Il indique donc à quel point le budget de la collectivité est pris en tenailles par les décisions antérieures en matière de politique d'endettement (à travers les charges d'intérêts nets) et de politique d'investissement (à travers les amortissements) ${ }^{164}$. L'indicateur rapporte l'effet de tenailles (le «service de la dette») aux revenus courants ${ }^{165}$ :

$$
\mathbf{I 1 3}^{\# \#}=\frac{\ll \text { service de la dette } »}{\text { revenus courants }} \times 100
$$

Un effet de tenailles important montre que la collectivité a beaucoup investi par le passé et s'est passablement endettée. Il présage de mauvaises perspectives d'avenir parce que les revenus courants consacrés aux amortissements et au paiement des intérêts ne seront pas disponibles pour financer les prestations publiques. Lorsque l'effet de tenailles dépasse $7,5 \%$, la note tombe en dessous de 4 (figure 49 ).

Un effet de tenailles inférieur à $0 \%$ indique que les intérêts créanciers sont supérieurs aux intérêts débiteurs et aux amortissements. La marge de manœuvre d'une telle collectivité ne subit pas de restriction liée à ses décisions antérieures. La note est donc de 6.

164 La dénomination donnée par le MCH2 à cet indicateur - «service de la dette» - est équivoque. En effet, les amortissements ne découlent pas de la dette, mais des investissements réalisés et autofinancés en totalité ou en partie. L'effet de tenailles fait l'objet d'une analyse plus poussée dans le chapitre 13 consacré aux limites de la situation financière.

165 Les modalités de calcul du «service de la dette» et des revenus courants sont précisées dans l'annexe 2. 
Figure 49 Évaluation de la part du "service de la dette» - I13"\#\#.

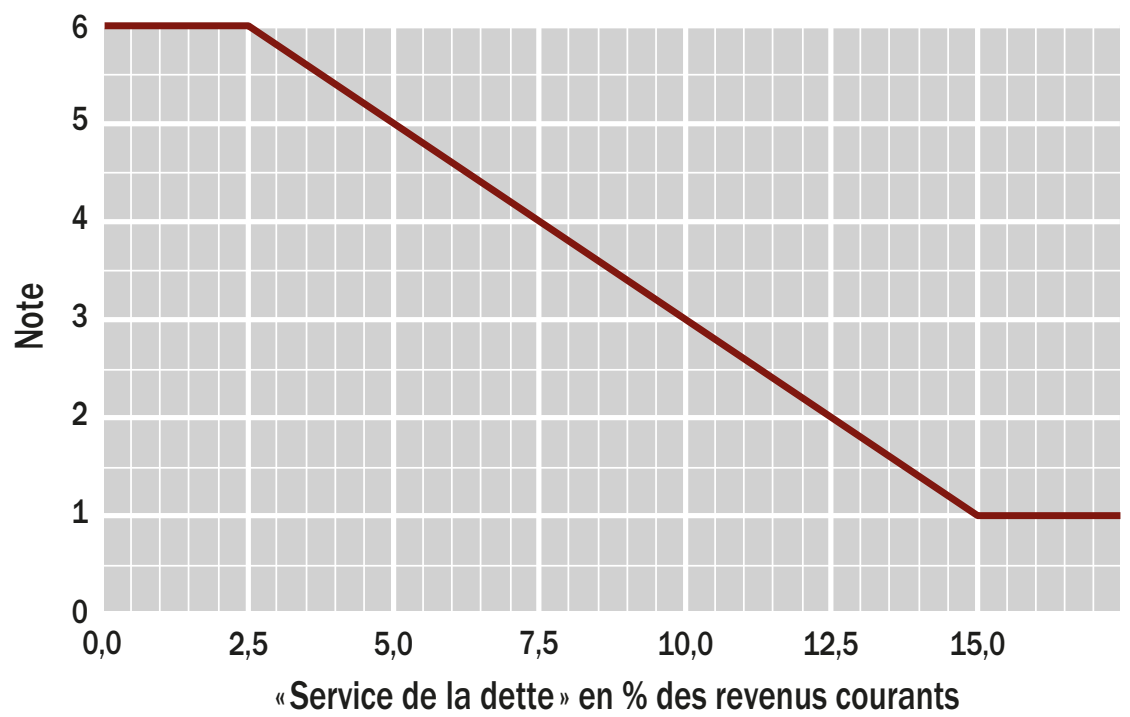

Avec des amortissements et des intérêts nets qui - ensemble - s'élèvent à KCHF 733 et des revenus courants de KCHF 14319, MaCollectivité présente un «service de la dette», un effet de tenailles, de 5,1\% et obtient ainsi une note de 5,0.

\section{Proportion des investissements bruts - I14 \#\#}

Indicateur $\mathrm{MCH} 2$ de deuxième priorité (\#\#), ce ratio jauge la politique d'investissement de la collectivité. Contrairement à l'indicateur de l'effort d'investissement (I6), cet indicateur-ci s'intéresse aux investissements bruts uniquement, sans tenir compte des contributions reçues. Par ailleurs, l'investissement est rapporté ici aux dépenses totales (incluant les dépenses courantes et d'investissement) plutôt qu'aux seules dépenses courantes ${ }^{166}$ :

$$
\mathbf{I 1 4}^{\# \#}=\frac{\text { investissements bruts }}{\text { dépenses totales }} \times 100
$$

Ne tenant pas compte des contributions d'investissement, ce ratio mesure imparfaitement l'effort d'investissement propre à la collectivité. En effet, il se peut qu'une partie importante des investissements soit dictée et financée par une entité tierce, canton ou Confédération.

La proportion des investissements bruts peut être trop élevée ou trop faible en regard des possibilités et des besoins de la collectivité. La figure 50 montre qu’il existe un volume idéal d'investissement. Une analyse historique des résultats des collectivités suisses nous indique que ce volume semble se situer entre $7,0 \%$ et 10,0\% par rapport aux dépenses totales. La note correspondante est 6 . 
Figure 50 Évaluation de la proportion des investissements bruts - I14\#\#.

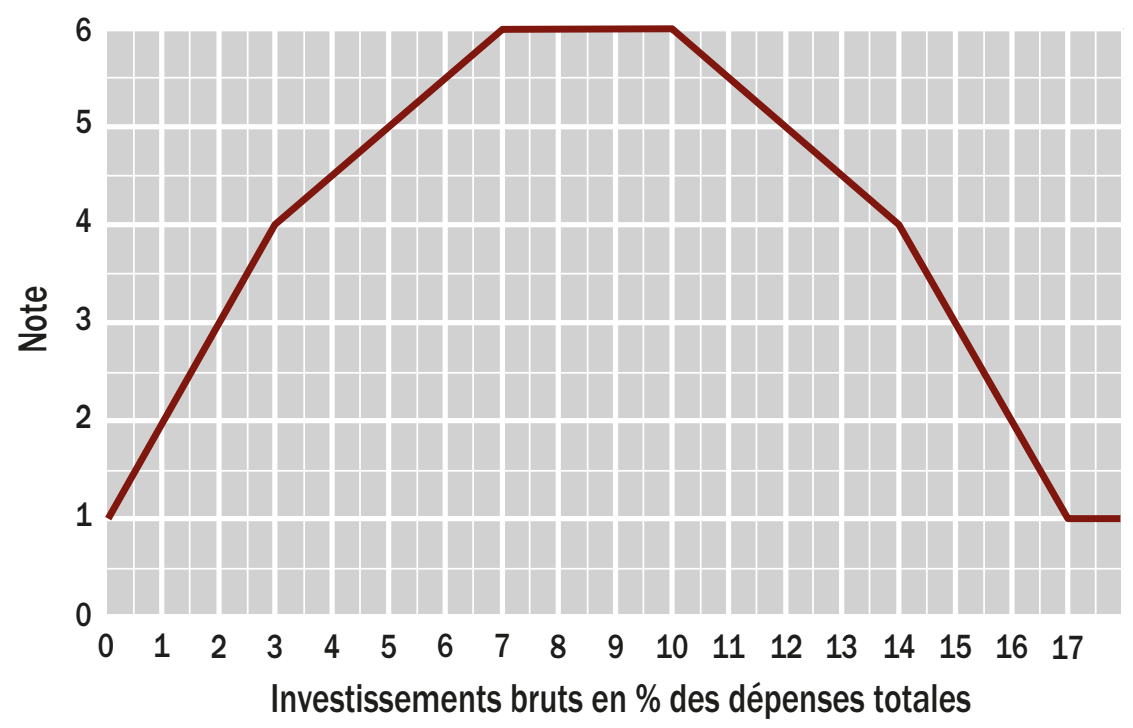

Lorsque la collectivité sous-investit - en deçà de 3,0\% - ou surinvestit - au-delà de $14,0 \%$-, la note tombe en dessous de 4.

Avec des investissements bruts de KCHF 1971 (Année t) et des dépenses totales de KCHF 14577, MaCollectivité présente un résultat de 13,5\% et obtient ainsi une note de 4,2 .

\section{Dette nette par habitant - I15 \#\#}

Indicateur $\mathrm{MCH} 2$ de deuxième priorité (\#\#), ce ratio est parfois utilisé pour mesurer l'importance de la dette. Sa valeur informative est toutefois limitée. En effet, la dette doit plutôt être mise en regard de la capacité financière des personnes physiques et morales établies sur le territoire de la collectivité. Cet indicateur est d'ailleurs le seul préconisé par le $\mathrm{MCH} 2$ et intégré dans ce tableau de bord à exprimer son résultat en francs par habitant plutôt qu'en pourcentage ${ }^{167}$ :

$$
\mathbf{I 1 5} 5^{\# \#}=\frac{\text { engagements nets }}{\text { population résidente permanente }}
$$

Comme on peut le constater dans la formule, on parle bien ici des engagements de la collectivité et pas seulement de son endettement. Le titre de l'indicateur prête donc un peu à confusion. Mais c'est le titre retenu par le MCH2. C'est pourquoi nous l'utilisons également ici.

Un résultat inférieur à CHF 0 indique que le stock d'engagements net des actifs financiers est nul, voire que la collectivité possède davantage d'actifs dans son patrimoine financier que d'engagements au passif de son bilan. Cette situation correspond à une note de 6 (figure 51).

167 Les modalités de calcul des engagements nets sont précisées dans l'annexe 2. 
Figure 51 Évaluation de la dette nette par habitant - I15\#\#.

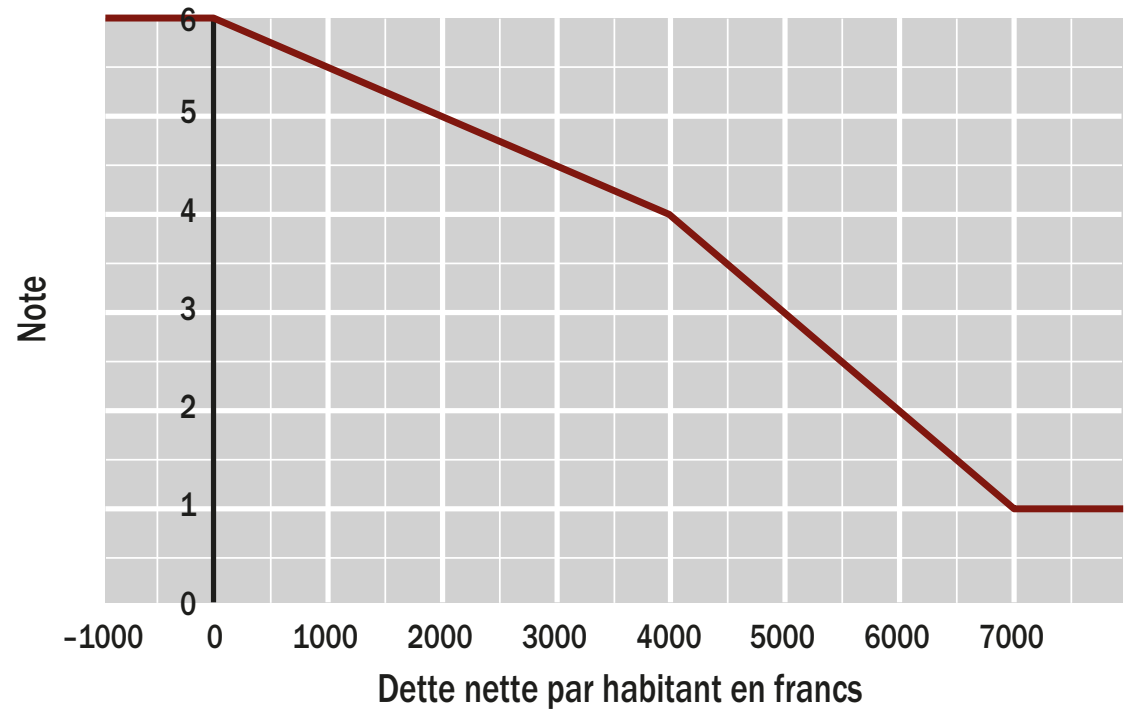

Par contre, dès que les engagements nets par habitant dépassent CHF 4000, la note tombe en dessous de 4 .

Avec des engagements nets de KCHF 2303 et une population de 3113 habitants, MaCollectivité présente une dette nette par habitant de CHF 740. Elle obtient donc une note de 5,6.

\subsubsection{Agrégation des résultats}

Pour agréger les résultats, il faut décider quel poids attribuer à chaque indicateur et à chaque groupe d'indicateurs. Calculer une simple moyenne arithmétique n'est pas satisfaisant. Certains indicateurs revêtent une plus grande importance que d'autres. C'est en particulier le cas lorsque la loi impose une règle budgétaire. Si cette règle vise à limiter les excédents de charges, alors l'indicateur de couverture des charges devrait être surpondéré (I1). Si la règle impose un autofinancement minimum, alors c'est l'indicateur d'autofinancement de l'investissement net qui devrait être surpondéré $\left(\mathrm{I} 2^{*}\right)$. Si encore la règle pose une limite au volume de la dette, un indicateur d'endettement devrait être surpondéré (par exemple I9" ou I10 ${ }^{\# \#) . ~ C ' e s t ~ p o u r-~}$ quoi nous encourageons tout utilisateur du dispositif à se livrer à sa propre réflexion et à déterminer sa propre pondération.

Afin d'initier la réflexion, nous offrons notre propre vision des choses. À l'intérieur de chacun des trois groupes d'indicateurs - équilibres budgétaires, qualité de la gestion financière, importance de l'endettement, nous nous sommes interrogés pour savoir quels seraient le ou les indicateurs que nous conserverions absolument s'il ne fallait en conserver qu'un ou deux. À ces indicateurs, nous avons 
donné un poids double (2). Pour les autres indicateurs, nous avons donné un poids simple (1). Les poids proposés sont rapportés dans le tableau 38. Dans le groupe qui s'intéresse aux équilibres budgétaires, nous donnons la priorité à l'information sur la couverture des charges (I1), sur l'autofinancement de l'investissement net (I2 ${ }^{\#}$ ) et sur les engagements supplémentaires (I3). La pondération de ces indicateurs est donc de 2. Dans le groupe centré sur la qualité de la gestion financière, nous donnons la priorité à l'information sur la maîtrise des dépenses courantes par habitant (I5) et sur l'effort d'investissement (I6). Dans le groupe relatif à l'importance de l'endettement, nous donnons la priorité à l'information sur le taux d'endettement net $\left(19^{\#}\right)$.

Notons que les indicateurs auxiliaires n'apparaissent pas dans l'agrégation. En effet, ces indicateurs viennent compléter l'information véhiculée par les autres indicateurs. Ils sont souvent redondants et corrélés avec ces autres indicateurs. Par conséquent, les inclure dans la synthèse biaiserait le constat d'ensemble en donnant un poids exagéré aux indicateurs concernés.

Concernant les groupes d'indicateurs, nous donnons la priorité à l'information sur les équilibres budgétaires et sur la gestion financière. La pondération de ces deux groupes est donc de 2. Par contre, nous considérons que l'importance de l'endettement doit être pondérée de manière moindre. En effet, cette information ne reflète que l'héritage du passé. Elle est donc moins importante que celle des autres groupes pour préparer l'avenir et pour gérer la collectivité. La pondération de ce groupe est donc de 1 . Ces poids permettent de dégager une note de synthèse globale reflétant la situation financière dans son ensemble.

Tableau 38 Pondération des indicateurs du tableau de bord financier.

\begin{tabular}{lllcc}
\hline Groupes & Indicateurs du tableau de bord financier & & \multicolumn{2}{c}{ Pondérations } \\
\cline { 3 - 5 } & & & Indicateurs Groupes \\
\hline Équilibres & Couverture des charges & 11 & 2 & 2 \\
budgétaires & Autofinancement de l'investissement net & $12^{\#}$ & 2 & \\
& Engagements nets supplémentaires & 13 & 2 & \\
& Poids des intérêts nets & 14 & 1 & \\
\hline Qualité de & Maîtrise des dépenses courantes par habitant & 15 & 2 & 2 \\
la gestion & Effort d'investissement & 16 & 2 & \\
financière & Exactitude de la prévision fiscale & 17 & 1 & \\
& Intérêt moyen de la dette & 18 & 1 & \\
\hline Importance de & Taux d'endettement net & $19^{\#}$ & 2 & 1 \\
l'endettement & Dette brute par rapport aux revenus & $10^{\# \#}$ & 1 & \\
\hline
\end{tabular}

\# Indicateur $\mathrm{MCH} 2$ de première priorité

\#\# Indicateur MCH2 de deuxième priorité 
Tableau 39 Notes de synthèse pour MaCollectivité à l'Année t.

\begin{tabular}{|c|c|c|c|c|c|c|}
\hline \multirow[t]{2}{*}{ Groupes } & \multicolumn{3}{|c|}{ Indicateurs } & \multicolumn{2}{|c|}{ Groupes } & \multirow{2}{*}{$\begin{array}{l}\text { Note de } \\
\text { synthèse } \\
\text { globale }\end{array}$} \\
\hline & Numéro & Note & Pondération & $\begin{array}{l}\text { Note de } \\
\text { synthèse }\end{array}$ & Pondération & \\
\hline \multirow{4}{*}{$\begin{array}{l}\text { Équilibres } \\
\text { budgétaires }\end{array}$} & 11 & 5,3 & 2 & \multirow{4}{*}{5,1} & \multirow{4}{*}{2} & \multirow{10}{*}{4,9} \\
\hline & $12^{\#}$ & 5,4 & 2 & & & \\
\hline & 13 & 4,2 & 2 & & & \\
\hline & 14 & 6,0 & 1 & & & \\
\hline \multirow{4}{*}{$\begin{array}{l}\text { Qualité de } \\
\text { la gestion } \\
\text { financière }\end{array}$} & 15 & 3,5 & 2 & \multirow{4}{*}{4,1} & \multirow{4}{*}{2} & \\
\hline & 16 & 3,4 & 2 & & & \\
\hline & 17 & 5,0 & 1 & & & \\
\hline & 18 & 6,0 & 1 & & & \\
\hline \multirow{2}{*}{$\begin{array}{l}\text { Importance de } \\
\text { l'endettement }\end{array}$} & $19^{\#}$ & 6,0 & 2 & \multirow{2}{*}{5,9} & \multirow{2}{*}{1} & \\
\hline & $110^{\# \#}$ & 5,6 & 1 & & & \\
\hline
\end{tabular}

\# Indicateur $\mathrm{MCH} 2$ de première priorité

\#\# Indicateur MCH2 de deuxième priorité

Compte tenu des résultats obtenus pour chaque indicateur et des poids proposés, le tableau 39 donne les notes de synthèse de MaCollectivité. Avec une note globale de 4,9, la situation financière de MaCollectivité apparaît plutôt satisfaisante. Ce résultat est en particulier dû à une situation d'endettement excellente (note de 5,9): les engagements nets représentent une faible proportion des revenus fiscaux (I9*); il

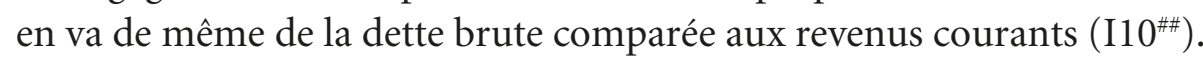

Le domaine où se situe le potentiel d'amélioration le plus important est celui de la gestion financière: la note de synthèse n'est que de 4,1. Cela s'explique par une faible maîtrise de l'évolution des dépenses au cours de l'Année t (I5) et un effort d'investissement très (trop) important par rapport à l'idéal (I6). Ces deux points devraient faire l'objet d'une vigilance particulière. Pour le reste, l'exactitude de la prévision fiscale (I7), comme la performance en matière de gestion de dette (I8) doivent être saluées.

Concernant les grands équilibres budgétaires, la note de synthèse de 5,1 reflète une situation satisfaisante. Ce résultat est toutefois marqué par un accroissement à surveiller des engagements nets au cours de l'Année t (I3). MaCollectivité devrait éviter que le phénomène se répète. 


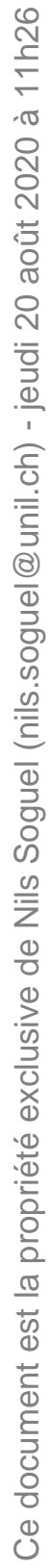




\section{Trouver les informations financières pour se comparer}

Les collectivités publiques ressentent de plus en plus souvent la nécessité de se comparer à d'autres, de se livrer à un benchmarking. Ce chapitre donne des pistes pour procéder à des comparaisons pertinentes. En effet, les documents financiers publiés par les collectivités, en particulier les comptes, n'offrent souvent pas les données appropriées pour comparer directement les collectivités entre elles. Après avoir rappelé les raisons et les enjeux de la comparaison, ce chapitre se penche sur les origines des difficultés qu'il y a à se comparer. Pour les administrations publiques suisses, la principale et la meilleure base de comparaison est offerte par la statistique financière établie par l'Administration fédérale des finances (AFF, portrait 6). Cette statistique est riche en informations, d'abord dans le fascicule publié chaque année et surtout dans les fichiers de données mis à disposition sur l'Internet (www.efv.admin.ch > statistique financière $>$ données). Ce chapitre explique comment utiliser cette statistique et selon quels principes elle est établie. Il compare également cette statistique aux autres sources d'informations à disposition, au plan suisse et au plan international.

\section{Portrait 6 Administration fédérale des finances}

L'Administration fédérale des finances (AFF) est l'office fédéral en charge de la préparation du budget, du plan financier et des comptes de la Confédération. Son rôle transversal l'amène à se prononcer sur tous les projets des départements fédéraux ayant une portée financière. Elle est également responsable du suivi de la politique budgétaire et de la péréquation financière fédérale, ainsi que de la gestion de la dette et de la trésorerie de la Confédération. C'est elle qui élabore la statistique des finances de l'ensemble des administrations publiques suisses.

Source: www.efv.admin.ch.

\subsection{Besoin de se comparer}

Objectiver la situation. Le besoin de se comparer a plusieurs origines et répercussions. D'abord, il reflète la volonté d'objectiver la situation dans laquelle la collectivité se trouve par rapport à d'autres ou par rapport à son propre passé. En effet, faute d'informations pertinentes, la situation financière risque d'être jugée de manière subjective, qu'il s'agisse de l'endettement, du résultat opérationnel, du coût de telle ou telle prestation, etc. Objectiver la situation permet donc de réduire la subjectivité et de fonder les discussions sur des bases plus solides. 
Disposer de données comparables. Se lancer dans une opération de comparaison nécessite de collecter des données. Cela oblige à rendre comparables les données des différentes entités avec lesquelles on souhaite se comparer. C'est là un des principaux aiguillons pour harmoniser les pratiques comptables des collectivités en Suisse, mais aussi à l'échelon international.

Déterminer les données pertinentes pour la gestion. D'emblée, il faut déterminer les données sur la base desquelles on souhaite se comparer. Cela n'a l'air de rien. Mais l'expérience nous montre que parfois des données que l'on considère finalement indispensables pour la gestion ne sont pas facilement disponibles, voire pas disponibles du tout. Vouloir se comparer fait apparaitre ces lacunes.

Identifier le potentiel d'amélioration. La comparaison permet de déterminer si des potentiels d'amélioration existent et, cas échéant, dans quels domaines. Le chapitre 9 proposait des indicateurs assortis d'échelles de notation pour analyser la situation financière. Ces échelles de notation peuvent être vues comme des normes de performance. Or ces échelles ne tiennent pas compte des situations particulières qui peuvent surgir: par exemple, une conjoncture économique particulièrement mauvaise, une évolution démographique singulièrement forte ou faible, une infrastructure publique accusant un retard spécialement important. Dans ces cas-là, il peut s'avérer utile pour une collectivité de se comparer à d'autres collectivités confrontées à des conditions similaires. La référence n'est plus alors absolue (une norme invariante), mais elle est relative à la situation d'autres entités. La marge d'amélioration peut ainsi être mesurée par rapport à des entités qui vivent la même réalité, avec lesquelles il est possible de dialoguer et d'échanger les expériences.

Fixer les objectifs. Connaissant le potentiel d'amélioration par rapport à d'autres, il devient plus aisé de fixer des objectifs. Après tout, si d'autres collectivités y parviennent, pourquoi notre collectivité n'y parviendrait-elle pas? La comparaison permet donc de légitimer les objectifs que la collectivité se fixe.

Se féliciter des réalisations. Toute comparaison débouche implicitement ou explicitement sur un classement, avec davantage de viennent-ensuite que de premiers. Si les viennent ensuite peuvent identifier le potentiel d'amélioration, le ou les premiers peuvent identifier leurs forces, leurs réalisations et s'en féliciter.

Maintenir la confiance envers les autorités. Se comparer ouvertement à d'autres collectivités est aussi une manière pour les autorités de montrer qu'elles s'engagent pour le maintien d'une situation financière saine. Cet effort d'ouverture et de transparence est de nature à maintenir et à renforcer la confiance de la population dans ses autorités élues. 


\subsection{Enjeux de la comparaison}

Comparaison de la situation générale. La comparaison peut s'intéresser à différents aspects. Elle peut s'intéresser à comparer la situation financière générale de la collectivité. Les indicateurs abordés au chapitre précédent visent précisément cet aspect. Pour ce type de comparaisons, les comptes et les budgets publiés par les collectivités elles-mêmes fournissent généralement une information suffisamment pertinente.

Comparaison des coûts et des revenus. La comparaison peut également s'intéresser au niveau des coûts et de leur structure. Ce type de comparaison peut concerner la globalité du portefeuille de prestations d'une collectivité ou concerner des prestations spécifiques (par exemple, le coût par habitant est-il comparable, globalement ou pour une prestation particulière?). En outre, la comparaison peut aussi porter sur le niveau et la structure des revenus (par exemple, la part des revenus fiscaux ou des taxes estelle comparable?). Pour ce type de comparaisons, les documents financiers publiés par les collectivités n'offrent généralement pas de données suffisamment comparables.

\subsection{Origines des difficultés de comparaison}

Les problèmes de comparabilité ont différentes origines. D'abord, les modèles et les plans comptables peuvent diverger. Par exemple, pendant quelques années entre 2008 et 2018, certaines collectivités utilisaient déjà le $\mathrm{MCH} 2$ alors que d'autres en restaient encore au MCH1. De ce fait, au cours de cette période, cette coexistence réduisait les possibilités de comparaison. Même si l'ensemble des collectivités suisses a fini par adopter le $\mathrm{MCH} 2$, la comparaison sur la base des documents publiés par les collectivités n'est ni aisée, ni suffisamment probante. En effet, ces documents ne sont pas encore assez harmonisés.

Ensuite, à supposer que modèles et plans comptables soient identiques, il faut être conscient que les attributions et les compétences peuvent différer entre collectivités. Ceci est un aspect particulièrement important dans le système fédéraliste que connaît la Suisse. La souveraineté des cantons en matière de répartition des tâches avec les communes implique que, d'un canton à l'autre, le catalogue de prestations et des activités fournies par l'échelon cantonal (le canton lui-même) varie, de même que le catalogue dont les communes sont responsables. Par conséquent, l'hétérogénéité dans la répartition verticale des tâches biaise les comparaisons de coûts ou de revenus qui sont menées sur la base des documents publiés par les collectivités. Par exemple, dans certains cantons, les communes salarient les enseignants; ailleurs, c'est l'échelon cantonal qui verse les salaires. Comparer le coût par élève en ne se référant qu'aux comptes des cantons fausserait les comparaisons. Pour réduire le risque de biais, on utilisera plutôt une agrégation des coûts et des revenus du canton et des communes (voir la section 10.6.2 ci-après). 
Enfin, même si la répartition des tâches était uniforme dans les différents cantons, on serait encore confronté à des dispositifs institutionnels potentiellement hétérogènes. Certains cantons externalisent certaines tâches ou autonomisent certaines institutions (universités par exemple). Ces institutions n’apparaissent alors plus dans leurs comptes. Certaines communes recourent à des intercommunalités pour fournir diverses prestations, tandis que d'autres continuent à les fournir ellesmêmes. Dans ces cas-là, les comptes n'offrent pas de données comparables.

\subsection{Des comptes à la statistique}

Si les comptes n'offrent pas de données comparables, vers quelles sources d'information se tourner pour pouvoir procéder à des comparaisons pertinentes? La réponse à cette question repose sur le lien existant entre les comptes présentés par les collectivités publiques et la statistique financière, notamment celle qui est élaborée par l'Administration fédérale des finances pour l'ensemble des collectivités publiques suisses.

Harmonisation des natures. Rares sont les personnes conscientes du fait que la présentation des comptes publics est tributaire de conventions statistiques élaborées à l'échelon international. Ces conventions sont notamment élaborées sous l'égide du Fonds monétaire international (FMI). Ce dernier a mis sur pied un manuel de statistiques de finances publiques (MSFP, Government Finance Statistics Manual, GFSM) dont la dernière édition a été publiée en 2014 (FMI 2014). Il offre en particulier une définition harmonisée des notions clés (par exemple, que faut-il classer sous le terme «impôts» ou sous le terme «contributions»?). Il offre également une classification harmonisée pour les charges, les revenus, les actifs et les passifs. Le plan comptable par nature du MCH2 est partiellement compatible avec cette classification, dans la mesure où le cadre du MSFP est plus exhaustif que celui qui est proposé par le $\mathrm{MCH} 2$.

Harmonisation des fonctions. Le plan comptable $\mathrm{MCH} 2$ exige des collectivités qu'elles présentent leurs charges et leurs revenus selon une classification fonctionnelle harmonisée, en plus d'une présentation selon la classification organique et par nature (section 3.3.9). La classification fonctionnelle est compatible avec la classification des fonctions des administrations publiques (Classification of the Functions of Government, COFOG, portrait 7). Cette dernière est appliquée internationalement. Elle offre un cadre qui devrait permettre de comparer les pays entre eux sur la base des sommes consacrées aux principales catégories de tâches étatiques ${ }^{168}$.

168 En pratique, les différences en termes organisationnels, institutionnels ou encore au niveau des priorités en matière de politique budgétaire ne permettent pas toujours de reconstruire les classes de fonctions de manière exactement comparable entre collectivités. 


\section{Portrait 7 Classification of the Functions of Government}

La classification des fonctions des administrations publiques (CFAP) (plus connue sous son acronyme anglais COFOG) est une nomenclature établie par l'Organisation de coopération et de développement économique (OCDE). Créée en 1993, elle a été révisée en 1999. Cette nomenclature est publiée par la Division des statistiques de l'Organisation des Nations Unies (ONU). Elle constitue la référence internationale lorsqu'il s'agit de catégoriser les fonctions et les tâches publiques. Elle est organisée selon trois niveaux de détail: (a) les divisions, (b) les groupes et (c) les classes. Les divisions couvrent les grandes missions de l'État. Elles sont au nombre de dix: (1) Services généraux des administrations publiques; (2) Défense; (3) Ordre et sécurité publics; (4) Affaires économiques; (5) Protection de l'environnement; (6) Logement et équipements collectifs; (7) Santé; (8) Loisirs, culture et culte; (9) Enseignement; (10) Protection sociale. Quant à eux, les groupes et les classes décrivent les prestations et les activités permettant d'exécuter la mission à laquelle ils sont attachés.

Source: Eurostat. ec.europa.eu/eurostat.

Harmonisation des périmètres et sectorisation. Afin de pouvoir comparer une commune à une autre, ou un canton à un autre, il est nécessaire d'harmoniser les périmètres. C'est ainsi que l'on peut s'assurer que derrière la notion de commune ou de canton se trouvent la même réalité, les mêmes fonctions. Le système européen des comptes (SEC 2010) offre à la statistique financière la base nécessaire pour harmoniser les périmètres là où c'est nécessaire (UE 2013) ${ }^{169}$. Pour y parvenir, le SEC catégorise d'abord les entités économiques en deux secteurs: le secteur public et le secteur privé. Le critère de distinction est celui du contrôle (ou de l'absence de contrôle) par l'État.

À l'intérieur du secteur public, le SEC introduit une distinction entre administrations publiques et entreprises publiques. C'est surtout le mode de financement qui détermine si une entreprise sous contrôle des autorités publiques fait statistiquement partie du périmètre de la Confédération, d'un canton ou d'une commune. Lorsqu'une telle entité finance plus de $50 \%$ de ses coûts de production par des taxes ou d'autres rétributions (y compris des recettes sur ventes), elle est catégorisée comme une entreprise (publique). Pour la statistique, cela signifie que l'essentiel des activités a un caractère commercial et donc que la logique décisionnelle est surtout dictée par le mécanisme des prix (même si les prix sont en partie administrés).

169 Le système des comptes de l'Union européenne (SEC) et le manuel de statistiques de finances publiques du FMI (MSFP-GFSM) se fondent tous deux sur le cadre établi pour la statistique économique sous les auspices des Nations Unies, de la Commission européenne, de l'Organisation de coopération et de développement économiques, du Fonds monétaire international et du Groupe de la Banque mondiale. Ce cadre constitue le Système de comptabilité nationale (SCN, System of National Accounts, SNA). Sa dernière mise à jour a été réalisée en 2008 avec la publication du SCN 2008 (ONU et al. 2013). 
C'est pourquoi la statistique financière exclut, par exemple, les hôpitaux, les établissements médico-sociaux, les maisons de retraite, les centrales électriques ou les stations d'épuration des eaux du périmètre des communes et des cantons qui les possèdent. Même si ces entités sont souvent incluses dans les comptes des communes et des cantons concernés, elles sont principalement financées par des taxes et d'autres rétributions. Ces entités sont donc catégorisées dans le secteur des entreprises publiques, comme le schématise la figure 52. Elles ne font dès lors pas partie du secteur des administrations publiques, qu'il s'agisse de la Confédération, des cantons ou des communes.

Si les coûts de l'entité sont couverts à 50\% ou à moins de 50\% par des taxes ou d'autres rétributions, cela signifie que l'entité est essentiellement financée par la fiscalité ou par des transferts. Dans ce cas, la logique marchande imposée par le prix de vente ne dicte pas majoritairement les décisions de l'entité. C'est pourquoi la statistique range l'entité concernée dans le périmètre de la Confédération, du canton ou de la commune qui la finance, autrement dit dans le secteur des administrations publiques. Ainsi, la statistique inclut les universités et les hautes écoles spécialisées dans le périmètre des cantons qui les financent et cela même dans les cas où ces hautes écoles sont indépendantes et ne sont pas incluses dans les comptes des cantons concernés.

Figure 52 Le périmètre statistique d'une administration publique en exclut les entreprises publiques.

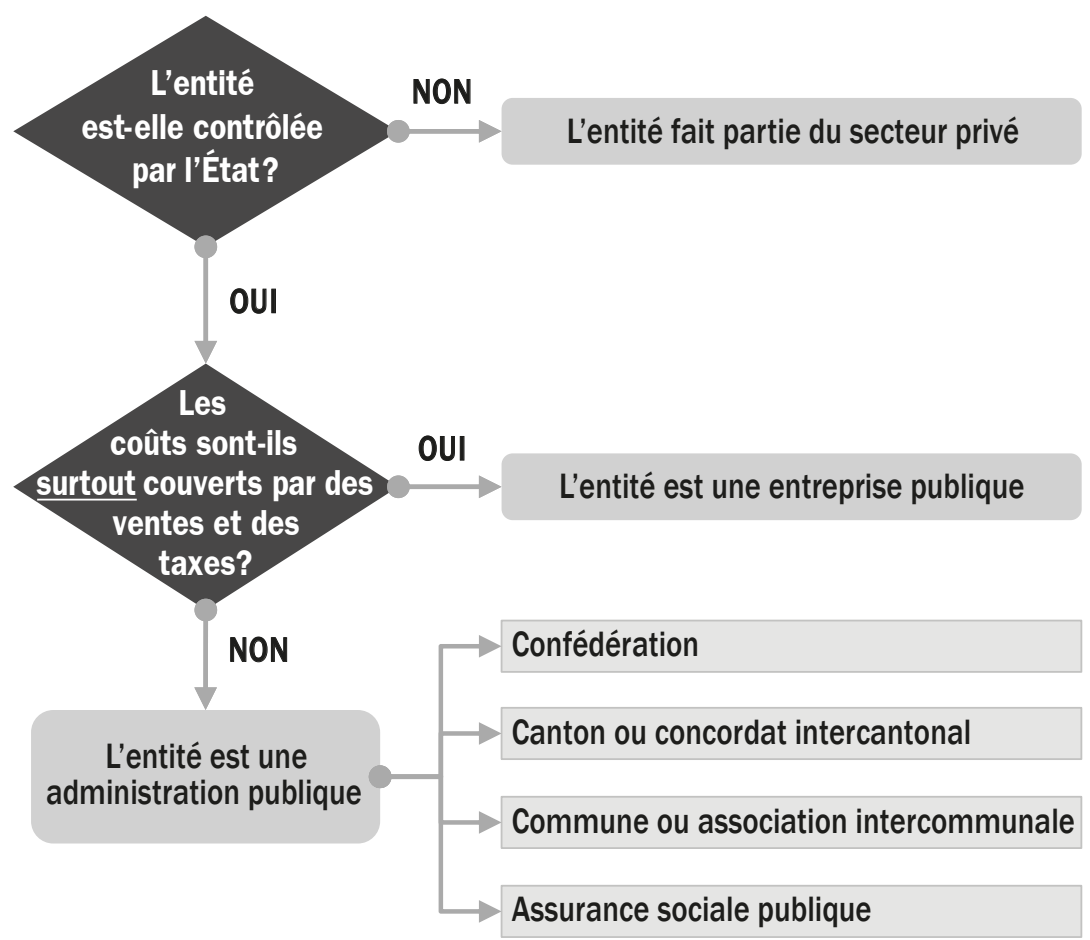


Compte tenu de l'organisation institutionnelle de la Suisse, la statistique financière établie par l'Administration fédérale des finances subdivise le secteur des administrations publiques en quatre sous-secteurs calqués sur la structure fédéraliste: (a) Confédération, (b) cantons ou concordats intercantonaux, (c) communes ou associations intercommunales ${ }^{170}$ et $(d)$ assurances sociales ${ }^{171}$. Le terme d'administrations publiques est donc un terme générique (portrait 8 ). Ce terme recouvre la notion de collectivités publiques que nous utilisons dans cet ouvrage. Cependant, le terme de collectivités publiques n'est pas utilisé en matière de statistique, pas plus qu'il ne connaît de définition universelle en droit suisse.

La statistique établie par l'AFF harmonise donc le périmètre des administrations publiques en utilisant la limite de $50 \%$ de financement par des ventes ou des taxes. Pour chaque canton et chaque ville, le fascicule de l'AFF renseigne sur les entreprises publiques qui ont été exclues de la statistique, alors qu'elles sont présentes dans les comptes de la collectivité concernée. Il renseigne également sur les entités qui ont été incluses dans la statistique, alors qu'elles ne sont pas dans les comptes (AFF 2019b). Cela explique en partie pourquoi des différences peuvent apparaître entre les chiffres publiés dans les comptes des collectivités et ceux de la statistique de l'AFF.

\section{Portrait 8 Administrations publiques}

Selon le Manuel de statistiques de finances publiques du FMI (2001, p. 7), "les administrations publiques d'un pays sont constituées des pouvoirs publics et de leurs organes d'exécution, qui sont des entités instituées par décision politique exerçant un pouvoir législatif, judiciaire ou exécutif sur un espace territorial donné. Les principales fonctions économiques des administrations publiques consistent (a) à fournir à la collectivité des biens et des services non marchands destinés à la consommation collective ou individuelle et (b) à redistribuer le revenu et la richesse au moyen de transferts. Les administrations publiques se caractérisent en outre par le fait que ces activités doivent être financées principalement par l'impôt ou par d'autres transferts obligatoires."

Suppression des comptes à double. Le chapitre 6 a montré l'importance et la variété des montants transférés entre les collectivités publiques. Certains transferts sont horizontaux, entre entités d'un même niveau, par exemple entre communes.

170 Les concordats intercantonaux sont des contrats qui règlent la collaboration entre cantons pour remplir une tâche publique spécifique. De tels concordats existent pour les offices régionaux de placement ou pour les hautes écoles spécialisées. Les associations intercommunales sont constituées par des communes afin de remplir une ou plusieurs tâches publiques dont elles sont responsables. Il en existe de nombreuses, par exemple dans le domaine scolaire ou dans celui de l'épuration des eaux.

171 Pour davantage de détails sur la sectorisation et la délimitation du secteur des administrations publiques, voir l'annexe A du Manuel MCH2, CDF (2008). 
D'autres sont verticaux entre entités de niveaux différents, par exemple entre un canton et les communes qui en font partie.

Si l'on veut avoir une image fiable des charges ou des revenus de l'échelon communal à l'intérieur d'un canton, il faut faire abstraction des transferts horizontaux entre communes. Imaginons un canton ayant deux communes dont MaCollectivité et que MaCollectivité verse à l'autre commune un dédommagement de KCHF 170. Ce montant apparaît dans les charges de MaCollectivité (dans la rubrique 361) et dans les revenus de l'autre commune (rubrique 461). Additionner simplement les charges et les revenus de ces deux communes pour connaître le total des charges et des revenus communaux dans le canton conduirait à une surestimation en raison d'un compte à double du montant de KCHF 170, à la fois dans les charges et dans les revenus.

Une surestimation des charges et des revenus à l'intérieur d'un canton se produirait également si l'on ne faisait pas abstraction des transferts verticaux. Pensons au cas de MaCollectivité qui reçoit une compensation du canton d'un montant de KCHF 82 pour le déneigement des routes communales (section 6.4). Ce montant figure dans les charges du canton (dans la rubrique 362) et dans les revenus de MaCollectivité (rubrique 462). Additionner simplement les charges et les revenus du canton et de MaCollectivité, et de l'autre commune, pour connaître le total des charges et des revenus de l'ensemble des administrations publiques sises dans le canton déboucherait aussi sur une surestimation. En effet, on compterait à double le montant de KCHF 82 (à la fois dans les charges et dans les revenus).

En établissant la statistique financière, l'AFF prend soin de neutraliser les transferts débiteurs et les transferts créditeurs pour éviter les comptes à double. De cette manière, la comparaison n'est pas faussée. À partir de là, on peut valablement comparer les charges, ou les revenus, (par habitant) entre cantons ou entre communes.

\subsection{Comparer les communes entre elles}

\subsubsection{Comparaison intra-cantonale}

Même réglementation financière cantonale. Les communes situées dans un même canton sont soumises à la même réglementation en matière financière. Par conséquent, cela réduit l'hétérogénéité des pratiques en matière de préparation et de présentation des comptes. Donc la comparaison des communes sur la base de leurs comptes donne des résultats relativement fiables. Évidemment, le degré de contrainte varie entre cantons. Mais, en général, les directives cantonales couvrent le plan comptable par nature et la classification fonctionnelle, les principes d'évaluation, ainsi que le périmètre de consolidation.

Même répartition des tâches et même régime fiscal. La comparaison des charges communales (par habitant) à l'intérieur d'un même canton offre des résultats 
utilisables. En effet, à l'intérieur d'un même canton, la répartition des tâches entre le canton et les communes est, par définition, identique pour toutes les communes. Il en va de même du régime fiscal: dans un canton donné, les impôts et les taxes que les communes sont autorisées à prélever - ou doivent prélever - sont identiques. Par conséquent, la comparaison des revenus (par habitant) est également utilisable.

Classifications fonctionnelle et par nature. Pour comparer les communes entre elles, il faut se référer soit à la classification fonctionnelle, soit à la classification par nature. Il est hasardeux d'utiliser la classification organique. En effet, les communes sont le plus souvent organisées différemment les unes des autres. Donc, la classification organique varie (voir sur ce point la section 3.3.9).

Sources statistiques cantonales. À l'exception du canton d'Appenzell RhodesIntérieures, toutes les administrations cantonales publient des informations relatives aux finances de leurs communes ${ }^{172}$. Selon les cantons, les données financières sont collectées auprès des communes et traitées par des unités administratives différentes. Il peut s'agir du service cantonal des communes ou de son équivalent, du service de la statistique s'il existe, du service des finances du canton, voire d'un secrétariat de département.

Les statistiques publiées couvrent le compte de résultats des communes avec un degré de détail qui s'étend à la deuxième position de la classification par nature et à la première position de la classification fonctionnelle. Les données du compte des investissements sont plus rarement disponibles, en particulier pour ce qui concerne la classification fonctionnelle. Les données du bilan sont publiées à peu près avec le même degré de détail que celles du compte de résultats. De plus, on trouve dans presque toutes les statistiques financières communales les résultats pour quelques indicateurs.

L'horizon temporel des données publiées varie. Les séries statistiques remontent souvent jusqu'en 2006. L'administration vaudoise offre les séries chronologiques les plus longues allant jusqu'en 1985 avec un haut degré de détail.

\subsubsection{Comparaison inter-cantonale}

Renoncer à utiliser directement les comptes communaux. Contrairement à la comparaison intra-cantonale, la comparaison des communes entre cantons ne peut guère être menée directement sur la base des comptes des communes. Il y a à cela différentes raisons: les réglementations financières varient entre cantons, de même que la répartition des tâches entre le canton et les communes, le régime fiscal ou

172 Bâle-Ville constitue un cas particulier. En effet, le canton ne compte que trois communes et la ville de Bâle est incluse dans le périmètre du canton. Les comptes de la Ville ne font qu’un avec ceux du canton. Par conséquent, des données financières purement communales ne sont disponibles que pour les deux communes restantes (Bettingen et Riehen). 
encore le périmètre comptable des communes elles-mêmes. Par conséquent, la comparabilité des communes de différents cantons n'est pas assurée. Il faut donc se tourner vers la statistique financière établie par l'Administration fédérale des finances afin de disposer d'une meilleure base de comparaison (modèle SF: communes). Sur la période qu'elle couvre, la statistique financière résout les problèmes de rupture de séries chronologiques engendrés par le passage du MCH1 au MCH2. Pour les villes et les chefs-lieux, elle résout également les problèmes de périmètres évoqués précédemment (section 10.4).

Cela dit, les chiffres publiés dans la statistique financière restent tributaires de la répartition des tâches entre le canton et les communes. À cet égard, il faut donc faire preuve de prudence dans les comparaisons.

Statistique financière agrégée pour l'ensemble des communes suisses. L'AFF publie sur l'Internet des données financières communales agrégées à l'échelon de l'ensemble du pays dans un fichier dit de «tableaux réguliers» (gdn.xlsx). Pour le bilan et le compte de résultats, ce fichier contient des séries remontant à 2008, structurées selon la classification par nature, avec un degré de détail qui s'étend à la quatrième position. Ce fichier contient également des séries plus longues, remontant à 1990, mais présentées selon la logique du compte de financement, tel qu'il est pratiqué par la Confédération (section 3.4). Ces données portent donc sur les dépenses et les recettes - courantes et d'investissement - présentées selon la classification par nature, à un degré de détail qui va jusqu'à la quatrième position. Les données portent également sur les dépenses et les recettes présentées selon la classification fonctionnelle et ce jusqu'à la deuxième position.

Pour l'ensemble des communes suisses, l'AFF publie également un fichier présentant les dépenses et les recettes - courantes et d'investissement - sur la base d'un croisement entre la classification par nature et la classification par fonction (gdn_fir_ art_funk.xlsx). On peut ainsi découvrir combien l'ensemble des communes suisses dépense pour la scolarité obligatoire (fonction 21) ou pour la formation professionnelle initiale (fonction 23) au titre des salaires des enseignants (nature 302). En effet, la décomposition par fonction va jusqu'à la deuxième position, tandis que celle par nature s'étend jusqu'à la troisième position. Ce fichier offre ce tableau croisé pour plusieurs années.

\section{Statistique financière agrégée pour l'ensemble des communes d'un canton.} L'AFF publie sur l'Internet des données financières communales agrégées par canton. Ainsi, pour chaque canton, un fichier de données est à disposition. Par exemple, le fichier du Valais (gdn_vs.xlsx) présente les données agrégées pour l'ensemble des communes valaisannes. Dans ces fichiers, les données sont structurées de la même manière, et avec le même niveau de détail, que dans le fichier présentant les données agrégées de l'ensemble des communes suisses (bilan et compte de résultats selon la classification par nature remontant à 2008, compte de financement selon la classification par nature et selon la classification fonctionnelle remontant à 1990). 
Un fichier additionnel offre, pour les communes de chaque canton, une statistique de la dette brute nominale et de la dette brute par habitant remontant à 1990 (gdn_schuld.xlsx).

Statistique financière individuelle pour les communes. L'AFF publie également des données financières non agrégées, c'est-à-dire individuelles, pour les chefs-lieux des cantons, les autres villes membres de l'Union des villes suisses, ainsi que les communes de plus de 5000 habitants. Ces données individuelles sont donc disponibles pour plus de cinquante communes. Deux fichiers séparés présentent les informations pour les chefs-lieux des cantons (stdt_kho_vgl.xlsx) et pour les villes (stdt_vgl. xlsx), et cela pour la dernière année statistique disponible. Dans ces fichiers, les données sont structurées de la même manière que dans le fichier présentant les données agrégées de l'ensemble des communes.

Deux fichiers additionnels offrent une statistique de la dette brute (nominale et par habitant) par ville selon qu'il s'agit d'un chef-lieu (stdt_kho_schuld.xlsx) ou d'une ville (stdt_schuld.xlsx).

Échantillon statistique. L'AFF établit la statistique financière à l'aide d'un échantillon de communes. Autrement dit, elle ne collecte pas les données financières communales de manière totalement exhaustive. À l'heure actuelle, l'échantillon comprend près de 1500 communes, représentant deux tiers des communes et plus de $80 \%$ de la population. À partir de là, l'AFF extrapole les données pour l'ensemble des communes, à l'échelon national et pour chaque canton lorsque c'est nécessaire. L'échantillon est révisé tous les cinq ans. À chaque révision, l'échantillon s'élargit, à tel point qu'un relevé exhaustif devait être atteint à terme. Actuellement les données sont collectées de manière exhaustive dans plus de la moitié des cantons (Appenzell Rhodes-Intérieures, Appenzell Rhodes-Extérieures, Bâle-Campagne, Bâle-Ville, Berne, Genève, Glaris, Lucerne, Neuchâtel, Nidwald, Obwald, Schaffhouse, Schwyz, Uri, Zoug, Zurich). Par ailleurs, l'échantillon comprend tous les chefs-lieux cantonaux et toutes les communes qui sont membres de l'Union des villes suisses.

\subsection{Comparer les cantons entre eux}

\subsubsection{Données des comptes cantonaux}

La Conférence des directrices et directeurs cantonaux des finances (CDF, voir le portrait 1) publie sur son site Internet des données comptables provenant des comptes des cantons (www.fdk-cdf.ch > Données des finances). Ces données ne font pas l'objet d'un redressement pour harmoniser le périmètre entre les cantons. Par conséquent, elles correspondent exactement à celles qui sont présentées par les cantons dans leurs propres comptes. Les données sont publiées pour chaque canton. 
Elles couvrent le compte de résultats, le compte des investissements et le bilan, selon la classification par nature. Le degré de détail est assuré jusqu’à la deuxième position de la classification. Pour certaines rubriques, il va jusqu'à la troisième position.

Les fichiers de données comprennent les comptes des deux dernières années. Ils comprennent également les données des deux derniers budgets. En cela, les informations disponibles auprès de la CDF se démarquent de celles qui sont disponibles auprès de l'AFF. Cette dernière n'établit la statistique financière que pour les comptes.

\subsubsection{Statistique financière pour comparer les cantons}

Statistique financière pour les seuls cantons. Comme pour les communes, la comparaison entre cantons ne peut guère être menée directement sur la base des comptes, d'abord en raison des différences dans la répartition des tâches avec les communes, ensuite en raison du périmètre comptable des cantons eux-mêmes. La statistique financière de l'Administration fédérale des finances pour les seuls cantons résout le problème du périmètre (modèle SF: cantons). Elle est structurée de la même manière que celle pour l'échelon communal et contient les informations suivantes dans des fichiers séparés ${ }^{173}$ :

- Une statistique financière agrégée pour l'ensemble des cantons suisses dans un fichier dit de «tableaux réguliers» (ktn.xlsx, équivalent dans sa structure et son contenu au fichier gdn.xlsx pour les communes présenté ci-dessus). Les données remontent à 1990.

- Un croisement entre la classification par nature et la classification par fonction pour les dépenses et les recettes selon la structure du compte de financement, pour plusieurs années (ktn_fir_art_funk.xlsx, équivalent au fichier gdn_fir_art_ funk.xlsx pour les communes). Le fichier existe également pour chaque canton.

- Une comparaison entre les vingt-six cantons pour la dernière année statistique uniquement (ktn_vgl.xlsx). Les données sont structurées de la même manière que dans le fichier présentant les données agrégées de l'ensemble des cantons (ou des communes).

- Une comparaison de la dette brute (nominale et par habitant) remontant à 1990 (ktn_schuld.xlsx).

- Des données financières non agrégées, c'est-à-dire individuelles, pour chaque canton et pour la dernière année statistique disponible. Chaque canton est présenté dans un fichier séparé (par exemple ktn_ju.xlsx pour le canton du Jura).

173 Une statistique financière est également disponible pour la Confédération (modèle SF: Confédération), une autre pour les assurances sociales publiques (modèle SF: assurances sociales). Finalement, une statistique pour l'ensemble des administrations publiques est aussi publiée sur le site Internet de l'AFF (modèle SF: secteur des administrations publiques avec et sans les assurances sociales publiques). Nous renonçons à en donner le détail ici. En effet, la structure de ces statistiques est la même que celle des statistiques financières des cantons et des communes. 
Statistique financière pour les cantons et leurs communes. Les données financières qui concernent l'échelon cantonal uniquement restent tributaires de la répartition des tâches. Pour s'affranchir de ce problème, il faut recourir aux données qui agrègent, pour chaque canton, le niveau cantonal et le niveau communal (modèle SF : cantons et leurs communes). C'est en particulier le cas si l'on souhaite effectuer une comparaison du niveau des charges et des dépenses (brutes ou nettes). Cette statistique est structurée de la même manière que celle pour l'échelon communal, également dans des fichiers séparés:

- Une statistique financière agrégée pour l'ensemble des cantons suisses et leurs communes dans un fichier dit de «tableaux réguliers» (ktn_gdn.xlsx).

- Un croisement entre la classification par nature et la classification par fonction pour les dépenses et les recettes (ktn_gdn_fir_art_funk.xlsx).

- Une comparaison entre les vingt-six cantons et leurs communes (ktn_gdn_vgl. xlsx).

- Une comparaison de la dette brute (nominale et par habitant, ktn_gdn_schuld. $\mathrm{xlsx}$ ).

- Des données financières non agrégées pour chaque canton avec ses communes. Chaque canton est présenté dans un fichier séparé (par exemple ktn_gdn_ne.xlsx pour le canton de Neuchâtel).

\subsection{Comparer la Suisse avec d'autres pays}

Modèle statistique différent. Le modèle utilisé par l'AFF pour offrir une base de comparaison aux collectivités suisses entre elles (modèle SF) ne correspond pas totalement au modèle nécessaire pour comparer les collectivités publiques suisses à leurs homologues étrangères. L'AFF publie donc une autre statistique selon un modèle compatible avec le manuel de statistiques de finances publiques du Fonds monétaire international (FMI 2014). Ce modèle additionnel porte l'abréviation SFP'74.

Outre le fait qu'il réponde aux besoins de comparaison internationale, le modèle SFP répond surtout à des enjeux de suivi macroéconomique des économies nationales. Il n'est pas conçu pour répondre aux impératifs de gestion financière des cantons ou des communes. Pour l'essentiel, le modèle SFP se distingue du modèle SF par une définition plus étroite des charges et des revenus, par l'absence de distinction entre éléments ordinaires et extraordinaires, ou encore par une différenciation entre les opérations découlant de transactions et celles relatives aux autres flux économiques (par exemple les pertes et gains dus aux variations de taux de change, éléments inclus dans les charges et revenus financiers par le $\mathrm{MCH} 2$ ).

174 L'abréviation SFP prête malheureusement à confusion avec l'abréviation SF qui correspond au modèle permettant la comparaison entre collectivités helvétiques uniquement. Une différenciation plus intuitive aurait sans doute été préférable. 
La statistique selon le modèle SFP est publiée sur le site Internet de l'AFF à différents niveaux d'agrégation: (a) pour la seule Confédération; (b) globalement pour l'ensemble des cantons; (c) pour l'ensemble des communes; $(d)$ pour l'ensemble des assurances sociales publiques; (e) globalement pour l'ensemble Confédération-cantons-communes; $(f)$ pour tout le secteur des administrations publiques (Confédération-cantons-communes-assurances sociales publiques). Dans tous les cas, les séries chronologiques remontent jusqu'à 1990.

L'AFF ne met pas à disposition les statistiques d'autres pays ${ }^{175}$. Pour comparer la Suisse à d'autres pays, il faut faire appel à d'autres sources, comme la statistique des recettes publiques publiée par l'OCDE (2018).

Statistique du FMI. Le Fonds monétaire international offre une statistique financière couvrant 135 pays dont la Suisse (data.imf.org > government finance). Les données sont structurées selon les classifications internationales, en particulier selon la classification COFOG (portrait 7). En ce qui concerne les niveaux étatiques, il est possible de distinguer le gouvernement central (en Suisse: la Confédération), les états fédérés (cantons), les gouvernements locaux (communes), ainsi que les assurances sociales. Les séries chronologiques remontent systématiquement jusqu'en 1990.

Statistique d'Eurostat. Eurostat, l'agence statistique de la Commission européenne, publie une statistique financière couvrant spécifiquement les pays membres de l'Union européenne ainsi que l'Islande, la Norvège et la Suisse (ec.europa.eu/eurostat/data/database $>$ economy and finance $>$ government statistics). Les données sont structurées sur la base des comptes nationaux (SEC 2010). Cette classification est compatible avec le cadre du MSFP (FMI 2014). Les données peuvent également être distinguées selon les différents niveaux étatiques. Les séries chronologiques remontent jusqu'à 1995.

175 L'AFF offre toutefois, dans certaines de ses publications, des informations statistiques relatives à d'autres pays que la Suisse. 


\section{Analyser l'origine des déficits: conjoncture ou structure}

Lorsqu'une collectivité présente un déficit de son compte de résultats, on s'interroge forcément sur l'origine du déséquilibre. Est-ce un problème passager? Ou le mal est-il plus profond? Dans la mesure où plusieurs positions budgétaires sont influencées par l'évolution de l'environnement socio-économique, il faut effectivement déterminer si les difficultés proviennent d'un tassement de la conjoncture économique ou si les difficultés sont structurelles. Si le diagnostic montre que le déficit est dû à la situation conjoncturelle, le problème se résorbera très vraisemblablement lorsque le cycle économique s'inversera. Il n'est alors pas véritablement nécessaire de prendre des mesures visant à rééquilibrer structurellement le budget de la collectivité.

Cette interrogation devrait aussi intervenir en cas d'excédent de revenus. Dans ce cas, il est tout aussi important de savoir si l'excédent est structurel, et donc durable, ou s'il est causé par un boom conjoncturel temporaire, avec le risque de voir l'excédent disparaître avec le prochain retournement du cycle économique. Si l'excédent est d'origine conjoncturelle, il serait imprudent de conclure qu'il permet une baisse d'impôts ou une extension de l'offre de prestations. Cela contribuerait à déséquilibrer structurellement les finances de la collectivité et celle-ci serait confrontée à des déficits dès lors que la conjoncture reviendrait à la normale.

Distinguer la composante conjoncturelle de la composante structurelle du solde budgétaire est possible pour autant que l'on procède par étapes. Ce chapitre donne une marche à suivre inspirée de celle qui est proposée par l'OCDE (Girouard et André 2005). Il présente en même temps les principales notions. D’abord, il est nécessaire d'évaluer dans quelle mesure la situation conjoncturelle s'écarte de la normale. Il faut donc jauger l'ampleur du creux ou du boom conjoncturel. Si l'économie connaît un creux, on peut ensuite déterminer le manque à gagner en termes de revenus, notamment fiscaux, lié à ce creux ou le surcroît de dépenses, notamment sociales, qui en découle. Si l'économie connaît un boom, on peut évaluer le surcroît de revenus ou dans quelle mesure les dépenses sont inférieures à la normale.

\subsection{Cycle conjoncturel et écart de production}

\subsubsection{PIB effectif et PIB potentiel}

On peut se prendre à rêver d'un monde où l'économie se développerait en suivant une tendance parfaitement linéaire, sans aléas conjoncturel, ni négatif, ni positif. La réalité est bien différente. La figure 53 l'illustre de manière stylisée. Elle permet 
de préciser les notions relatives au développement structurel de l'économie et aux cycles conjoncturels. Ces deux aspects sont liés puisque l'économie tend à osciller autour d'une tendance structurelle de long terme dictée essentiellement par la démographie (y compris le solde migratoire) et par l'évolution technologique. En réalité, ces oscillations ne sont pas forcément de même ampleur ni de même durée. Elles reflètent l'évolution cyclique ou conjoncturelle de l'économie. Elles découlent également de chocs aléatoires qui provoquent des écarts temporaires à la tendance. D’autres chocs - non représentés dans la figure - ont un caractère permanent et engendrent un changement de tendance. Ces chocs proviennent de changements durables dans l'évolution démographique et technologique.

L'axe horizontal porte le temps. L'axe vertical porte la mesure la plus courante de la performance d'une économie nationale, à savoir le produit intérieur brut (PIB). En l'occurrence, on représente le PIB réel, c'est-à-dire le PIB déflaté afin de neutraliser les effets de la hausse du niveau des prix. Rappelons que le PIB mesure la richesse produite par une économie nationale - c'est-à-dire par les ménages, les entreprises et les administrations publiques localisés, dans le cas qui nous occupe, en Suisse - au cours d'une période donnée (généralement pendant une année). Par richesse, il faut entendre la valeur des biens et des services produits, pour autant que ces biens et services ne soient pas des intrants, c'est-à-dire ne soient pas des biens et services ayant servi à produire d'autres biens et services dans le pays. C'est pourquoi le PIB correspond à la seule valeur ajoutée dans le pays au cours de la période considérée.

Figure 53 Principales phases et situations à l'intérieur d'un cycle conjoncturel.

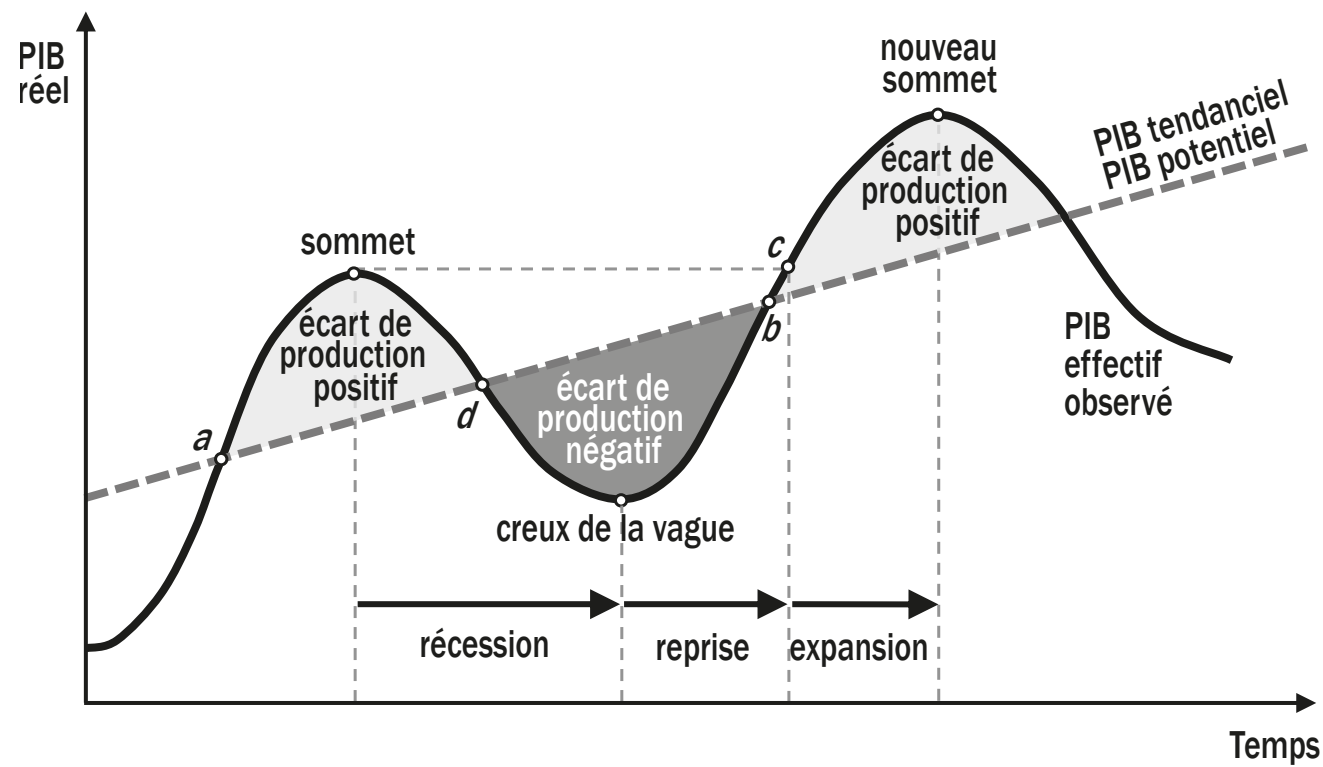

Source: Soguel (2011, p. 10). 
La courbe à l'allure sinusoïdale retrace l'évolution effective de la richesse produite. C'est celle qui est observée et documentée dans les statistiques officielles. La droite croissante en trait discontinu correspond à l'évolution tendancielle ou structurelle de cette richesse. Dit autrement, cette droite indique quelle serait potentiellement la richesse produite par l'économie nationale si tous les facteurs de production - le travail et le capital - étaient utilisés pleinement et normalement. C'est pourquoi on parle souvent de PIB potentiel ou de PIB tendanciel. En réalité, ce dernier n'évolue évidemment pas en suivant une droite, pas davantage que le PIB effectif évolue en suivant une sinusoïde. Il ne s'agit là que d'une représentation stylisée.

\subsection{2 Écart de production}

L'écart qui sépare la richesse effectivement générée par l'économie au cours d'une année donnée d'une part et la richesse qui aurait pu être potentiellement produite d'autre part nous renseigne sur l'ampleur de l'aléa conjoncturel. On parle ici d'écart de production (output gap).

À certains moments, l'économie génère moins de richesse qu'elle ne le ferait en temps normal. Par rapport à la tendance, on est en présence d'un écart de production négatif. On se trouve dans une phase de sous-emploi des facteurs de production ou de basse conjoncture marquée particulièrement par une remontée du chômage. Cette phase est également marquée par des revenus, des bénéfices, des ventes, etc. inférieurs à ce qu'ils auraient dû être tendanciellement. En outre, elle se caractérise par un taux de chômage supérieur à son niveau naturel.

À d'autres moments, l'économie génère davantage de production que si elle fonctionnait normalement. Par rapport au potentiel, on se trouve confronté à un écart de production positif. On dit que l'économie est en phase de surchauffe ou de haute conjoncture avec un risque de hausse généralisée des prix comme symptôme marquant. Cette phase coïncide avec des revenus, des bénéfices, des ventes, etc. supérieurs à la tendance. Quant au taux de chômage, il est inférieur à son niveau naturel.

Le passage d'un écart positif à un écart négatif correspond à une bascule conjoncturelle. Un cycle conjoncturel complet voit se succéder un écart de production positif et un écart négatif. Un cycle va par exemple du point $a$ au point $b$.

La figure permet également de préciser différentes notions. Techniquement, une phase de récession ne coïncide pas avec une phase de basse conjoncture. Elle s'insère entre le sommet d'un cycle et le creux qui lui succède. Au cours de cette phase, le taux de variation du PIB est négatif et le PIB diminue. L'économie est en décroissance. La phase de reprise s'étend du creux de la vague jusqu'au moment où le PIB réel regagne un niveau équivalent à celui qu'il connaissait à l'apogée du cycle précédent (point $c$ ). À cette phase de reprise succède une phase d'expansion: elle s'étend jusqu'au sommet conjoncturel suivant. Tant durant la phase de reprise que durant la phase d'expansion, le taux de variation du PIB est positif. L'économie est en croissance. 
On constate ainsi que les phases de récession, de reprise et d'expansion ne correspondent pas aux épisodes de basse conjoncture et de haute conjoncture. Au sommet, lorsque l'écart de production positif est à son maximum, le taux de variation du PIB s'inverse et devient négatif. L'économie entre en récession. Mais l'écart de production reste positif pour un temps, jusqu'à ce que le point $d$ soit dépassé. Ensuite, l'écart devient négatif et c'est au creux de la vague qu'il est le plus important. À ce moment-là, on assiste à un retournement conjoncturel car le taux de variation du PIB redevient positif. Toutefois, il faut attendre que la reprise atteigne le point $b$ pour s'extraire de l'écart de production négatif.

\subsubsection{Estimation de l'écart de production}

En principe, il est facile d'estimer l'écart de production. Il suffit pour cela de connaître le niveau de production effectif et le niveau potentiel. En pratique, deux difficultés apparaissent. D’abord, il faut estimer le niveau de production potentiel. En effet, le PIB potentiel correspond à une situation virtuelle dans laquelle tous les facteurs de production seraient pleinement utilisés. Il n'est donc pas observable. Ensuite, s'agissant du niveau effectif, en Suisse, l'Office fédéral de la statistique publie des chiffres pour l'ensemble du pays et pour chaque canton. Par contre, il n'existe pas de statistique à l'échelon communal. Par conséquent, lorsque l'on souhaite déterminer la part structurelle et la part conjoncturelle du solde budgétaire d'une commune, il faut remédier à cette lacune. Pour surmonter ces deux difficultés, des solutions pragmatiques, simples existent.

Pour estimer le PIB potentiel, différentes approches existent. La plus accessible consiste à extraire la composante tendancielle du PIB à partir des données sur le PIB effectif observé, mesuré en termes réels ${ }^{176}$. Pour y parvenir, on peut utiliser une moyenne mobile, par exemple d'ordre 5. Avec cette solution, le PIB potentiel estimé pour l'Année $t$ correspond à la moyenne du PIB réel effectif observé des Années t-2, $\mathrm{t}-1, \mathrm{t}, \mathrm{t}+1$ et $\mathrm{t}+2$; donc à une moyenne du PIB sur cinq ans :

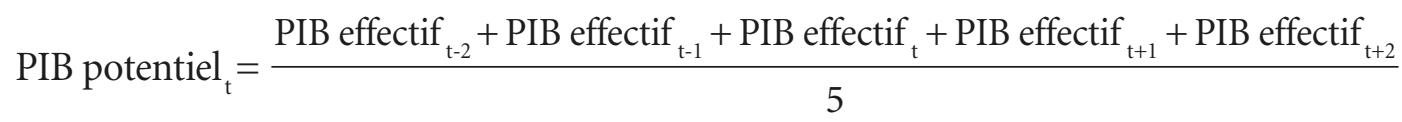

La majorité des analyses empiriques suivent cette approche, parfois avec des raffinements techniques. C'est le cas de la Confédération. Cette dernière utilise un filtre basé également sur une moyenne mobile, mais dans laquelle les années les plus récentes sont surpondérées par rapport aux années les plus anciennes ${ }^{177}$.

176 Une approche alternative est utilisée par l'Organisation de coopération et de développement économiques (OCDE) ou la Banque nationale suisse. Elle consiste d'abord à modéliser la manière dont l'économie nationale mobilise le travail et le capital dans le pays (modélisation d'une fonction de production). Elle consiste ensuite à utiliser ce modèle pour estimer le volume de production pouvant être potentiellement généré avec le travail et le capital disponible dans le pays à un moment donné.

177 Le filtre utilisé par la Confédération est une version modifiée du filtre proposé par Hodrick et Prescott (1980). 
Plus le lissage s'effectue sur un nombre important d'années, plus la technique de filtrage est gourmande en données. Il faut alors disposer d'une série du PIB sur quelques années. On en dispose pour l'économie suisse dans son ensemble depuis longtemps. C'est aussi le cas pour les cantons puisqu'une statistique est publiée depuis 2008 sur le PIB de chacun des cantons. Nous ne disposons cependant d'aucune statistique pour les communes. Il faut toutefois se rappeler que la Suisse est une petite économie et que cette économie est ouverte. Par conséquent, de légères différences peuvent certes exister dans le calendrier des différentes phases du cycle conjoncturel et dans l'importance de l'écart de production. Cependant, on peut raisonnablement postuler qu'un canton ou une commune ne va pas durablement être confronté à un écart de production négatif alors que la collectivité voisine - ou l'économie nationale - connaît un écart positif. C'est pourquoi une solution de repli consiste à estimer le PIB de la collectivité qui nous intéresse - canton avant 2008 ou commune - sur la base du PIB national. Toutefois, il faut tenir compte de l'évolution de l'effectif de population. En effet, la croissance démographique est, avec l'évolution technologique, le facteur qui détermine le développement du PIB potentiel. Or la démographie cantonale peut évoluer différemment de la démographie nationale et la démographie communale différemment de la démographie cantonale. Il s'agit donc d'en tenir compte. On y parvient en multipliant le PIB national par habitant par le nombre d'habitants dans la collectivité qui nous intéresse (ou alternativement pour une commune en multipliant le PIB cantonal par habitant par le nombre d'habitants de la commune). Ce faisant, on postule que l'économie de la collectivité tire parti de l'évolution technologique dans la même mesure que l'économie nationale (ou cantonale).

$$
\underset{\text { pour MaCollectivité }}{\text { PIB effectif estimé }}=\underset{(\text { ou cantonal }) \text { par habitant }}{\text { PIB effectif national }} \times \begin{gathered}
\text { Nombre d'habitants } \\
\text { de MaCollectivité }
\end{gathered}
$$

La technique de filtrage pose également problème si l'Année t est l'année la plus récente. On ne dispose alors d'aucune observation pour les années à venir, c'està-dire $t+1$ et $t+2$. Il faut alors faire une hypothèse sur la manière dont va évoluer l'économie à l'avenir afin de prolonger la série du PIB effectif observé à l'aide de prévisions. Cette hypothèse peut par exemple reprendre la tendance conjoncturelle pronostiquée par les organismes spécialisés, notamment le Groupe d'experts de la Confédération pour les prévisions conjoncturelles.

En connaissant le PIB potentiel pour l'année qui nous intéresse, l'écart de production par rapport au PIB effectif s'estime facilement par soustraction. En pratique, l'écart est estimé en pourcentage, c'est-à-dire en rapportant l'écart au PIB potentiel. L'écart de production EP en pour cent correspond donc à

$$
\mathrm{EP}=\frac{\text { PIB effectif }- \text { PIB potentiel }}{\text { PIB potentiel }}=\frac{\text { PIB effectif }}{\text { PIB potentiel }}-1
$$


Le tableau 40 montre comment estimer le PIB potentiel et l'écart de production pour MaCollectivité. Nous nous situons au début de l'Année $t+1$ au moment où nous bouclons les comptes de l'Année $t$ et où nous cherchons à déterminer la part structurelle et conjoncturelle du résultat. Nous postulons ici que l'Année t correspond à 2017. Donc au début de l'Année $t+1$, nous sommes en 2018. À ce moment-là, la statistique du PIB effectif national, celle de la population suisse et de la population de MaCollectivité pour 2017 sont déjà disponibles (ainsi que celles des années précédentes, notamment 2015 et 2016) ${ }^{178}$. Ces informations permettent d'estimer le PIB effectif pour MaCollectivité. À ce moment-là, le pronostic de croissance du PIB national pour les années 2018 et 2019 est également disponible ${ }^{179}$.

Tableau 40 Estimation du PIB potentiel et de l'écart de production.

Exemple pour MaCollectivité, considérant que l'Année t est 2017.

\begin{tabular}{|c|c|c|c|c|c|}
\hline \multirow[t]{2}{*}{ Année } & 2015 & 2016 & 2017 & 2018 & 2019 \\
\hline & $\mathrm{t}-2$ & $\mathrm{t}-1$ & $\mathrm{t}$ & $t+1$ & $t+2$ \\
\hline PIB effectif national (CHF mio) & 661248,7 & 671836,8 & 682698,3 & & \\
\hline Population suisse & 8327126 & 8419550 & 8484130 & & \\
\hline $\begin{array}{l}\text { PIB effectif national, } \\
\text { par habitant (CHF) }\end{array}$ & 79409,0 & 79794,9 & 80467,7 & & \\
\hline Population de MaCollectivité & 3002 & 3097 & 3113 & & \\
\hline $\begin{array}{l}\text { PIB effectif estimé pour } \\
\text { MaCollectivité (CHF mio) }\end{array}$ & 238,4 & 247,1 & 250,5 & & \\
\hline $\begin{array}{l}\text { Pronostic de croissance du PIB } \\
\text { pour les années à venir }\end{array}$ & & & & $2,4 \%$ & $2,0 \%$ \\
\hline $\begin{array}{l}\text { PIB effectif pronostiqué pour } \\
\text { MaCollectivité (CHF mio) }\end{array}$ & & & & 256,5 & 261,6 \\
\hline $\begin{array}{l}\text { PIB potentiel pour MaCollectivité } \\
\text { à l'Année t (CHF mio) }\end{array}$ & & & 250,8 & & \\
\hline Écart de production (CHF mio) & & & $-0,3$ & & \\
\hline $\begin{array}{l}\text { Écart de production en } \% \\
\text { par rapport au PIB potentiel }\end{array}$ & & & $-0,13 \%$ & & \\
\hline
\end{tabular}

178 La statistique du PIB effectif national sous l'angle de la production en termes réels (aux prix courants) est disponible auprès du Secrétariat d'État à l'économie: www.seco.admin.ch. La statistique de la population résidante permanente est disponible auprès de l'Office fédéral de la statistique: www.bfs.admin.ch.

179 Les prévisions conjoncturelles et le pronostic de croissance du PIB sont disponibles auprès du Secrétariat d'État à l'économie: www.seco.admin.ch. 
On s'attend à une croissance de 2,4\%, puis de 2,0\%. Sur la base de cette information, le PIB effectif peut être pronostiqué pour MaCollectivité. La moyenne mobile du PIB effectif estimé pour les années 2015, 2016 et 2017 et du PIB effectif pronostiqué pour les années 2018 et 2019 permet de dégager le PIB potentiel pour MaCollectivité à l'Année t (2017). Ce PIB potentiel s'élève à CHF 250,8 mio alors que le PIB effectif se monte à CHF 250,5 mio. Nous sommes donc en présence d'un écart de production négatif: l'économie locale a produit $\mathrm{CHF}$ 0,3 mio de moins que ce qu'elle aurait potentiellement pu. L'écart de production se monte ainsi à $-0,13 \%$ du PIB potentiel, autrement dit la production est inférieure de $0,13 \%$ à son potentiel.

Une telle démarche d'estimation peut paraître fastidieuse ou ne pas être à la portée de toutes les collectivités. Pour parer au plus pressé, on peut s'en remettre à l'écart de production estimé chaque année par la Confédération pour appliquer le frein à l'endettement prévu par la Constitution fédérale. Les comptes - et le budget - de la Confédération mentionnent la valeur d'un «facteur conjoncturel» reflétant, comme son nom l'indique, l'état de la conjoncture ${ }^{180}$. L'Administration fédérale des finances calcule ce facteur conjoncturel $k$ pour l'Année $t$ en rapportant le PIB potentiel estimé au PIB effectif:

$$
\mathrm{k}=\frac{\text { PIB potentiel }}{\text { PIB effectif }}
$$

Lorsque le PIB effectif est inférieur au PIB potentiel, la valeur du facteur $k$ est supérieure à 1,00 . Cela signale un écart de production négatif. Par contre, lorsque la valeur de $k$ est inférieure à l'unité, cela dénote un écart de production positif: le PIB effectif est supérieur au PIB potentiel.

La figure 54 illustre l'évolution du facteur $k$ depuis l'introduction du frein à l'endettement en 2003. Visuellement, on voit se dégager le mouvement d'oscillation du PIB effectif, symbolisé par les barres verticales noires, autour du PIB potentiel, normé ici à 1,00 et représenté par la ligne horizontale discontinue. Attention, l'axe vertical est inversé! Par conséquent, lorsque la valeur de k est supérieure à l'unité, la barre noire entre dans la zone foncée signalant un écart de production négatif. C'est le cas en 2017 qui est notre Année t puisque la Confédération a estimé que k est égal à 1,0090 (valeur indiquée dans la barre). L'écart de production n'a pas toujours été négatif. Entre 2004 et 2008, k est inférieur à 1,00 et les barres noires s'arrêtent dans la zone légèrement grisée correspondant à un écart de production positif. Ces années-là, l'économie nationale surperformait et produisait davantage que la normale. C'est aussi ce qui est prévu pour 2018 et 2019, ce qui est cohérent avec le pronostic conjoncturel mentionné ci-dessus (tableau 40).

180 La valeur du facteur conjoncturel est publiée chaque année dans le Compte d’État de la Confédération, plus précisément dans le Tome 1 «Rapport sur le Compte d'État» au chapitre 22 «Frein à l'endettement» (AFF 2019a). 
Figure 54 Valeurs annuelles du facteur conjoncturel k estimé pour appliquer le frein fédéral à l'endettement.

De 2003 à 2018 pour les comptes, 2019 pour le budget (B).

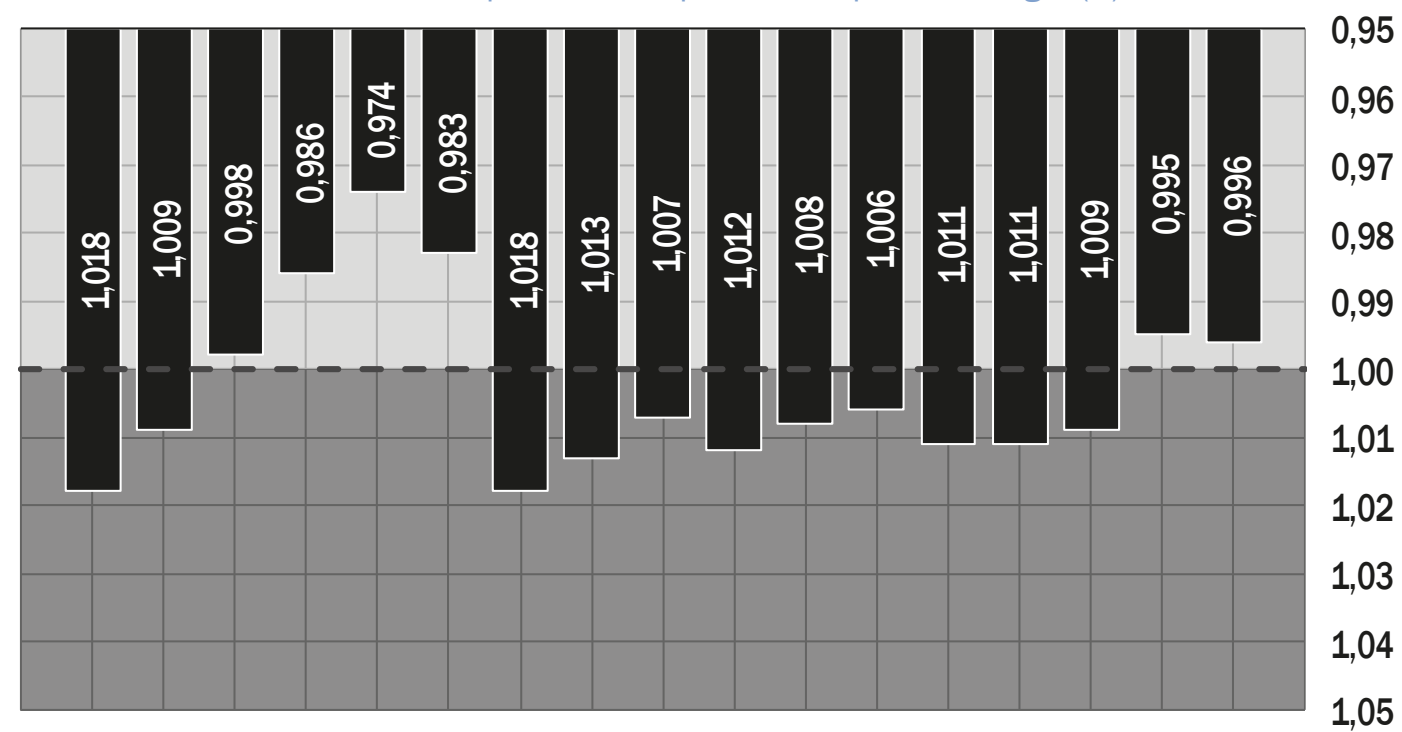

$\begin{array}{lllllllllllllllll}03 & 04 & 04 & 05 & 07 & 08 & 09 & 10 & 11 & 12 & 13 & 14 & 15 & 16 & 17 & 18 & \text { B19 }\end{array}$

écart de production négatif

- PIB potentiel par rapport à l'effectif $(k)$

Source des données: AFF (2018, 2019a). écart de production positif

- - PIB potentiel normé à 1,00

La formule de calcul du facteur k est différente de la formule de calcul habituelle de l'écart de production que nous avons mentionnée précédemment. Toutefois, le facteur $k$ peut être mis en lien avec l'écart de production EP exprimé en \%:

$$
\mathrm{k}=\frac{\mathrm{PIB} \text { potentiel }}{\mathrm{PIB} \text { effectif }}=\frac{1}{\mathrm{EP}+1}
$$

Sur la base des informations présentées dans le tableau 40, le facteur k pour l'Année t peut être estimé égal à 1,0013, comme le montre le tableau 41. Selon cette estimation, l'écart de production est inférieur à celui qu'a estimé la Confédération pour appliquer le frein à l'endettement $(\mathrm{k}=1,009)$. La différence s'explique par le fait que nous avons estimé un PIB effectif et un PIB potentiel pour MaCollectivité en extrapolant les données disponibles à l'échelon national. Elle s'explique aussi par le fait que nous avons utilisé une moyenne mobile avec un ordre relativement faible (ordre de 5) pour extraire le PIB potentiel, alors que la Confédération utilise un autre filtre. Cela montre que l'estimation du PIB potentiel est fortement tributaire de la méthode utilisée. Malgré cela, dans les deux cas, le constat demeure que l'Année t est marquée par un écart de production négatif. 
Tableau 41 Estimation du facteur conjoncturel k sur la base du PIB potentiel calculé. Exemple pour MaCollectivité, considérant que l'Année t est 2017.

\begin{tabular}{lc} 
Année & 2017 \\
& $t$ \\
\hline PIB potentiel pour MaCollectivité à l'Année t (CHF mio) & 250,8 \\
PIB effectif estimé pour MaCollectivité (CHF mio) & 250,5 \\
Facteur k estimé pour l'Année t & 1,0013
\end{tabular}

\subsection{Fluctuations budgétaires automatiques, solde conjoncturel et stabilisateurs automatiques}

\subsubsection{Fluctuations automatiques et solde conjoncturel}

Fluctuations automatiques. Pour analyser l'impact de la conjoncture sur les finances publiques, connaître le signe de l'écart de production (positif ou négatif) et son ampleur est déterminant. En effet, savoir si le PIB est supérieur ou inférieur à la normale - et de combien - permet implicitement de savoir où se situent d'autres variables par rapport à leur niveau normal, en particulier des variables directement en lien avec les recettes ou avec les dépenses des administrations publiques. On sait depuis longtemps que la conjoncture provoque des fluctuations automatiques de certaines recettes et de certaines dépenses; qu'un écart de production négatif augmente la probabilité d'un déficit et qu'un écart positif accroît celle d'un surplus (Hart 1945). L'automatisme provient des dispositions juridiques en vigueur.

Un exemple parlant est donné par les impôts dont le rendement évolue au gré du cycle économique et des fluctuations des bases imposables (revenus, bénéfices, consommation, etc.). Une haute conjoncture augmente ces bases, tandis qu'une mauvaise conjoncture les réduit. Évidemment, si le barème d'imposition est progressif, l'effet sur le rendement de l'impôt est plus que proportionnel. Autrement dit, lorsqu'une récession se développe, le rendement diminue plus fortement que la contraction de l'assiette fiscale. En phase de boom, l'inverse se produit. Le rendement de l'impôt augmente plus rapidement que la base d'imposition. Avec un barème proportionnel, la relation entre la base d'imposition et le rendement est, elle aussi, proportionnelle. Mais quel que soit le barème, l'effet sur les recettes fiscales est automatique. Aucune modification de la législation n'est nécessaire pour que le rendement de l'impôt augmente ou diminue. D'autres exemples de fluctuations automatiques sont présentés ci-après (voir les stabilisateurs automatiques) ${ }^{181}$.

181 Ces diverses prescriptions légales, en particulier lorsqu'elles sont constitutionnelles, constituent des compétences liées au sens du droit administratif. En effet, le Législatif - et l'Exécutif - sont obligés de les utiliser, de s'y plier, qu'ils le veuillent ou non. 
Solde conjoncturel. L'effet des variations conjoncturelles de l'environnement macroéconomique sur les budgets des administrations publiques est schématisé dans la figure 55. Ces variations expliquent une partie du solde opérationnel et du solde de financement présenté dans la comptabilité d'une collectivité182. Il s'agit de la composante conjoncturelle du solde, qualifiée de solde conjoncturel pour faire court. Ce solde sera un surplus conjoncturel lorsque l'écart de production est positif; il sera un déficit conjoncturel lorsque l'écart de production est négatif. Par conséquent, en théorie, au cours d'un cycle économique, le surplus conjoncturel compense le déficit conjoncturel. L'équilibre à long terme du budget n'est donc pas menacé par la composante conjoncturelle du solde, qu'il s'agisse d'un surplus ou d'un déficit.

Figure 55 Fluctuations budgétaires automatiques et solde conjoncturel, impact budgétaire des impulsions discrétionnaires et solde structurel.

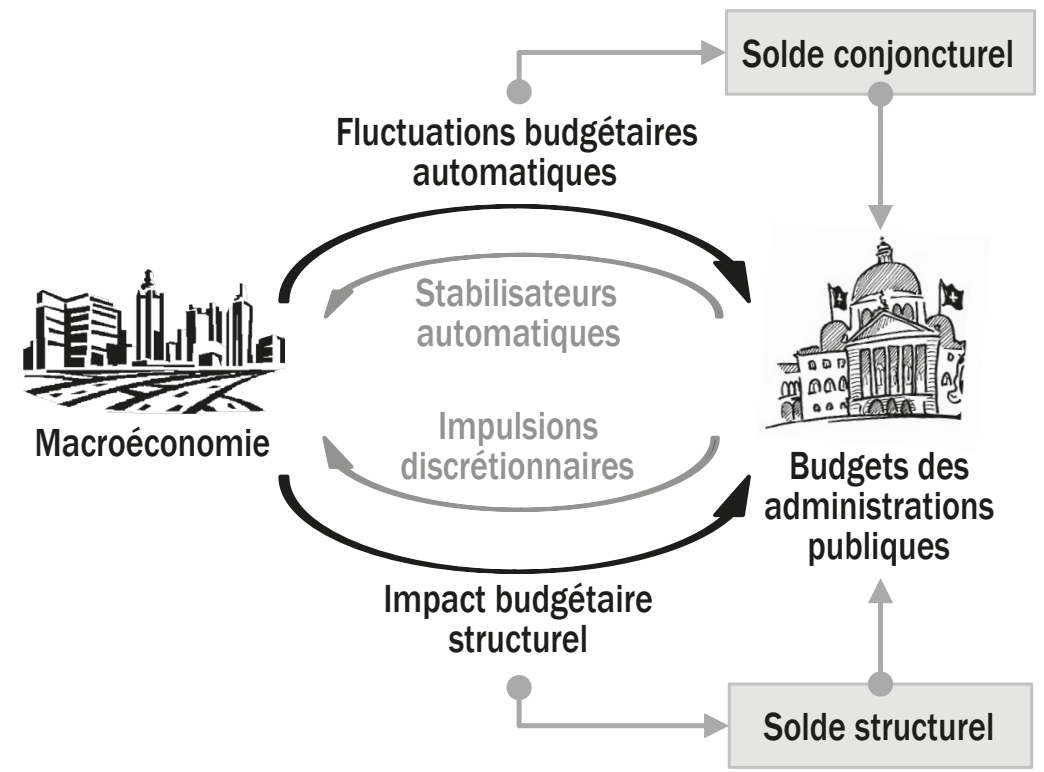

\subsubsection{Stabilisateurs automatiques}

Les fluctuations budgétaires automatiques engendrent à leur tour un phénomène spontané, celui des stabilisateurs automatiques. Ce sont « des mécanismes qui, même en l'absence de modifications délibérées des politiques économiques, tendent spontanément à freiner ou à stimuler l'activité» (Ammann 2009, p. 6). Lorsque l'écart de

182 Par contre, l'évolution conjoncturelle n'influence pas automatiquement le solde extraordinaire. Les charges et les revenus extraordinaires qui composent ce dernier sont purement discrétionnaires lorsqu'ils découlent de la tactique politique en matière de présentation des comptes. Pour ce qui est des charges et des revenus véritablement extraordinaires, ils sont totalement découplés de l'activité économique. 
production est négatif, le mécanisme réduit certains prélèvements fiscaux et laisse davantage de pouvoir d'achat aux ménages et aux entreprises. L'effet est amplifié par un barème progressif. Inversement, en cas d'écart positif, le mécanisme accroît spontanément le montant prélevé par les impôts et réduit le pouvoir d'achat.

Les prestations en cas de chômage constituent un autre mécanisme de stabilisation automatique. Ce mécanisme agit à travers les finances des administrations publiques qui en sont responsables. En Suisse, il touche donc essentiellement l'assurance-chômage $^{183}$. Il agit à la fois du côté des dépenses (prestations globalement moindres en haute conjoncture et plus élevées en basse conjoncture) et du côté des recettes (primes prélevées plus élevées au total lorsque l'écart de production est positif que lorsqu'il est négatif). Ici aussi, le mécanisme agit globalement sur le pouvoir d'achat des ménages. Il le maintient en phase de basse conjoncture, contribuant à éviter une chute plus importante. Il le freine en période de haute conjoncture, aidant à contenir la surchauffe de l'économie.

\subsubsection{Estimation de la part conjoncturelle des recettes et des dépenses et estimation du solde conjoncturel}

Élasticité des recettes. La conjoncture n'affecte automatiquement et de manière significative qu'un nombre restreint de postes budgétaires. Pour l'essentiel, cela ne concerne que les recettes fiscales. Afin d'estimer le volume de recettes apporté par un écart de production positif ou celui qui manque en raison d'un écart de production négatif, il faut pouvoir évaluer la sensibilité des recettes aux variations du PIB. Dans le jargon, on parle de l'élasticité des recettes en fonction du PIB. Concrètement, il s'agit de la variation des recettes, mesurée en pourcentage, provoquée par une variation du PIB de 1\%. Cette sensibilité, cette élasticité $\alpha$, correspond donc à l'expression suivante:

$$
\text { Élasticité } \alpha=\frac{\text { variation des recettes en } \%}{\text { variation du PIB en } \%}
$$

L'estimation de la valeur de a nécessite des méthodes impliquant un savoirfaire économétrique ${ }^{184}$. Ce savoir-faire n'est pas toujours disponible. Pour parer au plus pressé, on peut s'en remettre aux estimations réalisées par l'OCDE (Price

183 En vertu des conventions de la statistique financière, les assurances sociales obligatoires - dont l'assurancechômage - font partie du cercle des administrations publiques (section 10.4).

184 L'estimation de a s'effectue par une analyse de régression dans laquelle les recettes sont la variable dépendante et le PIB la variable indépendante. Il faut disposer d'une série historique la plus longue possible. Au cours de l'histoire, l'évolution des recettes est soumise à bien d'autres aléas que la seule évolution du PIB. Cela peut être une modification du taux d'imposition ou de la définition de l'assiette fiscale, l'introduction d'une correction de la progression à froid, le passage d'un système prenumerando à un système postnumerando, etc. L'influence de ces éléments sur les recettes doit être neutralisée. On y parvient en ajoutant diverses variables de contrôle aux côtés du PIB. 
et al. 2015) ${ }^{185}$. Pour l'ensemble des collectivités publiques suisses, ces estimations indiquent une élasticité de 1,93 pour les recettes de l'impôt sur le revenu des personnes physiques et de 1,80 pour les recettes de l'impôt sur le bénéfice des personnes morales. Autrement dit, lorsque le PIB augmente (se contracte) de 1,00\%, les recettes de l'impôt sur le revenu s'accroissent (diminuent) de 1,93\% et celles de l'impôt sur le bénéfice de 1,80\%.

Le fait que les recettes réagissent plus que proportionnellement est logique. D'abord parce que le barème d'imposition est progressif, en particulier s'agissant de l'impôt sur le revenu, ensuite parce que la substance fiscale, et singulièrement le bénéfice, est très sensible aux variations du PIB. D'ailleurs pour les recettes des impôts indirects - dont le barème est souvent proportionnel et dont l'assiette fiscale est peu sensible aux fluctuations du PIB, Price et al. (2015) estiment que l'élasticité se limite à 1,09 .

Part conjoncturelle dans les recettes. La part conjoncturelle dans les recettes s'obtient en déduisant des recettes effectives la part qui ne varie pas en fonction du cycle conjoncturel. Il faut donc déduire dans les recettes effectives les recettes structurelles:

recettes conjoncturelles $=$ recettes effectives - recettes structurelles

Les recettes structurelles sont celles dont bénéficierait la collectivité si le PIB se situait à son niveau normal, c'est-à-dire à son niveau potentiel. Pour les obtenir, il faut neutraliser l'effet de l'écart de production (concrètement l'effet du facteur k) associé à l'élasticité, en fonction de l'expression suivante, proposée par Luck (2011):

Donc

$$
\text { recettes structurelles }=\mathrm{k}^{\mathrm{a}} \times \text { recettes effectives }
$$

recettes conjoncturelles $=$ recettes effectives $-\left(k^{a} \times\right.$ recettes effectives $)$

et par conséquent

recettes conjoncturelles $=\left(1-\mathrm{k}^{a}\right) \times$ recettes effectives

Sur cette base, le tableau 42 montre comment les recettes fiscales conjoncturelles de l'Année t peuvent être estimées pour MaCollectivité. Pour tester la sensibilité des résultats à l'estimation de l'écart de production, nous recourons à deux scénarios. Le premier se fonde sur le facteur k estimé par la Confédération pour son frein à l'endettement $(\mathrm{k}=1,009)$; le second sur le facteur $\mathrm{k}$ estimé à l'aide de notre méthode pragmatique $(\mathrm{k}=1,0013)$. On aboutit ainsi à deux évaluations des recettes

185 L'OCDE publie de temps à autre de nouvelles estimations des élasticités. Avec le développement des bases et des outils statistiques, la précision de ces estimations s'améliore. Une marge d'erreur d'estimation subsiste. Il faut donc conserver une distance critique face aux chiffres publiés. Cependant, les estimations mentionnées ici nous semblent correspondre à une certaine réalité cantonale et communale en Suisse. 
conjoncturelles, l'une haute, l'autre basse. Rappelons que l'impôt sur les personnes physiques provient essentiellement de l'impôt sur le revenu et que l'impôt sur les personnes morales provient surtout de l'impôt sur le bénéfice. D'après les informations figurant dans les comptes, le premier apporte des recettes pour KCHF 6231 et le second pour KCHF 501. Nous utilisons les élasticités estimées par Price et al. (2015), soit 1,93 et 1,80. L'évaluation haute montre que l'écart de production négatif conduit à des recettes inférieures de KCHF -109 au niveau structurel des impôts sur les personnes physiques et inférieures de KCHF -8 s'agissant de l'impôt sur les personnes morales. Dans le cadre de l'évaluation basse, le manque à gagner se limite à $\mathrm{KCHF}-16$ pour les personnes physiques et à $\mathrm{KCHF}-1$ pour les personnes morales.

Tableau 42 Estimation des recettes fiscales conjoncturelles.

Exemple pour MaCollectivité, Année t, deux scénarios.

\begin{tabular}{|c|c|c|c|c|}
\hline \multirow[b]{3}{*}{ Facteur k } & \multicolumn{4}{|c|}{ Impôt sur les personnes ... } \\
\hline & \multicolumn{2}{|c|}{... physiques } & \multicolumn{2}{|c|}{... morales } \\
\hline & 1,009 & 1,0013 & 1,009 & 1,0013 \\
\hline Recettes effectives (KCHF) & \multicolumn{2}{|c|}{6231} & \multicolumn{2}{|r|}{501} \\
\hline Élasticité $\alpha$ & \multicolumn{2}{|c|}{1,9300} & \multicolumn{2}{|r|}{1,8000} \\
\hline$k^{\alpha}$ & 1,0174 & 1,0025 & 1,0163 & 1,0023 \\
\hline $1-k^{\alpha}$ & $-0,0174$ & $-0,0025$ & $-0,0163$ & $-0,0023$ \\
\hline Recettes conjoncturelles (KCHF) & -109 & -16 & -8 & -1 \\
\hline
\end{tabular}

Élasticité des dépenses. Nous l'indiquions ci-dessus: l'essentiel des dépenses des collectivités publiques suisses s'avère indépendant du cycle conjoncturel. Certes, les prestations allouées en cas de chômage sont fréquemment considérées comme fluctuant avec la conjoncture. Toutefois en Suisse, elles sont, avec d'autres prestations de ce type, versées par les assurances sociales - ces dernières sont des entités indépendantes des collectivités publiques, qu'il s'agisse de la Confédération, des cantons ou des communes. Ceci explique pourquoi l'élasticité des dépenses publiques estimée par Price et al. (2015) est pour ainsi dire insignifiante:-0,13. Autrement dit, lorsque le PIB diminue (augmente) de 1,00\%, les dépenses des collectivités publiques s'accroissent (se contractent) de $0,13 \%$ sous l'effet spécifique des fluctuations automatiques.

Part conjoncturelle dans les dépenses. À partir de là, les dépenses conjoncturelles s'estiment également en neutralisant l'effet de l'écart de production (concrètement du facteur k) associé à l'élasticité, sur les dépenses effectives, en fonction de l'expression suivante:

dépenses conjoncturelles $=\left(1-\mathrm{k}^{\beta}\right) \times$ dépenses effectives 
Le tableau 43 montre comment les dépenses conjoncturelles de l'Année t peuvent être estimées pour MaCollectivité. À nouveau, les deux estimations concurrentes de l'écart de production sont utilisées pour tester la sensibilité des résultats $(\mathrm{k}=1,009$ et $\mathrm{k}=1,0013)$. Puisque nous ne disposons que d'une estimation de l'élasticité pour l'ensemble des dépenses $(-0,1300)$, nous l'appliquons à l'ensemble des charges opérationnelles, en prenant soin d'en exclure les amortissements, ainsi que les attributions aux financements spéciaux et aux fonds; d'abord parce que ces opérations sont uniquement comptables n'entraînant pas de décaissement et ensuite parce qu'elles ne sont en aucun cas tributaires automatiquement de la conjoncture. Le volume des dépenses considérées comme potentiellement influencées par le cycle conjoncturel s'élève ainsi à KCHF 12614 . Selon l'évaluation haute, l'écart de production négatif conduit à des dépenses supérieures de KCHF 15 au niveau structurel des dépenses. Selon l'évaluation basse, le surcroît de dépenses se limite à KCHF 2. Ainsi que nous nous y attendions, l'effet concret est donc faible.

Tableau 43 Estimation des dépenses conjoncturelles.

Exemple pour MaCollectivité, Année t, deux scénarios.

\begin{tabular}{lcc} 
Facteur $k$ & 1,0090 & 1,0013 \\
Dépenses effectives (KCHF) & \multicolumn{2}{c}{12614} \\
Élasticité $\beta$ & \multicolumn{2}{c}{$-0,1300$} \\
$\mathrm{k}^{\beta}$ & 0,9988 & 0,9998 \\
$1-\mathrm{k}^{\beta}$ & 0,0012 & 0,0002 \\
Dépenses conjoncturelles (KCHF) & 15 & 2
\end{tabular}

Solde conjoncturel. Connaissant le volume des dépenses et des recettes conjoncturelles, il devient possible de dégager l'importance du solde conjoncturel:

solde conjoncturel $=$ recettes conjoncturelles - dépenses conjoncturelles

L'écart de production, parce qu’il est négatif, détériore le résultat en raison du manque à gagner en matière de recettes fiscales et en raison du surcroît de dépenses. Globalement, par rapport à une situation où la situation conjoncturelle serait équilibrée, avec un PIB effectif équivalent au niveau potentiel, l'écart de production négatif détériore le résultat de $\mathrm{KCHF}-132$ selon l'estimation haute et de KCHF -19 selon l'estimation basse (tableau 44). Cet écart de production conduit donc à un excédent conjoncturel de charges ou, vu autrement, à une insuffisance conjoncturelle de revenus. 
Tableau 44 Estimation du solde conjoncturel.

Exemple pour MaCollectivité, Année t, deux scénarios.

\begin{tabular}{lrr}
\hline Facteur k & 1,0090 & 1,0013 \\
\hline Recettes conjoncturelles (KCHF) & -117 & -17 \\
Dépenses conjoncturelles (KCHF) & 15 & 2 \\
Solde conjoncturel (KCHF) & -132 & -19
\end{tabular}

\subsection{Mesures discrétionnaires et solde structurel}

\subsubsection{Impulsions et mesures discrétionnaires}

Impulsions discrétionnaires. Les impulsions discrétionnaires correspondent aux décisions prises spécifiquement et ponctuellement par les administrations publiques. Au cours de l'histoire récente, la Confédération a par exemple lancé divers programmes d'impulsion pour lutter contre les épisodes de basse conjoncture. Elle a également pris des mesures pour lutter contre le renchérissement en période de surchauffe (Ammann 2011). Le substantif «impulsion» traduit le fait qu'une variation du niveau des dépenses publiques ou des prélèvements obligatoires influence la demande globale (les commandes), puis la production et l'emploi. L'épithète « discrétionnaire» provient aussi bien du droit administratif que du langage commun. Il indique qu'une collectivité, et singulièrement son Législatif, font usage de la marge plus ou moins grande de liberté que leur laisse le cadre juridique existant.

Mesures discrétionnaires. Par extension, une modification du cadre légal ou une décision souveraine de la collectivité qui conduit à une modification du niveau des dépenses ou des recettes est également une mesure discrétionnaire. Un changement de l'échelle de traitement de la fonction publique constitue une mesure discrétionnaire qui change le niveau des dépenses. Une baisse ou une hausse de la fiscalité constitue une mesure discrétionnaire qui modifie le niveau des recettes, qu'elle touche la définition de l'assiette fiscale (par exemple en introduisant de nouvelles déductions fiscales ou en les supprimant) ou le taux d'imposition (hausse ou baisse du taux ou du coefficient d'imposition). Ces mesures, qu'on le veuille ou non, influencent la demande globale.

\subsubsection{Impact budgétaire des mesures discrétionnaires et solde structurel}

Impact budgétaire structurel. Ces décisions discrétionnaires ont un impact structurel sur le budget (et sur les comptes) de la collectivité concernée. En effet, elles modifient l'équilibre budgétaire. Cependant, contrairement aux fluctuations automatiques qui compensent un déficit conjoncturel par un excédent conjoncturel ultérieur, l'impact budgétaire se fera sentir tant que la mesure discrétionnaire n’aura pas été levée. 
Solde structurel. Les mesures discrétionnaires modifient la structure du budget. Elles sont ainsi à l'origine de la composante structurelle du solde budgétaire (figure 55). Les mesures discrétionnaires qui augmentent les dépenses ou réduisent les recettes détériorent le solde structurel. Elles creusent un déficit structurel préexistant ou réduisent un excédent structurel préexistant. Les mesures discrétionnaires qui réduisent les dépenses et augmentent les recettes augmentent l'excédent structurel préexistant ou réduisent un déficit structurel préexistant.

\subsubsection{Estimation du solde structurel}

Connaissant la composante conjoncturelle du résultat présenté dans les comptes (tableau 44), il devient aisé d'estimer la composante structurelle. On y parvient en déduisant le solde conjoncturel du résultat effectif:

$$
\text { solde structurel }=\text { solde effectif }- \text { solde conjoncturel }
$$

Solde opérationnel structurel. Dans les cantons et dans les communes, on s'intéressera avant tout à la part structurelle du solde opérationnel du compte de résultats. En effet, ce dernier est au centre du dispositif. Dans la plupart des cantons, le solde du compte de résultats est la grandeur budgétaire prise pour cible par les dispositions légales visant à freiner ou lutter contre l'endettement. Il s'agit également de garantir que les charges opérationnelles (c'est-à-dire sans les éléments extraordinaires) soient couvertes par les revenus opérationnels. Il serait faux de s'intéresser aux charges et aux revenus extraordinaires et de les intégrer dans la réflexion sur la composante structurelle. Rappelons en effet que les éléments extraordinaires se composent en réalité essentiellement d'opérations de tactique politique sans réalité économique. Les opérations ayant effectivement une réalité économique extraordinaires sont, par définition, à la fois rares et tout à fait ponctuelles. Par conséquent, les éléments extraordinaires n'ont rien à voir avec la structure du budget et la persistance de cette dernière à travers le temps. La gestion financière devrait donc viser à lutter contre les déséquilibres opérationnels structurels. On dégage la composante structurelle du solde opérationnel en affinant le calcul ci-dessus:

solde opérationnel structurel $=$ solde opérationnel effectif - solde conjoncturel

Le solde opérationnel effectif de MaCollectivité à l'Année t correspond à un excédent opérationnel de revenus de KCHF 1034 (tableau 17). Ce solde est repris dans le tableau 45 pour lui soustraire le solde conjoncturel que nous avons dégagé par rapport aux charges et aux revenus opérationnels. Selon l'estimation haute (donnée par le facteur $\mathrm{k}=1,0090$ ), l'écart de production négatif détériore le résultat de KCHF -132. Si la conjoncture n'apportait pas cette détérioration, le solde opérationnel serait d'autant plus élevé. Par conséquent, le résultat opérationnel structurel 
montrerait un excédent de revenus de KCHF 1166. L'estimation basse $(\mathrm{k}=1,0013)$ de l'écart de production montre un excédent structurel de revenus opérationnels de KCHF 1053. L'estimation de KCHF 1166, respectivement KCHF 1053, correspond au solde opérationnel qu'enregistrerait MaCollectivité si la conjoncture était équilibrée, c'est-à-dire s'il n'y avait aucun écart de production, ni négatif, ni positif. Donc un déséquilibre structurel positif du budget de MaCollectivité existe. Compte tenu de ce résultat, et avec prudence, MaCollectivité pourrait envisager soit de revoir à la hausse ses prestations (et ses charges), soit de revoir à la baisse sa fiscalité.

\section{Tableau 45 Estimation du solde opérationnel structurel. \\ Exemple pour MaCollectivité, Année t, deux scénarios.

\begin{tabular}{lcr|}
\hline Facteur k & 1,0090 & 1,0013 \\
\hline Solde opérationnel effectif (KCHF) & \multicolumn{2}{c}{1034} \\
Solde conjoncturel (KCHF) & -132 & -19 \\
Solde opérationnel structurel (KCHF) & 1166 & 1053
\end{tabular}

Il en irait tout autrement si l'analyse montrait un déséquilibre négatif, c'est-àdire un excédent opérationnel structurel de charges. Pour illustrer ce cas et pour les besoins de la démonstration, imaginons qu'à l'Année t, l'écart de production ne soit pas légèrement négatif comme nous l'avons estimé précédemment ( $\mathrm{EP}=-0,13 \%)$, mais massivement positif $(\mathrm{EP}=+10,00 \%)^{186}$. Le tableau 46 présente ce scénario et, en même temps, récapitule la démarche d'estimation du solde structurel. Si le PIB effectif était supérieur de 10\% du PIB potentiel, le facteur $\mathrm{k}$ s'élèverait à 0,91 [=1/ $(0,10+1)]$. En utilisant les mêmes élasticités que précédemment, le solde conjoncturel estimé s'élèverait à $\mathrm{KCHF}+1283$. Donc le résultat d'exploitation serait supérieur de $\mathrm{KCHF}+1283$ à ce qu'il serait en l'absence d'écart de production $(\mathrm{k}=1)$. En neutralisant cet effet, on aboutit à un solde opérationnel structurel de KCHF -249. Autrement dit, la situation conjoncturelle extrêmement favorable dissimulerait la réalité: alors que les comptes montreraient un excédent opérationnel de revenus de KCHF 1034, le budget de MaCollectivité serait en réalité structurellement déséquilibré avec un excédent structurel de charges. Dans un tel cas, MaCollectivité devrait s'abstenir de toute hausse des prestations ou de toute baisse de la fiscalité. Au contraire, elle devrait plutôt envisager de réduire ses charges et d'accroître ses revenus.

La leçon à tirer de l'estimation du solde opérationnel structurel est qu'il ne suffit pas de constater que les comptes présentent un solde opérationnel positif - et cela même de manière récurrente - pour conclure qu'il existe un excédent structurel de revenus. Il faut en plus tenir compte de la situation conjoncturelle et estimer le solde structurel comme nous l'avons montré ci-dessus.

186 Nous utilisons ce chiffre pour les besoins de la démonstration. Un tel écart de production positif est en réalité quasi inconcevable. 
Tableau 46 Solde opérationnel structurel si l'écart de production était positif. Exemple pour MaCollectivité, Année t.

\begin{tabular}{|lr|}
\hline Écart de production en \% du PIB potentiel & $10 \%$ \\
\hline Facteur k & 0,9091 \\
\hline Impôt sur les personnes physiques & \\
\hline Recettes effectives (KCHF) & 6231 \\
\hline Élasticité $\alpha$ & 1,9300 \\
\hline $1-k^{\alpha}$ & 0,1680 \\
\hline Recettes conjoncturelles (KCHF) & 1047 \\
\hline Impôt sur les personnes morales & \\
\hline Recettes effectives (KCHF) & 501 \\
\hline Élasticité $\alpha$ & 1,8000 \\
\hline $1-k^{\alpha}$ & 0,1576 \\
\hline Recettes conjoncturelles (KCHF) & 79 \\
\hline Dépenses & \\
\hline Dépenses effectives (KCHF) & 12614 \\
\hline Élasticité $\beta$ & $-0,1300$ \\
\hline $1-k^{\beta}$ & $-0,0125$ \\
\hline Dépenses conjoncturelles (KCHF) & -157 \\
\hline Solde conjoncturel (KCHF) & 1283 \\
\hline Solde opérationnel effectif (KCHF) & 1034 \\
\hline Solde opérationnel structurel (KCHF) & -249 \\
\hline
\end{tabular}

L'expérience vécue par les collectivités qui ont abaissé significativement leur fiscalité, entre grosso modo 2004 et 2008, l'illustre. Au cours de ces années, les collectivités bénéficiaient d'un excédent conjoncturel de revenus, d'un solde conjoncturel positif, lié à un écart de production positif. La figure 54 le montre bien: le facteur k estimé par les services de la Confédération est inférieur à l'unité; il signale que le PIB effectif est supérieur au PIB potentiel. Or certaines collectivités ont saisi l'opportunité des recettes fiscales additionnelles générées grâce à une performance économique au-dessus de la normale pour réduire leur fiscalité. Elles ont ainsi participé à l'essor de la concurrence fiscale qui s'est manifestée de manière particulièrement visible pendant ces années-là. Toutefois, réduire la fiscalité modifie structurellement les équilibres budgétaires. Dans les faits, quelques collectivités ont créé un déficit structurel ou l'ont aggravé. Cette situation n'est devenue apparente qu'ultérieurement, lorsque la conjoncture a basculé, passant d'un écart de production positif à un écart négatif (dès 2009 dans la figure 54). De ce fait, l'excédent conjoncturel de revenus s'est transformé en un excédent conjoncturel de charges. Le solde conjoncturel a alors détérioré le solde présenté dans les comptes - alors qu'auparavant il l'améliorait, faisant apparaittre ici et là des excédents structurels de charges significatifs. 
La figure 56 offre un diagramme décisionnel qui souligne l'importance du diagnostic à poser avant toute mesure discrétionnaire, pour autant que l'on vise à préserver un équilibre budgétaire. Des mesures provoquant une baisse structurelle des recettes courantes (par exemple suite à une baisse de la fiscalité) ou une hausse structurelle des dépenses courantes ne devraient être envisagées que si l'on est sûr d'être en présence d'un excédent structurel significatif de revenus ${ }^{187}$. A contrario, si le diagnostic montre la présence d'un excédent structurel significatif de charges, des mesures de rééquilibrage devraient être envisagées: soit une hausse structurelle des recettes courantes (par exemple en relevant la fiscalité), soit une baisse structurelle des dépenses courantes ${ }^{188}$. Si le diagnostic montre que le solde opérationnel est proche de zéro, alors mieux vaut éviter de prendre des mesures asymétriques qui déséquilibreraient le budget. Par contre, des mesures symétriques structurelles peuvent être envisagées: une hausse structurelle des dépenses devrait s'accompagner d'une hausse structurelle des recettes (autrement dit, «le financement des nouvelles dépenses devrait être assuré») et une baisse structurelle des recettes devrait aller de pair avec une baisse structurelle des dépenses.

Figure 56 Solde opérationnel structurel et mesures discrétionnaires.

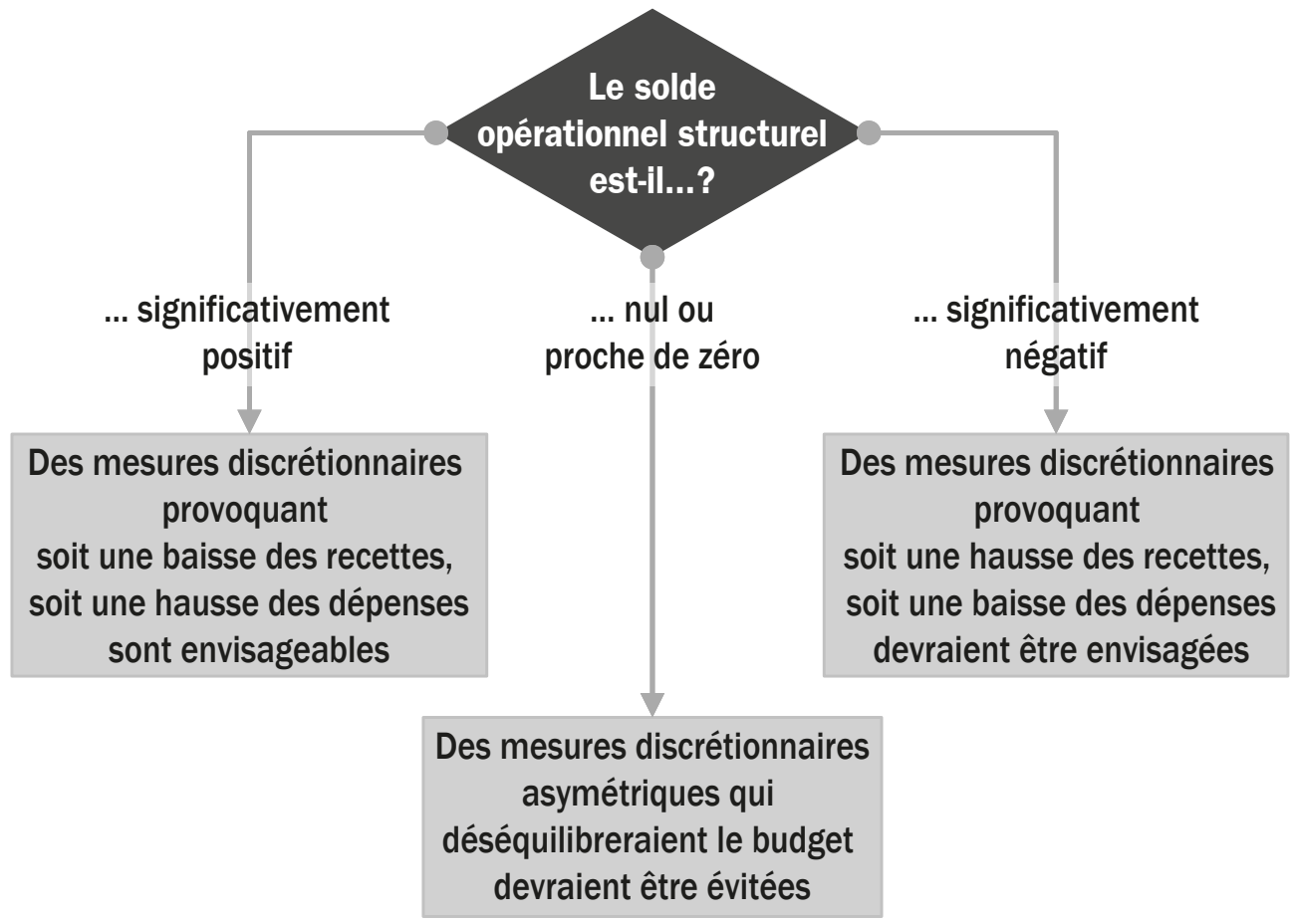

187 Le niveau à partir duquel considérer qu'un excédent structurel de revenus est significatif est sujet à appréciation. Dans l'évaluation de l'indicateur de couverture des charges, nous considérons qu'au-delà de 10,00\% un excédent opérationnel de revenus devient excessif: la note évaluative passe en dessous de 5,00 (section 9.3.1). Un excédent structurel de revenus de cet ordre-là, s'il se répète, devrait assurément conduire à rééquilibrer la structure du budget.

188 Dans l'évaluation de l'indicateur de couverture des charges, un excédent opérationnel de charges supérieur à $1,00 \%$ conduit déjà à une note évaluative inférieure à 5,00. Et un excédent de charges de 2,50\% à une note de 4,00 (cf. section 9.3.1). Sur cette base, on peut considérer qu'un excédent structurel récurrent de charges, même de relativement faible importance, devrait alerter sur la nécessité de rééquilibrer la structure du budget. 
Solde de financement structurel. Nous nous sommes ci-dessus intéressé au résultat opérationnel, puisque le compte de résultats est au centre des préoccupations des collectivités locales dans leur propre gestion financière. Cependant, il est également possible d'estimer le solde de financement structurel. Ce solde correspond à la différence entre les recettes totales et les dépenses totales qui apparaîtrait si la conjoncture était équilibrée. Cela implique de neutraliser l'effet du solde conjoncturel sur le solde de financement effectif.

Rappelons que les recettes totales englobent à la fois les recettes enregistrées dans le compte de résultats (les recettes courantes) et celles qui sont enregistrées dans le compte des investissements (les recettes d'investissement). De leur côté, les dépenses totales englobent les dépenses courantes et les dépenses d'investissement. Le solde de financement indique donc dans quelle mesure une collectivité doit recourir à l'emprunt pour financer l'ensemble de ses dépenses ou si, au contraire, ses recettes sont suffisantes.

Le solde de financement ne sert toutefois pas qu'à renseigner sur le volume emprunté ou remboursé. Il permet aussi de porter une appréciation sur l'impact macroéconomique du budget de la collectivité. En effet, tant les dépenses que les recettes influencent la demande globale. Or la demande globale est un des éléments clés qui sous-tend l'activité économique et donc le PIB. Les dépenses des collectivités contribuent à cette demande. De leur côté, les recettes des collectivités publiques sont essentiellement le résultat des prélèvements opérés auprès des ménages et des entreprises. Par conséquent, les recettes des collectivités privent les ménages et les entreprises d'une partie de leur pouvoir d'achat. Cela contribue négativement à la demande globale. Connaître la différence entre les dépenses et les recettes totales d'une collectivité offre une première indication sur sa contribution à la demande globale.

Le compte de financement utilisé par la Confédération apporte directement cette information. Il n'est toutefois pas utilisé par les cantons et les communes. Mais les informations contenues dans le compte de résultats et celles qui sont contenues dans le compte des investissements permettent de dégager le solde de financement. Pour cela, il faut se concentrer sur les rubriques qui concernent des recettes ou des dépenses. Les opérations non monétaires, purement comptables, telles que les amortissements, les régularisations (actifs et passifs transitoires), la constitution ou la dissolution de provisions, sont donc laissées de côté.

Le tableau 47 présente la situation de MaCollectivité. Il montre les dépenses et les recettes courantes et d'investissement à prendre en considération conformément au $\mathrm{MCH} 2^{189}$ afin de dégager le solde de financement. Pour l'Année t, ce dernier correspond à un déficit de KCHF -264. Ce déficit s'explique par de fortes dépenses d'investissement ${ }^{190}$.

189 CDF (2008), annexe C (Indicateurs financiers: Définitions et calculs).

190 C'est d'ailleurs ce que mettent en évidence l'indicateur de l'effort d'investissement (I6) et celui de la proportion des investissements bruts (I14 ${ }^{\# \#)}$ (section 9.3). 
Tableau 47 Calcul du solde de financement.

\section{MaCollectivité, Année t, en KCHF.}

\begin{tabular}{|c|c|c|c|c|c|}
\hline $\mathrm{N}^{\circ}$ & Libellé & $\mathrm{KCHF}$ & $\mathrm{N}^{\circ}$ & Libellé & KCHF \\
\hline+30 & Charges de personnel & 2020 & +40 & Revenus fiscaux & 7651 \\
\hline+31 & $\begin{array}{l}\text { Charges de biens et services } \\
\text { et autres charges d'exploitation }\end{array}$ & 4076 & +41 & Patentes et concessions & 12 \\
\hline-3180 & Réévaluations sur créances & -8 & +42 & Taxes et redevances & 5076 \\
\hline+34 & Charges financières & 125 & +430 & Revenus d'exploitation divers & 0 \\
\hline-344 & $\begin{array}{l}\text { Réévaluations des immobilisations } \\
\text { du patrimoine financier }\end{array}$ & 0 & +439 & $\begin{array}{l}\text { Autres revenus (avec incidence } \\
\text { sur les liquidités) }\end{array}$ & 0 \\
\hline+36 & Charges de transferts & 6393 & +44 & Revenus financiers & 167 \\
\hline-364 & Réévaluations de prêts du PA & 0 & -444 & $\begin{array}{l}\text { Réévaluations des immobilisations } \\
\text { du patrimoine financier }\end{array}$ & -9 \\
\hline-365 & $\begin{array}{l}\text { Réévaluations de participations } \\
\text { du PA }\end{array}$ & 0 & -4490 & $\begin{array}{l}\text { Réévaluations du patrimoine } \\
\text { administratif }\end{array}$ & 0 \\
\hline-366 & $\begin{array}{l}\text { Amortissements des subventions } \\
\text { d'investissement }\end{array}$ & 0 & +46 & Revenus de transferts & 1373 \\
\hline+380 & $\begin{array}{l}\text { Charges extraordinaires } \\
\text { de personnel }\end{array}$ & 0 & -466 & $\begin{array}{l}\text { Dissolutions des subventions } \\
\text { d'investissement portées au passif }\end{array}$ & 0 \\
\hline+381 & $\begin{array}{l}\text { Charges extraordinaires } \\
\text { de biens et services et } \\
\text { charges d'exploitation }\end{array}$ & 0 & +481 & $\begin{array}{l}\text { Revenus extraordinaires } \\
\text { de patentes, concessions }\end{array}$ & 0 \\
\hline+3840 & $\begin{array}{l}\text { Charges financières } \\
\text { extraordinaires }\end{array}$ & 0 & +482 & Contributions extraordinaires & 0 \\
\hline \multirow[t]{3}{*}{+386} & $\begin{array}{l}\text { Charges extraordinaires } \\
\text { de transferts }\end{array}$ & 0 & +483 & Revenus extraordinaires divers & 0 \\
\hline & & & +484 & Revenus financiers extraordinaires & 0 \\
\hline & & & +486 & Revenus extraordinaires de transferts & 0 \\
\hline \multicolumn{2}{|c|}{ Dépenses courantes } & 12606 & \multicolumn{2}{|c|}{ Recettes courantes } & 14270 \\
\hline+50 & Immobilisations corporelles & 1971 & +60 & $\begin{array}{l}\text { Transferts d'immobilisations } \\
\text { corporelles dans le PF }\end{array}$ & 0 \\
\hline+51 & $\begin{array}{l}\text { Dépenses d'investissement } \\
\text { pour le compte de tiers }\end{array}$ & 0 & +61 & $\begin{array}{l}\text { Remboursements de dépenses d'in- } \\
\text { vestissement pour le compte de tiers }\end{array}$ & 0 \\
\hline \multirow[t]{2}{*}{+52} & Immobilisations incorporelles & 0 & +62 & $\begin{array}{l}\text { Transferts d'immobilisations incor- } \\
\text { porelles dans le PF }\end{array}$ & 0 \\
\hline & & & +63 & $\begin{array}{l}\text { Subventions d'investissement } \\
\text { acquises }\end{array}$ & 43 \\
\hline+54 & Prêts & 0 & +64 & Remboursements de prêts & 0 \\
\hline+55 & Participations et capital social & 0 & +65 & Transferts de participations & 0 \\
\hline+56 & $\begin{array}{l}\text { Propres subventions } \\
\text { d'investissement }\end{array}$ & 0 & +66 & $\begin{array}{l}\text { Remboursements de propres } \\
\text { subventions d'investissement }\end{array}$ & 0 \\
\hline+58 & $\begin{array}{l}\text { Dépenses d'investissement } \\
\text { extraordinaires }\end{array}$ & 0 & +68 & $\begin{array}{l}\text { Recettes d'investissement } \\
\text { extraordinaires }\end{array}$ & 0 \\
\hline \multicolumn{2}{|c|}{ Dépenses d'investissement } & 1971 & \multicolumn{2}{|c|}{ Recettes d'investissement } & 43 \\
\hline \multicolumn{2}{|c|}{ Dépenses totales } & 14577 & \multicolumn{2}{|c|}{ Recettes totales } & 14313 \\
\hline \multicolumn{6}{|c|}{ Solde de financement total (- Dépenses totales + Recettes totales), ici déficit de financement -264} \\
\hline
\end{tabular}


Comme le solde opérationnel, le solde de financement est influencé par un éventuel écart de production et doit être corrigé du solde conjoncturel pour dégager le solde structurel:

solde de financement structurel $=$ solde de financement effectif - solde conjoncturel

Le tableau 48 montre qu'en l'absence d'écart de production, le solde de financement structurel de l'Année $t$ se situerait à KCHF -132 selon l'estimation haute et à $\mathrm{KCHF}-245$ selon l'estimation basse. Le fait que l'écart de production soit négatif cette année-là péjore le solde de financement. Neutraliser l'impact conjoncturel a pour effet de réduire le déficit de financement. Par conséquent, MaCollectivité aurait moins dû recourir à l'emprunt si la conjoncture avait été équilibrée.

On notera que pour déterminer le solde de financement structurel, la même estimation du solde conjoncturel est utilisée (KCHF -132, respectivement KCHF -19) que celle qui est utilisée pour calculer le solde opérationnel structurel. Rien de plus normal! En effet, les dépenses et les recettes d'investissement ne sont pas influencées automatiquement par l'évolution du cycle conjoncturel. Leur niveau dépend de mesures intentionnellement prises par les collectivités publiques.

Tableau 48 Estimation du solde de financement structurel.

Exemple pour MaCollectivité, Année t, deux scénarios.

\begin{tabular}{lcr} 
Facteur $k$ & 1,0090 & 1,0013 \\
\hline Solde de financement effectif (KCHF) & \multicolumn{2}{c}{-264} \\
\hline Solde conjoncturel (KCHF) & -132 & -19 \\
\hline Solde de financement structurel (KCHF) & -132 & -245
\end{tabular}

\subsubsection{Anticyclicité et procyclicité}

Face au constat d'un déséquilibre structurel de son solde opérationnel ou de son solde de financement, une collectivité doit s'interroger sur la nécessité d’y remédier. Si elle décide d'y remédier, elle devrait également s'interroger sur le moment approprié pour le faire. Elle devrait tenir compte dans sa réflexion des implications macroéconomiques de sa décision. En effet, remédier à un éventuel déséquilibre structurel implique des mesures discrétionnaires modifiant le niveau des recettes ou des dépenses. Ces mesures donnent une impulsion à la demande globale, donc à l'activité économique.

Impulsion expansive ou restrictive. Les mesures discrétionnaires peuvent être expansives ou restrictives, comme le précise le tableau 49. Elles donnent une impulsion expansive lorsque la collectivité détériore son solde de financement structurel, soit en accroissant ses dépenses ou en diminuant ses prélèvements, notamment en réduisant sa charge fiscale. Alors la demande globale est stimulée, contribuant ainsi à doper la croissance. Les mesures donnent au contraire une impulsion restrictive 
lorsque la collectivité améliore son solde structurel en réduisant ses dépenses ou en relevant les prélèvements. Dans ce cas, la demande globale est réduite et la croissance est freinée.

Les adjectifs «expansif» ou «restrictif» ne suffisent pas à conclure que l'action de la collectivité est adéquate ou inadéquate du point de vue de la politique conjoncturelle. Pour en juger, il faut tenir compte de la situation qui prévaut au moment de l'impulsion discrétionnaire.

Impulsion anticyclique. Une impulsion s'avère adéquate, sous l'angle de la politique conjoncturelle, lorsqu' elle est expansive en basse conjoncture, alors que l'écart de production est négatif. En effet, elle stimule la demande globale et la croissance à point nommé. L'impulsion est également appropriée si elle est restrictive en haute conjoncture avec un écart de production positif, contribuant à freiner la demande globale et la croissance. Dans les deux cas, l'impulsion est anticyclique - ou contra-cyclique parce qu'elle contribue à atténuer les fluctuations de l'activité économique. Rappelons ici que les stabilisateurs automatiques ont par définition un effet anticyclique.

Impulsion procyclique. À l'opposé, une impulsion est inadéquate, du point de vue de la politique conjoncturelle, quand elle est restrictive en basse conjoncture, alors que l'écart de production est négatif. En effet, elle altère la demande globale et la croissance au mauvais moment. L'impulsion est aussi inappropriée si elle s'avère expansive en haute conjoncture, puisqu'elle stimule la demande alors que l'écart de production est déjà positif. Dans un cas comme dans l'autre, l'impulsion est procyclique puisqu'elle amplifie les fluctuations de l'activité économique.

Compatibilité des objectifs. Le tableau 49 montre ainsi qu'un objectif de réduction d'un déficit structurel n'est temporellement compatible avec une politique budgétaire anticyclique que si l'on est en présence d'un écart de production positif. En effet, une baisse des dépenses ou une hausse des prélèvements donne alors une impulsion restrictive à la demande globale. Par contre, vouloir combattre un déficit structurel lorsque l'écart de production est négatif correspond à un comportement procyclique. Un tel comportement n'est pas compatible avec le mandat constitutionnel donné à la Confédération, aux cantons et aux communes de fixer «leur politique budgétaire en prenant en considération la situation conjoncturelle» (art. 100, al. 4, voir l'extrait 4). Il en va de même lorsqu'une collectivité laisse intentionnellement filer son déficit structurel alors que la conjoncture est haute.

C'est là une attitude fréquemment observée en Suisse et en particulier à l'échelon des cantons (Soguel 2006; Martin 2011). Ces derniers privilégient souvent la lutte contre les déficits, quelle que soit la situation conjoncturelle. Cela les conduit à se comporter de manière procyclique lorsque l'écart de production est négatif. La «bouffée d'air» apportée par un écart de production positif est, quant à elle, souvent saisie pour relâcher les efforts déployés pour équilibrer structurellement le budget. Dans ce cas également, le comportement est procyclique. 
L'évolution récente des règles budgétaires cantonales ne fait que renforcer le phénomène: le degré de contrainte s'est accru sans que les dispositifs prévoient de tenir compte systématiquement de la situation conjoncturelle ${ }^{191}$, contrairement à ce que prévoit le frein à l'endettement de la Confédération.

Nous avons vu que le solde opérationnel structurel de MaCollectivité à l'Année t consiste en un excédent de revenus. Par conséquent, elle pourrait prudemment envisager soit de revoir à la hausse ses prestations (et ses charges), soit de revoir à la baisse sa fiscalité. Si elle le fait déjà au cours de l'Année $t$, son comportement serait anticyclique. En effet, l'écart de production est négatif cette année-là. Elle contribuerait ainsi, certes modestement, à stimuler l'activité économique. Par contre, si MaCollectivité procède au rééquilibrage structurel de son budget ultérieurement, elle risque d'adopter un comportement procyclique puisque l'écart de production devrait redevenir positif (facteur k inférieur à l'unité, figure 54).

Tableau 49 Impulsions procycliques et anticycliques.

\begin{tabular}{lccc}
\cline { 2 - 3 } & Impulsion expansive & Impulsion restrictive \\
\hline Dépenses de la collectivité publique & Hausse & Baisse \\
\hline Prélèvements de la collectivité publique & Baisse & Hausse \\
\hline Impact sur le solde structurel & Détérioration & Amélioration \\
\hline Impact sur la demande globale & Stimulation & Réduction \\
\hline $\begin{array}{l}\text { Si l'écart de } \\
\text { production est... }\end{array}$ & ... négatif & Impulsion anticyclique & Impulsion procyclique \\
\cline { 2 - 4 } & ... positif & Impulsion procyclique & Impulsion anticyclique \\
\hline
\end{tabular}

\section{Extrait 4 Constitution fédérale, art. 100 "Politique conjoncturelle"}

“ ${ }^{1}$ La Confédération prend des mesures afin d'assurer une évolution régulière de la conjoncture et, en particulier, de prévenir et combattre le chômage et le renchérissement.

2 Elle prend en considération le développement économique propre à chaque région. Elle collabore avec les cantons et les milieux économiques.

3 Dans les domaines du crédit et de la monnaie, du commerce extérieur et des finances publiques, elle peut, au besoin, déroger au principe de la liberté économique.

4 La Confédération, les cantons et les communes fixent leur politique budgétaire en prenant en considération la situation conjoncturelle.

$[\ldots] "$

Source: Constitution fédérale de la Confédération suisse du 18 avril 1999 (état au 1er janvier 2018).

191 Les législations cantonales prévoient en effet qu’il n'est possible de déroger à la règle budgétaire que si la situation économique est exceptionnellement difficile. Une dérogation requiert l'accord d'une majorité qualifiée du Législatif. 


\section{Analyser la dynamique de l'endettement: cercle vicieux ou vertueux}

\subsection{Soutenabilité de l'endettement}

La problématique de la soutenabilité de l'endettement est souvent évoquée en lien avec la santé des finances publiques. On s'interroge généralement sur ce qu'il faut entendre par «soutenabilité». La politique budgétaire est soutenable si la collectivité est capable de maintenir dans le long terme les principaux paramètres financiers à leur niveau actuel, notamment ses dépenses et sa pression fiscale, tout en restant solvable, en étant capable de faire face à ses engagements et en pouvant offrir les prestations publiques qu'elle a promises. Partant de là, le FMI (2002, p. 4) définit la soutenabilité de l'endettement comme une situation où la collectivité débitrice est capable de financer le service de sa dette sans devoir corriger à l'avenir un déséquilibre majeur entre ses recettes et ses dépenses.

Partant de cette définition, la soutenabilité de l'endettement n'est pas assurée lorsque, par exemple:

- une restructuration de la dette est dès à présent nécessaire ou devient nécessaire;

- la collectivité accroît continuellement son endettement et cela à un rythme plus soutenu que sa capacité à honorer le service de cette dette;

- la collectivité vit au-dessus de ses moyens en contractant de nouveaux emprunts tout en sachant qu' elle devra procéder tôt ou tard à un important assainissement budgétaire pour en financer les intérêts passifs (et cela même en l'absence de modification de l'environnement légal ou socio-économique).

Ces exemples montrent d'abord à quel point le coût du financement est un facteur déterminant de la dynamique de l'endettement (ou du désendettement). Ils montrent ensuite que la soutenabilité est en lien avec les questions de solvabilité et de liquidité. Les problèmes de liquidités surviennent en cas d'impasse de trésorerie à court terme et de difficulté à renouveler les emprunts arrivant à échéance parce que les bailleurs de fonds potentiels rechignent à prêter de l'argent frais. Le droit suisse prévoit la poursuite pour dettes contre les communes et autres collectivités de droit public cantonal, tout en excluant la possibilité d'une faillite de ces entités (portrait 9).

Les problèmes de solvabilité apparaissent lorsque l'endettement s'accumule à un rythme qui dépasse la croissance des capacités économiques de la collectivité. Les exemples ci-dessus montrent enfin que la solvabilité et la soutenabilité sont directement liées au taux d'endettement et à l'évolution du taux à travers le temps. 
Portrait 9 Poursuite pour dettes contre les collectivités de droit public cantonal

La loi fédérale du 11 avril 1889 sur la poursuite pour dettes et faillite prévoit, à son article 30, qu'elle ne s'applique pas à l'exécution forcée contre les cantons, districts et communes s'il existe des lois fédérales ou cantonales en la matière (LP, RS 281.1). Pour ces collectivités locales, la loi fédérale du 4 décembre 1947 réglant la poursuite pour dettes contre les communes et autres collectivités de droit public cantonal s'applique (LPDC, RS 282.11). Cette loi ne s'applique pas contre les cantons eux-mêmes, ni contre la Confédération. Cette dernière n'est d'ailleurs pas mentionnée à l'art. 30 LP.

L'exécution forcée est limitée à la saisie et à la réalisation de gage. La faillite est donc exclue, de même que le séquestre (art. 2 LPDC). Le patrimoine administratif, ainsi que les créances d'impôts ne peuvent pas être saisis (art. 9). Seuls les biens appartenant au patrimoine financier de la collectivité concernée sont saisissables (art. 7). La LPDC ne prévoit pas d'acte de défaut de biens, mais une attestation de découvert. Cette attestation vaut reconnaissance de dette et permet d'intenter l'action révocatoire (art. 2).

Si la LPDC exclut la mise en faillite, elle prévoit en dernier ressort l'institution d'une gérance (art. 2). La gérance consiste à retirer aux autorités de la collectivité concernée la compétence d'administrer les finances et à la confier à des personnes extérieures. L'objectif est de garantir l'exécution des tâches publiques et de payer le plus rapidement possible les dettes échues. La mise en gérance a pour effet de suspendre toutes les poursuites dirigées contre la collectivité en question (art. 41). La décision de mise en gérance est prononcée par l'autorité cantonale de surveillance (art. 34). La gérance est décrétée, sur demande d'un intéressé, lorsque la collectivité se déclare insolvable (gérance obligatoire, art. 28) ou si la collectivité elle-même en fait la demande afin de suspendre la poursuite (gérance facultative, art. 29). L'autorité cantonale nomme un ou plusieurs gérants (art. 31-33). Ceux-ci ont le pouvoir d'établir un plan financier, de recouvrer les créances, de réaliser les actifs du patrimoine financier, ainsi que d'augmenter les impôts et les taxes (art. 34-37). Les droits politiques ne peuvent être invoqués à l'encontre des mesures prises par les gérants (art. 39). La gérance est instituée pour trois ans au plus et peut être prolongée une seule fois pour trois ans au plus (art. 30). Par conséquent, la gérance ne peut durer plus de six ans au total.

En excluant la possibilité d'une faillite, le législateur reconnaît l'impossibilité de liquider la collectivité, comme il en adviendrait d'une entreprise. II reconnaît également la nécessité que la collectivité, même poursuivie, continue à fonctionner et à fournir les prestations exigées par la loi.

\subsection{Taux d'endettement}

Le taux d'endettement est un ratio couramment utilisé dans les comparaisons internationales. Il rapporte l'endettement au produit intérieur brut des pays (PIB), autrement dit à la richesse générée par les économies nationales, typiquement au cours d'une année. De par sa nature, le PIB reflète la capacité économique des pays à faire face à l'endettement de leurs administrations publiques. Ces dernières regroupent 
l'ensemble des collectivités publiques. En Suisse, le périmètre englobe la Confédération, les cantons, les concordats intercantonaux, les communes, les associations intercommunales et les assurances sociales publiques (AVS, AI, ...) (section 10.4).

La figure 57 nous rappelle quels sont les taux d'endettement des pays de l'Union européenne (UE). Les barres grisées montrent pour 2018 que la moitié des pays membres affiche un taux d'endettement supérieur à la norme de $60 \%$ fixée par le traité de Maastricht. En comparaison, les administrations publiques suisses connaissent une situation favorable. Leurs engagements bruts ne représentent que 27,7\% du PIB national. Seuls trois pays de l'Union se situent en-deça (Bulgarie, Luxembourg, Estonie). Si l'on considère l'ensemble géographique plus vaste des pays de l'OCDE, à ces trois pays, s'ajoute uniquement la Nouvelle-Zélande.

Figure 57 Évolution du taux d'endettement brut des pays de l'Union européenne et de la Suisse.

Engagements bruts des administrations publiques en \% du PIB, 2008, 2014 et 2018.

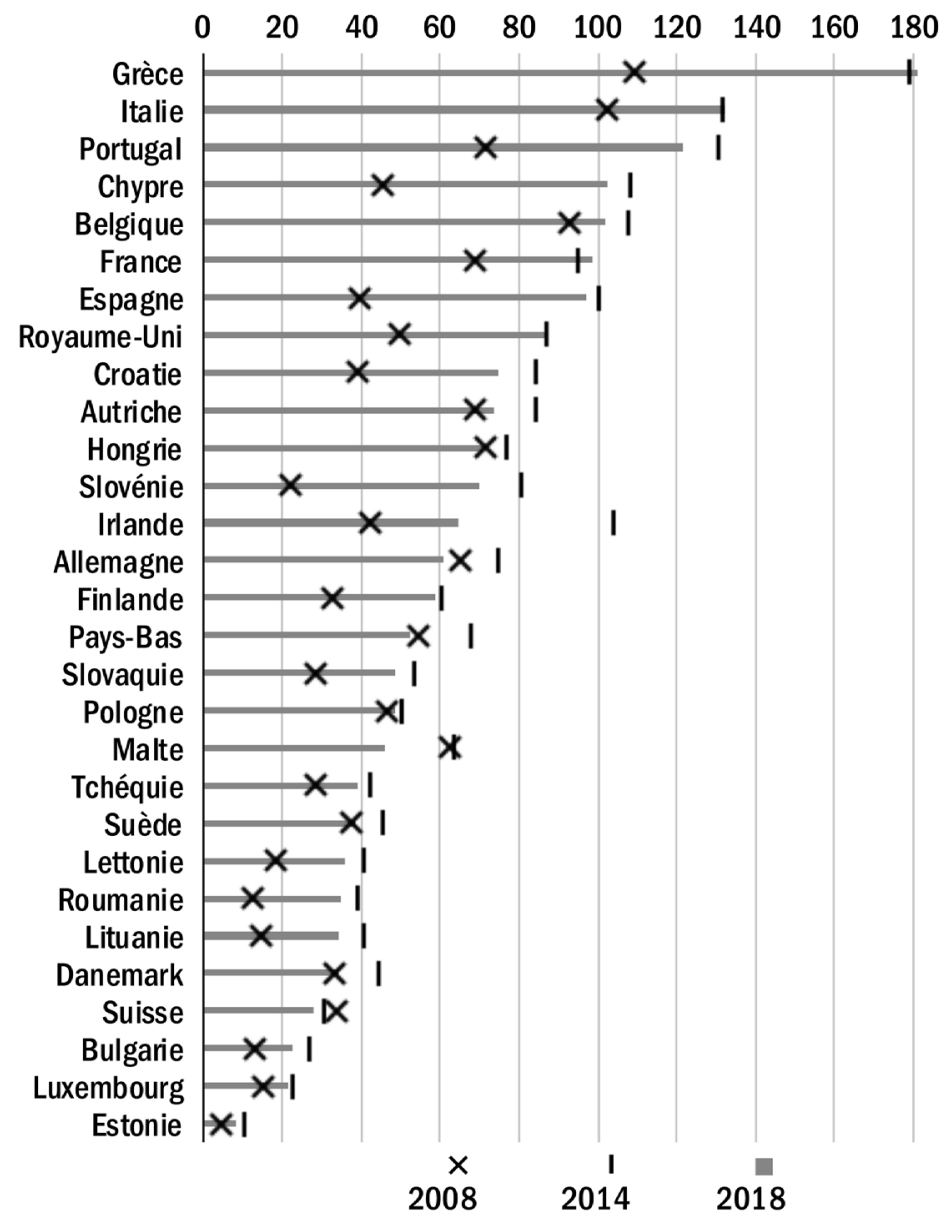

Source des données: Eurostat pour les pays de l'Union européenne et Administration fédérale des finances pour la Suisse. 
Sans perdre de vue la situation de 2018, la figure offre également une perspective dynamique en indiquant quel était le taux d'endettement en 2014 par des croix $(\times)$ et en 2008 par des segments verticaux (|). 2008 marque l'année de la dernière crise financière majeure, tandis que 2014 marque l'apogée du taux d'endettement dans les pays de l'Union européenne et de l'OCDE. Cela permet de voir que le taux d'endettement des administrations suisses s'est réduit entre 2008 et 2014, puis à nouveau entre 2014 et 2018. Cette double baisse consécutive ne s'est produite dans aucun pays de l'UE. Elle témoigne d'une solvabilité et d'une soutenabilité accrues de l'endettement public helvétique. Partout dans l'Union européenne, entre 2008 (X) et $2014(\mid)$, la soutenabilité s'est détériorée; certains pays enregistrant d'ailleurs une augmentation particulièrement importante du taux d'endettement alors que leur taux se situait déjà en 2008 au-delà de 60\% (surtout le Portugal et la Grèce). Depuis 2014, la soutenabilité de l'endettement s'est améliorée pour tous les pays européens, à l'exception de trois pays. La Grèce, l'Italie et la France présentent un taux d'endettement plus élevé en 2018 qu'en 2014. Cela dit, la progression y est moins forte qu'entre 2008 et 2014.

Les taux d'endettement mentionnés ici sont ceux qui sont calculés par Eurostat. D'autres organismes publient également des chiffres à ce sujet, par exemple l'OCDE ou le FMI. Ces organisations fondent leurs statistiques sur les informations transmises par les différents pays. Malheureusement, les informations varient souvent en fonction des ministères qui communiquent avec les organisations internationales. Cela affecte à la fois la mesure de l'endettement et la mesure du PIB. Parfois, les périmètres statistiques diffèrent légèrement. Par exemple, l'Union européenne se contente de la seule dette brute, car c'est elle qui est visée par le critère de Maastricht. D'autres, en fonction des informations communiquées, élargissent la notion de dette pour y inclure d'autres engagements. Une autre source de différences réside dans le principe d'évaluation. Eurostat recourt à la valeur nominale - faciale - de la dette. L'OCDE et le FMI s'en remettent aux principes d'évaluation utilisés par les pays eux-mêmes, sachant que certains se fondent sur la valeur de marché de la dette. Par conséquent, le taux d'endettement publié par l'OCDE, sur la base des chiffres de l'endettement et du PIB qui lui sont communiqués par les pays, est généralement supérieur d'environ un cinquième par rapport à celui qui est publié par Eurostat. Par exemple, pour 2018, le taux pour la France s'élève à $98,5 \%$ selon Eurostat (figure 57) et à 122,7\% selon l'OCDE. Pour les administrations publiques suisses, l'OCDE indique un taux de 41,6\%. En s'appuyant sur la définition d'Eurostat, l'Administration fédérale des finances mesure pour sa part un taux de 29,7\%, dont la moitié est le fait de la Confédération, un bon tiers des cantons et un petit tiers des communes.

Notons que les indicateurs financiers prescrits par le $\mathrm{MCH} 2$ s'intéressent eux aussi à la thématique de l'endettement et de la soutenabilité de ce dernier. Un indicateur intitulé «taux d'endettement net» met d'ailleurs en relation les engagements 
nets avec les revenus fiscaux. Un autre indicateur compare la dette brute avec les revenus courants (voir la section 9.3.3 consacrée aux indicateurs d'importance de l'endettement).

On constate que sous la dénomination de taux d'endettement se cachent des grandeurs statistiques différentes. Les uns mobilisent strictement une statistique de la dette là où d'autres recourent à une statistique plus large. Certains indicateurs se réfèrent à des grandeurs brutes, d'autres à des grandeurs nettes. À l'échelle internationale, ces grandeurs sont rapportées au produit intérieur brut. Mais, comme mesure des capacités économiques, les indicateurs du MCH2 utilisent les revenus fiscaux ou les revenus courants.

\subsection{Grandeurs brutes ou grandeurs nettes}

Les comparaisons internationales se fondent essentiellement sur des grandeurs brutes. Cela signifie qu'elles ignorent - ou feignent d'ignorer - qu'une collectivité peut en parallèle détenir des actifs à caractère de placements. Plusieurs raisons expliquent cela.

La première raison est purement pratique. Le modèle comptable des collectivités publiques suisses offre une distinction claire entre les placements dans le patrimoine financier et les actifs productifs de prestations publiques dans le patrimoine administratif (section 3.3.3). Une telle partition de l'actif n'existe pas dans les autres pays. Il n'est donc pas possible de savoir quels actifs les collectivités pourraient aliéner pour rembourser leur dette, sans nuire à la réalisation des tâches dont elles sont responsables. En admettant que cette distinction puisse exister à l'étranger également, encore faudrait-il que les principes d'évaluation des placements soient les mêmes partout. Dans leur bilan, les collectivités suisses doivent quant à elles présenter la plupart des actifs appartenant au patrimoine financier à leur valeur vénale. Tant l'OCDE qu'Eurostat préfèrent contourner ces difficultés pratiques. Ces organisations se concentrent sur les capitaux étrangers figurant au passif du bilan des collectivités. Elles recourent donc à une approche brute du niveau de l'endettement. Qu'elle soit fondée sur la valeur faciale ou la valeur de marché, l'évaluation des seuls capitaux étrangers s'avère plus aisée.

Une deuxième raison est également d'ordre pratique. Les capitaux de tiers sont en lien direct avec les intérêts passifs dont une collectivité doit s'acquitter. Évidemment, cela fait fi des recettes qui sont générées par les placements. Mais cela simplifie aussi l'analyse.

Une troisième raison relève de la psychologie collective. Les grandeurs brutes sont logiquement supérieures aux grandeurs nettes. Par exemple, et pour reprendre le chiffre pour MaCollectivité, la valeur au bilan des capitaux de tiers s'élève à KCHF 6987 au 31 décembre de l'Année t. Mais si l'on soustrait le patrimoine financier 
de ces capitaux de tiers, on s'aperçoit que MaCollectivité a en fait des engagements nets de KCHF 2303. Présenter des grandeurs brutes permet donc d'amplifier les craintes vis-à-vis de l'endettement des administrations publiques.

L'analyse de l'endettement devrait chercher à être objective et rationnelle. Par conséquent, si les données statistiques concernant la valeur des placements des collectivités sont disponibles et sont fiables, alors il faudrait les utiliser. Une approche fondée sur des grandeurs nettes devrait être privilégiée. Dans le cas des collectivités suisses, ces informations sont disponibles et sont bien harmonisées. Par conséquent, il vaut mieux en faire usage pour améliorer la précision de l'analyse.

\subsection{Dette ou engagements}

Juridiquement, une dette correspond à une obligation contractée par une entité à l'égard d'un tiers, ayant pour contrepartie du point de vue de ce dernier une créance. La dette naît soit d'un acte bilatéral, soit d'une disposition légale. Elle s'éteint par l'exécution complète de l'obligation de remboursement. Par conséquent, une dette n'existe en droit que dès lors qu'une obligation existe réellement et effectivement.

Dans une perspective économique et financière, la notion d'endettement est plus large. Elle s'étend aux obligations potentielles et futures auxquelles l'entité peut raisonnablement s'attendre. Pour souligner cette extension de la notion d'endettement, on recourt volontiers à la notion d'engagements. Cette dernière souligne que l'on considère davantage que les strictes obligations financières effectives de l'État.

Toutefois, le langage courant tend à utiliser systématiquement le terme de dette. D'ailleurs, le titre de certains indicateurs financiers du $\mathrm{MCH} 2$ entretient le flou: il parle de dette ou d'endettement là où il faudrait parler d'engagements (voir l'indicateur du taux d'endettement net ou celui de dette nette par habitant). Pour éviter tout risque de confusion dans l'analyse de la dynamique de l'endettement, il est utile de se référer à la décomposition offerte par le plan comptable. C'est ce que propose le tableau 50. Ce tableau indique quelles sont les rubriques qui définissent la dette brute, la dette nette, les engagements bruts et les engagements nets. Ces définitions sont celles qui sont données par le $\mathrm{MCH} 2$ lui-même ${ }^{192}$.

Dette brute. La dette brute correspond à trois éléments figurant au passif du bilan de la collectivité, à savoir les engagements courants (rubrique 200), les engagements financiers à court terme (201) et à long terme (206). Pour être plus précis, et pour autant qu'ils existent, il faut déduire les instruments financiers dérivés à court terme (2016) et à long terme (2066), ainsi que les éventuelles subventions d'investissement inscrites au passif (2068). Pour MaCollectivité, la dette brute s'élève à KCHF 6321 au 31 décembre de l'Année $t$. 
Tableau 50 Dette et engagements, nets ou bruts.

MaCollectivité, Année t, en KCHF.

\begin{tabular}{|c|c|c|c|c|c|c|c|}
\hline $\mathrm{N}^{0}$ & Libellé & KCHF & $\mathrm{N}^{\circ}$ & Libellé & KCHF & \multirow{2}{*}{ Libellé } & \multirow[t]{2}{*}{ KCHF } \\
\hline \multirow[t]{2}{*}{100} & $\begin{array}{l}\text { Disponibilités et } \\
\text { placements à court terme }\end{array}$ & 783 & 200 & Engagements courants & 1721 & & \\
\hline & Créances & 1622 & 201 & $\begin{array}{l}\text { Engagements } \\
\text { financiers à court terme }\end{array}$ & 0 & & \\
\hline 102 & $\begin{array}{l}\text { Placements financiers } \\
\text { à court terme }\end{array}$ & 0 & & & & & \\
\hline 106 & $\begin{array}{l}\text { Marchandises, fournitures } \\
\text { et travaux en cours }\end{array}$ & $s$ & 206 & $\begin{array}{l}\text { Engagements } \\
\text { financiers à long terne }\end{array}$ & 4600 & & \\
\hline 107 & $\begin{array}{l}\text { Placements financiers } \\
\text { à long terme }\end{array}$ & 100 & & & & & \\
\hline 108 & $\begin{array}{l}\text { Immobilisations } \\
\text { corporelles PF }\end{array}$ & 2014 & & & & & \\
\hline & $\begin{array}{l}\text { Patrimoine financier } \\
\text { "disponible" }\end{array}$ & 4519 & & Dette brute* & 6321 & $\begin{array}{l}\text { Dette } \\
\text { nette }\end{array}$ & 1802 \\
\hline \multirow[t]{3}{*}{104} & Actifs de régularisation & 165 & 204 & Passifs de régularisation & 21 & & \\
\hline & & & 205 & Provisions à court terme & 323 & & \\
\hline & & & 208 & Provisions à long terme & 0 & & \\
\hline 109 & $\begin{array}{l}\text { Créances envers } \\
\text { les FSp et fonds sous CT }\end{array}$ & 0 & 209 & $\begin{array}{l}\text { Engagements envers } \\
\text { les FSp et fonds sous CT }\end{array}$ & 322 & & \\
\hline 10 & $\begin{array}{l}\text { Patrimoine financier } \\
{[\mathrm{PF}]}\end{array}$ & 4684 & 20 & $\begin{array}{l}\text { Capitaux de tiers } \\
\text { (= engagements bruts) }\end{array}$ & 6987 & $\begin{array}{l}\text { Engage- } \\
\text { ments nets }\end{array}$ & ${ }_{s}^{2303}$ \\
\hline
\end{tabular}

* Pour être tout à fait précis, la dette brute correspond aux rubriques 200+201+206, desquelles il faut déduire - s'ils existent - les instruments financiers dérivés à court terme (2016) et à long terme (2066), ainsi que les éventuelles subventions d'investissement inscrites au passif (2068).

Dette nette. La dette nette s'obtient en déduisant de la dette brute ce que nous appelons le patrimoine financier «disponible». Cela correspond aux disponibilités et placements à court terme (100), aux créances (101), aux placements financiers à court terme (102) et à plus long terme (107), aux marchandises, fournitures et travaux en cours (106), ainsi qu'aux immobilisations corporelles (108). En définitive, il s'agit de l'ensemble du patrimoine financier duquel on exclut les actifs de régularisation (104), puisque les passifs de régularisation ne sont pas intégrés dans la dette brute. On en exclut également les créances envers les financements spéciaux et les fonds considérés comme des capitaux de tiers (109), puisque les engagements envers les financements spéciaux et fonds ne sont pas non plus intégrés dans la dette brute. Pour MaCollectivité, le patrimoine financier «disponible» s'élève à KCHF 4519. Ce montant doit être porté en diminution de la dette brute afin de dégager une dette nette de KCHF 1802. 
Engagements bruts. Le MCH2 définit les engagements bruts comme l'ensemble des capitaux de tiers (rubrique 20 au passif du bilan). Par conséquent, il faut ajouter à la dette brute les obligations potentielles et futures de la collectivité, à savoir les passifs de régularisation (204), les provisions à court terme (205) et à long terme (208), ainsi que les engagements envers les financements spéciaux et les fonds considérés comme des capitaux de tiers (209). Pour MaCollectivité, les engagements bruts s'élèvent à KCHF 6987.

Engagements nets. Le MCH2 définit les engagements nets comme la différence entre les capitaux de tiers (20) et le patrimoine financier (10). Pour MaCollectivité, l'ensemble du patrimoine financier s'élève à KCHF 4684. Ce montant doit être porté en diminution des engagements bruts afin de dégager le montant net, soit KCHF 2303 à la fin de l'Année t.

Pour l'analyse de la dynamique de l'endettement, nous considérons les engagements nets. Certes, ces derniers reflètent des montants un peu plus élevés que les seuls engagements exigibles, reflétés quant à eux par la dette nette. Toutefois, ce sont les engagements nets qui sont considérés dans l'indicateur du taux d'endettement net préconisé par le $\mathrm{MCH} 2$, indicateur intégré dans le tableau de bord que nous proposons ici (19*, chapitre 9$)$.

\subsection{Solde primaire}

La soutenabilité de l'endettement dépend largement des intérêts que la collectivité doit supporter et de la capacité de cette dernière à y faire face à l'aide de ses recettes. La capacité de payer les intérêts de la dette dépend de la marge de manœuvre qui existe à l'intérieur du budget. Cette marge de manœuvre est représentée par le solde primaire et plus précisément par le solde de financement primaire ${ }^{193}$. Le solde primaire est le solde que présenterait la collectivité si elle n'avait pas d'intérêts à payer. C'est donc le solde hors intérêts.

Excédent primaire. Un excédent primaire correspond à une situation où des recettes restent disponibles pour le paiement des intérêts après que toutes les autres dépenses ont été honorées. Dans ce cas, une marge de manœuvre existe dans le budget pour le «service de la dette».

193 Le solde primaire du compte de résultats peut également être calculé. Il correspond au solde qu'afficherait le compte si la collectivité n'avait pas à supporter d'intérêts. Le résultat d'exploitation qui apparaît dans la présentation échelonnée du compte de résultats offre largement cette information. En effet, il ne tient pas compte des charges et des revenus financiers (voir à ce propos la section 3.3.4 consacrée au compte de résultats). Dans l'analyse de la dynamique de l'endettement, on doit cependant considérer le solde de financement primaire. En effet, c'est le solde de financement qui correspond directement à la hausse de l'endettement en cas de déficit ou à la baisse de l'endettement en cas d'excédent (voir à ce propos la section 3.4). 
Déficit primaire. À l'opposé, un déficit primaire témoigne d'une situation où les recettes ne suffisent pas à financer les dépenses, même hors intérêts. Cela signifie que la collectivité devrait s'endetter même si elle n'avait pas à s'acquitter du service de sa dette. Cela signifie aussi qu'elle doit accroître son endettement pour financer non seulement les intérêts, mais aussi ses autres dépenses, qu'elles soient courantes ou d'investissement. L'entité n'a donc aucune marge de manœuvre budgétaire. Le tableau 51 montre que MaCollectivité est confrontée à une telle situation à l'Année t dans la mesure où elle affiche un léger déficit primaire de KCHF -243.

Pour mettre en évidence le solde primaire, il ne suffit pas de s'intéresser aux recettes et aux dépenses courantes qui figurent dans le compte de résultats. Il faut $\mathrm{y}$ inclure les recettes et les dépenses d'investissement. On doit donc se tourner vers le compte de financement de la collectivité. Nous avons vu que la Confédération présente ses comptes sous cette forme (section 3.4). Les cantons et les communes ne connaissent pas de compte de financement. Cependant, les informations véhiculées par le compte administratif - c'est-à-dire par le compte de résultats et par le compte des investissements - permettent de pallier cette absence. On doit retenir dans le calcul les rubriques concernées par les recettes et les dépenses et exclure les rubriques qui enregistrent des opérations purement comptables.

Le tableau 51 offre l'exemple de MaCollectivité. Il dégage d'abord les dépenses et les recettes courantes, puis les dépenses et les recettes d'investissement. Les rubriques qui les composent sont données par le $\mathrm{MCH} 22^{194}$. Par addition, on parvient aux dépenses et aux recettes totales. La différence entre ces dernières correspond au solde de financement. Pour l'Année $t$, on voit apparaître un léger déficit de financement de KCHF -264. Ce déficit s'explique par des dépenses d'investissement particulièrement élevées ${ }^{195}$.

Pour aboutir au solde primaire, il faut encore retrancher du solde de financement les intérêts passifs et par extension les autres frais générés par l'endettement et par le patrimoine financier. Ces dépenses d' «intérêts» se montent à KCHF 125. Toutefois, elles sont en partie compensées par les recettes d' «intérêts» apportées par le patrimoine financier, soit KCHF 104. Ce montant inclut les revenus des intérêts, mais aussi les loyers apportés par les immeubles du patrimoine financier. En termes nets, les frais liés à l'endettement n'ont donc coûté que KCHF -21 (KCHF $104-125)$. Si MaCollectivité n'avait pas eu à les payer, son solde de financement se serait élevé à KCHF -243 (KCHF -264 + 21). C'est là son solde primaire - son déficit primaire pour l'Année t.

194 CDF (2008), annexe C (Indicateurs financiers: Définitions et calculs).

195 C'est d'ailleurs ce que mettent en évidence l'indicateur de l'effort d'investissement (I6) et celui de la proportion des investissements bruts (I14 ${ }^{\# \#) ~(v o i r ~ l a ~ s e c t i o n ~} 9.3$ consacrée aux indicateurs financiers). 
Tableau 51 Calcul du solde de financement primaire.

\section{MaCollectivité, Année t, en KCHF.}

\begin{tabular}{|c|c|c|c|c|c|c|}
\hline & $\mathrm{N}^{\circ}$ & Libellé & $\mathrm{KCHF}$ & $\mathbf{N}^{\circ}$ & Libellé & KCHF \\
\hline+ & 30 & Charges de personnel & 2020 & 40 & Revenus fiscaux & 7651 \\
\hline+ & 31 & $\begin{array}{l}\text { Charges de biens et services } \\
\text { et autres charges d'exploitation }\end{array}$ & 4076 & 41 & Patentes et concessions & 12 \\
\hline - & 3180 & Réévaluations sur créances & -8 & 42 & Taxes et redevances & 5076 \\
\hline+ & 34 & Charges financières & 125 & 430 & Revenus d'exploitation divers & 0 \\
\hline - & 344 & $\begin{array}{l}\text { Réévaluations des immobilisations } \\
\text { du patrimoine financier }\end{array}$ & 0 & 439 & $\begin{array}{l}\text { Autres revenus (avec incidence } \\
\text { sur les liquidités) }\end{array}$ & \\
\hline+ & 36 & Charges de transferts & 6393 & 44 & Revenus financiers & 167 \\
\hline - & 364 & Réévaluations de prêts du PA & 0 & 444 & $\begin{array}{l}\text { Réévaluations des immobilisations } \\
\text { du PF }\end{array}$ & $-\varsigma$ \\
\hline - & 365 & $\begin{array}{l}\text { Réévaluations de participations } \\
\text { du PA }\end{array}$ & 0 & 4490 & $\begin{array}{l}\text { Réévaluations du patrimoine } \\
\text { administratif }\end{array}$ & \\
\hline - & 366 & $\begin{array}{l}\text { Amortissements de subventions } \\
\text { d'investissement }\end{array}$ & 0 & 46 & Revenus de transferts & 1373 \\
\hline+ & 380 & Charges extraordinaires de personnel & 0 & 466 & $\begin{array}{l}\text { Dissolutions des subventions } \\
\text { d'investissement portées au passif }\end{array}$ & \\
\hline+ & 381 & $\begin{array}{l}\text { Charges extraordinaires de biens, } \\
\text { services et charges d'exploitation }\end{array}$ & 0 & 481 & $\begin{array}{l}\text { Revenus extraordinaires } \\
\text { de patentes, concessions }\end{array}$ & \\
\hline+ & 3840 & Charges financières extraordinaires & 0 & 482 & Contributions extraordinaires & 0 \\
\hline+ & 386 & Charges extraordinaires de transferts & 0 & 483 & Revenus extraordinaires divers & 0 \\
\hline & & & & 484 & Revenus financiers extraordinaires & \\
\hline & & & & 486 & Revenus extraordinaires de transferts & 0 \\
\hline Dép & enses & s courantes & 12606 & Recettes & courantes & 14270 \\
\hline+ & 50 & Immobilisations corporelles & 1971 & 60 & $\begin{array}{l}\text { Transferts d'immobilisations } \\
\text { corporelles dans le PF }\end{array}$ & 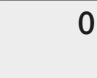 \\
\hline+ & 51 & $\begin{array}{l}\text { Dépenses d'investissement } \\
\text { pour le compte de tiers }\end{array}$ & 0 & 61 & $\begin{array}{l}\text { Remboursements de dépenses d'in- } \\
\text { vestissement pour le compte de tiers }\end{array}$ & 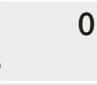 \\
\hline+ & 52 & Immobilisations incorporelles & 0 & 62 & $\begin{array}{l}\text { Transferts d'immobilisations } \\
\text { incorporelles dans le PF }\end{array}$ & 0 \\
\hline & & & & 63 & $\begin{array}{l}\text { Subventions d'investissement } \\
\text { acquises }\end{array}$ & 43 \\
\hline+ & 54 & Prêts & 0 & 64 & Remboursements de prêts & 0 \\
\hline+ & 55 & Participations et capital social & 0 & 65 & Transferts de participations & $c$ \\
\hline+ & 56 & Propres subventions d'investissement & 0 & 66 & $\begin{array}{l}\text { Remboursements de propres } \\
\text { subventions d'investissement }\end{array}$ & 0 \\
\hline+ & 58 & $\begin{array}{l}\text { Dépenses d'investissement } \\
\text { extraordinaires }\end{array}$ & 0 & 68 & $\begin{array}{l}\text { Recettes d'investissement } \\
\text { extraordinaires }\end{array}$ & 0 \\
\hline & enses & d'investissement & 1971 & Recettes & d'investissement & 43 \\
\hline & enses & totales & 14577 & Recettes & totales & 14313 \\
\hline don & 340 & Charges d'intérêts & 74 & dont 440 & Revenus des intérêts & 12 \\
\hline & 342 & Frais d'approvisionnement en capitaux & 10 & 442 & Revenus de participations & 0 \\
\hline & 343 & Charges pour bien-fonds du PF & 41 & 443 & Produits des immeubles du PF & 92 \\
\hline & & Total des dépenses d'« intérêts" & 125 & & Total des recettes d'« intérêts" & 104 \\
\hline & de & financement total (- Dépenses totales & s+ Rece & ttes totales & s), ici déficit de financement & -264 \\
\hline & térêts & nets (- Total des dépenses d'« intérêt & ts" + Tot & al des rece & ttes d'“intérêts") & $-(-21)$ \\
\hline & (d) & financement) primaire (Solde de finan & comen & Intónôte & ets), ici déficit primaire & -243 \\
\hline
\end{tabular}




\subsection{Boule de neige de l'endettement}

Le développement de l'endettement à travers le temps est sujet à des effets dynamiques. Ces effets peuvent être négatifs. La collectivité se trouve alors prise dans un cercle vicieux. Le cercle est vicieux parce que l'endettement s'autoalimente sous l'effet notamment des intérêts à payer. L'élévation du niveau de l'endettement conduit à une augmentation des intérêts qui, s'ils ne peuvent être financés par le budget courant, nécessitent un nouveau recours à l'emprunt. Ce risque d'explosion peut être renforcé si les bailleurs de fonds augmentent la prime de risque de la collectivité et qu'ainsi les taux d'intérêt débiteurs augmentent. Le taux d'endettement augmente. Le niveau d'endettement devient insoutenable.

Plusieurs conditions doivent être réunies pour en arriver là. Ces conditions ont trait au taux d'intérêt, à la croissance économique et à l'état du solde primaire de la collectivité. Un modèle relativement simple, dit modèle de la boule de neige, les met en lien (Soguel 1996). Il montre qu'une collectivité entre dans un cercle vicieux à deux conditions. D'abord, il faut que le taux de croissance soit inférieur au taux d'intérêt. La différence entre ces deux taux provoque un effet de levier négatif. Ensuite, la collectivité doit être confrontée à un déficit primaire, à l'instar de MaCollectivité pour l'Année $t$, c'est-à-dire que ses recettes sont inférieures aux dépenses avant le paiement des intérêts (intérêts nets). Lorsque ces deux conditions se cumulent, l'endettement fait boule de neige: il grossit sous l'effet de sa propre dynamique.

Mais la dynamique peut aussi être positive, déclenchant un cercle vertueux et une autoérosion de l'endettement. Pour bénéficier d'un cercle vertueux, les conditions inverses s'appliquent: un effet de levier positif des taux grâce à un taux de croissance supérieur au taux d'intérêt et/ou un excédent primaire. Le taux d'endettement se réduit, indiquant une plus grande soutenabilité de la dette.

L'évolution du taux d'endettement est donc un élément déterminant de la soutenabilité de la dette. Si ce taux reste identique d'une année à l'autre, la soutenabilité ne s'améliore pas, mais elle ne se détériore pas non plus. Nous avons déjà vu que le taux d'endettement est le rapport entre la dette D en fin d'année et la capacité économique de la collectivité à faire face à cette dette. La capacité économique est mesurée grâce au PIB lorsque ce dernier est disponible. En Suisse, l'Office fédéral de la statistique publie le PIB pour l'ensemble du pays et le PIB de chaque canton. Par contre, il n'existe pas de statistique à l'échelon communal. Qu'à cela ne tienne, les revenus fiscaux Y présentés dans les comptes fournissent une approximation de la capacité économique. Comme le formulent le $\mathrm{MCH} 2$ et l'indicateur $\mathrm{I}^{\#}$ de notre tableau de bord financier, le taux d'endettement net correspond donc au ratio D/Y. L'endettement est soutenable si, à la fin de l'Année t, l'indicateur affiche la même valeur qu'à la fin de l'Année t-1, voire une valeur inférieure. Arithmétiquement, la condition de soutenabilité se présente donc de la manière suivante: 


$$
\frac{D_{t}}{Y_{t}} \leq \frac{D_{t-1}}{Y_{t-1}}
$$

Dans le cas de MaCollectivité, les revenus fiscaux de l'Année $t$ s'élèvent à KCHF 7651 (tableau 51), contre KCHF 7538 pour l'Année t-1 (voir le tableau des comptes en annexe pour l'Année t-1), soit une hausse de 1,5\%. Pour que la soutenabilité de la dette reste identique, les engagements nets, qui étaient de KCHF 2080 à la fin de $\mathrm{t}-1$, auraient dû augmenter au maximum du même pourcentage. Ils n'auraient donc pas dû dépasser KCHF 2111 à la fin de l'Année t. Or ils ont atteint KCHF 2303 (tableau 50). Le taux d'endettement est donc passé de $27,6 \%$ en t-1 à $30,1 \%$ à la fin de l'Année t. La dette est donc devenue moins soutenable, puisque

$$
\frac{D_{t}}{Y_{t}}>\frac{D_{t-1}}{Y_{t-1}}
$$

La figure 58 illustre le phénomène tantôt de l'amélioration, tantôt de la détérioration de la soutenabilité de l'endettement de MaCollectivité au cours des vingt années précédant l'Année t. La zone grisée reflète l'évolution du taux d'endettement net. La valeur du taux d'endettement se lit sur l'axe vertical de droite qui va jusqu'à $100 \%$.

Figure 58 Évolution du taux d'endettement de MaCollectivité sous l'effet de la croissance économique et de la variation de l'endettement. De l'Année t-20 à l'Année t.

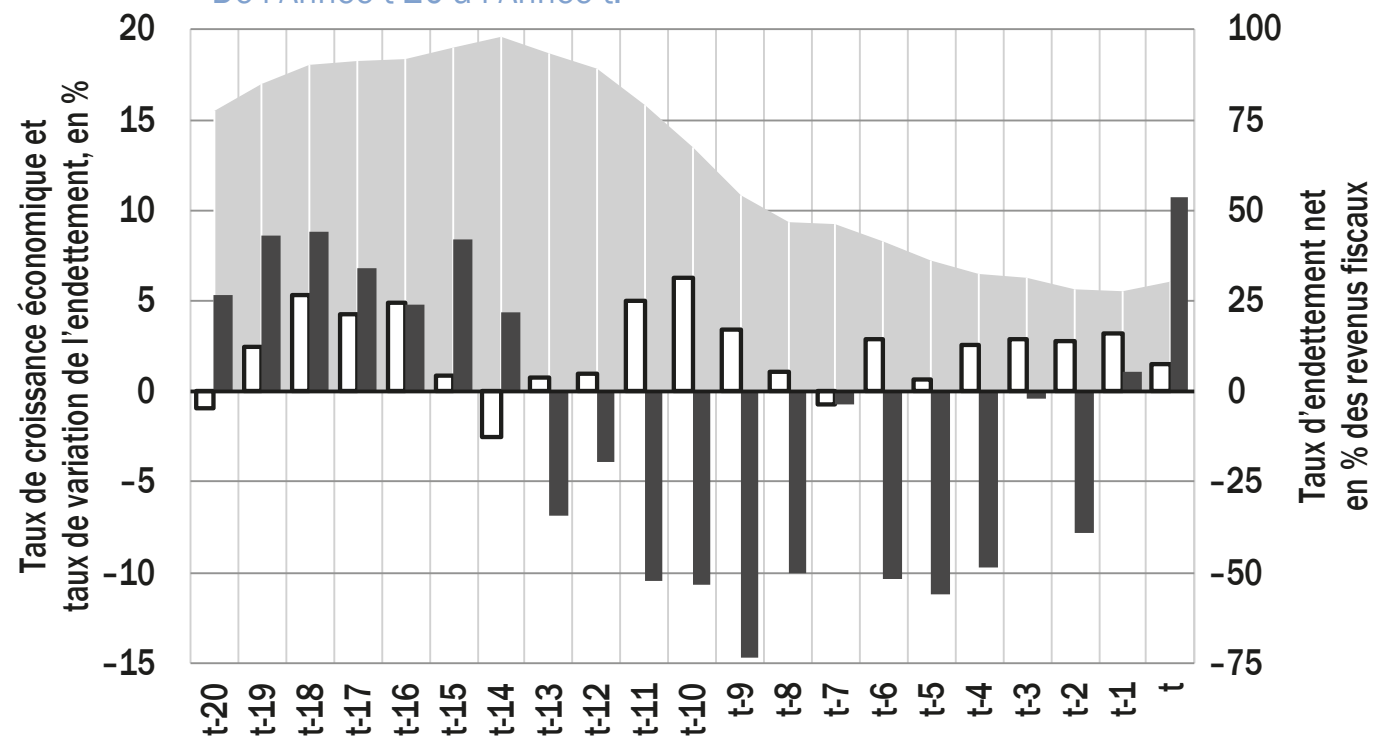

Taux d'endettement net (échelle de droite) $\square$ Taux de croissance économique - Variation des engagements nets 
La croissance économique et la variation de l'endettement se lisent sur l'axe vertical de gauche qui s'échelonne de $+20 \%$ à $-15 \%$. Les barres verticales blanches montrent une croissance économique - en l'occurrence la croissance des revenus fiscaux - sur l'année concernée lorsqu'elles pointent vers le haut et une décroissance économique lorsqu'elles pointent vers le bas. Les barres noires témoignent de la variation de l'endettement - plus précisément des engagements nets - entre le début et la fin de l'année. Si, pour une année donnée, la barre blanche et la barre noire sont de même longueur, indiquant une variation relative identique, le taux d'endettement reste stable. C'est le cas en t-16 (D et Y croissent tous deux d'un pourcentage quasi identique) et en t-7 (ils décroissent d'un même pourcentage). Ces deux années-là affichent un taux d'endettement identique à celui de l'année précédente: $91 \%$ en $\mathrm{t}-16$ comme en $\mathrm{t}-17 ; 46 \%$ en $\mathrm{t}-7$ comme en $\mathrm{t}-8$. Lorsque le taux de croissance est supérieur au taux de variation de la dette, le taux d'endettement diminue. L'Année t-10 en fournit un exemple puisque la barre blanche pointe vers le haut, tandis que la barre noire se dirige vers le bas. Le taux d'endettement chute de $79 \%$ en $\mathrm{t}-11$ à $67 \%$ en $\mathrm{t}-10$. Inversement, un taux de croissance inférieur au taux de variation de l'endettement implique une hausse du taux d'endettement. Un tel cas se produit au cours de l'Année t-14. La barre blanche est orientée vers le bas, alors que la noire pointe vers le haut, propulsant le taux d'endettement de 95\% en $\mathrm{t}-15$ à $98 \%$ en $\mathrm{t}-14$.

L'amélioration ou la détérioration de la soutenabilité de l'endettement s'explique évidemment par la situation budgétaire, y compris par le déficit primaire. Les engagements nets en fin d'année - notés $D_{t}$ - dépendent du stock d'engagements en début d'année $\mathrm{D}_{\mathrm{t}-1}$. Mais en cours d'année, les engagements $(a)$ se réduisent sous l'effet des recettes encaissées $\mathrm{R}_{\mathrm{t}}(b)$ augmentent en fonction des dépenses hors paiement des intérêts $G_{t}$ et $(c)$ s'accroissent en raison des intérêts à payer sur le stock d'engagements $\mathrm{i} \times \mathrm{D}_{\mathrm{t}-1}$ ( $\mathrm{i}$ étant le taux d'intérêt). Arithmétiquement, l'endettement en fin d'année correspond donc à l'expression suivante:

$$
D_{t}=D_{t-1}-R_{t}+G_{t}+i \times D_{t-1}
$$

Dans le cas de MaCollectivité, l'endettement à la fin de l'Année t s'élève à KCHF 2303. C'est KCHF 223 de plus qu'à la fin de l'Année t-1, lorsque l'endettement se situait à KCHF 2080. Cette variation correspond approximativement au déficit de financement de l'Année t (KCHF -264) découlant des recettes totales $\left(R_{t}=K C H F 14313\right)$, des dépenses totales hors intérêts nets $\left(G_{t}=K C H F\right.$ 14556) et des intérêts nets $\left(\mathrm{i} \times \mathrm{D}_{\mathrm{t}-1}=\mathrm{KCHF} 21\right)$ de l'Année $\mathrm{t}^{196}$.

196 Ici une différence subsiste entre la variation de l'endettement net (KCHF 223) et le solde de financement (KCHF 264). Cette différence est en particulier liée au fait que les flux de trésorerie associés aux dépenses et aux recettes courantes et d'investissement - telles que comptabilisées dans le compte de résultats et dans le compte des investissements - ne sont pas toujours rattachés exactement à l'exercice comptable concerné. 
Les dépenses représentées par $G_{t}$ sont les dépenses hors paiement des intérêts. Donc, la différence entre $R_{t}$ et $G_{t}\left(=R_{t}-G_{t}\right)$ correspond au solde primaire $S_{t}$ (KCHF -243). Par conséquent, l'expression précédente peut aussi s'écrire en faisant référence au solde primaire $S_{t}$ :

$\begin{array}{ll} & D_{t}=D_{t-1}-\left(R_{t}-G_{t}\right)+i \times D_{t-1} \\ \text { c'est-à-dire } & D_{t}=D_{t-1}-S_{t}+i \times D_{t-1} \\ \text { ou encore } & D_{t}=D_{t-1}+i \times D_{t-1}-S_{t} \\ \text { soit } & D_{t}=(1+i) \times D_{t-1}-S_{t}\end{array}$

Partant de là, le taux d'endettement de l'Année t peut être réécrit d'une part en remplaçant $D_{t}$ par cette dernière expression et d'autre part en exprimant la capacité économique de l'Année t comme étant celle de l'Année t-1, augmentée du taux de croissance r. On aboutit à cette nouvelle expression:

$$
\frac{D_{t}}{Y_{t}}=\frac{(1+i) \times D_{t-1}-S_{t}}{(1+r) \times Y_{t-1}}
$$

La condition de soutenabilité de l'endettement peut maintenant être réécrite en remplaçant le taux d'endettement de l'Année t par cette nouvelle expression. Elle devient ainsi:

$$
\frac{(1+i) \times D_{t-1}-S_{t}}{(1+r) \times Y_{t-1}} \leq \frac{D_{t-1}}{Y_{t-1}}
$$

En simplifiant et en réarrangeant, on peut dégager l'expression que nous recherchons:

$$
(\mathrm{r}-\mathrm{i}) \times \mathrm{D}_{\mathrm{t}-1}+\mathrm{S}_{\mathrm{t}} \geq 0
$$

Cela signifie que l'endettement reste soutenable, autrement dit le taux d'endettement reste constant voire diminue, si l'effet de levier des taux $(r-i)$ sur les engagements $\mathrm{D}_{\mathrm{t}-1}$ cumulé avec le solde primaire est égal ou supérieur à zéro. On retrouve donc ici les deux conditions mentionnées précédemment: celle de l'effet de levier et celle du solde primaire.

Les deux conditions sont combinées dans le tableau 52. Certaines circonstances sont claires: un effet de levier positif des taux associé à un excédent primaire déclenche un cercle vertueux, une baisse du taux d'endettement et donc un endettement soutenable (situation localisée dans la cellule A du tableau).

Si le taux de croissance économique est identique au taux d'intérêt, alors l'effet de levier est neutre. Dans ce cas, si la collectivité enregistre un excédent primaire, le taux d'endettement diminue et l'endettement reste soutenable (cellule B). Mais si un déficit primaire apparaît, le taux d'endettement augmente et l'endettement n'est pas soutenable (cellule $\mathrm{Y}$ ). 
Tableau 52 Combinaison des conditions de déclenchement d'un cercle vicieux ou d'un cercle vertueux de l'endettement.

Effet de levier des taux sur l'endettement

\begin{tabular}{|c|c|c|c|c|}
\hline & \multicolumn{3}{|c|}{ Effet de levier des taux sur l'endettement } \\
\hline & & $\begin{array}{l}\text { Effet positif } \\
\qquad(r-i)>0\end{array}$ & $\begin{array}{l}\text { Effet neutre } \\
\qquad(r-i)=0\end{array}$ & $\begin{array}{l}\text { Effet négatif } \\
\qquad(r-i)<0\end{array}$ \\
\hline & $\begin{array}{l}\text { Excédent primaire } \\
\qquad S>0\end{array}$ & $\begin{array}{c}\text { Cercle vertueux } \\
\text { Endettement } \\
\text { soutenable }(\mathrm{A})\end{array}$ & $\begin{array}{l}\text { Cercle vertueux } \\
\text { Endettement } \\
\text { soutenable (B) }\end{array}$ & $?(\mathbf{N})$ \\
\hline $\begin{array}{l}\text { Solde } \\
\text { primaire }\end{array}$ & $\begin{array}{l}\text { Équilibre primaire } \\
\qquad S=0\end{array}$ & $\begin{array}{l}\text { Cercle vertueux } \\
\text { Endettement } \\
\text { soutenable (C) }\end{array}$ & $\begin{array}{l}\text { Cercle neutre } \\
\text { Endettement } \\
\text { soutenable (D) }\end{array}$ & $\begin{array}{c}\text { Cercle vicieux } \\
\text { Endettement } \\
\text { non soutenable }(X)\end{array}$ \\
\hline & $\begin{array}{c}\text { Déficit primaire } \\
\qquad<<0\end{array}$ & $?(\mathrm{M})$ & $\begin{array}{c}\text { Cercle vicieux } \\
\text { Endettement } \\
\text { non soutenable (Y) }\end{array}$ & $\begin{array}{c}\text { Cercle vicieux } \\
\text { Endettement } \\
\text { non soutenable (Z) }\end{array}$ \\
\hline
\end{tabular}

L'endettement n'est pas soutenable non plus si l'effet de levier des taux est négatif comme le solde primaire (cellule Z). Le même constat se dégage si l'effet de levier est négatif avec un équilibre primaire (cellule $\mathrm{X}$ ).

Deux situations ne sont toutefois pas tranchées. Il s'agit du cas où l'effet de levier est positif, tandis que le solde primaire est négatif (cellule $\mathrm{M}$ ) et du cas où l'effet de levier est négatif alors que le solde primaire est positif (cellule N). Ces situations sont marquées par un point d'interrogation dans le tableau. Pour identifier l'existence d'un cercle vicieux respectivement d'un cercle vertueux, il faut s'interroger sur la marge de manœuvre dont dispose la collectivité avant que son endettement ne commence à faire boule de neige.

Si l'effet de levier positif des taux est suffisamment important pour compenser un solde primaire négatif, alors le taux d'endettement diminue et la soutenabilité de l'endettement s'améliore. En cas d'effet de levier négatif, le taux d'endettement peut aussi diminuer pour autant qu'un excédent primaire se présente et soit suffisamment important pour offrir une compensation. Par conséquent, il faut déterminer

$$
\begin{array}{lll}
\text { si } & (\mathrm{r}-\mathrm{i}) \times \mathrm{D}_{\mathrm{t}-1}+\mathrm{S}_{\mathrm{t}}>0 & \text { indiquant la présence d'un cercle vertueux } \\
\text { ou si } & (\mathrm{r}-\mathrm{i}) \times \mathrm{D}_{\mathrm{t}-1}+\mathrm{S}_{\mathrm{t}}<0 & \text { indiquant la présence d'un cercle vicieux }
\end{array}
$$

Un écart positif par rapport à zéro indique un cercle vertueux de l'endettement. Plus l'écart est important, plus la collectivité dispose d'une marge de manœuvre avant d'entrer dans un cercle vicieux de l'endettement, avant que son endettement ne commence à faire boule de neige. En revanche, un écart négatif par rapport à zéro nous indique l'importance de l'effort d'assainissement à accomplir pour échapper au cercle vicieux de l'endettement. La collectivité n'a aucune marge de manœuvre, bien au contraire. 
À l'Année t, la marge de manœuvre M correspond à l'expression suivante:

$$
\mathrm{M}_{\mathrm{t}}=(\mathrm{r}-\mathrm{i}) \times \mathrm{D}_{\mathrm{t}-1}+\mathrm{S}_{\mathrm{t}}
$$

Pour MaCollectivité, rappelons que le taux de croissance des recettes fiscales $\mathrm{r}$ s'élève à 1,5\% à l'Année t et le taux d'intérêt moyen (ou implicite) de la dette i à 1,2\% (indicateur I8, voir la section 9.3.2 consacrée aux indicateurs de qualité de la gestion financière). Compte tenu de l'endettement à la fin de l'Année $t-1$, l'effet de levier des taux sur l'endettement se limite à KCHF 6, soit $(0,015-0,012) \times \operatorname{KCHF} 2080$. Le solde primaire de l'Année $t$ atteignant KCHF -243, la marge de manœuvre est négative pour l'équivalent de KCHF -237. Autrement dit, pour sortir de la boule de neige vicieuse de l'endettement dans laquelle elle s'est trouvée au cours de l'Année t, MaCollectivité aurait dû améliorer son solde financier au moins de ce montant.

La figure 59 met précisément en évidence les éléments que nous venons de détailler: (a) l'effet de levier des taux de croissance et d'intérêt, reflété par les barres verticales noires; (b) le solde de financement primaire, schématisé par les barres blanches et (c) la marge de manœuvre correspondant à un cercle soit vicieux, soit vertueux de l'endettement, représentée à l'aide d'une courbe. Ces trois éléments sont mesurés sur l'axe vertical de gauche qui s'échelonne entre KCHF 1000 et -500 . Nous retrouvons la zone grisée reflétant l'évolution du taux d'endettement net dont la valeur se lit sur l'axe vertical de droite.

Figure 59 Évolution du taux d'endettement de MaCollectivité liée à l'effet de levier des taux et au solde primaire.

De l'Année t-20 à l'Année t.

1000

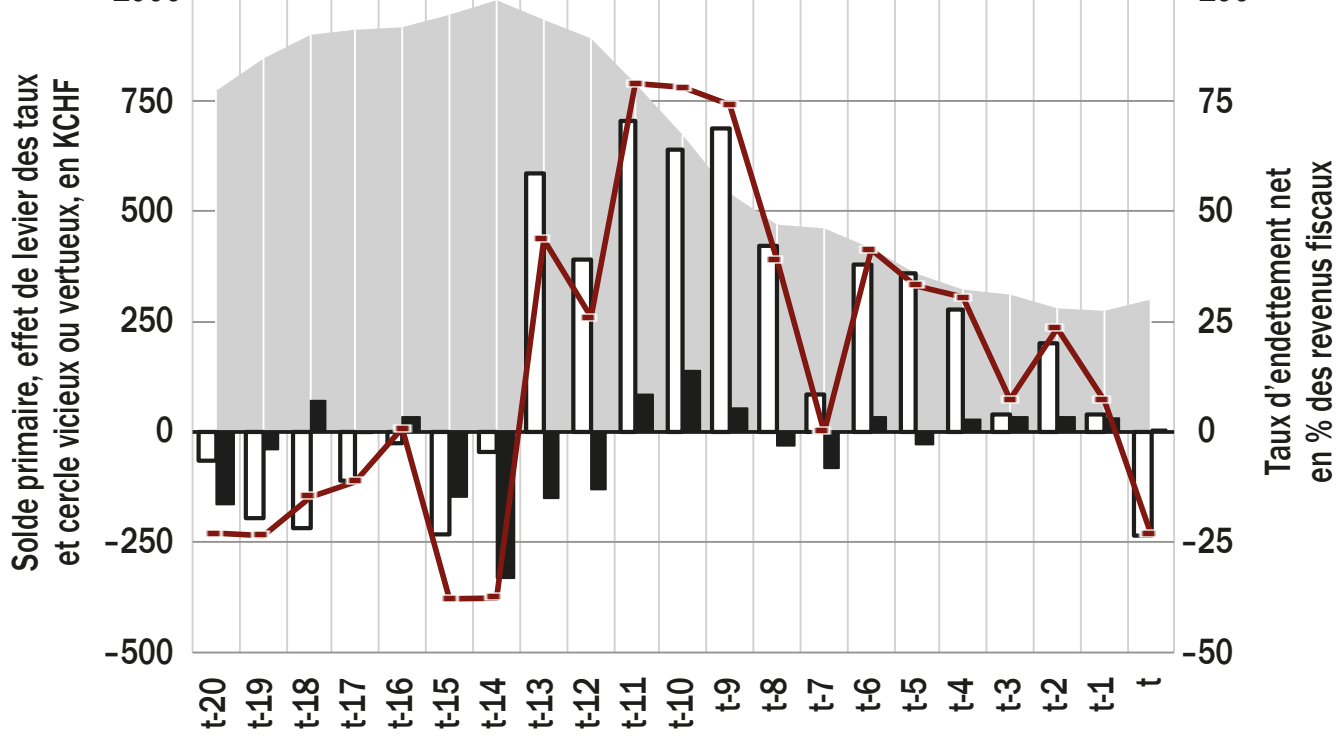

Taux d'endettement net (échelle de droite) $\square$ Solde de financement primaire

Effet de levier de $r$ - i sur l'endettement

--- Marge: cercle vicieux (-) ou vertueux (+) 
La figure présente la situation de MaCollectivité au cours des vingt années précédant l'Année t. Les barres blanches montrent un excédent primaire lorsqu'elles pointent vers le haut et un déficit primaire lorsqu'elles pointent, comme à l'Année t, vers le bas. Les barres noires montrent un effet de levier positif des taux sur l'endettement si elles sont orientées au nord, comme c'est légèrement le cas à l'Année t (et plus visiblement les années précédentes), et un effet négatif lorsqu'elles indiquent le sud.

En début de période, nous voyons clairement apparaître des années au cours desquelles toutes les conditions sont réunies pour que l'endettement fasse boule de neige et que le taux d'endettement s'accroisse significativement. La courbe de la marge de manœuvre est d'ailleurs toujours en zone négative, sauf en t-16.

Ensuite, la situation s'améliore en particulier sous l'effet d'un solde primaire qui redevient systématiquement positif, alors que l'effet de levier des taux est tantôt positif, tantôt négatif, mais reste de faible ampleur. Mais de manière cumulée, la courbe montre toutefois que la marge de manœuvre reste positive. L'Année t-7 fait exception: MaCollectivité dégage certes un excédent primaire, mais il suffit à peine à compenser l'effet de levier négatif des taux. Notons que l'Année t-16 présente le phénomène inverse: un déficit primaire compensé par un effet de levier positif. Finalement l'Année t bénéficie également d'un effet de levier positif des taux. Mais celui-ci est largement insuffisant pour compenser l'important déficit primaire lié en particulier à un effort d'investissement élevé. La figure montre donc la marge de manœuvre négative équivalant à KCHF -237 que nous avons détaillée ci-dessus.

\subsection{Lutte contre un cercle vicieux de l'endettement}

Il est bien souvent difficile de s'extraire d'un cercle vicieux de l'endettement. Les éléments qui viennent d'être mis en évidence permettent de s'en rendre compte. Mais ils permettent également d'identifier quelques solutions envisageables.

Une collectivité ne peut guère agir seule et à court terme sur le taux d'intérêt implicite pour le réduire (variablei). D'abord, le taux résulte des contrats d'emprunt qui sont en cours. Ensuite, il reflète le jugement porté par les bailleurs de fonds sur la solvabilité de la collectivité. Si ces bailleurs jugent négativement la solvabilité, ils imposeront des conditions plus sévères et donc un taux plus important, c'est-à-dire une prime de risque. L'effet de levier des taux s'en trouvera péjoré. Cela dit, une gestion de trésorerie plus rigoureuse permet souvent d'emprunter à de meilleures conditions et de réaliser ainsi des économies (voir à ce sujet le chapitre 7 consacré aux modalités d'emprunt).

Il est aussi difficile pour une collectivité d'influencer la croissance économique (variabler), du moins à court terme. Évidemment, si cette capacité est évaluée comme nous l'avons montré ici à l'aide des revenus fiscaux, il est plus aisé pour la collectivité de l'augmenter que si elle est mesurée avec un indicateur comme le PIB. 
La collectivité peut en effet accroître la pression fiscale sur les personnes physiques ou morales domiciliées sur son territoire. Cette relative flexibilité explique d'ailleurs que les bailleurs de fonds offrent des conditions d'emprunt plus favorables aux collectivités publiques qu'aux entités du secteur privé. Mais évidemment, dans un contexte où règne la concurrence fiscale entre collectivités, cette flexibilité doit être utilisée avec précaution. Faute de quoi, la hausse de la pression fiscale pourrait se solder par une évolution défavorable de l'assiette fiscale, et donc des impôts.

L’endettement hérité du passé s'apparente à un boulet. La variable $\mathrm{D}_{\mathrm{t}-1}$ constitue donc une donnée que l'on ne peut pas réduire. Il n'y a guère que dans des cas de boule de neige extrêmes, d'insolvabilité et d'impasse de trésorerie, que les créanciers d'une collectivité acceptent un rééchelonnement de la dette ou une remise de dette.

Reste la solution consistant à agir sur le solde primaire, en particulier s'il s'agit d'un déficit (variable $S_{t}$ ). La hausse de la pression fiscale évoquée ci-dessus peut également évidemment augmenter les recettes $\mathrm{R}$. Une autre manière de dynamiser les recettes, considérée parfois comme plus sournoise, consiste à réviser à la hausse les taxes et les redevances. Un recours plus systématique aux imputations internes permet de mieux démontrer comptablement le coût complet des prestations financées en totalité ou en partie selon le principe de l'utilisateur. Il peut contribuer à faire accepter l'idée d'une hausse des taxes et des redevances (voir le chapitre 4 consacré au mécanisme des imputations internes permettant de mieux refléter le coût des prestations et le chapitre 5 consacré au financement spécifique de certaines prestations).

Dans le cas de MaCollectivité, nous avons vu que le déficit primaire s'explique en partie par un effort d'investissement relativement élevé (indicateur I6 dans la section 9.3.2 et indicateur I1 ${ }^{\# \#}$ dans la section 9.3.4). Dans ces conditions, il faut s'interroger afin de savoir si les dépenses d'investissement vont continuer à se situer à un tel niveau à l'avenir. Si tel n'est pas le cas, la décrue attendue des dépenses d'équipement devrait garantir un retour à la normale et une sortie du cercle vicieux de l'endettement.

Par contre, si la collectivité présente un retard infrastructurel et doit persévérer dans son effort d'investissement, une analyse des dépenses courantes devient probablement incontournable. Dans le cas de MaCollectivité, l'indicateur I5 montre d'ailleurs que la maîtrise des dépenses pose quelque peu problème (section 9.3.2). L'éventail des mesures visant à mieux maîtriser les dépenses est vaste. Il sera en partie abordé au chapitre 14 présentant quelques pistes pour améliorer la situation financière à l'avenir. 


\section{Projeter la situation financière à la limite}

\subsection{Se projeter à la limite avec quelques hypothèses}

Dans les chapitres précédents, l'accent a été mis sur l'analyse de la situation financière existante. Il est temps de porter le regard vers le futur. En effet, la collectivité doit produire différents documents qui l'obligent à s'interroger sur son avenir (voir la section 2.5 relative au processus financier). L'élaboration du budget la confronte à son devenir immédiat; la planification financière à son futur à moyen terme. Toutefois, ces instruments sont inadaptés pour déterminer quelle pourrait être la situation financière à un horizon de trente ou cinquante ans.

Dans la vie courante, nous utilisons parfois la locution adverbiale «à la limite». Elle nous permet de signaler à notre interlocuteur que nous poussons un raisonnement et une manière de voir à leur extrême. Dans ce chapitre, nous appliquons ce raisonnement "à la limite» pour déterminer quelle serait la situation financière de la collectivité si l'on se projetait à un horizon temporel extrêmement lointain. À cet horizon, quel serait le taux d'endettement, le degré d'autofinancement ou encore l'importance de l'effet de tenailles des amortissements et des intérêts passifs sur les recettes? Pour cela, nous nous basons sur les mécanismes internes au modèle comptable harmonisé mis en évidence par Burgat et Jeanrenaud (1989 et 1991). Ces mécanismes sont ceux qui lient, à travers le tableau de financement, le compte de résultats, l'investissement net et le bilan (voir la section 3.3.7 relative également au tableau des flux de trésorerie). Ces mécanismes sont tels que les indicateurs du taux d'endettement, de l'autofinancement et de l'effet de tenailles tendent vers une valeur spécifique lorsque l'horizon temporel tend vers l'infini. Autrement dit, ces indicateurs tendent vers une valeur limite. Cela est vrai dès le moment où quelques hypothèses et paramètres fondamentaux sont posés.

I. Compte de résultats équilibré. Cette première hypothèse prévoit que les charges courantes sont toujours égales aux revenus courants. Autrement dit, la couverture des charges est égale à 100\% (indicateur financier I1). Le solde du compte de résultats est nul: il n'y a ni excédent de charges, ni excédent de revenus. Cette hypothèse reflète une des contraintes souvent évoquées dans la législation sur les finances: le compte de résultats doit être équilibré, si ce n'est annuellement, du moins à moyen terme. La conséquence mécanique d'un solde du compte de résultats égal à zéro est quadruple: (a) le niveau du capital propre au passif du bilan reste constant; (b) l'autofinancement dégagé par le fonctionnement correspond strictement aux amortissements; (c) le besoin de financement externe (et donc la variation des capitaux tiers, c'est-à-dire des engagements nets entre le début et la fin de chaque exercice) est donné par la différence entre l'investissement net et les seuls amortissements; (d) la variation des engagements nets (à la hausse ou à la baisse) est identique à celle du 
patrimoine administratif puisque ce dernier augmente avec les investissements nets et diminue avec l'amortissement ${ }^{197}$.

II. Taux d'amortissement unique et constant. Selon cette deuxième hypothèse, l'usure et l'obsolescence de l'infrastructure de la collectivité correspondent à une part constante du patrimoine administratif. Ce taux fixe s'applique à la valeur résiduelle du patrimoine administratif au bilan. Pour simplifier, ce taux est unique. Il peut être vu comme une moyenne pondérée des taux appliqués aux différents actifs productifs selon leur nature et leur durée d'utilisation. À travers cette hypothèse, on admet que le portefeuille des équipements ne change pas à travers le temps et que les nouveaux équipements ont la même durée de vie que les anciens. Ce taux $\mathrm{k}_{\mathrm{A}}$ est donc le rapport entre l'amortissement du patrimoine administratif et ce patrimoine en début d'exercice. Il détermine donc le montant de l'amortissement de l'année sur la base du patrimoine existant:

amortissements de l'année $=\mathbf{k}_{\mathrm{A}} \times$ patrimoine en début d'année

III. Effort d'investissement constant. Selon cette troisième hypothèse, l'effort d'investissement est constant sur toute la période. L'effort d'investissement est défini par analogie avec l'indicateur financier I6 qui rapporte l'investissement net aux dépenses courantes. Dans leur modèle, Burgat et Jeanrenaud considèrent toutefois le rapport $\mathrm{k}_{\mathrm{I}}$ entre l'investissement net et les revenus courants, plutôt que les dépenses courantes. $\mathrm{k}_{\mathrm{I}}$ détermine, pour chaque année, le montant de l'investissement à partir des revenus courants:

investissements nets de l'année $=\mathbf{k}_{\mathrm{I}} \times$ revenus courants de l'année

IV. Taux de croissance constant. Selon cette quatrième hypothèse, la capacité économique de la collectivité varie de manière constante à travers le temps. Plus précisément, le taux annuel de croissance ou de décroissance des revenus fiscaux (et courants) r est fixe. Donc r détermine le montant des revenus courants de l'année à partir de ceux de l'année précédente:

revenus courants de l'année $=(1+\mathbf{r}) \times$ revenus courants de l'année précédente

V. Taux d'intérêt constant. Selon cette cinquième hypothèse, le taux d'intérêt i à payer sur les engagements nets est constant à travers le temps. Il correspond à l'indicateur financier I8 qui rapporte les intérêts passifs à la moyenne de la dette brute entre le début et la fin de l'exercice. Ainsi i détermine le montant des intérêts à payer au cours de l'année à partir des engagements nets hérités de l'année précédente:

intérêts dus pour l'année $=\mathbf{i} \times$ engagements nets à la fin de l'année précédente

197 Notons que dans le cas de MaCollectivité et de l'Année t, cette hypothèse n'est ponctuellement pas vérifiée puisque le compte de résultats présente un excédent de revenus de près de CHF 1,0 mio, après neutralisation des opérations de cosmétique comptable. C'est d'ailleurs ce que nous indique l'indicateur I1: les charges sont couvertes à près de $108 \%$ par les revenus. 
VI. Part constante des revenus fiscaux. Selon cette sixième hypothèse, les revenus fiscaux représentent une part fixe à l'intérieur des revenus courants. Cette part $\mathrm{k}_{\mathrm{F}}$ correspond donc au rapport entre les revenus fiscaux et les revenus courants. Donc $\mathrm{k}_{\mathrm{F}}$ détermine le montant des revenus fiscaux en fonction des revenus courants ${ }^{198}$ :

revenus fiscaux de l'année $=\mathbf{k}_{\mathrm{F}} \times$ revenus courants de l'année

\subsection{Taux d'endettement net à la limite}

En se fondant sur les hypothèses ci-dessus, il devient possible de calculer quelle est la valeur limite prise par le taux d'endettement net. Le taux d'endettement net correspond à l'indicateur financier I9*, soit au rapport entre l'endettement net et les revenus fiscaux. La définition est donc la même que celle qui est utilisée dans le cadre de l'analyse de la dynamique de l'endettement menée au chapitre 12.

Burgat et Jeanrenaud (1989) établissent que la valeur limite du taux d'endettement I9" dépend de l'effort d'investissement $\mathrm{k}_{\mathrm{I}}$, du taux d'amortissement $\mathrm{k}_{\mathrm{A}}$ et du taux de croissance $r$, éléments auxquels il faut ajouter la part des revenus fiscaux dans les revenus courants $\mathrm{k}_{\mathrm{F}}$. Ces paramètres se combinent dans l'expression suivante:

$$
\text { limite de } I 9^{\#}=\left[\mathrm{k}_{\mathrm{l}} \times \frac{1+\mathrm{r}}{\mathrm{r}+\mathrm{k}_{\mathrm{A}}}\right] \times \frac{1}{\mathrm{k}_{\mathrm{F}}}
$$

Pour MaCollectivité, si l'on part du principe que les valeurs observées au cours de l'Année t peuvent être représentatives de l'avenir lointain, alors la valeur limite du taux d'endettement se situe à 277,3\%. En effet, à l'Année t, l'effort d'investissement $\mathrm{k}_{\mathrm{I}}$ (indicateur I6) s'élevait à $14,6 \%$; le taux de croissance $\mathrm{r}$ à $1,5 \%$; le taux d'amortissement $\mathrm{k}_{\mathrm{A}}$ à 8,7\% et la part des revenus fiscaux $\mathrm{k}_{\mathrm{F}}$ à 52,4\%. La figure 60 illustre la montée du taux d'endettement à travers les années, à partir du taux de $30,1 \%$ calculé sur la base des comptes de l'Année $t^{199}$. Elle montre que le taux d'endettement se rapproche graduellement de sa valeur limite calculée sur la base de l'expression ci-dessus (ligne grisée horizontale).

Un taux d'endettement au-dessus de 200\% est extrême et correspond à une note évaluative de 1,0 dans notre tableau de bord (voir section 9.3.3). Cela signifierait un changement drastique par rapport aux 30\% de l'Année t et à la note de 6,0 qui y correspond.

198 Contrairement aux autres paramètres, celui-ci est nécessaire pour une raison purement arithmétique. Burgat et Jeanrenaud (1989) s'intéressent à la valeur limite du taux d'endettement exprimé par rapport aux revenus courants. Par contre, le MCH2 s'intéresse à la valeur limite du taux d'endettement exprimé par rapport aux seuls revenus fiscaux. Le paramètre $\mathbf{k}_{\mathrm{F}}$ permet tout simplement de passer de l'un à l'autre.

199 À chaque nouvelle année, le niveau des engagements nets augmente sous l'effet des investissements nets (qui sont fonction de $\mathrm{k}_{\mathrm{I}}$ et des revenus courants) et diminue sous l'effet des amortissements (qui sont fonction de $\mathrm{k}_{\mathrm{A}}$ et du patrimoine administratif). De leur côté, les revenus augmentent sous l'effet du taux de croissance r. 
Pour éviter que le taux d'endettement projeté ne se concrétise, il faut entamer une réflexion sur la valeur que devraient prendre les paramètres entrant dans le calcul. Nous avons déjà mentionné que l'effort d'investissement de l'Année t était trop important (sanctionné par une note de 3,4). Le profil d'évaluation de l'indicateur financier I6 nous indique qu'un effort d'investissement idéal devrait représenter entre 7,0 et 10,0\% des dépenses courantes. En considérant une valeur de 7,5\% pour le paramètre $\mathrm{k}_{\mathrm{r}}$, un taux de croissance $\mathrm{r}$ plus élevé de 3,0\% et un taux d'amortissement $\mathrm{k}_{\mathrm{A}}$ de 10,0\% - un taux d'amortissement utilisé comme une valeur de référence par le $\mathrm{MCH} 1$-, la valeur limite du taux d'endettement net s'élèverait à 113,4\% avec une note évaluative sensiblement améliorée de $4,5^{200}$.

Figure 60 Évolution du taux d'endettement net (19\#) de l'Année t à l'Année t+50

en direction de sa valeur limite, en cas d'équilibre structurel.

Avec $k_{1}(=\mid 6)=14,6 \% ; r=1,5 \% ; k_{A}=8,7 \%$; et $k_{F}=52,4 \%$.

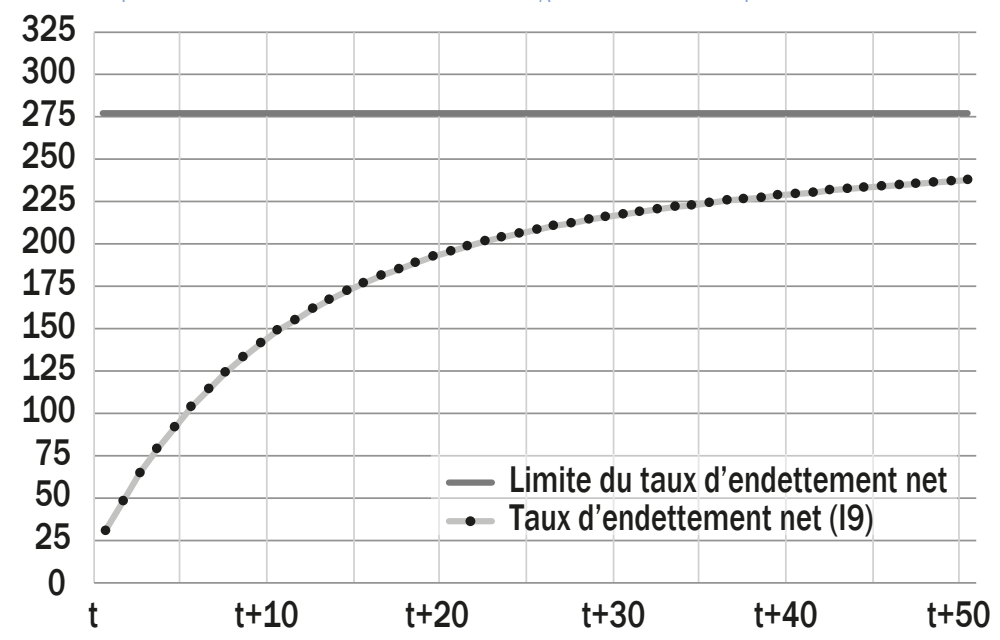

Le tableau 53 renseigne sur la limite du taux d'endettement net $19^{\#}$ en fonction du taux de croissance $\mathrm{r}(1,5 \%, 3,0 \%$ et $4,5 \%)$, de l'effort d'investissement $\mathrm{k}_{\mathrm{I}}$ (entre $12,5 \%$ et $5,0 \%)$ et du taux d'amortissement $(7,5 \%, 10,0 \%$ et $12,5 \%)$. Par hypothèse, les revenus fiscaux représentent la moitié des revenus courants $\left(\mathrm{k}_{\mathrm{F}}=50 \%\right)$. Il est important de noter que les valeurs figurant dans le tableau ne sont pas propres à MaCollectivité. En effet, elles sont valables pour n'importe quelle collectivité - canton ou commune - en fonction des paramètres affichés en tête de colonnes et de lignes et pour autant que les hypothèses posées précédemment soient vérifiées.

200 Le paramètre $\mathrm{k}_{\mathrm{F}}$ doit être considéré comme donné. En effet, Burgat et Jeanrenaud (1989) établissent leur relation sur la base d'un rapport d'endettement où la dette est exprimée en pourcentage des revenus courants. Pour utiliser la définition donnée par le $\mathrm{MCH} 2$ au taux d'endettement net, nous devons transformer le rapport pour passer des revenus courants aux revenus fiscaux. Cette transformation nécessite de recourir à un coefficient fixe, $\mathrm{k}_{\mathrm{F}}$. 
Tableau 53 Limite du taux d'endettement net (19\#) en fonction du taux de croissance $r$, du taux d'amortissement $k_{A}$ et de l'effort d'investissement $k_{l}(16)$.

Avec $k_{F}=50 \%$; paramètres et limites en $\%$; notes évaluatives entre parenthèses.

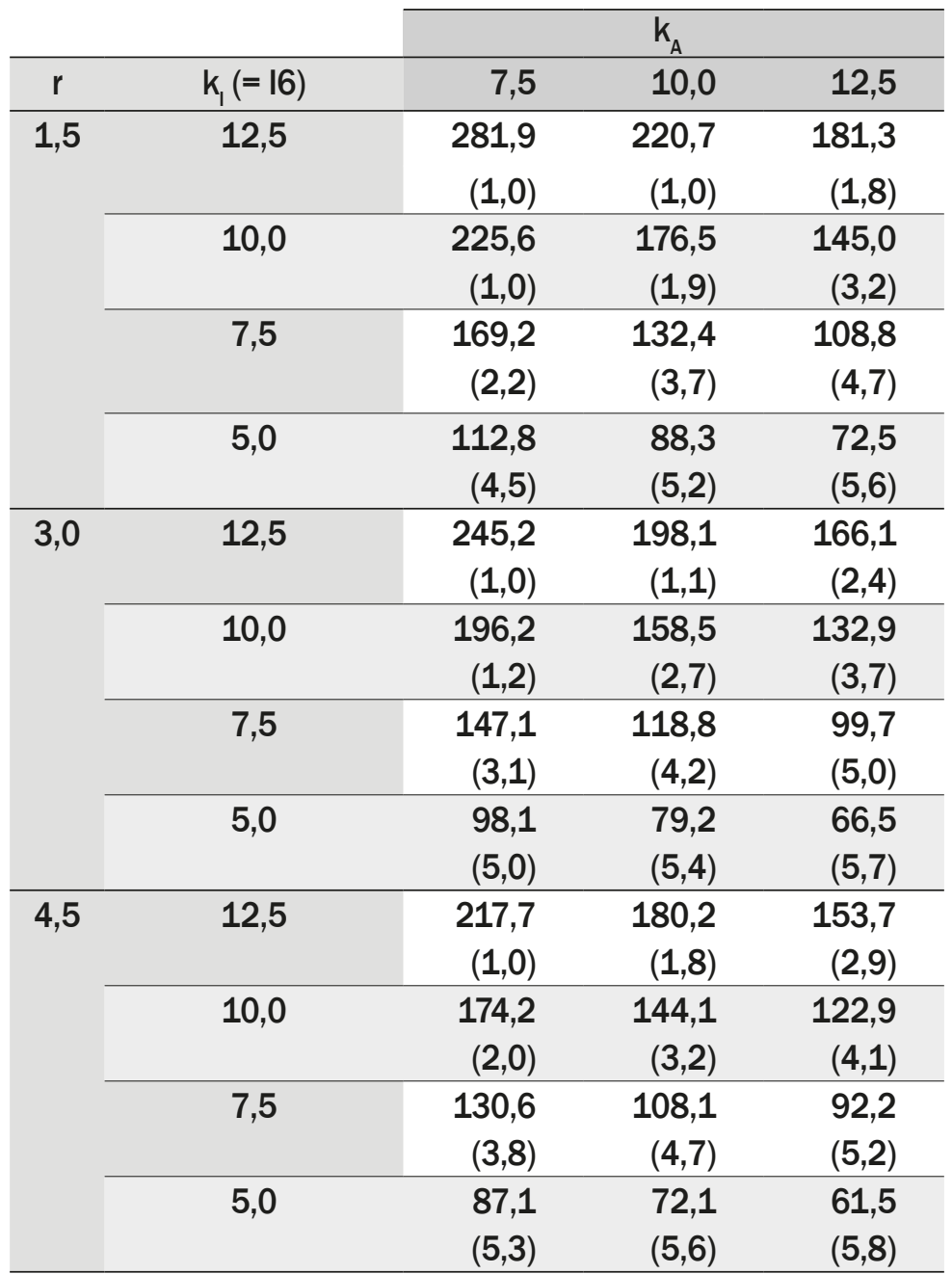

Ces chiffres confirment l'analyse que l'on peut directement tirer de l'expression arithmétique de la limite du taux d'endettement:

- D'abord, cette limite est proportionnelle à l'effort d'investissement $\mathrm{k}_{\mathrm{I}}$ : lorsque l'effort double (par exemple pour passer de 5,0\% à 10,0\%), le taux d'endettement double.

- Ensuite, la limite augmente si une collectivité réduit son taux d'amortissement $\mathrm{k}_{\mathrm{A}}$ (pour autant que parallèlement, elle présente toujours un compte de résultats parfaitement équilibré). Cela démontre qu'une stratégie qui vise, pour équilibrer le budget, à réduire les taux d'amortissement se solde par un taux d'endettement plus élevé. Dans ces conditions, équilibrer le budget ne suffit pas à se prémunir contre un endettement exagéré. 
- De plus, un développement économique soutenu, se traduisant par une croissance r élevée, contribue à abaisser la valeur limite du taux d'endettement.

- Finalement, et de manière mécanique, la limite du taux d'endettement est d'autant plus élevée que la part des revenus fiscaux dans le total des revenus est faible. Si $\mathrm{k}_{\mathrm{F}}$ diminue de moitié - passant par exemple de 50\% des revenus à $25 \%$ - la valeur limite du taux d'endettement net double. Cependant, cela n'est qu'un effet purement lié à l'arithmétique du ratio.

\subsection{Effet de tenailles à la limite}

Toujours en se fondant sur les hypothèses évoquées précédemment, une autre valeur limite peut être calculée: celle de l'effet de tenailles, autrement dit celle de l'indicateur financier I13 $3^{\# \#}$ de la part du «service de la dette», comme il est appelé dans le $\mathrm{MCH}$ 2. L'effet de tenailles correspond à la part des revenus courants absorbée par les charges d'intérêts nets et par les amortissements du patrimoine administratif.

Burgat et Jeanrenaud (1991) montrent que la valeur limite de l'effet de tenailles I1 $3^{\# \#}$ dépend de l'effort d'investissement $\mathrm{k}_{\mathrm{I}}$, du taux d'amortissement $\mathrm{k}_{\mathrm{A}}$, du taux de croissance $r$ et d'intérêt $i$ à travers cette nouvelle expression:

$$
\text { limite de } \mathrm{I} 13^{\#}=\mathrm{k}_{\mathrm{l}} \times \frac{\mathrm{i}+\mathrm{k}_{\mathrm{A}}}{\mathrm{r}+\mathrm{k}_{\mathrm{A}}}
$$

En faisant le calcul pour MaCollectivité à l'aide des valeurs observées au cours de l'Année $t$, on obtient une valeur limite de l'effet de tenailles de 14,2\%. Les paramètres $\mathrm{k}_{\mathrm{I}}, \mathrm{k}_{\mathrm{A}}$ et $\mathrm{r}$ ont la même valeur que précédemment et le taux d'intérêt moyen de la dette s'élève à 1,2\% (I8). La figure 61 met en évidence l'accroissement de l'effet de tenailles à travers le temps, partant du niveau de 5,1\% enregistré lors de l'Année $\mathrm{t}^{201}$. L'évolution asymptotique de l'effet de tenailles en direction de la valeur limite calculée à l'aide de l'expression ci-dessus se manifeste clairement. La valeur de $5,1 \%$ pour l'Année t correspond à la note de 5,0. Par contre, la valeur calculée pour la limite de 14,2\% serait excessive et conduirait à une note de 1,3 . En effet, à la limite, il ne resterait à la collectivité que $85,8 \%$ de ses revenus courants pour faire face à ses autres charges courantes; l'effet de tenailles serait donc très problématique.

Utiliser d'autres valeurs pour les paramètres que les valeurs observées à l'Année t permet d'envisager l'avenir avec davantage de sérénité. En tablant sur une valeur inférieure pour le paramètre $\mathrm{k}_{\mathrm{I}}$ par exemple $7,5 \%$, un taux de croissance $\mathrm{r}$ plus élevé de $3,0 \%$ et un taux d'amortissement $\mathrm{k}_{\mathrm{A}}$ de $10,0 \%$, la valeur limite de l'effet de tenailles tombe à $6,5 \%$ avec une note qui remonte à 4,4 .

201 À chaque nouvelle année, les intérêts sont calculés sur la base du taux i et du stock d'engagements nets hérités de l'année précédente. Les amortissements sont fonction de $\mathrm{k}_{\mathrm{A}}$ et du patrimoine administratif. Les revenus augmentent sous l'effet du taux de croissance $r$. 
Figure 61 Évolution de l'effet de tenailles (I13"\#) de l'Année t à l'Année t+50 en direction de sa valeur limite.

Avec $k_{l}(=\mid 6)=14,6 \%, r=1,5 \%, k_{A}=8,7 \%$ et $i=1,2 \%$.

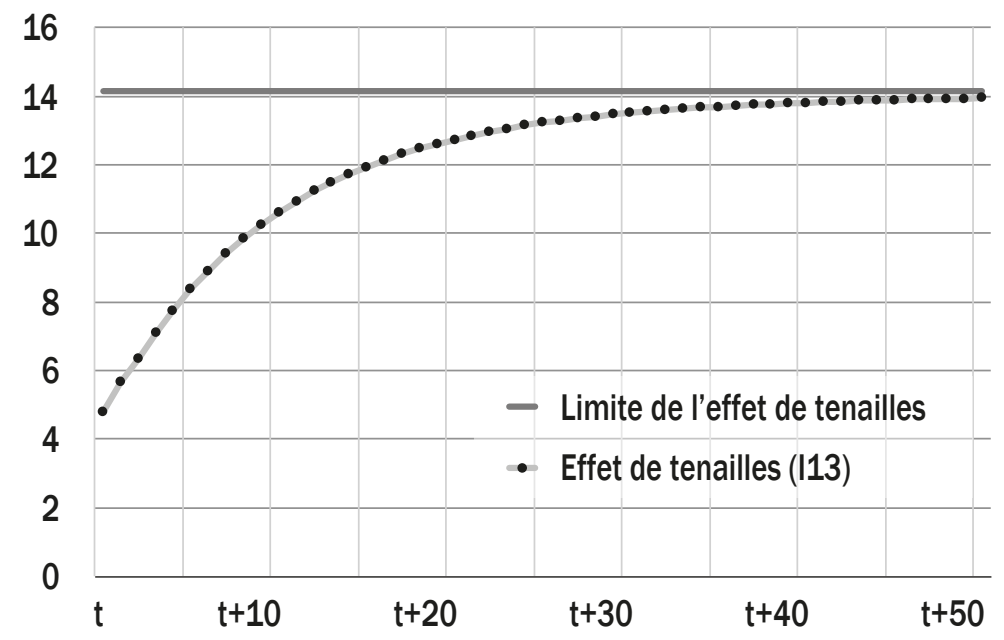

Le tableau 54 renseigne sur la limite de l'effet de tenailles en fonction du taux de croissance $r$ et d'intérêt i (1,5\%,3,0\% et 4,5\%), ainsi que de l'effort d'investissement $\mathrm{k}_{\mathrm{I}}$ (entre $12,5 \%$ et 5,0\%). Ici, l'impact du taux d'amortissement sur la valeur limite est réduit puisque $\mathrm{k}_{\mathrm{A}}$ se retrouve en dessus et en dessous de la barre de fraction. Par conséquent, le tableau se fonde sur une valeur unique de $10,0 \%$ pour $\mathrm{k}_{\mathrm{A}}$. Soulignons encore que les valeurs affichées dans le tableau ne sont pas propres à MaCollectivité. Elles sont valables quelle que soit la collectivité en fonction des paramètres figurant en tête de colonnes et de lignes et pour autant que les hypothèses posées précédemment soient respectées. Ces chiffres confirment l'analyse que l'on peut directement tirer de l'expression arithmétique de la limite de l'effet de tenailles:

- Ici aussi, l'effort d'investissement exerce une influence forte et proportionnelle sur la valeur limite. Doubler cet effort, par exemple de 5\% à 10\%, double la part du budget absorbée par les intérêts et les amortissements. Par conséquent, un effort d'investissement excessif pèse lourdement sur les revenus disponibles pour financer les activités opérationnelles de la collectivité et en particulier la fourniture des services publics.

- Lorsque la croissance des revenus $r$ (et donc du budget) est supérieur au taux d'intérêt i, l'effet de tenailles reste inférieur à l'effort d'investissement. Par exemple, si $\mathrm{r}$ et i s'élèvent à 3,0\% et que $\mathrm{k}_{\mathrm{I}}$ est égal à 10\%, l'effet de tenailles est également de $10 \%$. Mais si i s'amenuise et passe à $1,5 \%$, r dépasse i et l'effet de tenailles baisse à $8,8 \%$, soit au-dessous de l'effort d'investissement. Par contre, si i augmente pour atteindre $4,5 \%$, il dépasse $r$ et l'effet de tenailles atteint $11,2 \%$, surpassant ainsi l'effort d'investissement.

- Par conséquent, l'effet de levier des taux que nous avons découvert au chapitre précédent, soit la différence - positive ou négative - entre la croissance et le taux 
d'intérêt, influence également la part des revenus qui reste disponible dans le budget. Un effet de levier positif contribue à réduire l'effet de tenailles (en même temps qu'il permet de compenser un solde primaire négatif, voir le chapitre précédent). Un effet de levier négatif amplifie l'effet de tenailles.

Tableau 54 Limite de l'effet de tenailles $\left(I 13^{\# \#}\right)$ en fonction du taux de croissance $r$, du taux d'intérêt i et de l'effort d'investissement $k_{1}$ (I6).

$k_{A}=10,0 \%$; paramètres et limites en \%; notes évaluatives entre parenthèses.

\begin{tabular}{|c|c|c|c|c|}
\hline & & & i & \\
\hline$r$ & $\mathrm{k}_{1}(=16)$ & 1,5 & 3,0 & 4,5 \\
\hline 1,5 & 12,5 & 12,5 & 14,1 & 15,8 \\
\hline & & $(2,0)$ & $(1,3)$ & $(1,0)$ \\
\hline & 10,0 & 10,0 & 11,3 & 12,6 \\
\hline & & $(3,0)$ & $(2,5)$ & $(2,0)$ \\
\hline & 7,5 & 7,5 & 8,5 & 9,5 \\
\hline & & $(4,0)$ & $(3,6)$ & $(3,2)$ \\
\hline & 5,0 & 5,0 & 5,7 & 6,3 \\
\hline & & $(5,0)$ & $(4,7)$ & $(4,5)$ \\
\hline 3,0 & 12,5 & 11,1 & 12,5 & 13,9 \\
\hline & & $(2,6)$ & $(2,0)$ & $(1,4)$ \\
\hline & 10,0 & 8,8 & 10,0 & 11,2 \\
\hline & & $(3,5)$ & $(3,0)$ & $(2,5)$ \\
\hline & 7,5 & 6,6 & 7,5 & 8,4 \\
\hline & & $(4,3)$ & $(4,0)$ & $(3,7)$ \\
\hline & 5,0 & 4,4 & 5,0 & 5,6 \\
\hline & & $(5,2)$ & $(5,0)$ & $(4,8)$ \\
\hline 4,5 & 12,5 & 9,9 & 11,2 & 12,5 \\
\hline & & $(3,0)$ & $(2,5)$ & $(2,0)$ \\
\hline & 10,0 & 7,9 & 9,0 & 10,0 \\
\hline & & $(3,8)$ & $(3,4)$ & $(3,0)$ \\
\hline & 7,5 & 5,9 & 6,7 & 7,5 \\
\hline & & $(4,6)$ & $(4,3)$ & $(4,0)$ \\
\hline & 5,0 & 4,0 & 4,5 & 5,0 \\
\hline & & $(5,4)$ & $(5,2)$ & $(5,0)$ \\
\hline
\end{tabular}

\subsection{Autofinancement de l'investissement net à la limite}

Si l'on garde toujours à l'esprit les hypothèses énoncées au début de ce chapitre, une valeur limite peut également être calculée pour l'autofinancement de l'investissement 
net, c'est-à-dire pour l'indicateur I2 $2^{\#}$. Rappelons que cet indicateur renseigne sur la part de l'investissement net que la collectivité peut financer par ses propres moyens, sans avoir à recourir à l'emprunt.

Burgat et Jeanrenaud (1991) montrent que la valeur limite de l'autofinancement de l'investissement net $\mathrm{I}^{*}$ ne dépend, à l'extrême, que du taux d'amortissement $\mathrm{k}_{\mathrm{A}}$ et du taux de croissance $\mathrm{r}$; ces deux taux se combinant dans l'expression suivante:

$$
\text { limite de } \mathrm{I} 12^{\#}=\frac{\mathrm{k}_{\mathrm{A}}}{\mathrm{r}+\mathrm{k}_{\mathrm{A}}}
$$

En faisant le calcul pour MaCollectivité à l'aide des valeurs observées au cours de l'Année $t$ pour $\mathrm{k}_{\mathrm{A}}$ et $\mathrm{r}$, on obtient une valeur limite de 85,3\%. La figure 62 montre l'élévation de l'autofinancement de l'investissement net à travers le temps, partant du niveau de 34,8\% enregistré lors de l'Année $\mathrm{t}^{202}$. Notons que cette valeur de 34,8\% est sensiblement différente de celle qui est présentée pour l'indicateur I2* dans le tableau de bord (91,1\%, voir la section 9.3). Rien de plus normal puisque MaCollectivité enregistre cette année-là un excédent de revenus de 1,0 CHF mio - après neutralisation des opérations comptables relevant de la tactique politique. Cet excédent vient s'ajouter à l'amortissement pour renforcer l'autofinancement. Rappelons encore une fois que l'analyse des limites doit respecter l'hypothèse que le compte de résultats est équilibré et qu'il ne présente donc pas d'excédent, que ce soit de charges ou de revenus. Par conséquent, si l'on considère pour l'Année t que l'autofinancement est généré uniquement par les amortissements, soit KCHF 671 et que cet autofinancement est mis en regard des investissements nets de KCHF 1928, le ratio s'élève bien à 34,8\%.

Figure 62 Évolution de l'autofinancement de l'investissement net (I2\#) de l'Année t à l'Année t+50 en direction de sa valeur limite.

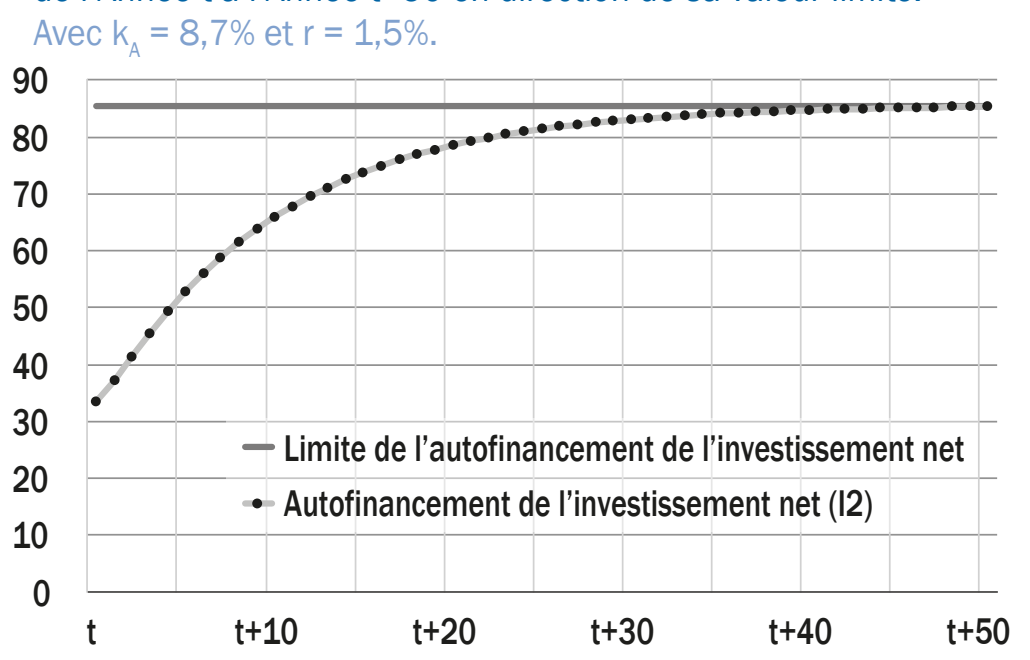

202 Les amortissements (et donc l'autofinancement) sont chaque année fonction de $\mathrm{k}_{\mathrm{A}}$ et du patrimoine administratif. L'investissement net dépend de $\mathrm{k}_{\mathrm{I}}$ et des revenus courants. Les revenus augmentent sous l'effet du taux de croissance $\mathrm{r}$. 
La figure souligne la convergence de la valeur de l'indicateur $2^{\#}$ vers la valeur limite de 85,3\%. La note pour une telle valeur est de 5,0. L'autofinancement se situerait donc à un bon niveau. En effet, à la limite, la collectivité ne devrait recourir à l'emprunt que pour financer $14,6 \%$ de ses investissements nets.

Le tableau 55 renseigne sur la limite de l'autofinancement selon le taux de croissance $\mathrm{r}$ et le taux d'amortissement $\mathrm{k}_{\mathrm{A}}$. À nouveau, relevons que les valeurs indiquées dans le tableau ne sont pas propres à MaCollectivité. Elles s'appliquent quelle que soit la collectivité, en fonction des paramètres figurant en tête de colonnes et de lignes et pour autant que les hypothèses nécessaires au calcul soient vérifiées.

Tableau 55 Limite de l'autofinancement (I2\#) en fonction du taux de croissance $r$ et du taux d'amortissement $\mathrm{k}_{\mathrm{A}}$.

Paramètres et limites en \%; notes évaluatives entre parenthèses.

\begin{tabular}{crrr}
\cline { 2 - 4 } & \multicolumn{3}{c}{$\mathrm{k}_{\mathrm{A}}$} \\
\hline $\mathrm{r}$ & 7,5 & 10,0 & 12,5 \\
\hline 1,5 & 83,3 & 87,0 & 89,3 \\
& $(4,9)$ & $(5,1)$ & $(5,3)$ \\
\hline 3,0 & 71,4 & 76,9 & 80,6 \\
& $(4,1)$ & $(4,5)$ & $(4,7)$ \\
\hline 4,5 & 62,5 & 69,0 & 73,5 \\
& $(3,5)$ & $(3,9)$ & $(4,2)$ \\
\hline
\end{tabular}

Ces chiffres illustrent l'analyse que l'on peut directement tirer de l'expression arithmétique de la limite de l'autofinancement de l'investissement net:

- La croissance économique, et singulièrement celle des revenus $r$, exerce une influence déterminante sur l'autofinancement. Une croissance nulle $(\mathrm{r}=0)$ conduit à un degré d'autofinancement de $100 \%$. En effet, elle implique une stagnation des revenus et une constance des montants investis puisque, compte tenu des hypothèses du modèle, ces derniers sont proportionnels aux revenus à travers le paramètre $\mathrm{k}_{\mathrm{r}}$.

- Par contre, un rythme de croissance des revenus strictement positif $(r>0)$ réduit la valeur limite du degré d'autofinancement. Une croissance provoque mécaniquement des dépenses d'investissement plus élevées d'année en année. Mais ces dépenses ne sont amorties que de manière décalée dans le temps parce que les amortissements sont fonction du patrimoine administratif à disposition en début d'année à travers le paramètre $\mathrm{k}_{\mathrm{A}}$. Dans ces conditions, le degré d'autofinancement est inférieur à $100 \%$. Plus le rythme de croissance est élevé, plus le degré d'autofinancement est faible, toutes choses égales par ailleurs.

- Le MCH1 recommandait un objectif de $60 \%$ en matière d'autofinancement en évoquant également un taux d'amortissement de 10\%. Le tableau 55 montre qu'un tel objectif ne peut être atteint dans la durée que si le taux de croissance des revenus s'établit et reste constant à un niveau élevé. Ce niveau est de 62/3\%. 
Le $\mathrm{MCH} 2$ recommande maintenant un degré d'autofinancement de $80 \%{ }^{203}$. La croissance nécessaire pour atteindre cet objectif se situe à un niveau plus réaliste de $2,5 \%$, avec un taux d'amortissement maintenu à $10 \%$.

- Intuitivement, on aurait pu penser que la valeur limite de l'autofinancement de l'investissement net dépendrait de l'effort d'investissement $\mathrm{k}_{\mathrm{r}}$. Ce n'est pas le cas. En revanche, la valeur limite dépend du taux d'amortissement $\mathrm{k}_{\mathrm{A}}$.

- Si le niveau de l'effort d'investissement n'influence pas en tant que tel la valeur limite du degré d'autofinancement, le contenu du programme d'investissement a des conséquences sur les perspectives de croissance. L'investissement influence donc sur le long terme le degré d'autofinancement, moins par son importance que par sa qualité. Certains investissements sont davantage porteurs que d'autres parce qu'ils servent directement à accompagner la croissance démographique, voire à la susciter, respectivement à promouvoir le développement des entreprises. C'est évidemment un élément à retenir pour améliorer la situation financière de la collectivité à l'avenir.

\subsection{Déséquilibre structurel et limite du taux d'endettement net}

Une collectivité peut vouloir réduire son taux d'endettement net à un niveau inférieur à celui qui est obtenu sur la base des hypothèses énoncées au début de ce chapitre. Pour y parvenir, elle peut notamment dégager un surplus sur le long terme, donc un surplus structurel. Burgat et Jeanrenaud (1993) complètent leur modèle afin de relâcher l'hypothèse d'un compte de résultats équilibré. Cela permet de calculer la valeur limite du taux d'endettement net en cas de déséquilibre du compte de résultats. L'expression proposée est complétée pour tenir compte du taux de déséquilibre $\mathrm{k}_{\mathrm{D}}$, en plus de l'effort d'investissement $\mathrm{k}_{\mathrm{I}}$, des taux d'amortissement $\mathrm{k}_{\mathrm{A}}$ et de croissance $r$, et de la part des revenus fiscaux dans les revenus courants $k_{\mathrm{F}}$. Ces paramètres se combinent selon l'expression suivante:

$$
\text { limite de I9 } 9^{\#} \text { en cas de déséquilibre }=\left[\mathrm{k}_{\mathrm{l}} \times \frac{1+\mathrm{r}}{\mathrm{r}+\mathrm{k}_{\mathrm{A}}}-\mathrm{k}_{\mathrm{D}} \times \frac{1+\mathrm{r}}{\mathrm{r}}\right] \times \frac{1}{\mathrm{k}_{\mathrm{F}}}
$$

Le taux de déséquilibre $\mathrm{k}_{\mathrm{D}}$ correspond au solde du compte de résultats rapporté aux revenus courants ${ }^{204}$. En cas d'équilibre, $\mathrm{k}_{\mathrm{D}}$ est égal à zéro et on en revient à la formule de la limite du rapport d'endettement net que nous avons présentée précédemment. Mais cette nouvelle expression montre qu'un excédent de revenus $\left(\mathrm{k}_{\mathrm{D}}>0\right)$ réduit la valeur limite, puisque $\mathrm{k}_{\mathrm{D}}$ est précédé d'un signe négatif. Par contre,

203 Recommandation 18, CDF (2008).

$204 \mathrm{k}_{\mathrm{D}}=$ (revenus courants - charges courantes)/revenus courants $\times 100$. Dans les grandes lignes, ce ratio est similaire à l'indicateur de couverture des charges I1 après soustraction de $100 \%: \mathrm{k}_{\mathrm{D}}=\mathrm{I} 1-100 \%$. Pour rappel $\mathrm{I} 1=$ revenus courants/charges courantes $\times 100$. 
un excédent de charges $\left(\mathrm{k}_{\mathrm{D}}<0\right)$ accroît la valeur limite. L'impact du déséquilibre - positif ou négatif - sur la valeur limite est d'autant plus fort que le taux de croissance $r$ est faible. En effet, la valeur de $(1+r) / r$ est d'autant plus élevée que $r$ est bas ${ }^{205}$.

Pour MaCollectivité, rappelons que, sous l'hypothèse d'un équilibre du compte de résultats, la valeur limite du taux d'endettement se situe à $277,3 \%$. Avec un excédent de revenus de $2 \%$, la valeur tombe à $19,0 \%\left(\right.$ avec $\mathrm{k}_{\mathrm{I}}=14,6 \% ; \mathrm{r}=1,5 \% ; \mathrm{k}_{\mathrm{A}}=$ $\left.8,7 \% ; \mathrm{k}_{\mathrm{F}}=52,4 \%\right)$ partant d'un taux de 30,1\% pour l'Année t. La figure 63 montre que, dans un premier temps, le taux d'endettement augmente car l'autofinancement ne suffit pas à couvrir l'investissement net ${ }^{206}$. Le fait que la collectivité dégage un excédent de revenus et bénéficie ainsi d'un autofinancement plus important, limite l'envolée du taux d'endettement. Ce dernier ne dépasse pas 125\%. De ce fait, la note évaluative ne tombe jamais en dessous de 4,0. À partir de l'Année t+20, le taux décroît parce que l'autofinancement devient supérieur à l'investissement net. Il se rapproche de la valeur limite de 19,0\%. Mais le rapprochement est très lent. C'est la raison pour laquelle, alors que les graphiques précédents couvraient un horizon temporel de cinquante années, celui-ci s'étend sur un horizon de cent années afin de mieux montrer la tendance asymptotique. Il faut maintenir le régime excédentaire durant près de 50 ans pour que le taux passe en-dessous de $100 \%$ et que la note remonte ainsi à 5,0. Après cent ans, le taux s'abaisse à 57,5\% pour une note de 5,9.

Figure 63 Évolution du taux d'endettement net (19\#) de l'Année t à l'Année t+100 en direction de sa valeur limite, en cas d'excédent structurel.

Avec $k_{I}(=\mid 6)=14,6 \% ; r=1,5 \% ; k_{A}=8,7 \% ; k_{D}(=\mid 1-100 \%)=2,0 \% ; k_{F}=52,4 \%$.

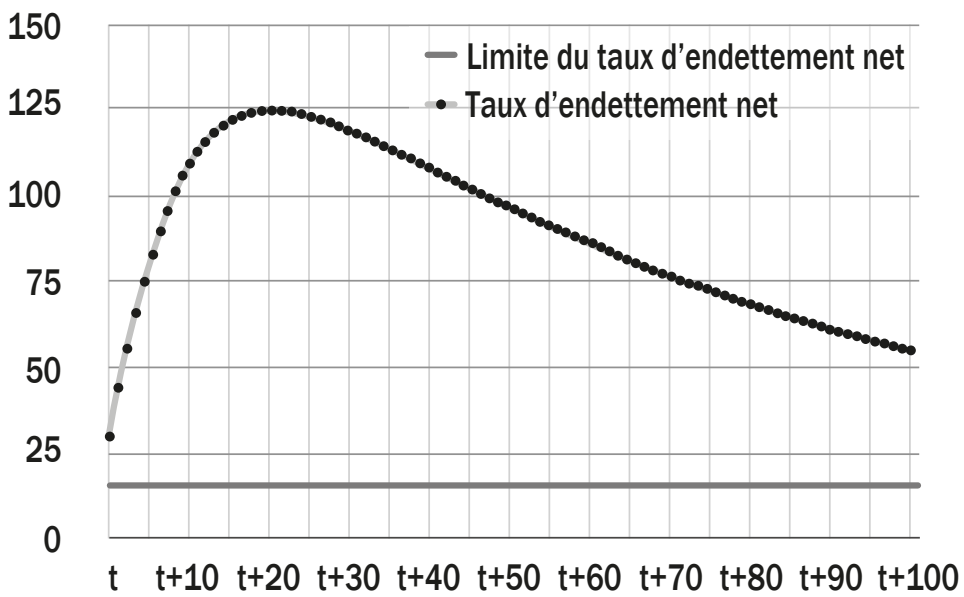

205 L'expression proposée ne tolère par une valeur de $\mathrm{r}=0$, puisque dans ce cas-là, l'opération $(1+\mathrm{r}) / \mathrm{r}$ est impossible. Pour des valeurs de $\mathrm{r}$ (positives ou négatives) proches de zéro (par exemple 0,1 ou $-0,1$ ), la valeur limite du taux d'endettement net est très élevée, ce qui n'est pas surprenant. Fixer une hypothèse de croissance hors de l'intervalle $[0,5 ;-0,5]$, voire $[1,0 ;-1,0]$ permet d'aboutir à des valeurs limites moins extrêmes.

206 À chaque nouvelle année, le niveau des engagements nets augmente sous l'effet des investissements nets (qui sont fonction de $\mathrm{k}_{\mathrm{I}}$ et des revenus courants), diminue sous l'effet des amortissements (qui sont fonction de $\mathrm{k}_{\mathrm{A}}$ et du patrimoine administratif) et de l'excédent de revenus (qui est fonction de $k_{D}$ et des revenus courants). De leur côté, les revenus augmentent sous l'effet du taux de croissance r. 
Le tableau 56 renseigne sur la limite du taux d'endettement en fonction du taux de croissance $\mathrm{r}(1,5 \%, 3,0 \%$ et $4,5 \%)$, de l'effort d'investissement $\mathrm{k}_{\mathrm{I}}$ (entre $12,5 \%$ et $5,0 \%$ ) et du taux de déséquilibre $\mathrm{k}_{\mathrm{D}}$ (entre $-2 \%$ et $2 \%$ ). Les valeurs limites sont calculées sur la base d'un taux d'amortissement $\mathrm{k}_{\mathrm{A}}$ de $10,0 \%$ et de revenus fiscaux équivalant à $50 \%$ des revenus courants, $\mathrm{k}_{\mathrm{F}}$. Il est important de noter que les valeurs figurant dans le tableau sont valables pour n'importe quelle collectivité - canton ou commune - en fonction des paramètres affichés en tête de colonnes et de lignes et pour autant que les hypothèses du modèle soient vérifiées.

Tableau 56 Limite du taux d'endettement net (19\#) en fonction du taux de croissance $r$, de l'effort d'investissement $k_{1}(16)$ et du taux de déséquilibre du compte de résultats $\mathrm{k}_{\mathrm{D}}(=\mathrm{I} 1-100 \%)$.

Avec $k_{A}=10 \%$ et $k_{F}=50 \%$; paramètres et limites en $\%$; notes évaluatives entre parenthèses.

\begin{tabular}{|c|c|c|c|c|c|c|}
\hline \multirow[b]{2}{*}{$r$} & \multirow[b]{2}{*}{$k_{1}(=16)$} & \multicolumn{5}{|c|}{$k_{\mathrm{D}}(=\mid 1-100 \%)$} \\
\hline & & $-2,0$ & $-1,0$ & 0,0 & 1,0 & 2,0 \\
\hline \multirow[t]{8}{*}{1,5} & 12,5 & 491,3 & 356,0 & 220,7 & 85,3 & $-50,0$ \\
\hline & & $(1,0)$ & $(1,0)$ & $(1,0)$ & $(5,3)$ & $(6,0)$ \\
\hline & 10,0 & 447,2 & 311,9 & 176,5 & 41,2 & $-94,1$ \\
\hline & & $(1,0)$ & $(1,0)$ & $(1,9)$ & $(6,0)$ & $(6,0)$ \\
\hline & 7,5 & 403,1 & 267,7 & 132,4 & $-2,9$ & $-138,3$ \\
\hline & & $(1,0)$ & $(1,0)$ & $(3,7)$ & $(6,0)$ & $(6,0)$ \\
\hline & 5,0 & 358,9 & 223,6 & 88,3 & $-47,1$ & $-182,4$ \\
\hline & & $(1,0)$ & $(1,0)$ & $(5,2)$ & $(6,0)$ & $(6,0)$ \\
\hline \multirow[t]{8}{*}{3,0} & 12,5 & 335,4 & 266,7 & 198,1 & 129,4 & 60,7 \\
\hline & & $(1,0)$ & $(1,0)$ & $(1,1)$ & $(3,8)$ & $(5,8)$ \\
\hline & 10,0 & 295,8 & 227,1 & 158,5 & 89,8 & 21,1 \\
\hline & & $(1,0)$ & $(1,0)$ & $(2,7)$ & $(5,2)$ & $(6,0)$ \\
\hline & 7,5 & 256,2 & 187,5 & 118,8 & 50,2 & $-18,5$ \\
\hline & & $(1,0)$ & $(1,5)$ & $(4,2)$ & $(6,0)$ & $(6,0)$ \\
\hline & 5,0 & 216,6 & 147,9 & 79,2 & 10,6 & $-58,1$ \\
\hline & & $(1,0)$ & $(3,1)$ & $(5,4)$ & $(6,0)$ & $(6,0)$ \\
\hline \multirow[t]{8}{*}{4,5} & 12,5 & 273,1 & 226,6 & 180,2 & 133,7 & 87,3 \\
\hline & & $(1,0)$ & $(1,0)$ & $(1,8)$ & $(3,7)$ & $(5,3)$ \\
\hline & 10,0 & 237,0 & 190,6 & 144,1 & 97,7 & 51,2 \\
\hline & & $(1,0)$ & $(1,4)$ & $(3,2)$ & $(5,0)$ & $(6,0)$ \\
\hline & 7,5 & 201,0 & 154,5 & 108,1 & 61,7 & 15,2 \\
\hline & & $(1,0)$ & $(2,8)$ & $(4,7)$ & $(5,8)$ & $(6,0)$ \\
\hline & 5,0 & 165,0 & 118,5 & 72,1 & 25,6 & $-20,8$ \\
\hline & & $(2,4)$ & $(4,3)$ & $(5,6)$ & $(6,0)$ & $(6,0)$ \\
\hline
\end{tabular}


284 Comprendre et gérer les finances de ma collectivité

Ces chiffres confirment l'analyse que l'on peut directement tirer de l'expression arithmétique de la limite du taux d'endettement en cas de déséquilibre. La valeur limite est fortement influencée par le taux de déséquilibre. Dès lors que la collectivité enregistre un excédent de revenus, la limite du taux d'endettement net affiche rarement des valeurs problématiques, sauf si la croissance $r$ est élevée ou si l'effort d'investissement est soutenu. 


\section{Améliorer la situation financière à l'avenir: quelques pistes}

Il n'existe pas une recette miracle pour améliorer une situation financière problématique, pour réduire un déficit structurel. S'il en existait une, nous serions heureux de pouvoir l'exposer ici. Le succès d'une telle opération découle généralement d'une conjonction de mesures, qui pour certaines n'ont rien de spectaculaire à court terme, mais produisent des effets à long terme.

Ce chapitre vise à donner quelques pistes. Ces pistes ne ciblent pas des prestations particulières. Privilégier une prestation plutôt qu'une autre relève d'un choix essentiellement politique. C'est donc un terrain sur lequel nous ne nous aventurerons pas.

Les pistes que nous décrivons ici sont essentiellement en lien avec la manière de gérer les finances et de concevoir le processus budgétaire. Elles portent sur l'accroissement de la marge de manœuvre financière, ainsi que sur une approche plus stratégique basée sur un effort d'investissement et un sentier de croissance des charges qui soient appropriés. Une autre idée est de repenser le processus budgétaire pour donner davantage la main à l'Exécutif et de simplifier l'organisation et l'information financières. De manière plus ciblée, nous attirons également l'attention sur les charges induites de l'investissement, avant de terminer sur un catalogue non exhaustif de mesures d'assainissement pouvant être envisagées si besoin.

\subsection{Améliorer la marge de manœuvre financière et réduire les charges liées}

Logique de court terme du budget. Les politiques publiques ont besoin de temps pour déployer leurs effets. Paradoxalement, la discussion du budget cristallise les débats. On est alors prisonnier d'une logique de court terme. C'est le moment où il faut plus ou moins équilibrer le budget en fonction des revenus prévus. Les charges doivent s'inscrire dans ce cadre, en particulier si des dispositions légales contraignantes comme un frein à l'endettement doivent être satisfaites. Or, à court terme, la marge de manœuvre est étroite.

Charges liées. Les collectivités publiques se plaignent souvent qu'une grande partie de leurs charges sont liées. Le terme de charges liées n'est pas consacré par les dispositions légales. Mais il évoque les difficultés de gestion engendrées par le fait qu'une part considérable des revenus prévus au budget doit être obligatoirement consacrée à financer des charges peu ou pas influençables à court terme.

Les charges sont liées lorsque des dispositions légales imposent d'affecter directement les revenus. Par exemple, la Constitution fédérale prévoit que les cantons bénéficient d'une part à l'impôt fédéral direct (art. 128, al. 4); cette part constitue 
une charge liée pour la Confédération. Les charges des cantons ou des communes au titre de la péréquation sont également des charges liées aux dispositions légales. Dans ces deux cas, les dispositions légales sont de rang supérieur. Par conséquent, les collectivités peuvent très difficilement intervenir pour les modifier. Lorsque les dispositions légales dépendent directement de la collectivité concernée par les dépenses liées, celles-ci peuvent plus facilement être révisées. Toutefois, cela demande du temps, en particulier lorsque la révision est soumise à référendum. C'est pourquoi une révision des dispositions légales n'est pas compatible avec le calendrier d'élaboration du budget. La Confédération considère que ce type de dépenses est fortement lié. Elle estime qu'il représente $57 \%$ des dépenses inscrites à son budget 2016, soit quelque CHF 38 mrd (AFF 2017).

D'autres charges sont liées aux engagements contractuels, parfois pluriannuels ou à durée indéterminée, pris par la collectivité concernée. Il s'agit notamment de contrats prévoyant le versement de contributions à des entités tierces. Les contrats de travail lient évidemment la collectivité aux membres de son personnel. Ils impliquent le versement des salaires et le paiement des cotisations sociales. Les contrats de prêts engagent la collectivité vis-à-vis de ses créanciers et l'obligent à leur verser des intérêts. Les investissements réalisés créent également des charges liées pour l'amortissement ou pour l'entretien. La Confédération considère que les dépenses de ce type sont faiblement liées ou non liées. Ces dépenses représentent le reste du budget: 43\% de ses dépenses d'après les estimations pour 2016, soit CHF 29 mrd (AFF 2017). Cette part de dépenses offre par conséquent une certaine marge de manœuvre dans le cadre de l'élaboration du budget. Certes, il est toujours délicat de dénoncer ou de renégocier ces contrats. Mais les contrats qui arrivent à échéance peuvent ne pas être systématiquement reconduits ou être réexaminés. Dans le domaine du personnel, en postulant succinctement, qu'une personne reste pour une durée moyenne de dix à vingt ans auprès de la même collectivité publique, cela représente un taux de rotation du personnel de quelque 5\% (100\%/20) à $10 \%$ $(100 \% / 10)$ par année. À l'heure actuelle, les charges de personnel représentent en moyenne environ un tiers des charges des cantons ou des communes (un peu plus de 10\% s'agissant de la Confédération) (AFF 2019a). Par conséquent, sous la seule influence de la rotation du personnel, une marge de manœuvre de 2 à $3 \%$ se fait jour en moyenne dans le budget d'un canton ou d'une commune. Évidemment, ce chiffre ne doit pas être interprété comme un montant devant être économisé. Il doit être compris comme un volant pour la préparation et la discussion du budget. Nous mentionnons ces éléments à titre exemplatif: d'autres volants, dans d'autres domaines, existent du côté des charges comme du côté des revenus.

Revenus à libre disposition. Toujours est-il que, dans la fenêtre temporelle couverte par le budget, l'essentiel des revenus est absorbé par des dépenses liées et peu de revenus sont à libre disposition. Mais la situation évolue si l'on anticipe et si l'on planifie, autrement dit, si l'on élargit l'horizon temporel. La figure 64 l'illustre. 
Figure 64 Élargir l'horizon temporel accroît la part des revenus à libre disposition.

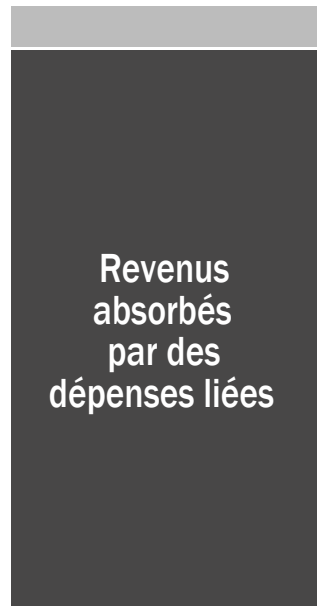

Court terme

Budget

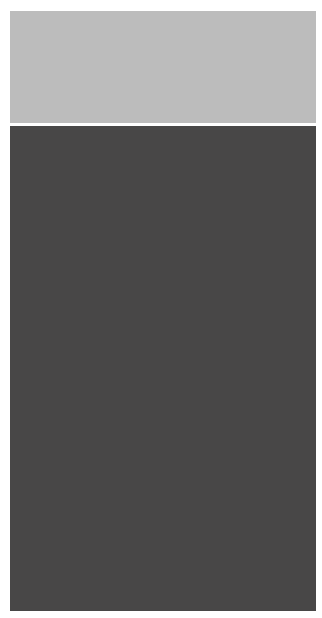

Moyen terme

Plan financier

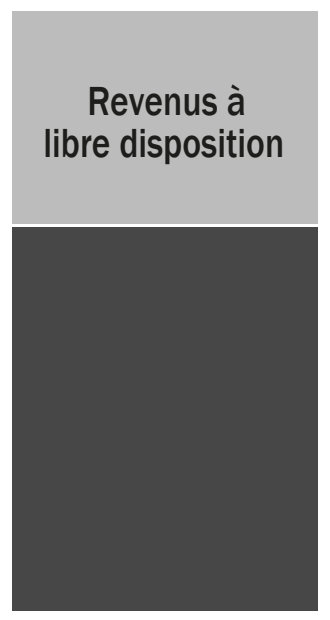

Long terme

Durée de vie des équipements

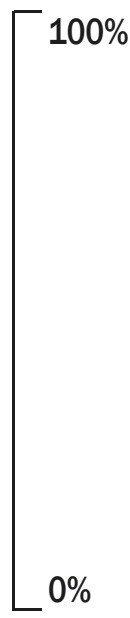

$0 \%$

L'horizon temporel du plan financier couvre le moyen terme, soit trois ou quatre années suivant le budget. Dans ce laps de temps, un volant de revenus à libre disposition plus important peut être dégagé. D’abord, les revenus, notamment fiscaux, peuvent s'accroître sensiblement sur la période, en raison d'une augmentation des assiettes fiscales. Ensuite, des contrats arrivent à échéance qu'il est possible de ne pas renouveler ou de renouveler à des conditions différentes. Enfin, on dispose de suffisamment de temps pour modifier les lois et règlements qui doivent l'être.

Si l'on allonge l'horizon de la réflexion pour prendre en compte la durée de vie des équipements, la marge de manœuvre devient considérable. Dans cette optique de long terme, les revenus qui doivent être consacrés aux amortissements des actifs concernés et au paiement des intérêts de la dette liée à ces équipements sont peu à peu libérés. L'effet de tenailles sur le budget se relâche graduellement (sections 9.3.4 et 13.3).

\subsection{Cadrer l'effort d'investissement et le sentier de croissance des charges}

Allonger l'horizon temporel pour penser au-delà du budget nécessite de modifier la manière d'approcher la planification. L'élaboration du budget se fonde presque toujours sur les revenus prévus pour l'année à venir. La prévision des revenus est un exercice difficile, en particulier parce qu'elle est réalisée plusieurs mois avant que l'année d'exécution du budget ne commence. On imagine bien que si cette prévision est difficile à court terme pour le budget, elle l'est plus encore à moyen terme pour le 
plan financier. Les incertitudes - entre autres macroéconomiques, démographiques, sociales, légales - sont telles que les prévisions revêtent un fort caractère aléatoire.

Faut-il pour autant renoncer à une réflexion et à une planification à moyen et à long terme? Sans doute pas! Mais il est conseillé de s'affranchir d'une approche centrée sur les revenus. Mieux vaut opter pour une approche plus stratégique. Le chapitre consacré à projeter la situation financière à la limite a montré à quel point l'effort d'investissement est un paramètre important (chapitre 13). Fixer cet effort à un niveau raisonnable garantit un taux d'endettement soutenable et limite l'effet de tenailles qui, sinon, met à mal la réalisation des politiques publiques. Un volume d'investissement net équivalent, en moyenne, à environ $8 \%$ des dépenses courantes met la collectivité à l'abri des mauvaises surprises. Il faut pour cela que la collectivité puisse bénéficier d'une croissance démographique et économique, même légère, lui garantissant une croissance de ses revenus de 1 à $2 \%$. Évidemment, un sentier de croissance des charges correspondant, en moyenne, à ces taux met la collectivité à l'abri des surprises. Le taux de croissance des charges et l'effort d'investissement à long terme devront toujours être réexaminés au moment de la révision du plan financier. Ce réexamen permet de s'assurer que ces paramètres n'excèdent pas les possibilités financières au vu des derniers comptes bouclés.

On pourrait croire qu'en se fixant une règle, c'est-à-dire un taux en matière d'effort d'investissement et de sentier de croissance des charges à moyen et à long terme, une collectivité se prive d'une partie de sa marge de manœuvre. Mais ce n'est pas le cas: la collectivité se dote ainsi d'une vision pour l'évolution de son infrastructure; une vision pour se positionner stratégiquement et afficher ouvertement le potentiel de développement qu'elle envisage. C'est à l'intérieur de ce potentiel, de cette enveloppe d'investissements et de charges possibles que la collectivité et ses membres disposent d'une latitude de choix.

Plus la réflexion s'engage tôt sur la manière d'utiliser cette marge de manœuvre, meilleurs sont les choix. Les trois ou quatre années du plan financier sont peu de chose en regard de la durée de vie de la plupart des équipements publics. Pensons aux écoles ou aux routes, dont la durée d'utilisation est au moins de trente ans. La mise au calendrier de ces investissements dans un horizon temporel long permet de prioriser les réalisations qui doivent l'être et de différer celles qui sont moins utiles.

\subsection{Réformer le processus budgétaire}

Les collectivités sont confrontées à des règles budgétaires de plus en plus contraignantes. Face à cette évolution, le processus budgétaire peut être repensé, à la fois pour en réduire le coût administratif et pour en améliorer le résultat. La budgétisation descendante (top-down budgeting) répond à ce besoin en rompant avec la traditionnelle approche ascendante (bottom-up budgeting). 
Budgétisation ascendante. Dans une approche ascendante, les unités administratives construisent leurs budgets avec un degré de liberté relativement élevé, sur la base de directives budgétaires. Ces directives sont généralement peu spécifiques, donc peu contraignantes. En pratique, les unités préparent leurs demandes en procédant à une simple adaptation du dernier budget qui leur a été alloué. Elles utilisent donc une méthode incrémentale (Wildavsky 1975). Elles soumettent ensuite leurs desiderata à la direction des finances. Ce faisant, elles ont tendance à présenter des requêtes supérieures à leurs vrais besoins, anticipant que les montants demandés feront l'objet de discussions à la baisse. L'addition des demandes de l'ensemble des unités aboutit immanquablement à un total dépassant les prévisions de revenus. Cela oblige la direction des finances à négocier pour réduire les prétentions. Plusieurs cycles de négociation sont généralement nécessaires. Toutefois, cela ne garantit pas qu'un accord soit trouvé. En finalité, il appartient aux membres de l'Exécutif d'aplanir les derniers différents et de «boucler» le budget, puis de le présenter au Législatif. Ce système confère évidemment un rôle central à la direction des finances. Cette dernière a pour mission de contrôler les requêtes budgétaires des autres unités et, cas échéant, de faire pression sur ces demandes pour tenter d'équilibrer le budget.

Plusieurs tentatives ont vu le jour pour remédier aux défauts de la budgétisation ascendante, en particulier cette tendance à la budgétisation incrémentale et à la surestimation tactique des besoins financiers. Parmi ces tentatives, relevons les plus souvent évoquées: la proposition d'un système associant planification, programmation et budgétisation (Planning, Programming, and Budgeting System, PPBS, portrait 10) et la proposition de préparer le budget sur une base zéro (Zero-Based Budgeting, ZBB, portrait 11). Ces diverses tentatives se sont soldées par des échecs en raison de la lourdeur administrative qu'elles impliquent, même si l'on retrouve des traces du PPBS, en Suisse et ailleurs, dans la mouvance de la «nouvelle gestion publique».

\section{Portrait 10 Planning, Programming, and Budgeting System}

Le système associant planification, programmation et budgétisation (plus connu sous son acronyme anglais PPBS) a été dans un premier temps introduit au département américain de la Défense en 1961, puis étendu au budget fédéral en 1965. L'idée en est que les objectifs des programmes gouvernementaux devraient être définis en amont, après quoi les programmes alternatifs permettant d'atteindre ces objectifs devraient être comparés en fonction de leurs coûts et de leurs avantages. En pratique, le PPBS n'a eu que peu d'impact sur la manière d'établir le budget du gouvernement fédéral. Deux raisons expliquent cet échec. D'abord, les objectifs concrets des programmes gouvernementaux sont difficiles à formuler. Ensuite, l'évaluation complète des programmes prend trop de temps. Le PPBS a été abandonné en 1971.

Source: Harlow (1973). 


\section{Portrait 11 Zero-Based Budgeting}

La budgétisation sur une base zéro (plus connue sous son acronyme anglais ZBB) est une méthode selon laquelle toutes les dépenses doivent être justifiées, si ce n'est dans le cadre de l'élaboration de chaque budget, du moins à intervalle régulier. Comme son nom l'indique, la démarche implique que l'on reparte de zéro pour repenser chaque dépense. Pour chaque unité administrative, les coûts sont comparés aux résultats obtenus et aux objectifs futurs. Cette comparaison permet aux échelons supérieurs, et en dernière analyse à l'Exécutif, d'affecter les moyens en fonction des besoins actuels plutôt que des dépenses historiques, comme c'est le cas avec la budgétisation incrémentale. Par conséquent, le budget de chaque unité peut être sensiblement plus élevé ou plus faible que le précédent.

Le ZBB a été initialement développé au sein de l'entreprise Texas Instruments. Alors qu'il était gouverneur de Géorgie, Jimmy Carter l'a adopté pour la préparation du budget 1973 de cet État. Élu à la présidence des États-Unis, il l'a introduit pour élaborer le budget 1977 du Parlement, de l'Exécutif et des agences gouvernementales au niveau de leur direction et des responsables de départements. Le ZBB a été officiellement abandonné par le gouvernement américain en 1981. Il y a deux raisons à cela. D'abord, la démarche s'est avérée trop lourde pour être valablement appliquée dans le cadre du calendrier budgétaire annuel. Ensuite, les informations à jour manquaient pour évaluer le niveau de la performance atteinte.

Source: Pyhrr (2012).

Budgétisation descendante. Le Danemark dès les années 1960 et le Canada dès les années 1970 font figure de précurseurs en abandonnant le paradigme de la budgétisation ascendante pour introduire celui de la budgétisation descendante (Kim et Park 2006). Ce faisant, ils mettent la priorité sur la maîtrise des dépenses et une stricte discipline budgétaire.

La budgétisation est dite descendante, car la première étape du processus consiste à prévoir le niveau global des recettes attendues et à fixer de manière contraignante l'objectif budgétaire. Cet objectif est souvent l'équilibre ou le quasi-équilibre. Par conséquent, le niveau prévu des recettes détermine l'enveloppe financière allouée aux différentes politiques publiques ou aux unités administratives. Cette décision d'allocation est donc prise en tout début de processus budgétaire par l'Exécutif au sein du collège gouvernemental. Cette allocation par politique ou par unité devient alors contraignante pour les directions d'unités et les directions en charge des diverses politiques publiques. Grâce à cela, la direction des finances dispose d'une décision préalable de l'Exécutif sur laquelle s'appuyer: les directions sont forcées de construire leur budget dans le respect de l'enveloppe qui leur est attribuée. De son côté, l'Exécutif peut ainsi maîtriser l'évolution des dépenses et donc le solde budgétaire. 
La budgétisation descendante nécessite un engagement initial plus important de l'Exécutif pour déterminer les enveloppes par unité ou par politique publique. L'effort à fournir en début de processus est aussi plus important pour la direction des finances qui doit soutenir l'Exécutif dans sa tâche. Mais ensuite, le processus est allégé, car les moult négociations que le processus ascendant impliquait ne sont plus nécessaires. Évidemment, cela suppose que l'on donne davantage de liberté et de flexibilité aux directions pour organiser leurs activités.

Les rôles sont ainsi plus clairement différenciés. L'Exécutif se concentre davantage sur son rôle stratégique avec l'appui de la direction des finances. Il n'intervient plus dans le détail du budget des unités. Ces dernières exercent leur rôle opérationnel pour remplir leur mission et atteindre les objectifs le plus souvent prescrits en amont par la législation, et cela en s'organisant à l'intérieur de l'enveloppe qui leur est dévolue. Finalement, l'approche descendante devrait améliorer la qualité du budget, d'où une efficacité supérieure. D'une part, le budget est en meilleure adéquation avec la stratégie de l'Exécutif. D'autre part, les directions développent le sentiment qu'elles sont propriétaires de l'enveloppe qui leur est allouée et se responsabilisent par rapport à l'usage qu'elles en font; l'efficience y trouve également son compte.

\subsection{Simplifier l'organisation et l'information financières}

Nous avons montré que les collectivités doivent présenter l'information financière sous trois angles: sous l'angle de la classification organique, sous l'angle de la classification par nature et sous celui de la classification fonctionnelle (voir la section 3.2.4 consacrée au plan comptable ${ }^{207}$. Nous avons également indiqué que la classification fonctionnelle a dû être instaurée pour remédier aux problèmes de comparabilité posés par la classification organique. En effet, cette dernière reflète l'organigramme de la collectivité. Or les organigrammes varient d'une organisation à l'autre, sans compter que l'organigramme d'une collectivité évolue au gré des restructurations.

Cette multiplication des manières de présenter le compte de résultats et le compte des investissements complique l'accès à l'information financière pour les non-initiés. Par ailleurs, elle est coûteuse parce qu'elle oblige l'administration à saisir, pour chaque opération, une clé d'imputation couvrant les trois classifications, puis à traiter l'information dans ces trois dimensions.

207 Certaines collectivités, essentiellement des cantons, ont encore accru la complexité en introduisant une présentation des budgets et des comptes selon un catalogue de prestations qui leur est propre et qui ne correspond pas à la classification fonctionnelle. 
Ces inconvénients peuvent être évités en calquant l'organisation de la collectivité sur la classification fonctionnelle. Cela revient à fonder l'organigramme sur la classification fonctionnelle comme le montre la figure 65. On y voit apparaître dix unités administratives (dicastères ou départements) subordonnées à l'Exécutif. Les unités 1 à 8 sont chacune en charge d'une des grandes fonctions de l'État, c'est-à-dire d'une des dix grandes prestations et politiques publiques. Les unités 0 et 9 constituent davantage des unités de support. À l'intérieur de chaque fonction, la classification fonctionnelle présente des sous-fonctions sur lesquelles calquer les subdivisions des unités administratives ${ }^{208}$.

Grâce à cela, la logique de présentation des budgets et des comptes ne repose plus que sur deux classifications: la classification par nature et la classification organico-fonctionnelle. Les états financiers sont plus condensés, donc plus clairs. En outre, cela ouvre de facto la voie à une gestion fondée sur les prestations.

Évidemment, tous les exécutifs ne comportent pas dix membres. Qu'à cela ne tienne! Si l'Exécutif compte un nombre plus limité de personnes - ce qui est généralement le cas -, il est possible de regrouper deux, voire trois unités, sous la responsabilité d'un membre du collège. Si d'aventure l'Exécutif est plus élargi, on peut répartir une fonction entre plusieurs membres en leur confiant la responsabilité de subdivisions.

De nombreuses communes ont déjà franchi le pas, depuis plusieurs années. De cette manière, leur organigramme reste stable à travers les législatures. Cependant, d'une législature à l'autre, le collège de l'Exécutif peut tout de même procéder à une nouvelle répartition des responsabilités des fonctions/unités entre ses membres afin de tenir compte des impératifs politiques, des compétences des élus et de leurs affinités.

Figure 65 Organigramme fondé sur la classification fonctionnelle.

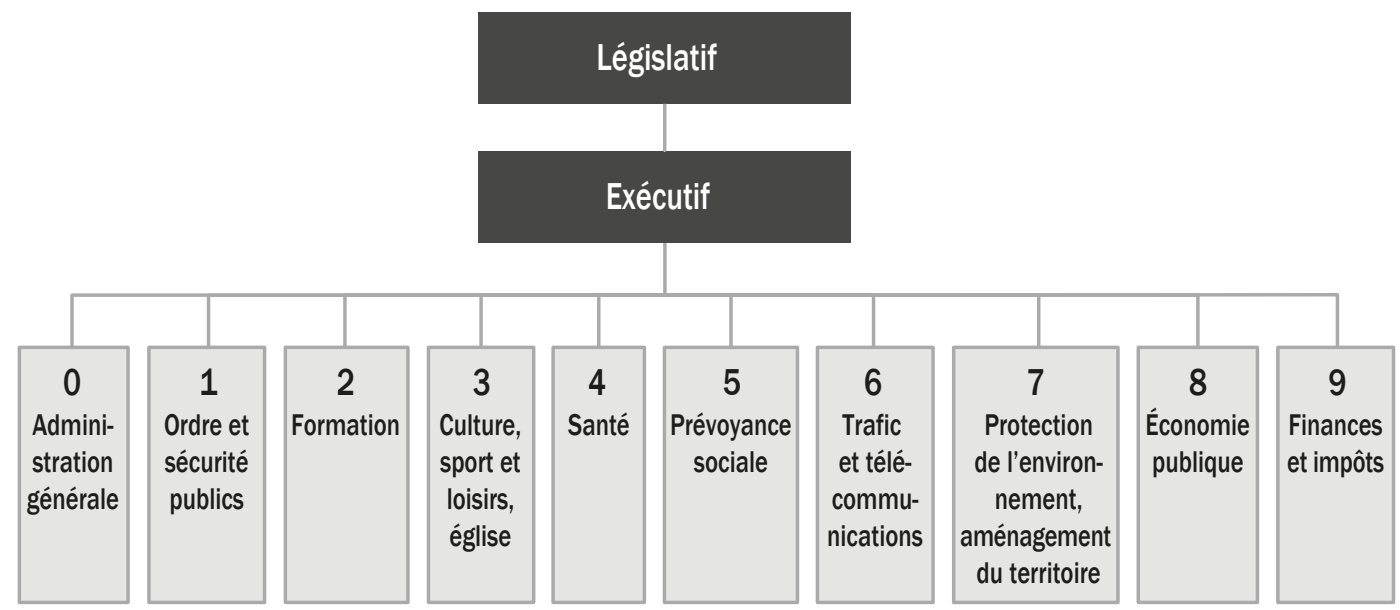

208 Pour les détails de la classification fonctionnelle, voir l'annexe B du Manuel MCH2, CDF (2008). 


\subsection{Penser aux charges induites de l'investissement}

Dans la réflexion sur les investissements à réaliser, il faut tenir compte des charges annuelles qui en découlent. Ces charges induites contribuent aux charges liées. Le tableau 57 présente les estimations réalisées par l'Office des affaires communales et de l'organisation du territoire du canton de Berne. Ces estimations portent sur plusieurs types de réalisations (OACOT 2001).

On peut voir que les charges induites par exemple pour une école correspondent à $2,5 \%$ de l'investissement brut, c'est-à-dire de la dépense avant déduction d'une quelconque subvention. Ces charges sont induites par l'entretien et la mise en valeur du bâtiment, par l'électricité, l'eau et le chauffage consommés dans l'établissement, ainsi que par les frais de conciergerie. Elles n'incluent pas l'amortissement. L'amortissement s'élève lui aussi à 2,5\%, s'il est linéaire et si l'on table sur une durée d'utilisation de quarante années. Pour un investissement brut de CHF 50 mio, ce sont donc CHF 2,5 mio qui doivent être mis annuellement à la charge du compte de résultats ( $5 \%$ sur CHF 50 mio). Il s'agit également de tenir compte des intérêts passifs à payer sur la part non autofinancée de l'investissement, des intérêts qui sont variables en fonction des conditions auxquelles il est possible d'emprunter (voir le chapitre 7 consacré aux modalités de financement des investissements et d'emprunt).

Tableau 57 Charges induites annuelles en pourcentage du montant de l'investissement brut. Entretien courant, mise en valeur, chauffage, eau, électricité et nettoyage, y compris conciergerie (hors intérêts et amortissements).

Type de réalisation $\%$ Ouvrage peu complexe

Halle de stockage, garage pour véhicules 1,0

Atelier, arsenal 1,5

Cimetière, place de sport, vestiaire, installation de tir, place de jeu, abris PC 2,0

\section{Ouvrage moyennement complexe}

Logement pour le troisième âge, jardin d'enfants, école, salle de gymnastique, $\quad 2,5$ halle polyvalente, centre de loisirs, bâtiment du service de défense contre l'incendie

Crèche, résidence pour personnes âgées (prodiguant ou non des soins), 3,5 école professionnelle, centre paroissial, bâtiment polyvalent, piscine couverte Immeuble administratif, bâtiment hospitalier standard 4,0

\section{Ouvrage complexe}

Bâtiment hospitalier affecté aux soins, clinique, musée, centre de conférence 4,5 Source: OACOT (2001). 
Tenir compte de ces charges induites - et d'éventuels revenus induits - dans la planification des charges permet d'éviter les mauvaises surprises. Or, dans la présentation des projets d'investissement, l'importance de ces éléments est souvent sous-estimée pour des raisons tactiques, afin de faciliter l'acceptation des projets. Ce manque de sincérité conduit à une hausse inattendue des charges quelque temps après la mise en fonction de la nouvelle infrastructure. Si la hausse met à mal l'équilibre budgétaire, elle nécessitera des mesures d'assainissement structurel.

\subsection{Choisir les mesures d'assainissement financier les plus opportunes}

Catalogue non exhaustif de mesures. Le catalogue des mesures d'assainissement financier est vaste. On peut imaginer des mesures visant à augmenter les revenus, à réduire les charges, à augmenter la productivité, à influencer l'environnement économique et démographique ou plus spécifiquement à améliorer le processus budgétaire.

Pour chacun de ces objectifs, nous nous bornons à mentionner dans le tableau 58, sans les détailler, quelques mesures possibles pour une commune. Nous ne prétendons pas à l'exhaustivité. Par contre, cette liste est dressée sans jugement de valeur, ni parti pris. Reconnaissons que le choix d'une mesure, plutôt qu'une autre, est le plus souvent dicté par des impératifs de faisabilité politique.

Effet des baisses de dépenses et des hausses de recettes. Plusieurs études se sont attachées à comparer les effets des mesures d'assainissement basées sur une baisse des dépenses aux effets des mesures basées sur une hausse des recettes et en particulier des recettes fiscales (Guichard et al. 2007; Alesina et Ardagna 2010). Ces études investiguent les conséquences au niveau des pays, plutôt que des collectivités locales. Elles évaluent les mesures en fonction de deux critères: (a) leur efficacité en termes de réduction du déficit et du taux d'endettement et (b) les éventuels effets négatifs sur la croissance économique. Selon ces études, à court terme, la réduction des déficits structurels pénalise généralement la demande globale et la croissance économique. Mais à long terme, une consolidation budgétaire est bénéfique à l'activité économique. Si l'effort d'assainissement est fondé sur une baisse des dépenses, ses effets à long terme sur l'activité économique sont plus favorables que si l'assainissement passe par une hausse des recettes, en particulier fiscales. Toutefois, cette conclusion n'est valable que si la baisse des dépenses concerne des dépenses de consommation et de transferts et que cette baisse épargne les dépenses les plus productives, telles les dépenses d'équipement. L'effet pénalisant à court terme peut être limité si les mesures de consolidation permettent de mettre fin à l'incertitude entourant la soutenabilité des finances publiques. En effet, ces mesures de consolidation contribuent à maintenir, voire rétablir la confiance des ménages et des entreprises. 
Tableau 58 Catalogue non exhaustif de mesures d'assainissement possibles.

\section{Augmenter les revenus}

Augmenter les revenus fiscaux (hausse des taux, lutte contre la fraude, suivi des débiteurs fiscaux)

Accroître les taxes et redevances (meilleure estimation du coût complet des prestations, hausse des tarifs)

Accroître le volume des biens et services vendus, et le taux d'utilisation des équipements tarifés

Augmenter les dédommagements facturés aux collectivités qui nous confient des tâches

Transférer au patrimoine financier les éléments du patrimoine administratif devenus inutiles pour l'accomplissement des tâches publiques

Accroître le rendement des placements grâce à une gestion plus dynamique

\section{Réduire les charges}

Allonger la durée d'utilisation des équipements pour étaler les amortissements Revoir à la baisse l'effort d'investissement, sélectionner plus drastiquement les projets Réexaminer le catalogue des prestations offertes et abandonner celles devenues peu utiles Limiter les travaux d'entretien des équipements à ce qui est vraiment nécessaire

Comprimer les frais généraux non directement liés aux prestations

Réduire le coût de la dette grâce à une gestion plus dynamique

Obtenir de meilleures conditions d'approvisionnement (achats groupés, appels d'offres)

Bloquer, voire réduire l'effectif de personnel, bloquer les salaires

\section{Augmenter la productivité}

Revoir l'organigramme et regrouper des unités trop petites pour travailler plus efficacement Recourir davantage aux nouvelles technologies lorsqu'elles permettent des gains de productivité

Simplifier les processus administratifs et supprimer les activités redondantes

Déléguer certaines activités/prestations à d'autres entités publiques ou à des mandataires privés

Rejoindre ou fonder des associations intercommunales ou des sociétés d'économie mixte Comparer plus systématiquement notre collectivité à d'autres par le benchmarking Inciter les bénévoles et la société civile à réaliser des activités à caractère public

\section{Influencer l'environnement économique et socio-démographique}

Favoriser l'implantation de nouvelles activités économiques

Favoriser la croissance ou au moins la stabilité démographique dans la collectivité

Favoriser l'entraide et la vie associative pour stimuler la cohésion sociale 


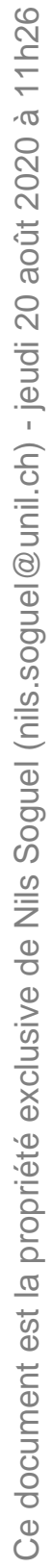




\section{Portée stratégique des finances de ma collectivité}

Ce chapitre aurait pu être le premier. Nous avons choisi qu'il referme l'ouvrage. Ce faisant, il doit nous rappeler que la gestion financière n'est pas une fin en soi. Elle n'est pas non plus qu'une affaire de spécialistes, formant un petit groupe au sein d'une unité administrative - la direction des finances - repliée sur elle-même. Les finances de la collectivité ont une dimension stratégique qui contribue à l'accomplissement de la plupart des missions de l'État.

Politique publique institutionnelle. La gestion financière est une véritable politique publique, au même titre que les autres politiques publiques, avec tout ce que cela implique de noblesse et de servitudes. Cependant, elle a ses particularités, car elle n'est pas une politique substantielle comme l'est la politique de la formation ou celle de la santé. Ces dernières fournissent des prestations directement aux ménages et, directement ou indirectement, aux entreprises.

Quant à la gestion financière, elle est une politique institutionnelle au sens où elle sert au bon fonctionnement de l'institution qu'est la collectivité publique. De fait, c'est à travers elle que les autres politiques publiques disposent des ressources nécessaires pour se déployer, qu'il s'agisse de ressources humaines, matérielles ou infrastructurelles. La gestion financière met ces ressources à disposition à travers le budget, elle en suit la consommation tout au long de l'année et elle en contrôle la bonne utilisation avec les comptes. Sans gestion financière adéquate, une politique publique substantielle, quelle qu'elle soit, serait bien en peine d'exister dans la durée.

Missions de l'État. Il ne faut pas réduire l'apport de la gestion financière à la seule mise à disposition de ressources au profit d'autres politiques publiques. En réalité, la gestion financière influence le bien-être collectif en contribuant directement aux grandes missions de l'État. À y regarder de plus près et en suivant Musgrave (1959), l'État doit intervenir pour remédier à trois types de problèmes non résolus ou créés par l'économie de marché: les problèmes d'allocation, ceux de redistribution et ceux de stabilisation.

Allocation. La première catégorie réunit les problèmes découlant de l'incapacité du marché à allouer, dans certains cas, correctement les ressources humaines, matérielles, infrastructurelles et environnementales entre les différentes activités, biens ou services dont, collectivement, nous avons besoin. Ces cas sont au nombre de trois. Le premier cas est celui des biens collectifs. Le second celui des monopoles naturels. Sur ces deux cas, nous ne nous attarderons pas, dans la mesure où le premier concerne des politiques publiques spécifiques (santé publique, sécurité publique, défense, etc.) et le deuxième cas concerne surtout l'activité de régulation par l'État des industries de réseau (eau, électricité, routes, chemins de fer, télécommunication, etc.) ${ }^{209}$. 
Par contre, le troisième cas, celui des externalités, mérite ici davantage d'attention, car il génère potentiellement et directement des encaissements ou des décaissements pour les collectivités.

En l'absence d'intervention de l'État, le système socio-économique consommerait parfois trop de ressources. Les nuisances à l'environnement sont un exemple de cette allocation inefficace générée par le marché. Dans leur jargon, les économistes parlent ici d'externalités négatives. Mais des externalités positives peuvent aussi apparaître. Alors le marché, laissé à lui-même, n’alloue pas suffisamment de ressources à certaines activités. Pensons ici à la formation. L'État peut dédier des moyens pour déployer des politiques publiques substantielles afin de lutter contre ces externalités: politique de l'environnement, politique de la formation dans le cas de nos exemples. Mais il peut également recourir à son régime fiscal et parafiscal. Les taxes dites d'internalisation sont là pour désinciter les agents économiques à générer des externalités négatives. Des subventions peuvent inciter ces mêmes agents à ne pas générer de telles externalités. Des subventions ou des déductions fiscales peuvent également être instituées pour rémunérer ceux qui créent des externalités positives ${ }^{210}$. Ces exemples montrent que les finances publiques peuvent influencer directement les décisions des individus et des entreprises et les comportements de ceux-ci.

Redistribution. La seconde catégorie regroupe les inégalités de situation physique ou de richesse non résolues ou créées par l'économie de marché. Les inégalités peuvent être interindividuelles, intergénérationnelles ou interrégionales. La société confère à l'État la mission de rétablir une certaine équité en fonction de la conception de justice sociale qui prévaut.

Les finances des collectivités constituent un levier direct d'intervention à travers les transferts sociaux ou à travers la péréquation financière, comme nous l'avons montré au chapitre 6. Mais les collectivités interviennent également à travers leur fiscalité directe, en particulier lorsqu'elle est progressive, et indirecte, en particulier lorsqu'elle frappe spécifiquement certains biens ou certaines activités.

Stabilisation. La dernière catégorie porte sur les fluctuations macroéconomiques créées ou non par l'économie de marché. Les agents économiques ont une responsabilité dans ces fluctuations en raison de leurs exubérances et de leurs déprimes successives. Des facteurs externes au marché jouent également souvent un rôle: désordres politiques, guerres, famines, crises dans l'approvisionnement en matières premières et en agents énergétiques. Dans ce domaine, on attend souvent de l'État qu'il veille à la performance de la macroéconomie, de l'économie dans son ensemble. Les objectifs assignés sont un haut niveau d'emploi, une relative stabilité des prix, un taux de croissance suffisant et, parfois, un équilibre du commerce extérieur.

210 Les économistes utilisent d'ailleurs le terme de dépenses fiscales plutôt que de déductions fiscales. Ils mettent ainsi l'accent sur le fait que l'État, en accordant une déduction pour encourager les comportements allant dans le sens de l'intérêt collectif, renonce à des recettes fiscales. 
L'emploi et l'inflation dépendent notamment de la capacité de la demande globale à absorber la production. Or cette demande est fonction de décisions d'un nombre considérable d'agents économiques en matière de consommation ou d'investissement. Ces décisions sont tributaires de nombreux facteurs et rien ne garantit que la demande globale s'inscrive à un niveau suffisant, en particulier si le mécanisme des prix et des salaires présente des rigidités qui ralentissent les ajustements. Des mesures expansionnistes anticycliques visant à stimuler la demande globale sont généralement réclamées. À d'autres moments, lorsque des pressions inflationnistes apparaissent, des mesures restrictives sont sollicitées. Dans une perspective keynésienne, à côté de la politique monétaire dont la banque centrale a la responsabilité, les finances des collectivités publiques ont un rôle à jouer, ainsi que nous l'avons montré au chapitre 11.

L'argent de l'État, celui des contribuables. On s'en rend compte, les attentes sociétales vis-à-vis de l'État sont grandes. Elles sont potentiellement sans limite. Mais une limite existe bel et bien. Cette limite dépend des ressources que la gestion financière parvient à mobiliser pour les allouer aux politiques publiques substantielles. En 1983, dans son discours devant le Congrès du parti conservateur britannique, Margaret Thatcher, alors Première ministre du Royaume-Uni, rappelait que «l'argent public n'existe pas, il n'y a que l'argent des contribuables». Que l'on partage ou non les convictions de la Dame de fer, il n'en demeure pas moins que l'État tire son financement de l'argent gagné par les ménages, et dans une moindre mesure, par les entreprises. Cela ne fait qu'accroître la responsabilité des collectivités publiques. Une collectivité et ses autorités sont responsables et redevables vis-à-vis des personnes auprès desquelles elles prélèvent l'impôt et auprès desquelles elles empruntent. Bien gérer les finances de ma collectivité n'en a donc que plus d'importance. 


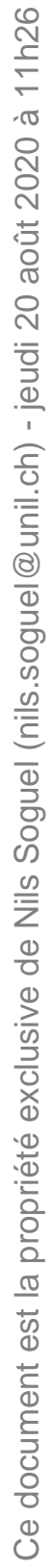




\section{Bibliographie}

AFC-Administration fédérale des contributions (2018, mars). «Distinction entre les impôts et les autres contributions publiques». Documentation et Information fiscale. Berne: CSI Conférence suisse des impôts.

AFF-Administration fédérale des finances (2006). NMC - Le nouveau modèle comptable de la Confédération. Berne: Publications fédérales.

AFF-Administration fédérale des finances (2017). Dépenses liées de la Confédération: Inventaire et pistes pour de futures réformes. Berne: Publications fédérales.

AFF-Administration fédérale des finances (2018). Confédération suisse. Rapport sur le budget 2019. Berne: Publications fédérales.

AFF-Administration fédérale des finances (2019a). Confédération suisse. Rapport sur le compte d'État 2018. Berne: Publications fédérales.

AFF-Administration fédérale des finances (2019b). Principes économiques, juridiques et organisationnels applicables à la gestion des finances. Berne: Publications fédérales.

AFF-Administration fédérale des finances (2019c). Statistique financière 2017 de la Suisse: Rapport annuel. Neuchâtel: OFS - Office fédéral de la statistique.

Alesina, A. et Ardagna, S. (2010). «Large Changes in Fiscal Policy: Taxes versus Spending». Tax Policy and the Economy, 24(1), 35-68.

Ammann, Y. (2009). Importance et utilisation des stabilisateurs automatiques, Papier de discussion $\mathrm{n}^{\circ} 17$, Berne: SECO-Secrétariat d'État à l'économie.

Ammann, Y. (2011). "Conjoncture et politique discrétionnaire de la Confédération». In Soguel N. (éd.), Des politiques au chevet de la conjoncture. Lausanne: PPUR - Presses polytechniques et universitaires romandes, 175-205.

Aschwanden, E. et Gerny, D. (2013, Août 15). «16 Kantone schnüren ein Sparpaket». Neue Zürcher Zeitung.

Burgat, P. et Jeanrenaud, C. (1989). «L'équilibre budgétaire et l'endettement des collectivités publiques locales». Revue suisse d'économie politique et de statistique, 125(4), 597-607.

Burgat, P. et Jeanrenaud, C. (1991). «"Effet de tenailles” et degré d'autofinancement des investissements dans le cas de l'équilibre budgétaire». Revue suisse d'économie politique et de statistique, 127(1), 35-53.

Burgat, P. et Jeanrenaud, C. (1993). Endettement des collectivités publiques: cas du canton de Genève. IRER working paper 9302. Neuchâtel: IRER-Institut de recherches économiques et régionales, Université de Neuchâtel.

Buschor, E. et Jéquier, R. (1985). Gestion et planification financières dans le secteur public. Genève: Banque hypothécaire du canton de Genève.

Busslinger, L. (2006, Décembre 6). «La liturgie du budget occupe un Grand Conseil presque serein ». 24 Heures, 27.

CDF-Conférence des directrices et directeurs cantonaux des finances (1982). Manuel de comptabilité publique. Berne: Éditions Paul Haupt.

CDF-Conférence des directrices et directeurs cantonaux des finances (2002). Handbuch Harmonisiertes Kosten- und Leistungsrechnungsmodell für die Kantone und Gemeinden (KOLIBRI). Schriftenreihe der Fachgruppe für kantonale Finanzfragen (FkF). Solothurn: Verlag der FkF, Amt für Finanzen des Kantons Solothurn. 
CDF-Conférence des directrices et directeurs cantonaux des finances (2008). Manuel - Modèle comptable harmonisé pour les cantons et les communes-MCH2. Berne: CDF.

Chatagny, F. et Soguel, N. (2012). «The Effect of Tax Revenue Budgeting Errors on Fiscal Balance: Evidence from Swiss cantons». International Tax and Public Finance, 19(3), 319-337.

Clémenceau, M. et Soguel, N. (2014). «Impact des imputations internes sur le niveau des déficits publics: Le cas des cantons suisses ». In Djouldem M., Tellier G. et de Visscher C. (éd.), Les réformes des finances publiques: Enjeux politiques et gestionnaires. Bruxelles: Éditions Bruylant, 187-216.

Clémenceau, M. et Soguel, N. (2015). «La présentation des comptes publics au service du politique: Enjeux et conséquences du recours à la cosmétique comptable dans les cantons suisses ». Expert Focus, 10|15, 703-707.

Clémenceau, M. et Soguel, N. (2017). «Does Personal Background Influence a Finance Minister to Cook the Books? An Investigation of Creative Accounting in Swiss Cantons». Applied economics, 49(10), 941-953.

Clémenceau, M. et Soguel, N. (2018). «How Does Depreciations Management Affect Subsequent Fiscal Performance? The Case of the Swiss Cantons». Swiss Journal of Economics and Statistics, 154(7), 1-15.

Christen, R. et Soguel, N. (2018). Comptes spéciaux dans les collectivités publiques. Rechnungswesen \& Controlling, 4I2018, 44-45.

Conseil fédéral (2000). Message sur le frein à l'endettement, du 5 juillet 2000. Berne: Feuille fédérale 20004295.

Conseil fédéral (2009). Kostentransparenz für staatliche Leistungen: Bericht des Bundesrates in Erfüllung des Postulates 04.3445 der Freisinnig-demokratischen Fraktion vom 21. September 2004. Berne: DFF- Département fédéral des finances.

Conseil fédéral (2015). Accroître la transparence des fonds spéciaux et des financements spéciaux. Berne: Département fédéral des finances.

Dafflon, B. (1994). La gestion des finances publiques locales. Paris: Economica.

Dafflon, B. (2007). Réforme de la péréquation intercommunale dans le canton de Fribourg. Fribourg: DFF - Département d'économie politique, Université de Fribourg.

Däniken, A. (2017, Mai 26). «Budget: Es geht um alles oder nichts». Luzerner Zeitung. des Robert, J.-F. et Colibert, J. (2008). Les normes IPSAS et le secteur public. Paris: Dunod.

Eurostat (1996). Système européen des comptes-SEC 1995. Luxembourg: OPOCE-Office des publications de l'Union européenne.

Feld, L. et Kirchgässner, G. (2008). «On the Effectiveness of Debt Brakes: The Swiss Experience». In Neck, R. et Sturm, J.-E. (éd.), Sustainability of Public Debt. Cambridge: MIT Press, 223-255.

FMI-Fonds monétaire international (2001). Manuel de statistiques de finances publiques. Washington D.C.: Fonds monétaire international, Département des statistiques.

FMI-Fonds monétaire international (2002, Mai 28). Assessing Sustainability. Washington D.C.: International Monetary Fund.

FMI-Fonds monétaire international (2014). Government Finance Statistics Manual 2014 (GFSM 2014). Washington D.C.: International Monetary Fund, Statistics Department.

Fourastié, J. et Kovacs, A. (1995). La comptabilité. Paris: PUF - Presses universitaires de France. Funk, P. et Gathmann, C. (2011). «Does Direct Democracy Reduce the Size of Government? New Evidence from Historical Data 1890-2000». The Economic Journal, 121, 1252-1280.

Gilles, W. (2009). Les principes budgétaires et comptables publics. Paris: LGDJ - Librairie générale de droit et de jurisprudence. 
Girouard, N. et André, C. (2005). Measuring Cyclically-adjusted Budget Balances for OECD Countries. OECD Economic Department Working Paper 434. Paris: OECD-Organisation for Economic Co-operation and Development.

Guichard, S., Kennedy, M., Wurzel, E. et André, C. (2007). What Promotes Fiscal Consolidation: OECD Country Experiences. OECD Economic Department Working Paper 553. Paris: OECD-Organisation for Economic Co-operation and Development.

Hart, A. (1945). «Model-building and fiscal policy». American Economic Review, 35(4), 531-558. Harlow, R.L. (1973). «On the Decline and Possible Fall of PPBS». Public Finance Quarterly, 1(1), 85-105.

Hodrick, R.J. et Prescott, E.C. (1980). Postwar U.S. Business Cycles: an Empirical Investigation. Mimeo. Pittsburgh P.A.: Carnegie-Mellon University.

Huet, G. (2010, Juin 3). Banques et collectivités locales: quelques règles de bon sens [Billet de blog]. Repéré à ACOFIL-Association des consultants en finances locales. http://acofil.canalblog.com/

Jeanrenaud, C. et Soguel, N. (1988). Un tableau de bord à l'usage des communes. Dossier 14. Neuchâtel: IRER - Institut de recherches économiques et régionales, Université de Neuchâtel.

Kim, J.M. et Park, C.-K. (2006). «Top-down Budgeting as a Tool for Central Resource Management». OECD Journal on Budgeting, 6(1), 87-125.

Lee, D.R. et Wagner, R.E. (1991). «The Political Economy of Tax Earmarking». In Wagner R.E. (éd.) Charging for Government: User Charges and Earmarked Taxes in Principle and Practice. New York: Routledge, 110-124.

Legay, M.-L. (éd.) (2010). Dictionnaire historique de la comptabilité publique. Rennes: PUR - Presses universitaires de Rennes.

Lienhard, A., Mächler, A. et Zielniewicz, A. (2017). Öffentliches Finanzrecht. Bern: Stämpfli Verlag.

Lienhard, A. et Marti Locher, F. (2015). «Art. $126 »$. In Waldmann B., Belser E.M. et Epiney A. (éd.), Bundesverfassung, Basler Kommentar. Basel: Helbing Lichtenhahn Verlag, 2025-2038.

Luck, S. (2011). Déficits structurels et conjoncturels des cantons, des communes et des assurances sociales publiques 1990-2009. Berne: DFF - Département fédéral des finances.

Mabut, J.-F. (2014, Décembre 12). «Abécédaire du budget 2015». Tribune de Genève.

Martin, M.-J. (2011). «Les dépenses publiques ont-elles exercé des impulsions procycliques ou anticycliques de 1970 et 2007 ?». In Soguel N. (éd.), Des politiques au chevet de la conjoncture. Lausanne: PPUR - Presses polytechniques et universitaires romandes, 27-50.

Müller-Marqués Berger, T. (2018). IPSAS Explained. A Summary of International Public Sector Accounting Standards. Chichester: John Wiley \& Sons Ltd.

Musgrave, R.A. (1959). The Theory of Public Finance. New York: McGraw-Hill.

Naser, K. et Pendlebury, M. (1992). "A Note on the Use of Creative Accounting». The British Accounting Review, 24(2), 111-118.

Nikitin, M. (2001). «The Birth of a Modern Public Sector Accounting System in France and Britain and the Influence of Count Mollien». Accounting History, 6(1), 75-101.

OACOT-Office des affaires communales et de l'organisation du territoire du canton de Berne (2001), Guide des finances communales. Berne: OACOT.

OCDE-Organisation de coopération et de développement économiques (2018). Statistiques des recettes publiques 2018, Paris: OECD-Organisation for Economic Co-operation and Development.

ONU-Organisation des Nations Unies, CE-Commission européenne, OCDE-Organisation de coopération et de développement économiques, FMI-Fonds monétaire international 
et BM-Banque mondiale (2013). Système de comptabilité nationale 2008 (SCN 2008). New York: ONU, CE, OCDE, FMI et BM.

Pasqualini, E. (2019). Comptabilité analytique de gestion. Limal: Anthémis.

Pfäffli, S. (2011). Budgetierung im öffentlichen Sektor: ein Handbuch für Studium und Praxis aus finanzwissenschaftlicher Sicht. Bern: Haupt Verlag AG.

Price, R.W.R., Dang, T.-T. et Botev, J. (2015). Adjusting Fiscal Balances for the Business Cycle: New Tax and Expenditure Elasticity Estimates for OECD Countries. OECD Economic Department Working Paper 1275. Paris: OECD-Organisation for Economic Co-operation and Development.

Pyhrr, P.A. (2012). «Zero-Based Budgeting». In Lalli W.R. (éd.), Handbook of Budgeting, Hoboken (NJ) : John Wiley \& Sons Ltd, 677-696.

Rühli, L., Frey, M. et Frey, R.L. (2013). Irrgarten Finanzausgleich. Wege zu mehr Effizienz bei der interkommunalen Solidarität. Zürich: Avenir Suisse.

Schwab Christe, N. (1996). Les communes de Suisse romande face à l'austérité budgétaire: processus décisionnels et stratégies adoptées. Neuchâtel: EDES - Éditions de la Division économique et sociale.

Soguel, N. (1996). «Faut-il s'inquiéter de l'augmentation de la dette de la Confédération?». Cahier des questions conjoncturelles, Berne: SECO - Secrétariat d'État à l'économie, 2/96, 3-8.

Soguel, N. (2004). Comprendre le fonds de péréquation intercommunal vaudois. Working paper de l'IDHEAP 7/2004. Lausanne: IDHEAP-Institut de hautes études en administration publique, Université de Lausanne.

Soguel, N. (2006). «Coordination et décentralisation des règles budgétaires dans une structure fédéraliste. Le cas des cantons suisses ». Revue d'économie régionale et urbaine, 1, 27-48.

Soguel, N. (2011). Des politiques au chevet de la conjoncture. Lausanne: PPUR - Presses polytechniques et universitaires romandes.

Soguel, N. et Munier, E. (2018). Vergleich der Kantons- und Gemeindefinanzen-Methodik 2.0. Comparatif des finances cantonales et communales-Méthodologie 2.0. Cahier de l'IDHEAP 304. Lausanne: IDHEAP-Institut de hautes études en administration publique, Université de Lausanne.

Stenghele, G.V. (2006). Gestion de la dette cantonale: Une étude sur les bonnes pratiques pour la gestion de la dette cantonale à l'exemple des cantons de Bâle-Ville, Berne, Genève, Vaud et Zurich. Working paper de l'IDHEAP 15/2006. Lausanne: IDHEAP-Institut de hautes études en administration publique, Université de Lausanne.

UE-Union européenne (2013). Système européen des comptes-SEC 2010. Luxembourg: OPUEOffice des publications de l'Union européenne.

Vlaeminck, J.-H. (1956). Histoire et doctrines de la comptabilité, Bruxelles: Éditions du Treurenber. Weber, L., Zarin-Nejadan, M. et Schönenberger, A. (2017). Économie et finances publiques. Paris : Economica.

Wildavsky, A. (1975). Budgeting: A Comparative Theory of Budgetary Processes. Boston et Toronto, Little, Brown and Co.

Yerly, N. (2014). «Les règles budgétaires entre souplesse et rigidité: la situation des cantons suisses». La Vie économique, 6, 38-40. 


\section{Annexes}

\section{Annexe 1 Comptes de MaCollectivité pour l'Année t}

Cette annexe contient le compte de résultats, le compte des investissements et le bilan de MaCollectivité pour l'Année t. Les sommes qui y figurent servent de base de calcul pour les exemples présentés dans les différents chapitres de cet ouvrage. Ces sommes correspondent à celles d'une commune d'environ 3000 habitants. Évidemment elles sont tributaires de la situation socio-économique et des choix budgétaires de la commune, ainsi que du canton dans lequel elle se situe, notamment en raison de la répartition des tâches entre le canton et les communes.

Diverses rubriques affichent des valeurs nulles. Nous avons choisi de les présenter malgré tout dans cette annexe. Notre idée est en effet de donner ici une vision d'ensemble du plan comptable harmonisé (état au $1^{\text {er }}$ janvier 2020). En réalité, les rubriques affichant des valeurs nulles ne sont généralement pas présentées dans les états financiers publiés par les collectivités. 


\section{Compte de résultats I Charges en KCHF}

3 Charges

30 Charges de personnel

300 Autorités, commissions et juges

301 Salaires du personnel administratif et d'exploitation

302 Salaires des enseignants

303 Travailleurs temporaires

304 Allocations

305 Cotisations patronales

306 Prestations de l'employeur

309 Autres charges de personnel

31 Charges de biens et services et autres charges d'exploitation

310 Charges de matières et de marchandises

311 Immobilisations ne pouvant être portées à l'actif

312 Alimentation et élimination des biens-fonds du PA

313 Prestations de service et honoraires

314 Gros entretien et entretien courant

315 Entretien des biens meubles et immobilisations incorporelles

316 Loyers, leasing, baux à ferme, frais d'utilisation

317 Dédommagements

318 Réévaluations sur créances

319 Autres charges d'exploitation

32 Charges d'armement (compte réservé à la Confédération)

33 Amortissements du patrimoine administratif

330 Amortissements des immobilisations corporelles du PA

332 Amortissements des immobilisations incorporelles du PA

34 Charges financières

340 Charges d'intérêts

341 Pertes réalisées sur le patrimoine financier

342 Frais d'approvisionnement en capitaux et frais administratifs

343 Charges pour biens-fonds du patrimoine financier

344 Réévaluations des immobilisations du patrimoine financier

349 Autres charges financières

35 Attributions aux financements spéciaux [FSp] et fonds

350 Attributions aux FSp et fonds sous capitaux de tiers

351 Attributions aux FSp et fonds sous capital propre

36 Charges de transferts

360 Parts de revenus destinées à des tiers

361 Dédommagements à des collectivités publiques

362 Péréquation financière et compensation des charges

363 Subventions à des collectivités publiques et à des tiers

364 Réévaluations de prêts du PA
Année t

Comptes Budget Comptes

$14546 \quad 14643 \quad 13741$

2020

175

2064

1986

1525

183

178

0

0

1542

1497

0

295

0

25

4076

1725

130

191

976

699

123

57

58

96

21

0

671

671

0

125

74

0

10

41

0

0

0

0

0

0

6393

0

3905
564

1924

0

0

0

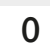

307

283

0

32

28

4595

4086

$1800 \quad 1755$

168

105

242

232

1196

913

$833 \quad 716$

169

154

48

50

56

57

79

26

25

0

0

709

677

$709 \quad 677$

0

0

219

135

$81 \quad 92$

0

92
0

65

73

9

34

0

0

0

0

0

$0 \quad 0$

6186

5924

0

3793

3541

$552 \quad 551$

$1841 \quad 1832$
[...] 


Compte de résultats I Charges [suite]
en KCHF $\quad$\begin{tabular}{c} 
Année t \\
\cline { 2 - 2 }
\end{tabular}

[...]

\begin{tabular}{|llrrr|}
\hline 365 & Réévaluations de participations du PA & 0 & 0 & 0 \\
366 Amortissements des subventions d'investissement & 0 & 0 & 0 \\
369 Autres charges de transferts & 0 & 0 & 0 \\
\hline 37 & Subventions redistribuées & 0 & 0 & 0 \\
\hline 370 Subventions redistribuées & 0 & 0 & 0 \\
\hline 38 & Charges extraordinaires [e.o.] & 991 & 613 & 645 \\
\hline 380 Charges extraordinaires de personnel & 0 & 0 & 0 \\
381 Charges e.o. de biens, services et charges d'exploitation & 0 & 0 & 0 \\
383 Amortissements supplémentaires [AS] & 0 & 0 & 0 \\
384 Charges financières extraordinaires & 0 & 0 & 0 \\
386 Charges extraordinaires de transferts & 0 & 0 & 0 \\
\hline 387 AS de prêts, participations, subventions d'investissement & 0 & 0 & 0 \\
\hline 389 Attributions au capital propre & 991 & 613 & 645 \\
\hline 39 & Imputations internes & 270 & 257 & 288 \\
\hline 390 Approvisionnement en matériel et marchandises & 0 & 0 & 0 \\
\hline 391 & Prestations de service & 144 & 134 & 160 \\
\hline 392 Baux à ferme, loyers, frais d'utilisation & 0 & 0 & 0 \\
\hline 393 Frais administratifs et d'exploitation & 116 & 108 & 118 \\
\hline 394 Intérêts et charges financières théoriques & 10 & 15 & 10 \\
\hline 395 & Amortissements planifiés et non planifiés & 0 & 0 & 0 \\
\hline 398 Virements comptables & 0 & 0 & 0 \\
\hline 399 & Autres imputations internes & 0 & 0 & 0
\end{tabular}




\section{Compte de résultats I Revenus en KCHF}

\begin{tabular}{r} 
\\
\hline Comp \\
76 \\
62 \\
5 \\
8 \\
8
\end{tabular}

Année t

4 Revenus

40 Revenus fiscaux

7651

6231

501

898

403 Impôts sur la propriété et sur les charges

41 Patentes et concessions

410 Patentes

411 Banque nationale suisse

412 Concessions

413 Parts de revenus à des loteries, Sport-Toto, paris

42 Taxes et redevances

420 Taxes de compensation

421 Émoluments pour actes administratifs

422 Taxes pour hôpitaux et établissements médicaux...

423 Frais d'écolage et taxes de cours

424 Taxes d'utilisation et prestations de service

425 Recettes sur ventes

426 Remboursements

427 Amendes

429 Autres taxes

43 Revenus divers

430 Revenus d'exploitation divers

431 Activations de prestations propres

432 Variations de stocks

439 Autres revenus

44 Revenus financiers

440 Revenus des intérêts

441 Gains réalisés sur le patrimoine financier

442 Revenus de participations du patrimoine financier

443 Produits des immeubles du patrimoine financier

444 Réévaluations des immobilisations du patrimoine financier

445 Revenus financiers de prêts et de participations du PA

446 Revenus financiers d'entreprises publiques

447 Produits des immeubles du patrimoine administratif

448 Revenus immeubles loués

449 Autres revenus financiers

45 Prélèvements sur les financements spéciaux [FSp] et fonds

450 Prélèvements sur les FSp et fonds sous capitaux de tiers

451 Prélèvements sur les FSp et fonds sous capital propre

[...]

$21 \quad 19 \quad 22$

$12 \quad 12 \quad 12$

$0 \quad 0 \quad 0$

$0 \quad 0 \quad 0$

$12 \quad 12 \quad 12$

$0 \quad 0 \quad 0$

$5076 \quad 5097 \quad 4852$

$192 \quad 175 \quad 195$

$\begin{array}{lll}73 & 61 & 57\end{array}$

$0 \quad 0 \quad 0$

$0 \quad 0 \quad 0$

$4651 \quad 4717 \quad 4406$

$92 \quad 88 \quad 96$

$\begin{array}{lll}68 & 56 & 98\end{array}$

$0 \quad 0 \quad 0$

$0 \quad 0 \quad 0$

$17 \quad 0 \quad 0$

$0 \quad 0 \quad 0$

$17 \quad 0 \quad 0$

$0 \quad 0 \quad 0$

$\begin{array}{lll}0 & 0 & 0\end{array}$

$167 \quad 155 \quad 160$

$12 \quad 20 \quad 16$

$0 \quad 0 \quad 0$

$\begin{array}{lll}0 & 0 & 0\end{array}$

$92 \quad 85 \quad 90$

903

$\begin{array}{lll}8 & 8 & 8\end{array}$

$0 \quad 0 \quad 0$

$46 \quad 42 \quad 43$

$0 \quad 0 \quad 0$

$0 \quad 0 \quad 0$

$23 \quad 0 \quad 3$

$23 \quad 0 \quad 3$

$0 \quad 0$

0
0
12
0
2
5
0
0
6
0
9
0
0
0
0
0
0
0
0
16
0
0
0
0
0
0
0
0
0
0



en KCHF

[...]

46 Revenus de transferts

460 Parts à des revenus de tiers

461 Dédommagements de collectivités publiques

462 Péréquation financière et compensation des charges

463 Subventions de collectivités publiques et de tiers

466 Dissolutions des subventions d'investissement portées au passif

\section{Compte de résultats I Revenus [suite]}

\begin{tabular}{|c|c|c|}
\hline \multicolumn{2}{|c|}{ Année t } & Année t-1 \\
\hline Comptes & Budget & Comptes \\
\hline 1373 & 1373 & 1366 \\
\hline 0 & 0 & 0 \\
\hline 645 & 639 & 623 \\
\hline 610 & 657 & 663 \\
\hline 117 & 76 & 79 \\
\hline 0 & 0 & 0 \\
\hline
\end{tabular}

$1 \quad 1 \quad 1$

$0 \quad 0 \quad 0$

$0 \quad 0 \quad 0$

$21 \quad 126 \quad 17$

48 Revenus extraordinaires

481 Revenus extraordinaires de patentes, concessions

482 Contributions extraordinaires

483 Revenus extraordinaires divers

484 Revenus financiers extraordinaires

486 Revenus extraordinaires de transferts

487 Dissolutions supplémentaires des subventions d'investissement au passif

489 Prélèvements sur le capital propre

$\begin{array}{lll}21 & 126 & 17\end{array}$

49 Imputations internes

270

490 Approvisionnement en matériel et marchandises

491 Prestations de service

492 Baux à ferme, loyers, frais d'utilisation

493 Frais administratifs et d'exploitation

494 Intérêts et charges financières théoriques

495 Amortissements planifiés et non planifiés

498 Virements comptables

499 Autres imputations internes

4-3 Résultat du compte de résultats

$\begin{array}{rrr}0 & 0 & 0 \\ 144 & 134 & 160 \\ 0 & 0 & 0 \\ 116 & 108 & 118 \\ 10 & 15 & 10 \\ 0 & 0 & 0 \\ 0 & 0 & 0 \\ 0 & 0 & 0\end{array}$

1
0
0
0
0
0
0
0
0
0
0
0
0
0




\section{Compte des investissements I Dépenses} en KCHF

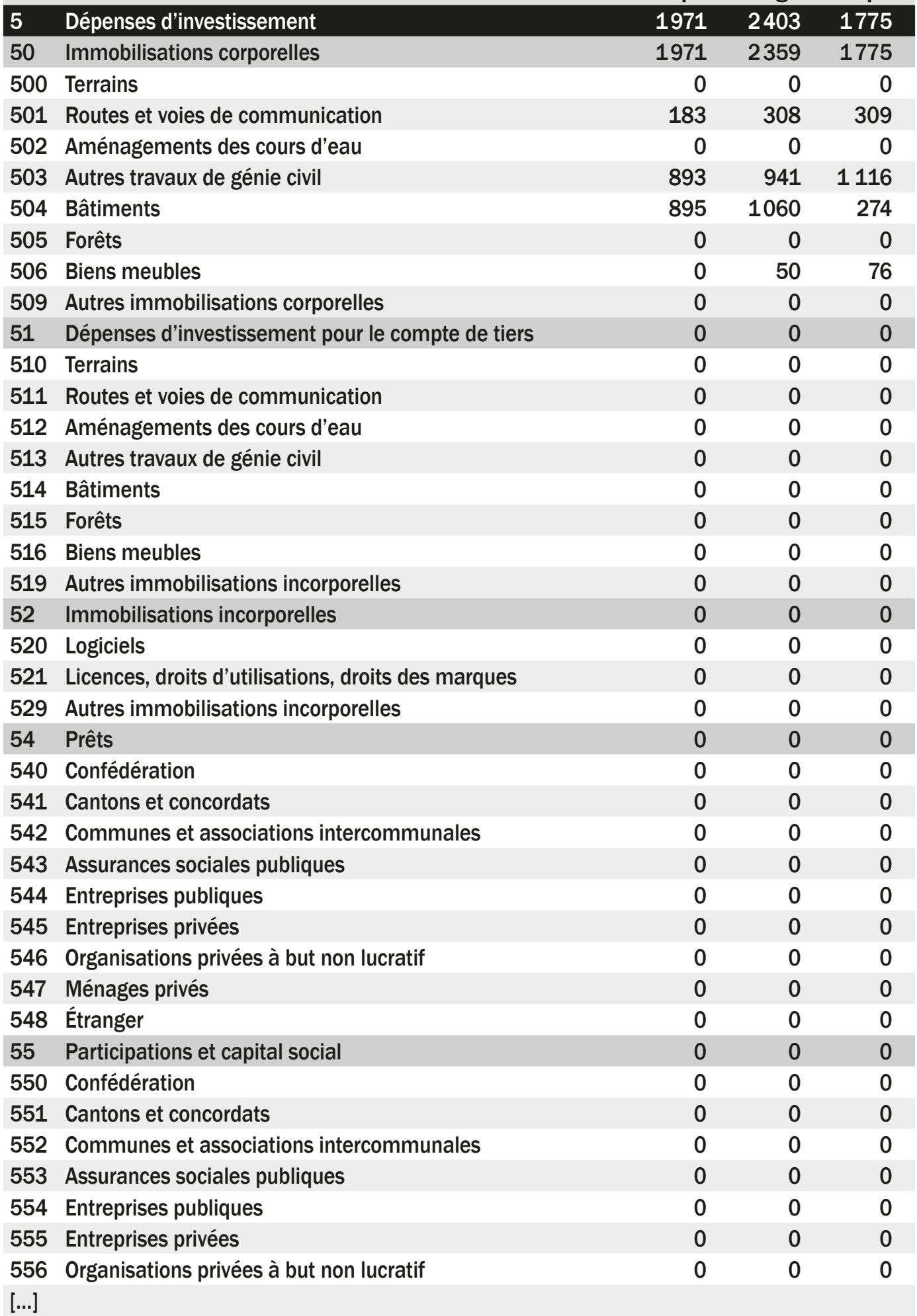




\begin{tabular}{|c|c|}
\hline \multirow{2}{*}{$\begin{array}{l}\text { Compte des investissements I Dépenses [suite] } \\
\text { en KCHF }\end{array}$} & Année t \\
\hline & Comptes Budget \\
\hline
\end{tabular}

[...]

\begin{tabular}{|c|c|c|c|c|}
\hline 557 & Ménages privés & 0 & 0 & 0 \\
\hline 558 & Étranger & 0 & 0 & 0 \\
\hline 56 & Propres subventions d'investissement & 0 & 44 & 0 \\
\hline 560 & Confédération & 0 & 0 & 0 \\
\hline 561 & Cantons et concordats & 0 & 0 & 0 \\
\hline 562 & Communes et associations intercommunales & 0 & 44 & 0 \\
\hline 563 & Assurances sociales publiques & 0 & 0 & 0 \\
\hline 564 & Entreprises publiques & 0 & 0 & 0 \\
\hline 565 & Entreprises privées & 0 & 0 & 0 \\
\hline 566 & Organisations privées à but non lucratif & 0 & 0 & 0 \\
\hline 567 & Ménages privés & 0 & 0 & 0 \\
\hline 568 & Étranger & 0 & 0 & 0 \\
\hline 57 & Subventions d'investissement redistribuées & 0 & 0 & 0 \\
\hline 570 & Confédération & 0 & 0 & 0 \\
\hline 571 & Cantons et concordats & 0 & 0 & 0 \\
\hline 572 & Communes et associations intercommunales & 0 & 0 & 0 \\
\hline 573 & Assurances sociales publiques & 0 & 0 & 0 \\
\hline 574 & Entreprises publiques & 0 & 0 & 0 \\
\hline 575 & Entreprises privées & 0 & 0 & 0 \\
\hline 576 & Organisations privées à but non lucratif & 0 & 0 & 0 \\
\hline 577 & Ménages privés & 0 & 0 & 0 \\
\hline 578 & Étranger & 0 & 0 & 0 \\
\hline 58 & Dépenses d'investissement extraordinaires [e.o.] & 0 & 0 & 0 \\
\hline 580 & Dépenses d'investissement e.o. immobilisations corporelles & 0 & 0 & 0 \\
\hline 582 & Dépenses d'investissement e.o. immobilisations incorporelles & 0 & 0 & 0 \\
\hline 584 & Dépenses d'investissement e.o. pour des prêts & 0 & 0 & 0 \\
\hline 585 & Dépenses d'investissement e.o. participations, capital social & 0 & 0 & 0 \\
\hline 586 & Subventions d'investissement e.o. & 0 & 0 & 0 \\
\hline 589 & Autres dépenses d'investissement e.o. & 0 & 0 & 0 \\
\hline
\end{tabular}




\section{Compte des investissements I Recettes} en KCHF

\section{Année t \\ Comptes Budget Comptes

$43 \quad 500 \quad 10$

60 Transferts d'immobilisations corporelles dans le PF

600 Transferts de terrains

601 Transferts de routes et voies de communication

602 Transferts d'aménagements des cours d'eau

603 Transferts d'autres travaux de génie civil

604 Transferts de bâtiments

605 Transferts de forêts

606 Transferts de biens meubles

609 Transferts d'autres immobilisations corporelles

61 Remboursements de dépenses d'investissement pour le compte de tiers

610 Terrains

611 Routes et voies de communication

612 Aménagements des cours d'eau

613 Autres travaux de génie civil

614 Bâtiments

615 Forêts

616 Bien meubles

619 Autres immobilisations corporelles

62 Transferts d'immobilisations incorporelles dans le PF

620 Logiciels

621 Licences, droits d'utilisations, droits des marques

629 Autres immobilisations incorporelles

63 Subventions d'investissement acquises

630 Confédération

631 Cantons et concordats

632 Communes et associations intercommunales

633 Assurances sociales publiques

634 Entreprises publiques

635 Entreprises privées

636 Organisations privées à but non lucratif

637 Ménages privés

638 Étranger

64 Remboursements de prêts

640 Confédération

641 Cantons et concordats

642 Communes et associations intercommunales

643 Assurances sociales publiques

644 Entreprises publiques

645 Entreprises privées

646 Organisations privées à but non lucratif

647 Ménages privés

648 Étranger

[...]

\section{0}

0

0

0

$0 \quad 0$

$0 \quad 0$

$0 \quad 0$

$0 \quad 0 \quad 0$

$\begin{array}{lll}0 & 0 & 0\end{array}$

$\begin{array}{lll}0 & 0 & 0\end{array}$

$\begin{array}{lll}0 & 0 & 0\end{array}$

$\begin{array}{lll}0 & 0 & 0\end{array}$

$\begin{array}{lll}0 & 0 & 0\end{array}$

$\begin{array}{lll}0 & 0 & 0\end{array}$

\section{0}

0

\section{0}

0

\section{0}

\section{0}

\section{0}

0

0

\section{0}

0

0

43

0

0

0

0

0

3

40

0

0

\section{0}

0

0

\section{0}

\section{0}

\section{0}

0

\section{0}

\section{0}

0

0

$0 \quad 0$

$0 \quad 0$

$0 \quad 0$

$0 \quad 0$

$0 \quad 0$

$0 \quad 0$

$0 \quad 0$

$0 \quad 0$

$0 \quad 0$

$0 \quad 0$

$0 \quad 0$

$500 \quad 10$

$0 \quad 0$

$100 \quad 0$

$0 \quad 0$

$0 \quad 0$

$0 \quad 10$

$0 \quad 0$

$\begin{array}{lll}0 & 0\end{array}$

$0 \quad 0$

$0 \quad 0$

$400 \quad 0$

$\begin{array}{lll}0 & 0 & 0 \\ 0 & 0 & 0 \\ 0 & 0 & 0 \\ 0 & 0 & 0 \\ 0 & 0 & 0 \\ 0 & 0 & 0 \\ 0 & 0 & 0 \\ 0 & 0 & 0 \\ 0 & 0 & 0 \\ 0 & 0 & 0\end{array}$




\section{Compte des investissements I Recettes [suite]} en KCHF
Année t Comptes Budget
Année t-1

Comptes

[...]

$\begin{array}{lllll}65 & \text { Transferts de participations } & 0 & 0 & 0 \\ 650 \text { Confédération } & 0 & 0 & 0 \\ 651 & \text { Cantons et concordats } & 0 & 0 & 0 \\ 652 & \text { Communes et associations intercommunales } & 0 & 0 & 0 \\ 653 & \text { Assurances sociales publiques } & 0 & 0 & 0 \\ 654 & \text { Entreprises publiques } & 0 & 0 & 0 \\ 655 & \text { Entreprises privées } & 0 & 0 & 0 \\ 656 & \text { Organisations privées à but non lucratif } & 0 & 0 & 0 \\ 657 & \text { Ménages privés } & 0 & 0 & 0 \\ 658 \text { Étranger } & 0 & 0 & 0\end{array}$

66 Remboursements de propres subventions d'investissement $\quad 0 \quad 000$

660 Confédération

661 Cantons et concordats

$\begin{array}{lll}0 & 0 & 0\end{array}$

662 Communes et associations intercommunales

$0 \quad 0$

0

663 Assurances sociales publiques

$0 \quad 0 \quad 0$

664 Entreprises publiques

$0 \quad 0 \quad 0$

665 Entreprises privées

$\begin{array}{lll}0 & 0 & 0\end{array}$

666 Organisations privées à but non lucratif

0

667 Ménages privés

$0 \quad 0$

668 Étranger

67 Subventions d'investissement à redistribuer

670 Confédération

$\begin{array}{lll}0 & 0 & 0\end{array}$

671 Cantons et concordats

0

0

0

0

672 Communes et associations intercommunales

673 Assurances sociales publiques

674 Entreprises publiques

675 Entreprises privées

676 Organisations privées à but non lucratif

677 Ménages privés

0

678 Étranger

68 Recettes d'investissement extraordinaires [e.o.]

680 Recettes d'investissement e.o. immobilisations corporelles

682 Recettes d'investissement e.o. immobilisations incorporelles

683 Subventions d'investissement extraordinaires acquises

684 Remboursements extraordinaires de prêts

685 Cessions extraordinaires de participations

686 Remboursements e.o. de propres subventions d'investissement

689 Autres recettes d'investissement extraordinaires

$\begin{array}{lll}0 & 0 & 0 \\ 0 & 0 & 0\end{array}$

$0 \quad 0 \quad 0$

$\begin{array}{lll}0 & 0 & 0\end{array}$

$0 \quad 0 \quad 0$

$0 \quad 0 \quad 0$

$\begin{array}{lll}0 & 0 & 0\end{array}$

$0 \quad 0 \quad 0$

$0 \quad 0 \quad 0$

$0 \quad 0 \quad 0$

$0 \quad 0 \quad 0$

$0 \quad 0 \quad 0$

$\begin{array}{lll}0 & 0 & 0\end{array}$

$\begin{array}{lll}0 & 0 & 0\end{array}$

$0 \quad 0 \quad 0$

$0 \quad 0 \quad 0$




\begin{tabular}{|c|c|c|c|c|c|}
\hline \multirow{2}{*}{\multicolumn{2}{|c|}{$\begin{array}{l}\text { Bilan I Actif } \\
\text { en KCHF }\end{array}$}} & \multirow{3}{*}{$\begin{array}{c}01.01 \\
\text { Année t } \\
13274\end{array}$} & \multicolumn{2}{|c|}{ Variation en cours d'année } & \multirow{3}{*}{$\begin{array}{c}31.12 \\
\text { Année t } \\
13676\end{array}$} \\
\hline & & & \multirow{2}{*}{$\begin{array}{c}\text { Augmentation } \\
41303\end{array}$} & \multirow{2}{*}{$\begin{array}{c}\text { Diminution } \\
40901\end{array}$} & \\
\hline & Actif & & & & \\
\hline & Patrimoine financier [PF] & 5539 & 39332 & 40187 & 4684 \\
\hline 100 & Disponibilités et placements à court terme & 1605 & 16094 & 16916 & 783 \\
\hline 101 & Créances & 1733 & 23064 & 23175 & 1622 \\
\hline 102 & Placements financiers à court terme & 0 & 0 & 0 & 0 \\
\hline 104 & Actifs de régularisation & 96 & 165 & 96 & 165 \\
\hline 106 & Marchandises, fournitures et travaux en cours & 0 & 0 & 0 & 0 \\
\hline 107 & Placements financiers à long terme & 91 & 9 & 0 & 100 \\
\hline 108 & Immobilisations corporelles PF & 2014 & 0 & 0 & 2014 \\
\hline 109 & Créances envers les FSp et fonds sous CT & 0 & 0 & 0 & 0 \\
\hline & Patrimoine administratif [PA] & 7735 & 1971 & 714 & 8992 \\
\hline 140 & Immobilisations corporelles PA & 7419 & 1971 & 714 & 8676 \\
\hline 142 & Immobilisations incorporelles PA & 0 & 0 & 0 & 0 \\
\hline 144 & Prêts PA & 0 & 0 & 0 & 0 \\
\hline 145 & Participations, capital social PA & 316 & 0 & 0 & 316 \\
\hline 146 & Subventions d'investissement & 0 & 0 & 0 & 0 \\
\hline 148 & Amortissements supplémentaires cumulés & 0 & 0 & 0 & 0 \\
\hline
\end{tabular}

\section{Bilan I Passif en KCHF}

01.01 Variation en cours d'année Année t Augmentation Diminution Année t

\section{4}

20 Capitaux de tiers [CT]

200 Engagements courants

201 Engagements financiers à court terme

204 Passifs de régularisation

205 Provisions à court terme

206 Engagements financiers à long terme

208 Provisions à long terme

209 Engagements envers les FSp et les fonds sous CT

29 Capital propre [CP]

290 Financements spéciaux [FSp] sous CP

291 Fonds sous CP

292 Réserves des enveloppes budgétaires

293 Préfinancements

294 Réserve de politique budgétaire

295 Réserve liée au retraitement du PA (introduction $\mathrm{MCH} 2$ )

296 Réserves liées au retraitement du PF

298 Autres capitaux propres

299 Excédent ou découvert du bilan

7619

1

1461

$\begin{array}{rrrr}33 & 21 & 33 & 21 \\ 277 & 82 & 36 & 323 \\ 5500 & 600 & 1500 & 4600 \\ 0 & 0 & 0 & 0 \\ 348 & 0 & 26 & 322\end{array}$

$\begin{array}{rrrr}5655 & 2961 & 1927 & 6689 \\ 1670 & 130 & 76 & 1724 \\ 0 & 0 & 0 & 0 \\ 0 & 0 & 0 & 0 \\ 1126 & 991 & 21 & 2096 \\ 120 & 0 & 0 & 120 \\ 0 & 0 & 0 & 0 \\ 0 & 0 & 0 & 0 \\ 0 & 0 & 0 & 0 \\ 2739 & 1840 & 1830 & 2749\end{array}$




\section{Annexe 2 Calcul des indicateurs financiers}

Les indicateurs portant la mention " sont recommandés en première priorité par le $\mathrm{MCH} 2$. Ceux qui portent la mention ${ }^{\# \#}$ sont recommandés en deuxième priorité.

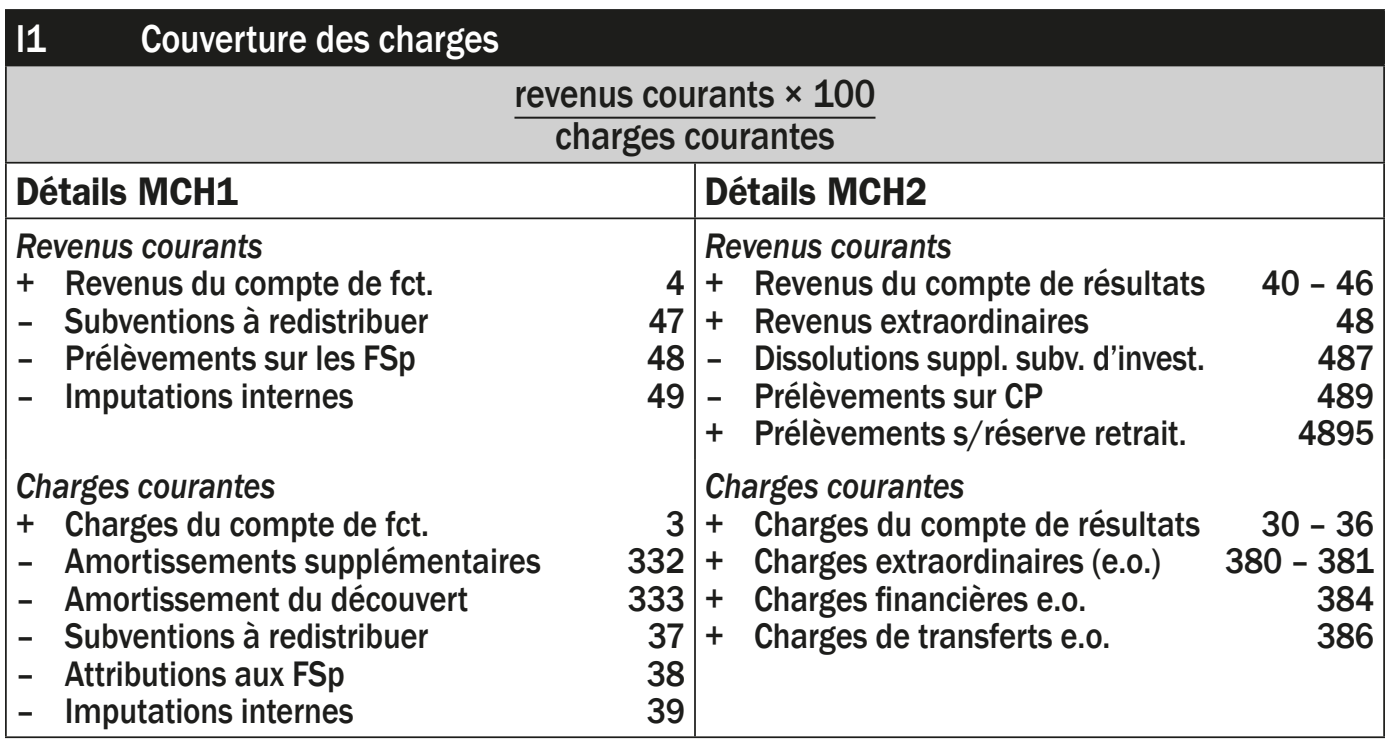

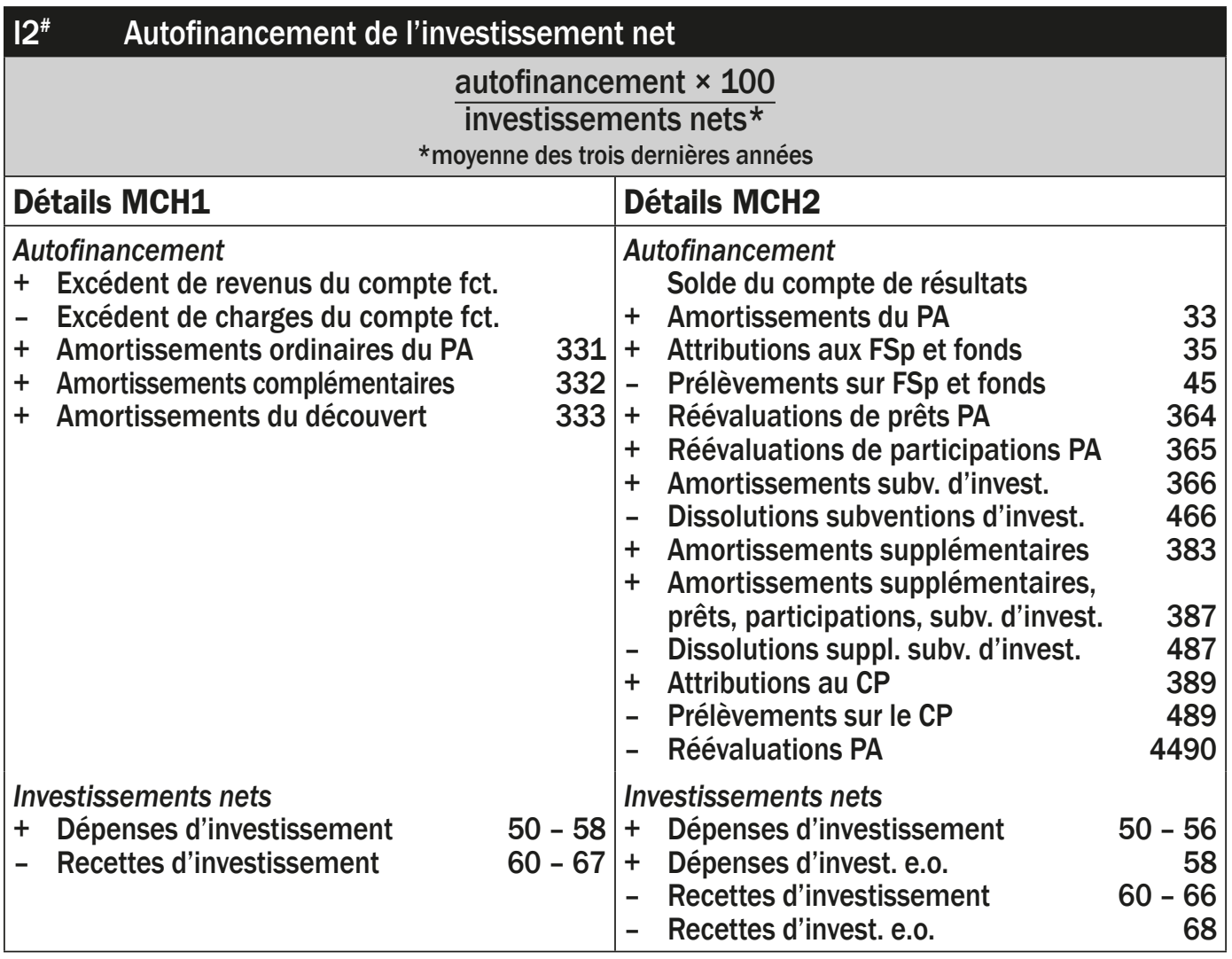




\begin{tabular}{|c|c|c|c|}
\hline \multicolumn{4}{|c|}{$\frac{\text { (engagements nets au 31.12. - engagements nets au 01.01.) } \times 100}{\text { dépenses courantes de l'exercice }}$} \\
\hline Détails MCH1 & & Détails MCH2 & \\
\hline $\begin{array}{l}\text { Engagements nets } \\
+\quad \text { Engagements } \\
\text { - Patrimoine financier } \\
\text { Dépenses courantes } \\
+\quad \text { Charges du compte de fct. } \\
\text { - Amortissements } \\
\text { - Subventions redistribuées } \\
\text { - Attributions aux FSp } \\
\text { - Imputations internes }\end{array}$ & $\begin{array}{r}3 \\
33 \\
37 \\
38 \\
39\end{array}$ & 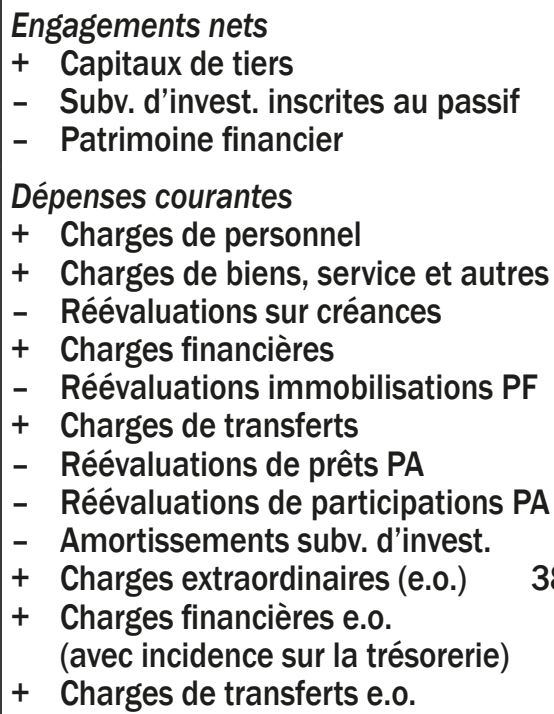 & $\begin{array}{r}30 \\
31 \\
3180 \\
34 \\
344 \\
36 \\
364 \\
365 \\
366 \\
-381 \\
3840 \\
386\end{array}$ \\
\hline
\end{tabular}

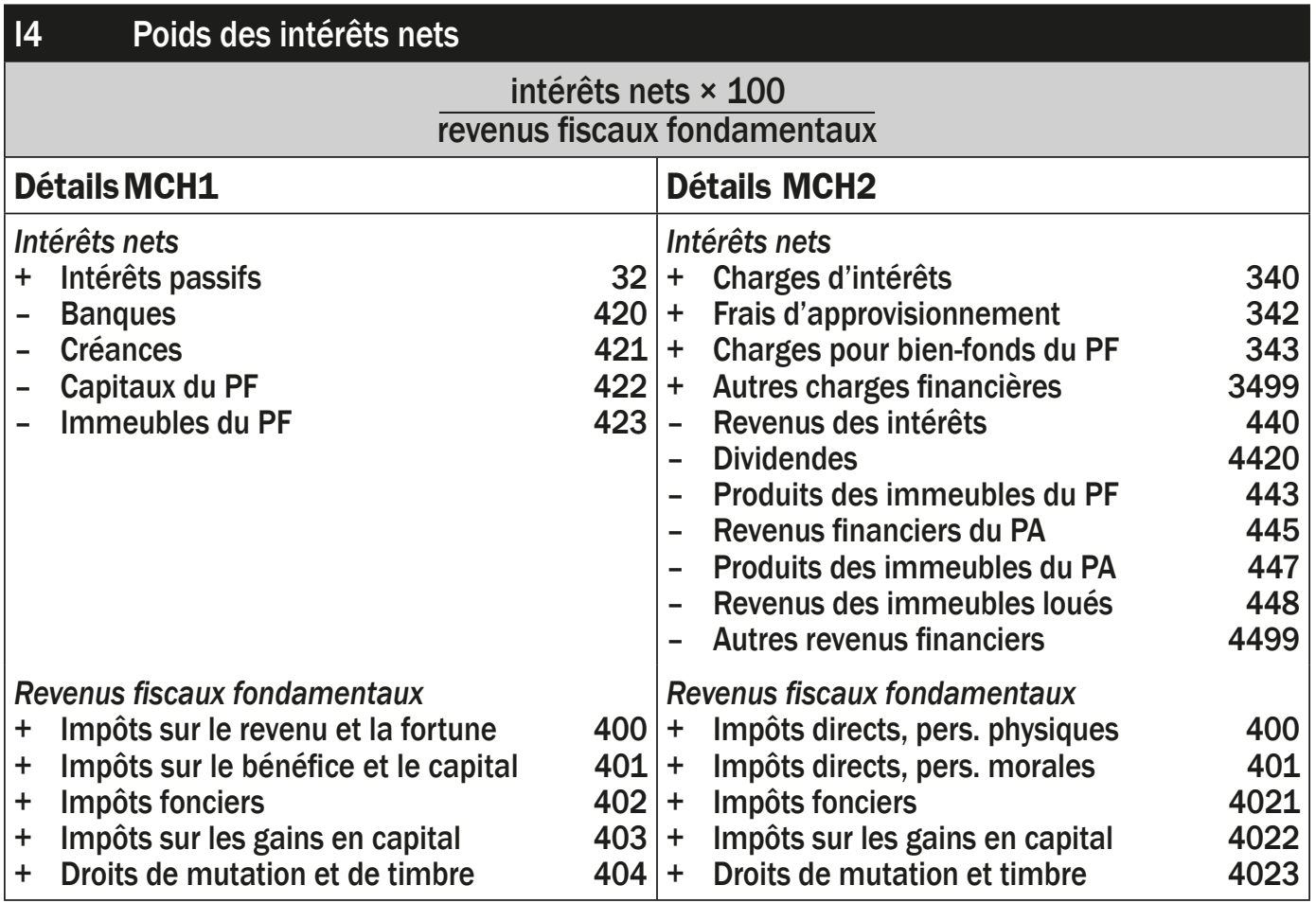




\section{I5 Maîtrise des dépenses courantes}

\begin{tabular}{|c|c|c|c|}
\hline \multicolumn{4}{|c|}{$\frac{\text { (dépenses de l'exercice }- \text { dépenses de l'exercice précédent) } \times 100}{\text { dépenses de l'exercice précédent }}$} \\
\hline Détails MCH1 & & Détails MCH2 & \\
\hline $\begin{array}{l}\text { Dépenses courantes } \\
+\quad \text { Charges du compte de fct. } \\
\text { - Amortissements } \\
\text { - } \quad \text { Subventions redistribuées } \\
\text { - Attributions aux FSp } \\
\text { - Imputations internes }\end{array}$ & $\begin{array}{r}3 \\
33 \\
37 \\
38 \\
39\end{array}$ & $\begin{array}{ll}\text { Dépenses courantes } \\
+\quad \text { Charges de personnel } \\
+\quad \text { Charges de biens, service et autres } \\
-\quad \text { Réévaluations sur créances } \\
+\quad \text { Charges financières } \\
-\quad \text { Réévaluations immobilisations PF } \\
+\quad \text { Charges de transferts } \\
-\quad \text { Réévaluations de prêts PA } \\
-\quad \text { Réévaluations de participations PA } \\
-\quad \text { Amortissements subv. d'invest. } \\
+\quad \text { Charges extraordinaires (e.o.) } \\
+\quad \text { Charges financières e.o. } \\
& \text { (avec incidence sur la trésorerie) } \\
+ & \text { Charges de transferts e.o. }\end{array}$ & $\begin{array}{r}30 \\
31 \\
3180 \\
34 \\
344 \\
36 \\
364 \\
365 \\
366 \\
-381 \\
3840 \\
386\end{array}$ \\
\hline
\end{tabular}

\section{Effort d'investissement}

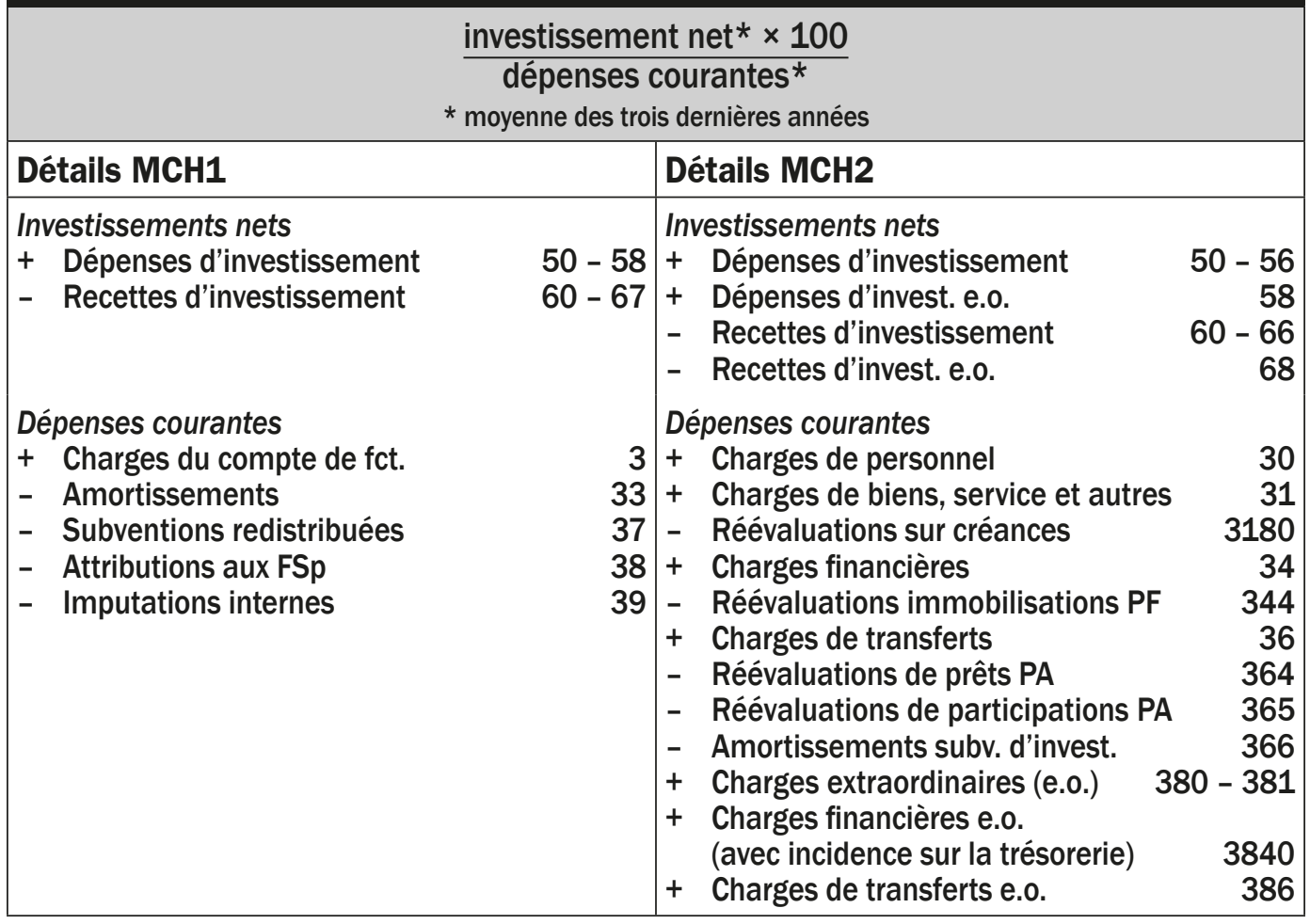




\begin{tabular}{|l|l|l|}
\hline 17 & \multicolumn{2}{l|}{ Exactitude de la prévision fiscale } \\
\hline \multicolumn{4}{|c|}{ (revenus fiscaux fondamentaux budgétés - revenus fiscaux effectifs) $\times 100$} \\
\hline \multicolumn{2}{|c|}{ revenus fiscaux fondamentaux effectifs } \\
\hline Détails MCH1 & Détails MCH2 \\
\hline $\begin{array}{l}\text { Revenus fiscaux fondamentaux } \\
+ \text { Impôts sur le revenu et la fortune } \\
+ \text { Impôts sur le bénéfice et le capital }\end{array}$ & 400 & $\begin{array}{l}\text { Revenus fiscaux fondamentaux } \\
+\quad \text { Impôts directs, pers. physiques }\end{array}$ \\
\hline
\end{tabular}

\section{Intérêt moyen de la dette}

\begin{tabular}{|c|c|c|c|}
\hline \multicolumn{4}{|c|}{$\frac{\text { intérêts passifs } \times 100}{\text { moyenne de la dette brute en début et en fin d'exercice }}$} \\
\hline Détails MCH1 & & Détails MCH2 & \\
\hline $\begin{array}{l}\text { Intérêts passifs } \\
+\quad \text { Dettes à court terme } \\
+\quad \text { Dettes à moyen et à long terme } \\
+\quad \text { Dettes envers des entités particulières }\end{array}$ & $\begin{array}{l}321 \\
322 \\
323\end{array}$ & $\begin{array}{l}\text { Intérêts passifs } \\
+\quad \text { Intérêts passifs } \\
\text { des engagements financiers }\end{array}$ & 3401 \\
\hline $\begin{array}{l}\text { Dette brute portant intérêt } \\
+ \text { Dettes à court terme } \\
+ \text { Dettes à moyen et à long terme } \\
+\quad \text { Engagements envers } \\
\quad \text { des entités particulières }\end{array}$ & $\begin{array}{l}21 \\
22 \\
23\end{array}$ & 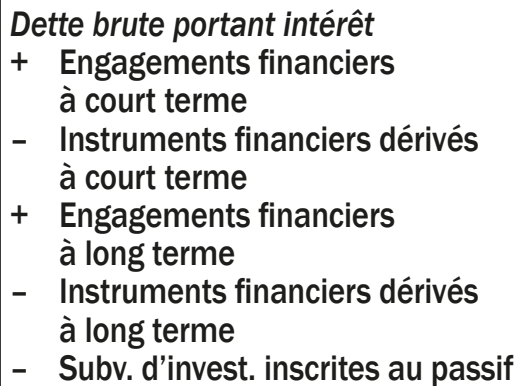 & $\begin{array}{r}201 \\
2016 \\
206 \\
2066 \\
2068\end{array}$ \\
\hline
\end{tabular}

\section{9\# Taux d'endettement net}

\begin{tabular}{|lr|lr|}
\hline \multicolumn{4}{c|}{ engagements nets $\times 100$} \\
\cline { 2 - 3 } & \multicolumn{2}{c|}{ revenus fiscaux } \\
\hline Détails MCH1 & & Détails MCH2 \\
\hline Engagements nets & $20-25$ & Engagements nets \\
$+\quad$ Engagements & $10-13$ & - Subitaux de tiers & 20 \\
$-\quad$ Patrimoine financier & & - Patrimoine financier & 2068 \\
& 40 & Revenus fiscaux & 10 \\
Impôts & & 40 \\
\hline
\end{tabular}


110"\# Dette brute par rapport aux revenus

\begin{tabular}{|c|c|c|c|}
\hline \multicolumn{4}{|c|}{$\frac{\text { dette brute } \times 100}{\text { revenus courants }}$} \\
\hline Détails MCH1 & & Détails MCH2 & \\
\hline $\begin{array}{l}\text { Dette brute } \\
+ \text { Engagements courants } \\
+ \text { Dettes à court terme } \\
+ \text { Dettes à moyen et à long terme } \\
+ \text { Engagements envers } \\
\quad \text { des entités particulières }\end{array}$ & $\begin{array}{l}20 \\
21 \\
22 \\
\\
23\end{array}$ & $\begin{array}{ll}\text { Dette brute } \\
+\quad \text { Engagements courants } \\
+\quad \text { Engagements financiers } \\
& \text { à court terme } \\
- & \text { Instruments financiers dérivés } \\
& \text { à court terme } \\
+ & \text { Engagements financiers } \\
& \text { à long terme } \\
- & \text { Instruments financiers dérivés } \\
& \text { à long terme } \\
- & \text { Subv. d'invest. inscrites au passif } \\
\text { Revenus courants } \\
+ & \text { Revenus du compte de résultats } \\
+ & \text { Revenus extraordinaires } \\
- & \text { Dissolutions suppl. subv. d'invest. } \\
- & \text { Prélèvements sur CP } \\
+ & \text { Prélèvements s/réserve retrait. }\end{array}$ & $\begin{array}{r}200 \\
201 \\
2016 \\
\\
206 \\
\\
2066 \\
2068\end{array}$ \\
\hline
\end{tabular}

\section{I11"\# Taux d'autofinancement}

\begin{tabular}{|c|c|c|c|}
\hline \multicolumn{4}{|c|}{$\frac{\text { autofinancement } \times 100}{\text { revenus courants }}$} \\
\hline Détails MCH1 & & Détails MCH2 & \\
\hline $\begin{array}{l}\text { Autofinancement } \\
+ \text { Excédent de revenus du compte fct. } \\
- \text { Excédent de charges du compte fct. } \\
+\quad \text { Amortissements ordinaires du PA } \\
+\quad \text { Amortissements complémentaires } \\
+ \text { Amortissements du découvert }\end{array}$ & $\begin{array}{l}331 \\
332 \\
333\end{array}$ & $\begin{array}{ll}\text { Autofinancement } & \text { Solde du compte de résultats } \\
& \text { + } \\
+ & \text { Amortissements du PA } \\
\text { - } & \text { Prélèvementions aux FSp et fonds } \\
+ & \text { Réévaluations de prêts PA } \\
+ & \text { Réévaluations de participations PA } \\
+ & \text { Amortissements subv. d'invest. } \\
- & \text { Dissolutions subventions d'invest. } \\
+ & \text { Amortissements supplémentaires } \\
+ & \text { Amortissements supplémentaires, } \\
& \text { prêts, participations, subv. d'invest. } \\
- & \text { Dissolutions suppl. subv. d'invest. } \\
+ & \text { Attributions au CP } \\
- & \text { Prélèvements sur le CP } \\
- & \text { Réévaluations PA }\end{array}$ & $\begin{array}{r}33 \\
35 \\
45 \\
364 \\
365 \\
366 \\
466 \\
383 \\
\\
387 \\
487 \\
389 \\
489 \\
4490\end{array}$ \\
\hline $\begin{array}{l}\text { Revenus courants } \\
+ \text { Revenus du compte de fct. } \\
\text { - Subventions à redistribuer } \\
\text { - Prélèvements sur les FSp } \\
-\quad \text { Imputations internes }\end{array}$ & $\begin{array}{r}4 \\
47 \\
48 \\
49\end{array}$ & $\begin{array}{l}\text { Revenus courants } \\
+ \text { Revenus du compte de résultats } \\
+ \text { Revenus extraordinaires } \\
\text { - } \text { Dissolutions suppl. subv. d'invest. } \\
-\quad \text { Prélèvements sur CP } \\
+\quad \text { Prélèvements s/réserve retrait. }\end{array}$ & $\begin{array}{r}40-46 \\
48 \\
487 \\
489 \\
4895\end{array}$ \\
\hline
\end{tabular}




\section{2" Part des charges d'intérêts}

\begin{tabular}{|c|c|c|c|}
\hline \multicolumn{4}{|c|}{$\frac{\text { intérêts nets } \times 100}{\text { revenus courants }}$} \\
\hline Détails MCH1 & & \multicolumn{2}{|l|}{ Détails MCH2 } \\
\hline Intérêts nets & \multirow[b]{2}{*}{32} & Intérêts nets & \\
\hline + Intérêts passifs & & + Charges d'intérêts & 340 \\
\hline - Banques & 420 & - Revenus des intérêts & 440 \\
\hline - Créances & 421 & & \\
\hline - Capitaux du PF & 422 & & \\
\hline Revenus courants & & Revenus courants & \\
\hline+ Revenus du compte de fct. & 4 & + Revenus du compte de résultats & $40-46$ \\
\hline - Subventions à redistribuer & 47 & + Revenus extraordinaires & 48 \\
\hline - Prélèvements sur les FSp & 48 & - Dissolutions suppl. subv. d'invest. & 487 \\
\hline - Imputations internes & 49 & - Prélèvements sur CP & 489 \\
\hline & & + Prélèvements $s /$ réserve retrait. & 4895 \\
\hline
\end{tabular}

\section{I13\#\# Part du "service de la dette"}

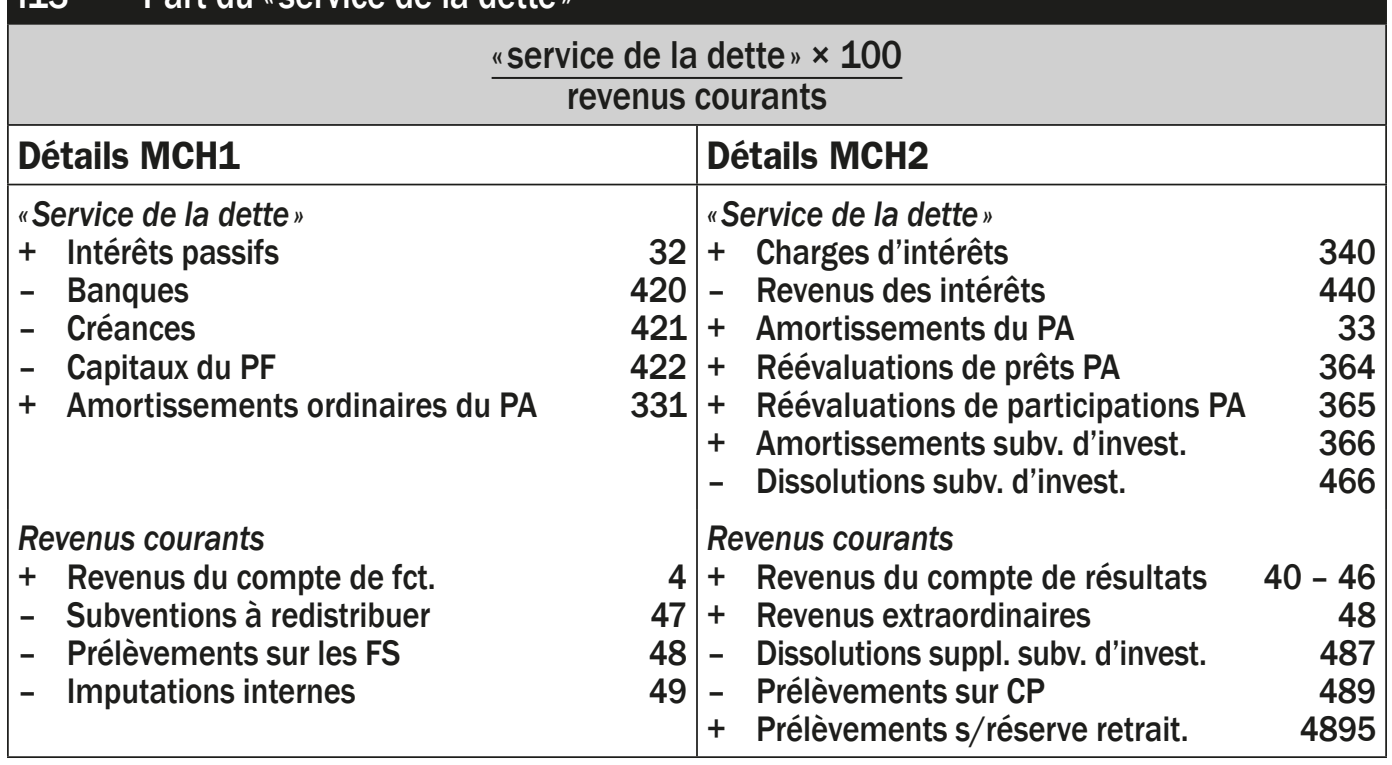




\section{Proportion des investissements bruts}

\begin{tabular}{|c|c|c|c|}
\hline \multicolumn{4}{|c|}{$\frac{\text { investissements bruts } \times 100}{\text { dépenses totales }}$} \\
\hline Détails MCH1 & & Détails MCH2 & \\
\hline $\begin{array}{l}\text { Investissements bruts } \\
+\quad \text { Dépenses d'investissement } \\
\text { Dépenses totales } \\
+\quad \text { Charges du compte de fct. } \\
\text { - Amortissements } \\
\text { - Subventions redistribuées } \\
-\quad \text { Attributions aux FSp } \\
-\quad \text { Imputations internes } \\
+\quad \text { Dépenses d'investissement }\end{array}$ & $\begin{array}{r}50-58 \\
3 \\
33 \\
37 \\
38 \\
39 \\
50-58 \\
\end{array}$ & $\begin{array}{l}\text { Investissements bruts } \\
+\quad \text { Dépenses d'investissement } \\
+\quad \text { Dépenses d'invest. e.o. } \\
\text { Dépenses totales } \\
+\quad \text { Charges de personnel } \\
+\quad \text { Charges de biens, service et autres } \\
-\quad \text { Réévaluations sur créances } \\
+\quad \text { Charges financières } \\
-\quad \text { Réévaluations immobilisations PF } \\
+\quad \text { Charges de transferts } \\
-\quad \text { Réévaluations de prêts PA } \\
-\quad \text { Réévaluations de participations PA } \\
-\quad \text { Amortissements subv. d'invest. } \\
+\quad \text { Charges extraordinaires } \\
+\quad \text { Charges financières e.o. } \\
\quad \text { (avec incidence sur la trésorerie) } \\
+\quad \text { Charges de transferts e.o. } \\
+\quad \text { Dépenses d'investissement } \\
+\quad \text { Dépenses d'invest. e.o. }\end{array}$ & $\begin{array}{r}50-56 \\
58 \\
\\
30 \\
31 \\
3180 \\
34 \\
344 \\
36 \\
364 \\
365 \\
366 \\
80-381 \\
3840 \\
386 \\
50-56 \\
58\end{array}$ \\
\hline
\end{tabular}

\section{I15 ${ }^{\# \# ~ D e t t e ~ n e t t e ~ p a r ~ h a b i t a n t ~}$}

engagements nets population résidente permanente

\begin{tabular}{|lr|lr|}
\hline Détails MCH1 & & Détails MCH2 \\
\hline Engagements nets & & Engagements nets \\
+ Engagements & $20-25$ & + Capital de tiers & 20 \\
- Patrimoine financier & $10-13$ & - Subv. d'invest. inscrites au passif & 2068 \\
& & - Patrimoine financier & 10 \\
\hline
\end{tabular}




\section{Annexe 3 Dynamique de l'endettement pour les Années t-20 à t}

\begin{tabular}{|c|c|c|c|c|c|c|c|c|c|c|c|c|}
\hline & $r_{t}$ & $Y_{t}$ & $\mathbf{R}_{\mathrm{t}}$ & $\mathrm{G}_{\mathrm{t}}$ & $D_{t}$ & $i_{t}$ & $r_{t}-i_{t}$ & $\left(r_{t}-i_{t}\right) D_{t-1}$ & $\begin{array}{c}I_{t}= \\
i_{t} \times D_{t-1}\end{array}$ & $\begin{array}{c}S_{t}= \\
R_{t}-G_{t}\end{array}$ & $\begin{array}{c}S F_{t}= \\
R_{t}-G_{t}-I_{t}\end{array}$ & $\begin{array}{c}B_{t}= \\
\left(r_{t}-i_{t}\right) D_{t-1}+S_{t}\end{array}$ \\
\hline & $\begin{array}{l}\text { Taux de } \\
\text { croissance } \\
\text { éco- } \\
\text { nomique }\end{array}$ & $\begin{array}{l}\text { Revenus } \\
\text { fiscaux }\end{array}$ & $\begin{array}{c}\text { Recettes } \\
\text { totales }\end{array}$ & $\begin{array}{c}\text { Dépenses } \\
\text { hors } \\
\text { intérêts }\end{array}$ & $\begin{array}{l}\text { Engage- } \\
\text { ments } \\
\text { nets }\end{array}$ & $\begin{array}{c}\text { Taux } \\
\text { d'intérêt }\end{array}$ & $\begin{array}{l}\text { Effet de } \\
\text { levier } \\
\text { des taux... }\end{array}$ & $\begin{array}{c}\text {... sur } \\
\text { la dette }\end{array}$ & $\begin{array}{l}\text { Intérêts } \\
\text { nets }\end{array}$ & $\begin{array}{c}\text { Solde } \\
\text { primaire }\end{array}$ & $\begin{array}{c}\text { Solde } \\
\text { financier }\end{array}$ & $\begin{array}{c}\text { Présence (-), } \\
\text { absence (+) } \\
\text { d'un effet } \\
\text { boule- } \\
\text { de-neige }\end{array}$ \\
\hline & $\%$ & KCHF & KCHF & KCHF & KCHF & $\%$ & $\%$ & KCHF & KCHF & KCHF & KCHF & KCHF \\
\hline $1996 \mathrm{t}-21$ & 0,49 & 4928 & 8871 & 9000 & 3632 & 4,00 & $-3,51$ & & & -129 & & \\
\hline 1997 t-20 & $-0,96$ & 4953 & 8786 & 8850 & 3826 & 3,59 & $-4,55$ & -165 & 130 & -64 & -194 & -229 \\
\hline $1998 \mathrm{t}-19$ & 2,49 & 4905 & 9005 & 9200 & 4155 & 3,50 & $-1,01$ & -39 & 134 & -195 & -329 & -234 \\
\hline 1999 t-18 & 5,31 & 5027 & 9483 & 9700 & 4523 & 3,63 & 1,69 & 70 & 151 & -217 & -368 & -147 \\
\hline 2000 t-17 & 4,29 & 5294 & 9890 & 10000 & 4829 & 4,33 & $-0,04$ & -2 & 196 & -110 & -306 & -112 \\
\hline $2001 \mathrm{t}-16$ & 4,90 & 5521 & 10375 & 10400 & 5058 & 4,22 & 0,68 & 33 & 204 & -25 & -229 & 7 \\
\hline $2002 t-15$ & 0,90 & 5792 & 10468 & 10700 & 5482 & 3,79 & $-2,89$ & -146 & 192 & -232 & -424 & -378 \\
\hline $2003 t-14$ & $-2,52$ & 5844 & 10205 & 10250 & 5720 & 3,52 & $-6,04$ & -331 & 193 & -45 & -238 & -376 \\
\hline 2004 t-13 & 0,81 & 5697 & 10287 & 9700 & 5330 & 3,45 & $-2,64$ & -151 & 197 & 587 & 390 & 436 \\
\hline $2005 \mathrm{t}-12$ & 1,01 & 5743 & 10391 & 10000 & 5124 & 3,45 & $-2,45$ & -130 & 184 & 391 & 207 & 260 \\
\hline 2006 t-11 & 4,95 & 5801 & 10905 & 10200 & 4587 & 3,30 & 1,65 & 85 & 169 & 705 & 536 & 790 \\
\hline 2007 t-10 & 6,29 & 6088 & 11591 & 10950 & 4096 & 3,26 & 3,03 & 139 & 149 & 641 & 492 & 780 \\
\hline 2008 t-9 & 3,43 & 6471 & 11989 & 11300 & 3495 & 2,15 & 1,28 & 52 & 88 & 689 & 601 & 741 \\
\hline 2009 t-8 & 1,10 & 6693 & 12121 & 11700 & 3143 & 2,00 & $-0,89$ & -31 & 70 & 421 & 352 & 390 \\
\hline 2010 t-7 & $-0,70$ & 6767 & 12037 & 11952 & 3120 & 1,95 & $-2,65$ & -83 & 61 & 85 & 23 & 1 \\
\hline $2011 \mathrm{t}-6$ & 2,85 & 6720 & 12380 & 12000 & 2796 & 1,78 & 1,07 & 33 & 56 & 380 & 324 & 413 \\
\hline 2012 t-5 & 0,64 & 6911 & 12459 & 12100 & 2482 & 1,62 & $-0,98$ & -27 & 45 & 359 & 314 & 332 \\
\hline $2013 \mathrm{t}-4$ & 2,54 & 6956 & 12776 & 12500 & 2240 & 1,41 & 1,14 & 28 & 35 & 276 & 241 & 304 \\
\hline 2014 t-3 & 2,84 & 7133 & 13139 & 13100 & 2231 & 1,33 & 1,50 & 34 & 30 & 39 & 9 & 72 \\
\hline 2015 t-2 & 2,77 & 7335 & 13502 & 13300 & 2057 & 1,26 & 1,51 & 34 & 28 & 202 & 174 & 236 \\
\hline $2016 \mathrm{t}-1$ & 3,21 & 7538 & 13935 & 13923 & 2080 & 1,69 & 1,51 & 31 & -29 & 41 & 70 & 72 \\
\hline $2017 \mathrm{t}$ & 1,50 & 7651 & 14313 & 14556 & 2303 & 1,20 & 0,30 & 6 & 21 & -243 & -264 & -237 \\
\hline
\end{tabular}




\section{Index}

Actif $47,55,57,71,73,170$

circulant 58

immobilisé 58

Activité

de financement $101,104,171$

de placement 101, 104

d'investissement 101, 102

opérationnelle 101

Administration fédérale des finances 215

Administrations publique 219

Aliénabilité 73

Allocation 297

Amortissement 56

comptable $96,173,183$

dégressif 92

du découvert 187

économique 95, 173

financier 95, 173

linéaire 92

supplémentaire $20,85,120,187$

Annexe aux comptes 105

Annualité 16

Antériorité 16, 31

Article 46

Association intercommunale 221

Audit

de conformité 36

de performance 37

Autofinancement 60, 99, 170

de l'investissement net 196, 278, 315

Autonomie 11

Avance à terme fixe 171

Avoir 42

Barème péréquatif 161

Benchmarking 69, 215

Besoins liés à la

composition sociale 150

topographie 150

Biens collectifs 297

Bilan 47, 57, 70, 71, 99, 184

Boule de neige 263
Budget $31,33,35$

Budgétisation

ascendante 289

descendante 290

incrémentale 289

Capacité contributive 128,153

Capital 57

productif 74

Capitaux

de tiers 75,99

étrangers 58, 257

propres 57,88

Centres

opérationnels 121

prestataires 121

Centres de coûts

homogènes 121

non homogènes 121

Charges 49

affectées 119, 133

de préférence 125,131

directes 119

extraordinaires 84,189

incorporables 120

indirectes 119

induites 293

liées 285, 293

non incorporables 120

par nature 83, 106

Clarté 19,183

Classification

administrative (voir classification

organique) 106

fonctionnelle $70,107,113,119,133,218$, 223, 291

institutionnelle (voir classification

organique) 106

of the Functions of Government (COFOG)

218

organique 106, 218, 291

par nature 106, 218, 223, 291 
Clé d'imputation 109, 133, 291

Comparabilité 20, 217, 291

Comparatif des finances cantonales et communales 192

Compensation des charges 147, 150, 151, 164

Comptabilité analytique 113

camérale 40,64

de caisse $18,40,111,113,183,187$

de gestion 113

d'exercice 18, 35, 65, 111, 113, 183, 187

d'exploitation 113

en partie double 20,41, 44, 64

générale 113

simple 40

Compte

administratif 76

de financement 110

de fonctionnement 76

de gestion 50

de patrimoine 44

de résultats $70,76,82,184,271$

des investissements 70,78, 184

de tiers 42

de valeurs 44

financier 64, 110

Comptes annuels

approbation 37

préparation 35

présentation 17

vérification 36

Concordat 221

Conférence des autorités cantonales de surveillance des finances communales 79

Conférence des directrices et directeurs cantonaux des finances 64

Conseil suisse de présentation des comptes publics 70

Consolidation 222

budgétaire 294

Continuité de l'exploitation 18

Contribution causale 117, 123, 124

Correction de valeur 97

Cosmétique comptable 185,187

Coût

de fonctionnement 82,117 de production 150

standard 167

Couverture

des charges $82,88,195,315$

directe $91,112,171$

préalable 91

ultérieure 91

Crédit 42

additionnel 25

bloqué 22

budgétaire $22,31,34,35,73$

cadre 25

d'engagement 23, 30, 34, 73, 79

d'objet 25

en compte courant 172

supplémentaire 22,35

Cycle conjoncturel 229

Débit 42

Découvert 59, 88

Dédommagements 146

Déficit primaire 261

Demande globale 243, 248, 250, 294, 299

Démocratie 28, 129

Dépassement de crédit 22

Dépenses 76

conjoncturelles 241

courantes $76,83,172$

d'investissement $76,79,91,112,172$

pour le fonctionnement 76

Dépréciation (du patrimoine administratif) 51, 56, 91

Déséquilibre 229, 244, 250, 253, 281

Dette brute 75, 225, 226, 227, 256, 258 par rapport aux revenus 205, 319

Dette nette 75,259

par habitant 210, 321

Différences endogènes 149

Disparités endogènes 148

exogènes 149

Doit 42

Dotation minimale 161

Double détermination du résultat 47

Droit d'initiative 27 
Droits constatés 18,65

Durée d'utilisation 77, 91, 93

Écart de production 231

Échéance 18

Effectivité 13

Effet de levier 268

des taux 263, 268, 277

Effet de tenailles 208, 276

Efficience 13

Effort d'investissement 272, 287, 317

Élasticité des dépenses 241

des recettes 239

Émoluments 124

Emploi 57 économe des fonds 13

efficace des fonds 13

ménager des fonds 13

Emprunt

à taux fixe 174

à taux variable 174

bancaire 172

d'impulsion 176

obligataire 175

par contrat de gré à gré 172

privé 172

Endettement 25, 75, 253, 273

Engagements

acceptés 31

bruts 255,260

nets 97,260

nets supplémentaires 197,316

Entreprises publiques 219

Équilibre (budgétaire) 25, 33, 184, 193, 195, 238, 243, 247

EURIBOR 174

Exactitude de la prévision fiscale 202, 318

Excédent

de charges $82,88,89,100$

de revenus 88

primaire 260

Externalités

négatives 298

positives 298
Facteur conjoncturel 235

Fiabilité 19, 183, 185

Financement 77

participatif 177

spécial 133

Fluctuations automatiques 237

Fonds 133

de roulement 60

Grand livre 46

Importance 18

de l'endettement 193, 204, 257

Impôt 124

Impulsion

anticyclique 251

discrétionnaire 243

expansive 250

procyclique 251

restrictive 250

Imputation interne 114

Indicateur 191, 315

Indicateurs auxiliaires 193, 206

Indice des ressources 153,159

Intégralité 14

Intérêt moyen de la dette 203, 318

Inventaire 52, 57

Investissement 73,79

IPSAS 65,186

Journal 46

Justice sociale 68,298

Légalité 13

LIBOR 174

Ligne de crédit 171

Limite (à la...) 271

Limite d'activation 78,189

Maîtrise des dépenses courantes $\quad$ 199, 317

Maturité 173 
Mésestimations 190

Mesures d'assainissement 294

Missions de l'État 297

Modèle comptable de la Confédération 110 harmonisé de $1^{\text {re }}$ génération 64 harmonisé de $2^{\mathrm{e}}$ génération 66

Modèle statistique 227

SF 227

SFP 227

Neutralité 19

Non-affectation des impôts 14,130

Non-contraction 17

Nouvelle gestion publique 32, 65, 289

Numérotation 108, 115, 133

Opération non monétaire 95

Opérations de bilan à bilan 189

Part des charges d'intérêts 207, 320

Part du «service de la dette» 208, 320

Partenariat public-privé (PPP) 177

Parts 145

Passif 47,57

Patrimoine administratif $72,75,91$ financier $73,75,97$

Péréquation des charges $150,151,164$ des ressources 150, 152 financière 147, 298

Périmètres 219

Périodicité 18

Permanence des méthodes 20

Pertes et profits 45,82

PIB

effectif 229

potentiel 229

tendanciel 231

Placement 74

Plan comptable $50,62,70,106,305$

financier 30

Planning, Programming, and Budgeting

System 289

Poids des intérêts nets 198,316

Politique budgétaire 85, 185, 251, 253

Politique publique institutionnelle 297 substantielle 297

Pollueur-payeur 126

Potentiel fiscal 152

Préférences locales 149

Préfinancements 85, 88, 187

Présentation échelonnée 88

Prime de risque 175, 263

Principe

d'équivalence 126

d'évaluation 75

Prix 126

Processus budgétaire 28, 288

Produit 49

brut 17,82

net 17,82

Programme de législature 30

Proportion des investissements bruts 209, 321

Provisions 48, 56, 183

Prudence 15

Qualité de la gestion financière 193, 199

Recettes

conjoncturelles 240

d'investissement 79

Recommandations $64,67,70,187$

Redistribution 298

Référendum financier 27

Règles budgétaires 25

Régularisation 56, 183

Régularité 20, 185

Report de crédit 23

Réserves 48, 57, 85, 187

Ressources 57 
Résultat

brut d'exploitation 55

d'exploitation 89

extraordinaire 90

financier 90

opérationnel $90,113,189$

total 90,187

Revenu fiscal harmonisé 156

Revenus 49

affectés 133

à libre disposition 286

extraordinaires 87,189

par nature 86

Réviseur 36

Révision 186, 187

Sectorisation 219, 221

Sentier de croissance des charges 287

Sincérité 19

Solde

conjoncturel 238, 242

de financement 111, 238, 248

de financement primaire 260, 262

de financement structurel 248

du compte de résultats 88

opérationnel 238

opérationnel structurel 244

primaire 260

structurel 244

Solvabilité 98, 175, 253

Soutenabilité 253, 260, 263, 294

Spécialisation 15

Spécialité 15, 21

qualitative 15

quantitative 16

temporelle 16

Stabilisateurs automatiques 238

Stabilisation 239, 298

Statistique financière 37,215

Structure de financement 62,149

Substance fiscale 149, 156

Subventions 147

au sens économique 147

d'investissement 91
Tableau

de bord 191

de financement 98

des flux de trésorerie 101,184

Taux

d'amortissement 93, 272

d'autofinancement 206, 319

de croissance 263,272

d'endettement 254, 263, 273

d'endettement net 204, 318

d'intérêt 174, 272

Taxe

de base 132

de raccordement 131

d'exploitation 132

d'internalisation 298

Transferts 145

entre les patrimoines 76

péréquatifs 163

sociaux 298

Transitoires 56

Transparence des coûts 117

Unité 15

Universalité 14

Urgence 13

Utilisateur-payeur 15, 78, 91, 124, 126

Valeur

d'acquisition 75,91

d'exploitation 58

disponible 58

réalisable 58

vénale 75

Variation des liquidités 104

Vérification des comptes 46

Zero-Based Budgeting (ZBB) 289

Zone neutre 163 


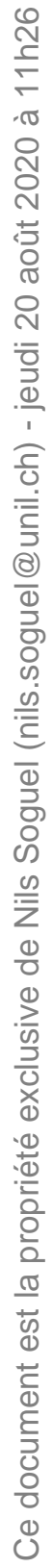




\section{Listes}

\section{Liste des figures}

Figure 1 Articulation des principes financiers. 12

Figure 2 Début de l'année budgétaire. 17

Figure 3 Articulation des crédits. $\quad 21$

Figure 4 Articulation entre crédit d'engagement et crédits budgétaires. 24

Figure 5 Processus financier et organes décisionnels. 29

Figure 6 L'entreprise: un patrimoine et des échanges. 49

Figure 7 Une comptabilité, cinq séries de comptes. 55

Figure 8 Représentation de l'actif et du passif par grandes masses. 58

Figure 9 La capitalisation de la perte aboutit à un découvert. 59

Figure 10 La règle fondamentale d'équilibre du financement. 60

Figure 11 L'autofinancement, c'est l'épargne de l'entreprise. 62

Figure 12 Introduction du $\mathrm{MCH} 2$ dans les cantons et dans les communes. 67

Figure 13 Un plan comptable méthodique est au cœur du modèle.

Figure 14 Distinction au bilan entre patrimoine administratif et patrimoine financier. $\quad 72$

Figure 15 Critère de l'aliénabilité pour distinguer le patrimoine administratif $\begin{array}{ll}\text { du patrimoine financier. } & 74\end{array}$

Figure 16 Critère de la durée d'utilisation pour distinguer entre dépenses $\begin{array}{ll}\text { courantes et dépenses d'investissement. } & 77\end{array}$

Figure 17 Les charges extraordinaires sont de nature très différente. 85

Figure 18 Procédure d'amortissement avec couverture ultérieure. 92

Figure 19 Valeur résiduelle au bilan selon que l'amortissement est linéaire ou dégressif. $\quad 94$

Figure 20 Tableau de financement selon MCH1. 100

Figure 21 L'information comptable répond à diverses interrogations de gestion. 110

Figure 22 Distinction entre une charge directe et une charge indirecte. 120

Figure 23 Imputation interne avec une unité d'œuvre ou avec une clé de répartition selon que le centre est homogène ou non.

Figure 24 Distinction entre impôts et contributions causales.

Figure 25 Arbre de décision permettant de choisir entre un financement fondé sur le principe d'équivalence ou un financement fondé sur le principe de la capacité contributive.

Figure 26 Selon la législation auquel il est soumis, un financement spécial ou un fonds doit être considéré comme du capital propre ou comme du capital de tiers.

Figure 27 L'attribution de l'excédent de revenus du domaine de la gestion des déchets augmente le financement spécial correspondant.

Figure 28 Décomposition du résultat en tenant compte des variations des financements spéciaux (FSp). 
Figure 29 Seules les disparités exogènes justifient une péréquation.

Figure 30 Échelonnement des collectivités en fonction de l'indice des ressources. 159

Figure 31 Deux solutions péréquatives extrêmes: système inopérant et système uniformisant.

Figure 32 Exemple de barème péréquatif

Figure 33 Sources de financement des investissements à la disposition des collectivités.

Figure 34 Arbre de décision permettant de choisir un outil de financement.

Figure 35 Modes de présentation des faits économiques en fonction du référentiel comptable.

Figure 36 Amortissements supplémentaires et préfinancements selon les cantons. 188

Figure 37 Évaluation de la couverture des charges - I1.

Figure 38 Évaluation de l'autofinancement de l'investissement net - 12".

Figure 39 Évaluation des engagements nets supplémentaires - 13.

Figure 40 Évaluation du poids des intérêts nets - 14.

Figure 41 Évaluation de la maîtrise des dépenses courantes par habitant - 15.

200

Figure 42 Évaluation de l'effort d'investissement - 16.

Figure 43 Évaluation de l'exactitude de la prévision fiscale - 17.

202

Figure 44 Évaluation de l'intérêt moyen de la dette - 18.

203

Figure 45 Évaluation du taux d'endettement net - 19\#.

204

Figure 46 Évaluation de la dette brute par rapport aux revenus - I10"\#\#.

206

Figure 47 Évaluation du taux d'autofinancement - I11 ${ }^{\# \# . ~}$

Figure 48 Évaluation de la part des charges d'intérêts - I12\#.

Figure 49 Évaluation de la part du «service de la dette»-I13"\#.

209

Figure 50 Évaluation de la proportion des investissements bruts - $114^{\# \#}$.

Figure 51 Évaluation de la dette nette par habitant - I15\#\#.

Figure 52 Le périmètre statistique d'une administration publique en exclut les entreprises publiques.

Figure 53 Principales phases et situations à l'intérieur d'un cycle conjoncturel.

Figure 54 Valeurs annuelles du facteur conjoncturel k estimé pour appliquer le frein fédéral à l'endettement.

Figure 55 Fluctuations budgétaires automatiques et solde conjoncturel, impact budgétaire des impulsions discrétionnaires et solde structurel.

Figure 56 Solde opérationnel structurel et mesures discrétionnaires.

Figure 57 Évolution du taux d'endettement brut des pays de l'Union européenne et de la Suisse.

Figure 58 Évolution du taux d'endettement de MaCollectivité sous l'effet de la croissance économique et de la variation de l'endettement.

Figure 59 Évolution du taux d'endettement de MaCollectivité liée à l'effet de levier des taux et au solde primaire.

Figure 60 Évolution du taux d'endettement net (19\#) de l'Année t à l'Année t+50 en direction de sa valeur limite, en cas d'équilibre structurel.

Figure 61 Évolution de l'effet de tenailles (I13"\#\#) de l'Année t à l'Année t+50 en direction de sa valeur limite. 
Figure 62 Évolution de l'autofinancement de l'investissement net (I2\#) de l'Année t à l'Année t+50 en direction de sa valeur limite.

Figure 63 Évolution du taux d'endettement net (19") de l'Année t à l'Année t+100 en direction de sa valeur limite, en cas d'excédent structurel.

Figure 64 Élargir l'horizon temporel accroît la part des revenus à libre disposition. 287

Figure 65 Organigramme fondé sur la classification fonctionnelle.

\section{Liste des portraits}

Portrait 1 Conférence des directrices et directeurs cantonaux des finances 65

Portrait 2 International Public Sector Accounting Standards 66

Portrait 3 Conseil suisse de présentation des comptes publics $\quad 71$

Portrait 4 Conférence des autorités cantonales de surveillance $\begin{array}{ll}\text { des finances communales } & 79\end{array}$

Portrait 5 Comparatif des finances cantonales et communales de I'IDHEAP 192

Portrait 6 Administration fédérale des finances 215

Portrait 7 Classification of the Functions of Government 219

Portrait 8 Administrations publiques $\quad 221$

Portrait 9 Poursuite pour dettes contre les collectivités de droit public cantonal 254

Portrait 10 Planning, Programming, and Budgeting System 289

Portrait 11 Zero-Based Budgeting 290

\section{Liste des extraits}

Extrait 1 Constitution fédérale, art. 126 "Gestion des finances" 27

Extrait 2 Au mois de décembre, la messe est dite... 33

Extrait 3 La cosmétique comptable a lieu au vu et au sus de tous 186

Extrait 4 Constitution fédérale, art. 100 "Politique conjoncturelle" 252 


\section{Liste des tableaux}

Tableau 1 Les recettes et les dépenses des Thénardier. 40

Tableau 2 Une colonne pour les recettes, une autre pour les dépenses. 41

Tableau 3 Compte du Débiteur X. $\quad 42$

Tableau 4 Le débiteur rembourse sa dette: deux comptes sont touchés. 43

Tableau 5 L'entreprise rembourse son créancier. 43

Tableau 6 Le compte de Pertes et profits enregistre la variation de valeur. 45

Tableau $7 \quad$ Quelques articles d'un journal. $\quad 46$

Tableau 8 On reporte au bilan les soldes des comptes de patrimoine et de PP. $\quad 47$

Tableau 9 Les provisions et les réserves figurent aussi au bilan. 48

Tableau 10 Les soldes des comptes de patrimoine sont reportés à nouveau. 51

Tableau 11 Les comptes de gestion sont virés, y compris le PP. 52

Tableau 12 Procédure de bouclement des comptes: un cas d'école. 53

Tableau 13 Journal de l'an 1820.

Tableau 14 Limite d'activation de la dépense. 79

Tableau 15 Rubriques du compte des investissements selon la classification $\begin{array}{ll}\text { par nature. } & 81\end{array}$

Tableau 16 Rubriques du compte de résultats selon la classification par nature. 84

Tableau 17 Présentation échelonnée du compte de résultats. 87

Tableau 18 Durée d'utilisation et taux d'amortissement. 93

Tableau 19 Tableau des flux de trésorerie préconisé par le MCH2. 103

Tableau 20 Compte de résultats et compte des investissements selon $\begin{array}{ll}\text { la classification fonctionnelle. } & 108\end{array}$

Tableau 21 Systématique de numérotation des transactions. 109

Tableau 22 Compte de financement de la Confédération. 111

Tableau 23 Importance des imputations internes dans le total des charges et des revenus.

Tableau 24 Imputations internes dans les unités administratives et dans les catégories fonctionnelles.

Tableau 25 Importance des revenus fiscaux, ainsi que des taxes et redevances dans le total des revenus.

Tableau 26 Catégorisation des charges pour la gestion des déchets.

Tableau 27 La gestion des déchets dégage un excédent de revenus, avant son attribution au capital propre.

Tableau 28 Catégorisation des revenus pour la gestion des déchets.

Tableau 29 Financements spéciaux et fonds au passif du bilan.

Tableau 30 Opération de clôture du domaine de la gestion des déchets.

Tableau 31 Équilibre dans le domaine de la gestion des déchets après attribution du solde au capital propre.

Tableau 32 Importance des transferts dans le total des charges et des revenus.

Tableau 33 Revenus à intégrer dans l'estimation du potentiel fiscal.

Tableau 34 Estimation du potentiel fiscal à travers le revenu fiscal harmonisé. 
Tableau 35 Effet d'un barème progressif sur le potentiel fiscal à substance fiscale équivalente entre collectivités.

Tableau 36 Transferts péréquatifs à recevoir et à payer.

Tableau 37 Tableau de bord financier proposé et lien avec les indicateurs recommandés par le Manuel $\mathrm{MCH} 2$.

Tableau 38 Pondération des indicateurs du tableau de bord financier.

Tableau 39 Notes de synthèse pour MaCollectivité à l'Année t.

Tableau 40 Estimation du PIB potentiel et de l'écart de production.

Tableau 41 Estimation du facteur conjoncturel $k$ sur la base du PIB potentiel calculé.

Tableau 42 Estimation des recettes fiscales conjoncturelles.

Tableau 43 Estimation des dépenses conjoncturelles.

Tableau 45 Estimation du solde opérationnel structurel.

Tableau 46 Solde opérationnel structurel si l'écart de production était positif.

Tableau 47 Calcul du solde de financement.

Tableau 48 Estimation du solde de financement structurel. Impulsions procycliques et anticycliques.

Tableau 52 Combinaison des conditions de déclenchement d'un cercle vicieux ou d'un cercle vertueux de l'endettement.

Tableau 53 Limite du taux d'endettement net (19\#) en fonction du taux de croissance $r$, du taux d'amortissement $\mathrm{k}_{\mathrm{A}}$ et de l'effort d'investissement $\mathrm{k}_{\mathrm{l}}(\mathrm{I6})$.

Tableau 54 Limite de l'effet de tenailles (I13"\#) en fonction du taux de croissance $r$, du taux d'intérêt i et de l'effort d'investissement $\mathrm{k}_{1}(\mathrm{I6})$.

Tableau 55 Limite de l'autofinancement (I2\#) en fonction du taux de croissance $r$ et du taux d'amortissement $k_{A}$.

Tableau 56 Limite du taux d'endettement net (19\#) en fonction du taux de croissance $r$, de l'effort d'investissement $k_{1}(16)$ et du taux de déséquilibre du compte de résultats $k_{D}(=11-100 \%)$.

Tableau 57 Charges induites annuelles en pourcentage du montant de l'investissement brut.

Tableau 58 Catalogue non exhaustif de mesures d'assainissement possibles. 


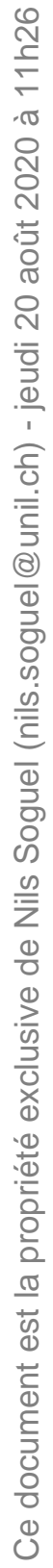




\section{Abréviations}

AFC

AFF

al.

art.

AS

ATF

BM

BNS

CDF

CE

CHF

$\mathrm{CP}$

CT

COFOG

e.o.

EURIBOR

FMI

fct.

Fds

FSp

GFSM

GMEB

IDHEAP

invest.

IPSAS

KCHF

LEaux

LIBOR

LIM

LP

LPDC

LPE

LPR

$\mathrm{MCH} 1$

$\mathrm{MCH} 2$

MSFP

mio

mrd
Administration fédérale des contributions

Administration fédérale des finances

Alinéa

Article

Amortissements supplémentaires

Arrêts du Tribunal fédéral suisse

Banque mondiale

Banque nationale suisse

Conférence des directrices et directeurs cantonaux des finances

Commission européenne

Francs suisses

Capital propre

Capitaux de tiers

Classification of the Functions of Government

Extraordinaires (charges ou revenus)

Euro interbank offered rate

Fonds monétaire international

Fonctionnement

Fonds

Financements spéciaux

Government Finance Statistics Manual

Gestion par enveloppe et mandat de prestation

Institut de hautes études en administration publique

Investissement

International Public Sector Accounting Standards

Millier de francs suisses

Loi fédérale sur la protection des eaux

London interbank offered rate

Loi fédérale sur l'aide aux investissements dans les régions de montagne

Loi fédérale sur la poursuite pour dettes et faillite

Loi fédérale réglant la poursuite pour dettes contre les communes et autres collectivités de droit public cantonal

Loi fédérale sur la protection de l'environnement

Loi fédérale sur la politique régionale

Modèle comptable harmonisé de première génération

Modèle comptable harmonisé de deuxième génération

Manuel de statistiques de finances publiques

Million

Milliard 
NMC Nouveau modèle comptable de la Confédération

NPR Nouvelle politique régionale

OCDE Organisation de coopération et de développement économiques

OFR Ordonnance sur les fonds propres

ONU Organisation des Nations Unies

PA Patrimoine administratif

PC Protection civile

pers. Personnes

PF Patrimoine financier

PIB Produit intérieur brut

PP Compte de Pertes et profits

PPP Partenariat public-privé

NPR Nouvelle politique régionale

retrait. Retraitement

RS Recueil systématique du droit fédéral

SNA System of National Accounts

SCN Système de comptabilité nationale

SEC Système européen des comptes

SRS-CSPCP Schweizerisches Rechnungslegungsgremium für den öffentlichen Sektor

- Conseil suisse de présentation des comptes publics

subv. Subvention

UE Union européenne 


\section{Table des matières}

Sommaire $\quad 5$

$\begin{array}{ll}\text { Deux mots... } & 7\end{array}$

$1 \quad$ Introduction pour prendre l'ouvrage en main 9

$2 \quad$ Identifier les acteurs et les fondements de la gestion financière 11

2.1 Principes financiers 12

2.2 Droit des crédits $\quad 21$

2.3 Règles budgétaires 25

2.4 Référendum financier et droit d'initiative 27

2.5 Processus financier et principaux acteurs 28

3 Décrypter comment les comptes et les budgets sont organisés 39

3.1 Approche de la comptabilité dans les pas de son histoire 39

3.2 Détour par la comptabilité de l'entreprise 49

3.3 Modèle comptable des cantons et des communes suisses 63

3.4 Modèle comptable de la Confédération 110

$4 \quad$ Utiliser les imputations internes pour refléter les coûts des prestations 113

4.1 Comptabilité générale et comptabilité de gestion 113

$\begin{array}{ll}4.2 & 1 m p u t a t i o n s \\ \text { internes } & 114\end{array}$

$\begin{array}{ll}4.3 \text { Éléments d'analyse des coûts } & 119\end{array}$

$5 \quad$ Comprendre le financement spécifique de certaines prestations $\quad 123$

5.1 Distinction entre impôts et contributions causales 123

5.2 Principe d'équivalence et ses conditions de mise en œuvre 126

$\begin{array}{ll}5.3 \text { Affectation tactique des recettes } & 129\end{array}$

5.4 Dispositions légales 130

5.5 Modalités comptables des financements spéciaux et des fonds $\quad 132$

6 Connaître les mécanismes de transferts et de péréquation financière 145

6.1 Types de transferts selon la classification par nature 145

6.2 Disparités et péréquation 148

6.3 Péréquation des ressources 152

6.4 Compensation (ou péréquation) des charges 164

$7 \quad$ Identifier les modalités de financement des investissements et d'emprunt 169

7.1 Éventail des sources de financement des investissements 169

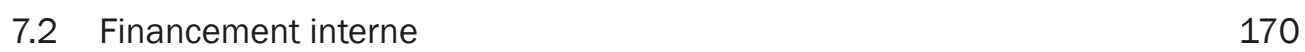

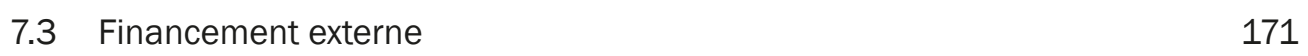

7.4 Quelques règles à garder en tête 178 
8 Déceler la tactique politique en matière de présentation des comptes

8.1 Une volonté de fiabilité et de clarté

8.2 Une transparence qui comporte des risques

8.3 Tension entre fiabilité et régularité

8.4 Cosmétique comptable

9 Porter un diagnostic sur la situation financière à l'aide d'indicateurs

9.1 Notion d'indicateur et de tableau de bord

9.2 Tableau de bord financier proposé

9.3 Indicateurs

10 Trouver les informations financières pour se comparer

10.1 Besoin de se comparer

10.2 Enjeux de la comparaison

10.3 Origines des difficultés de comparaison

10.4 Des comptes à la statistique

10.5 Comparer les communes entre elles

10.6 Comparer les cantons entre eux

10.7 Comparer la Suisse avec d'autres pays

11 Analyser l'origine des déficits: conjoncture ou structure

11.1 Cycle conjoncturel et écart de production

11.2 Fluctuations budgétaires automatiques, solde conjoncturel et stabilisateurs automatiques

11.3 Mesures discrétionnaires et solde structurel

12 Analyser la dynamique de l'endettement: cercle vicieux ou vertueux

12.1 Soutenabilité de l'endettement

12.2 Taux d'endettement

12.3 Grandeurs brutes ou grandeurs nettes

257

12.4 Dette ou engagements

258

12.5 Solde primaire

260

12.6 Boule de neige de l'endettement

263

12.7 Lutte contre un cercle vicieux de l'endettement

13 Projeter la situation financière à la limite

13.1 Se projeter à la limite avec quelques hypothèses

13.2 Taux d'endettement net à la limite

13.3 Effet de tenailles à la limite

13.4 Autofinancement de l'investissement net à la limite 278

13.5 Déséquilibre structurel et limite du taux d'endettement net 
14 Améliorer la situation financière à l'avenir : quelques pistes

14.1 Améliorer la marge de manœuvre financière et réduire les charges liées

14.2 Cadrer l'effort d'investissement et le sentier de croissance des charges

14.3 Réformer le processus budgétaire

14.4 Simplifier l'organisation et l'information financières

14.5 Penser aux charges induites de l'investissement

14.6 Choisir les mesures d'assainissement financier les plus opportunes

15 Portée stratégique des finances de ma collectivité

Bibliographie

301

Annexes

305

Annexe 1 - Comptes de MaCollectivité pour l'Année t

305

Annexe 2 - Calcul des indicateurs financiers

315

Annexe 3 - Dynamique de l'endettement pour les Années t-20 à t

Index

Listes

Liste des figures

Liste des portraits

Liste des extraits

Liste des tableaux

Biographie 


\section{Biographie}

Nils Soguel est professeur ordinaire de finances publiques à l'Institut de hautes études en administration publique (IDHEAP) de l'Université de Lausanne. Il est également président du Conseil suisse de présentation des comptes publics, l'instance de normalisation des états financiers pour toutes les collectivités publiques suisses. Son expertise est fréquemment sollicitée par les administrations fédérales, cantonales et communales en matière de gestion financière, d’organisation, de fusion, de péréquation et de répartition des tâches, ainsi que dévaluations des biens non marchands. Dans ces domaines, il a publié plusieurs ouvrages et de nombreux articles dans des revues scientifiques internationales. En outre, il dirige la publication annuelle du Comparatif des finances cantonales et communales de l'IDHEAP. 